\title{
High Transverse Momentum \\ Direct Photon Production aT \\ Fermilab Fixed-Target Energies
}

By

Leonard Apanasevich

\section{A DISSERTATION}

Submitted to

Michigan State University

in partial fulfillment of the requirements

for the degree of

DOCTOR OF PHILOSOPHY

Department of Physics and Astronomy

2005 



\section{Abstract \\ High Transverse Momentum \\ Direct Photon Production at \\ Fermilab Fixed-TARGet EnERgies}

By

Leonard Apanasevich

This thesis describes a study of the production of high transverse momentum direct photons and $\pi^{0}$ mesons by proton beams at 530 and $800 \mathrm{GeV} / \mathrm{c}$ and $\pi^{-}$ beams at $515 \mathrm{GeV} / \mathrm{c}$ incident on beryllium, copper, and liquid hydrogen targets. The data were collected by Fermilab experiment E706 during the 1990 and 1991-92 fixed target runs. The apparatus included a large, finely segmented lead and liquid argon electromagnetic calorimeter and a charged particle spectrometer featuring silicon strip detectors in the target region and proportional wire chambers and drift tubes downstream of a large aperture analysis magnet. The inclusive cross sections are presented as functions of transverse momentum and rapidity. The measurements are compared with next-to-leading order perturbative QCD calculations and to results from previous experiments. 



\section{Acknowledgments}

I would like to take the opportunity to thank some of the people who helped make this dissertation possible. Foremost, I thank my adviser, Carl Bromberg, for his guidance and support over these many years. His insights and enthusiasm for the subject have been a continuing source of inspiration for me and I feel lucky to have had him as my adviser.

The analysis presented here is built upon the work of many collaborators who invested significant time and effort into making this experiment a success. I thank them for their dedication and hope this thesis reflects their work in a positive light. There are several collaborators I would like to thank in particular. George Ginther has been a mentor and a second adviser to me. I am grateful for his advice and encouragement more than he could possibly know. I thank Paul Slattery for his heroic efforts in keeping the analysis moving forward, for standing by me, and for providing financial support in the end. I would also like to thank Marek Zieliński and Joey Huston for their always valuable comments and suggestions. I thank Michael Begel for many spirited and enlightening discussions over the years and for his technical assistance in putting this document together. Finally, I'd like to thank Steve Blusk, Casey Hartman, Chris Lirakis, Rob Roser, Nikos Varelas and all the other students and post-docs on E706 for their help during the early years of my involvement with this experiment.

The road to this Ph.D. has been a long one and I would like to thank some people outside of the experiment who have helped me along the way. I thank Walsh Brown, Stacey Hamlin, Rick Kwarciany, Noah Wallace and Steve Worm for helping keep me sane during the pursuit of this degree. I especially thank Kate Frame and Jordan Poler for their constant support and encouragement. It has meant a lot to me. And also thanks to Larry Servidio. There are not too many 
vi Acknowledgments

people in this world who would be willing to spend their Saturday afternoons in a dingy bar on the lower east side of Manhattan. For those Saturdays and for everything else, I am eternally grateful.

Finally, I would like to thank my parents and my brother. Without their love and support, I could not have completed this thesis. 


\section{Table of Contents}

Abstract

Acknowledgments

List of Tables _ xi

List of Figures

Chapter 1 Introduction 1

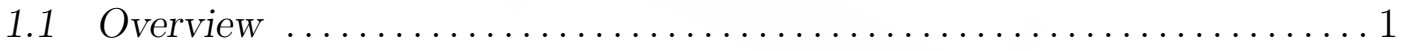

1.2 Quantum Chromodynamics ................................ 1

1.3 Phenomenology of High Transverse Momentum Interactions . ..........3

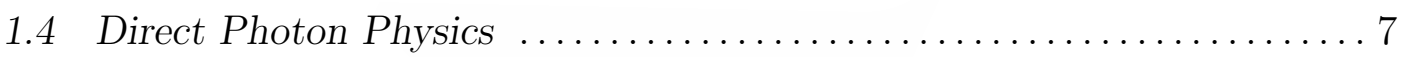

1.5 Experimental Challenges and Techniques ..................... 11

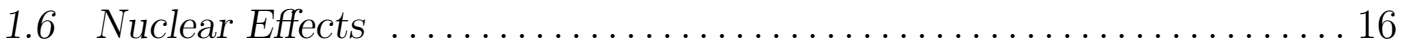

1.7 Initial State Parton Transverse Momentum Effects ................ 17

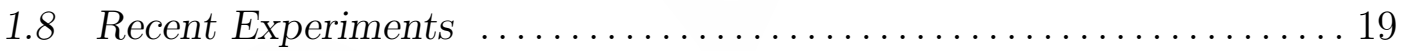

Chapter 2 The Meson West Spectrometer 23

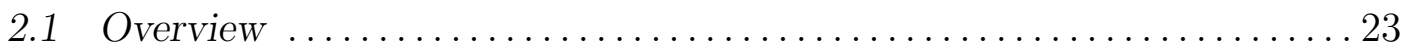

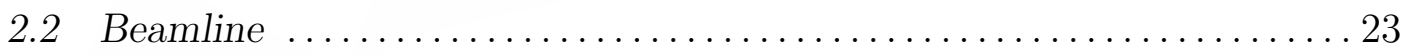

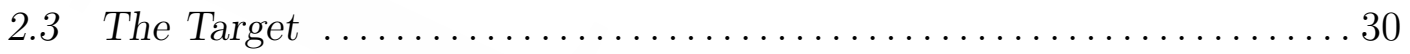

2.4 The Beam and Interaction Detectors $\ldots \ldots \ldots \ldots \ldots \ldots \ldots \ldots \ldots \ldots . \ldots . \ldots . \ldots$

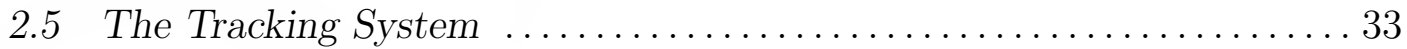

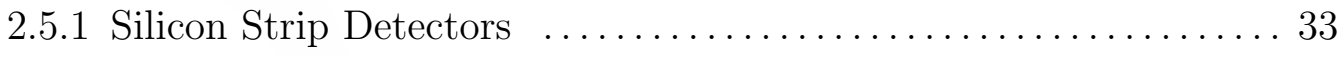

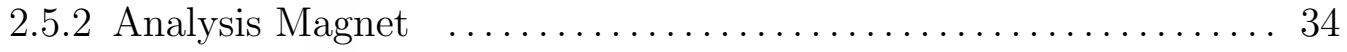

2.5.3 Proportional Wire Chambers $\ldots \ldots \ldots \ldots \ldots \ldots \ldots \ldots \ldots \ldots$

2.5.4 Straw Tube Drift Chambers $\ldots \ldots \ldots \ldots \ldots \ldots \ldots \ldots \ldots \ldots . \ldots . \ldots . \ldots$ 
viii Table of Contents

2.6 Liquid Argon Calorimeter ................................ 43

2.6.1 Electromagnetic Calorimeter $\ldots \ldots \ldots \ldots \ldots \ldots \ldots \ldots \ldots \ldots$

2.6.2 Hadronic Calorimeter $\ldots \ldots \ldots \ldots \ldots \ldots \ldots \ldots \ldots . \ldots \ldots$

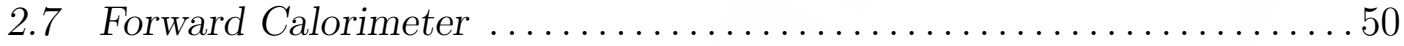

Chapter 3 Trigger and Data Acquisition 53

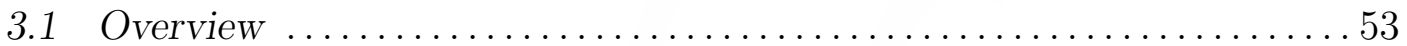

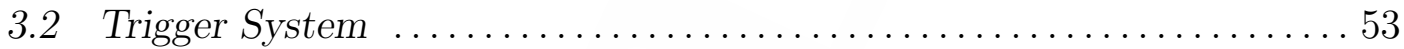

3.2.1 Beam and Interaction Requirement $\ldots \ldots \ldots \ldots \ldots \ldots \ldots$

3.2 .2 Pretrigger Requirement $\ldots \ldots \ldots \ldots \ldots \ldots \ldots \ldots \ldots \ldots \ldots$

3.2 .3 The Local Triggers $\ldots \ldots \ldots \ldots \ldots \ldots \ldots \ldots \ldots \ldots \ldots \ldots \ldots \ldots \ldots \ldots \ldots$

3.2.4 The Global Triggers $\ldots \ldots \ldots \ldots \ldots \ldots \ldots \ldots \ldots \ldots \ldots \ldots \ldots \ldots$

3.2.5 The Two Gamma Trigger $\ldots \ldots \ldots \ldots \ldots \ldots \ldots \ldots \ldots \ldots \ldots \ldots \ldots \ldots \ldots$

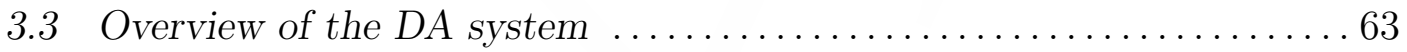

Chapter 4 Event Reconstruction 67

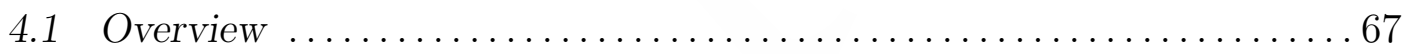

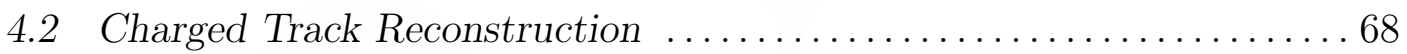

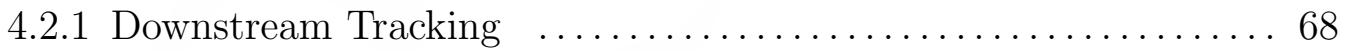

4.2.2 Upstream View Tracking and Linking $\ldots \ldots \ldots \ldots \ldots \ldots \ldots \ldots$

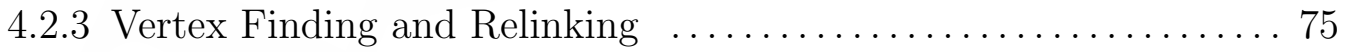

4.2.4 Beam Tracking $\ldots \ldots \ldots \ldots \ldots \ldots \ldots \ldots \ldots \ldots \ldots \ldots \ldots \ldots \ldots \ldots \ldots \ldots \ldots \ldots$

4.2.5 Charged Track Momentum Determination $\ldots \ldots \ldots \ldots \ldots . \ldots 8$

4.3 Electromagnetic Shower Reconstruction ................... 80

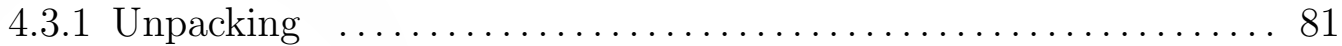

4.3.2 Group and Peak Finding $\ldots \ldots \ldots \ldots \ldots \ldots \ldots \ldots \ldots \ldots$

4.3.3 GAMMA and PHOTON reconstruction $\ldots \ldots \ldots \ldots \ldots \ldots \ldots$

4.4 Discrete Logic Reconstruction $\ldots \ldots \ldots \ldots \ldots \ldots \ldots$ 
Chapter 5 Data Analysis _ 93

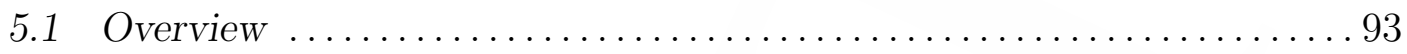

5.2 Target Fiducial Requirement .............................. 93

5.3 EMLAC Fiducial Requirement ............................. 97

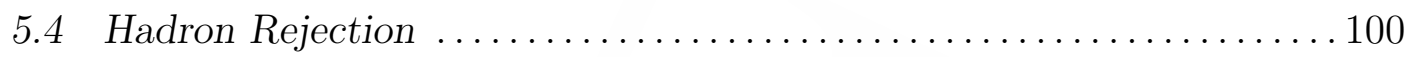

5.5 Rejection of Beam Halo Muons ............................... 103

5.5.1 Veto Wall Requirement ........................... 105

5.5.2 Directionality Requirement $\ldots \ldots \ldots \ldots \ldots \ldots \ldots \ldots \ldots \ldots$

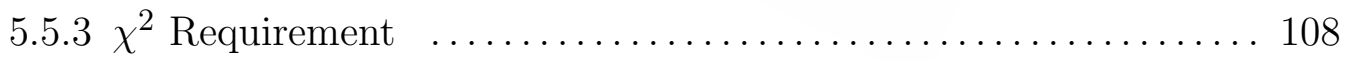

5.5.4 Balanced $p_{T}$ Requirement $\ldots \ldots \ldots \ldots \ldots \ldots \ldots \ldots \ldots \ldots \ldots \ldots$

5.5.5 Corrections for Muon Requirements $\ldots \ldots \ldots \ldots \ldots \ldots \ldots . \ldots 111$

$5.6 \quad \pi^{0}$ and $\eta$ Energy Asymmetry Requirement ....................113

5.7 Photon Conversion Correction .............................. 114

5.8 Electron Studies ....................................... 115

5.9 EMLAC Energy Scale Calibration ........................... 118

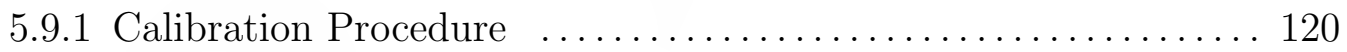

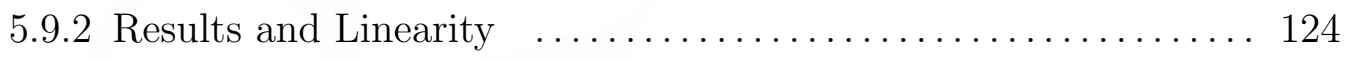

5.10 Trigger Analysis ....................................... 125

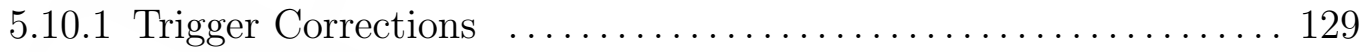

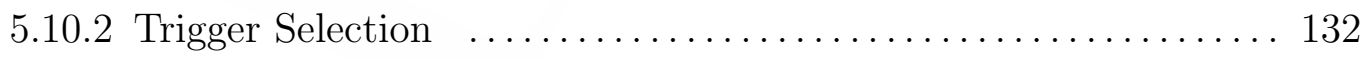

$5.11 \pi^{0}$ and $\eta$ Signal Determination $\ldots \ldots \ldots \ldots \ldots \ldots \ldots \ldots \ldots \ldots \ldots \ldots \ldots \ldots$

5.12 Direct Photon Candidate Definition ......................... 138

5.13 Beam Normalization .................................. 141

Chapter 6 Monte Carlo Simulation _ 145

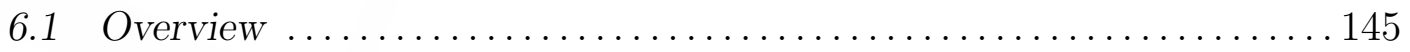


6.2 Parameterized Monte Carlo Studies ........................ 146

6.2.1 EMLAC Resolution Effects $\ldots \ldots \ldots \ldots \ldots \ldots \ldots \ldots \ldots \ldots . \ldots \ldots$

6.3 The Detailed Geant Simulation .......................... 155

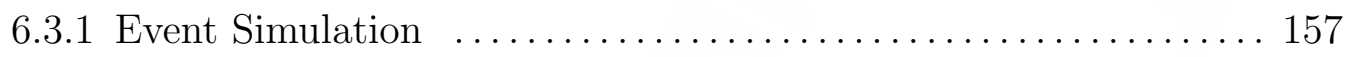

6.3.2 Simulation of Detector Response $\ldots \ldots \ldots \ldots \ldots \ldots \ldots \ldots \ldots \ldots$

6.3.3 Monte Carlo Spectrum Weighting $\ldots . \ldots \ldots \ldots \ldots \ldots \ldots$

6.3.4 Monte Carlo and Data Comparisons $\ldots . \ldots \ldots \ldots \ldots \ldots \ldots . \ldots . \ldots 172$

6.3.5 Evaluation of Reconstruction Efficiencies ................. 181

6.3.6 Vertex Reconstruction Efficiency ...................... 190

6.3.7 Background Photon to $\pi^{0}$ Ratio $\ldots \ldots \ldots \ldots \ldots \ldots \ldots \ldots \ldots . \ldots \ldots$

Chapter 7 Results and Conclusions _ 201

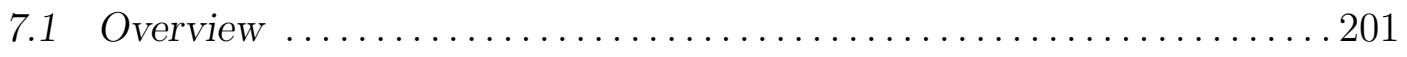

7.2 Cross Section Calculation ................................ 201

$7.3 \pi^{0}$ Cross Sections ..................................... 202

7.4 Direct Photon Cross Sections ............................ 203

7.5 Summary of Systematic Uncertainties . ....................... 211

7.6 Nuclear Dependence ....................................... 223

7.7 Comparisons with NLO Calculations . ...................... 226

7.7.1 Evidence for Initial State Parton $k_{T} \ldots \ldots \ldots \ldots \ldots \ldots \ldots \ldots$

7.7.2 Comparisons with $k_{T}$-enhanced NLO Theory $\ldots \ldots \ldots \ldots \ldots 240$

7.8 Comparisons Between Other Experiments and NLO Theory ....... 244

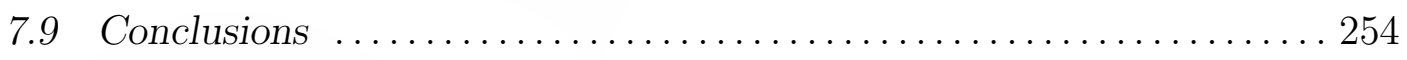

Appendix A Tabulated $\pi^{0}$ Cross Sections 259

Appendix B Tabulated Direct Photon Cross Sections _ 273

Appendix C $\quad \eta$ Cross Sections 283

REFERENCES 295 


\section{List of Tables}

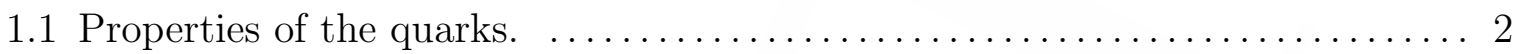

1.2 Valence quark content of some common hadrons. $\ldots \ldots \ldots \ldots \ldots \ldots \ldots \ldots$

1.3 Recent direct photon experiments. $\ldots \ldots \ldots \ldots \ldots \ldots \ldots \ldots \ldots \ldots \ldots \ldots \ldots \ldots \ldots \ldots \ldots \ldots \ldots \ldots \ldots$

2.1 Beam composition of the secondary beams. $\ldots \ldots \ldots \ldots \ldots \ldots \ldots \ldots \ldots \ldots$

2.2 SSD beam chamber geometrical parameters. $\ldots \ldots \ldots \ldots \ldots \ldots \ldots \ldots \ldots \ldots$

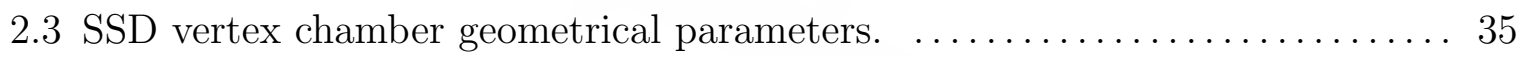

2.5 Orientation and positions of the garlands. The positions are relative to the center of the chamber. Note that within a chamber, the garlands are arranged

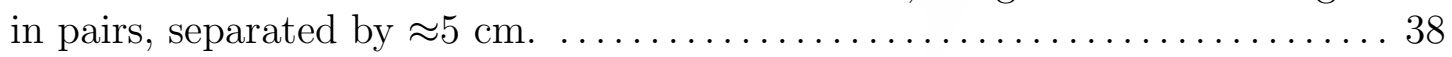

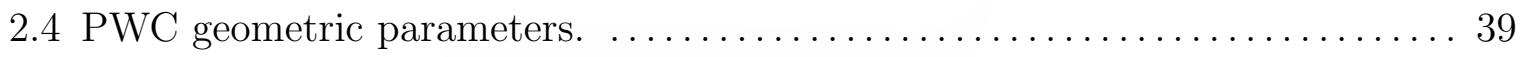

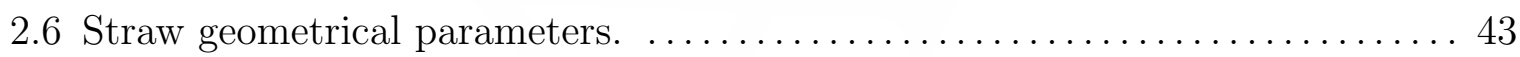

3.1 Trigger characteristics during the 1990 fixed target run. Many events satisfied more than one trigger. Some prescale factors changed during the run. . ... 56

5.1 Data summary for the 1990 and 1991-92 fixed target runs. ............ 94

5.2 Peak and sideband mass regions used in the $\pi^{0}$ and $\eta$ meson analysis. ... 134

5.3 Average values of the beam absorption correction. $\ldots \ldots \ldots \ldots \ldots \ldots \ldots \ldots$

6.1 Number of generated HERWIG filter 2 and filter 3 direct photon background Monte Carlo events (in thousands) as a function of the $p_{T}^{G E N}$ threshold. . . 168

6.2 Number of generated HERWIG Monte Carlo direct photon events (in thousands) as a function of the $p_{T}^{G E N}$ threshold. $\ldots \ldots \ldots \ldots \ldots \ldots \ldots \ldots \ldots$

7.1 Average value of corrections applied to the data. . . . . . . . . . . . . 202

7.2 Measured $\alpha$ values for $\pi^{0}$ and direct photon production as determined from

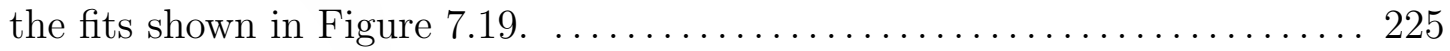

A.1 Invariant differential cross sections per nucleon for $\pi^{0}$ production by 530 and $800 \mathrm{GeV} / c$ proton beams and $515 \mathrm{GeV} / c \pi^{-}$beam on Be targets, for

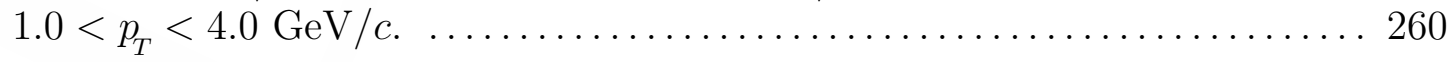


A.2 Invariant differential cross sections per nucleon for $\pi^{0}$ production by 530 and $800 \mathrm{GeV} / c$ proton beams and $515 \mathrm{GeV} / c \pi^{-}$beam on Be targets, for

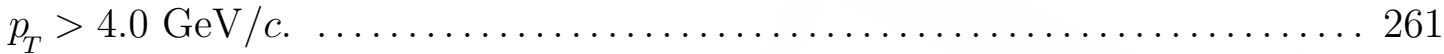

A.3 Invariant differential cross sections per nucleon for $\pi^{0}$ production by 530 and $800 \mathrm{GeV} / c$ proton beams and $515 \mathrm{GeV} / c \pi^{-}$beam on $\mathrm{Cu}$ targets. ....... 262

A.4 Invariant differential cross sections for $\pi^{0}$ production by 530 and $800 \mathrm{GeV} / c$ proton beams and $515 \mathrm{GeV} / c \pi^{-}$beam on proton targets. ........... 263

A.5 Invariant differential cross section per nucleon for $\pi^{0}$ production by $530 \mathrm{GeV} / c$ proton beam on a Be target as a function of $p_{T}$ and rapidity. . ......... 264

A.6 Invariant differential cross section per nucleon for $\pi^{0}$ production by $800 \mathrm{GeV} / c$ proton beam on a Be target as a function of $p_{T}$ and rapidity. .......... 265

A.7 Invariant differential cross section per nucleon for $\pi^{0}$ production by $515 \mathrm{GeV} / c$ $\pi^{-}$beam on a Be target as a function of $p_{T}$ and rapidity. $\ldots \ldots \ldots \ldots \ldots 266$

A.8 Invariant differential cross section for $\pi^{0}$ production by $530 \mathrm{GeV} / c$ proton beam on a $\mathrm{Cu}$ target as a function of $p_{T}$ and rapidity. $\ldots \ldots \ldots \ldots \ldots \ldots 267$

A.9 Invariant differential cross section for $\pi^{0}$ production by $800 \mathrm{GeV} / c$ proton beam on a $\mathrm{Cu}$ target as a function of $p_{T}$ and rapidity. $\ldots \ldots \ldots \ldots \ldots \ldots 268$

A.10 Invariant differential cross section for $\pi^{0}$ production by $515 \mathrm{GeV} / c \pi^{-}$beam on a $\mathrm{Cu}$ target as a function of $p_{T}$ and rapidity. ................. 269

A.11 Invariant differential cross section for $\pi^{0}$ production by $530 \mathrm{GeV} / c$ proton beam on a $p$ target as a function of $p_{T}$ and rapidity. ............... 270

A.12 Invariant differential cross section for $\pi^{0}$ production by $800 \mathrm{GeV} / c$ proton beam on a $p$ target as a function of $p_{T}$ and rapidity. $\ldots \ldots \ldots \ldots \ldots \ldots \ldots \ldots$

A.13 Invariant differential cross section for $\pi^{0}$ production by $515 \mathrm{GeV} / c \pi^{-}$beam on a $p$ target as a function of $p_{T}$ and rapidity. $\ldots \ldots \ldots \ldots \ldots \ldots \ldots \ldots \ldots \ldots \ldots \ldots \ldots \ldots$

B.1 Invariant differential cross sections per nucleon for direct photon production by 530 and $800 \mathrm{GeV} / c$ proton beams and $515 \mathrm{GeV} / c \pi^{-}$beam on Be targets. 274

B.2 Invariant differential cross sections per nucleon for direct photon production by 530 and $800 \mathrm{GeV} / c$ proton beams and $515 \mathrm{GeV} / c \pi^{-}$beam on $\mathrm{Cu}$ targets. 275

B.3 Invariant differential cross sections for direct photon production by 530 and $800 \mathrm{GeV} / c$ proton beams and $515 \mathrm{GeV} / c \pi^{-}$beam on proton targets. .... 276 
List of Tables xiii

B.4 Invariant differential cross section per nucleon for the inclusive reaction $\mathrm{pBe} \rightarrow \gamma \mathrm{X}$ at $530 \mathrm{GeV} / c$ as a function of rapidity for several $p_{T}$ bins. $\ldots 277$

B.5 Invariant differential cross section per nucleon for the inclusive reaction $\mathrm{pBe} \rightarrow \gamma \mathrm{X}$ at $800 \mathrm{GeV} / c$ as a function of rapidity for several $p_{T}$ bins $\ldots \ldots 277$

B.6 Invariant differential cross section per nucleon for the inclusive reaction $\pi^{-} \mathrm{Be} \rightarrow \gamma \mathrm{X}$ at $515 \mathrm{GeV} / c$ as a function of rapidity for several $p_{T}$ bins $\ldots 278$

B.7 Invariant differential cross section per nucleon for the inclusive reaction $\mathrm{pCu} \rightarrow \gamma \mathrm{X}$ at $530 \mathrm{GeV} / c$ as a function of rapidity for several $p_{T}$ bins. $\ldots 278$

B.8 Invariant differential cross section per nucleon for the inclusive reaction $\mathrm{pCu} \rightarrow \gamma \mathrm{X}$ at $800 \mathrm{GeV} / c$ as a function of rapidity for several $p_{T}$ bins. . . 279

B.9 Invariant differential cross section per nucleon for the inclusive reaction $\pi^{-} \mathrm{Cu} \rightarrow \gamma \mathrm{X}$ at $515 \mathrm{GeV} / c$ as a function of rapidity for several $p_{T}$ bins. $\ldots 279$

B.10 Invariant differential cross section for the inclusive reaction $p p \rightarrow \gamma \mathrm{X}$ at 530 $\mathrm{GeV} / c$ as a function of rapidity for several $p_{T}$ bins. $\ldots \ldots \ldots \ldots \ldots \ldots \ldots$

B.11 Invariant differential cross section for the inclusive reaction $p p \rightarrow \gamma \mathrm{X}$ at 800 $\mathrm{GeV} / c$ as a function of rapidity for several $p_{T}$ bins. $\ldots \ldots \ldots \ldots \ldots \ldots \ldots$

B.12 Invariant differential cross section for the inclusive reaction $\pi^{-} p \rightarrow \gamma \mathrm{X}$ at $515 \mathrm{GeV} / c$ as a function of rapidity for several $p_{T}$ bins. $\ldots \ldots \ldots \ldots \ldots \ldots$

C.1 Invariant differential cross sections per nucleon for $\eta$ production by 530 and $800 \mathrm{GeV} / c$ proton beams and $515 \mathrm{GeV} / c \pi^{-}$beam on Be targets. ........ 287

C.2 Invariant differential cross sections per nucleon for $\eta$ production by 530 and $800 \mathrm{GeV} / c$ proton beams and $515 \mathrm{GeV} / c \pi^{-}$beam on $\mathrm{Cu}$ targets. ....... 287

C.3 Invariant differential cross sections for $\eta$ production by 530 and $800 \mathrm{GeV} / c$ proton beams and $515 \mathrm{GeV} / c \pi^{-}$beam on proton targets. ........... 288

C.4 Invariant differential cross section per nucleon for $\eta$ production by $530 \mathrm{GeV} / c$ proton beam on Be target as a function of $p_{T}$ and rapidity. $\ldots \ldots \ldots \ldots \ldots 289$

C.5 Invariant differential cross section per nucleon for $\eta$ production by $800 \mathrm{GeV} / c$ proton beam on Be target as a function of $p_{T}$ and rapidity. $\ldots \ldots \ldots \ldots \ldots 290$

C.6 Invariant differential cross section per nucleon for $\eta$ production by $515 \mathrm{GeV} / c$ $\pi^{-}$beam on Be target as a function of $p_{T}$ and rapidity. $\ldots \ldots \ldots \ldots \ldots \ldots 291$ 
xiv Table of Contents

C.7 Invariant differential cross section per nucleon for $\eta$ production by $530 \mathrm{GeV} / c$ proton beam on $\mathrm{Cu}$ target as a function of $p_{T}$ and rapidity. $\ldots \ldots \ldots \ldots \ldots 29$

C.8 Invariant differential cross section per nucleon for $\eta$ production by $800 \mathrm{GeV} / c$ proton beam on $\mathrm{Cu}$ target as a function of $p_{T}$ and rapidity. $\ldots \ldots \ldots \ldots \ldots 292$

C.9 Invariant differential cross section per nucleon for $\eta$ production by $515 \mathrm{GeV} / c$ $\pi^{-}$beam on $\mathrm{Cu}$ target as a function of $p_{T}$ and rapidity. $\ldots \ldots \ldots \ldots \ldots 292$

C.10 Invariant differential cross section for $\eta$ production by $530 \mathrm{GeV} / c$ proton beam

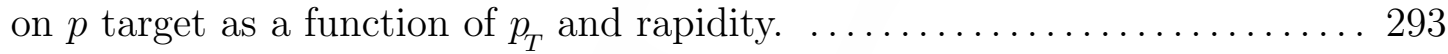

C.11 Invariant differential cross section for $\eta$ production by $800 \mathrm{GeV} / c$ proton beam on $p$ target as a function of $p_{T}$ and rapidity. $\ldots \ldots \ldots \ldots \ldots \ldots \ldots \ldots \ldots \ldots \ldots$

C.12 Invariant differential cross section per nucleon for $\eta$ production by $515 \mathrm{GeV} / c$ $\pi^{-}$beam on $p$ target as a function of $p_{T}$ and rapidity. $\ldots \ldots \ldots \ldots \ldots \ldots 293$ 


\section{List of Figures}

1.1 Schematic illustration of a high $p_{T}$ hadronic interaction. $\ldots \ldots \ldots \ldots \ldots \ldots$

1.2 Left: Parton distribution functions for the proton as a function of $x$. Right: Probability that a parton will fragment into a $\pi^{0}$ as a function of $z$. $\ldots \ldots 7$

1.3 Lowest order Feynman diagrams for direct photon production. $\ldots \ldots \ldots \ldots .9$

1.4 Relative contribution of the annihilation and Compton diagrams to the leading order direct photon cross section for $\pi^{-}$and proton beams as a function of the photon $p_{T}$. GRV92 LO parton distribution function is used for the $\pi^{-}$ calculation, and CTEQ6.1L is used for the proton calculation. The theory

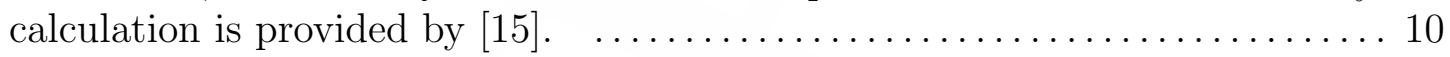

1.5 Top: Gluon distribution uncertainty as characterized by the CTEQ6.1M eigenvector basis sets. Bottom: Fractional uncertainty in the NLO prediction for direct photon production in pBe interactions at $530 \mathrm{GeV} / \mathrm{c}$ due to the

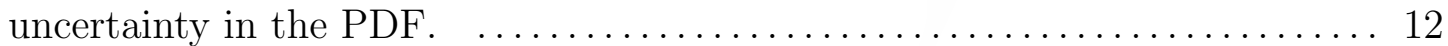

1.6 Top: Definition of $\pi^{0}$ energy asymmetry, $A$. Bottom: $\pi^{0}$ opening angle in the lab frame versus energy asymmetry. $\ldots \ldots \ldots \ldots \ldots \ldots \ldots \ldots \ldots \ldots \ldots \ldots \ldots \ldots \ldots$

1.7 Average $p_{T}$ of muon, photon, and jet pairs produced in hadronic collisions

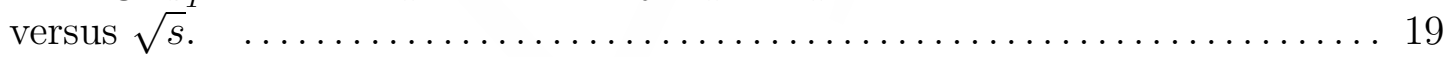

2.1 Schematic view of the Meson West spectrometer. $\ldots \ldots \ldots \ldots \ldots \ldots \ldots \ldots . \ldots \ldots$

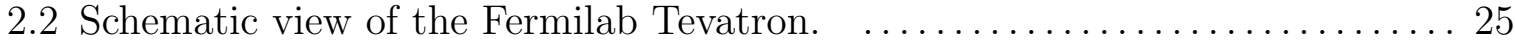

2.3 A schematic drawing of the beamline Cerenkov counter. Each ring of photomultiplier tubes is labeled according to the particle it was designed to

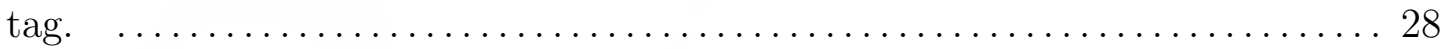

2.4 Pressure curves for the proton, kaon, and pion tags with incident $+530 \mathrm{GeV} / \mathrm{c}$ beam. Peaks/plateaus are labeled by the associated particle type. The solid vertical line represents a typical Čerenkov operating pressure. . . . . . . . . 29

2.5 Layout of the target region during the 1990 and $1991-92$ runs. . . . . . . . 32

2.6 Exploded view of a single PWC chamber. $\ldots \ldots \ldots \ldots \ldots \ldots \ldots \ldots \ldots \ldots \ldots \ldots \ldots$

2.7 Relative chamber efficiency versus distance from the center of a garland pair for (a) zero voltage applied to the garland wire, and (b) 1500 volts applied to

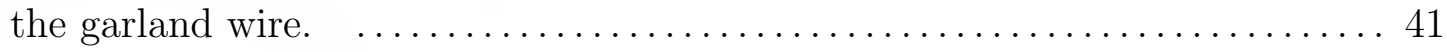

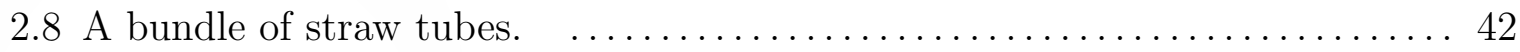

2.9 Side view of the LAC gantry and cryostat. $\ldots \ldots \ldots \ldots \ldots \ldots \ldots \ldots \ldots \ldots \ldots \ldots \ldots \ldots \ldots \ldots$

2.10 Detailed view of the electromagnetic calorimeter. $\ldots \ldots \ldots \ldots \ldots \ldots \ldots \ldots 4$

2.11 Schematic diagram of a single LACAMP module. $\ldots \ldots \ldots \ldots \ldots \ldots \ldots \ldots$ 
2.12 Exploded view of a HALAC cookie. $\ldots \ldots \ldots \ldots \ldots \ldots \ldots \ldots \ldots \ldots \ldots \ldots \ldots \ldots \ldots \ldots \ldots \ldots$

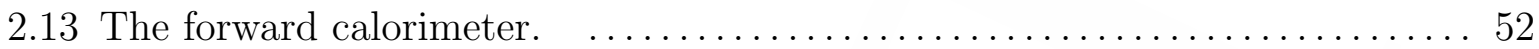

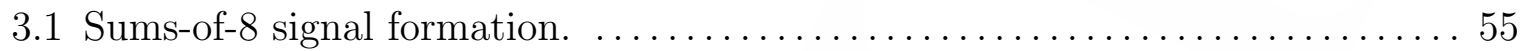

3.2 Number of photon pairs with mass in the $\pi^{0}$ region per $p_{T}$ bin versus $p_{T}$ for several different trigger types. No corrections have been applied to the data

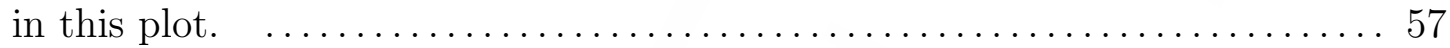

3.3 Block diagram of a local discriminator module. $\ldots \ldots \ldots \ldots \ldots \ldots \ldots \ldots . \ldots 2$

3.4 Block diagram of the E706 DA system. $\ldots \ldots \ldots \ldots \ldots \ldots \ldots \ldots \ldots \ldots \ldots \ldots \ldots \ldots \ldots \ldots \ldots$

4.1 Distribution of PWC hits per space track. $\quad \ldots \ldots \ldots \ldots \ldots \ldots \ldots \ldots \ldots \ldots \ldots \ldots \ldots \ldots$

$4.2 \Delta X, \Delta Y$, and $\Delta Y_{S L}$ linking resolution as a function of the track momentum. The dotted lines indicate the functions used to determine the size of the linking

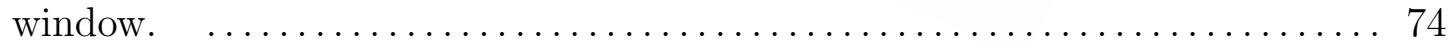

$4.3 \Delta Z_{X Y}$ distribution for vertices in the $1990 \pi^{-}$data. $\ldots \ldots \ldots \ldots \ldots \ldots \ldots 7$

4.4 Reconstructed $K_{s}^{0}$ and $J / \psi$ masses. The means are within $0.1 \%$ of the world

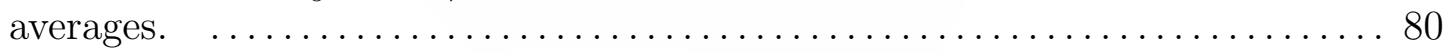

4.5 Schematic drawing of $R$ and $\phi$-boards showing the left-right $\mathrm{R}$ and inner-outer

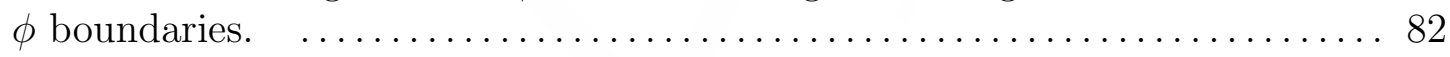

4.6 Energy deposition in the EMLAC. The dotted lines in the $R$ view ( $\phi$ view) plots indicate the location of the inner-outer $\phi$ (left-right $R$ ) boundary. The pair of high energy depositions in the left $R$ and outer $\phi$ views are most likely

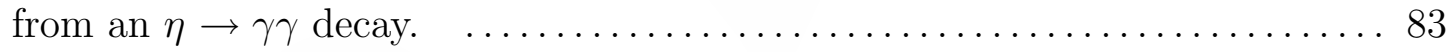

4.7 Time dependence of the response of the EMLAC. $\ldots \ldots \ldots \ldots \ldots \ldots \ldots \ldots$

4.8 Another example of energy deposition in the EMLAC. The high energy deposition in the right $R$ view and the pair of high energy depositions in the outer $\phi$ view are most likely from a $\pi^{0} \rightarrow \gamma \gamma$ decay. $\ldots \ldots \ldots \ldots \ldots . \ldots 9$

$5.1 \gamma \gamma$ invariant mass distribution in the $530 \mathrm{GeV} / c$ proton data. . . . . . . . 95

$5.2 Z$ position of vertices in the $1990515 \mathrm{GeV} / c \pi^{-}$and $1991800 \mathrm{GeV} / c$ proton data for events containing $\gamma \gamma$ pairs with invariant mass within the $\pi^{0}$ signal region and $p_{T}>4 \mathrm{GeV} / c$. The events are corrected for beam absorption and

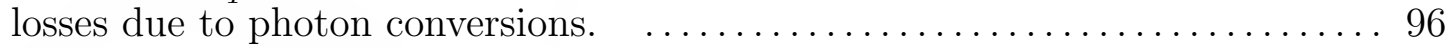

$5.3 X-Y$ distribution of vertices in the Copper and Beryllium targets in the 1990 $515 \mathrm{GeV} / c \pi^{-}$data and the $1991530 \mathrm{GeV} / c$ proton beam data for events containing $\gamma \gamma$ pairs with invariant mass within the $\pi^{0}$ signal region and with $p_{T}>3.5 \mathrm{GeV} / c$. The vertices outside the $\mathrm{Cu}$ and Be target area in the 1990 data are primarily due to interactions in the Rohacell target stand. 
$5.4 X-Y$ position of photons contained within the EMLAC fiducial volume. The photons are from $\gamma \gamma$ pairs with invariant mass within the $\pi^{0}$ signal region and $p_{T}>5 \mathrm{GeV} / c$ in the $1990 \pi^{-}$data.

5.5 Geometric acceptance for single photons, $\pi^{0}$ 's, and $\eta$ 's versus $y_{\text {lab }}$ for several

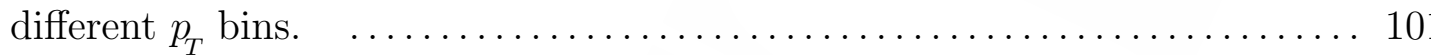

5.6 The distance to nearest track distribution for showers reconstructed in the

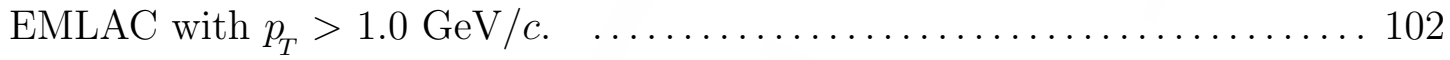

$5.7 E_{\text {front }} / E_{\text {total }}$ distributions for electromagnetic showers and hadronic show-

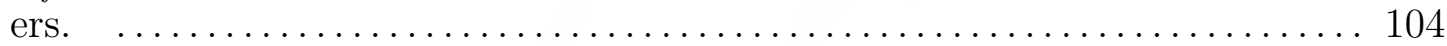

5.8 The effect of the muon rejection requirements on the $\gamma \gamma$ invariant mass distribution in the vicinity of the $\pi^{0}$ for $515 \mathrm{GeV} / c \pi^{-}$beam data. Each subsequent plot includes all the requirements from the previous plots. ... 106

5.9 Use of focusing of the EMLAC radial strips to discriminate against showers induced by muons from the beam halo. $\ldots \ldots \ldots \ldots \ldots \ldots \ldots \ldots \ldots \ldots \ldots \ldots$

5.10 Directionality distributions for beam halo muons and photons in the 1990 $515 \mathrm{GeV} / c \pi^{-}$data for showers with $p_{T}>5 \mathrm{GeV} / c$. The beam halo muon sample was obtained by requiring the veto wall requirement to fail in the quadrant of the reconstructed shower. The photon sample was obtained by imposing all the muon rejection criteria on the showers, with the exception of

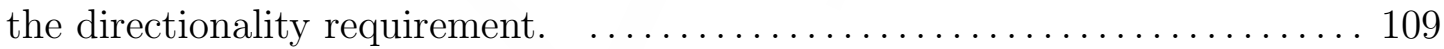

$5.11 \chi_{R}^{2} /$ E distributions for beam halo muons and photons in the $1990515 \mathrm{GeV} / c$ $\pi^{-}$data for showers with $p_{T}>5 \mathrm{GeV} / c$. The beam halo muon sample was obtained by requiring the veto wall requirement to fail in the quadrant of the reconstructed shower. The photon sample was obtained by imposing all the muon rejection criteria on the showers, with the exception of the $\chi^{2}$

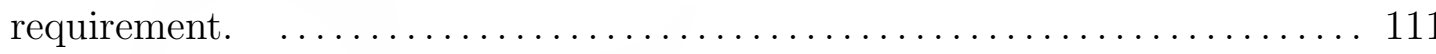

$5.12 P_{T}^{\text {away }} / p_{T}$ distributions for beam halo muons and photons in the 1990515 $\mathrm{GeV} / c \pi^{-}$data for showers with $p_{T}>5 \mathrm{GeV} / c$. The beam halo muon sample was obtained by requiring the veto wall requirement to fail in the quadrant of the reconstructed shower. The photon sample was obtained by imposing all the muon rejection criteria on the showers, with the exception of the balanced

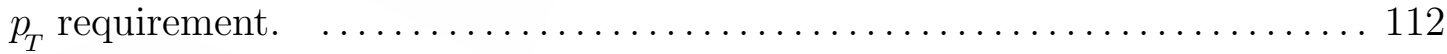

5.13 The $\gamma \gamma$ invariant mass distribution in the region of the $\pi^{0}$ for several energy asymmetry ranges. The number of $\pi^{0}$ 's was obtained using the sideband subtraction technique described in Section 5.11. .................. 115

5.14 Average value of the photon non-conversion probability versus $Z$ for the 1990 (top) and 1991 (bottom) fixed target runs. Superimposed on the figures are the corresponding primary vertex distributions for these runs. $\ldots \ldots \ldots \ldots 116$ 


\section{xviii Table of Contents}

5.15 Effect of ZMP cuts on the $\Delta S_{y}$ and $Z X_{\text {int }}$ distributions of oppositely charged track pairs. The $\Delta S_{y}$ cut is $\pm 3 \mathrm{mr}$ and the $Z X_{\text {int }}$ cut is $\pm 10 \mathrm{~cm}$ from the magnet center.

5.16 EMLAC efficiency for reconstructing an electron as a function of electron momentum. The solid curve represents a fit to these data.

5.17 The resultant systematic uncertainty in the $\pi^{0}$ cross-section due to a $0.5,1.0$, and $2.0 \%$ uncertainty in the energy scale. This is for $\pi^{-}$Be interactions at 515 $\mathrm{GeV} / c$.

5.18 The $\gamma \gamma$ mass distribution in the region of the $\pi^{0}$ and $\eta$ and the $\pi^{0} \gamma$ mass distribution in the region of the $\omega$ for mass combinations with $p_{T}>5.0 \mathrm{GeV} / c$. The mean values for these masses agree with the world values to better than

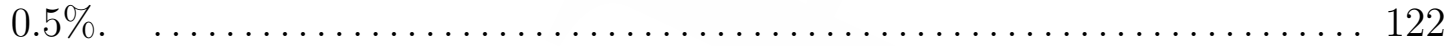

5.19 Average energy lost in the material upstream of the EMLAC for photons and electrons as a function of reconstructed energy.

5.20 Radial dependence of the reconstructed $\pi^{0}$ and $\eta$ masses (normalized to their world values) and the $E / P$ ratio for ZMP electrons from the 1991 data sample. Inset: Radial dependence of the reconstructed $\pi^{0}$ mass for several choices of charge integration time.

5.21 The mean $\eta$ mass (relative to the world value) as a function of the $\eta$ energy (top) and $p_{T}$ (bottom) from the 1991 data sample. ................ 126

5.22 The $\gamma e^{+} e^{-}$mass distribution from the combined 1990 and 1991 data samples.

5.23 Ratio of the reconstructed $\gamma e^{+} e^{-}(\bullet)$ and $\gamma \gamma(\circ)$ masses to the $\pi^{0}$ world value versus the number of radiation lengths traversed in the target. The lines are

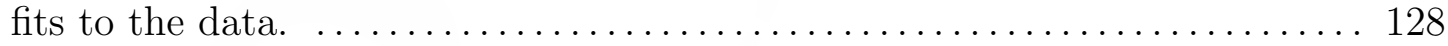

5.24 Ratio of the reconstructed $\gamma e^{+} e^{-}$mass to the $\eta$ and $\pi^{0}$ world values versus the energy of the unconverted photon. $\ldots \ldots \ldots \ldots \ldots \ldots \ldots \ldots \ldots \ldots \ldots \ldots \ldots \ldots \ldots$

5.25 LOCAL_HI and LOCAL_LO discriminator turn-on curves for typical discriminators in the inner and outer regions of the EMLAC versus trigger- $p_{T}$ for the

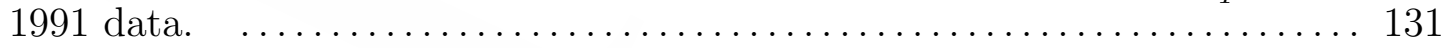

5.26 Composite trigger maps used for the $800 \mathrm{GeV} / c$ proton beam data. .... 133

5.27 Invariant mass distributions for $\gamma \gamma$ pairs in the region of the $\pi^{0}$ for several $p_{T}$

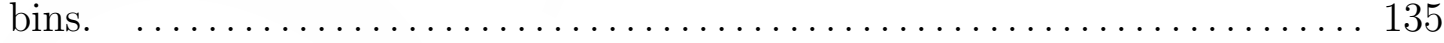

5.28 Invariant mass distributions for $\gamma \gamma$ pairs in the region of the $\eta$ for several $p_{T}$

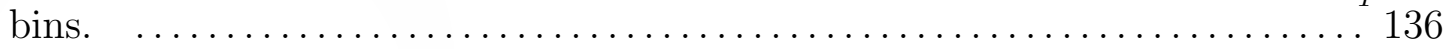

5.29 Invariant mass distributions for $\gamma \gamma$ pairs with $p_{T}>3.5 \mathrm{GeV} / c$ in the region of the $\pi^{0}$ and $\eta$. The arrows indicate the boundaries of the peak and sideband regions. 
5.30 Illustration of the various fits and fit ranges used extract the signal in the bin $1.0<p_{T}<1.2 \mathrm{GeV} / c$. The resultant fits are drawn over the fit range of the histogram.

5.31 Asymmetry distributions for $\gamma \gamma$ mass combinations in the $\pi^{0}$ signal region, sideband region, and for the sideband subtracted $\pi^{0}$ signal. $\ldots \ldots \ldots \ldots \ldots 140$

6.1 The $\pi^{0}$ cross section versus $p_{T}$ and rapidity (inset) for $530 \mathrm{GeV} / c$ proton beam incident on beryllium. The error bars are statistical only. Overlaid on the plots are the results of the fit used in the PMC (solid curve) and a fit to Equation 6.1 (dotted curve), integrated over the appropriate $p_{T}$ and rapidity

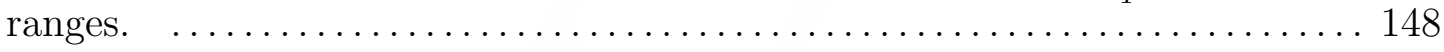

6.2 Background subtracted $\pi^{0}$ (left) and $\eta$ (right) mass distributions from the data compared to the energy resolution smeared $\pi^{0}$ and $\eta$ distributions from the PMC. Here, and in future plots where the y-axis values are unspecified, the histograms are normalized to unity.

6.3 Comparison between the sideband subtracted $\pi^{0}$ energy asymmetry distribution in the data and the energy smeared PMC. $\ldots \ldots \ldots \ldots \ldots \ldots \ldots \ldots \ldots \ldots \ldots$

6.4 Left: Contribution to $\gamma_{b} / \pi^{0}$ from $\pi^{0}$ decays in the $75 \mathrm{~S}$ candidate photon definition. Also shown is the contribution from each of the sources described in the text. Right: Contribution to $\gamma_{b} / \pi^{0}$ from $\pi^{0}$ 's, $\eta$ 's, $\omega$ 's and $\eta^{\prime}$ 's. In these curves, the background photons have not been corrected for effects due to energy resolution.

6.5 Illustration showing the effect of energy resolution on a steeply falling $p_{T}$ spectrum. For any given reconstructed $p_{T}$ bin, the number of entries entering from bins with lower true $p_{T}$ outnumber the losses out of the $p_{T}$ bin, leading to a net shift in the observed uncorrected $p_{T}$ spectrum. $\ldots \ldots \ldots \ldots \ldots \ldots 154$

6.6 a) $E_{\text {smear }} / E$ versus $p_{T}$ in the PMC before rescaling the photon energies and b) after rescaling the photon energies.

6.7 Comparison between the number of reconstructed photons in the triggering octant and the total number of reconstructed tracks in PYTHIA, HERWIG and the data for events containing $\pi^{0}$ 's with $p_{T}>3.5 \mathrm{GeV} / c$. The triggering octant

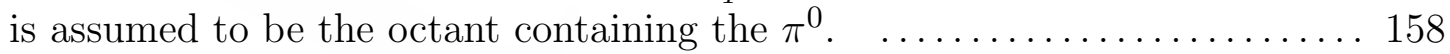

6.8 Comparison of $E / P$ distributions in the data and the Monte Carlo. ..... 165

6.9 Comparison between the the total number of reconstructed tracks and the number of reconstructed photons in the triggering $1 / 2$ octant in the $515 \mathrm{GeV} / c$ $\pi^{-}$beam data and the $530 \mathrm{GeV} / c$ proton beam data for events containing $\pi^{0}$ 's

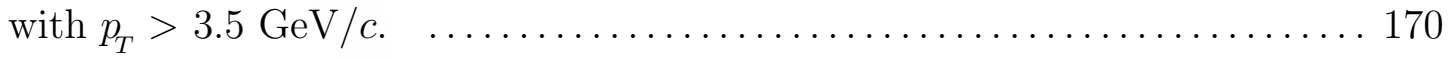

6.10 Comparison between the weighted and unweighted HERWIG $\pi^{0} p_{T}$ spectra for the $p_{T}^{G E N}=3.0 \mathrm{GeV} / c \pi^{-}$sample. The weighting surface is normalized to unity at $p_{T}=p_{T}^{G E N}, y_{c m}=0$. 
6.11 Ratio of the measured $\eta$ to $\pi^{0}$ production cross sections versus $p_{T}$ for $515 \mathrm{GeV} / c$ $\pi^{-}$beam and 530 and $800 \mathrm{GeV} / c$ proton beams. The error bars represent only statistical errors.

6.12 Comparison of $\pi^{0}$ energy distributions in the data and the DGS. The solid points are the DGS and the histogram is the data. $\ldots \ldots \ldots \ldots \ldots \ldots \ldots \ldots \ldots$

6.13 Comparison of $E_{R}-E_{\phi}$ distributions in the data and the DGS for the 530 $\mathrm{GeV} / c$ proton beam. The black dots are the DGS and the histogram is the data.

6.14 Comparison of the $\pi^{0}$ and $\eta$ mass distributions in the data (histogram) and the DGS $(\bullet)$ for the $800 \mathrm{GeV} / c$ p beam.

6.15 Comparison of $E_{\text {front }} / E_{\text {total }}$ distributions in the data and the DGS for the 515 $\mathrm{GeV} / c \pi^{-}$beam. The histogram is the data, and the points are the DGS. 178

6.16 Comparisons of background subtracted $\pi^{0}$ energy asymmetry distributions in data (histogram) and the DGS $(\bullet)$. Comparisons are shown for two $p_{T}$ intervals, $4.0<p_{T}<5.5 \mathrm{GeV} / c$ and $5.5<p_{T}<7.0 \mathrm{GeV} / c$. Also shown are energy asymmetry distributions from the PMC (dotted curves). . . . . . . 179

6.17 Comparison of background subtracted $\pi^{0}$ energy asymmetry distributions in the data (histogram) and the DGS $(\bullet)$ for backward, central, and forward

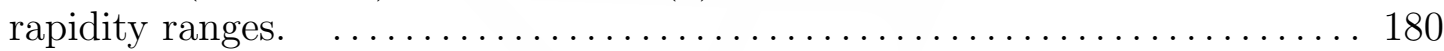

6.18 Comparison between the number of reconstructed photons in half-octants containing a $\pi^{0}$ candidate with $p_{T}>3.5 \mathrm{GeV} / c$ in the DGS and the data. . 182

$6.19 \pi^{0}$ reconstruction efficiency for the $530 \mathrm{GeV} / c$ proton beam as a function of $p_{T}$ for several rapidity ranges. The dotted curves in the figure indicate the contribution from EMLAC resolution smearing alone. . . . . . . . . . . . . . 184

$6.20 \eta$ reconstruction efficiency for the $530 \mathrm{GeV} / c$ proton beam as a function of $p_{T}$ for several rapidity ranges. The dotted curves in the figure indicate the contribution from EMLAC resolution smearing alone.

6.21 SINGLE LOCAL HIGH direct photon reconstruction efficiencies for the $90 \mathrm{~N}, 75 \mathrm{~N}$ and $75 \mathrm{~S}$ candidate definitions as functions of $p_{T}$ for several rapidity ranges. The dotted curves in the figure indicate the contribution from EMLAC resolution smearing alone.

6.22 Reconstruction probability for $\pi^{0}$ 's as a function of the number of generated photons in the $1 / 2$ octant of the generated $\pi^{0}$. The curves represent fits to the Monte Carlo points. No trigger requirement has been placed on the Monte Carlo data. . . . . . . . . . . . . . . . . . . . . . . . . . . . . . . . . . . . 189

6.23 Comparison between reconstruction probabilities obtained using HERWIG and

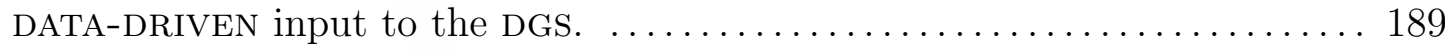

6.24 Comparison between the $\mathrm{Z}$ positions of primary vertices in the DGS (points) and the data (histogram) for events containing a $\pi^{0}$ candidate with $p_{T}>4.0$ $\mathrm{GeV} / c$. 
6.25 The ratio $\gamma_{b} / \pi^{0}$ for each direct photon candidate definition for the three major beam types as functions of $p_{T}$. The dotted curves represent the results of the background fits integrated over the indicated rapidity ranges. . .......... 194

6.26 The ratio $\gamma_{b} / \pi^{0}$ as a function of $p_{T}$ for backward, central and forward rapidities for the $530 \mathrm{GeV} / c$ proton beam. The dotted curves represent the results of the background fits integrated over the indicated rapidity ranges. . . . . . . 195

6.27 Difference between $\gamma_{b} / \pi^{0}$ in copper and beryllium versus $p_{T}$ for the 1991 $800 \mathrm{GeV} / c \mathrm{p}$ beam data. Also shown is the difference between $\gamma_{b} / \pi^{0}$ in hydrogen and beryllium. The dotted lines represent fits to the differences, integrated over the rapidity range $-1.0<y<0.5$.

6.28 Ratio of background photons to charged pions for the $515 \mathrm{GeV} / c \pi^{-}$data. The background photons are from interactions in the EMLAC by charged pions. The dotted line represents a fit to the Monte Carlo data. (Note $\gamma_{b} / \pi^{ \pm}$

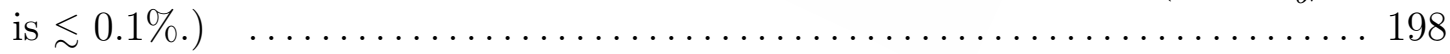

6.29 Comparison of $\gamma_{b} / \pi^{0}$ from the PMC and the DGS simulations for the $75 \mathrm{~S}$ photon candidate definition.

6.30 Comparison of $\gamma_{b} / \pi^{0}$ from the DGS using input events from HERWIG $(\bullet)$ and from data (o) for $90 \mathrm{~N}, 75 \mathrm{~N}$ and $75 \mathrm{~S}$ photon definitions.

$7.1 \pi^{0}$ production cross sections per nucleus as functions of $p_{T}$ for $530 \mathrm{GeV} / c$ proton beam on copper, beryllium, and hydrogen targets. . . . . . . . . . 204

$7.2 \pi^{0}$ production cross sections per nucleus as functions of $p_{T}$ for $800 \mathrm{GeV} / c$ proton beam on copper, beryllium, and hydrogen targets. $\ldots \ldots \ldots \ldots \ldots 205$

$7.3 \pi^{0}$ production cross sections per nucleus as functions of $p_{T}$ for $515 \mathrm{GeV} / c \pi^{-}$ beam on copper, beryllium, and hydrogen targets. $\ldots \ldots \ldots \ldots \ldots \ldots \ldots$

$7.4 \pi^{0}$ cross section per nucleon as a function of rapidity for $530 \mathrm{GeV} / c$ proton beam on beryllium for several $p_{T}$ intervals. $\ldots \ldots \ldots \ldots \ldots \ldots \ldots \ldots \ldots \ldots \ldots \ldots$

$7.5 \pi^{0}$ cross section per nucleon as a function of rapidity for $800 \mathrm{GeV} / c$ proton

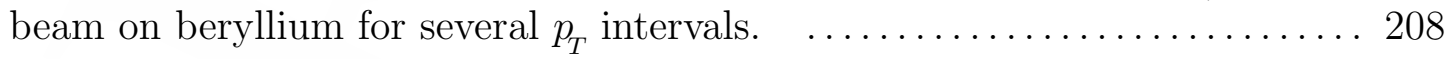

$7.6 \pi^{0}$ cross section per nucleon as a function of rapidity for $515 \mathrm{GeV} / c \pi^{-}$beam

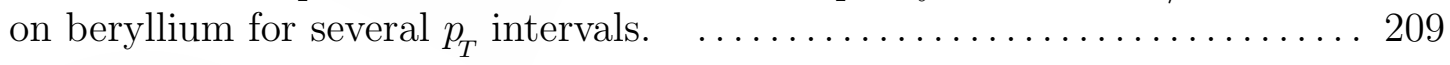

7.7 Single (unsubtracted) photon to $\pi^{0}$ ratio for $530 \mathrm{GeV} / c$ proton beam on beryllium. The error bars represent the statistical uncertainties only. Also shown is $\gamma_{b} / \pi^{0}$.

7.8 Single (unsubtracted) photon to $\pi^{0}$ ratio for $800 \mathrm{GeV} / c$ proton beam on beryllium. The error bars represent the statistical uncertainties only. Also shown is $\gamma_{b} / \pi^{0}$. 
7.9 Single (unsubtracted) photon to $\pi^{0}$ ratio for $515 \mathrm{GeV} / c \pi^{-}$beam on beryllium. The error bars represent the statistical uncertainties only. Also shown is $\gamma_{b} / \pi^{0}$.

7.10 Direct photon cross sections per nucleus versus $p_{T}$ for $530 \mathrm{GeV} / c$ proton beam on copper, beryllium, and hydrogen targets. $\ldots \ldots \ldots \ldots \ldots \ldots \ldots \ldots \ldots \ldots \ldots \ldots \ldots$

7.11 Direct photon cross sections per nucleus versus $p_{T}$ for $800 \mathrm{GeV} / c$ proton beam

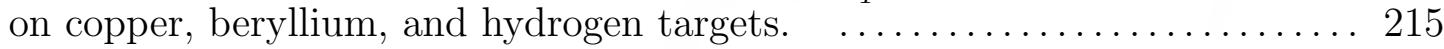

7.12 Direct photon cross sections per nucleus versus $p_{T}$ for $515 \mathrm{GeV} / c \pi^{-}$beam on copper, beryllium, and hydrogen targets. $\ldots \ldots \ldots \ldots \ldots \ldots \ldots \ldots \ldots \ldots \ldots \ldots \ldots \ldots \ldots \ldots$

7.13 Direct photon cross section per nucleon as a function of rapidity for $530 \mathrm{GeV} / \mathrm{c}$ proton beam on beryllium for several $p_{T}$ intervals. $\ldots \ldots \ldots \ldots \ldots \ldots \ldots 217$

7.14 Direct photon cross section per nucleon as a function of rapidity for $800 \mathrm{GeV} / c$ proton beam on beryllium for several $p_{T}$ intervals. $\ldots \ldots \ldots \ldots \ldots \ldots \ldots 218$

7.15 Direct photon cross section as a function of rapidity for $515 \mathrm{GeV} / c \pi^{-}$beam on beryllium for several $p_{T}$ intervals.

7.16 Relative systematic uncertainties for direct photon (top) and $\pi^{0}$ (bottom)

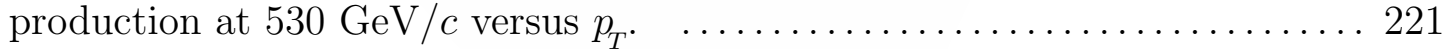

7.17 Ratios of direct photon and $\pi^{0}$ production cross sections by $515 \mathrm{GeV} / c \pi^{-}$ beam on Be obtained from the 1991-92 fixed target run to those obtained from the 1990 run. The error bars reflect statistical uncertainties only. . . . . . 222

7.18 Ratio of $90 \mathrm{~N}$ to $75 \mathrm{~S}$ and $75 \mathrm{~N}$ to $75 \mathrm{~S}$ direct photon cross sections for the $515 \mathrm{GeV} / \mathrm{c} \pi^{-}$and 530 and $800 \mathrm{GeV} / \mathrm{c}$ proton beams. .............. 224

7.19 The nuclear dependence parameter $\alpha$ measured using $\mathrm{Cu}$ and Be targets versus $p_{T}$ for direct photon and $\pi^{0}$ production by incident 530 and $800 \mathrm{GeV} / c$ proton

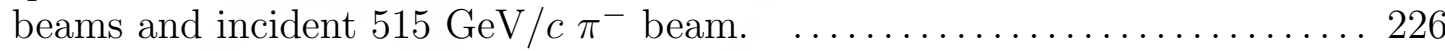

7.20 The ratio of inclusive $\pi^{0}$ production cross sections per nucleon on $\mathrm{Cu}$ to those on Be as functions of $\pi^{0} p_{T}$. Also shown are shape comparisons with HIJING. The error bars represent only statistical uncertainties.

7.21 The ratio of inclusive direct photon production cross sections per nucleon on $\mathrm{Cu}$ to those on $\mathrm{Be}$ as functions of direct photon $p_{T}$. Also shown are shape comparisons with HIJING. The error bars represent only statistical uncertainties.

7.22 The ratio of inclusive $\pi^{0}$ cross sections per nucleon on Be target to those on $p$ target as functions of $\pi^{0} p_{T}$. Also shown are shape comparisons with HIJING. The error bars represent only statistical uncertainties.

7.23 The ratio of inclusive direct photon cross sections per nucleon on Be target to those on $p$ target as function of direct photon $p_{T}$. Also shown are shape comparisons with HIJING. The error bars represent only statistical uncertainties. 
7.24 Direct photon cross section versus $p_{T}$ for $515 \mathrm{GeV} / c \pi^{-}$beam on beryllium compared to NLO pQCD results for several choices of scales. Also shown is the $\pi^{0}$ comparison (scaled down by a factor of 1000).

7.25 Comparison between a threshold resummed pQCD calculation and NLO pQCD for scale choices of $\frac{1}{2} p_{T}$ and $2 p_{T}$. Figure from Ref. [31]. . . . . . . 233

7.26 Direct photon and $\pi^{0}$ cross sections versus $p_{T}$ for $530 \mathrm{GeV} / c$ proton beam on beryllium compared to NLO pQCD results for several choices of parton distribution functions.

7.27 Direct photon and $\pi^{0}$ cross sections versus $p_{T}$ for $800 \mathrm{GeV} / c$ proton beam on beryllium compared to NLO pQCD results using MRST2001E PDF. The shaded band indicates the uncertainty associated with the PDF. . ...... 236

$7.28 p_{O U T}, \Delta \phi$ and $Q_{T}$ distributions for high-mass direct photon pairs in the $515 \mathrm{GeV} / c \pi^{-}$data. The data are compared to NLO pQCD (dashed), resummed NLO (solid) and $k_{T}$ enhanced PYTHIA (dotted) results. Figure from Ref. [27].

$7.29 p_{\text {OUT }}$ distributions for high-mass $\gamma \gamma, \pi^{0} \pi^{0}, \gamma \pi^{0}$, and $\pi^{0} \eta$ pairs in $\pi^{-}$Be interactions at $515 \mathrm{GeV} / c$. Figure from Ref. [27]

7.30 Away-side fragmentation function for jets recoiling against isolated $\gamma$ 's with

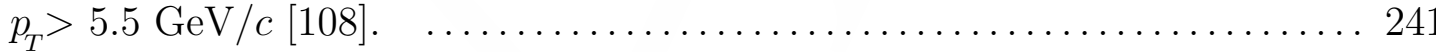

$7.31 \mathrm{D}^{ \pm}$cross section per nucleon versus $p_{T}$ for $515 \mathrm{GeV} / c \pi^{-}$-Nucleon collisions

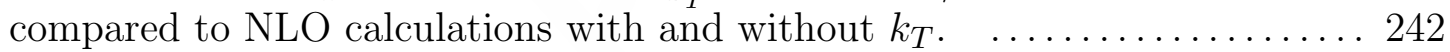

7.32 Direct photon cross section for pBe collisions at $530 \mathrm{GeV} / c$ compared to NLO (dotted), threshold resummed (dashed) and joint threshold and recoil resummed (solid) calculations. Figure from $[111] . \quad \ldots \ldots \ldots \ldots \ldots \ldots \ldots . \ldots . \ldots . \ldots$.

$7.33 k_{T}$-enhancement factors for direct photon and $\pi^{0}$ production cross sections by $530 \mathrm{GeV} / c$ proton beam for several values of $\left\langle k_{T}\right\rangle . \ldots \ldots \ldots \ldots \ldots \ldots \ldots \ldots$

7.34 Direct photon and $\pi^{0}$ cross sections for $530 \mathrm{GeV} / c$ proton beam on beryllium compared to $k_{T}$-enhanced NLO pQCD calculations. Also shown is the quantity (Data - Theory)/Theory for the direct photon cross section. . .. 246

7.35 Direct photon and $\pi^{0}$ cross sections for $800 \mathrm{GeV} / c$ proton beam on beryllium compared to $k_{T}$-enhanced NLO pQCD calculations. Also shown is the quantity (Data - Theory)/Theory for the direct photon cross section. . . . 247

7.36 Direct photon and $\pi^{0}$ cross sections for $515 \mathrm{GeV} / c \pi^{-}$beam on beryllium compared to $k_{T}$-enhanced NLO pQCD calculations. Also shown is the quantity (Data - Theory)/Theory for the direct photon cross section. . . . 248

7.37 Data/Theory for proton induced direct photon data from various experiments as a function of $x_{T}$. The theory calculations use CTEQ5M PDF and scale $\mu=\frac{1}{2} p_{T}$. 
7.38 Data/Theory for proton induced $\pi^{0}$ data from various experiments as a function of $x_{T}$. The theory calculations use CTEQ5M PDF, KKP FF, and scale $\mu=\frac{1}{2} p_{T}$.

7.39 Direct-photon and $\pi^{0}$ cross sections from experiments WA70 and UA6, compared to $k_{T}$-enhanced NLO calculations. $\ldots \ldots \ldots \ldots \ldots \ldots \ldots \ldots \ldots \ldots \ldots \ldots \ldots \ldots \ldots$

7.40 Isolated direct photon cross sections from CDF and D $\varnothing$ at $\sqrt{s}=1.8 \mathrm{TeV}$ (top) and $\sqrt{s}=0.63 \mathrm{TeV}$ (bottom) compared to NLO predictions. The predictions for CDF (DØ) use CTEQ5M (CTEQ4M) PDF. The solid curves represent the expected enhancement to the predictions from parton- $k_{T}$. The data from CDF at $\sqrt{s}=1.8 \mathrm{TeV}$ have been scaled up by $10 \%$ to facilite a shape comparision. 253

7.41 Isolated direct photon cross section from ZEUS. The NLO predictions are by Gordon (LG) and by Krawczyk and Zembruski (K\&W). . . . . ....... 255

7.42 The ratio between $530 \mathrm{GeV} / \mathrm{c}$ and $800 \mathrm{GeV} / \mathrm{c}$ p beam direct photon cross sections as functions of $p_{T}$. Overlayed on the plots are NLO predictions with

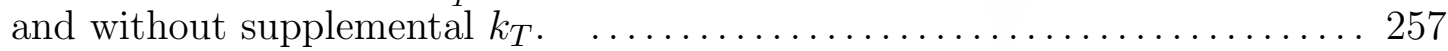

C.1 $\eta$ production cross sections per nucleus as functions of $p_{T}$ for $530 \mathrm{GeV} / c$ proton beam on copper, beryllium, and liquid hydrogen targets. . . . . . . . . 284

C.2 $\eta$ production cross sections per nucleus as functions of $p_{T}$ for $800 \mathrm{GeV} / c$ proton beam on copper, beryllium, and liquid hydrogen targets. . ............ 285

C.3 $\eta$ production cross sections per nucleus as functions of $p_{T}$ for $515 \mathrm{GeV} / c \pi^{-}$ beam on copper, beryllium, and liquid hydrogen targets. 286 


\section{Chapter 1 Introduction}

\section{$1.1 \quad$ Overview}

This thesis describes a study of the production of high transverse momentum $\left(p_{T}\right)$ direct photons by proton and $\pi^{-}$beams on beryllium, copper, and liquid hydrogen targets. The data were recorded by Fermilab experiment E706 during the 1990 and 1991-92 fixed target runs. The results presented here have been published previously by the author [1-3]. Presented here is a compilation of these results and of the various methods used to extract the direct photon production cross sections. To achieve the smallest possible systematic uncertainty, which limited the direct photon measurement, extensive detector evaluations were required and alternative analyses were explored. This necessarily led to a relatively long document.

In this chapter, the motivations for investigating direct photons, the experimental techniques and challenges associated with this study, and an overview of other experiments that have reported direct photon results are presented.

\subsection{Quantum Chromodynamics}

In 1969, a deep inelastic scattering experiment performed at the Stanford Linear Accelerator Center (SLAC) $[4,5]$ provided the first direct evidence that hadrons, the particles which experience the strong force, are comprised of pointlike constituents called partons $[6,7]$. Partons have since been identified as massive spin $1 / 2$ fermions, quarks, and massless spin 1 gauge bosons, gluons. There are six types (or flavors) of quarks and eight gluons. Table 1.1 lists some of the physical properties of the quarks. 
Table 1.1 Properties of the quarks.

\begin{tabular}{|c|c|c|c|c|c|c|}
\hline Generation & \multicolumn{2}{|c|}{1} & \multicolumn{2}{c|}{2} & \multicolumn{2}{c|}{3} \\
\hline Quark & $\mathrm{u}(\mathrm{up})$ & $\mathrm{d}($ down $)$ & $\mathrm{c}($ charm $)$ & $\mathrm{s}($ strange $)$ & $\mathrm{t}($ top $)$ & $\mathrm{b}$ (bottom) \\
\hline Electric Charge & $+2 / 3$ & $-1 / 3$ & $+2 / 3$ & $-1 / 3$ & $+2 / 3$ & $-1 / 3$ \\
\hline Mass $\left(\mathrm{MeV} / \mathrm{c}^{2}\right)$ & $\sim 300$ & $\sim 300$ & $\sim 1500$ & $\sim 500$ & $\sim 175000$ & $\sim 5000$ \\
\hline
\end{tabular}

The interactions of quarks and gluons are described by a non-Abelian gauge theory called Quantum Chromodynamics (QCD) [8, 9]. In QCD, quarks are assigned a quantum number called color charge, which is analogous to the electric charge in quantum electrodynamics. Color charge has three degrees of freedom. Gluons also carry color charge and can consequently interact with each other.

Colored objects are not expected to exist as free particles. This is called color confinement and is manifested in high energy scattering experiments by the outgoing partons fragmenting into collimated jets of particles traveling in roughly the same direction as the scattered partons. The hadrons are colorless combinations of bound quarks. Until recently, only two classes of bound quark states have been observed: mesons and baryons. Mesons are formed from quark-antiquark pairs and baryons are formed from three quark (or antiquark) combinations. ${ }^{1}$ The quarks which form these combinations are called the valence quarks. The valence quarks in a hadron describe gross features such as the hadron charge and spin. However, the interactions of these valence quarks give rise to a dynamic sea of quarks and gluons within the hadrons as well. The valence quark content of some common hadrons are listed in Table 1.2.

One important result from QCD is that the strong coupling constant, $\alpha_{s}$, becomes weaker as the momentum transfer, $Q^{2}$, of the interaction between

1 Evidence for pentaquarks, hadrons comprised of 4 quarks and one antiquark, was first reported by the LEPS collaboration in 2002 [10]. 
Table 1.2 Valence quark content of some common hadrons.

\begin{tabular}{|c|c|c|c|c|}
\hline Hadron & $\mathrm{p}$ & $\mathrm{n}$ & $\pi^{+}$ & $K^{+}$ \\
\hline Quark Content & uud & udd & $u \bar{d}$ & $u \bar{s}$ \\
\hline
\end{tabular}

partons increases. In the literature, this is commonly referred to as the running of the coupling constant. The dependence of $\alpha_{s}$ on $Q^{2}$ is given, in the leading log approximation, by [11]

$$
\alpha_{s}\left(Q^{2}\right)=\frac{12 \pi}{\left(33-2 n_{f}\right) \ln \left(Q^{2} / \Lambda^{2}\right)},
$$

where $n_{f}$ is the number of quark flavors, and $\Lambda$ is a constant.

Equation 1.1 indicates that as $Q^{2} \rightarrow \infty, \alpha_{s} \rightarrow 0$. This phenomenon is known as asymptotic freedom. If the momentum transfer in an interaction is large enough, $\alpha_{s}$ may be sufficiently small to allow the effective application of perturbative methods to make quantitative calculations about the interaction. Conversely, for $Q^{2} \sim \Lambda^{2}, \alpha_{s}$ becomes large, and the suitability of perturbative techniques becomes questionable. The parameter $\Lambda$ may be regarded as the limit where the "strong interactions become strong" [12]. This is also thought to be the region where the confining forces of QCD set in.

\subsection{Phenomenology of High Transverse Momentum Interactions}

Consider the inclusive reaction $A+B \rightarrow C+X$, where $A$ and $B$ are colliding hadrons, $C$ represents the outgoing observed hadron of interest, and $X$ represents everything else produced in the reaction. This process is shown schematically in Figure 1.1. If emerging hadron $C$ has large transverse momentum (high $p_{T}$ ), it is likely that the underlying partonic subprocess, $a+b \rightarrow c+d$, involved a large $Q^{2}$, and thus the use of perturbative techniques to evaluate the cross section for 
the subprocess may be justified [13]. However, although the cross section for the partonic hard scatter may be properly evaluated, the challenge of relating this quantity to the experimentally measured hadronic cross section remains.

This challenge is addressed via the ansatz of factorization [14]. Factorization asserts that the cross section calculation can be separated into a short-distance, or high momentum transfer, piece describing the partonic subprocess, and longdistance pieces describing the momentum distributions of partons within hadrons and the fragmentation of partons into hadrons. Assuming the initial state partons are traveling collinearly with their respective hadrons, the cross section for particle production can be written as [15]:

$$
\begin{aligned}
E_{C} \frac{d^{3} \sigma}{d p_{C}^{3}}( & A+B \rightarrow C+X)=\sum_{a b c d} \int d x_{a} d x_{b} d z_{c} \delta(\hat{s}+\hat{t}+\hat{u}) \frac{\hat{s}}{z_{c}^{2} \pi} \\
& \times G_{a / A}\left(x_{a}, Q^{2}\right) G_{b / B}\left(x_{b}, Q^{2}\right) \frac{d \sigma}{d \hat{t}}(a b \rightarrow c d) D_{C / c}\left(z_{c}, Q^{2}\right),
\end{aligned}
$$

where $\hat{s}, \hat{t}$ and $\hat{u}$ are the parton level Mandelstam variables, defined as $\hat{s}=$ $\left(p_{a}+p_{b}\right)^{2}, \hat{t}=\left(p_{a}-p_{c}\right)^{2}$, and $\hat{u}=\left(p_{a}-p_{d}\right)^{2} ; p_{i}$ being the 4-momentum of parton $i, \frac{d \sigma}{d \hat{t}}(a b \rightarrow c d)$ is the parton level hard scattering cross section, $G_{i / I}\left(x_{i}, Q^{2} / \mu_{F}^{2}\right)$ represents the probability of finding parton $i$ with fraction $x_{i}$ of the longitudinal momentum of hadron $I$, and $D_{C / c}\left(z_{c}, Q^{2} / m_{F}^{2}\right)$ represents the probability of finding hadron $C$ with the fraction $z_{c}$ of the momentum of the outgoing parton $c$.

$G\left(x, Q^{2} / \mu_{F}^{2}\right)$ is called the parton distribution function (PDF) and $D\left(z, Q^{2} / m_{f}^{2}\right)$ is called the fragmentation function $(\mathrm{FF})$. The variables $\mu_{F}$ and $m_{F}$ are called the factorization and fragmentation scales, respectively. These scales are arbitrary, non-physical parameters which serve to define the separation between long and short-distance phenomena. Typically, the values chosen for these scales are related to some experimental observable such as transverse momentum, although 


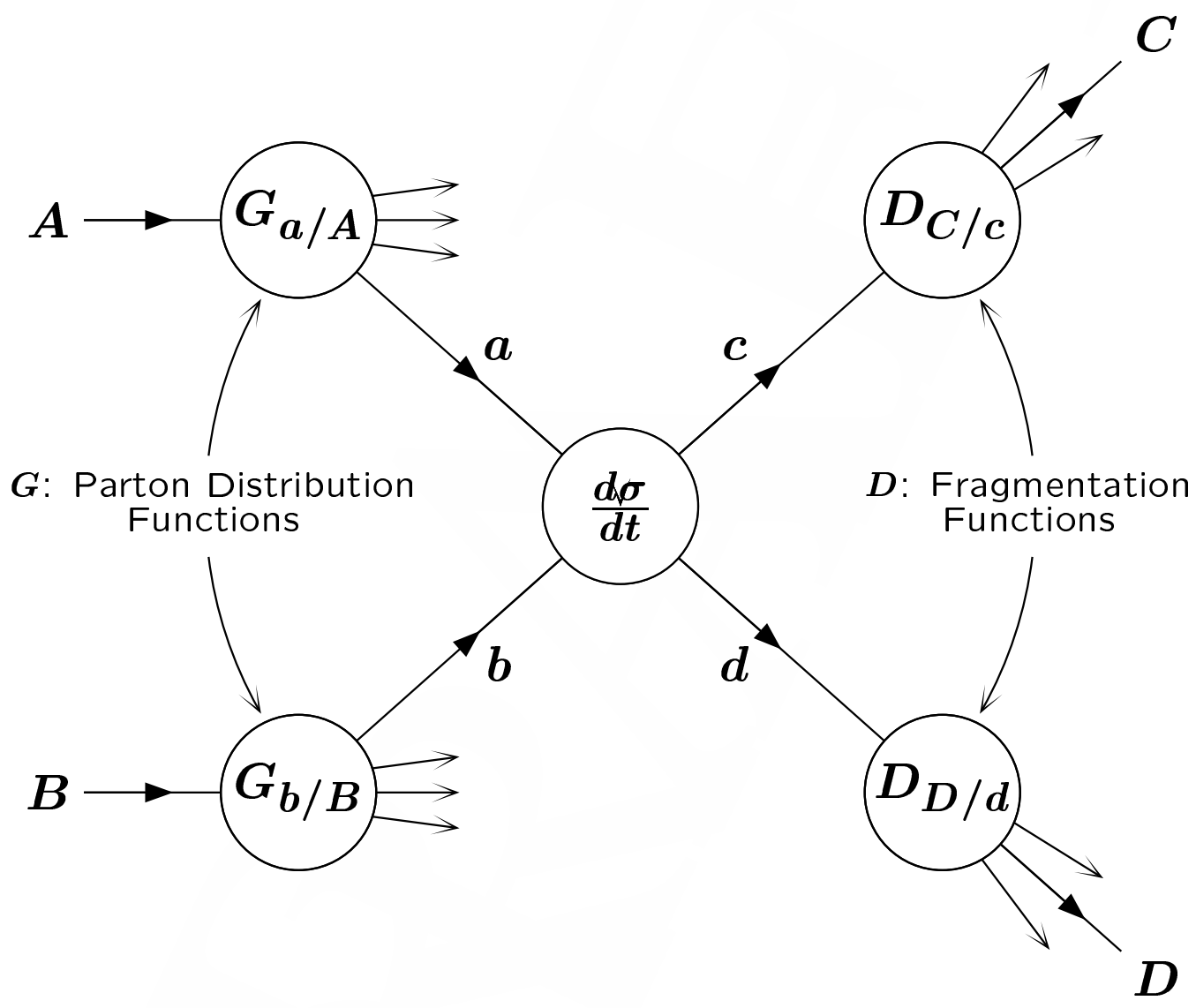

Figure 1.1 Schematic illustration of a high $p_{T}$ hadronic interaction. 


\section{Introduction}

other, more sophisticated choices are sometimes made [16, 17]. Note that the cross section, being a physical quantity, cannot depend upon these arbitrary parameters. Therefore, any significant scale dependence in the prediction is usually taken as an indication of the need for higher order diagrams in the perturbative calculation.

As the $G\left(x, Q^{2} / \mu_{F}^{2}\right)$ and $D\left(z, Q^{2} / m_{f}^{2}\right)$ functions describe the long-distance interactions of partons bound within hadrons, perturbative techniques cannot be used to calculate them, and thus these functions are presently determined from experimental data. However, they are universal in the sense that they are presumed to be independent of the process used to measure them. Furthermore, although these functions have an explicit $Q^{2}$ dependence, this dependence is calculable using perturbative QCD (pQCD). ${ }^{2}$ Thus it is possible to evaluate $G\left(x, Q^{2} / \mu_{F}^{2}\right)$ or $D\left(x, Q^{2} / m_{F}^{2}\right)$ from some process at some $Q_{0}^{2}$ and use it in the calculation of the cross section for other processes at other $Q^{2}$ values. Graphs of proton PDFs (as determined by CTEQ [18]), and $\pi^{0}$ FFs (determined by KKP [19]), are shown in Figure 1.2.

Calculations for the parton level hard scattering matrix elements have been carried out to next-to-leading order (NLO) precision for many processes, including direct photon and $\pi^{0}$ production. At NLO, another scale is introduced into the perturbative calculation, the renormalization scale $\mu_{R}$. This scale is used during renormalization, which is a technique used to regulate divergences encountered while evaluating diagrams containing loops. It is typical in practice to choose the scales $\mu_{R}, \mu_{F}$, and $m_{F}$ to be equal to each other, though they need not be equal in general.

\footnotetext{
2 Typically, the distribution or fragmentation function is extracted at some scale, $Q_{0}$, and then evolved to a different scale using DGLAP [20], or their equivalent, equations.
} 

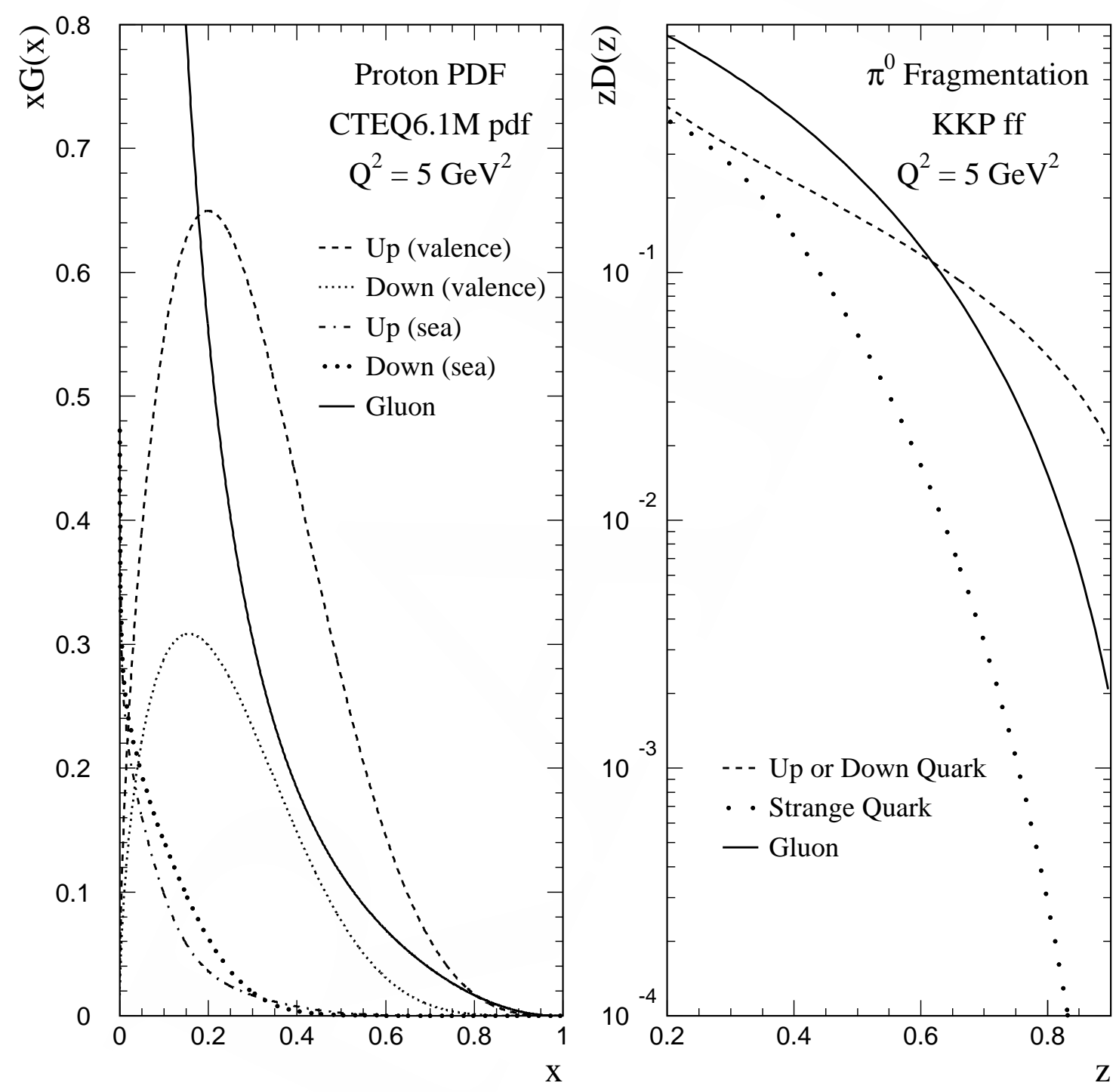

Figure 1.2 Left: Parton distribution functions for the proton as a function of $x$. Right: Probability that a parton will fragment into a $\pi^{0}$ as a function of $z$. 


\subsection{Direct Photon Physics}

The study of direct photon production has attractive aspects from both experimental and theoretical viewpoints. Direct photons are photons produced in parton interactions, rather than as the result of the electromagnetic decay of hadrons. At leading order, they emerge carrying the full $p_{T}$ of the hard scatter, and thus offer a clean probe of the underlying quark-quark and quark-gluon dynamics. Although the study of jet production also offers this opportunity, the 4-momentum of the parent parton may be difficult to reconstruct accurately since there are many particles that must be simultaneously detected and measured. There are also experimental and theoretical ambiguities present when assigning final state particles to particular jets. With direct photons, there is only one particle to measure, and the measurement can be made with good accuracy using electromagnetic calorimeters.

It has been proposed $[21,22,23]$ that the study of direct photon production can provide important information about the partonic structure of hadrons. At leading order, only two processes contribute to direct photon production in hadronic interactions. These processes are illustrated in Figure 1.3. In protonproton $(p p)$ reactions, the cross section is expected to be dominated by the Compton process since there are no valence anti-quarks present. This is shown quantitatively in Figure 1.4. Note that since the Compton process is initiated by quark-gluon scattering, direct photon production in $p p$ interactions is sensitive to the gluon content of the proton. Consequently, direct photon data have been anticipated to provide constraints on the gluon distribution function which, particularly at moderate to large values of $x$, is not very well determined. To

illustrate, gluon distributions at $Q^{2}=5 \mathrm{GeV}^{2}$ for the 40 CTEQ6.1M eigenvector basis sets are shown at the top of Figure 1.5. These sets represent the positive 


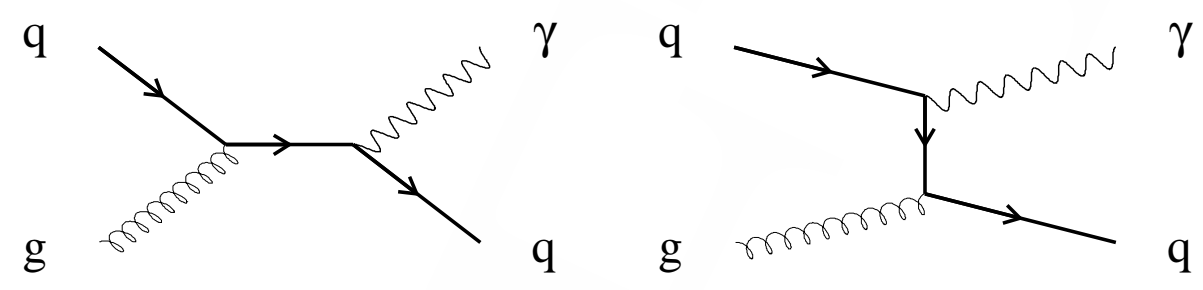

\section{Compton Diagrams}

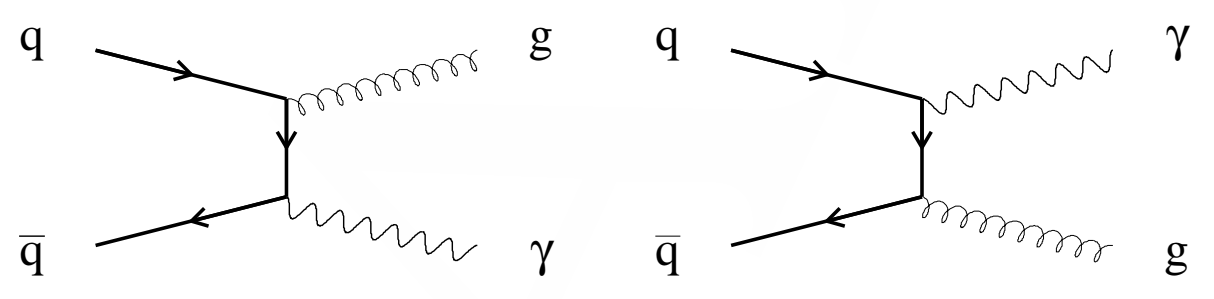

\section{Annihilation Diagrams}

Figure 1.3 Lowest order Feynman diagrams for direct photon production. 


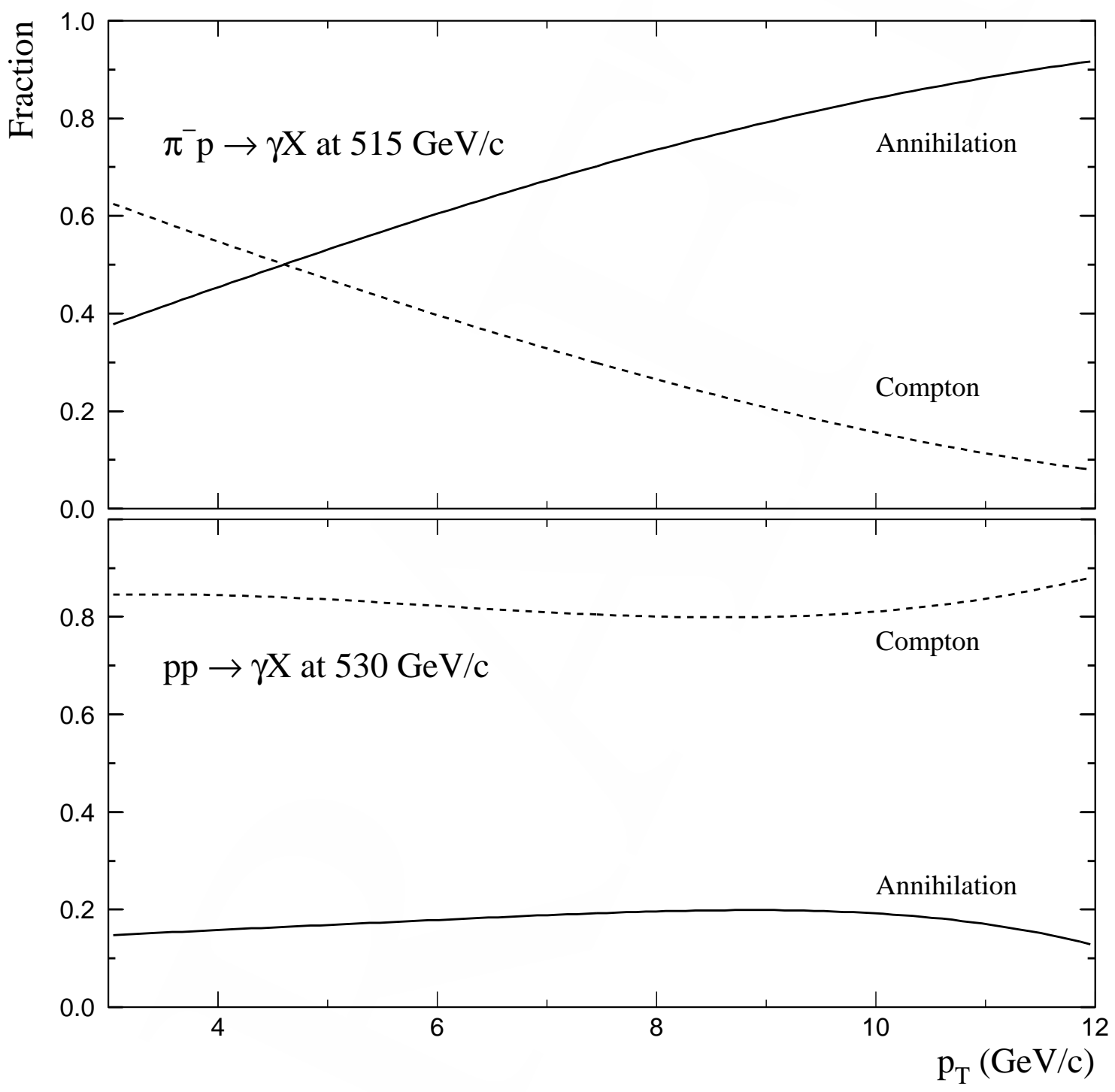

Figure 1.4 Relative contribution of the annihilation and Compton diagrams to the leading order direct photon cross section for $\pi^{-}$and proton beams as a function of the photon $p_{T}$. GRV92 LO parton distribution function is used for the $\pi^{-}$calculation, and CTEQ6.1L is used for the proton calculation. The theory calculation is provided by [15]. 
and negative displacements, within some allowed tolerance, about each of the 20 independent parameters used in the determination of the PDF. The spread in these distributions gives some measure of the gluon uncertainty. The uncertainty in the NLO prediction for direct photon production at $530 \mathrm{GeV} / \mathrm{c}$ due to the PDF uncertainty is shown at the bottom of Figure 1.5. ${ }^{3}$ As expected, the uncertainty in the prediction, which is dominated by the uncertainty in the gluon distribution, is large.

\subsection{Experimental Challenges and Techniques}

Due to the weakness of the electromagnetic coupling constant relative to the strong coupling constant, the direct photon cross section is $\approx 10^{-3}$ times smaller than the jet cross section. Thus the electromagnetic decay of hadrons in jets can become significant sources of background to the direct photon signal. The biggest contributor to this background comes from the decay of $\pi^{0}$ mesons. $\pi^{0}$ s are produced copiously in hadronic reactions and decay to two photons nearly $100 \%$ of the time. Because of the small $\pi^{0}$ mass, the opening angle in the laboratory between the photons from the $\pi^{0}$ decay tends to be small. This is shown in Figure 1.6, where the opening angle is plotted as a function of the energy asymmetry of the photons from the $\pi^{0}$ decay;

$$
A \equiv \frac{\left|E_{1}-E_{2}\right|}{E_{1}+E_{2}},
$$

where $E_{1}$ and $E_{2}$ are the energies of the photons. Unless the photon detector has sufficient granularity, the two photons often appear as a single shower in the detector, mimicking the direct photon signal. Also, even for detectors with enough transverse segmentation to efficiently resolve the photons from $\pi^{0}$ decays, substantial background may result from highly asymmetric decays in which the detector failed to detect the low energy photon.

3 The uncertainty is calculated using Eq. 2.5 of [18]. 

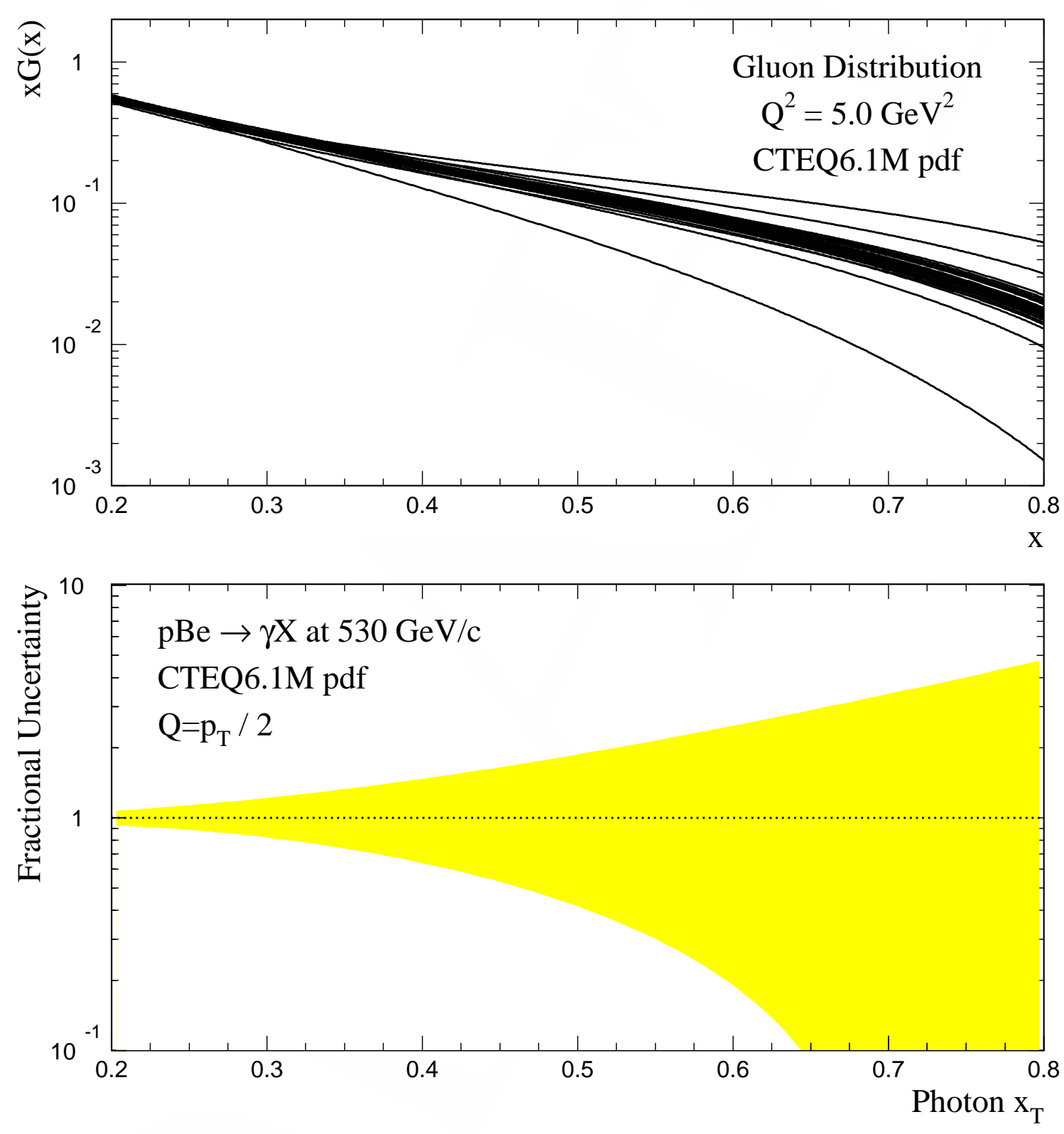

Figure 1.5 Top: Gluon distribution uncertainty as characterized by the CTEQ6.1M eigenvector basis sets. Bottom: Fractional uncertainty in the NLO prediction for direct photon production in pBe interactions at $530 \mathrm{GeV} / \mathrm{c}$ due to the uncertainty in the PDF. 


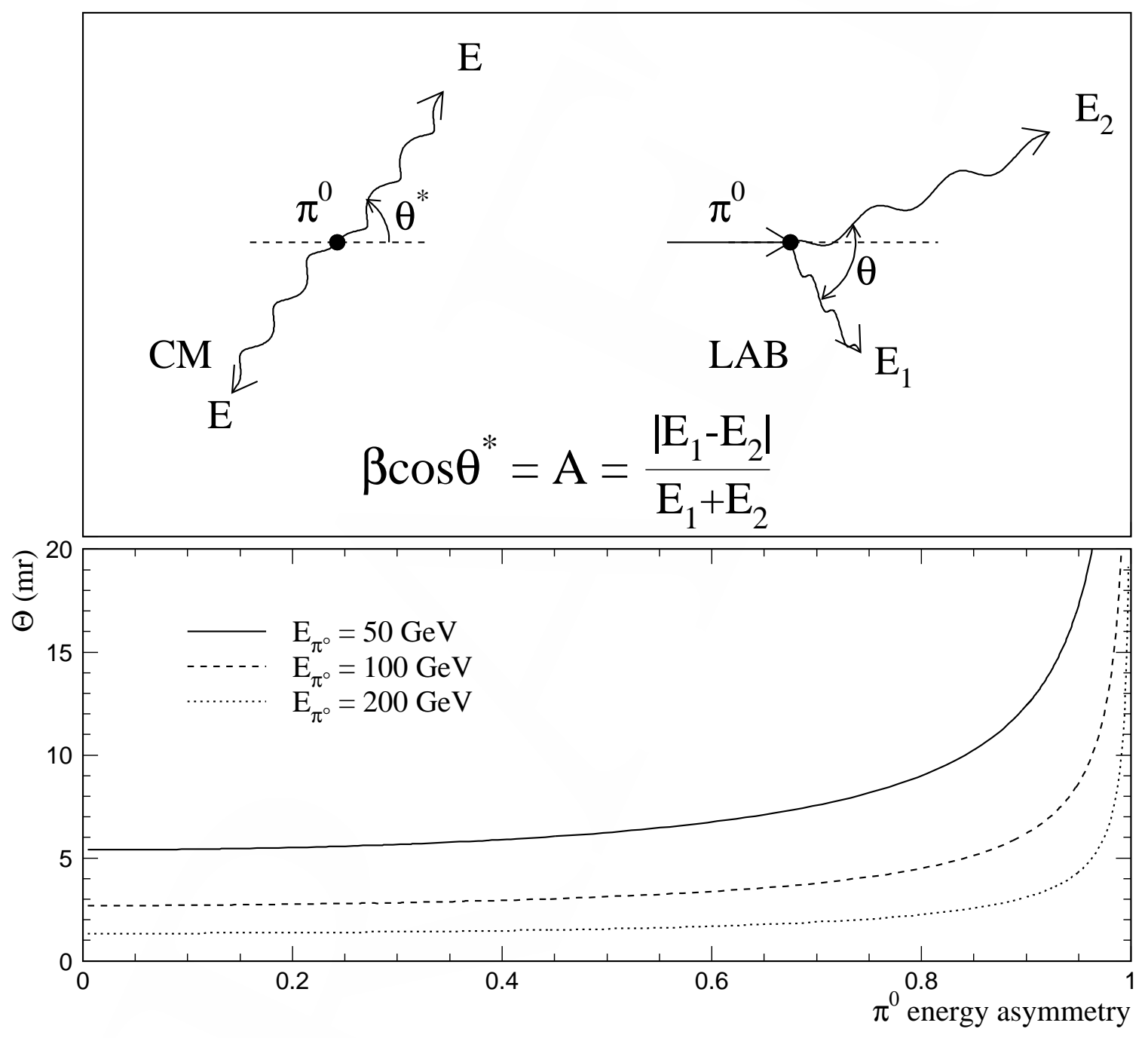

Figure 1.6 Top: Definition of $\pi^{0}$ energy asymmetry, $A$. Bottom: $\pi^{0}$ opening angle in the lab frame versus energy asymmetry. 
After the $\pi^{0}$, the next leading contributor to the direct photon background comes from $\eta$ meson decays. The $\eta$ 's contribution is roughly $20 \%$ that of the $\pi^{0}$ 's, since the production rate of the $\eta$ is approximately half that of the $\pi^{0}$ and the only decay mode that significantly contributes is $\eta \rightarrow \gamma \gamma$ which has a $39 \%$ branching ratio. ${ }^{4}$ As will be shown later, the $\pi^{0}$ and $\eta$ decays contribute nearly $100 \%$ of the direct photon background.

Although the overall signal-to-background ratio may be small, it is expected to increase with $p_{T}$. The chief reason for this increase is attributed to the fact that at leading order, direct photons emerge from the hard scatter carrying off the full momentum of the collision, while $\pi^{0}$ 's and $\eta$ 's are fragments of jets. At medium to large values of $x$, the fragmentation functions get softer as the $Q^{2}$ of the interaction increases ${ }^{5}[24]$, which leads to a steepening of the $\pi^{0}$ and $\eta p_{T}$ spectra relative to the parent jet $p_{T}$ spectra. Therefore, there are fractionally fewer $\pi^{0}$ 's and $\eta$ 's contributing to the background at high $p_{T}$ than at low $p_{T}$.

There are several experimental techniques used to statistically separate the direct photon signal from the background. The most common of these techniques are described below.

\section{$\underline{\text { Reconstruction }}$}

In this method, photons from $\pi^{0}$ 's and $\eta$ 's are identified and eliminated from the direct photon sample through the measurement of the two photon invariant mass. The remaining background, resulting from inefficiencies in identifying photons from these decays, as well as background from other sources, is usually evaluated using Monte Carlo simulations and then statistically subtracted from

\footnotetext{
4 Contributions from decay modes such as $\eta \rightarrow 3 \pi^{0}$ are automatically included in the $\pi^{0}$ contribution.

5 In other words, the momentum fractions carried off by the jet fragments shift to lower values as the $p_{T}$ of the hard scatter increases.
} 
the direct photon sample. To minimize the background contributions, the photon detector should have good spatial resolution in order to separate the two photons from the $\pi^{0}$ decay and should be able to efficiently reconstruct low energy photons. This is the method used by E706.

$\underline{\text { Isolation }}$

From the first order diagrams, it is expected that direct photons should emerge from the hard scatter unaccompanied by other particles. $\pi^{0}$ 's and $\eta$ 's come from jets, and are usually accompanied by other particles. Therefore, a significant reduction of the background can be obtained by imposing some isolation requirement on the direct photon candidates.

One drawback to this method is that in higher order production diagrams, such as the quark bremsstrahlung diagram, direct photons are no longer expected to appear as isolated particles, and therefore a portion of the cross section is excluded from this isolated cross section measurement. The theoretical expectations must be adjusted accordingly to make meaningful comparisons with the data.

$\underline{\text { Shower }} \underline{\text { Profile }}$

For detectors whose granularity is too coarse to efficiently resolve the individual photons from $\pi^{0}$ decays, some background discrimination is still possible through a comparison of transverse and/or longitudinal electromagnetic shower profiles. Showers resulting from $\pi^{0}$ s are expected to be broader in the transverse view and to peak sooner in the longitudinal view than showers from direct photons. Typically, shower profiles for $\pi^{0}$ 's and direct photons are determined using Monte Carlo simulations. Then, by comparing shower profiles in the data with Monte Carlo profiles containing both $\pi^{0}$ 's and direct photons, the fraction of direct photons in the data can be evaluated. 
$\underline{\text { Conversion }}$

The conversion method relies on the fact that it is more probable to observe a photon conversion into an $e^{+} e^{-}$pair from a $\pi^{0}$ or an $\eta$ than it is for a direct photon (simply because there are two photons in the final state rather than one). Typically, a thin piece of material, called a converter, is positioned upstream of an electromagnetic calorimeter and scintillators are placed immediately upstream and downstream of the converter. The scintillators are used to count the number of photon conversions in the converter. By comparing the conversion fraction in the data to the fraction expected from a pure $\pi^{0}$ and $\eta$ sample, the fraction of direct photons in the data can be extracted.

\subsection{Nuclear Effects}

E706 is one of relatively few high energy experiments to use multiple targets. Data were taken on beryllium, copper, and liquid hydrogen targets. Thus the nuclear dependence of the direct photon and $\pi^{0}$ production cross sections may be studied. In a simple view of high $p_{T}$ particle production using nuclear targets, the observed high $p_{T}$ particle is believed to result from a single hard-scatter between the beam and target nucleon constituents. Consequently, the cross-section per nucleus is expected to be proportional to the number of nucleons in the target. This can be expressed mathematically as

$$
\sigma_{A}=\sigma_{o} A^{\alpha}
$$

where $\sigma_{o}$ is the cross section for a free nucleon, $A$ is the atomic mass of the nuclear target, and $\alpha$ is a parameter which is equal to one in this naive view. However, as early as 1975, it was discovered that this simple view of hard scattering within nuclei did not explain the experimental data, and that the parameter $\alpha$ is a function of $p_{T}$ and for meson production is somewhat larger than one at high 
$p_{T}[25]$. This nuclear enhancement is presumed to be due to the multiple scattering of partons as they travel through nuclear matter. In high- $p_{T}$ hadron production, multiple scattering may take place in the initial and/or final states. However, in direct photon production, multiple scattering is expected to occur only in the initial state since direct photons do not interact strongly.

The nuclear targets employed by E706 were copper and beryllium. Since the $Z / A$ ratio ( $Z$ is the atomic number) is similar for these materials, the parameter $\alpha$ can be extracted for direct photon production as well as for meson production. As E706 is the only direct photon experiment that used more than one nuclear target, its data provides a unique measurement of $\alpha$ for direct photon production. In addition, a theoretical calculation for the nuclear enhancement of direct photon production is available [26] and can be compared to the E706 measurement.

\subsection{Initial State Parton Transverse Momentum Effects}

In the theoretical description of high $p_{T}$ particle production presented thus far, the effects of transverse motion in the initial state partons were assumed to be negligible. However, measurements of the average $p_{T}\left(\left\langle p_{T}\right\rangle\right)$ in dimuon and diphoton pairs indicate instead the presence of significant initial state parton transverse momentum $\left(k_{T}\right)$. A collection of these measurements spanning a wide range of center of mass energies is presented in Figure 1.7 [27-30]. The amount of $k_{T}$ is significantly larger than that expected from hadron confinement alone, ${ }^{6}$ and is currently attributed to soft-gluon emissions prior to the hard scatter. Also shown in Figure 1.7 is the $\left\langle p_{T}\right\rangle$ of dijet pairs, $\left\langle p_{T}\right\rangle_{\text {pair }}$. Note that $\left\langle p_{T}\right\rangle_{\text {pair }}$ in these measurements is somewhat higher than corresponding $\left\langle p_{T}\right\rangle_{\text {pair }}$ measurements for

6 Since partons are confined within hadrons, the uncertainty principle dictates that they have an intrinsic transverse momentum spread of order $0.4 \mathrm{GeV} / c$ (assuming a hadron size of order $0.5 \mathrm{fm}$ ). This is commonly referred to as Fermi motion. 
dimuon pairs. This is expected because in dijet production soft-gluon emissions can occur in the final state as well.

Theoretically, soft-gluon emissions have been treated formally for certain processes using Sudakov resummation calculations. ${ }^{7}$ However, for inclusive direct photon production no such calculation currently exists, although progress in this area is being made [31-34]. In lieu of a rigorous treatment of soft-gluon radiation, the effects of $k_{T}$ can be approximated by assuming a Gaussian $k_{T}$ distribution and convoluting it with the cross section either analytically [35], or through Monte Carlo methods [15]. The width of the Gaussian is usually obtained through the measurement of kinematic distributions sensitive to initial state $k_{T}$.

It is expected that the inclusion of parton $k_{T}$ will enhance the predicted single inclusive differential cross section. This can be understood qualitatively through the following argument. On an event by event basis, $k_{T}$ can either add to or subtract from the $p_{T}$ from the underlying partonic interaction. If the cross section for particle production was a flat function of $p_{T}$, then for any given value of observed photon $p_{T}$, the number of instances in which there was a net $p_{T}$ gain will cancel with the number of instances with a net $p_{T}$ loss. However, the cross section for particle production is a steeply falling function of $p_{T}$, falling roughly an order of magnitude per $1 \mathrm{GeV}$ of $p_{T}$ at fixed target beam energies. Therefore, there will be many more cases of interactions with lower partonic $p_{T}$ receiving a $k_{T}$ boost than vice versa, leading to a net increase in the differential cross section at high $p_{T}$.

7 For example, in Drell-Yan [36, 37] and diphoton production [38, 39]. 


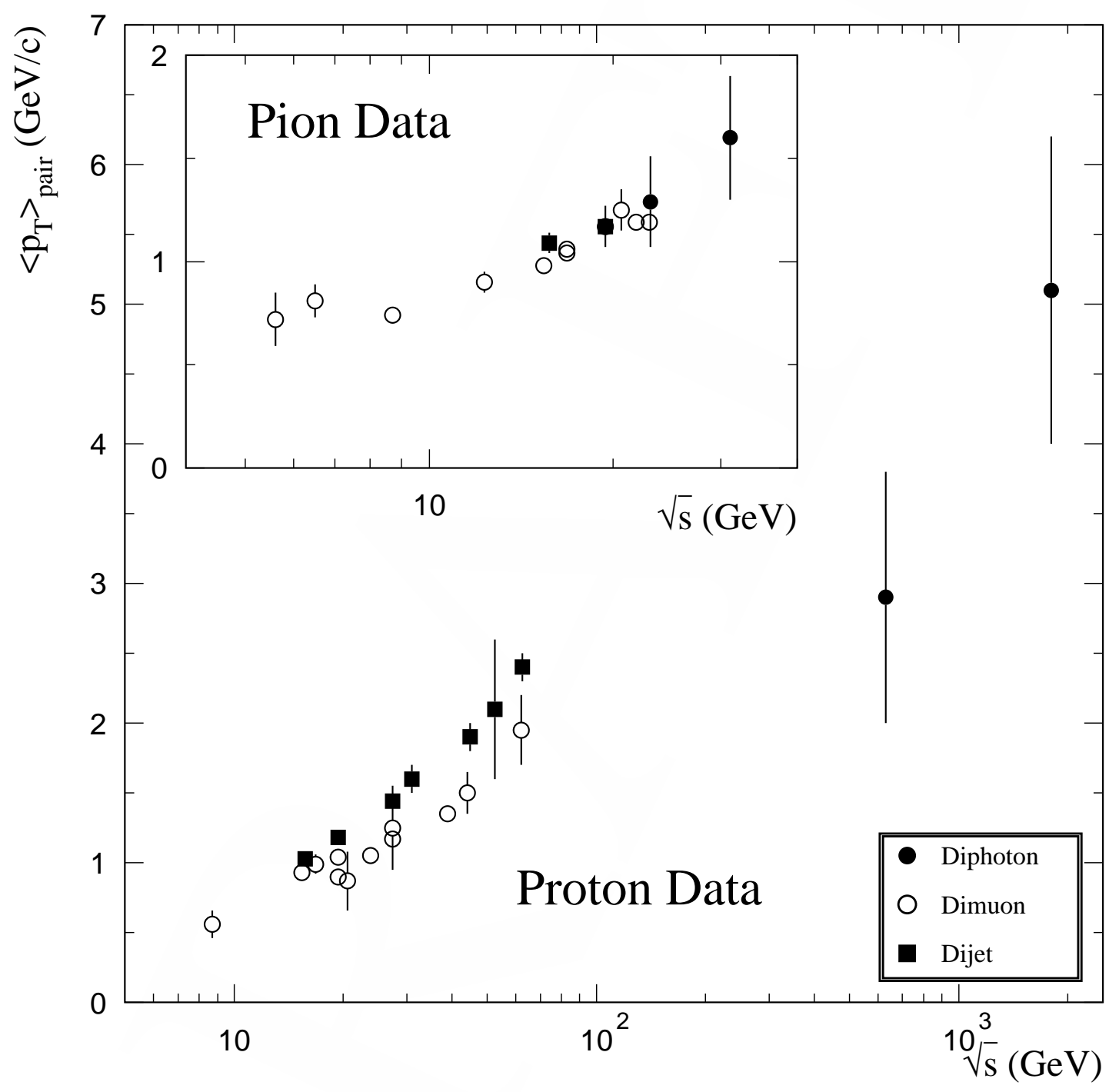

Figure 1.7 Average $p_{T}$ of muon, photon, and jet pairs produced in hadronic collisions versus $\sqrt{s}$. 


\subsection{Recent Experiments}

Direct photon production in hadronic collisions has been studied extensively over the past 20 years. The earliest results came from the CERN ISR machine [40-44]. Though the results were subject to large statistical and systematic uncertainties, they provided clear evidence for the existence of direct photons. A review of the early experimental results can be found in [45].

Table 1.3 shows a summary of recent experiments that have published results on direct photon production. Note that the E706 data, in addition to spanning a wide range in $x_{T}$, explores the largest $x_{T}$ values of any direct photon experiment. 
Table 1.3 Recent direct photon experiments.

\begin{tabular}{|c|c|c|c|c|c|c|}
\hline Experiment & Beam & Target & Machine & $\begin{array}{c}\sqrt{s} \\
(\mathrm{GeV})\end{array}$ & Method & $\begin{array}{c}x_{T} \\
\left(=2 p_{T} / \sqrt{s}\right)\end{array}$ \\
\hline \multirow{2}{*}{ UA1 [46] } & \multirow{2}{*}{$\bar{p}$} & \multirow{2}{*}{$p$} & \multirow{2}{*}{$\mathrm{S} p \bar{p} \mathrm{~S}$} & 546 & Isolation, & $.006 \rightarrow .17$ \\
\hline & & & & 630 & Shower Profile & $.005 \rightarrow .29$ \\
\hline \multirow{2}{*}{ UA2 [47] } & \multirow{2}{*}{$\bar{p}$} & \multirow{2}{*}{$p$} & \multirow{2}{*}{$\mathrm{S} p \bar{p} \mathrm{~S}$} & 546 & Isolation, & NA \\
\hline & & & & 630 & Conversion & $.04 \rightarrow .26$ \\
\hline CDF [48] & $\bar{p}$ & $p$ & Tevatron & 1800 & $\begin{array}{c}\text { Isolation, } \\
\text { Conversion/Profile }\end{array}$ & $.01 \rightarrow .13$ \\
\hline $\mathrm{D} \varnothing[49]$ & $\bar{p}$ & $p$ & Tevatron & 1800 & $\begin{array}{c}\text { Isolation, } \\
\text { Conversion,Profile }\end{array}$ & $.01 \rightarrow .11$ \\
\hline R108 [43] & $p$ & $p$ & ISR & 63 & Conversion & $.16 \rightarrow .38$ \\
\hline R110 [44] & $p$ & $p$ & ISR & 63 & Conversion & $.14 \rightarrow .32$ \\
\hline \multirow{3}{*}{ R806 [50] } & \multirow{3}{*}{$p$} & \multirow{3}{*}{$p$} & \multirow{3}{*}{ ISR } & 31 & \multirow{3}{*}{ Reconstruction } & $.05 \rightarrow .32$ \\
\hline & & & & 45 & & $.05 \rightarrow .32$ \\
\hline & & & & 63 & & $.12 \rightarrow .37$ \\
\hline R807 [51] & $p$ & $p$ & ISR & 63 & Reconstruction & $.15 \rightarrow .33$ \\
\hline $\mathrm{R} 808$ [52] & $p, \bar{p}$ & $p$ & ISR & 53 & Reconstruction & $.11 \rightarrow .23$ \\
\hline E629 [53] & $p, \pi^{+}$ & $\mathrm{C}$ & Tevatron & 19.4 & Reconstruction & $.25 \rightarrow .5$ \\
\hline E704 [54] & $p$ & $\mathrm{Be}$ & Tevatron & 19.4 & Reconstruction & $.25 \rightarrow .4$ \\
\hline UA6 [55] & $p, \bar{p}$ & $p$ & $\mathrm{~S} p \bar{p} \mathrm{~S}$ & 24.3 & Reconstruction & $.34 \rightarrow .50$ \\
\hline NA3 [56] & $p, \pi^{ \pm}$ & $\mathrm{C}$ & SPS & 19.4 & $\begin{array}{c}\text { Conversion/ } \\
\text { Reconstruction }\end{array}$ & $.30 \rightarrow .52$ \\
\hline NA24 [57] & $p, \pi^{ \pm}$ & $p$ & SPS & 23.7 & Reconstruction & $.27 \rightarrow .50$ \\
\hline WA70 $[58,59]$ & $p, \pi^{ \pm}$ & $p$ & SPS & 22.9 & Reconstruction & $.36 \rightarrow .54$ \\
\hline \multirow{2}{*}{ E706 } & $p$ & $\mathrm{Be}, \mathrm{Cu}, \mathrm{H}_{2}$ & \multirow{2}{*}{ Tevatron } & 38.8 & \multirow{2}{*}{ Reconstruction } & $.18 \rightarrow .53$ \\
\hline & $p, \pi^{ \pm}$ & $\mathrm{Be}, \mathrm{Cu}, \mathrm{H}_{2}$ & & $31.1,31.6$ & & $.22 \rightarrow .65$ \\
\hline
\end{tabular}





\section{Chapter 2 The Meson West Spectrometer}

\section{$2.1 \quad$ Overview}

The Meson West spectrometer was designed and built to perform experiment E706. The spectrometer was located in the Meson West experimental hall at the Fermi National Accelerator Laboratory (Fermilab). This spectrometer was also used for experiment E672, a dimuon experiment which ran concurrently with E706. The physical layout of the Meson West spectrometer is shown in Figure 2.1.

The experiment used a right-handed coordinate system. The $Z$-axis was oriented along the nominal beam direction and pointed away from the source of the beam and the $Y$-axis pointed upward. The $X$-axis therefore pointed to the left when viewed from along the $Z$-axis. The origin of this coordinate system was located approximately $9 \mathrm{~cm}$ downstream ${ }^{1}$ of the spectrometer target and was roughly centered on the beam.

Various elements of the Meson West spectrometer are described in this chapter. A description of the downstream dimuon identifier can be found elsewhere[60].

\subsection{Beamline}

During the 1990 and 1991-92 fixed target runs, the Fermilab Tevatron (Figure 2.2) operated on a 58 second cycle. During the first 35 seconds of the cycle, protons were accelerated to an energy of $800 \mathrm{GeV}$. The remaining 23 seconds constituted the spill, during which time the beam was extracted and directed to the various fixed target experiments. Within each spill, beam particles were bunched within 1 ns buckets separated by 19 ns. The 19 ns period was a consequence of the accelerator's RF frequency. During normal operation, the Tevatron beam intensity was $\approx 10^{13}$ protons per spill.

1 The term downstream means along the direction of increasing $Z$. 


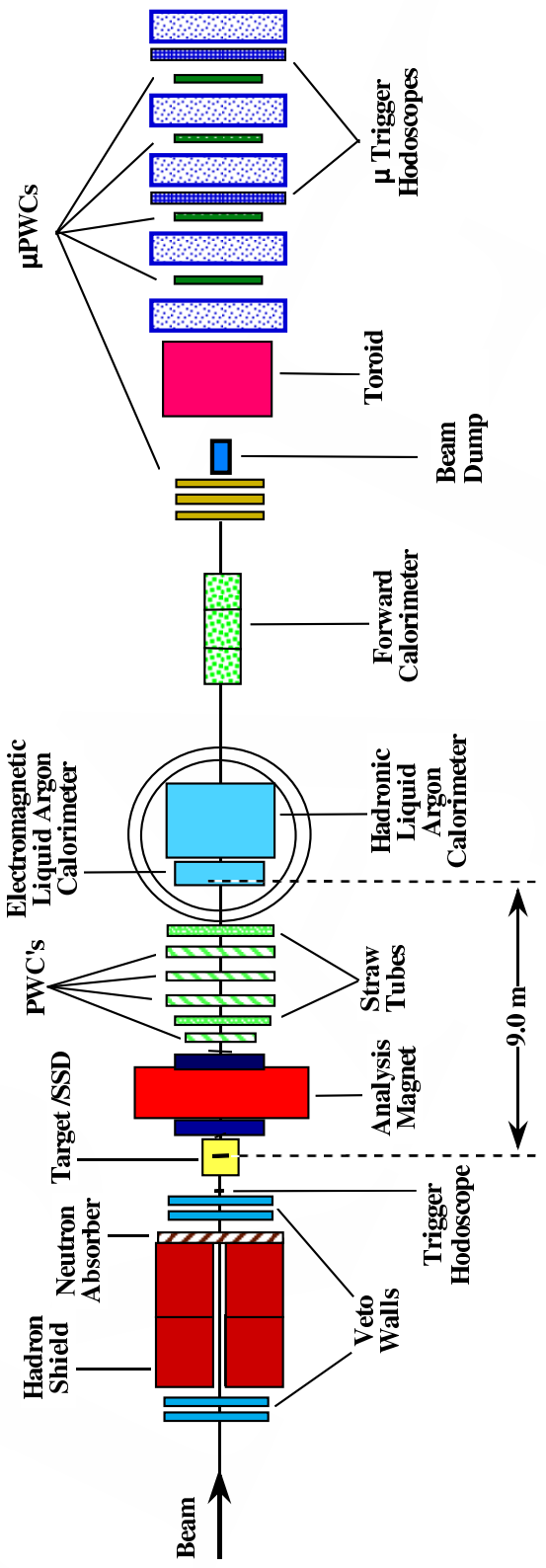

Figure 2.1 Schematic view of the Meson West spectrometer. 


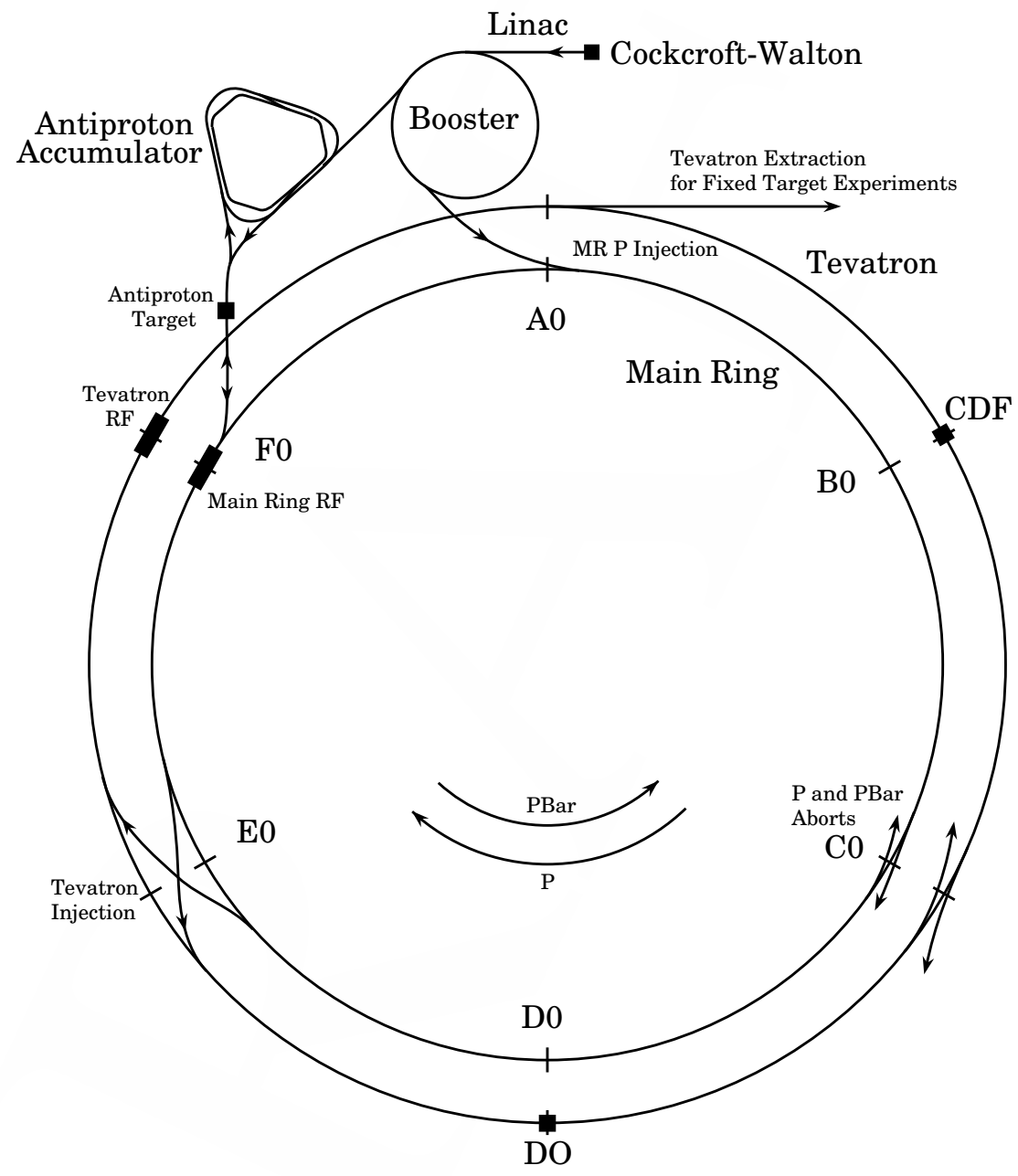

Figure 2.2 Schematic view of the Fermilab Tevatron. 
Beam particles were transported to the experimental hall via the Fermilab Meson West (MWEST) beamline. The beamline was designed to transport negative or positive charged beams, with momenta ranging from 25 to 1000 $\mathrm{GeV} / c$. During the 1990/91 runs, the beamline delivered three types of particle beams to the experiment: an $800 \mathrm{GeV} / c$ primary proton beam, a $515 \mathrm{GeV} / c$ secondary negative beam (primarily $\pi^{-}$'s), and a $530 \mathrm{GeV} / c$ secondary positive beam (primarily protons). For each of these beams, the beam intensity at the spectrometer target was about $2 \times 10^{8}$ particles per spill. The intensity limit was set by radiation safety requirements and by the rate limitations of the data acquisition system of the experiment.

To generate the secondary beams, a beryllium production target was inserted into the beamline $\approx 300 \mathrm{~m}$ upstream of the spectrometer target. The production target was 1.14 interaction lengths in 1990, and 0.75 interaction lengths in 199192. A dipole magnet was located just downstream of the production target. By adjusting the current and polarity of the dipole magnet, a beam of secondary particles with the desired charge and mean momentum was directed down the beamline. To obtain the desired secondary beam intensity, the Tevatron beam intensity at the production target was attenuated to $5 \times 10^{12}$ protons per spill for the negative beam, and to $2 \times 10^{12}$ protons per spill for the positive beam. The beam attenuation was accomplished using two long pinhole collimators located in the beamline, and monitored using two Secondary Emission Monitors (SEMs).

The particle content of the secondary beams was evaluated using a $43 \mathrm{~m}$ long helium filled Čerenkov counter located $\approx 100 \mathrm{~m}$ upstream of the spectrometer target. A spherical mirror at the downstream end of the counter was used to reflect the light emitted by the beam particles back to an array of photomultiplier tubes located at the upstream end of the counter. This array consisted of three concentric rings of photomultiplier tubes, with each ring containing six tubes. A 
schematic diagram of the Čerenkov counter is shown in Figure 2.3. Various logical combinations of signals from the photomultiplier tubes were used to identify, or tag, the incident beam particles. For example, the $\pi 2 \overline{\mathrm{P} 2}$ pion tag required signals from two or more $\pi$-ring phototubes along with NOT two or more signals from the $p$-ring phototubes. In Figure 2.4, the tag probability is shown as a function of pressure for three incident particle tags. The solid vertical line in the figure represents a typical Čerenkov operating pressure. Note that this line passes through the desired peaks for tagging $\pi^{+}, K^{+}$, and protons with the appropriate coincidence logic, thus enabling the counter to tag these three particles simultaneously. From a study of these curves, the secondary beam composition was extracted [61]. The secondary beam composition for the 1990 and 1991-92 runs is shown in Table 2.1.

Table 2.1 Beam composition of the secondary beams.

\begin{tabular}{|c|c|c|c|c|c|c|}
\hline Beam & \multicolumn{2}{|c|}{$-515 \mathrm{GeV} / c$} & \multicolumn{2}{c|}{+530} & $\mathrm{GeV} / c$ \\
\hline Particle Type & $\pi^{-}$ & $K^{-}$ & $\bar{p}$ & $\pi^{+}$ & $K^{+}$ & $\mathrm{p}$ \\
\hline Beam Fraction & $97 \%$ & $2.9 \%$ & $0.1 \%$ & $2.8 \%$ & $0.5 \%$ & $96.7 \%$ \\
\hline
\end{tabular}

The beamline was equipped with a series of dipole and quadrupole magnets to focus and direct the beam onto the spectrometer target. The beam position and $X$ and $Y$ profile was monitored using a series of Segmented Wire Ion Chambers (SWICs) positioned at various locations along the beamline.

The beamline also contained a series of spoiler magnets which were designed to sweep away beam halo particles. ${ }^{2}$ A $4.3 \times 4.7 \times 3.7 \mathrm{~m}^{3}$ hadron shield composed

2 Beam halo particles are particles produced in conjunction with the secondary beams that travel alongside and approximately parallel to the beam. They are mainly hadrons produced at the production target, and muons, which arise from the subsequent in-flight decay of these hadrons. 

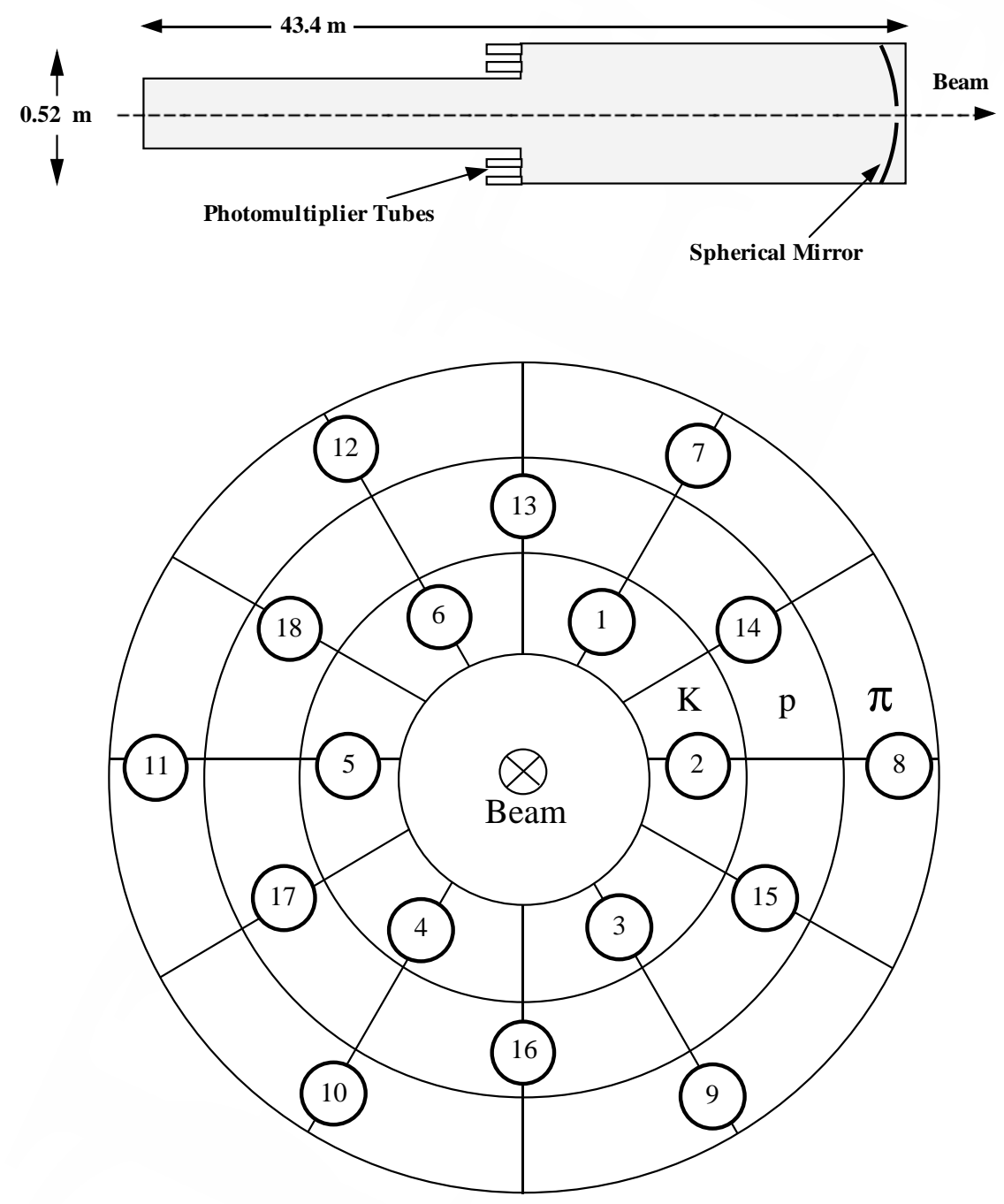

Figure 2.3 A schematic drawing of the beamline Čerenkov counter. Each ring of photomultiplier tubes is labeled according to the particle it was designed to tag. 


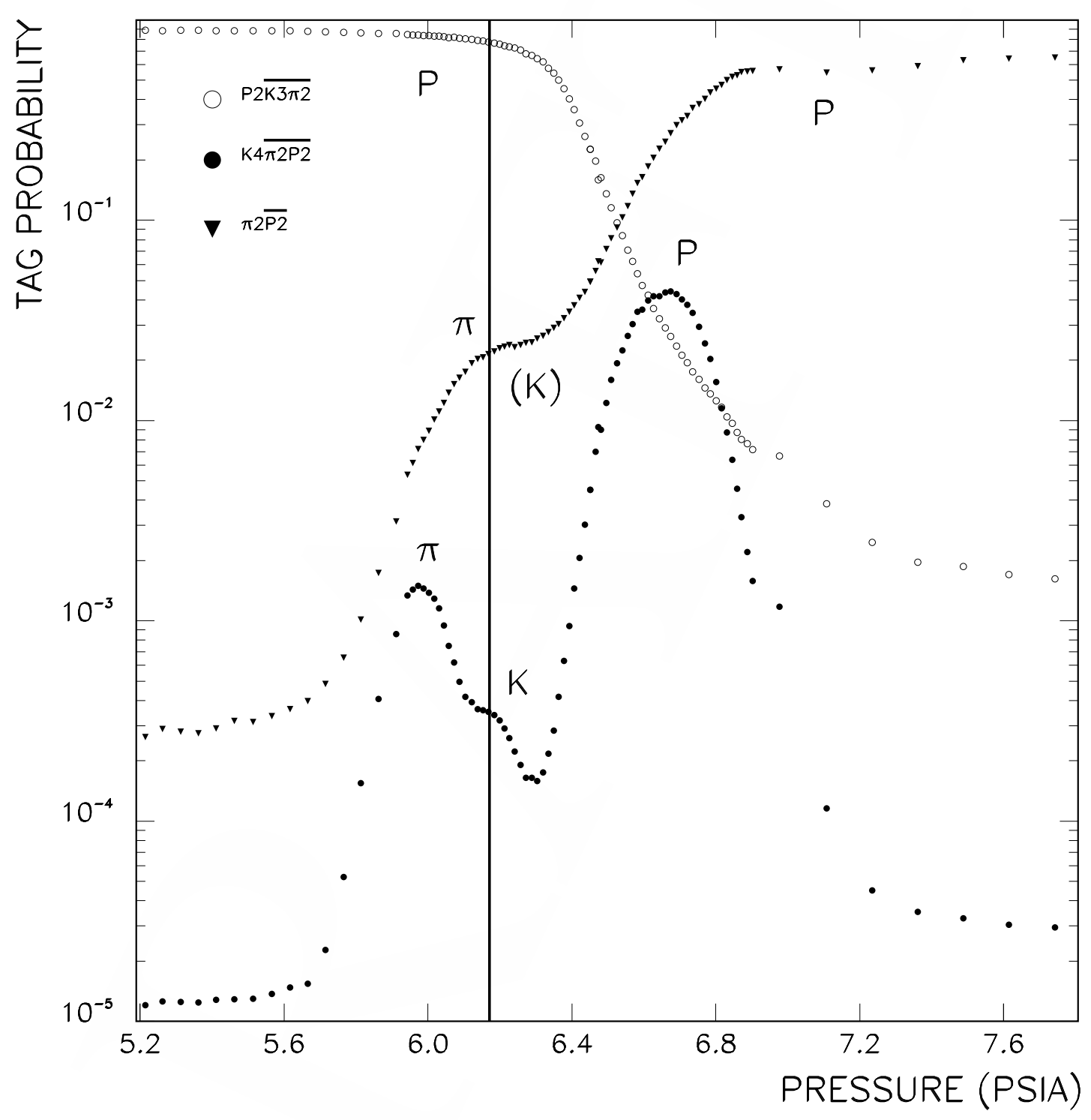

Figure 2.4 Pressure curves for the proton, kaon, and pion tags with incident $+530 \mathrm{GeV} / \mathrm{c}$ beam. Peaks/plateaus are labeled by the associated particle type. The solid vertical line represents a typical Čerenkov operating pressure. 
of steel was installed at the end of the beamline to further reduce the number of halo particles incident on the spectrometer. A tank of distilled water was located at the downstream end of the shield to absorb neutrons produced in interactions in the hadron shield. Finally, charged particles not absorbed by the shield (muons) were detected by arrays of scintillation counters called veto walls. During the 1990 run, there were three veto walls, one located just upstream of the hadron shield, and two located just downstream. For the 1991-92 run, a fourth wall was constructed and placed adjacent to the veto wall upstream of the hadron shield.

\subsection{The Target}

During the 1990 run the experiment used two beryllium and two copper targets. The beryllium targets were cylindrical in shape, with radii of $1 \mathrm{~cm}$. The upstream beryllium target had a length of $3.7 \mathrm{~cm}$, while the downstream target had a length of $1.1 \mathrm{~cm}$. The copper targets were located upstream of the beryllium targets. The copper targets were $0.08 \mathrm{~cm}$ thick. They were formed from $2.54 \mathrm{~cm}$ diameter cylindrical disks that had two diametrically opposite arcs sliced off. This gave the targets a cross-sectional shape that was circular on top and bottom and rectangular in the middle. In the rectangular region, the copper targets were $2 \mathrm{~cm}$ wide in the $X$ view.

In 1991, the target configuration was changed to include a liquid hydrogen target. The liquid hydrogen was contained in a $15.3 \mathrm{~cm}$ long mylar flask. The flask was cylindrical with tori-spherical endcaps. It had a diameter of $6.4 \mathrm{~cm}$, and each endcap had a crown radius of $6.4 \mathrm{~cm}$ and a knuckle radius of $1.6 \mathrm{~cm}$. The flask was housed within a stainless steel vacuum shell. The vacuum shell was cylindrical in shape and was oriented with the cylindrical axis along the $Z$ axis. At the upstream and downstream ends of the vacuum shell were beryllium 
windows. The upstream window was $8.25 \mathrm{~cm}$ in diameter and $0.25 \mathrm{~cm}$ thick while the downstream window was $9.52 \mathrm{~cm}$ in diameter and $0.28 \mathrm{~cm}$ thick.

The 1991-92 target configuration also included two $0.08 \mathrm{~cm}$ thick copper targets and a $2.54 \mathrm{~cm}$ thick beryllium target. The copper targets were located approximately two centimeters upstream of the vacuum shell. The copper targets were cylindrical in shape with diameters of $2.5 \mathrm{~cm}$. The beryllium target was placed adjacent to the downstream end of the vacuum shell. This target was also cylindrical in shape and had a diameter of $2.54 \mathrm{~cm}$.

The physical configurations of the target during the 1990 and 1991-92 runs are illustrated in Figure 2.5. Also shown in the figure are the positions of the silicon strip detectors, described in Section 2.5.1.

\subsection{The Beam and Interaction Detectors}

Beam particles were detected using the beam hodoscope. The beam hodoscope consisted of three planes of scintillators and was located $\approx 1.5 \mathrm{~m}$ upstream of the target region. Each plane contained 12 scintillator elements. Each element was $2 \mathrm{~mm}$ thick and $35 \mathrm{~mm}$ long. The width of the elements varied depending upon their location relative to the center of the hodoscope plane. The central eight scintillator elements were $1 \mathrm{~mm}$ wide, the elements adjacent to the central eight were $2 \mathrm{~mm}$ wide, and the outermost elements were $5 \mathrm{~mm}$ wide. The hodoscope planes were arranged in $X, Y$ and $U$ views, with the $U$ view making a 45 degree angle with the horizontal.

A second scintillating plastic device, called the beam hole counter, was used to help ensure that the beam was incident on the target, and to help eliminate interactions from particles belonging to the beam halo. In the 1990 run, the beam

hole counter was simply a $4 \times 4 \times \frac{1}{8}$ inch $^{3}$ piece of scintillator with a $3 / 8$ inch 

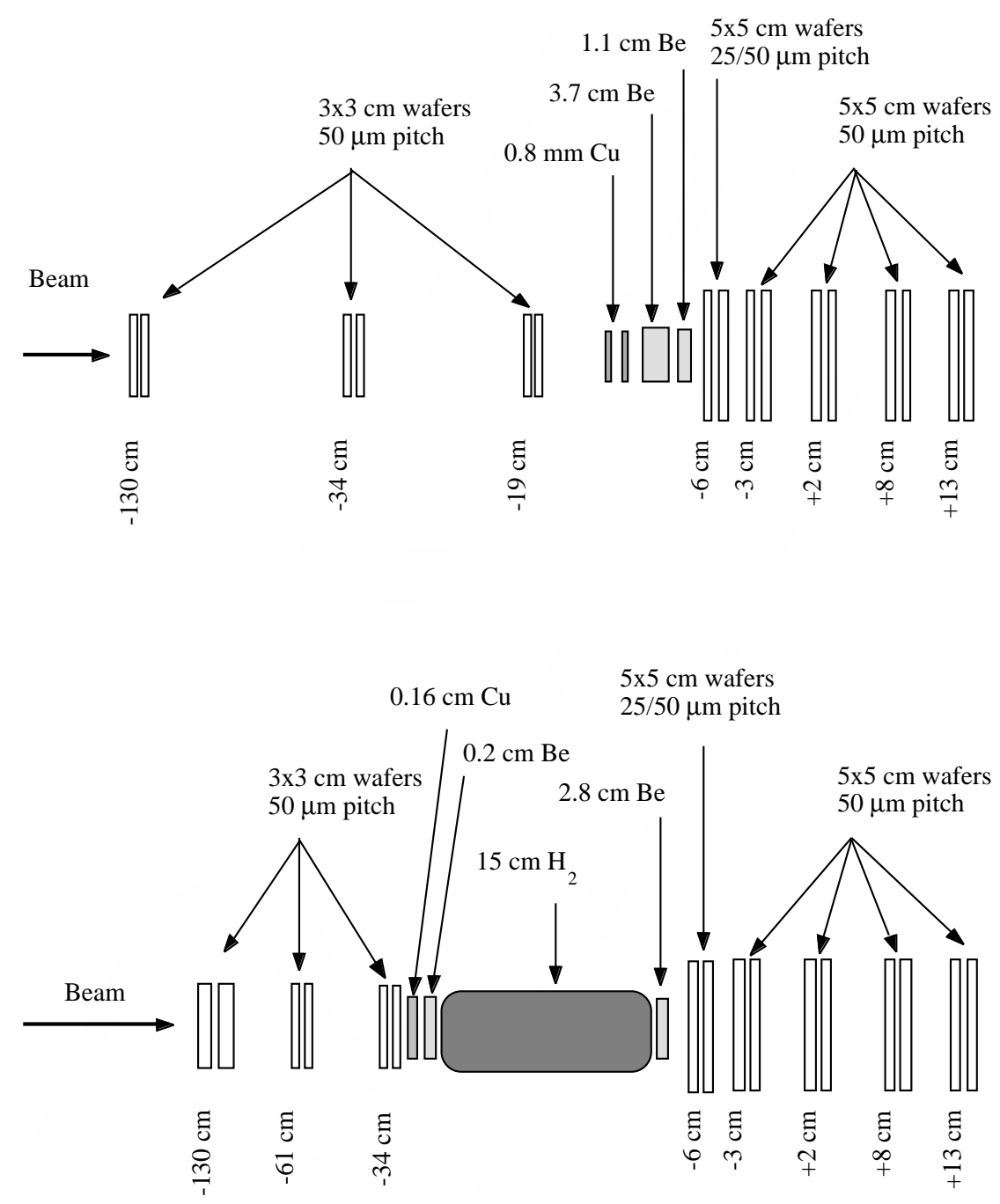

Figure 2.5 Layout of the target region during the 1990 and 1991-92 runs. 
diameter hole in the center. In 1991, the single scintillator was replaced by a set of four $2 \frac{5}{8} \times 2 \frac{5}{8} \times \frac{1}{4}$ inch $^{3}$ scintillators. Each scintillator had a circular piece removed from of one its corners. The piece was $3 / 8$ inch in diameter and centered on the corner. When installed, the four counters formed a square with a $3 / 8$ inch diameter hole in the center, essentially reproducing the geometry of the single counter used in the 1990 run.

An interaction was detected through the use of two sets of two scintillation counters, one set located a few centimeters upstream of the magnet, and the other set located a few centimeters downstream of the magnet. The upstream counters measured $3 \times 6 \times \frac{1}{16}$ inch $^{3}$ while the downstream counters measured $4 \times 8 \times \frac{1}{16}$ inch $^{3}$. Each counter had a circular piece removed from it whose center was located at the middle of the edge of one of the longer sides. The diameter of the hole was $3 / 4$ inch in the upstream counters, and $1 \frac{3}{4}$ inch in the downstream counters. When installed, the upstream counters formed a $6 \times 6$ inch $^{2}$ square with a $3 / 4$ inch hole in the center. The downstream counters formed a $8 \times 8$ inch $^{2}$ square with a $1 \frac{3}{4}$ inch diameter hole in the center.

\subsection{The Tracking System}

Charged particles were detected using sixteen Silicon Strip Detectors (SSDs), four sets of Proportional Wire Chambers (PWCs) and two sets of Straw Drift Tubes (STRAWs). A large aperture analyzing dipole magnet was used to evaluate the momenta of the charged particles. The following sections briefly describe each of these elements.

\subsubsection{Silicon Strip Detectors}

The SSD system consisted of 16 planes of silicon wafers. The planes were arranged in 8 modules. Each module contained two planes, with the strips on the 
upstream plane oriented along the $Y$ direction ( $X$-plane) and the strips on the downstream plane oriented along the $X$ direction ( $Y$-plane). Three of the eight modules were located upstream of the target and were used to measure the position and trajectory of the beam particles. These were called the beam chambers and they contained wafers measuring $3 \times 3 \mathrm{~cm}^{2}$. The five modules located downstream of the target were called the vertex chambers and they contained wafers measuring $5 \times 5 \mathrm{~cm}^{2}$. All the silicon wafers were $\approx 300 \mu \mathrm{m}$ thick. On 7 of the 8 modules, the width of the individual strips on the planes was $50 \mu \mathrm{m}$. On the other module, located just downstream of the target, the strips were $25 \mu \mathrm{m}$ wide in the central region, and $50 \mu \mathrm{m}$ wide in the outer region. The geometric parameters of the SSD system are given in Tables 2.2 and 2.3. The SSD system had an angular resolution of $\approx 0.06 \mathrm{mr}$. The angular acceptance of the SSD system was $\approx \pm 125 \mathrm{mr}$.

The signals from each strip were amplified by Rel-Lab IO 323-C charge sensitive pre-amplifiers. The outputs from the pre-amps were then transported via $\approx 20$ feet of twisted pair cable to Nanometeric[62] N-277 amplifier cards for further amplification and discrimination. The outputs from the amplifiers were then transmitted to Nanometeric N-278 latches, which were housed within standard CAMAC[63] crates. These latches stored the hit status of each strip in a buffer while awaiting a trigger decision to be reached ${ }^{3}$. In all, the SSD system included 8192 instrumented channels. Detailed information regarding the SSD electronics can be found in [64].

\subsubsection{Analysis Magnet}

The analysis magnet used in this experiment was a 350 ton iron core dipole electromagnet whose aperture measured 127.0 x 91.4 x $167.64 \mathrm{~cm}^{3}$. At the upstream and downstream ends of the magnet $20 \mathrm{~cm}$ thick iron mirror

\footnotetext{
3 The trigger system is described in Chapter 3.
} 
Table 2.2 SSD beam chamber geometrical parameters.

\begin{tabular}{|c|c|c|c|c|}
\hline Module & $\begin{array}{c}\text { Number of } \\
\text { Instrumented Strips }\end{array}$ & $\begin{array}{c}\text { Active Region } \\
(\mathrm{cm})\end{array}$ & $\begin{array}{c}Z \text { Position (1990) } \\
(\mathrm{cm})\end{array}$ & $\begin{array}{c}Z \text { Position (1991) } \\
(\mathrm{cm})\end{array}$ \\
\hline $1 X$ & 256 & 1.28 & -130.2 & -130.5 \\
\hline $1 Y$ & 256 & 1.28 & -129.3 & -129.6 \\
\hline $2 X$ & 256 & 1.28 & -34.2 & -61.8 \\
\hline $2 Y$ & 256 & 1.28 & -33.3 & -60.9 \\
\hline $3 X$ & 256 & 1.28 & -19.2 & -34.4 \\
\hline $3 Y$ & 256 & 1.28 & -18.3 & -33.5 \\
\hline
\end{tabular}

Table 2.3 SSD vertex chamber geometrical parameters.

\begin{tabular}{|c|c|c|c|}
\hline Module & $\begin{array}{c}\text { Number of } \\
\text { Instrumented Strips }\end{array}$ & $\begin{array}{c}\text { Active Region } \\
(\mathrm{cm})\end{array}$ & $\begin{array}{c}Z \text { Position } \\
(\mathrm{cm})\end{array}$ \\
\hline $1 X$ & 640 & 2.08 & -6.3 \\
\hline $1 Y$ & 640 & 2.08 & -5.3 \\
\hline $2 X$ & 512 & 2.56 & -3.7 \\
\hline $2 Y$ & 512 & 2.56 & -2.8 \\
\hline $3 X$ & 704 & 3.52 & 1.8 \\
\hline $3 Y$ & 704 & 3.52 & 2.7 \\
\hline $4 X$ & 832 & 4.16 & 7.3 \\
\hline $4 Y$ & 832 & 4.16 & 8.2 \\
\hline $5 X$ & 1000 & 5.00 & 12.8 \\
\hline $5 Y$ & 1000 & 5.00 & 13.7 \\
\hline
\end{tabular}


plates were installed to reduce the fringe field. The upstream mirror plate had a 35.6 x $25.4 \mathrm{~cm}^{2}$ hole in its center while the downstream plate had a $127.0 \times 91.4 \mathrm{~cm}^{2}$ hole. A helium filled polyethylene bag was placed inside the magnet aperture to help minimize the effects of multiple scattering.

The magnetic field was mapped using the ZIPTRACK[65] system developed at Fermilab. At an operating current of approximately 1050 amperes, the maximum field strength was 6.2 kilogauss. This field imparted a $450 \mathrm{MeV} / \mathrm{c}$ momentum impulse in the horizontal plane to charged particles.

\subsubsection{Proportional Wire Chambers}

The PWC system consisted of 4 modules, with each module containing 4 planes of anode sense wires. In each module, the anode wires in each successive plane made the following angles with the horizontal: $-90^{\circ}$ ( $X$ plane), $0^{\circ}$ ( $Y$ plane), $36.9^{\circ}(U$ plane $),-53.1^{\circ}(V$ plane $)$. Each anode plane was sandwiched between two sheets of graphite coated mylar, which served as cathode planes. The distance between the anode and cathode planes was $5.74 \mathrm{~mm}$. The cathodes had three electrically independent regions, referred to as the beam, diffractive and main regions. The main and diffractive regions were held at a high negative voltage of $\approx 3000$ volts. In the beam region, current limiting resistors were installed between the cathode and the voltage supply. These resistors reduced the voltage on the cathode by an amount proportional to the beam current in that region. This desensitized the beam region, and thus prevented wires intersecting the beam region from continuously registering hits. An exploded view of a PWC chamber is shown in Figure 2.6.

The PWC modules varied in size in order to maintain an approximate constant solid angle acceptance. The first module measured $1.63 \times 1.22 \mathrm{~m}^{2}$. The second and third modules measured $2.03 \times 2.03 \mathrm{~m}^{2}$. The fourth module measured 


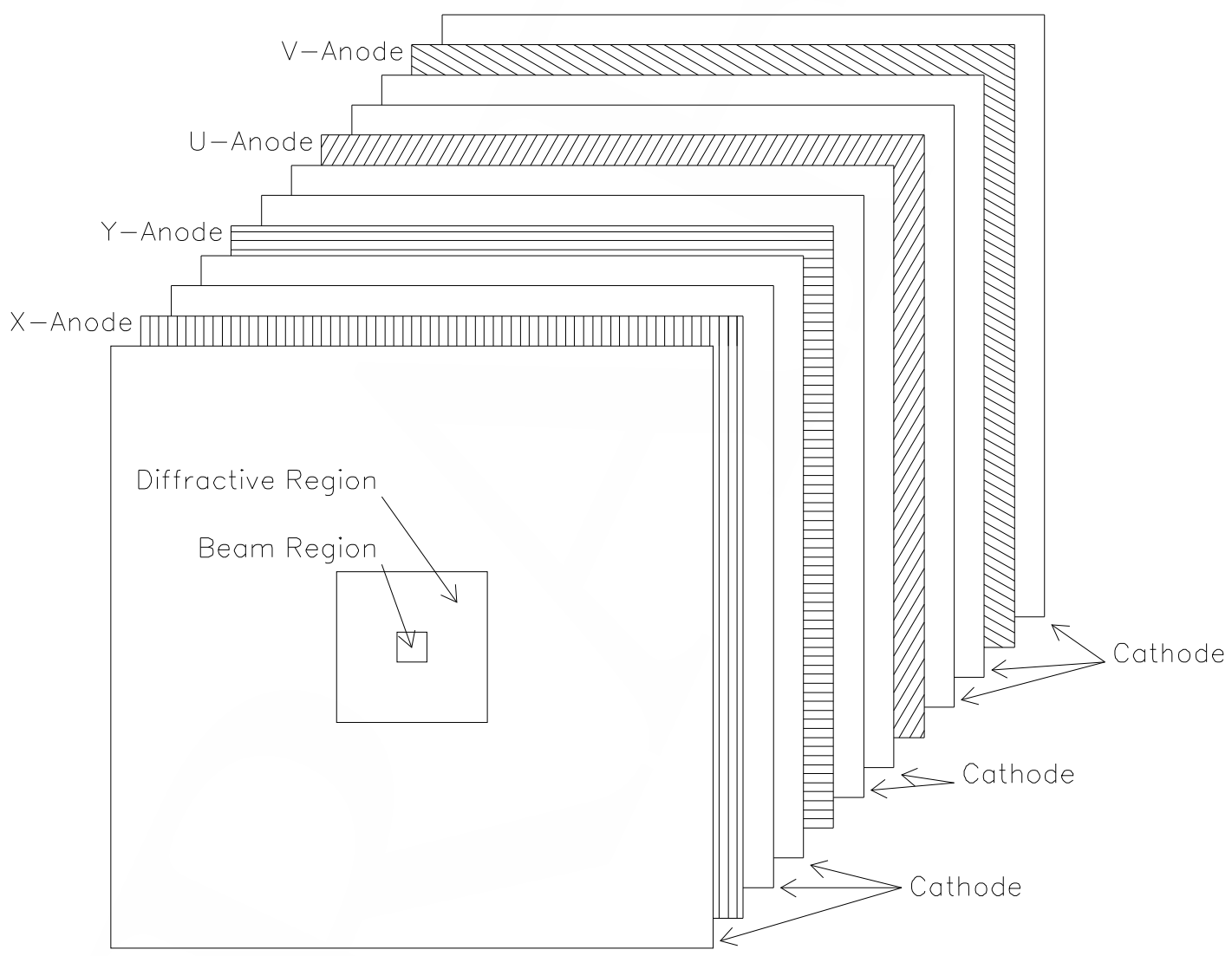

Figure 2.6 Exploded view of a single PWC chamber. 
$2.44 \times 2.44 \mathrm{~m}^{2}$. Table 2.4 gives the number of wires, wire orientation, and nominal $Z$ position for each of the 16 PWC planes.

The sense wires were made of $20 \mu \mathrm{m}$ diameter gold-plated tungsten wires. In each plane, the wires were spaced $2.54 \mathrm{~mm}$ apart and were strung to a tension of 40 grams prior to being fastened into place. To help maintain a constant distance between the anode and cathode planes, zigzagged strips of Kapton, called garlands, were installed in pairs at various positions along the length of the cathodes. Table 2.5 shows the location and orientation of the garland supports. The presence of these garlands disturbed the electric field locally. To compensate for this, an insulated field-restoring wire was strung along each garland on the side adjacent to the anodes. Figure 2.7 shows how the efficiency of the plane varies with respect to distance from the the center of a garland pair for two cases: (a) with no garland voltage applied, and (b) with the operating voltage of $1500 \mathrm{~V}$ applied to the garland wire.

Table 2.5 Orientation and positions of the garlands. The positions are relative to the center of the chamber. Note that within a chamber, the garlands are arranged in pairs, separated by $\approx 5 \mathrm{~cm}$.

\begin{tabular}{|c|c|c|c|}
\hline Module & View & Orientation & Positions $(\mathrm{cm})$ \\
\hline 1 & $X, U$ & Horizontal & $\pm 2.54, \pm 38.1, \pm 43.2$ \\
\hline 1 & $Y, V$ & Vertical & $\pm 17.8, \pm 22.9, \pm 58.4, \pm 63.5$ \\
\hline 2,3 & $X, U$ & Horizontal & $\pm 12.7, \pm 17.8, \pm 43.2, \pm 48.3, \pm 73.7, \pm 78.7$ \\
\hline 2,3 & $Y, V$ & Vertical & $\pm 12.7, \pm 17.8, \pm 43.2, \pm 48.3, \pm 73.7, \pm 78.7$ \\
\hline 4 & $X, U$ & Horizontal & $\pm 15.2, \pm 20.3, \pm 50.8, \pm 55.9, \pm 86.4, \pm 91.4$ \\
\hline 4 & $Y, V$ & Vertical & $\pm 15.2, \pm 20.3, \pm 50.8, \pm 55.9, \pm 86.4, \pm 91.4$ \\
\hline
\end{tabular}


Table 2.4 PWC geometric parameters.

\begin{tabular}{|c|c|c|c|}
\hline Module & Number of Wires & Angle (degrees) & $Z$ Position $(\mathrm{cm})$ \\
\hline $1 X$ & 640 & -90.0 & 379.0 \\
\hline $1 Y$ & 480 & 0.0 & 380.8 \\
\hline $1 U$ & 704 & -53.1 & 382.5 \\
\hline $1 V$ & 672 & -36.9 & 384.2 \\
\hline $2 X$ & 800 & -90.0 & 472.3 \\
\hline $2 Y$ & 800 & 0.0 & 474.0 \\
\hline $2 U$ & 896 & -53.1 & 475.8 \\
\hline $2 V$ & 896 & -36.9 & 477.5 \\
\hline $3 X$ & 800 & -90.0 & 567.4 \\
\hline $3 Y$ & 800 & 0.0 & 569.1 \\
\hline $3 U$ & 896 & -53.1 & 570.9 \\
\hline $3 V$ & 896 & -36.9 & 572.6 \\
\hline $4 X$ & 960 & -90.0 & 660.1 \\
\hline $4 Y$ & 960 & 0.0 & 661.9 \\
\hline $4 U$ & 1120 & -53.1 & 663.7 \\
\hline $4 V$ & 1120 & -36.9 & 665.4 \\
\hline
\end{tabular}


In all, the PWC system contained 13,440 instrumented channels. The readout electronics for the PWC system was the same as for the SSD system with the exception that the preamp boards were unnecessary, and therefore not used. For a detailed description regarding the construction of the PWC chambers, the reader is referred to [66].

\subsubsection{Straw Tube Drift Chambers}

Two straw chambers were installed downstream of the magnet prior to the start of the 1990 fixed target run to improve the resolution of the downstream tracking system. The first chamber was located in between the first and second PWC modules. The second chamber was located just downstream of the last PWC module. Each chamber consisted of eight planes of straw tubes; four in the $X$ view, followed by four in the $Y$ view. The tubes in each view were placed adjacent to one another and glued together to form a bundle. The first two planes in each view were offset by $1 / 2$ of the straw tube diameter and glued together. The last two planes in each view were glued together in the same manner. These two pairs of planes were then glued to opposite sides of a thin sheet of mylar and offset with respect to each other by $1 / 4$ of the straw tube diameter (see Figure 2.8). This offset helped minimize the number of left/right hit ambiguities ${ }^{4}$ in the straw tubes.

The diameter of the tubes in the first chamber was $10.3 \mathrm{~mm}$, while in the second chamber, the diameter of the tubes was $15.9 \mathrm{~mm}$. Each tube was constructed from two spiral wrapped layers of $75 \mu \mathrm{m}$ thick mylar. The inner surface of each tube was coated with a $8 \mu \mathrm{m}$ thick layer of aluminum. In addition, the central four tubes in each plane had $7.5 \mathrm{~cm}$ long mylar inserts glued to their inner surfaces at the midpoints to desensitize the tubes in the beam region. The

\footnotetext{
4 This is discussed in the straw tracking portion of Section 4.2.1.
} 


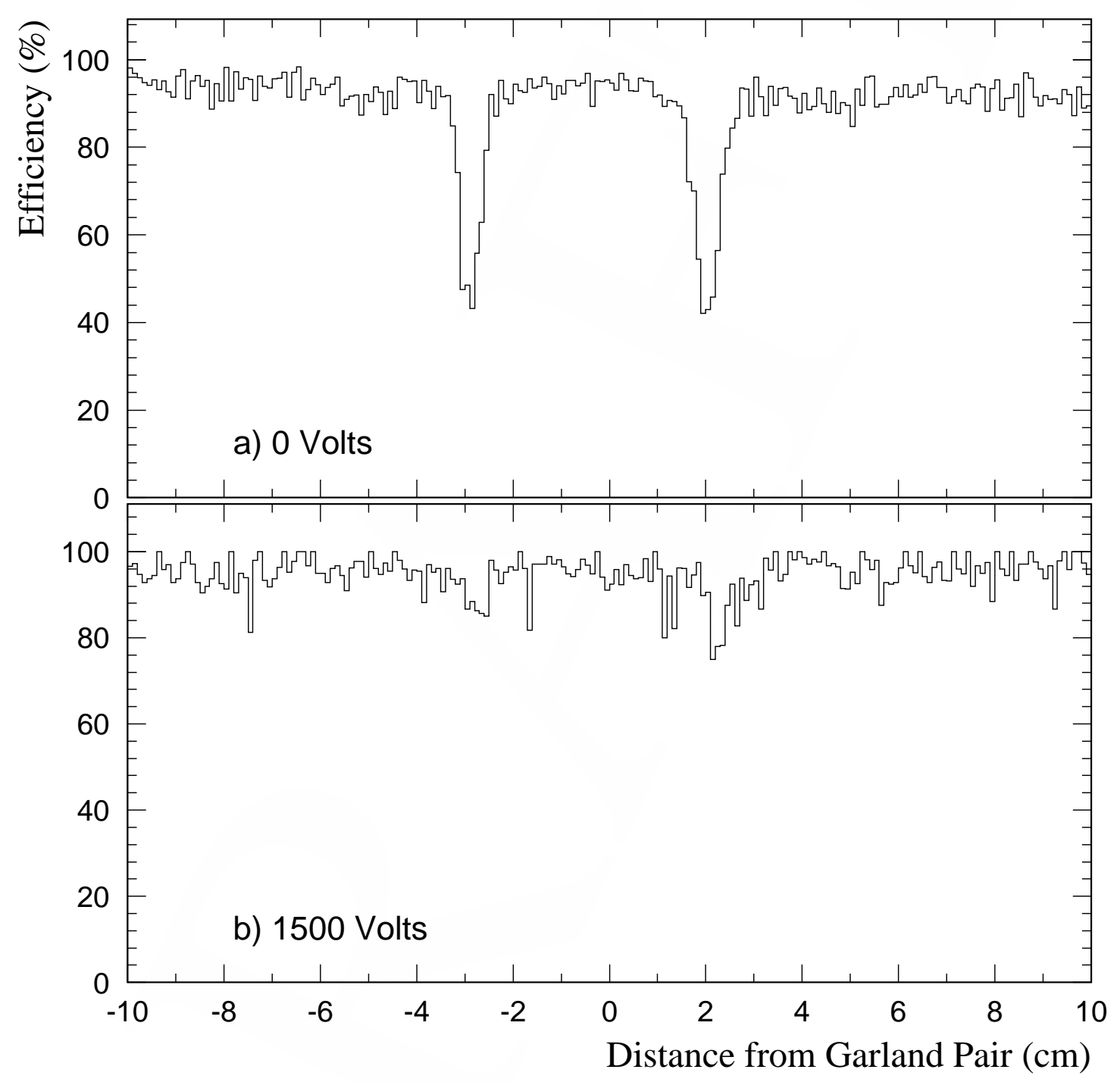

Figure 2.7 Relative chamber efficiency versus distance from the center of a garland pair for (a) zero voltage applied to the garland wire, and (b) 1500 volts applied to the garland wire. 
tubes in the upstream chamber were $1.67 \mathrm{~m}$ long in the $X$ view, and $1.26 \mathrm{~m}$ long in the $Y$ view. The downstream chamber's tubes were $2.80 \mathrm{~m}$ long in both views. The STRAW chambers geometric parameters are given in Table 2.6. The anode wires were made from $20 \mu \mathrm{m}$ diameter gold plated tungsten wire, and were strung at a tension of $50 \mathrm{~g}$. During operation, the anodes were held at a voltage of $\approx 1800 \mathrm{~V}$.

The signals from the straw tubes were amplified and discriminated using N277 amplifier cards. The signals from the cards were then sent to time-todigital converters (TDCs) [67] via $\approx 23 \mathrm{~m}$ of twisted pair cable. From the time measurement, a drift-time to drift-distance conversion was determined. The straw chamber resolution was $\approx 250 \mu \mathrm{m}$ per tube.

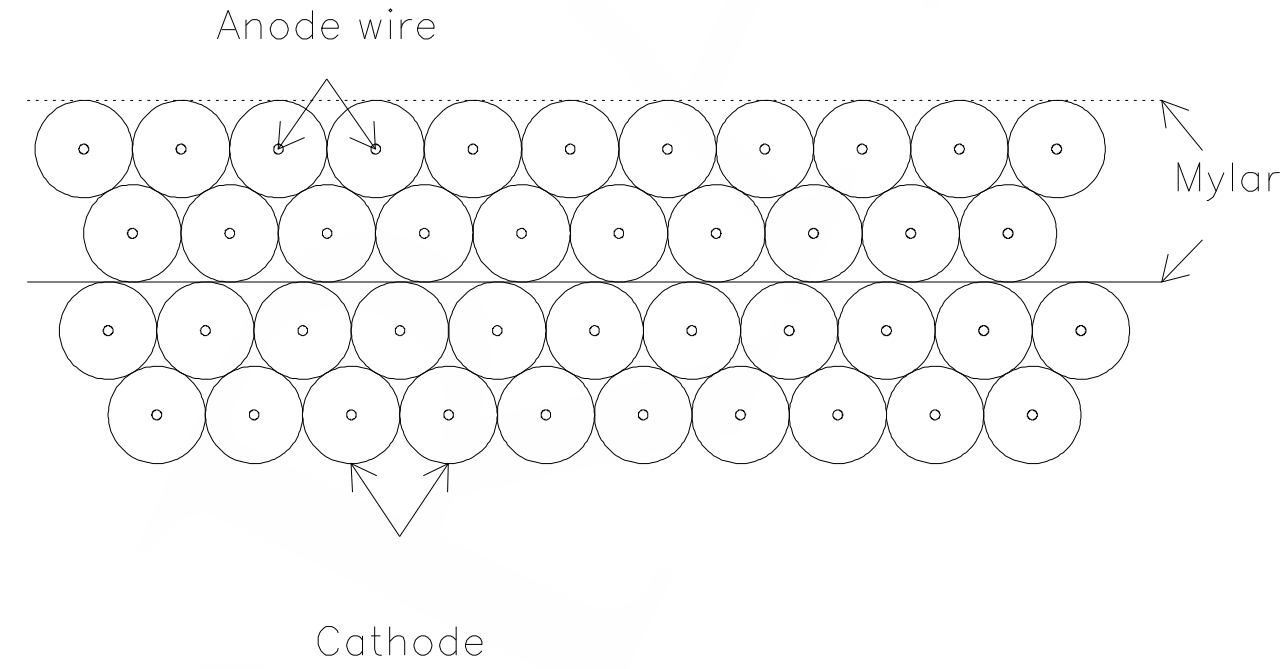

Figure 2.8 A bundle of straw tubes. 
Table 2.6 Straw geometrical parameters.

\begin{tabular}{|c|c|c|c|}
\hline Module & Number of Wires & Tube Diam. $(\mathrm{cm})$ & $Z$ Position $(\mathrm{cm})$ \\
\hline $1 X$ & 160 & 1.04 & 426.2 \\
\hline $1 X$ & 160 & 1.04 & 427.1 \\
\hline $1 X$ & 160 & 1.04 & 428.1 \\
\hline $1 X$ & 160 & 1.04 & 429.0 \\
\hline $1 Y$ & 128 & 1.04 & 434.0 \\
\hline $1 Y$ & 128 & 1.04 & 434.9 \\
\hline $1 Y$ & 128 & 1.04 & 435.9 \\
\hline $1 Y$ & 128 & 1.04 & 436.8 \\
\hline $2 X$ & 160 & 1.59 & 743.9 \\
\hline $2 X$ & 160 & 1.59 & 745.3 \\
\hline $2 X$ & 160 & 1.59 & 747.0 \\
\hline $2 X$ & 160 & 1.59 & 748.4 \\
\hline $2 Y$ & 160 & 1.59 & 750.3 \\
\hline $2 Y$ & 160 & 1.59 & 751.8 \\
\hline $2 Y$ & 160 & 1.59 & 753.4 \\
\hline $2 Y$ & 160 & 1.59 & 754.8 \\
\hline
\end{tabular}




\subsection{Liquid Argon Calorimeter}

The Liquid Argon Calorimeter (LAC), refers collectively to two independent calorimeters, an electromagnetic calorimeter (EMLAC) and a hadronic calorimeter (HALAC). These were sampling calorimeters that used liquid argon as the ionizing medium, and lead (in the EMLAC), and steel (in the HALAC), as the absorber. The EMLAC and HALAC were housed in a common steel cryostat, which was suspended from a movable gantry, as shown in Figure 2.9. The cryostat was made of $1.6 \mathrm{~cm}$ thick stainless steel and held $\approx 17,000$ gallons of liquid argon. The cryostat was encased in $\approx 25 \mathrm{~cm}$ of fiberglass and polyurethane foam for thermal insulation. A low density filler vessel was placed between the cryostat wall and the front face of the EMLAC to help minimize the development of electromagnetic showers before the EMLAC. This vessel had $1.6 \mathrm{~mm}$ thick steel walls and was filled with Rohacell foam.

\subsubsection{Electromagnetic Calorimeter}

Photons were detected by the EMLAC, a large lead and liquid argon sampling calorimeter. A sampling calorimeter was chosen because it offers fine position resolution at an affordable cost. Liquid argon was used as the sampling medium because it can sustain relatively high interaction rates and has good energy resolution. Lead was used as the absorber because it has a relatively small radiation length and a relatively large interaction length. These properties allow electromagnetic showers to develop relatively early in the calorimeter, while minimizing the development of hadronic showers. For a detailed discussion of the design criteria of the EMLAC, the reader is referred to [68].

The front face of the EMLAC was located approximately 9 meters downstream of the target. The EMLAC was cylindrical in shape, with the cylindrical axis 


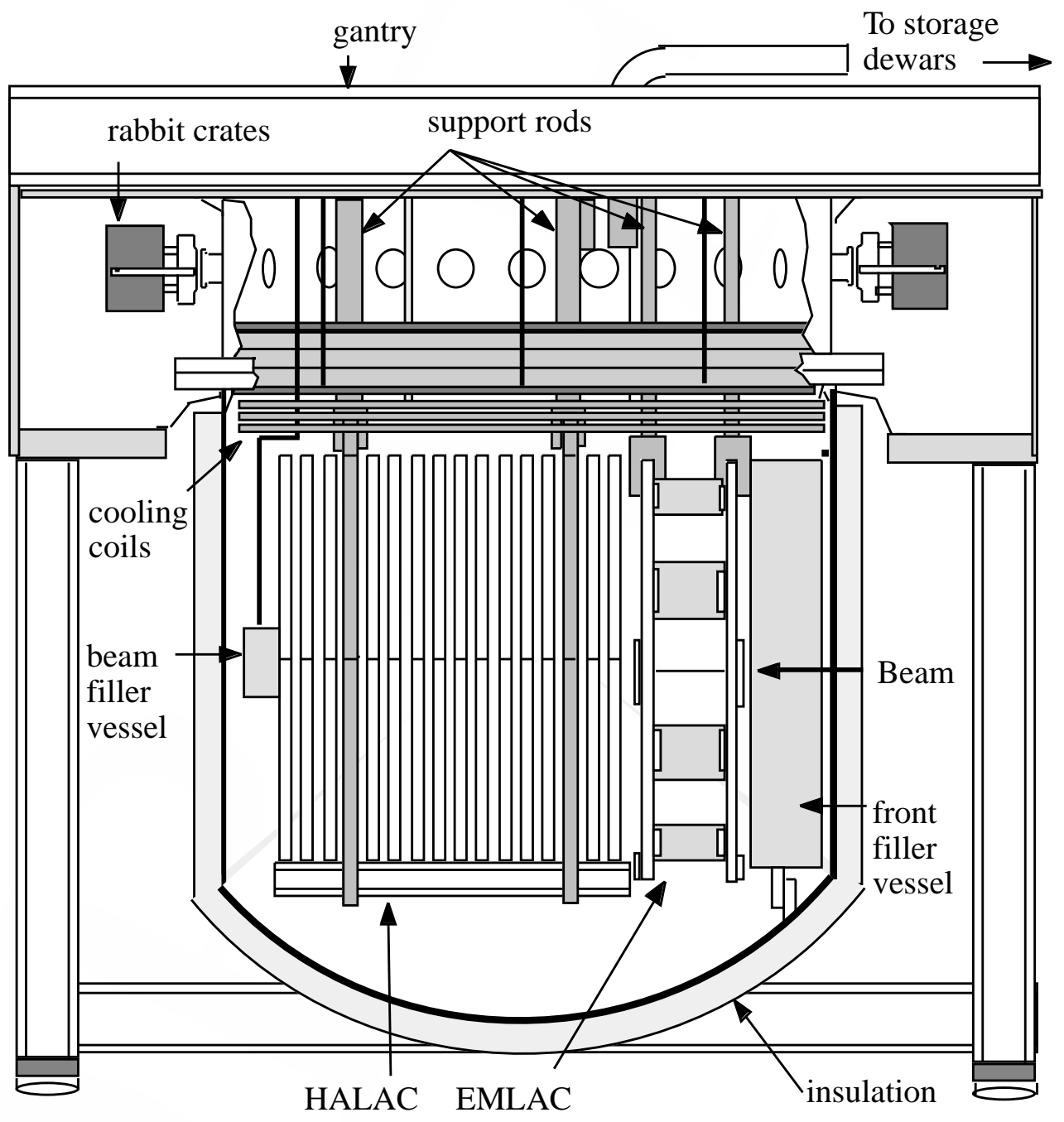

Figure 2.9 Side view of the LAC gantry and cryostat. 
oriented parallel to the beamline. It had an inner radius of $20 \mathrm{~cm}$ and an outer radius of $150 \mathrm{~cm}$. The hole at the center contained a second filler vessel filled with gaseous helium to minimize the interactions beam and forwardly produced particles in this region. The filler vessel was $40 \mathrm{~cm}$ in diameter, and was made from stainless steel. In the case of the $530 \mathrm{GeV} / c$ proton beam, the angular coverage of the EMLAC was from $40^{\circ}$ to $138^{\circ}$ in the center of mass frame, corresponding to $\approx 77 \%$ of the total $4 \pi$ solid angle.

The EMLAC was divided into four independent quadrants. Each quadrant was divided longitudinally into 66 layers of alternating lead absorber sheets ${ }^{5}$ and copper clad G-10 6 boards. The lead absorber sheets were $2 \mathrm{~mm}$ thick while the G-10 boards were $1.59 \mathrm{~mm}$ thick. They were separated by $2.5 \mathrm{~mm}$ gaps filled with liquid argon. The width of the EMLAC was $71 \mathrm{~cm}$, or $\approx 26.5$ radiation lengths.

The lead absorbers also served as high voltage cathode planes. During operation, the cathodes were maintained at a voltage of $-2.5 \mathrm{kV}$. The $\mathrm{G}-10$ boards served as the anodes. The anodes were segmented into electrically isolated strips of either constant radius $(R$-board) or constant phi ( $\phi$-board). The strips on the $R$ boards were split azimuthally down the center of each quadrant, effectively dividing each quadrant into two electrically isolated octants. The $R$ and $\phi$ boards were interleaved, with the first board being an $R$-board. The structure of the EMLAC with an exploded view of one of its quadrants is shown in Figure 2.10.

On the first $R$-board, the inner boundary of the first $r$-strip was located $20.3 \mathrm{~cm}$ from the cylindrical axis. The strips on this board were $0.55 \mathrm{~cm}$ wide. On each successive $r$-board after the first board, the strip width increased slightly, as did the distance from the cylindrical axis to the first strip. The strip width

\footnotetext{
5 To be precise, the absorber sheet in the first layer was made from aluminum.

${ }^{6}$ G-10 is the industrial name for a type of fiberglass-epoxy laminate.
} 


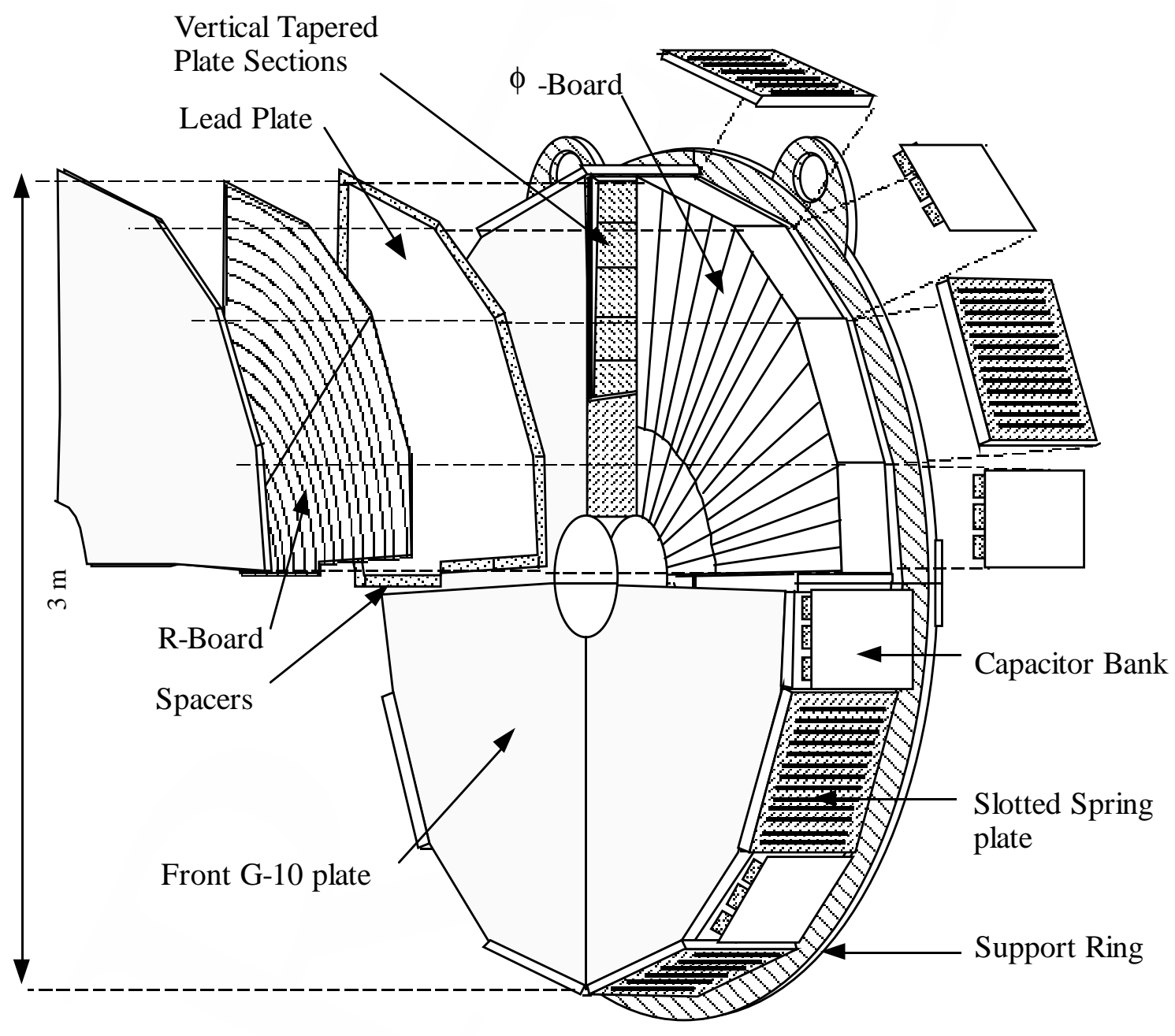

Figure 2.10 Detailed view of the electromagnetic calorimeter. 
increased in such a manner that the line of flight of photons emanating from the target region intersects the same sequential $r$-strip on each $R$-board. This is referred to as the focusing of the EMLAC.

The $\phi$-boards were divided into two sections, called inner and outer $\phi$. The boundary between the two sections occurred at a radius of $40.2 \mathrm{~cm}$. The inner $\phi$ strips each subtended an angle of $\pi / 192 \mathrm{rad}$, while the outer $\phi$ strips subtended an angle of $\pi / 384 \mathrm{rad}$.

The EMLAC was read out in two sections, a front and a back section. Corresponding strips from first $11 R$-boards were ganged together along the quadrant boundaries with braided copper wire connector strings. Similarly, the first 11 inner (outer) $\phi$-boards were ganged together along the inner (outer) edge of the quadrants. The connector strings were then attached to one of several readout boards located at the front of the calorimeter. This formed the front section. The back section was formed by applying a similar wiring procedure to the last $22 R$ and $\phi$ boards, but in this case running the connecting strings to readout boards located at the back of the calorimeter.

The signals from the readout boards were transmitted through the top of the cryostat via low impedance cable to specially designed LAC amplifier cards, called LACAMPs. The LACAMPs were designed to operated within the RABBIT $[69]^{7}$ system, developed at Fermilab. Each LACAMP card handled 16 detector channels. The LACAMPs provided three types of output: a fast estimate of the energy based upon $\mathrm{a} \approx 180 \mathrm{~ns}$ charge integration time, a more precise energy measurement based upon a longer $(\approx 800 \mathrm{~ns})$ charge integration time, and a timeof-arrival measurement. A schematic diagram of a LACAMP card is shown in Figure 2.11.

\footnotetext{
7 RABBIT stands for Redundant Analog-Bus Based Information Transfer
} 


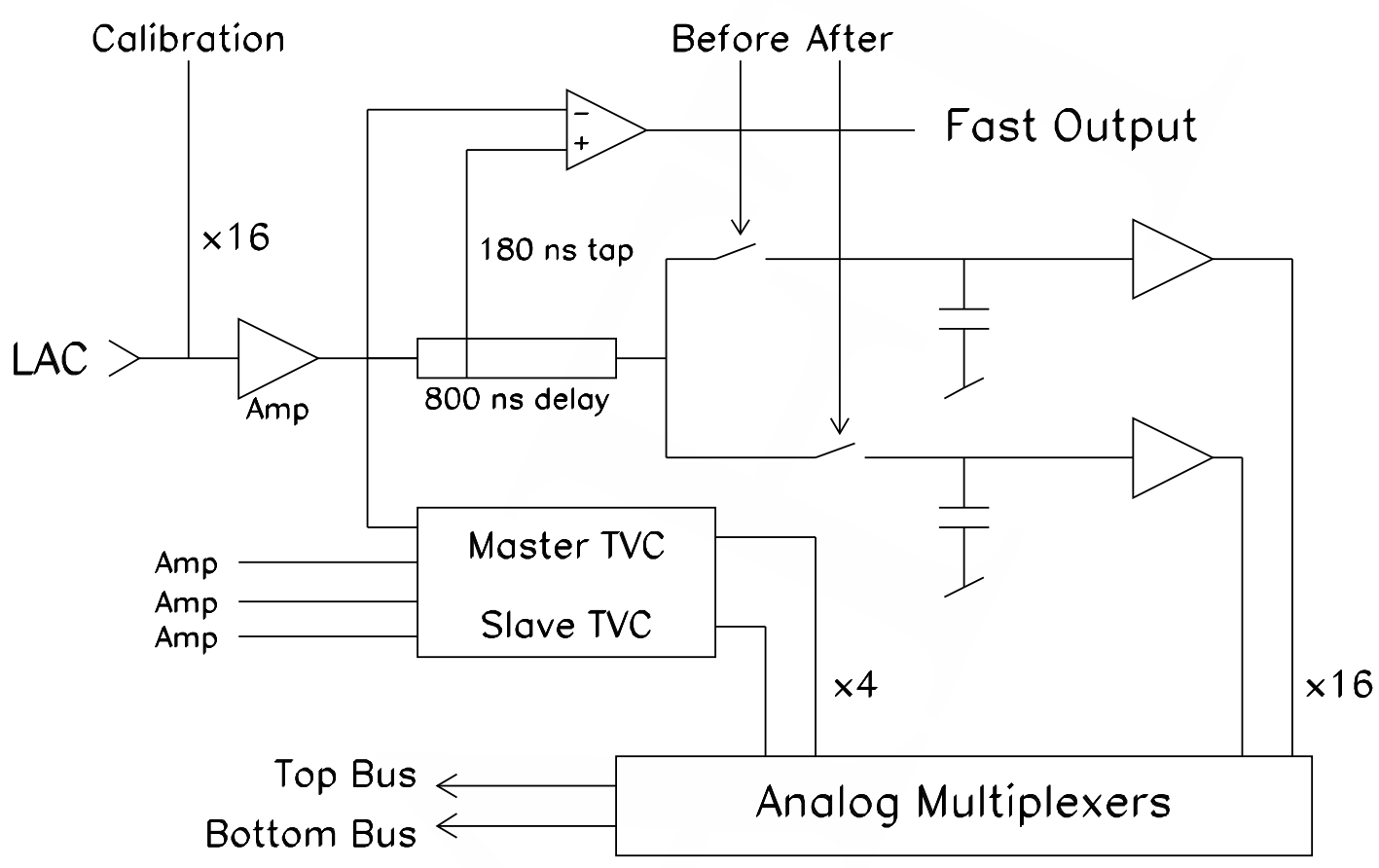

Figure 2.11 Schematic diagram of a single LACAMP module.

\subsubsection{Hadronic Calorimeter}

The HALAC was located directly downstream of the EMLAC. It consisted of 53 sampling cells, or cookies, separated by $2.54 \mathrm{~cm}$ thick steel plates. The cookies consisted of four layers of $0.8 \mathrm{~mm}$ thick copper clad G-10 boards. The two outermost layers of G-10 had copper cladding on both sides. The outsides of these two layers were held grounded, while the insides were held at high voltage and served as the cathode planes. The inner two G-10 boards were the anode planes. They were copper clad on only one side, the side nearest the cathode 
plane. Etched in the copper were horizontal rows of equilateral triangles that served as the readout pads. The signals from the readout pads were channeled to the edge of the plane via signal traces that ran horizontally between the rows. The anode and cathode planes were kept separated by $3 \mathrm{~mm}$ thick strips of G-10. These strips were oriented along the direction of the readout pads, and covered the area in between the readout pads. The G-10 spacer strips also displaced the liquid argon in front of the signal traces which preventing any current from being induced directly on the signal traces. The height of the rows containing the readout pads was equal to the height of the rows containing the signal traces, so that only half of each anode plane was instrumented. The two anode planes were aligned so that the rows of readout pads on one anode shadowed the rows of signal traces on the other, so that when taken together, the two anodes left no area uninstrumented. The size of the triangular readout pads increased in each successive cell. This served to focus the pads on the target. The height of the rows ranged from $11 \mathrm{~cm}$ to $14 \mathrm{~cm}$. An exploded view of a cookie is shown in Figure 2.12.

As with the EMLAC, the HALAC was divided longitudinally into a front and a back section. The front section consisted of 14 sampling cells, while the back section consisted of 39 cells. Corresponding pads in the front section were connected together and read out into the same amplifier channel. The back section was read out in a similar manner. The HALAC employed readout electronics similar to those used for the EMLAC.

\subsection{Forward Calorimeter}

The forward calorimeter, or FCAL, was designed to measure the energy and mean $p_{T}$ of the beam jet. It was located downstream of the LAC gantry, approximately 15 meters from the target. The FCAL consisted of three nearly 


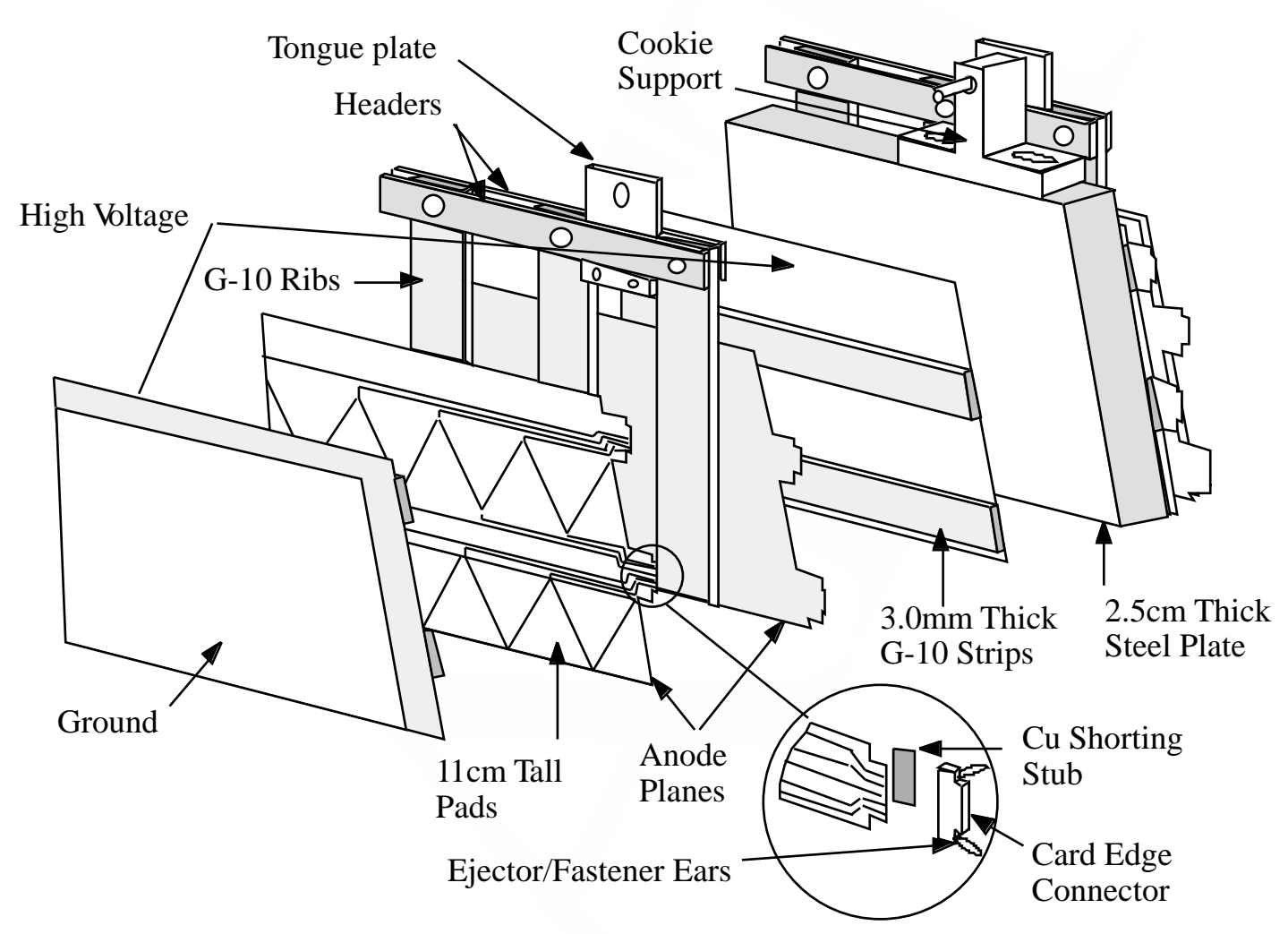

Figure 2.12 Exploded view of a HALAC cookie.

identical modules. Each module was composed of interleaved sheets of $1.9 \mathrm{~cm}$ thick steel absorber and $4.8 \mathrm{~mm}$ thick acrylic scintillator and measured $114 \mathrm{~cm}$ in diameter, with a $3.2 \mathrm{~cm}$ diameter hole in the center. The upstream and middle modules contained 28 steel plates and 29 scintillator sheets, while the downstream module contained 32 steel plates and 33 scintillator sheets. These three modules gave the forward calorimeter a thickness of 10.5 interaction lengths.

To collect the light produced by the scintillator, $601.0 \mathrm{~cm}$ diameter wave shifter rods, arranged in a $11.5 \mathrm{~cm}$ grid, were placed through each module. A photomultiplier tube was located at one end of each rod. The signals read out 
from the photomultipliers were proportional to the light collected by the wave shifters, which in turn was proportional to the energy deposited in the scintillator sheets. A more detailed discussion of the FCAL can be found in reference [70].

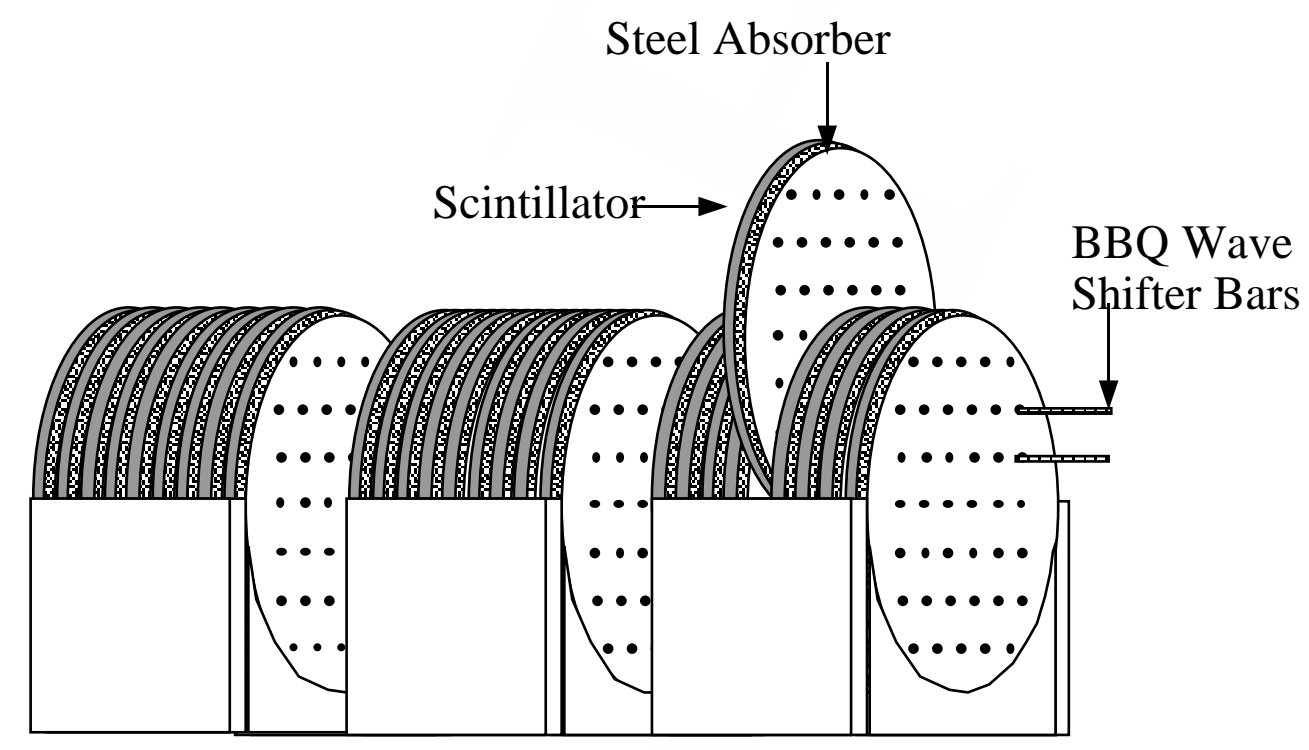

Figure 2.13 The forward calorimeter. 


\section{Chapter 3 Trigger and Data Acquisition}

\subsection{Overview}

The primary physics goal of this experiment was to study the production of direct photons at high $p_{T}$. However, since the majority of strong interactions are soft (i.e. produced at low $p_{T}$ ), an online trigger system was developed that predominately selected the high $p_{T}$ interactions, or events, of interest for further study, and rejected the rest. This trigger system selected approximately 1 out of every $10^{5}$ interactions that occurred in the data. Once an interaction was selected by the trigger system, a signal was sent to the data acquisition (DA) system, and the event was read out and written to $8 \mathrm{~mm}$ magnetic tape.

This chapter provides a brief description of the E706 trigger and DA systems. For a complete discussion of the trigger system, the reader is referred to [71]. Complete discussions of the DA system can be found in references $[72,73]$.

\subsection{Trigger System}

The signature of an interaction containing a high $p_{T}$ direct photon is the localized deposition of high $p_{T}$ electromagnetic energy in the EMLAC. To select such events, the trigger used a dedicated system of electronics, called the " $p_{T}$ system". The $p_{T}$ system, which operated within the RABBIT standard, used the fast output lines from the $r$-view LACAMPs to form fast estimates of the $p_{T}$ in the EMLAC. To form these fast estimates, the signals from the fast outputs of adjacent $r$-strips were added together and attenuated by a factor proportional to

$\sin \left(\theta_{i}\right)$, where $\theta_{i}$ was the angle between the $i^{t h} r$-strip and the beam direction. The attenuated signals from groups of eight consecutive $r$-strips were summed together to form sums-of-8 signals (see Figure 3.1). Because of the $\sin (\theta)$ weighting, these 
signals represented, to a first approximation, the $p_{T}$ deposited in a given radial region of an octant. These sums-of- 8 signals formed the basis from which most of the trigger decisions were made.

Since the $\pi^{0}$ and direct photon $p_{T}$ spectra fall rapidly with $p_{T}$, the experiment used several different trigger definitions to populate different $p_{T}$ regions. To populate the low end of the $p_{T}$ spectrum, simple triggers based upon the detection of beam particles and interacting beam particles were used. To keep these triggers from overwhelming the DA system, only a certain fraction of them were selected for further processing. This is referred to as trigger prescaling. At moderate values of $p_{T}$, LAC-based prescaled triggers with relatively low thresholds were used, and at high $p_{T}$, LAC-based triggers with relatively high thresholds were used. In Table 3.1 the primary trigger definitions used by the experiment, the fraction of events selected by these triggers, and the prescale factors associated with these triggers is shown. The $p_{T}$ regimes of these triggers is illustrated in Figure 3.2. At this point, no corrections have been applied to the data.

The formation of the trigger took place in a series of three increasingly complex steps. The first step is the beam and interaction determination. This was followed by a preliminary pretrigger determination, and finally, the trigger determination. In the following sections, these steps are described.

\subsubsection{Beam and Interaction Requirement}

The lowest level of the trigger formation is the beam and interaction requirement. The beam hodoscope planes (Section 2.4) were used to detect beam particles. If at least two hodoscope planes registered at least one hit cluster each ${ }^{1}$, then a BM signal was produced. If in addition, not more than one hodoscope plane

\footnotetext{
1 A cluster is defined as one hodoscope element registering a hit or two adjacent elements registering hits.
} 


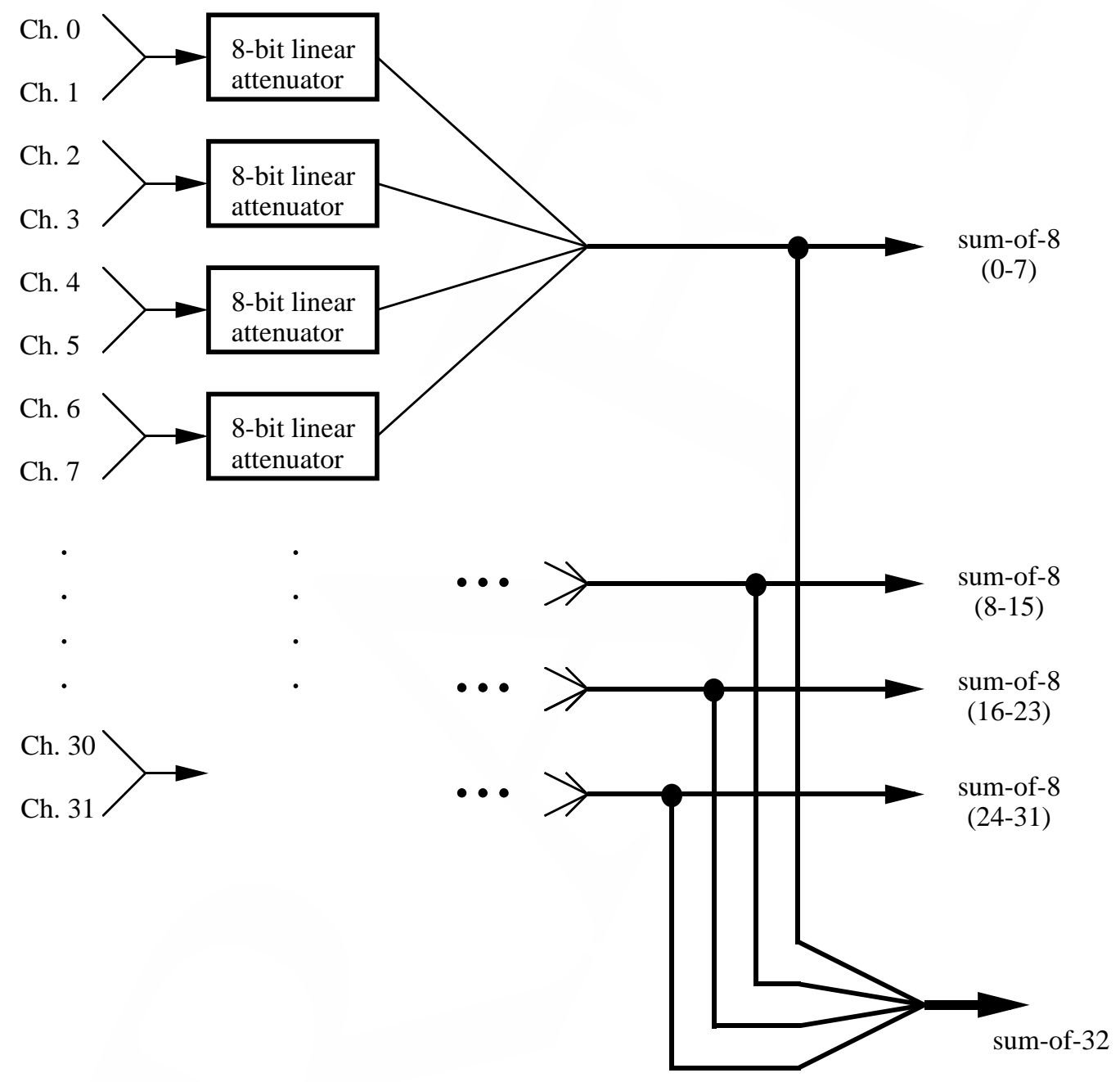

Figure 3.1 Sums-of-8 signal formation. 
Table 3.1 Trigger characteristics during the 1990 fixed target run. Many events satisfied more than one trigger. Some prescale factors changed during the run.

\begin{tabular}{|c|c|c|}
\hline Trigger & $\begin{array}{c}\text { Prescale } \\
\text { Factor }\end{array}$ & $\begin{array}{c}\text { Fraction of } \\
\text { Events }(\%)\end{array}$ \\
\hline BEAM & $15^{6}$ & 2 \\
$15^{5}$ & 3 \\
\hline INTERACTION & 2925 & 7 \\
\hline PINGLE LOCAL LOW & 40 & 18 \\
SINGLE LOCAL HIGH & 1 & 40 \\
LOCAL GLOBAL LOW & 40 & 20 \\
LOCAL GLOBAL HIGH & 1 & 35 \\
\hline TWO GAMMA & 1 & 20 \\
\hline DIMUON & 1 & 20 \\
\hline
\end{tabular}

identified two or more hit clusters, then a second signal, called BM1 was produced. To ensure that these signals were produced in phase with the bucket structure of the beam, two timing signals from the main accelerator were used to form the final beam definition. These were BMGATE, which was a $\approx 23$ sec pulse generated during the spill cycle of the accelerator, and RF_CLOCK, which was a $\approx 52 \mathrm{MHz}$ pulser signal that produced a train of $1 \mathrm{~ns}$ wide pulses in phase with the bucket structure of the beam. With these signals, the following beam definitions were made:

$$
\mathrm{BEAM} \equiv \mathrm{BM} \otimes \mathrm{BMGATE} \otimes \mathrm{RF}_{-} \mathrm{CLOCK}
$$

and

$$
\mathrm{BEAM} 1 \equiv \mathrm{BM} 1 \otimes \mathrm{BMGATE} \otimes \mathrm{RF} \_\mathrm{CLOCK},
$$




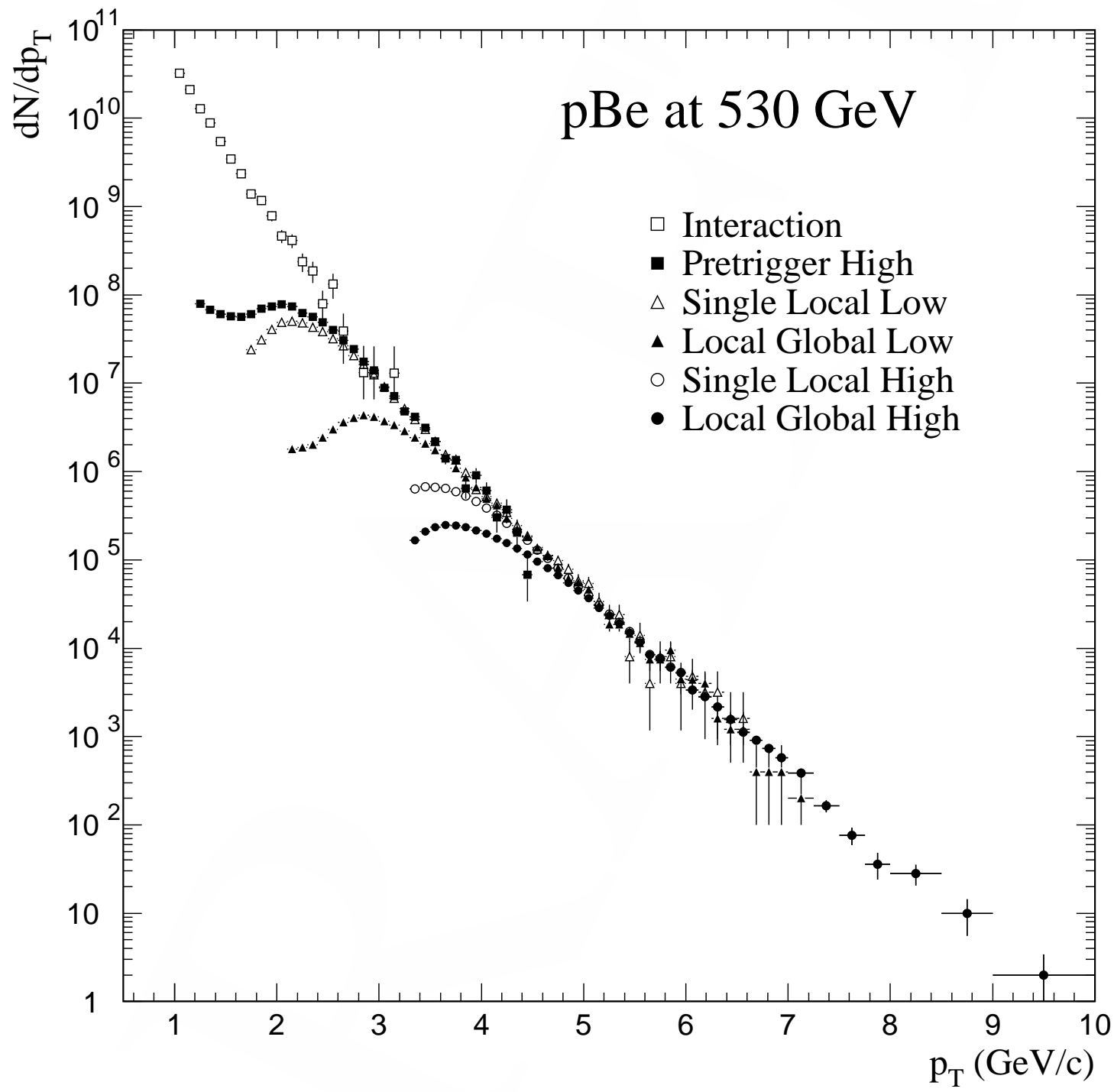

Figure 3.2 Number of photon pairs with mass in the $\pi^{0}$ region per $p_{T}$ bin versus $p_{T}$ for several different trigger types. No corrections have been applied to the data in this plot. 
where $\otimes$ is the symbol for the logical AND. The BEAM1 definition was the definition primarily used by the experiment since it guarded against the presence of multiple beam particles within the same RF bucket.

The indication of an interaction came from the interaction counters. An INTERACTION signal was generated when at least two of the four interaction counters fired in coincidence with the BEAM signal. However, before an interaction could be considered for triggering purposes, there were several additional criteria imposed on the event. To ensure that the interactions occurred within the target region, a veto was imposed on signals from the beam hole counter, $\overline{\mathrm{BH}}$. In addition, a CLEAN signal, which was generated when there was no INTERACTION signal within \pm 3 RF buckets of the current RF bucket, was required. This was necessary because the pretrigger logic units needed time to reset themselves after receiving the final interaction signals. Also, since the tracking electronics had a timing gate of $\approx 100 \mathrm{~ns}$, this helped eliminate overlapping events in the tracking system. Finally, a signal from the DA system, CMPRDY, was used to indicate that the DA system was ready to accept data. The final interaction signal, LIVE_INT1, was given by

$$
\text { LIVE_INT } 1=\mathrm{INT} \otimes \mathrm{BEAM} 1 \otimes \overline{\mathrm{BH}} \otimes \mathrm{CMPRDY} \otimes \mathrm{CLEAN} .
$$

\subsubsection{Pretrigger Requirement}

The next step in the formation of the trigger was the pretrigger requirement. The pretrigger was designed to quickly reject the bulk of the low- $p_{T}$ interactions. To form the pretrigger signal, the sums-of- 8 signals from each octant were sent to specially designed biased $p_{T}$ adder cards. There were two such cards for each octant, one for the inner $128 r$-strips and one for the outer $128 r$-strips. The biased $p_{T}$ adder cards added together the signals from the sums-of- 8 to produce an output corresponding to the total $p_{T}$ in each half octant of the EMLAC. These cards only 
summed signals from the sums-of- 8 that were above a certain threshold ${ }^{2}$. This was done to suppress the effects of image-charge induced signals on strips located in regions of the EMLAC that did not have any associated shower activity [71].

To temporally match the signals from the biased $p_{T}$ adder cards with the signals from the beam and interaction counters, the outputs from the $p_{T}$ adder cards were sent to two sets of zero-crossing discriminators. One set had a higher threshold than the other, and was used to produce the PRETRIGGER HIGH signal. The other set was used to produce the PRETRIGGER LOW signal. In the 1990 run, the PRETRIGGER HIGH threshold corresponded to $\approx 2 \mathrm{GeV} / c p_{T}$ deposited in a half-octant. The PRETRIGGer LOW threshold was $\approx 0.5 \mathrm{GeV} / c$ lower than the PRETRIGGER HIGH threshold. In the 1991-92 run, the corresponding thresholds were somewhat higher.

Once the pretrigger $p_{T}$ requirement for a given octant was met, several other conditions had to be satisfied before a PRETRIGGER HIGH or PRETRIGGER LOW signal was sent to the next level of the trigger system. A LIVE_INT1 signal was required to ensure the presence of a usable interaction. In addition, to protect against triggers resulting from interactions of beam halo muons in the EMLAC, the status of the veto wall quadrants shadowing the pretrigger octant was checked. If an event satisfied the following logic:

$$
\left(V W_{1} \oplus V W_{2}\right) \otimes V W_{3}
$$

in 1990 , or

$$
\left(V W_{1} \oplus V W_{2}\right) \otimes\left(V W_{3} \oplus V W_{4}\right)
$$

in 1991, where $V W_{i}$ indicates that veto wall $i$ registered a hit within $\pm 3 \mathrm{RF}$ buckets of the current RF bucket in the quadrant shadowing the pretrigger octant

\footnotetext{
2 The threshold was of the order of a few hundred $\mathrm{MeV}$.
} 
and $\oplus$ and $\otimes$ are the symbols for the logical OR and AND respectively, then the event was rejected. Also, since the signals from the EMLAC have a rise time of the order of $300 \mathrm{~ns}$, signals from interactions occurring in close time proximity may overlap, creating the impression of a high- $p_{T}$ event. To avoid these "pile-up" events, the pretrigger vetoed events in which the pretrigger octant had significant $p_{T}$ in one of its half-octants within the previous $\approx 300$ ns. This was called the early$p_{T}$ requirement. Finally, the power supplies for the LAC electronics generated a characteristic $400-\mathrm{Hz}$ noise spike. These noise spikes affected the signals from the LACAMPs, and therefore events occurring in time coincidence with these noise spikes were vetoed.

\subsubsection{The Local Triggers}

The local triggers were designed to select events that deposited large amounts of $p_{T}$ over relatively confined regions of the EMLAC. This made them well suited for efficiently selecting events containing high- $p_{T}$ direct photons or $\pi^{0}$ 's. There were two local triggers, one with a relatively high $p_{T}$ threshold called the SINGLE LOCAL HIGH, and one with a lower threshold called the SINGLE LOCAL LOW. To keep the SINGLE LOCAL LOW trigger from dominating the data sample, the SINGLE LOCAL LOW trigger was prescaled by a factor of 40 during the 1990 run. During the 1991 run, the prescale factor was 200 for all but the last part of the run, where the prescale factor was 280 .

To form the local triggers, the sum-of- 8 signals from each octant were sent into local discriminator modules. There were two modules for each octant, one for the SINGLE LOCAL HIGH and one for the SINGLE LOCAL LOW. In these modules, overlapping sums-of-16 were formed by combining the signals from neighboring sums-of-8 (see Figure 3.3). This summing eliminated the likelihood of inefficiency in the trigger due to showers that were centered near the boundaries 
of neighboring sums-of-8's. If the signals from any of the sums-of-16, called the trigger- $p_{T}$, exceeded the modules threshold, either a LOCAL_HI or LOCAL_LO logic signal was produced (depending on the module). The final local triggers were then formed from the logical AND of the LOCAL_HI Or LOCAL_LO signals with the PRETRIGGER HIGH signal, viz.

$$
\text { SINGLE LOCAL HIGH } \equiv \text { LOCAL_HI } \otimes \text { PRETRIGGER HIGH }
$$

and

$$
\text { SINGLE LOCAL LOW } \equiv \text { LOCAL_LO } \otimes \text { PRETRIGGER HIGH. }
$$

\subsubsection{The Global Triggers}

Although the local triggers were well suited for selecting events containing high $p_{T}$ direct photons and $\pi^{0}$ 's, they were not well suited for selecting events containing other high $p_{T}$ particles such as the $\omega$ and $\eta$, since the showers resulting from the decay products of these particles are typically separated by distances greater than the width of a single sum-of-16. For this reason, the experiment also used another class of triggers, called global triggers. The global triggers were formed from the analog sum of the signals from the inner and outer biased $p_{T}$ adder cards in an octant. As with the local triggers, there was a high threshold global trigger, called the GLOBAL_HI, and a low threshold GLOBAL_LO trigger. To suppress global triggers due to coherent noise in the EMLAC and/or image charge effects, the final global trigger definitions also contained a LOCAL_LO requirement:

$$
\text { LOCAL GLOBAL HIGH } \equiv \text { GLOBAL_HI } \otimes \text { LOCAL_LO } \otimes \text { PRETRIGGER HIGH }
$$

and

LOCAL GLOBAL LOW $\equiv$ GLOBAL_LO $\otimes$ LOCAL_LO $\otimes$ PRETRIGGER HIGH. (3.9) During the 1990 and 1991 runs, the LOCAL GLOBAL LOW triggers were prescaled by various amounts ranging between 10 and 70 . 


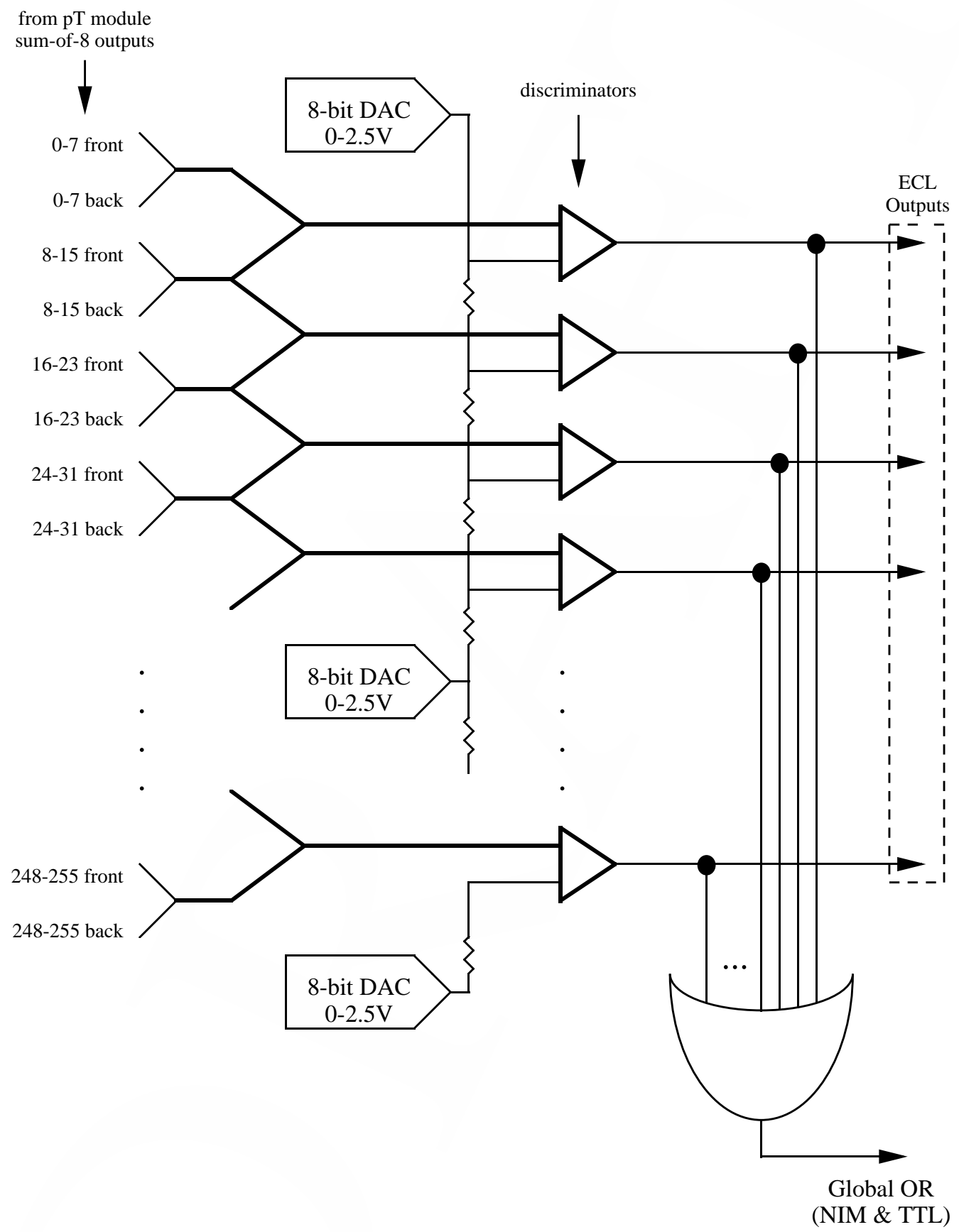

Figure 3.3 Block diagram of a local discriminator module. 


\subsubsection{The TwO GAMma Trigger}

In addition to studying the inclusive production of direct photons, the experiment also sought to study the production of high-mass pairs of direct photons. The signature of such a pair is significant $p_{T}$ deposition in opposite hemispheres of the EMLAC. The TWO GAMMA trigger was used to select such events. This trigger was formed by coincidences of the LOCAL_LO and PRETRIGGER LOW logic signals from a given octant and in one of the three octants opposing it. There was no need for a prescale factor for the TWO GAMMA trigger, since it is relatively rare for opposing octants to have $p_{T}$ depositions satisfying the LOCAL_LO requirement

\subsection{Overview of the DA system}

Data acquisition was controlled by a $\mathrm{DEC}^{3} \mu \mathrm{VAX}$ computer. Linked to the $\mu$ VAX were three DEC PDP-11 computers, referred to as ROCH, NEU, and MU, and a FASTBUS[74] system. Each of these systems was responsible for the readout of one or more of the experiment's detectors. ROCH and MU read out the CAMAC crates connected to the forward calorimeter and the E672 downstream dimuon system, respectively. NEU read out the CAMAC crates connected to the SSD's and the PWC's. This PDP-11 also recorded the state of the trigger logic and the Cerenkov information. The FASTBUS system read out the STRAW TDC's and the LAC RABBIT crates. A block diagram of the DA system is shown in Figure 3.4.

Once a trigger was satisfied, an INTERRUPT signal was sent to each of the front-end systems indicating that the data were to be read out. While the data were being transmitted from each system to the host $\mu$ VAX, a BUSY gate was

\footnotetext{
3 Digital Equipment Corporation
} 


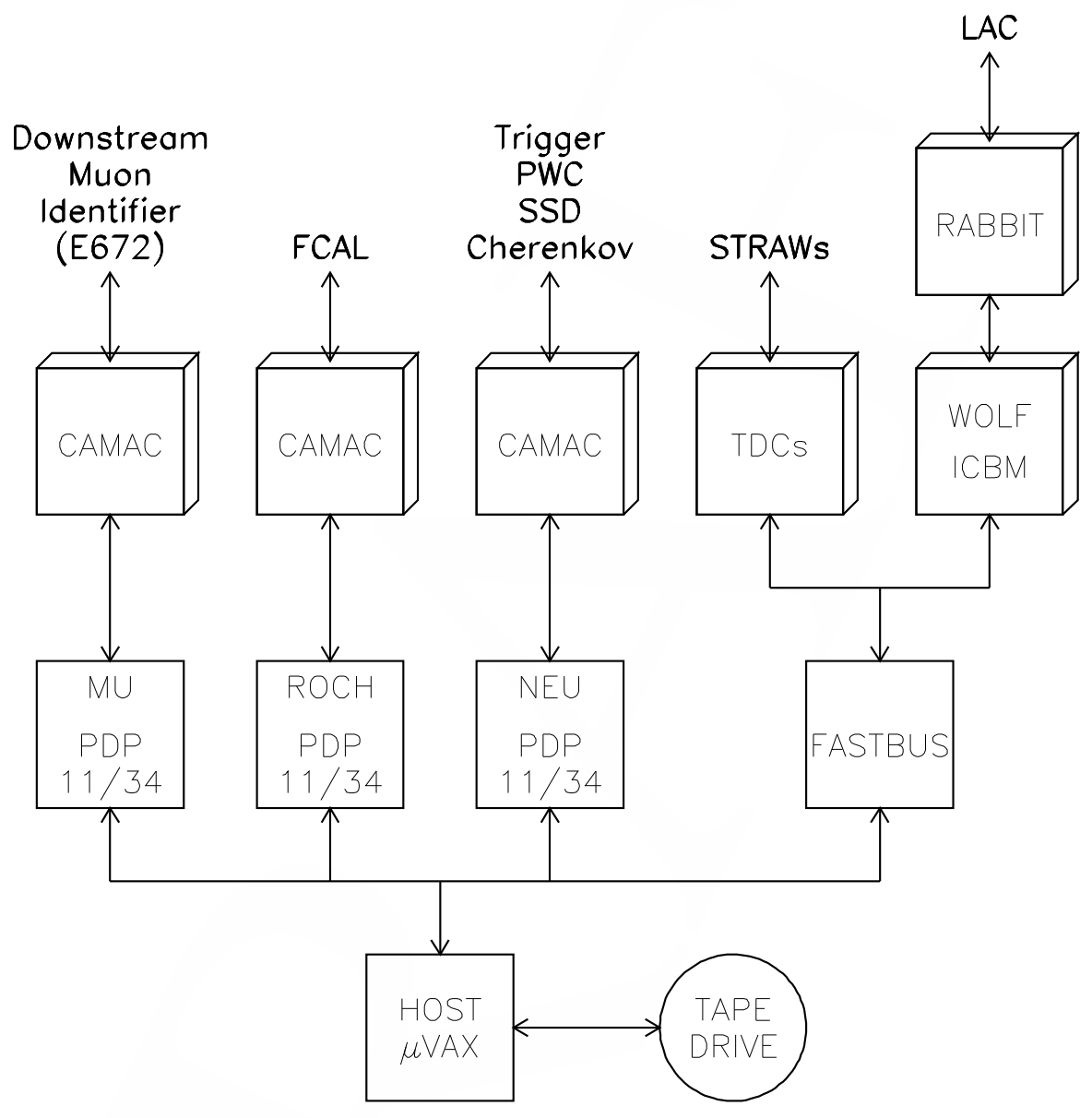

Figure 3.4 Block diagram of the E706 DA system. 
generated which remained enabled until all the data had been read out. Once all the BUSY gates were disabled, a CMPRDY signal was sent to the trigger, indicating that the DA system was ready to accept more data. The complete readout of a typical event took $\approx 8 \mathrm{~ms}$.

The software used for data acquisition was VAXONLINE [75], a DA software package developed at Fermilab. The VAXONLINE package consisted of 4 major components: EVENT_BUILDER, OUTPUT, RUN_CONTROL, and BUFFER_MANAGER. EVENT_BUILDER was responsible for the concatenation of the data received from each subsystem. The data were checked for consistency and collected into a single event buffer. The size of an event was $\approx 25$ kilobytes. OUTPUT was responsible for writing the buffered events to the output media. During normal running conditions, events were written to a pair of $8 \mathrm{~mm}$ tape drives, with events being sent to each drive in alternating order. Events were written out in sets known as runs, with each run containing $\approx 65000$ events. RUN_CONTROL managed the above processes, and also performed any initialization necessary to begin the data acquisition. BUFFER_MANAGER was used to send a copy of some of the events to various computers for online monitoring of the data. 



\section{Chapter 4 Event Reconstruction}

\subsection{Overview}

Over the course of the 1990 and 1991 fixed target runs, E706 recorded $\approx 71$ million events onto $\approx 1250$ eight $\mathrm{mm}$ magnetic tapes. To reconstruct this large data sample, the experiment used a number of $\mathrm{SGI}^{1}$ computer farms located at the Feynman Computing Center at Fermilab. Each farm consisted of a cluster of $\approx 10$ central processing units (CPU's). Within each farm, one CPU was the host, or I/O node, while the other CPU's were the worker nodes. The host node read events from the raw data tapes, and distributed (farmed) single events to the worker nodes. The worker nodes reconstructed the events, and then returned the results to the host node, which then wrote these results to eight mm tape. These tapes were called Data Summary Tapes, or DST's.

The raw data were reconstructed using a software package called MAGIC [76]. MAGIC was written in FORTRAN 77 and utilized the ZEBRA [77] memory management system. Technically, the main source code was not written in proper FORTRAN, but was converted into FORTRAN through the use of the PATCHY [78] code management package. The use of the PATCHY package allowed essentially the same piece of computer code to produce executable programs on a variety of system platforms. MAGIC was successfully run on DEC VAX, SGI INDIGO, and $\mathrm{IBM}^{2}$ RISC machines.

The various detectors of the spectrometer had their data reconstructed through calls to their respective reconstruction subroutines. These were:

\footnotetext{
1 Silicon Graphics, Inc.

2 International Business Machines
} 
- PLREC - Charged Track Reconstruction;

- EMREC - Electromagnetic Shower Reconstruction;

- DLREC - Discrete Logic Reconstruction;

- HCREC - Hadronic Shower Reconstruction;

- FCREC - Forward Calorimeter Reconstruction.

In the following sections, a brief description of PLREC, EMREC, and DLREC will be provided, as they were the reconstructors of most direct relevance to this analysis. Readers interested in the details of the HCREC and FCREC reconstruction programs are referred to [79] and [70], respectively.

\subsection{Charged Track Reconstruction}

The planes reconstruction subroutine, PLREC, was responsible for reconstructing the positions and momenta of the particles detected by the charged particle tracking system, and for finding the location of the primary interaction vertex. The trajectories of the charged particles, called tracks, were determined independently upstream and downstream of the magnet. The upstream and downstream tracks were then linked together, and the track momenta were calculated. The major elements of PLREC are highlighted below. For a more complete discussion, see $[80]$.

\subsubsection{Downstream Tracking}

The paths of charged particles downstream of the magnet were reconstructed using information from the PWCs and straw drift tubes. View tracks were formed from the wire locations with a latched signal (hits) observed in each PWC view $(X, Y, U$ or $V)$. The view tracks were then correlated to form three dimensional space tracks. Space track parameters (slope and intercept) could then be improved 
using information from the straw drift tubes. Because of the high efficiency, redundancy, and low noise in the PWC system, and in spite of the high average track multiplicity (about 30), tracking was rather straightforward in all but a small region surrounding the beam trajectory. Since the direct-photon, dimuon, and heavy-quark production physics of the experiment did not rely on tracking in this region, the tracking hardware and software were not intended to be used for these very forward tracks. Nevertheless, attempts were made to handle this region and complicated the details of the tracking code. These complications will largely be omitted in the discussion below in order to focus on the features of the tracking code relevant to the physics of the experiment.

\section{$\underline{\text { PWC Tracking }}$}

In the high multiplicity environment of this experiment, Monte Carlo studies showed that the highest efficiency for finding true tracks while keeping the number spurious tracks to a minimum, was obtained not by a simple cut on a fit $\chi^{2}$ (per degree of freedom) but by cuts on the nature of the track constraint class, i.e., the number of hits or hits shared with another track. For example, outside of the forward region, it was rare for more than two tracks to share a large number of hits between them. Therefore, the first level of the tracking code performed a search for four hit tracks with a straight line fit $\chi^{2}<3.0$, in each of the four views. Hits were assigned to tracks if they were contained within a window of \pm 1.0 wire spacings. If two or more tracks shared three hits, only the lowest $\chi^{2}$ solution was retained. Also, if two or more tracks shared one or two hits, only the two tracks with the lowest $\chi^{2}$ solutions were retained. This was followed by a pass searching for three hit tracks with a straight line fit $\chi^{2}<2.0$, that shared at most one hit with a four hit track. These were then subjected to sharing cuts to select one or two tracks from a cluster. 
Using the view tracks as a guide, space tracks were identified that had at least 13 hits (out of the 16 possible hits in the four views) and were consistent with a straight line fit to a common track with appropriate $\chi^{2}$ cuts. A hitsharing cut removed tracks that shared nine or more hits with another track of higher hit count. To capture tracks with large $\chi^{2}$ due to multiple scattering or other anomalies, hits not used in the tracks found above were searched for tracks with 12 hits if they projected through all four PWC modules, or 10 hits if they projected through only three modules. To further increase the acceptance for low momentum particles, wide-angle tracks that lay outside of the acceptance of the last two PWC modules were identified. These tracks had to have at least 6 hits and show correlated activity in the $X$ view Straw Drift Tubes and point back to the target in the $Y$ view.

Figure 4.1 shows the number of hits per space track from a representative sample of the $800 \mathrm{GeV} / c$ data. Assuming all losses of hits are due to the intrinsic efficiency of the PWC planes, such a hit distribution implies an average PWC plane efficiency of $\approx 96 \%$. Note that this is a lower limit, since losses can result from multiple scattering within the PWC planes and from biases in the track reconstruction algorithm.

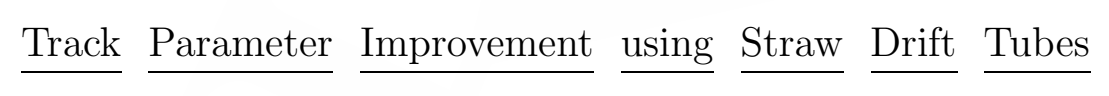

The downstream tracking system included two modules of straw drift tubes, with each view containing four $X$ and four $Y$ planes. Since the drift tubes have a spatial resolution approximately 3 times smaller than that of a PWC, the drift tube hits were used to improve the PWC track parameters. Closely spaced drift tubes, however, have a weak pattern recognition capability. Therefore, previously found PWC space tracks were projected to the straw drift tubes, and the tubes searched for hits (both left and right solutions for each) within a $3.5 \mathrm{~mm}$ window. 


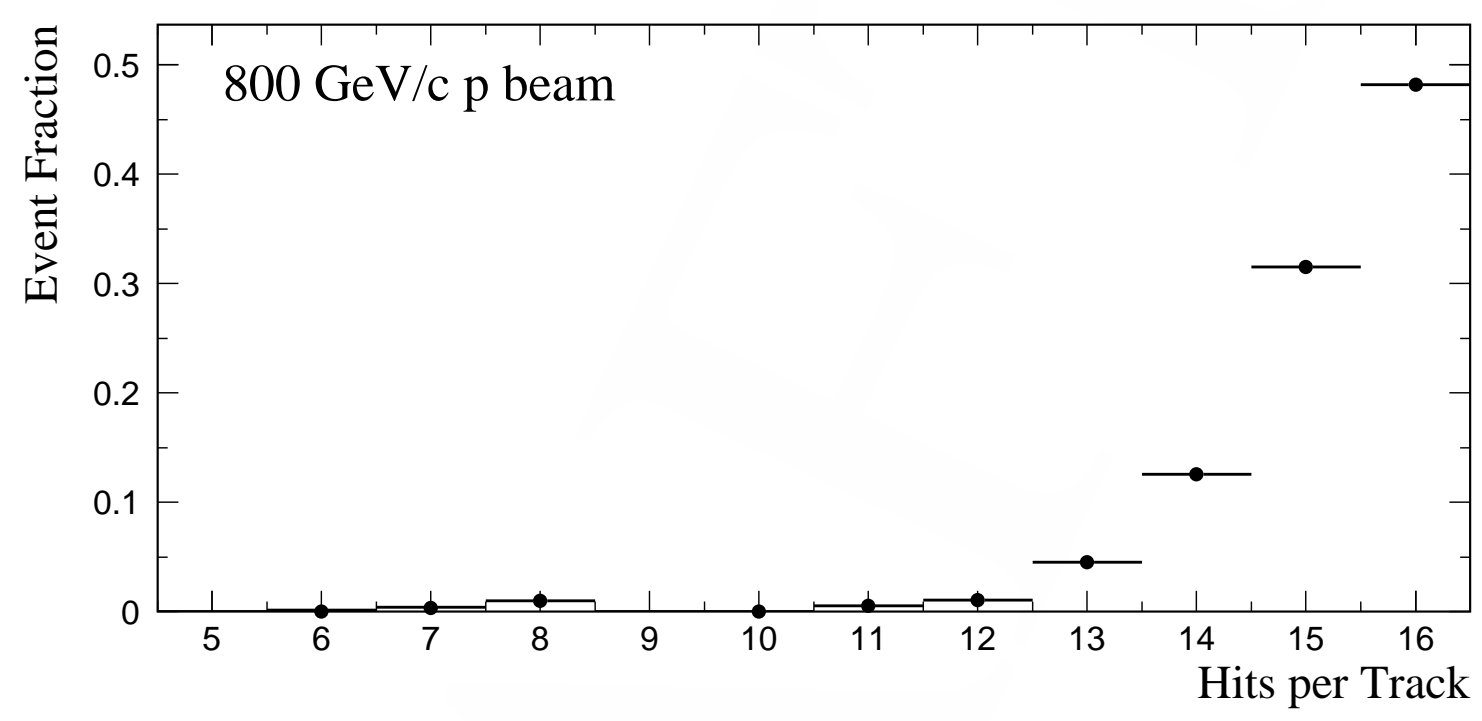

Figure 4.1 Distribution of PWC hits per space track.

In a number of iterations straw tube hits were removed that were not consistent with the PWC track parameters. As a final step, hits in the straws (minimum of four hits with at least one in each straw drift tube module) and those of the PWC were used in a combined fit of the space tracks. Space tracks using only PWC information were called PWC tracks, while space tracks incorporating both PWC and straw drift tube information were called sTRAW tracks. Approximately $75 \%$ of the space tracks were STRAW tracks.

\subsubsection{Upstream View Tracking and Linking}

Charged particle trajectories upstream of the magnet were reconstructed using 
the SSD vertex chambers ${ }^{3}$. Note only $X$ and $Y$ view tracks were found, since the SSD vertex chambers did not have a rotated view with which to correlate the view tracks.

The SSD view tracking was carried out in two stages. In the first stage, only four and five hit view tracks were found. The procedure for finding view tracks was analogous to the PWC view track finding. Two planes were chosen as seed planes, and the three remaining planes were used as search planes. The window for finding hits in the search planes was $75 \mu \mathrm{m}$ wide. To find all possible track combinations, two passes were made through the SSD hits, using different planes as the seed planes in each pass. View track candidates were fit to straight lines, and only view tracks with $\chi^{2}$ 's of 5 or less, for the five hit tracks, and 4 or less, for the four hit tracks, were saved. Any pair of view tracks was allowed to share a maximum of three hits between them. If a pair shared more than three hits, then the track with the fewer number of hits was dropped. In cases where both tracks had the same number of hits, the track with the larger $\chi^{2}$ was dropped.

Once all the four and five hit view tracks were found, they were then correlated, or linked, with downstream space tracks. To link the tracks, each downstream space track was projected to the center of the magnet in the $X$ and $Y$ views. The upstream view tracks were also projected to the center of the magnet, and the difference between these projections in each view, $\Delta X$ and $\Delta Y$, as well as the difference in the slope in the $Y$ view, $\Delta Y_{S L}$, was calculated. Corrections to $\Delta X, \Delta Y$, and $\Delta Y_{S L}$ were included to account for the effects of the magnet on the trajectories of charged particles[81]. If these quantities fell within their respective linking windows, the SSD view track was considered linked. To determine the size of the linking windows, the widths of the $\Delta X, \Delta Y$, and $\Delta Y_{S L}$

3 These were the five SSD $X-Y$ modules located downstream of the target. 
distributions were determined as functions of the track momentum. Separate functions were determined for PWC and STRAW tracks. These distributions are shown in Figure 4.2. The broadening of the resolution at low momentum is due to the increased importance of the effects of the magnet's fringe field and multiple scattering on the particle trajectories in this regime. A width of $3.3 \sigma$ was assigned to the linking window for each of these distributions. Also, an additional $0.1 \mathrm{~mm}$, for the $\Delta X$ and $\Delta Y$ windows, and $0.015 \mathrm{mr}$, for the $\Delta Y_{S L}$ window, was added to the linking windows to accommodate small variations in the alignment of the PWC and STRAW chambers over the course of the run.

Often, several SSD tracks would link to a given downstream space track (particularly in the case of PWC space tracks). To determine the best link in these situations, a "linking $\chi^{2}$ " was defined:

$$
\chi^{2}=\left(\Delta X / \sigma_{\Delta X}\right)^{2}
$$

in the $\mathrm{X}$ view, and

$$
\chi^{2}=\left(\Delta Y / \sigma_{\Delta Y}\right)^{2}+\left(\Delta Y_{S L} / \sigma_{\Delta Y_{S L}}\right)^{2}
$$

in the $\mathrm{Y}$ view, where $\sigma_{\Delta X}, \sigma_{\Delta Y}$, and $\sigma_{\Delta Y_{S L}}$ were the expected uncertainties in $\Delta X, \Delta Y$, and $\Delta Y_{S L}$, respectively. The link with the smallest linking $\chi^{2}$ was called the best link to the downstream track. In addition, up to four extra links were stored for each downstream track. In cases where the downstream track had more than five links, the five links with the lowest linking $\chi^{2}$ were saved.

After the linking, all unlinked SSD view tracks, with the exception of isolated view tracks ${ }^{4}$, were removed. Isolated view tracks were generally formed by low momentum particles that were swept out of the acceptance of the downstream

\footnotetext{
4 These are tracks that did not share any hits with other tracks.
} 


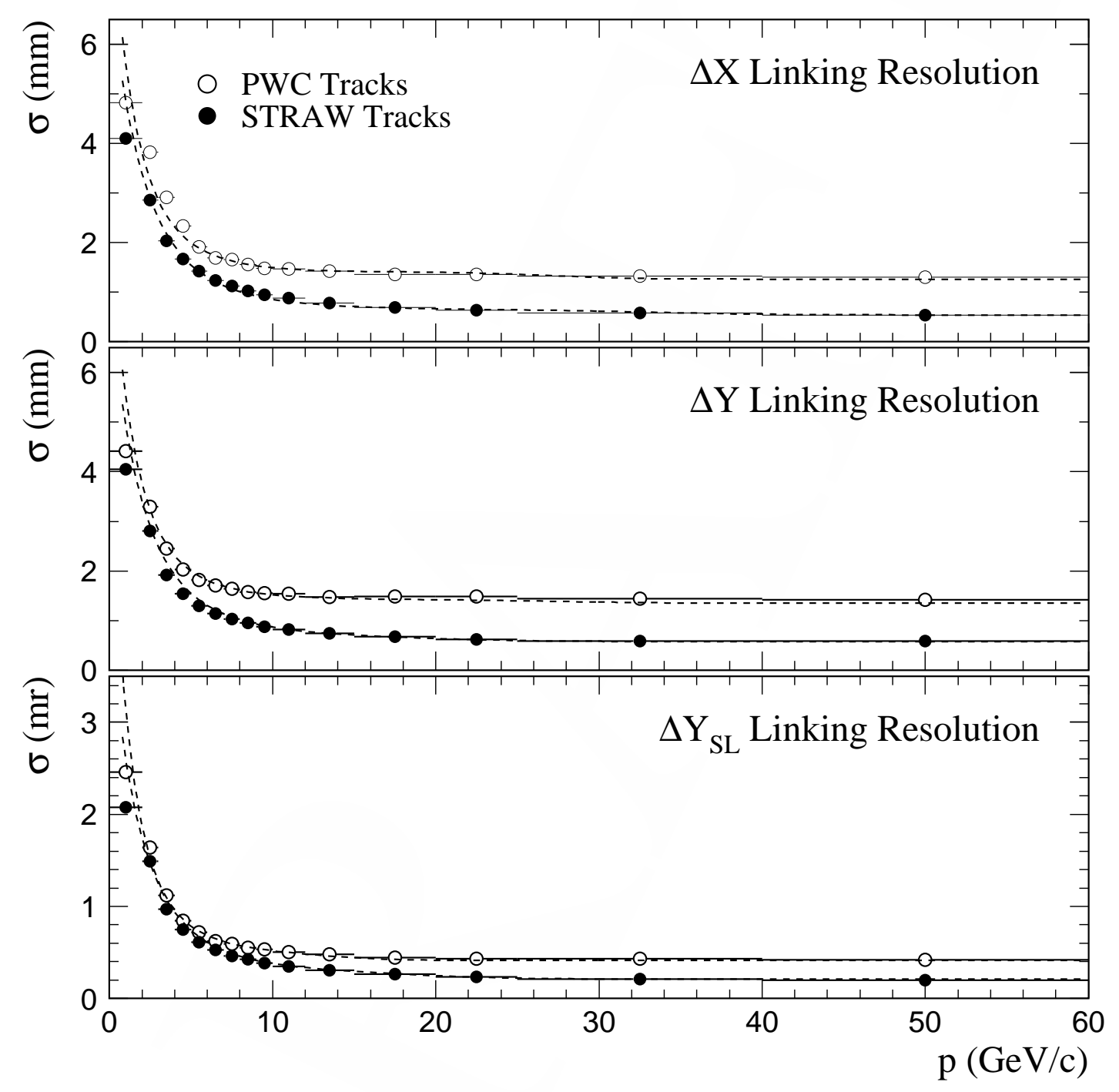

Figure 4.2 $\Delta X, \Delta Y$, and $\Delta Y_{S L}$ linking resolution as a function of the track momentum. The dotted lines indicate the functions used to determine the size of the linking window. 
tracking system by the magnet. These were saved to aid in the finding of the primary interaction vertex in cases where there were few linked tracks.

In the second stage of SSD view tracking, three hit view tracks were found. In this stage, all the hits associated with previously found view tracks were removed from consideration. To find all possible combinations of three hit tracks, there were four passes made through the SSD hits, with each pass using a different set of SSD seed planes. The tracks were fit to straight lines, and only tracks with $\chi^{2}$ values of less than 2.0 were saved. To save these tracks, it was also required that they linked to downstream space tracks that were previously unlinked.

\subsubsection{Vertex Finding and Relinking}

The location of the interaction vertex (primary vertex) was reconstructed using the SSD view tracks. Vertices were found in the $X$ and $Y$ views independently. At first, only SSD tracks that were best links to downstream tracks were used by the vertex finding algorithm. If no vertex was found, then SSD tracks that were extra links, and, if necessary, unlinked were used. The vertex finding algorithm was based upon an impact parameter minimization (IPM) scheme which is described in detail in reference [82]. For a given vertex position, a $\chi^{2}$ was defined,

$$
\chi^{2}=\sum_{k=1}^{n} b_{k} / \sigma_{k},
$$

where $b_{k}$ is the impact parameter of track $k^{5}$, and $\sigma_{k}$ is the projection uncertainty of track $k$. The vertex position was found by minimizing this $\chi^{2}$. Once the minimum was found, the vertex algorithm calculated the average impact parameter of the input tracks. If the average impact parameter was less than

5 The impact parameter is defined as the shortest distance between the track and the vertex. 
$20 \mu \mathrm{m}$, or the largest impact parameter was less than $50 \mu \mathrm{m}$, the vertex candidate was retained. Otherwise, the track with the highest impact parameter was excluded from the fit and the vertex position was re-evaluated.

After the view vertices were found, they were correlated based upon the difference in the $Z$ positions, $\Delta Z_{X Y}$, of the vertices. View vertices with $\Delta Z_{X Y}<5 \mathrm{~mm}$, or with $\sigma_{\Delta Z} / \Delta Z_{X Y}<8$, where $\sigma_{\Delta Z}$ was the estimated uncertainty in $\Delta Z_{X Y}$, were called matched vertices. In cases where multiple combinations of view vertices satisfied the matched vertex criterion, the view vertices with the smallest $\Delta Z_{X Y}$ became matched vertices. The $Z$ position of the matched vertex was then given by the weighted average of the $Z$ positions found in the two views. Finally, in cases where several matched vertices were found, the matched vertex located furthest upstream was assumed to be the primary vertex.

The difference $\Delta Z_{X Y}$ provides a measure of the vertex resolution in $Z$, since the view vertices are determined independently. The $\Delta Z_{X Y}$ distribution is shown in Figure 4.3 for the $1990 \pi^{-}$data. The half width at half maximum (HWHM) $0.6 \mathrm{~mm} .{ }^{6}$ Given that the uncertainties in the $Z$ position in the two views are approximately equal, the uncertainty in $Z$ for a given view is $\mathrm{HWHM} / \sqrt{2}$, or $0.4 \mathrm{~mm}$.

Once the location of the primary vertex was established, the assignment of the best SSD links to the downstream space tracks was performed again using the position of the vertex as an added constraint. The linking $\chi^{2}$ was redefined to include terms proportional to the SSD track's impact parameter with the primary vertex. The SSD track with the smallest "relinking $\chi^{2}$ " was subsequently reassigned as the best link.

${ }^{6}$ The HWHM is being used to characterize the width of the distribution, since the distribution is non-Gaussian. 


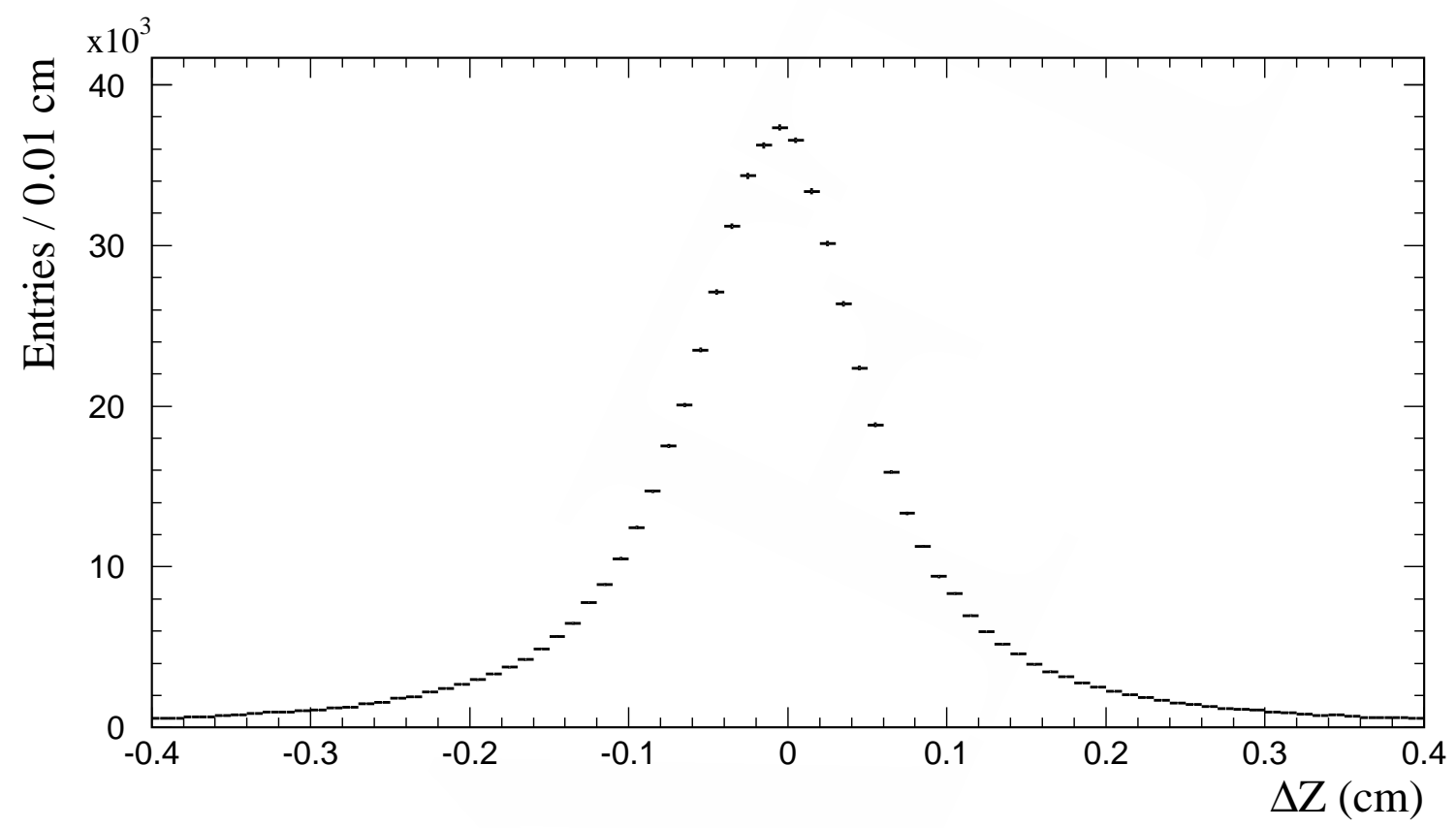

Figure 4.3 $\Delta Z_{X Y}$ distribution for vertices in the $1990 \pi^{-}$data.

\subsubsection{Beam Tracking}

The SSD planes located upstream of the target were used to measure the trajectories of the incoming beam particles. There were six SSD planes upstream of the target - three in the $X$ view and three in the $Y$ view. Beam view tracks were reconstructed in each of these views. To reconstruct the view tracks, two passes were made through the hits in the SSD beam planes. In the first pass, beam tracks were reconstructed requiring hits in all three SSD view planes. Two planes were chosen as the seed planes. Candidate beam tracks were constructed by forming all possible combinations of pairs of hits from the two seed planes. These tracks were then projected to the third (search) plane, and if the search 
plane contained a hit within $75 \mu \mathrm{m}$ (1.5 strips), a least squares straight line fit was performed. If the $\chi^{2}$ of the fit was less than 3.0, then the three hit combination was considered a beam view track. In the second pass, all SSD hits used to make tracks in the first pass were removed from consideration. The remaining hits were used to form two hit view track candidates. These candidates were retained if their slopes were less than $2.0 \mathrm{mr}$.

The closest beam track within $100 \mu \mathrm{m}$ of the primary vertex in each view was assumed to be the trajectory of the beam particle that produced the event. If no view track was found within $100 \mu \mathrm{m}$ of the primary vertex in either, or both views, then the interacting beam particle was assumed to travel parallel to the Z-axis. In later stages of the analysis, the measurement of $p_{T}$ for each particle was made with respect to the direction of the interacting beam particle.

\subsubsection{Charged Track Momentum Determination}

The determination of the charged particle momenta required a knowledge of the particle trajectories upstream and downstream of the magnet. The downstream trajectories were obtained from the downstream space tracks. The upstream trajectories were obtained from the best linked SSD view tracks. For downstream tracks that did not contain a link in the $X$-view, the upstream $X$-view trajectory was obtained by construction, assuming that the particle originated at the primary vertex. For downstream tracks that did not have a link in the upstream $Y$-view $(\sim 5 \%)$, the upstream $Y$-view trajectory was assumed to be the same as it was downstream.

The track momentum was calculated using the effective field approximation. In the effective field approximation, the real magnetic field is replaced by a dipole field with an effective field strength, $B_{0}$, and an effective length, L. The track 
momentum, $p$, and charge, $q$, were then calculated from the following equations:

$$
\begin{aligned}
q & =\operatorname{sign}\left(\theta_{1}-\theta_{2}\right) \cdot \operatorname{sign}\left(B_{0}\right) \\
\sqrt{p_{x}^{2}+p_{y}^{2}} & =\frac{p_{T}^{k i c k}}{\sin \theta_{1}-\sin \theta_{2}}, \quad p_{T}^{k i c k}=q B_{0} L \\
\frac{p_{x}}{p_{z}} & =\tan \theta_{1} \\
\frac{p_{y}}{p_{z}} & =\tan \theta_{y}
\end{aligned}
$$

where $\theta_{1}$ is the angle between the charged particle trajectory in the $X$ view and the $Z$ axis upstream of the magnet, $\theta_{2}$ is the angle between the charged particle trajectory in the $X$ view and the $Z$ axis downstream of the magnet, and $\theta_{y}$ is the angle between the charged particle trajectory in the $Y$ view and the $Z$ axis upstream of the magnet. The nominal value of $p_{T}{ }^{k i c k}$ was $450 \mathrm{MeV}$. After studying signals in the data, this value was adjusted slightly so that the reconstructed $K_{s}^{0}$ and $J / \psi$ masses measured via the tracking system were consistent with the world values. In Figure 4.4, the $\pi^{+} \pi^{-}$and $\mu^{+} \mu^{-}$invariant mass distributions in the regions of the $K_{s}^{0}$ and $J / \psi$ are shown. The mean value of the peaks in these distributions are within $0.1 \%$ of the accepted world average of the $K_{s}^{0}$ and $J / \psi$. Note that in these samples, the momenta of tracks from $J / \psi$ decays tended to be much larger than the momenta of tracks from $K_{s}^{0}$ decays.

The momentum resolution for charged particles was measured using the Monte Carlo simulation of the spectrometer described in Chapter 6. For particles produced in the target region, the average momentum resolution was found to be

$$
\sigma_{p} / p \approx 0.0076+0.0026 p
$$

where $p$ is the momentum measured in $\mathrm{GeV} / c$. 

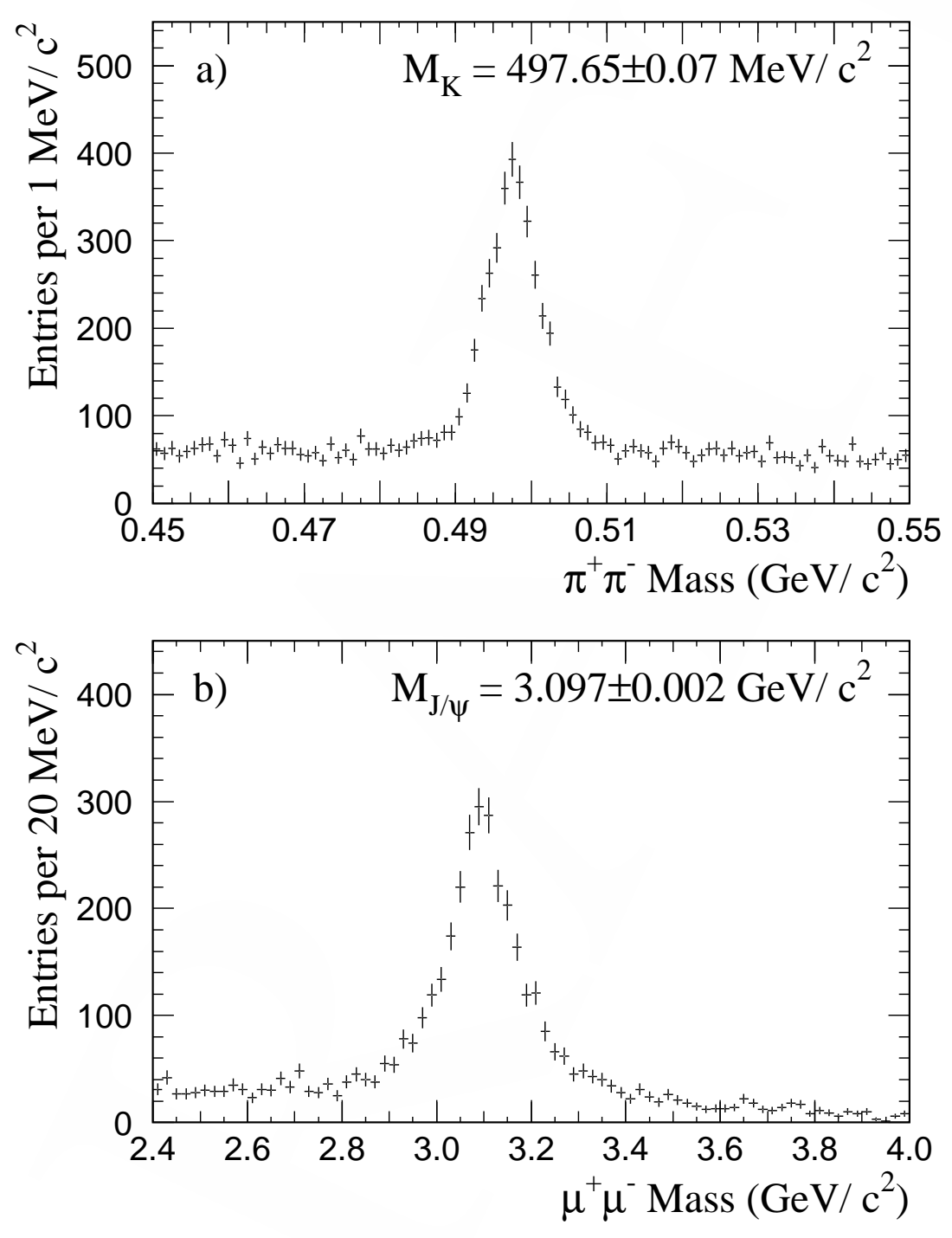

Figure 4.4 Reconstructed $K_{s}^{0}$ and $J / \psi$ masses. The means are within $0.1 \%$ of the world averages. 


\subsection{Electromagnetic Shower Reconstruction}

Showers in the electromagnetic calorimeter were reconstructed using the EMREC subroutine package. EMREC reconstructed showers on a quadrant by quadrant basis. Within each quadrant, showers were reconstructed independently in the $R$ and $\phi$ views of the EMLAC. Furthermore, in each quadrant, the $R$ view was subdivided into left and right $R$ views, and the $\phi$ view was subdivided into inner and outer $\phi$ views. The boundary between the $R$ views occurred at $\phi=45^{\circ}$, while the boundary between the $\phi$ views occurred at $R=40.2 \mathrm{~cm}$ (see Figure 4.5). The showers reconstructed in each of these four views were called GAMMAS. An example of the energy deposition and the associated GAMMAS in an EMLAC quadrant for a typical high $p_{T}$ event is shown in Figure 4.6. The GAMmaS from the different views were correlated based upon their energies and positions to form the final reconstructed showers, which were called PHOTONs.

In the following sections, the major features of the EMREC reconstruction algorithm are described; a more complete description can be found in reference [83].

\subsubsection{Unpacking}

The first task of the reconstructor was to convert the digitized pulse heights recorded for each EMLAC channel into units of energy. The energy of the $i^{\text {th }}$ strip, $E_{i}$, was given by

$$
E_{i}=A_{e m} G_{i} B(t)\left[N_{i}-N_{i}^{o}\right]
$$

where:

- $A_{e m}$ was the factor used to convert ADC counts to energy (determined from electron data to be $\approx 3.1 \mathrm{MeV} /$ count);

- $G_{i}$ was the relative gain of the amplifier for channel $i$; 

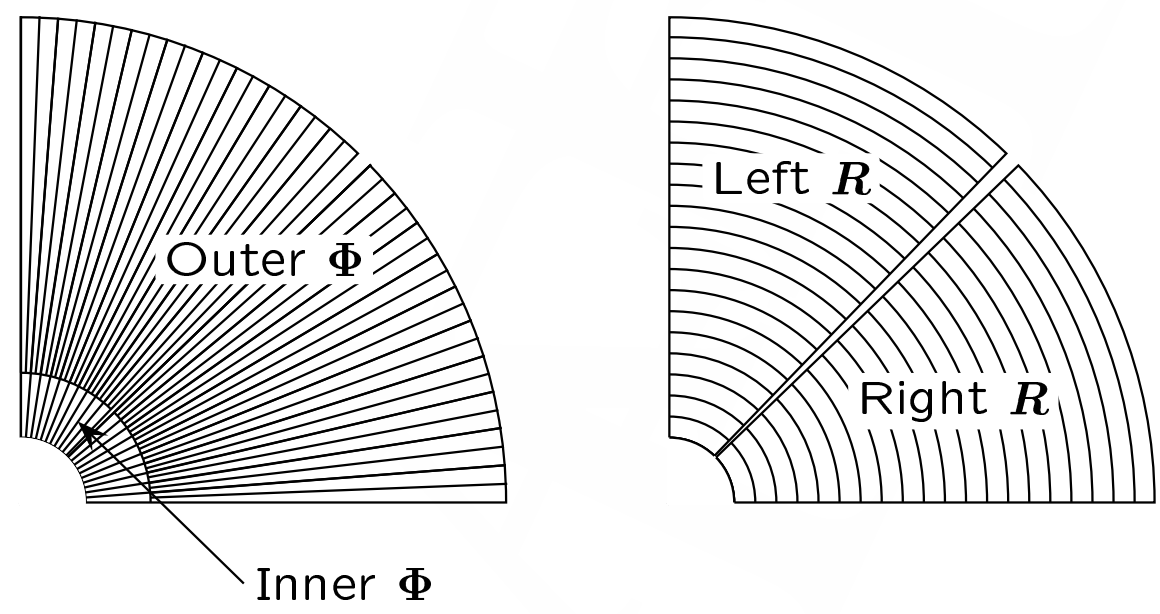

Figure 4.5 Schematic drawing of $R$ and $\phi$-boards showing the left-right $\mathrm{R}$ and inner-outer $\phi$ boundaries.

- $B(t)$ was a correction factor for the observed time dependence of the response of the EMLAC;

- $N_{i}$ was the ADC pulse height in channel $i$;

- $N_{i}^{o}$ was the pedestal (in ADC counts) for channel $i$.

The channel pedestal was the mean response of a channel when there was no energy deposited into the EMLAC. Initial values of the channel pedestals (mean and RMS) were calculated and stored between spills during data acquisition at $\approx 8$ hour intervals. These values were later modified offline using prescaled beam triggered events $[73]^{7}$.

7 Prescaled beam events were used since they are expected to have a minimal amount of shower activity in the EMLAC. Channels associated with reconstructed showers were excluded from this offline analysis. 
Run 14876 Event 15777 Quadrant 3
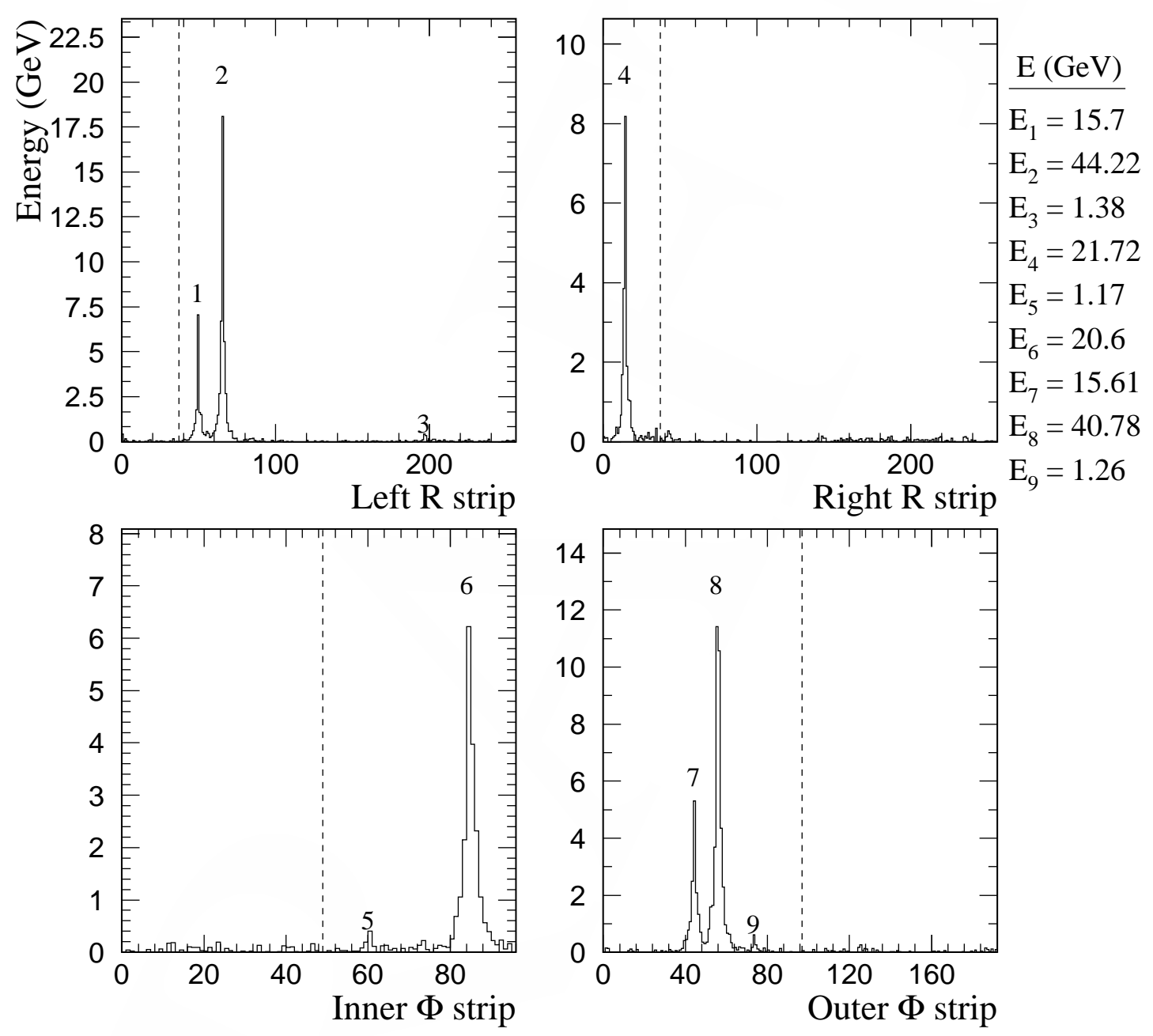

Figure 4.6 Energy deposition in the EMLAC. The dotted lines in the $R$ view ( $\phi$ view) plots indicate the location of the inner-outer $\phi$ (left-right $R$ ) boundary. The pair of high energy depositions in the left $R$ and outer $\phi$ views are most likely from an $\eta \rightarrow \gamma \gamma$ decay. 
The channel gains were also measured between spills during the data acquisition phase of the experiment. Each LACAMP channel was equipped with calibration hardware that included a charge injection capacitor. This provided the means for each channel amplifier to be pulsed with a known charge distribution and then read out. The channel output was then plotted as a function of the input charge and fit to a straight line. The slope of the line was the gain. The gains were found to be very stable over time, typically varying by less than $0.2 \%$ over the course of each of the 1990 and 1991 fixed target runs.

Although the response of the EMLAC electronics appeared to be stable over time, the overall response of the EMLAC was observed to change with time. This time dependence is illustrated in Figure 4.7, which shows the dependence of the mean uncorrected $\pi^{0}$ and $\eta$ masses on the number of beam days. Days in which the EMLAC high voltage was turned off ${ }^{8}$ are not included in the beam day count as the EMLAC response did not appear to change during these periods. Possible sources for this behavior, such as impurities in the liquid argon, have been investigated, but no satisfactory explanation for this behavior has been found. For a detailed discussion regarding this effect, the reader is referred to [84].

\subsubsection{Group and Peak Finding}

After the ADC to energy conversion, the energies from corresponding channels in the front and back sections of the EMLAC were added together to form the summed section. The reconstruction algorithm began by searching the summed section in each view for contiguous clusters of strips with energies above 80 $\mathrm{MeV}(95 \mathrm{MeV}$ in outer $\phi)$. If such a cluster satisfied the following additional requirements:

- It was at least 3 strips (2 strips in outer $\phi)$ wide;

\footnotetext{
8 Generally, the high voltage was turned off during periods of extended accelerator down time.
} 


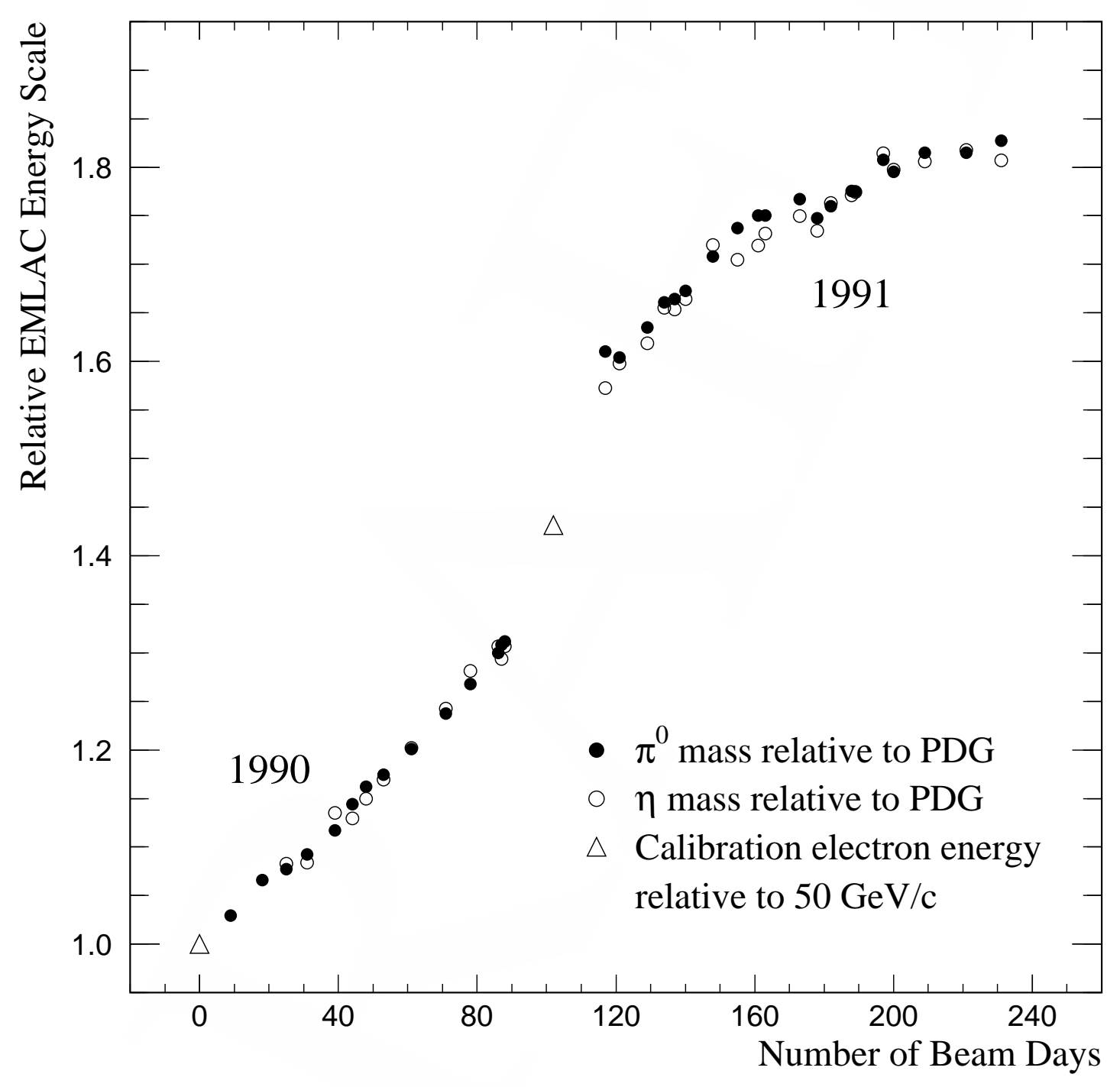

Figure 4.7 Time dependence of the response of the EMLAC. 
- It had at least one channel with energy greater than $300 \mathrm{MeV}$ (350 $\mathrm{MeV}$ in outer $\phi)$;

- Its total energy exceeded $600 \mathrm{MeV}$.

then that cluster was identified as a group.

After the group finding was done, each group was searched for peaks. This was done in an effort to resolve showers in close spatial proximity to one another ${ }^{9}$. To find the peaks, each group was scanned from left to right for local minima and maxima. If a local maximum was found bounded by two minima, a peak candidate was obtained. To eliminate peaks found due to simple energy fluctuations within the strips, the significance of the peak was evaluated using the nominal EMLAC energy resolution function,

$$
\sigma^{2}(E)=A^{2}+B^{2} E+C^{2} E^{2}
$$

where $A=0.22 \mathrm{GeV}, B=0.16 \mathrm{GeV}^{1 / 2}$, and $C=0.01$. If the height of the peak relative to the minima was consistent with energy fluctuations to within $2.5 \sigma$, then the candidate was discarded and the search continued for another peak. If a peak was considered significant, initial estimates of its energy and position were made. The peak energy was given by the sum of the energies of the strips between the peak minima, while the peak position was given by midpoint of the peak strip offset by an amount determined by the energies in the two strips adjacent to the peak strip.

Once a peak was found in the summed section, the strips in the group in the front and back sections were searched for corresponding peaks. Often, two showers which had coalesced to form a single peak in the summed section were resolved into two peaks in the front section. (This is because the shower profiles

\footnotetext{
9 E.g., the two showers resulting from the decay $\pi^{0} \rightarrow \gamma \gamma$.
} 
in the front section are narrower than the corresponding profiles in the summed section.) In these cases, the summed section peak was split in two and energies were assigned to the new peaks according to the relative energy fractions seen in the front view. The positions of the new peaks were assigned the corresponding positions of the front view peaks.

\subsubsection{GAMMA and PHOTON reconstruction}

After the peak finding was completed, a more precise calculation of each peak's position and energy was made by fitting the peak to a parameterized shower shape [72]. The fitted peaks were referred to as GAMmAs. The shower shape was parameterized as a function of the radial distance from the shower centroid. To determine the shower shape, a sample of single photon showers emanating from the target region was generated using a Monte Carlo simulation of the EMLAC's response to photon showers ${ }^{10}$. This shape was compared to the shape derived from isolated photons in the data and the two were found to be consistent.

The fitting procedure is simplest to describe in the case of single peak groups in the $R$ view. In this case, the fit energy was given by the energy, $E$, that minimized the $\chi^{2}$,

$$
\chi^{2}=\sum_{i} \frac{\left(E_{i}-z_{i} E\right)^{2}}{\sigma_{i}^{2}},
$$

where $E_{i}$ is the energy in strip $i, z_{i}$ is the fraction of energy in strip $i$ predicted from the shower shape, and $\sigma_{i}$ is the standard deviation of the energy for the $i^{t h}$ strip (Equation 4.10). After the fit energy was determined, the energy in the tails of the shower was calculated from

$$
E_{\text {tail }}=E\left(1-\sum_{i} z_{i}\right)
$$

10 The details of this simulation are given in Section 6.3.2. 
If the $\chi^{2}$ was less than five, then the energy stored for the GAMMA was the fit energy. However, if the $\chi^{2}$ was greater than five, then the sum energy was stored,

$$
E_{\text {sum }}=\sum_{i} E_{i}+E_{\text {tail }}
$$

In the case of the single peak $\phi$ groups, the situation was complicated by the fact that distance from the strip to the shower centroid is not well known due to the radial dependence of the strip width. To proceed, an estimate of the radial position of the shower was made based upon the energy and width of the peak. Later, when the radial position of the shower was better determined, the $\phi$ peaks were refit.

Once all the GAMMAS were reconstructed, the next task was to correlate the GAMMAS from the different views to form PHOTONS. The correlation routines looked to match GAMmas from the $R$ and $\phi$ views based upon the difference in GAMMA energies and $E_{\text {front }} / E_{\text {total }}$ ratios $^{11}$. To illustrate the general procedure for correlating GAMMAS, consider the event shown in Figure 4.6. In the quadrant shown, there are nine reconstructed Gammas. Based upon the similarities in GAMMA energies, GAMMAS 1 and 7,2 and 8,4 and 6, and 3 and 9, all appear to be good candidates for correlation. Note that GAMMAS 3 and 5 are not considered for correlation since their respective locations are not compatible; GAMMA 3 is located on the outside of the detector (large $R$ ), while GAMMA 5 is located on the inside of the detector (inner $\phi$ view). To correlate GAMmAs, the differences between the $R$ and $\phi$ view energies and $E_{\text {front }} / E_{\text {total }}$ ratios were calculated in units of $\sigma$, where $\sigma$ was the standard deviation of the total energy (Equation 4.10). If these differences fell within a preassigned window, the GAMMAS were considered correlated and a РнотоN of energy $E=E_{r}+E_{\phi}$ was obtained.

11 Recall that the EMLAC's $R$ and $\phi$ boards were interleaved. Therefore, the energy and longitudinal development of correlated GAMMAs in the $R$ and $\phi$ views should be nearly equal. 
Often, two showers would overlap in one view to form a single GAMMA (this happened frequently in $\pi^{0} \rightarrow \gamma \gamma$ decays). An example of such an occurrence is shown in Figure 4.8. In this event, the energy of $R$ view GAMMA 5 is roughly equal to the sum of the energies of $\phi$ view GAMMAs 9 and 10, which implies that GAMMA 5 was comprised of two showers that strongly overlapped in the $R$ view. EMREC contained specific routines designed to correlate GAMMAS in these situations. In this case, the two $R$ view GAMMAs were summed together and compared to the $\phi$ view GAMMA. If the differences in energies and $E_{\text {front }} / E_{\text {total }}$ ratios were within the correlation window, then two PHOTONS, with relative energies assigned according to the relative energies of the $\phi$ view GAMMAs, were obtained. Note also a similar situation involving GAMMAS 3, 4 and 6, although in this case, the overlapping showers occurred in the $\phi$ view.

EMREC also contained a set of routines designed to correlate showers that developed near the view boundaries. In such cases, the showers were often split into multiple GAMMAS, with each GAMMA located in a different view. For example, a shower that develops near the inner-outer $\phi$ boundary can result in three reconstructed GAMMAS - one in the $R$ view and two, one in inner $\phi$ and one in outer $\phi$, in the $\phi$ view. To correlate these GAmMAs, the inner and outer $\phi$ view GAMMAS were summed together and compared to candidate $R$ view GAMMAs.

The correlation process was repeated twice. After the first pass, the $\phi$ view GAMMAS that were correlated were refit to obtain a better measurement of their energy. A second correlation pass was then performed.

\subsection{Discrete Logic Reconstruction}

The discrete logic reconstructor, DLREC, was used to reconstruct the status

of the trigger, veto wall elements, Čerenkov counter, beam counters, interaction counters, beam hodoscope elements, and the hole counter. With the exception 

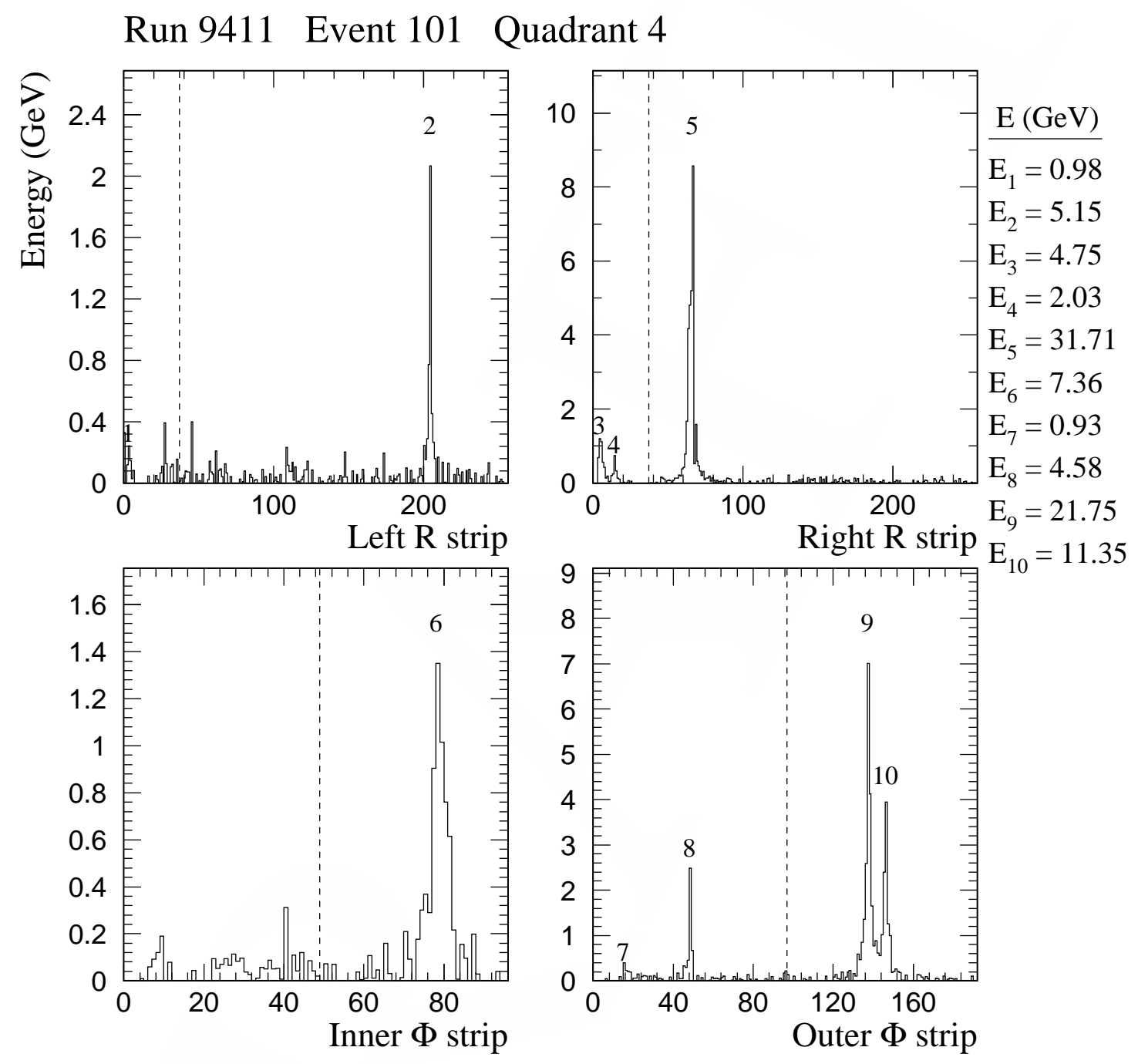

Figure 4.8 Another example of energy deposition in the EMLAC. The high energy deposition in the right $R$ view and the pair of high energy depositions in the outer $\phi$ view are most likely from a $\pi^{0} \rightarrow \gamma \gamma$ decay. 
of the trigger, the status of these devices was latched and read out using custom made CAMAC modules called "Minnesota Latches". The Minnesota Latches stored information in a buffer at the RF_CLOCK rate, thus storing the status of the counters for each beam bucket. When an event was selected, an INTERRUPT signal was sent to the latches, and the information stored in the $15 \mathrm{RF}$ buckets centered roughly on the time of the triggering interaction were read out.

The status of the trigger for each event was latched by LeCroy 4508 Programmable Logic Units and Nanometric N278 latches. The 4508's stored the status of the PRETRIGGER HIGH, PRETRIGGER LOW, LOCAL_HI, LOCAL_LO, GLOBAL_HI, and GLOBAL_LO logic signals for each octant. The Nanometric N278 latches stored the status of each of the local discriminators.

DLREC provided two main summary banks of information regarding the state of the discrete data. This information was stored in the bits of integer words. The first bank contained four words of quality information. In these words, a summary of the status of the trigger, veto walls, and Čerenkov elements was contained. In addition, bits were set that indicated if there were CAMAC readout failures, and/or inconsistencies in the trigger logic.

The second bank contained $\approx 40$ integer words. These words stored the status of each of the triggers for each octant and the status of each of the trigger discriminators. From these words, the efficiency for each trigger could be evaluated. The bank also contained a summary of the data from the Minnesota Latches, from which the time history of the various counters could be obtained. 



\section{Chapter 5 Data Analysis}

\subsection{Overview}

The data used in this analysis, sorted by beam and target type, are summarized in Table 5.1. To facilitate the analysis of this large data sample, object oriented ntuples ${ }^{1}$ were created from the DST tapes and stored on disk. Independent ntuples were written for the direct photon and neutral meson $\left(\pi^{0}\right.$ and $\eta$ ) analyses, as well as for most other analyses. The direct photon ntuples stored information on a per photon basis. In the neutral meson analyses, $\pi^{0}$ 's and $\eta$ 's were reconstructed via their decay into two photons. Therefore, the neutral meson ntuples stored information on a per $\gamma \gamma$ basis. Most of the data analysis was based upon the information stored in these ntuples.

This chapter describes the various requirements on the events and showers used in the direct photon and neutral meson analyses. As an illustration of the effect of these requirements, a $\gamma \gamma$ invariant mass spectrum for photon pairs with total $p_{T}>3.5 \mathrm{GeV} / c$ that landed in the same EMLAC octant is shown in Figure 5.1. The solid line shows the spectrum for all photon pairs, while the dashed line shows the spectrum after applying the photon and photon pair requirements. Although the $\pi^{0}$ and $\eta$ signals are clearly evident in both cases, the background is significantly reduced after the requirements are applied.

\subsection{Target Fiducial Requirement}

Figure 5.2 shows the reconstructed $Z$ position of vertices for events containing $\gamma \gamma$ pairs with invariant mass within the $\pi^{0}$ signal region (defined in Section 5.11) and $p_{T}>4 \mathrm{GeV} / c$ in the $515 \mathrm{GeV} / c \pi^{-}$(1990) and $800 \mathrm{GeV} / c$ proton (1991) beam

\footnotetext{
1 Ntuples are $n \times m$ arrays, where $n$ is the number of objects stored and $m$ is the number of variables stored for each object.
} 
Table 5.1 Data summary for the 1990 and 1991-92 fixed target runs.

\begin{tabular}{|c|c|c|c|c|}
\hline Run & Interaction & $\begin{array}{l}\text { Beam Momentum } \\
\qquad(\mathrm{GeV} / \mathrm{c})\end{array}$ & $\begin{array}{l}\text { Number of Recorded Events } \\
\text { (millions) }\end{array}$ & $\begin{array}{l}\text { Sensitivity } \\
\text { (events/pb) }\end{array}$ \\
\hline \multirow[b]{2}{*}{1990} & $\pi^{-} \mathrm{Be}$ & \multirow[b]{2}{*}{515} & \multirow[b]{2}{*}{30} & 8.6 \\
\hline & $\pi^{-} \mathrm{Cu}$ & & & 1.4 \\
\hline \multirow{9}{*}{1991} & $\mathrm{pBe}$ & \multirow{3}{*}{800} & \multirow{3}{*}{23} & 7.3 \\
\hline & $\mathrm{pCu}$ & & & 1.8 \\
\hline & $\mathrm{pH}$ & & & 1.5 \\
\hline & $\left(\mathrm{p}, \pi^{+}\right) \mathrm{Be}$ & \multirow{3}{*}{530} & \multirow{3}{*}{14} & 6.4 \\
\hline & $\left(\mathrm{p}, \pi^{+}\right) \mathrm{Cu}$ & & & 1.6 \\
\hline & $\left(\mathrm{p}, \pi^{+}\right) \mathrm{H}$ & & & 1.3 \\
\hline & $\pi^{-} \mathrm{Be}$ & \multirow{3}{*}{515} & \multirow{3}{*}{4} & 1.4 \\
\hline & $\pi^{-} \mathrm{Cu}$ & & & 0.3 \\
\hline & $\pi^{-} \mathrm{H}$ & & & 0.3 \\
\hline
\end{tabular}




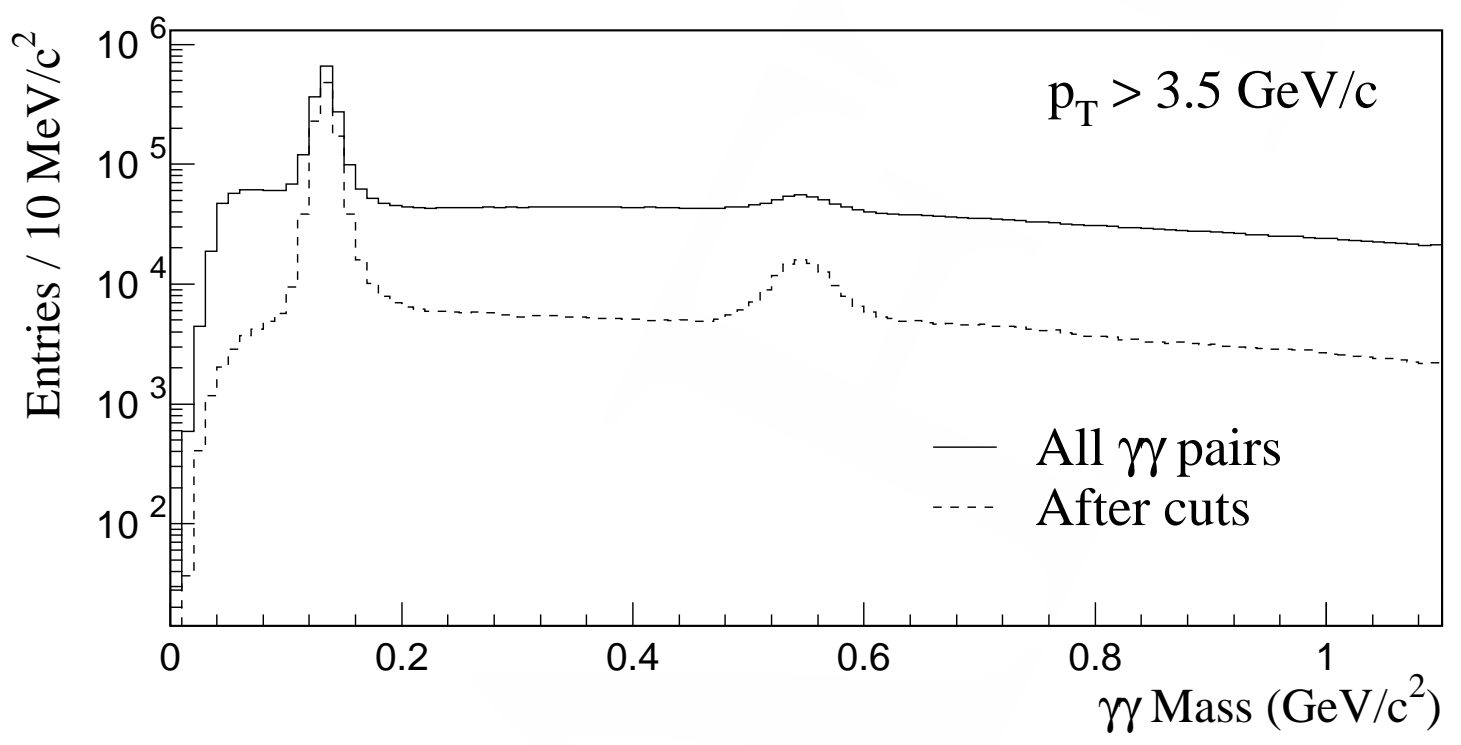

Figure $5.1 \gamma \gamma$ invariant mass distribution in the $530 \mathrm{GeV} / c$ proton data.

data. From plots such as these, target locations were determined and longitudinal fiducial regions for each target were defined. The events used in this analysis were required to have a reconstructed primary vertex within the fiducial region of the $\mathrm{Be}, \mathrm{Cu}$, or Liquid $\mathrm{H}_{2}$ targets.

Transverse target fiducial volumes for the $\mathrm{Be}, \mathrm{Cu}$, and Liquid $\mathrm{H}_{2}$ targets were also defined. The transverse location of vertices with $Z$ position inside the $\mathrm{Cu}$ and Be fiducial regions is shown in Figure 5.3 for the $1990515 \mathrm{GeV} / c \pi^{-}$beam data and the $1991530 \mathrm{GeV} / c$ proton beam data. The transverse positions of the $\mathrm{Be}$ and $\mathrm{Cu}$ targets are indicated by the solid line. Also shown are the boundaries of the instrumented regions of the upstream SSD wafers (dashed line segments) and the location of the beam hole counter during the 1990 run (dotted circle). 


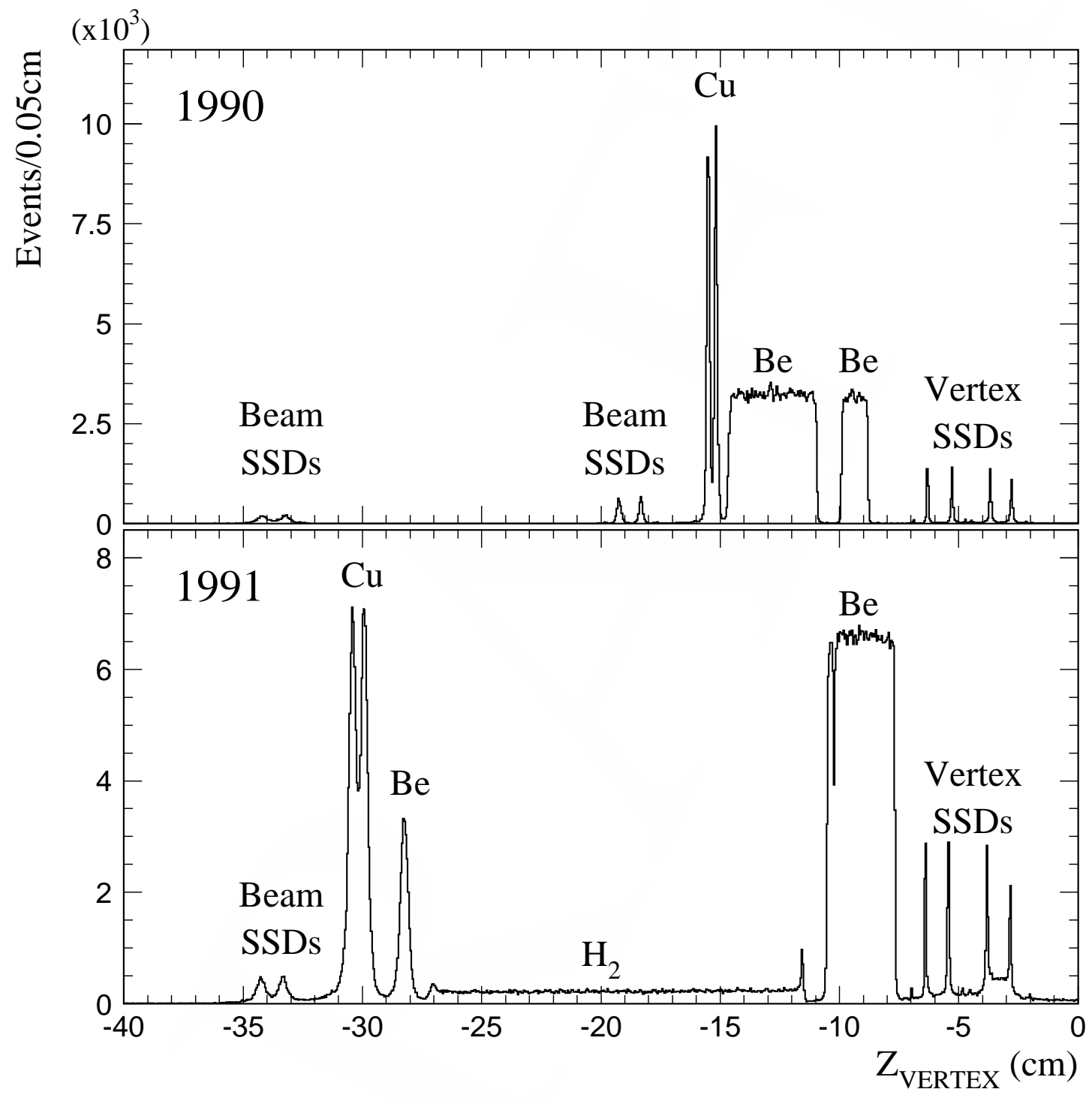

Figure 5.2 $Z$ position of vertices in the $1990515 \mathrm{GeV} / c \pi^{-}$and $1991800 \mathrm{GeV} / c$ proton data for events containing $\gamma \gamma$ pairs with invariant mass within the $\pi^{0}$ signal region and $p_{T}>4 \mathrm{GeV} / c$. The events are corrected for beam absorption and losses due to photon conversions. 
Note that in the 1990 run, the beam was not centered on the target. ${ }^{2}$ To account for the fraction of the beam that missed the $\mathrm{Cu}$ and Be targets, corrections were determined using interactions in the upstream SSD wafers. These corrections were evaluated as the fraction of vertices contained within each target's transverse fiducial volume.

\subsection{EMLAC Fiducial Requirement}

It is difficult to accurately measure the energy and position of photons near the quadrant boundaries of the EMLAC since portions of the resultant electromagnetic showers are deposited in non-instrumented regions. An EMLAC fiducial volume was defined for this reason. The boundaries of the fiducial volume were located far enough away from the EMLAC's physical boundaries that such losses were minimal. Figure 5.4 shows the $X-Y$ position of photons contained within the EMLAC fiducial volume. Note that a small region between octants within a quadrant was also excluded from the fiducial volume, even though it was fully instrumented. This was done to simplify the trigger analysis. Recall, the trigger selected events based upon the energy deposited in individual EMLAC octants. By cutting away from the octant boundaries, the trigger analysis did not have to account for energy leakage into other octants.

An additional fiducial requirement was placed upon the contributing photon pairs in the neutral meson analysis - both photons were required to land inside the same EMLAC octant. Again, this requirement was imposed to simplify the trigger analysis.

\footnotetext{
2 This offset was caused by a manufacturing flaw in the target stand used during
} the 1990 run. 


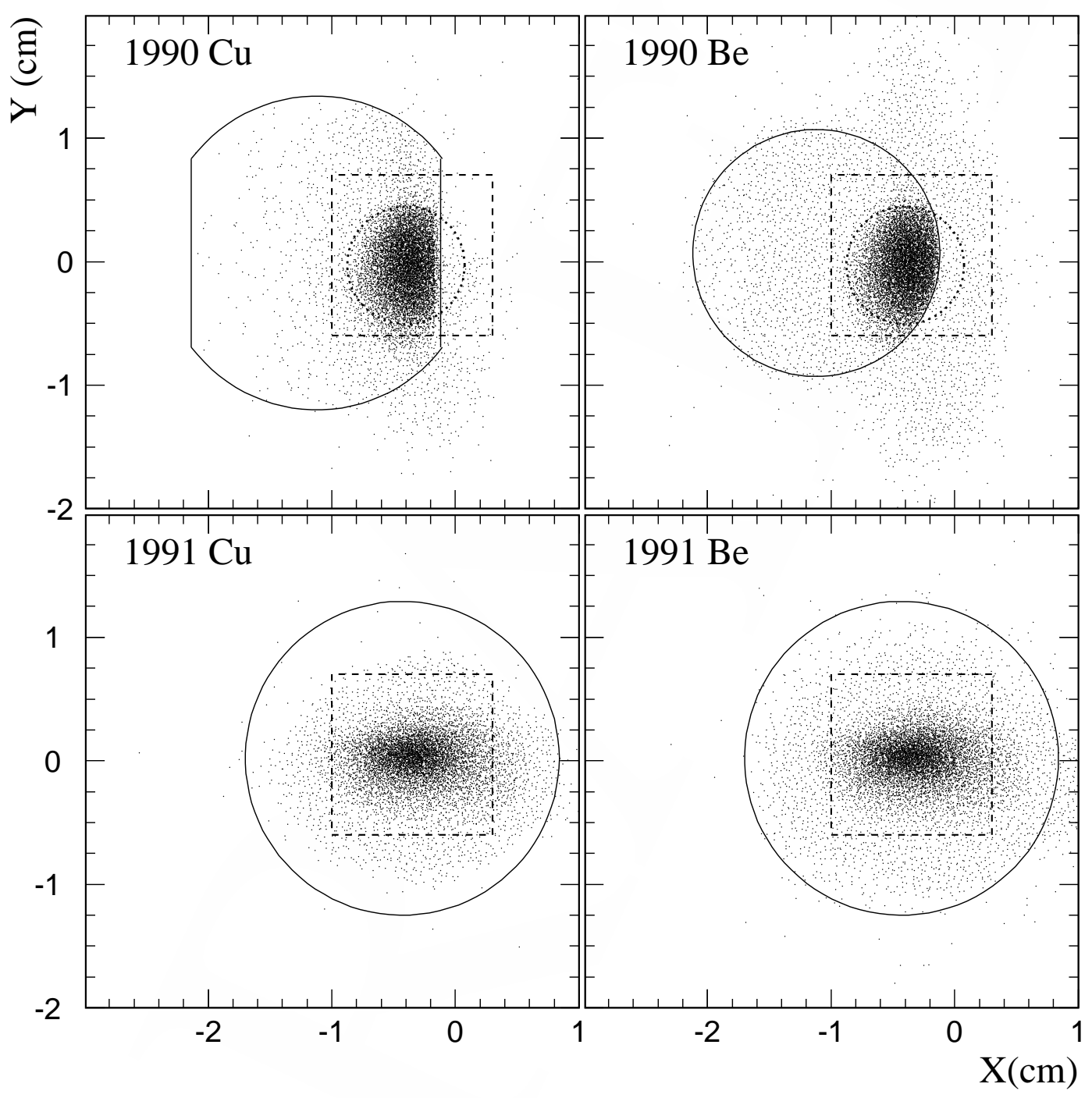

Figure 5.3 $X-Y$ distribution of vertices in the Copper and Beryllium targets in the $1990515 \mathrm{GeV} / c \pi^{-}$data and the $1991530 \mathrm{GeV} / c$ proton beam data for events containing $\gamma \gamma$ pairs with invariant mass within the $\pi^{0}$ signal region and with $p_{T}>3.5 \mathrm{GeV} / c$. The vertices outside the $\mathrm{Cu}$ and Be target area in the 1990 data are primarily due to interactions in the Rohacell target stand. 


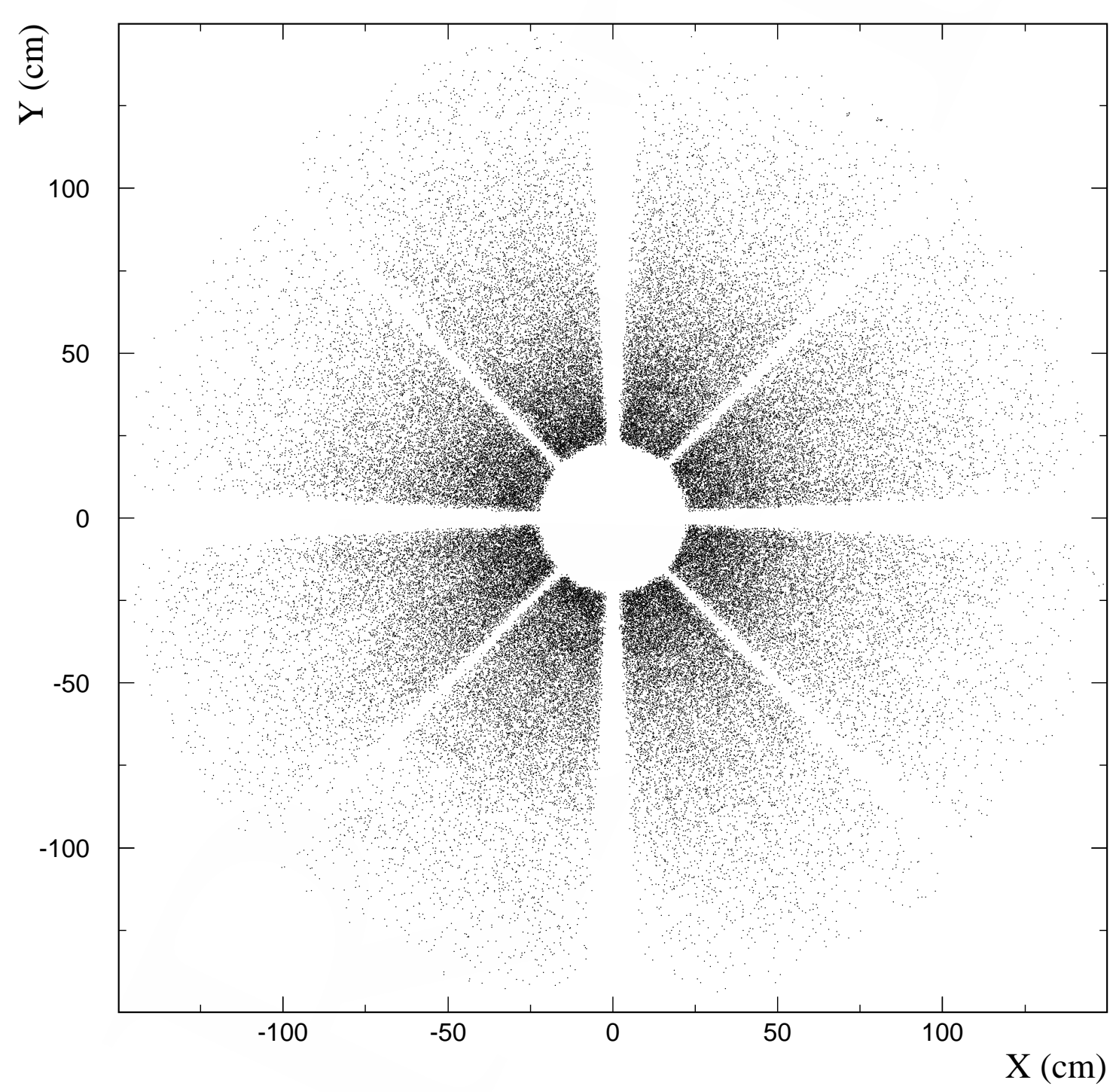

Figure 5.4 $X-Y$ position of photons contained within the EMLAC fiducial volume. The photons are from $\gamma \gamma$ pairs with invariant mass within the $\pi^{0}$ signal region and $p_{T}>5 \mathrm{GeV} / c$ in the $1990 \pi^{-}$data. 
The correction for the fiducial requirement was called the EMLAC geometric acceptance correction. The EMLAC's geometric acceptance was determined from a simple geometrical Monte Carlo simulation. For $\pi^{0}$ 's, a sample of $\pi^{0} \rightarrow \gamma \gamma$ decays was generated on a $p_{T}$ and rapidity ${ }^{3}$ grid. Only $\pi^{0}$ 's whose photons had decay energy asymmetry (Equation 1.3) less than 0.75 were included in the simulation. ${ }^{4}$ The photons from the $\pi^{0}$ decay were projected to the front face of the EMLAC and checked to see if they satisfied the EMLAC fiducial requirement. The ratio of the number of $\pi^{0}$ 's in which both photons passed the fiducial requirement to the total number of generated $\pi^{0}$ 's is the EMLAC's geometric acceptance. An analogous procedure was used to evaluate the geometric acceptance for $\eta$ 's and single photons. The geometric acceptance for $\pi^{0}$ 's, $\eta$ 's, and single photons is shown in Figure 5.5 as a function of $y_{l a b}$ for several $p_{T}$ intervals for the $800 \mathrm{GeV} / c$ $\mathrm{p}$ beam data. The acceptance is similar for the other data samples.

\subsection{Hadron Rejection}

Background contributions to the direct photon signal due to interactions of charged hadrons (and electrons) in the EMLAC were suppressed by imposing a distance to nearest track (DTRK) requirement on direct photon candidates. The DTRK distribution for reconstructed showers with $p_{T}>1.0 \mathrm{GeV} / c$ is shown in Figure 5.6. Showers with DTRK $<1.0 \mathrm{~cm}$ were considered to be likely from charged particles and were excluded from the direct photon candidate sample.

3 The rapidity, $y$, is defined as $y \equiv \frac{1}{2} \ln \left(\frac{E+p_{z}}{E-p_{z}}\right)$, where $E$ is the particle energy and $p$ is the momentum. In this thesis, unless otherwise specified, $y$ refers to the rapidity in the center of mass frame of reference. The relation between the rapidity in the center of mass and laboratory frames of reference is given by $y=y_{\text {lab }}-y_{\text {boost }}$, where $y_{\text {boost }}=3.50$, for the $515 \mathrm{GeV} / c \pi^{-}$beam, and 3.51 (3.72), for the 530 (800) $\mathrm{GeV} / c$ p beam.

4 The motivation behind this asymmetry requirement is discussed in Section 5.6. 


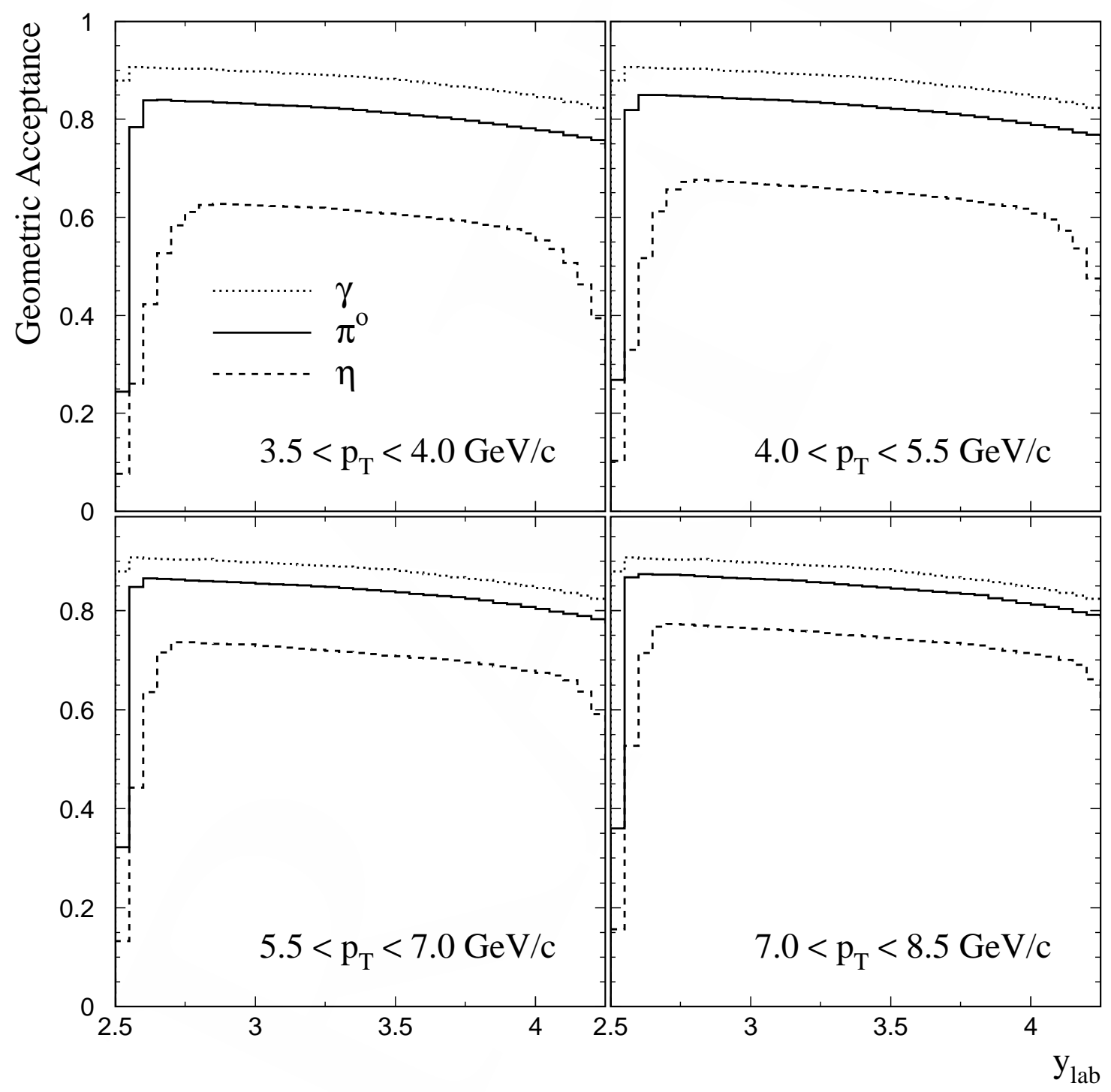

Figure 5.5 Geometric acceptance for single photons, $\pi^{0}$ 's, and $\eta$ 's versus $y_{l a b}$ for several different $p_{T}$ bins. 


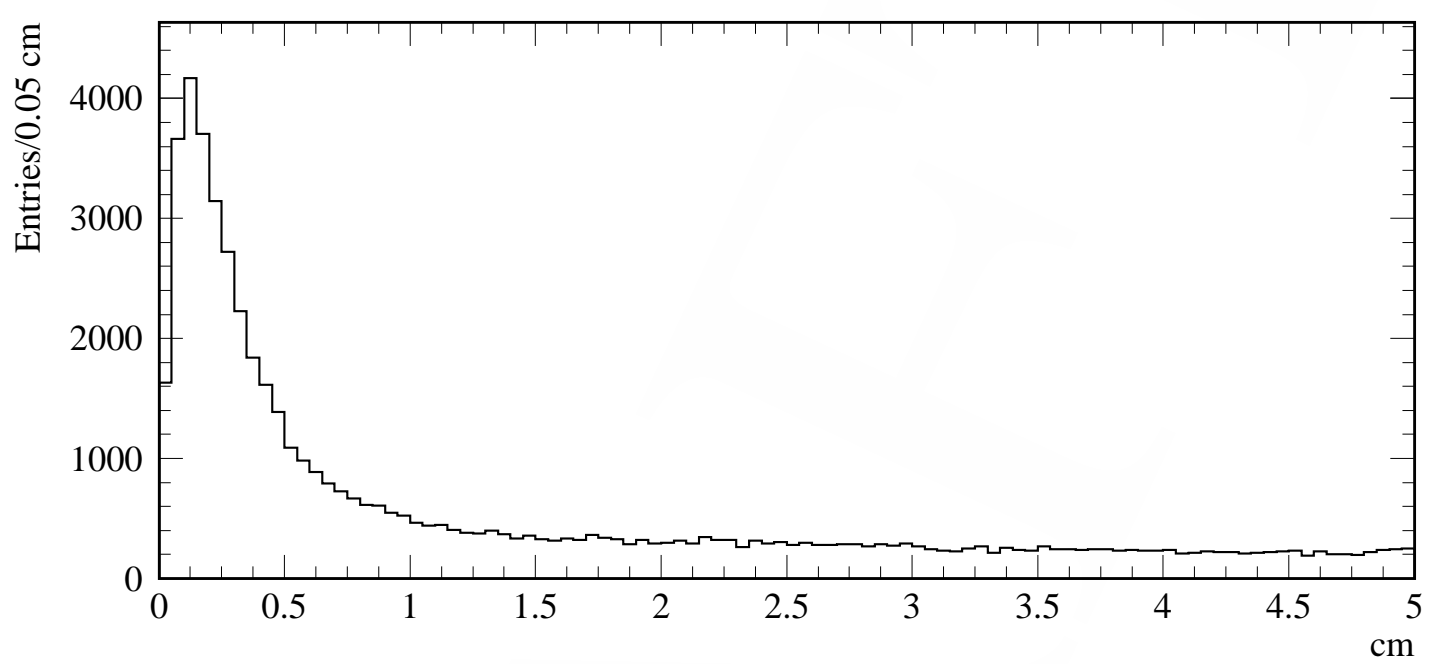

Figure 5.6 The distance to nearest track distribution for showers reconstructed in the EMLAC with $p_{T}>1.0 \mathrm{GeV} / c$.

The correction for the DTRK requirement was evaluated using the $\pi^{0}$ signal, since any losses resulting from the application of the DTRK requirement can be attributed to incidental track and shower overlap. The ratio of the $\pi^{0}$ cross section measured with the DTRK requirement to the cross section measured without it provided the correction factor for $\pi^{0}$ 's. This ratio varied with rapidity, since the spacial density of particles increases as the rapidity increases. In backward regions of rapidity the correction fractor was $\approx 2 \%$. At forward rapidity, it increased to $\approx 4 \%$. The correction for the losses incurred to direct photons was taken as the square root of the correction for $\pi^{0}$ 's.

Information on the longitudinal development of the shower was used to suppress the background from long lived neutral hadrons such as neutrons and 
$K_{L}^{0}$ 's. The ratio of the energy in the front section of the EMLAC to the total energy due to an individual incident particle, $E_{\text {front }} / E_{\text {total }}$, is shown in Figure 5.7 for electromagnetic showers and for hadronic showers. The electromagnetic showers are from a sample of electrons. ${ }^{5}$ The hadronic showers were selected by using the signal $K_{S}^{0} \rightarrow \pi^{+} \pi^{-}$, where the $K_{S}^{0}$ was identified through the invariant mass of the $\pi^{+} \pi^{-}$pair as measured by the tracking system. The tracks were then projected to the EMLAC and spatially matched with showers. Candidate photons contributing to the direct photon and neutral meson analysis were required to have $E_{\text {front }} / E_{\text {total }}>0.2$. The correction for this requirement was determined using a detailed Monte Carlo simulation of the EMLAC response (Section 6.3.2) and was found to range from $\approx 1 \%$, at moderate values of $p_{T}$, to $\approx 1.5 \%$, at high values of $p_{T}$. This correction was absorbed into the direct photon and neutral meson reconstruction efficiencies (Section 6.3.5).

\subsection{Rejection of Beam Halo Muons}

A major source of background to the direct photon signal in the $515 \mathrm{GeV} / \mathrm{c}$ $\pi^{-}$and the $530 \mathrm{GeV} / c$ proton beam data was due to bremsstrahlung radiation from beam halo muons. These muons were typically produced far upstream of the target region and tended to travel approximately parallel to the beam. Therefore, when they interacted in the EMLAC, they were often misidentified as high $p_{T}$ showers, since the $p_{T}$ calculation assumed that the particle that produced the shower emanated from the target region. Beam halo muons were also a source of background in the $\pi^{0}$ analysis. The reason for this follows. Since beam halo muons do not emanate from the target region, muon induced showers are distorted relative to showers from the target region. Due to this distortion, muon showers

\footnotetext{
${ }^{5}$ The identification of electrons in the data is described in Section 5.8.
} 


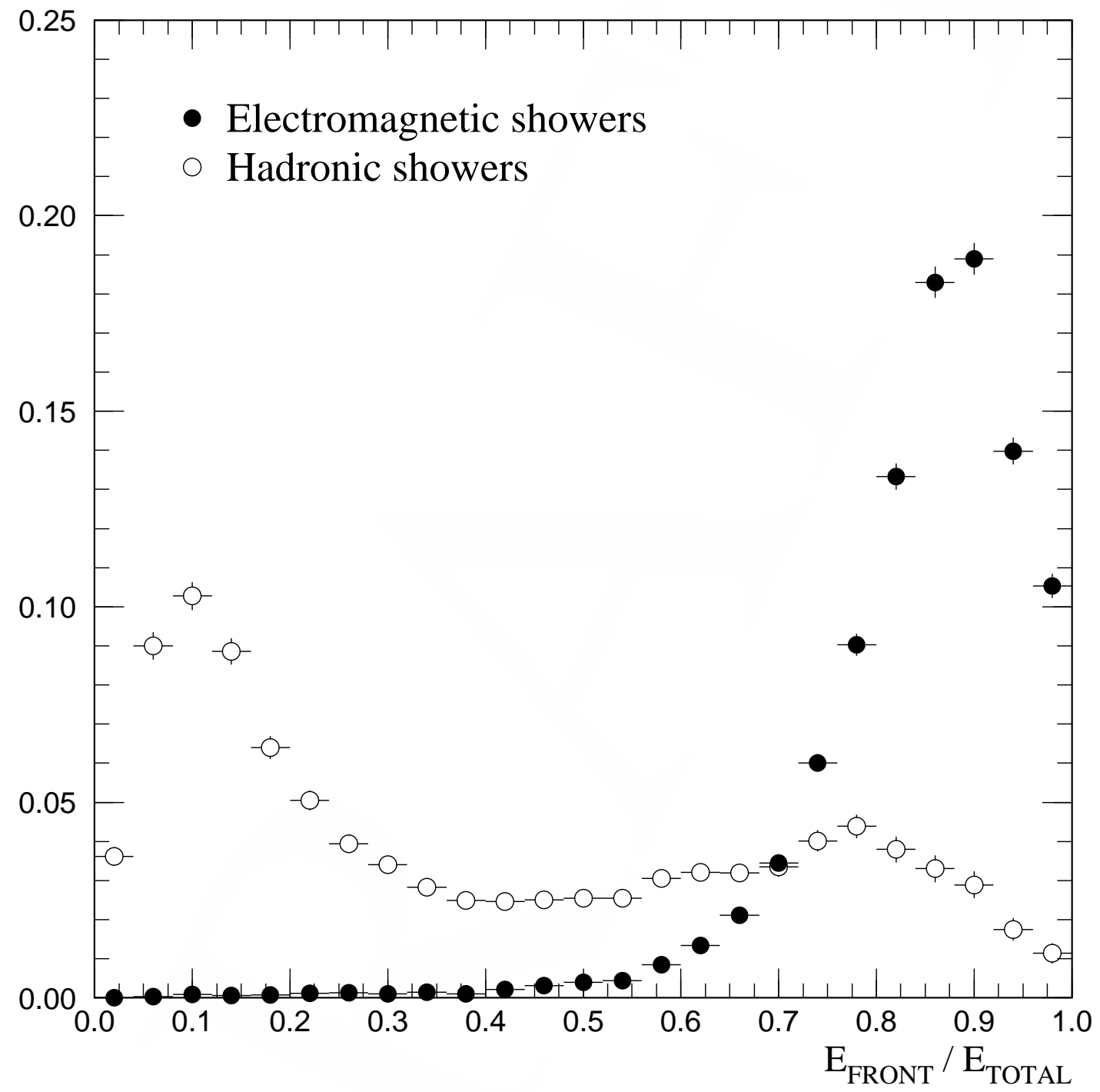

Figure 5.7 $E_{\text {front }} / E_{\text {total }}$ distributions for electromagnetic showers and hadronic showers. 
were occasionally split by EMREC into two closely separated GAMMA's, which tended to form low mass $\gamma \gamma$ pairs.

Although the Meson West beamline was equipped with spoiler magnets to deflect muons, and the pretrigger logic used the signals from the veto walls to reject events associated with beam halo muons, additional measures were required in the offline analysis to completely eliminate beam halo muons from the data sample. In Figure 5.8, the low mass region of the $\gamma \gamma$ invariant mass spectrum is shown both before and after the application of these measures. Figure 5.8a shows the $\gamma \gamma$ invariant mass distribution in the region of the $\pi^{0}$ for $\gamma \gamma$ mass pairs satisfying the target and EMLAC fiducial requirements, with energy asymmetry less than 0.75 , and with $p_{T}$ in the range $7<p_{T}<10 \mathrm{GeV} / c$. There is a large peak at low mass and the $\pi^{0}$ mass peak is barely discernable. However, as the beam halo muon rejection criteria are applied (Figures 5.8b-f), the low mass peak disappears and a very significant $\pi^{0}$ mass peak is revealed. These muon rejection criteria are described below.

\subsubsection{Veto Wall Requirement}

Although the pretrigger vetoed events in which the veto walls registered coincidences in the triggering quadrant (Section 3.2.2), there was some inefficiency in the online veto wall requirement due to the relatively tight timing windows imposed on the coincidence of the signals from the veto walls (and the challenge of establishing the proper timing). Therefore, to improve the rejection efficiency, the veto wall requirement was recreated offline to allow for a $\pm 1 \mathrm{RF}$ bucket jitter between the signals from the veto wall elements. In addition, it was found that further beam halo muon rejection was possible if the timing window for the veto wall signals with respect to the interaction time was expanded from the online requirement of $\pm 3 \mathrm{RF}$ buckets to $\pm 5 \mathrm{RF}$ buckets. 


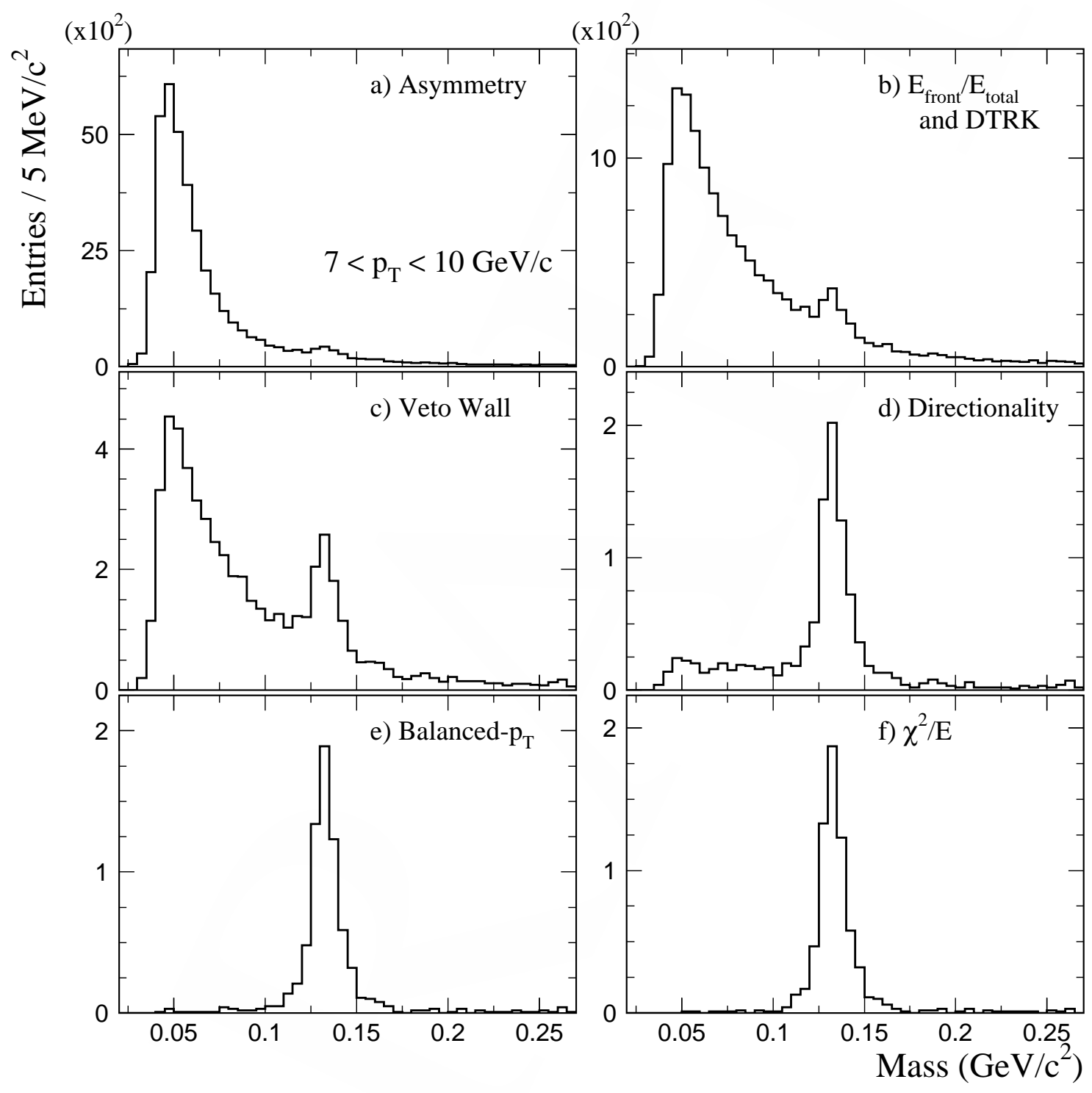

Figure 5.8 The effect of the muon rejection requirements on the $\gamma \gamma$ invariant mass distribution in the vicinity of the $\pi^{0}$ for $515 \mathrm{GeV} / c \pi^{-}$beam data. Each subsequent plot includes all the requirements from the previous plots. 


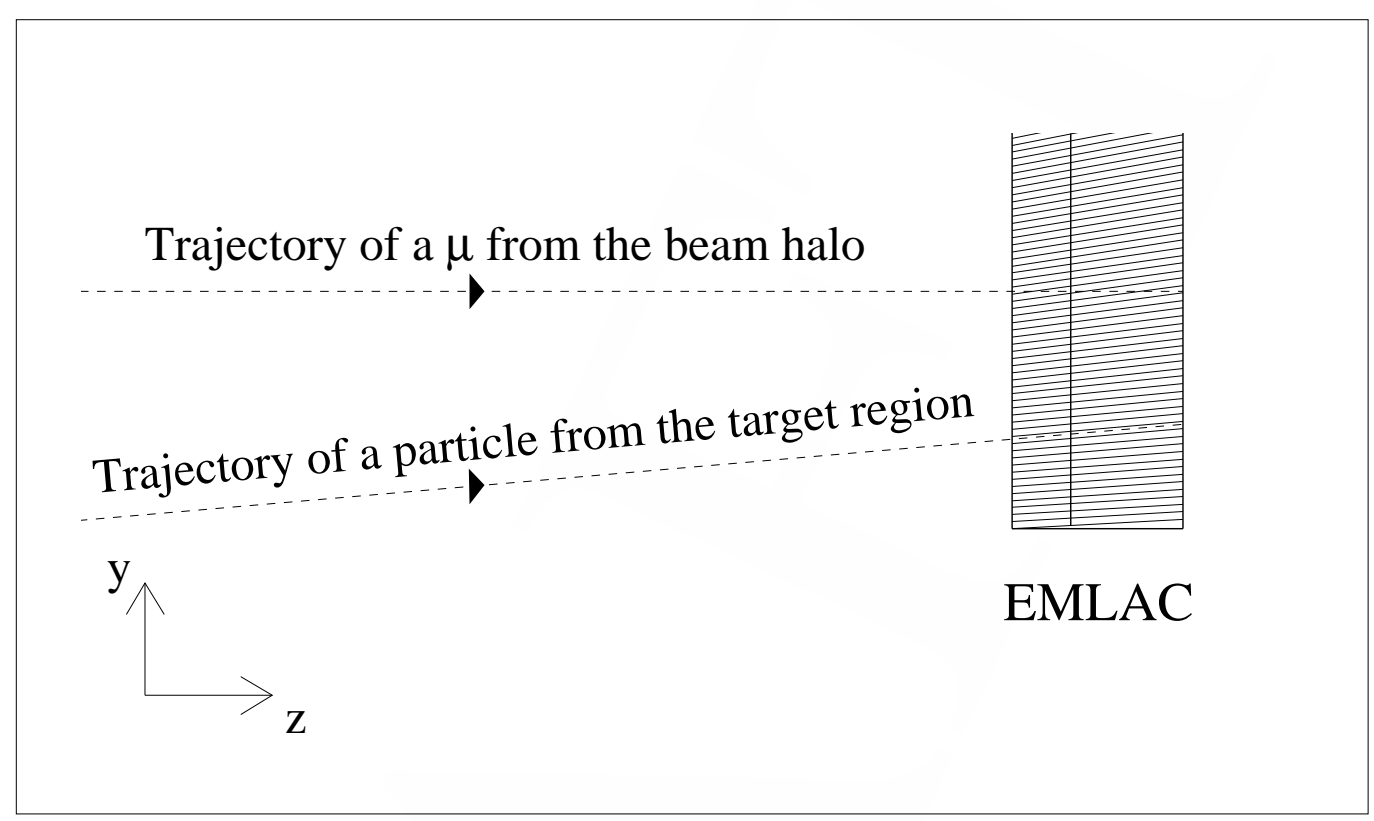

Figure 5.9 Use of focusing of the EMLAC radial strips to discriminate against showers induced by muons from the beam halo.

\subsubsection{Directionality Requirement}

The separate readout and offline reconstruction of the signals from the front and back sections of the EMLAC was used to discriminate against showers produced by beam halo muons. Because the radial strips of the EMLAC are focused upon the target region, showers resulting from particles emanating from this region will be roughly centered on the same sequential radial strip in the front and back sections. This is not expected to be the case for showers from beam halo muons, since their trajectories do not follow the focusing of the EMLAC radial strips (see Figure 5.9). 
To quantify this characteristic, a directionality parameter was defined,

$$
\delta \equiv R_{f}-\frac{Z_{f}^{L A C}}{Z_{b}^{L A C}} R_{b},
$$

where $R_{f}\left(R_{b}\right)$ is the radial position of the shower as measured in the front (back) section, and $Z_{f}^{L A C}\left(Z_{b}^{L A C}\right)$ is the $Z$ position of the first EMLAC cell in the front (back) section. Showers produced by particles coming from the target region are expected to have distributions in $\delta$ centered about zero, while showers produced by beam halo muons are expected to have distributions in $\delta$ centered about a value of delta greater than zero. Directionality distributions for two classes of showers with $p_{T}>5 \mathrm{GeV} / c$ are shown in Figure 5.10. One class is a beam halo muon enriched sample, which was obtained by requiring the veto wall requirement to fail in the quadrant containing the sample. The other class of showers is a photon enriched sample. It was obtained by applying all the muon rejection criteria, except the directionality requirement, on the showers.

The directionality requirement was a function of radius. Showers with directionality

$$
\begin{array}{ll}
\delta>0.193 & \text { for } R<40.175 \mathrm{~cm}, \text { or } \\
\delta>0.0048 \times R & \text { for } R \geq 40.175 \mathrm{~cm},
\end{array}
$$

were considered to be likely from beam halo muons, and thus were excluded from the direct photon and neutral meson candidate samples.

\subsection{3 $\chi^{2}$ Requirement}

The fact that beam halo muons do not emanate from the target region can be exploited further. The electromagnetic shower shape was determined for photons coming from the target region. Consequently, showers resulting from beam halo muons are poorly fit by the shower shape, particularly in the R-view. The $\chi^{2}$ of 

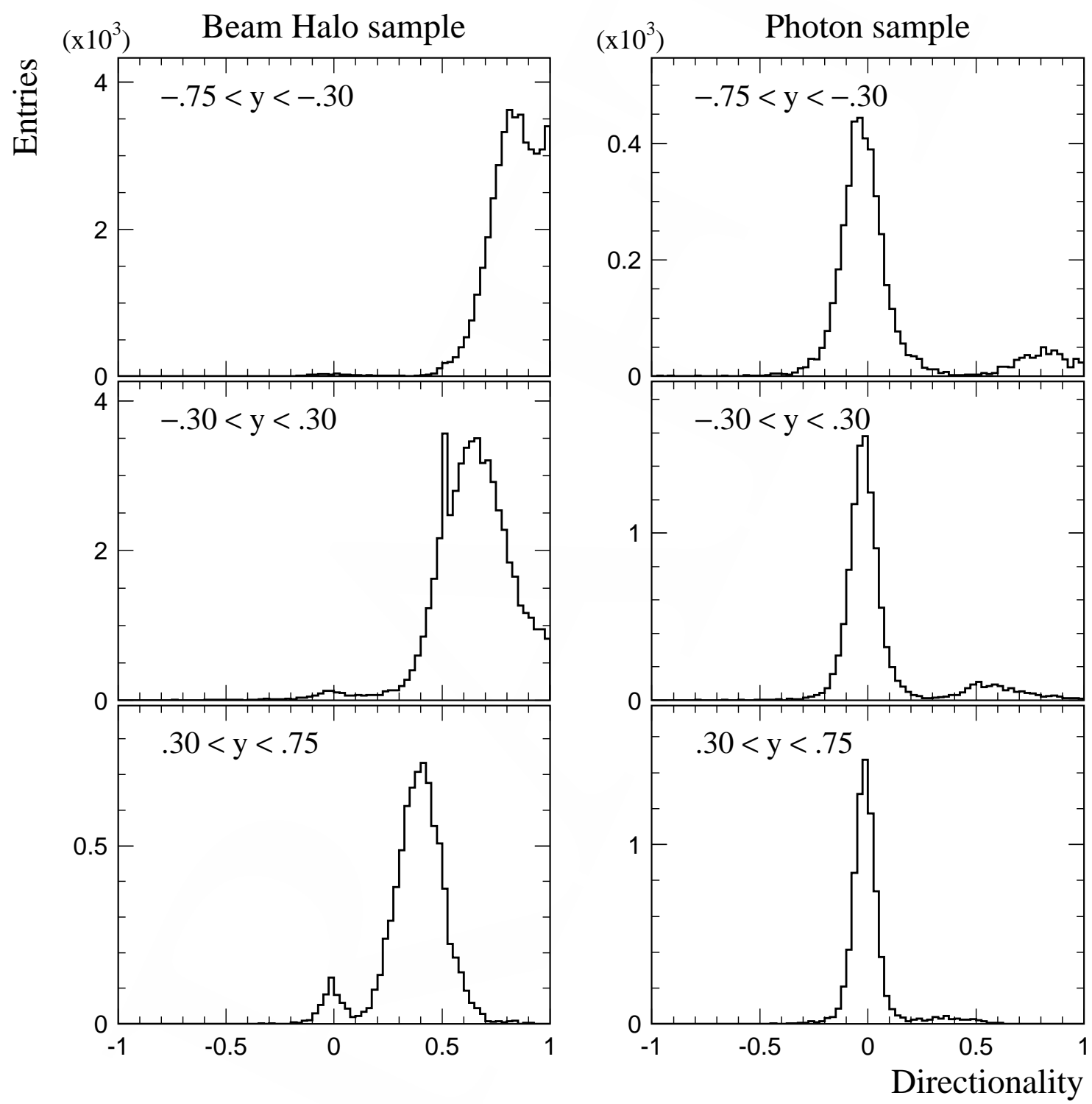

Figure 5.10 Directionality distributions for beam halo muons and photons in the $1990515 \mathrm{GeV} / c \pi^{-}$data for showers with $p_{T}>5 \mathrm{GeV} / c$. The beam halo muon sample was obtained by requiring the veto wall requirement to fail in the quadrant of the reconstructed shower. The photon sample was obtained by imposing all the muon rejection criteria on the showers, with the exception of the directionality requirement. 
the fit in the R-view therefore provided further discrimination against beam halo muons. Figure 5.11 shows the $\chi_{R}^{2} / \mathrm{E}$ distributions for a muon rich sample and a photon rich sample for three rapidity ranges. Candidates with $\chi_{R}^{2} / \mathrm{E}>0.1$ were attributed to beam halo muons and were rejected.

\subsubsection{Balanced $p_{T}$ Requirement}

The last requirement used to reject beam halo muons from the direct photon and neutral meson data samples was the balanced $p_{T}$ requirement. In real high $p_{T}$ interactions, the net $p_{T}$ in the trigger hemisphere of the EMLAC should be roughly balanced by the net $p_{T}$ in the opposite, or away-side, hemisphere. However, for events triggered by beam halo muons, the $p_{T}$ in the trigger hemisphere is expected to be much larger than the $p_{T}$ in the away-side hemisphere, since the event accompanying the beam halo muon is typically a soft (low $p_{T}$ ) interaction uncorrelated with the beam halo muon responsible for generating the trigger $p_{T}$.

The away-side $p_{T}, P_{T}^{\text {away }}$, was calculated by summing the $p_{T}$ 's of all the reconstructed tracks and photons which landed inside a $120^{\circ}$ cone opposite the direct photon or meson candidate. The reconstructed tracks and photons used in this calculation were required to have $p_{T}>0.3 \mathrm{GeV} / c$. The reconstructed tracks were also required to have total momentum $<250 \mathrm{GeV} / c$. The fraction $P_{T}^{\text {away }} / p_{T}$, where $p_{T}$ represents the $p_{T}$ of the direct photon or meson candidate, should be near zero for triggers induced by beam halo muons, and should be near one for

triggers induced by real direct photons or mesons. Figure 5.12 shows the $P_{T}^{a w a y} / p_{T}$ distribution for a muon rich sample and a photon rich sample for three rapidity ranges. Candidates where $P_{T}^{\text {away }} / p_{T}<0.3$ were considered likely to be beam halo muons and were rejected. 

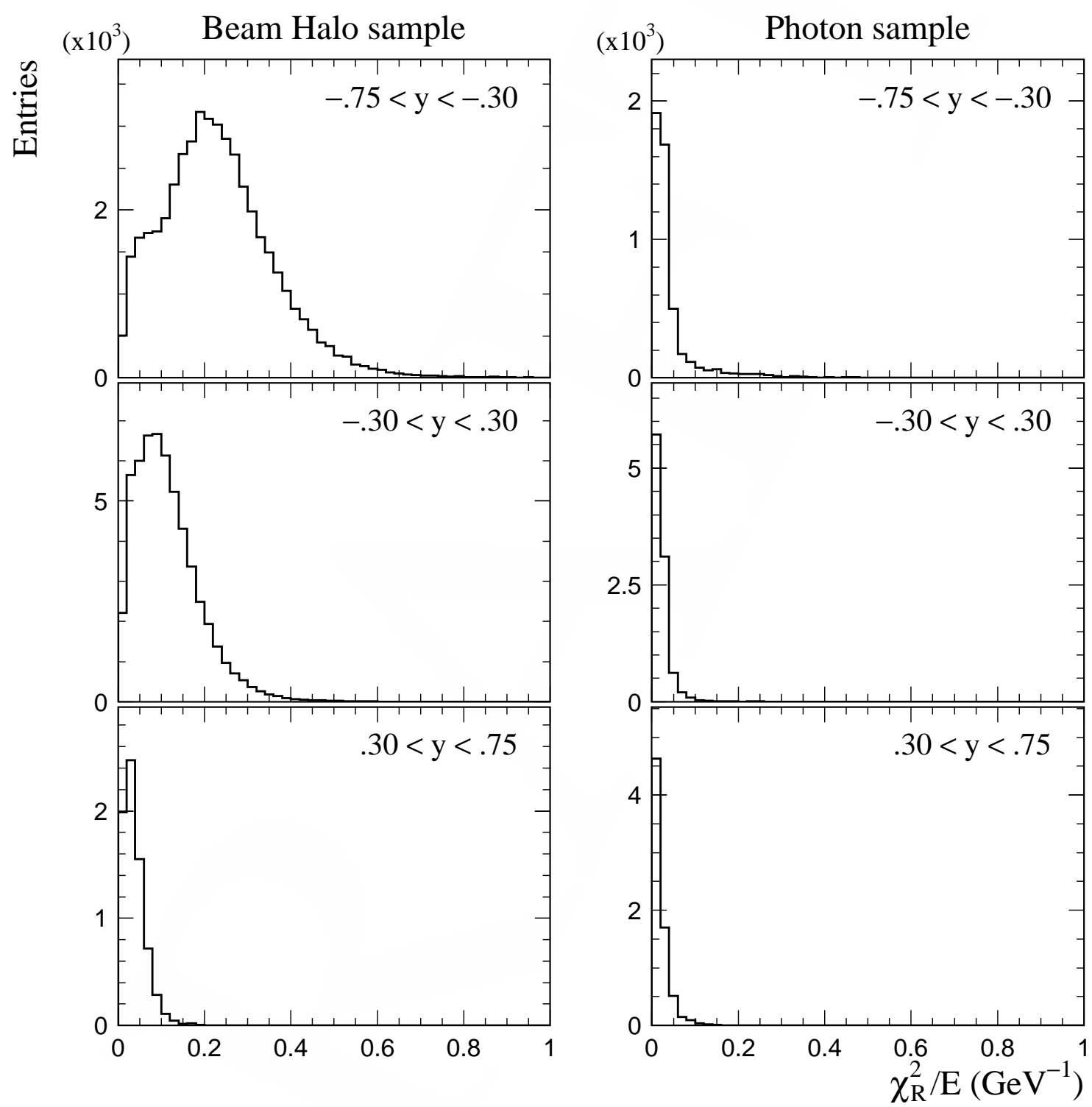

Figure $5.11 \chi_{R}^{2} / \mathrm{E}$ distributions for beam halo muons and photons in the 1990515 $\mathrm{GeV} / c \pi^{-}$data for showers with $p_{T}>5 \mathrm{GeV} / c$. The beam halo muon sample was obtained by requiring the veto wall requirement to fail in the quadrant of the reconstructed shower. The photon sample was obtained by imposing all the muon rejection criteria on the showers, with the exception of the $\chi^{2}$ requirement. 

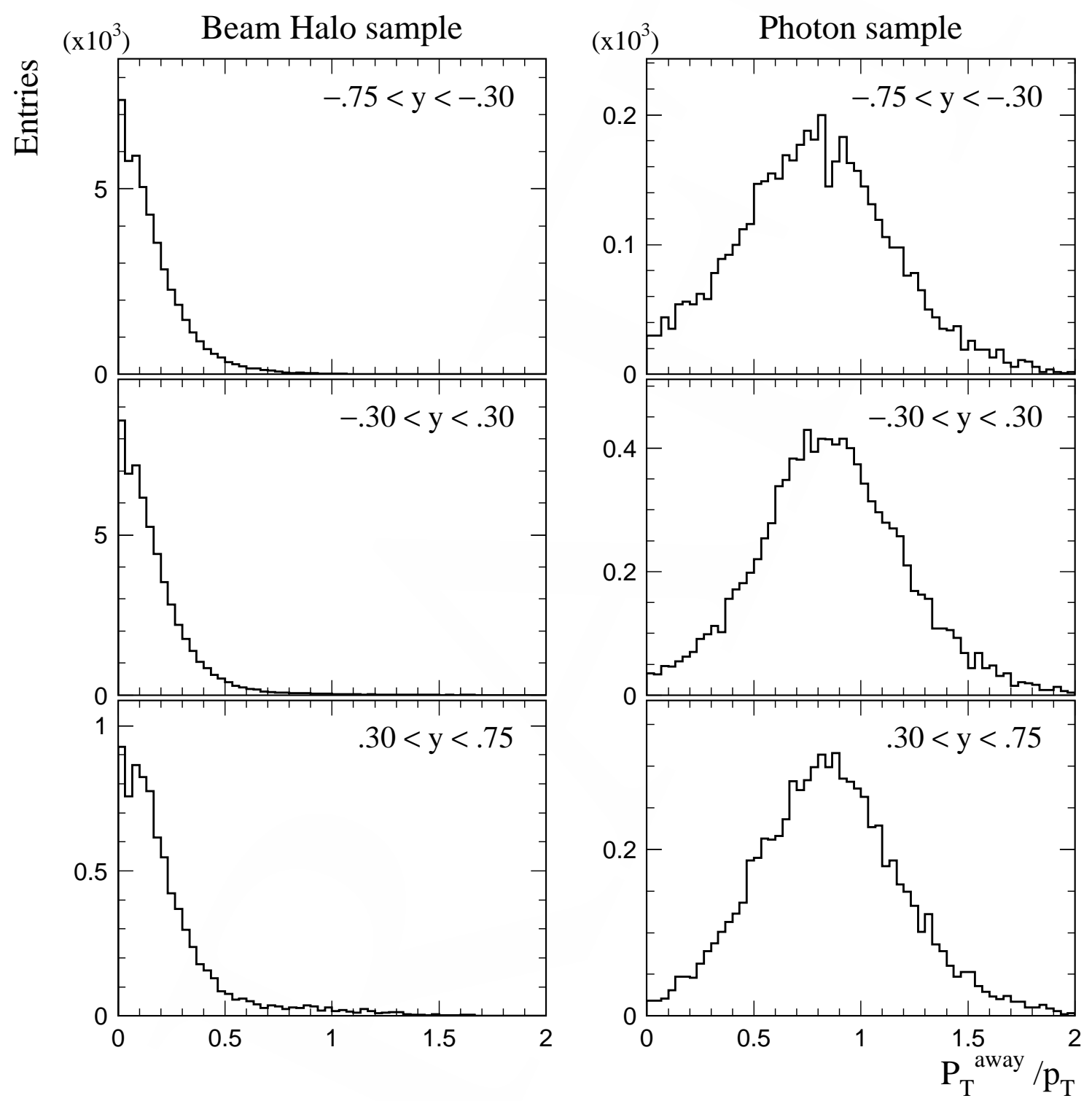

Figure 5.12 $P_{T}^{\text {away }} / p_{T}$ distributions for beam halo muons and photons in the 1990 $515 \mathrm{GeV} / c \pi^{-}$data for showers with $p_{T}>5 \mathrm{GeV} / c$. The beam halo muon sample was obtained by requiring the veto wall requirement to fail in the quadrant of the reconstructed shower. The photon sample was obtained by imposing all the muon rejection criteria on the showers, with the exception of the balanced $p_{T}$ requirement. 


\subsubsection{Corrections for Muon Requirements}

The application of the beam halo muon rejection requirements also resulted in rejection of some real direct photons and neutral mesons. To account for these losses, a separate correction factor was determined for each of the muon rejection requirements. In the case of the veto wall requirement, the same correction was used for single photons and neutral mesons. Each EMLAC quadrant had its own veto wall correction factor, and these factors varied somewhat for each data sample. For the other requirements, separate corrections for single photons and neutral mesons were necessary. These corrections were determined from a "pure" single photon or neutral meson sample which was obtained by applying harsh versions of all the muon rejection requirements except for the requirement in question. The fraction of signal lost by the application of the requirement was taken as the correction for that requirement. These corrections were functions of the single photon, or neutral meson, $p_{T}$ and rapidity. In the case of neutral mesons, the product of the corrections for the muon rejection criteria was $\approx 1.08$ at $p_{T}=4 \mathrm{GeV} / c$ and increased to $\approx 1.10$ at $p_{T}=7 \mathrm{GeV} / c$. For single photons, the product of the corrections was $\approx 1.08$ at $p_{T}=4 \mathrm{GeV} / c$ and decreased to $\approx 1.02$ at $p_{T}=7 \mathrm{GeV} / c$.

\section{6 $\pi^{0}$ and $\eta$ Energy Asymmetry Requirement}

The $\pi^{0}$ and $\eta$ are pseudoscalar mesons. As such, they decay uniformly in $\cos \theta^{*}$, where $\theta^{*}$ is the angle between the line of flight of one of the photons from the meson decay in the rest frame of the meson and the line of flight of the meson (Figure 1.6). At high energy $(\beta \approx 1)$, this implies that the energy asymmetry distribution of the two photons from the decay is flat. However, experimentally it is difficult to measure $\pi^{0}$ 's and $\eta$ 's over the entire energy asymmetry range because of the difficulty of efficiently detecting extremely low 
energy photons (see Figure 5.16). To illustrate, Figure 5.13 shows the $\gamma \gamma$ invariant mass in the region of the $\pi^{0}$ for several energy asymmetry intervals. The number of reconstructed $\pi^{0}$ 's is fairly constant over the energy asymmetry range $0.0<A<0.8$. However, for energy asymmetries above 0.8 , the number of reconstructed $\pi^{0}$ 's drops substantially. In addition, for $A>0.8$, the $\pi^{0}$ mass peak is significantly broader and the signal-to-background ratio is considerably diminished. At large radius, the degradation of the $\pi^{0}$ signal begins at $A>0.75$. For this reason, photon pairs were required to have an $A<0.75$ to be considered as $\pi^{0}$ or $\eta$ candidates. Because of the flatness of the energy asymmetry distribution, this requirement has a simple $1 / 0.75$ correction factor.

\subsection{Photon Conversion Correction}

Photons with energy above $2 m_{e}$, where $m_{e}$ is the mass of the electron, can convert into an electron-positron pair in the presence of matter. The probability for conversion, $P_{c o n v}$, is approximately constant for photon energies above $1 \mathrm{GeV}$ and is given by [85]

$$
P_{\text {conv }}=1-e^{-7 X / 9}
$$

where $X$ is the thickness of the material in radiation lengths. This formula was used to correct for losses resulting from photon conversions. For each photon used in the neutral meson and direct photon analyses, the amount of material (in radiation lengths) the photon passed through was calculated. The probability of non-conversion, $1-P_{\text {conv }}$, was then calculated. The average value of the nonconversion probability for photons versus the $Z$ location of the photon production point is shown in Figure 5.14 for the 1990 and 1991 fixed target runs. The inverse of the non-conversion probability is the conversion correction and was applied to each photon as a weight. 

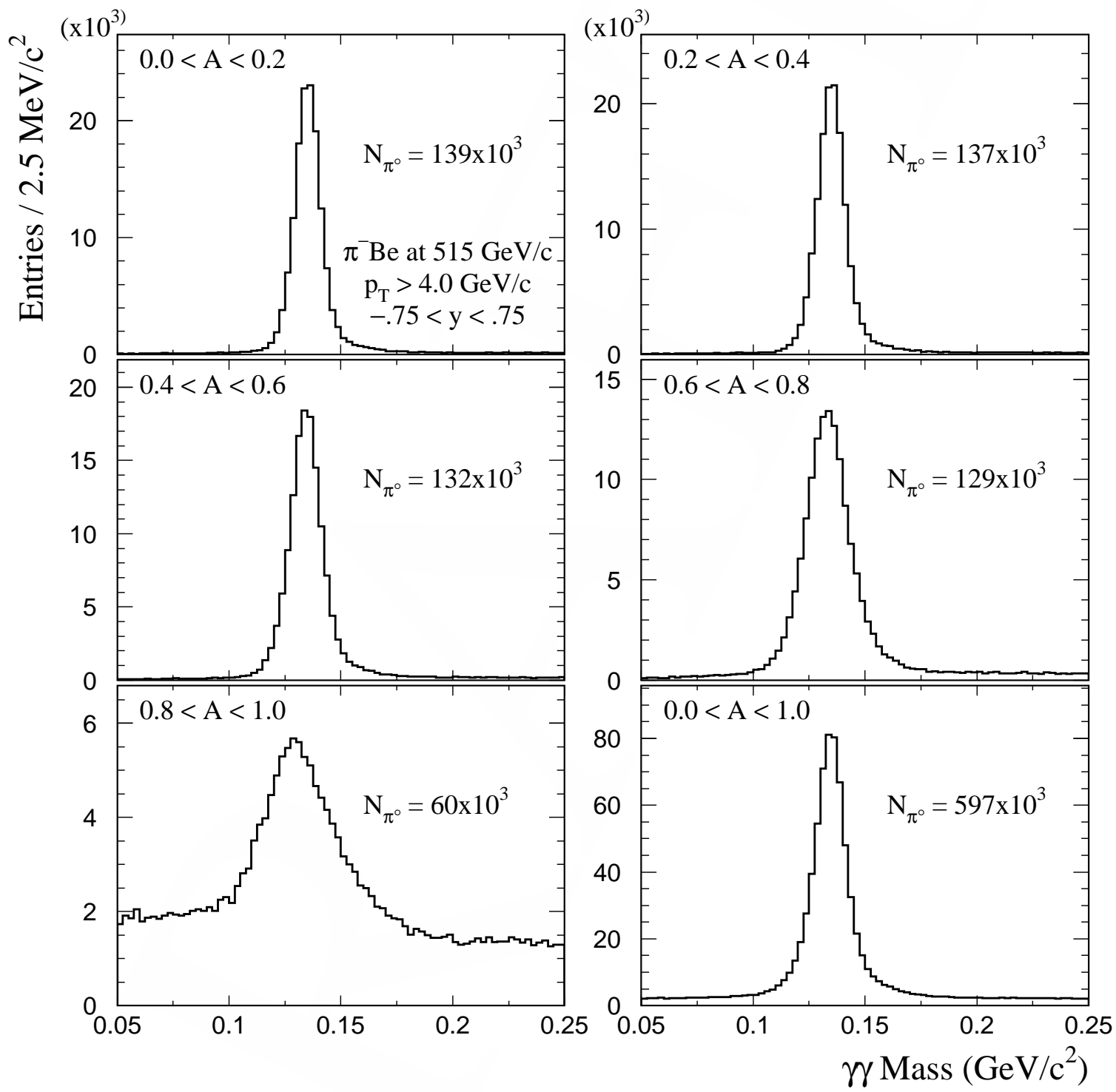

Figure 5.13 The $\gamma \gamma$ invariant mass distribution in the region of the $\pi^{0}$ for several energy asymmetry ranges. The number of $\pi^{0}$ 's was obtained using the sideband subtraction technique described in Section 5.11. 

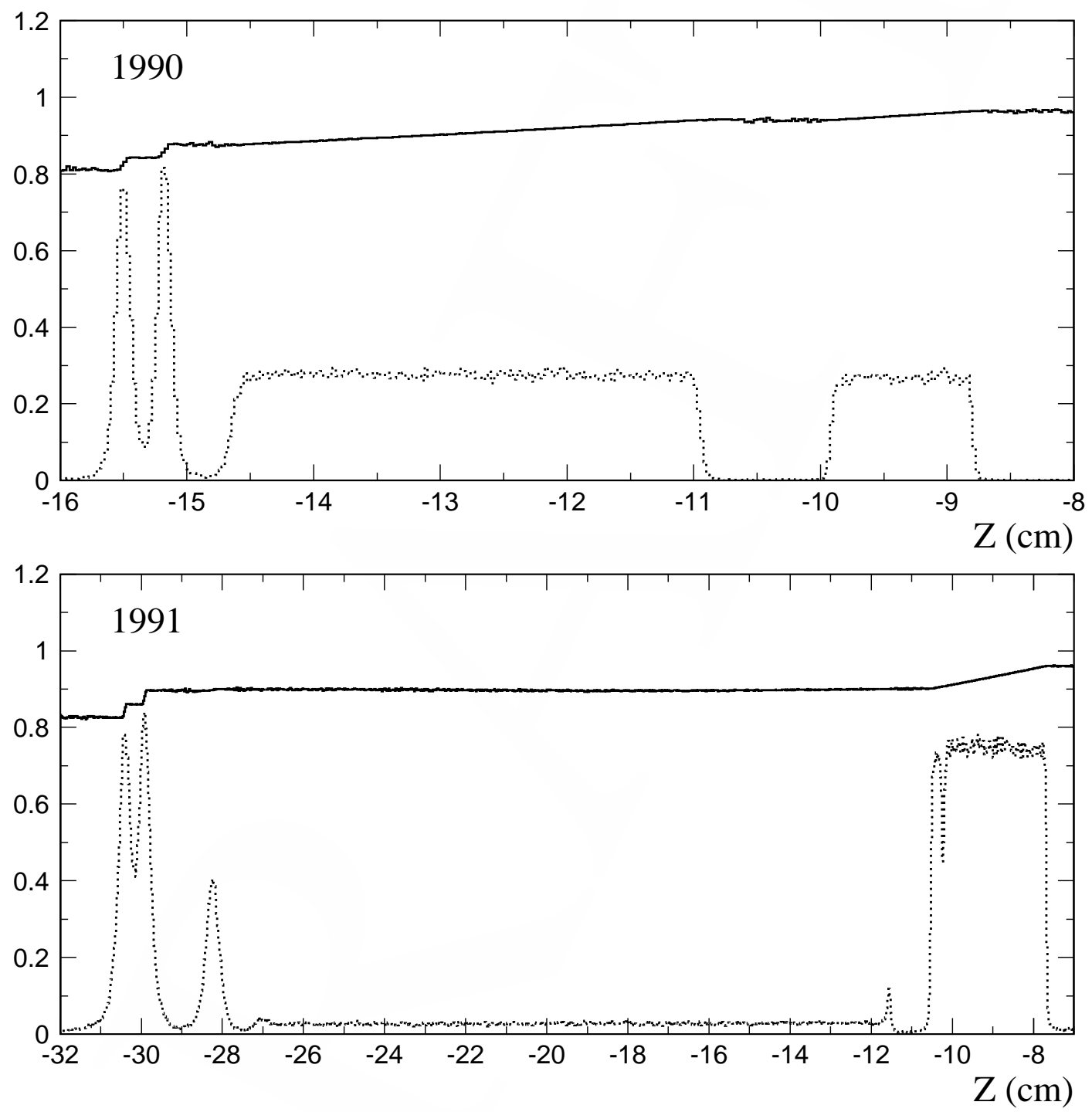

Figure 5.14 Average value of the photon non-conversion probability versus $Z$ for the 1990 (top) and 1991 (bottom) fixed target runs. Superimposed on the figures are the corresponding primary vertex distributions for these runs. 


\subsection{Electron Studies}

The study of electrons ${ }^{6}$ in the data can yield important information about the response of the EMLAC to photons since electromagnetic showers initiated by electrons have similar characteristics to showers initiated by photons, and the tracking system provides an independent measurement of the electron momentum.

The primary source of electrons in the data is from the two photon decay modes of the $\pi^{0}$ and $\eta$, where one of the photons from the decay converts into an electron-positron pair in the target. These electrons are typically referred to as zero mass pairs, or ZMP's, because of the small $\left(\approx 2 m_{e}\right)$ invariant mass of the pair. As a result of this low invariant mass, the opening angle between the ZMP electrons in the laboratory is very small, typically smaller than the angular resolution of the SSD system. This characteristic feature allows ZMP's to be readily identified in the data.

The algorithm for identifying a ZMP began by selecting pairs of oppositely charged physics tracks. Since the ZMP electrons travel nearly parallel to each other, and are not bent significantly in the $Y$ view by the magnet, the $Y$-view slopes of the tracks should be nearly equal. Therefore, the difference in $Y$-slope, $\Delta S_{y}$, of the pair was required to be less than $\pm 3 \mathrm{mr}$. Also, in the impulse approximation, the track trajectories downstream of the magnet in the $X$ view should intercept each other at the center of the magnet. The $Z$ position of the intersection, $Z X_{\text {int }}$, was therefore required to be less than $10 \mathrm{~cm}$ from the magnet center. In Figure 5.15, the effect of these cuts on pairs of oppositely charged tracks is shown. Also shown in the figure is the effect of requiring both tracks to match with showers in the EMLAC. A track was considered to match with a shower if

${ }^{6}$ In the context of this section, the term electron refers collectively to both electrons and positrons. 
its projection to the front face of the EMLAC came within one centimeter of a reconstructed shower's position.

One use of ZMP electrons is to estimate the EMLAC efficiency for detecting electromagnetic showers. To determine this efficiency, ZMP electrons were selected using the $\Delta S_{y}$ and $Z X_{i n t}$ requirements on pairs of charged tracks described above. Since most of these electrons come from the conversion of photons from $\pi^{0}$ decays, spurious ZMP background was reduced by using the $\gamma e^{+} e^{-}$invariant mass. Mass combinations were made between each ZMP candidate and the photons in the octant that the ZMP candidate intercepted. If the mass of the combination was within the $\pi^{0}$ signal region, the ZMP candidate was retained. Further reduction in the background was achieved by requiring one of the electrons to match with an electromagnetic shower. In addition, the shower was required to have strong electromagnetic characteristics - a high value of $E_{\text {front }} / E_{\text {total }}$ and $E / P$, where $E$ is the shower energy and $P$ is the track momentum, close to one. The efficiency was then determined by taking the other electron and seeing if it was spatially matched with a reconstructed shower. The efficiency measured in this manner is shown in Figure 5.16.

\subsection{EMLAC Energy Scale Calibration}

A precise calibration of the EMLAC energy response (energy scale) was important to this experiment because relatively small uncertainties in the EMLAC energy scale result in relatively large uncertainties in the measurement of the inclusive direct photon and neutral meson cross sections due to the steep $p_{T}$ dependence of these cross sections. To illustrate, the uncertainty in the $\pi^{0}$ cross section is shown in Figure 5.17 as a function of $p_{T}$ for three values of systematic energy scale uncertainty. 
$\left(\mathrm{x} 10^{3}\right)$

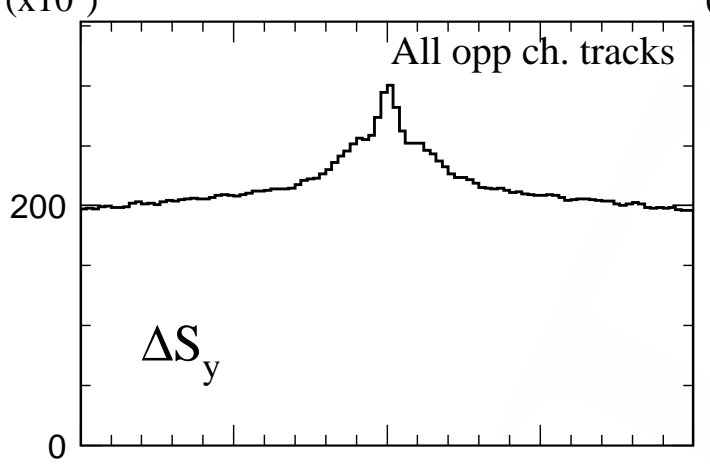

100

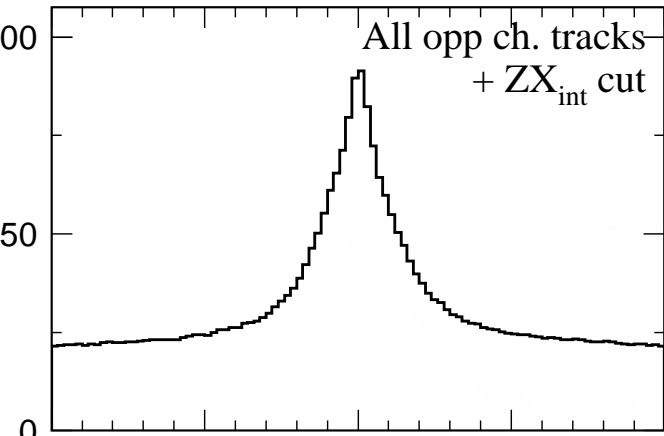

20

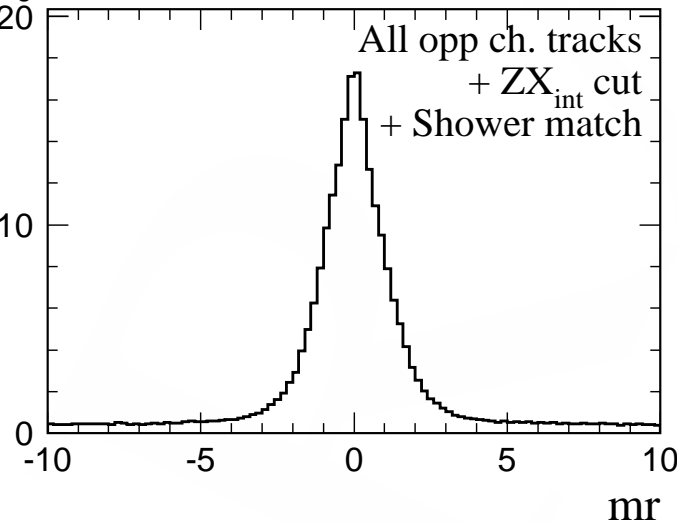

$\left(\mathrm{x} 10^{3}\right)$
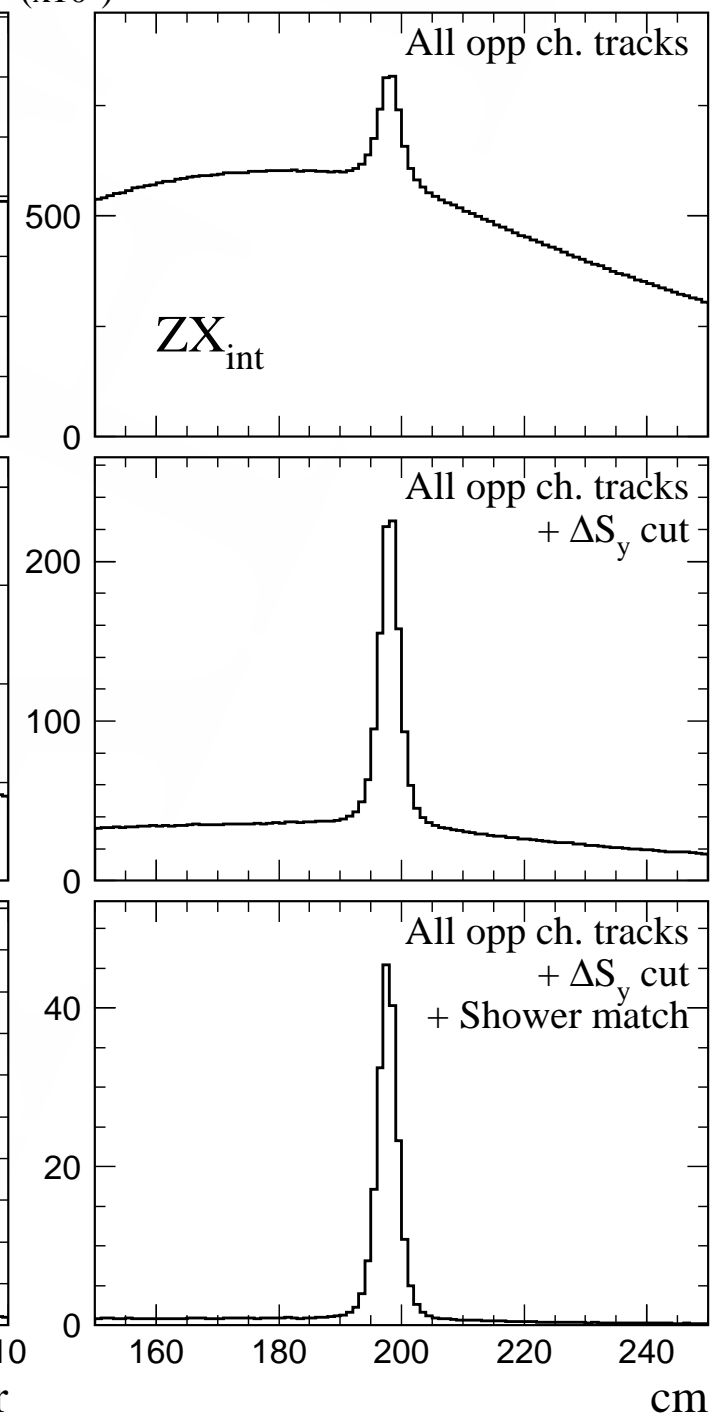

Figure 5.15 Effect of ZMP cuts on the $\Delta S_{y}$ and $Z X_{\text {int }}$ distributions of oppositely charged track pairs. The $\Delta S_{y}$ cut is $\pm 3 \mathrm{mr}$ and the $Z X_{\text {int }}$ cut is $\pm 10 \mathrm{~cm}$ from the magnet center. 


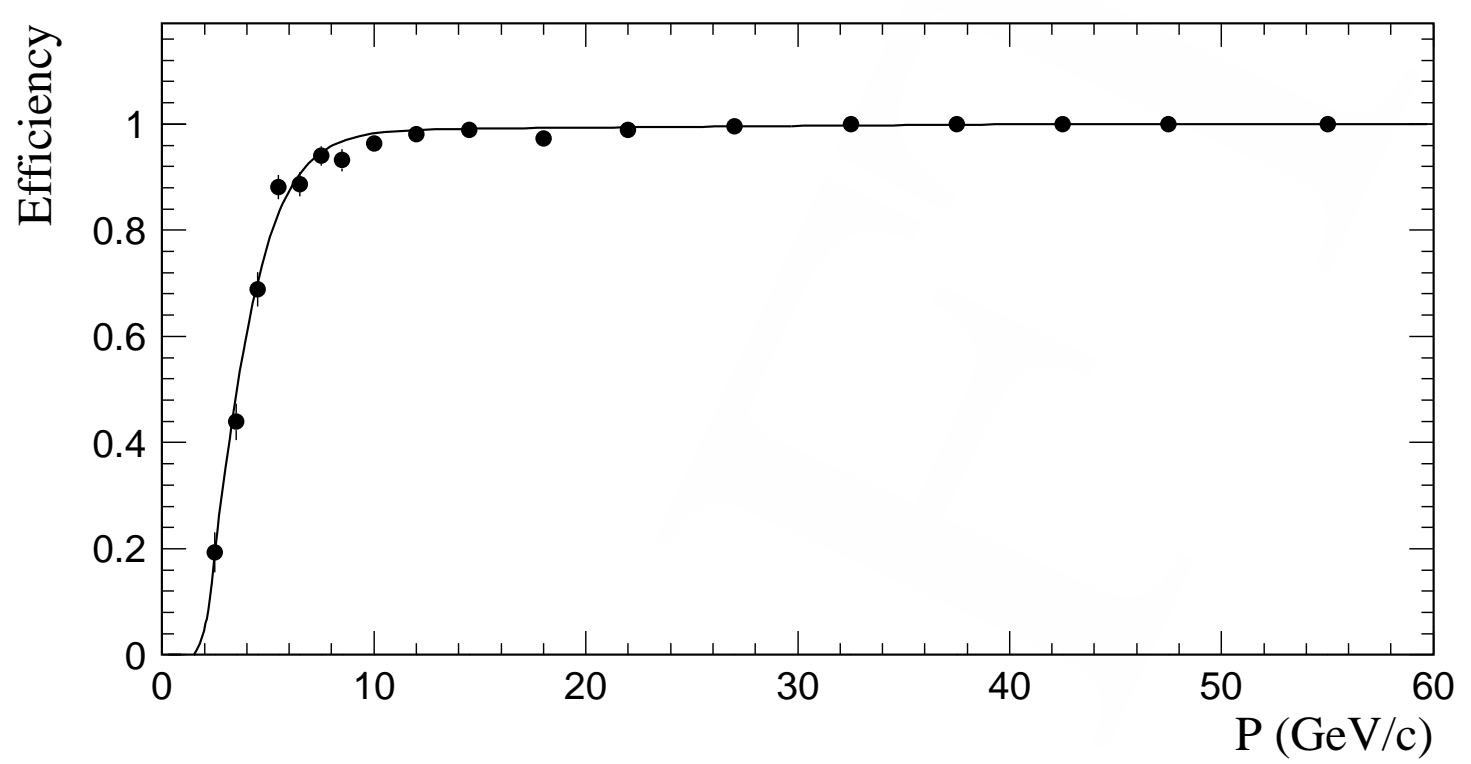

Figure 5.16 EMLAC efficiency for reconstructing an electron as a function of electron momentum. The solid curve represents a fit to these data.

As a result of a detailed analysis of the EMLAC energy scale [84, 86, 27], the systematic uncertainty in the energy scale was determined to be less than $0.5 \%$. The calibrated $\pi^{0}, \eta$, and $\omega$ masses are shown in Figure 5.18 for the 1991 data. The mean values of the masses agree with the world values [87] to well within the quoted EMLAC energy scale uncertainty. In the following sections, the salient features of the energy scale analysis are presented.

\subsubsection{Calibration Procedure}

After setting the initial scale for the EMLAC and correcting for the observed time dependence of the EMLAC response (Section 4.3.1), octant-to-octant variations in the EMLAC response were corrected. These variations $(\approx 5 \%)$ were attributed to differences in the construction and operation of each octant, and 


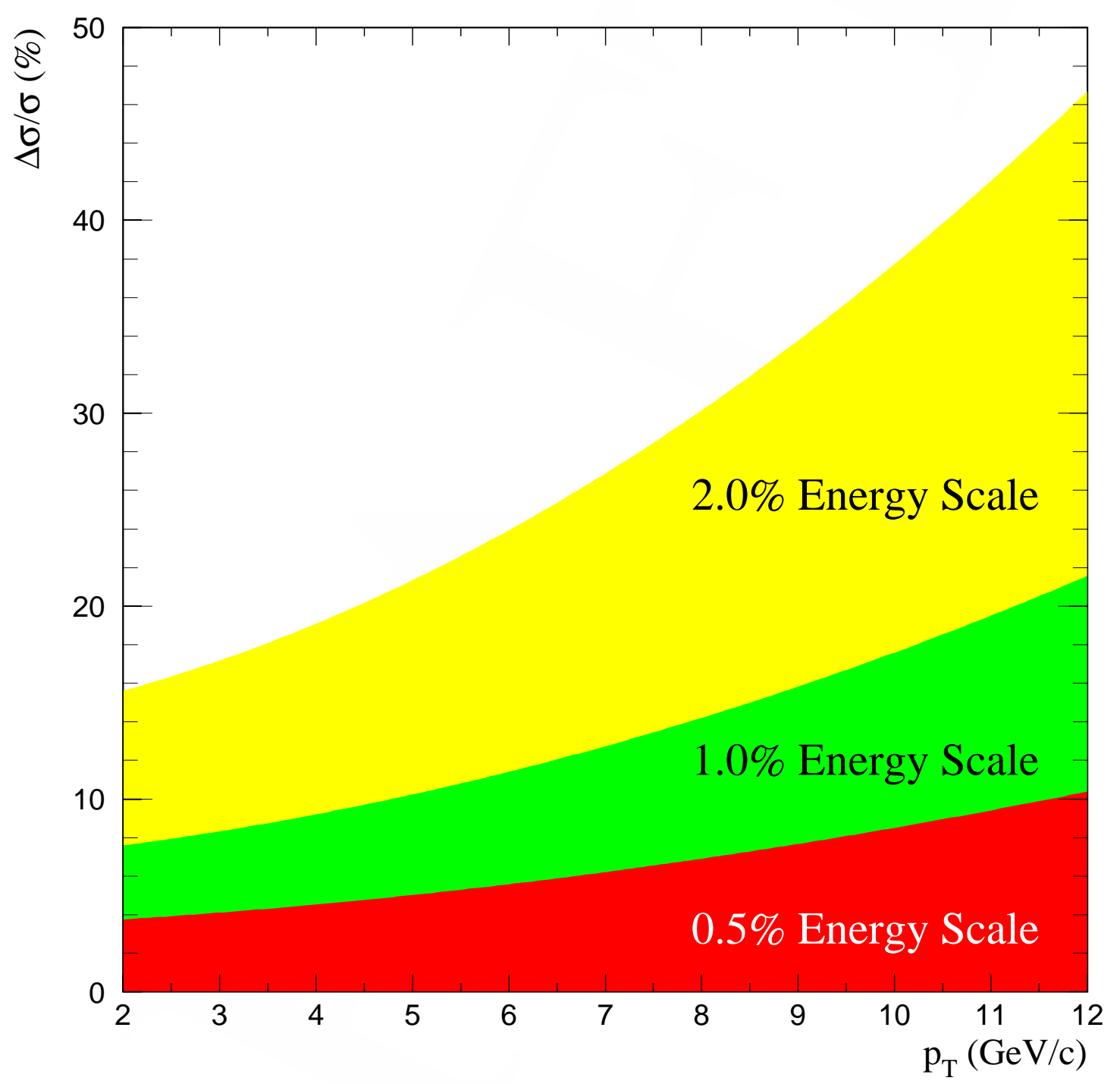

Figure 5.17 The resultant systematic uncertainty in the $\pi^{0}$ cross-section due to a $0.5,1.0$, and $2.0 \%$ uncertainty in the energy scale. This is for $\pi^{-} \mathrm{Be}$ interactions at $515 \mathrm{GeV} / c$. 

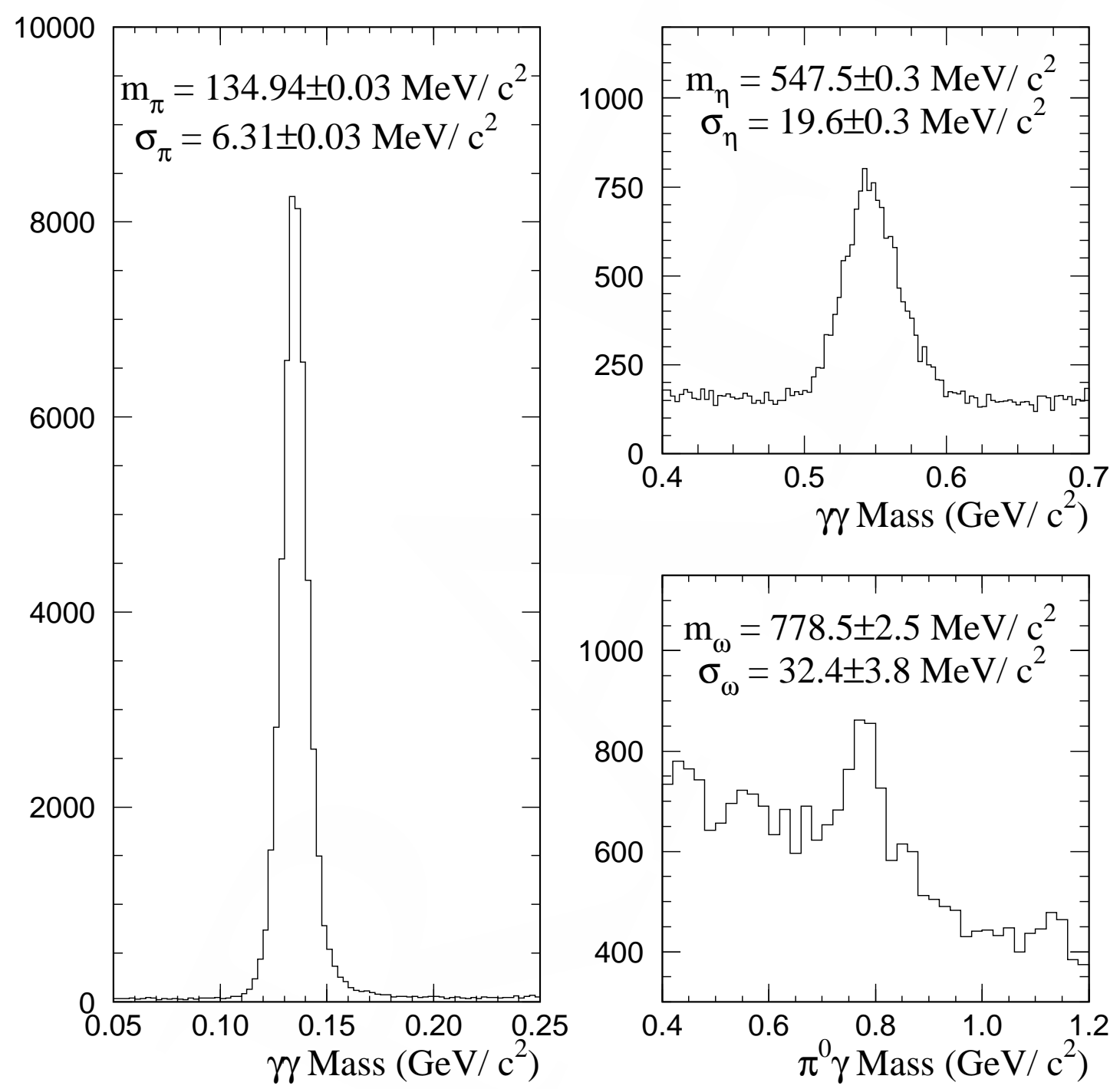

Figure 5.18 The $\gamma \gamma$ mass distribution in the region of the $\pi^{0}$ and $\eta$ and the $\pi^{0} \gamma$ mass distribution in the region of the $\omega$ for mass combinations with $p_{T}>5.0 \mathrm{GeV} / c$. The mean values for these masses agree with the world values to better than $0.5 \%$. 


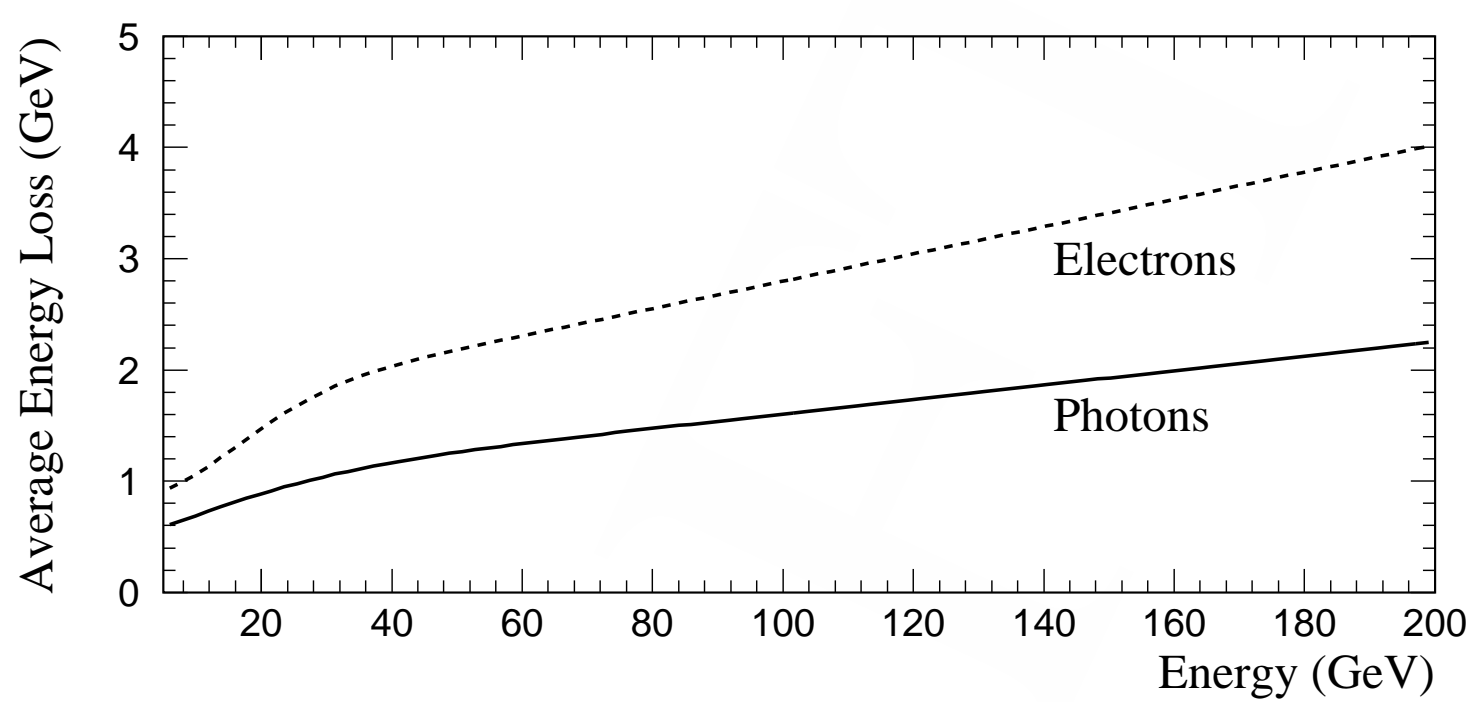

Figure 5.19 Average energy lost in the material upstream of the EMLAC for photons and electrons as a function of reconstructed energy.

to biases in the reconstruction algorithm. The corrections were determined using low $p_{T} \pi^{0} \rightarrow \gamma \gamma$ decays, and adjusting the photon energies so that the mean $\pi^{0}$ mass corresponded to the world value.

The next step corrected for the average energy lost in the inactive material in front of the EMLAC. A GEANT full shower Monte Carlo simulation (described in Section 6.3.2) was used to evaluate corrections for the energy lost for photon and electron showers as functions of the reconstructed shower energy. These functions are shown in Figure 5.19.

The EMLAC response was found to vary as a function of radius. This radial dependence is shown in Figure 5.20. Although the details of the radial dependence are sensitive to event structure and biases in the reconstruction algorithm, the gross effect was strongly correlated with the choice of charge integration time for the LAC amplifiers, as shown in the inset of Figure 5.20. The radial dependence of 
the response observed from each octant was parametrized using the reconstructed mass from low $p_{T} \pi^{0} \rightarrow \gamma \gamma$ decays. These parameterizations were then used to correct individual photon energies.

\subsubsection{Results and Linearity}

The calibrated masses of the $\pi^{0}, \eta$, and $\omega$ have been shown in Figure 5.18. The linearity of the energy scale is illustrated in Figure 5.21, where the reconstructed mass from $\eta \rightarrow \gamma \gamma$ decays is shown as a function of the $\eta$ energy and $p_{T}$.

ZMP electrons were used extensively to cross-check the calibration procedure. Figure 5.22 shows the $\gamma e^{+} e^{-}$mass distribution. The momenta of the $e^{+} e^{-}$pair was measured by the tracking system. The mean $\pi^{0}$ and $\eta$ masses measured in this mode are $\approx 1 \%$ lower than their respective world values. This drop, however, is expected since the conversion electrons lose energy as they travel through the target material via bremsstrahlung. This energy loss is demonstrated in Figure 5.23, where the mean $\pi^{0}$ mass measured in this mode is shown as a function

of the number of radiation lengths traversed through the target. The $\gamma e^{+} e^{-}$mass ratio approaches unity in the limit of zero radiation lengths traversed, and drops uniformly with the amount of target material traversed. For comparison, the $\gamma \gamma$ mass ratio, which is not expected to show any radiation length dependence, is also shown.

The $\gamma e^{+} e^{-}$sample also provides another important cross-check on the energy scale calibration. The unconverted photon in the $\gamma e^{+} e^{-}$sample is typically isolated from the electrons since the electrons are deflected away from the photon by the magnet. Therefore, by plotting the $\gamma e^{+} e^{-}$mass relative to the $\eta$ and $\pi^{0}$ world values versus the energy of the unconverted photon, the isolated photon energy scale is investigated. This is shown in Figure 5.24. The ratio is flat versus energy and $\approx 1 \%$ low, as expected from Figure 5.22 


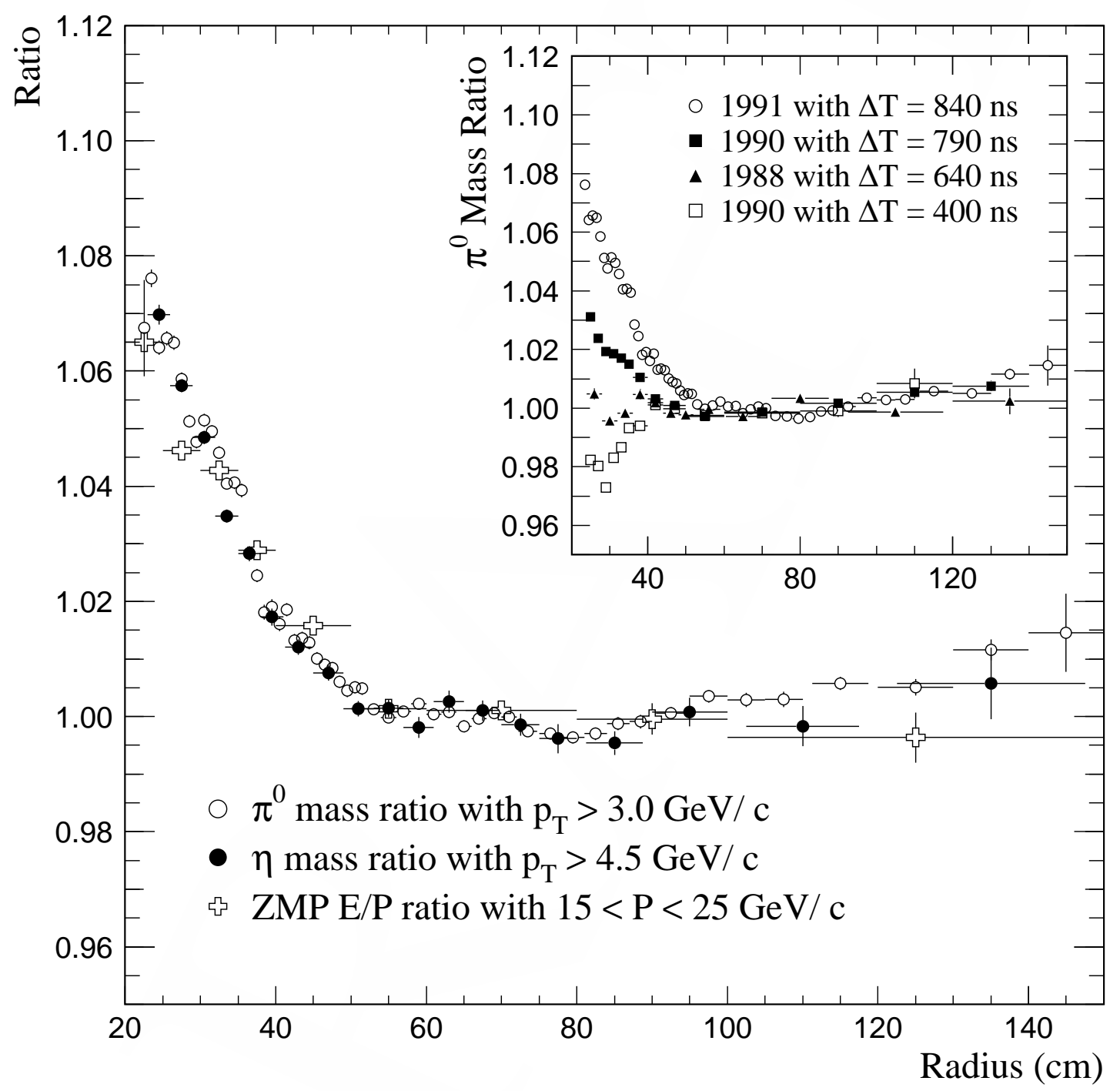

Figure 5.20 Radial dependence of the reconstructed $\pi^{0}$ and $\eta$ masses (normalized to their world values) and the $E / P$ ratio for ZMP electrons from the 1991 data sample. Inset: Radial dependence of the reconstructed $\pi^{0}$ mass for several choices of charge integration time. 

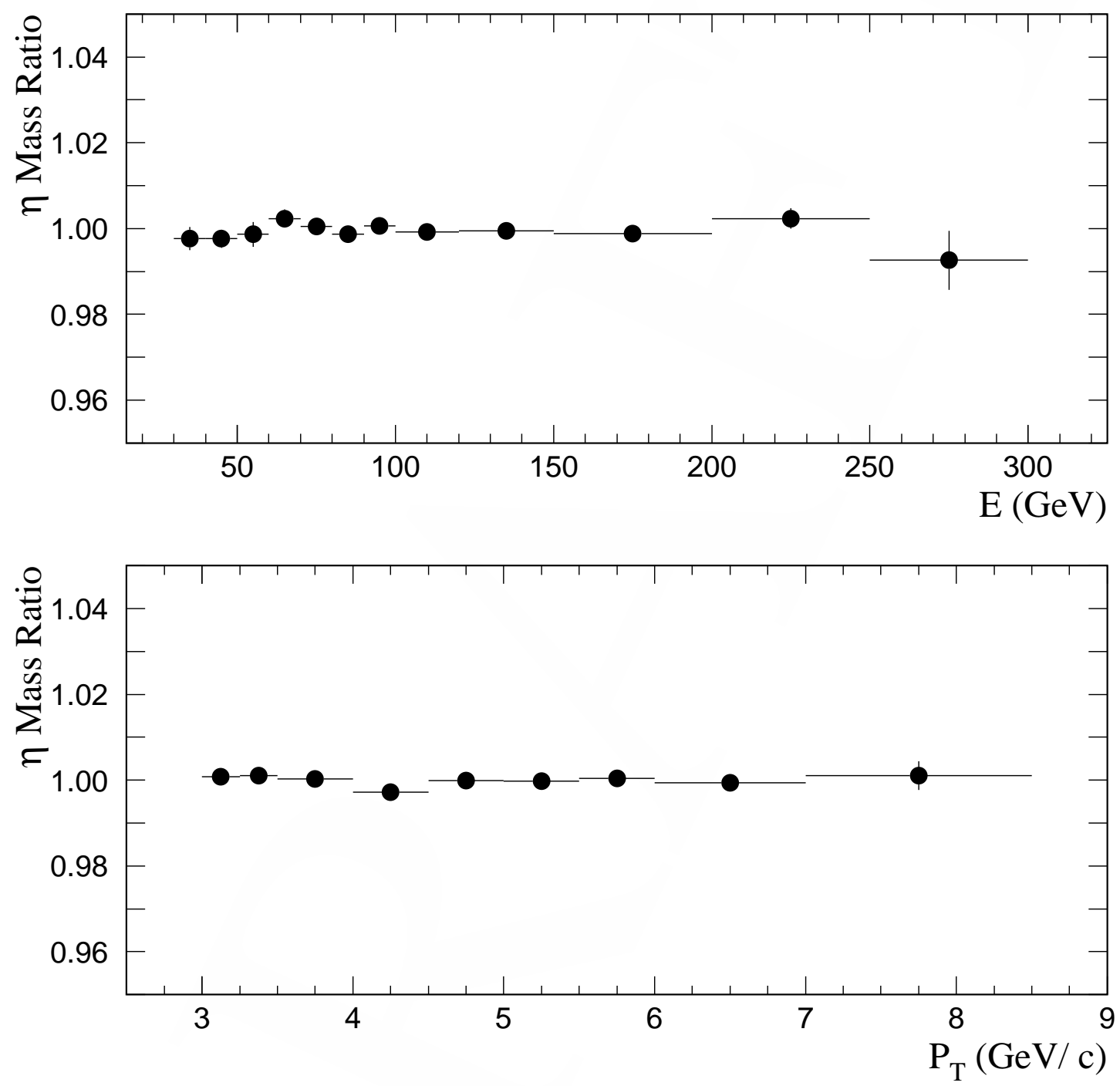

Figure 5.21 The mean $\eta$ mass (relative to the world value) as a function of the $\eta$ energy (top) and $p_{T}$ (bottom) from the 1991 data sample. 


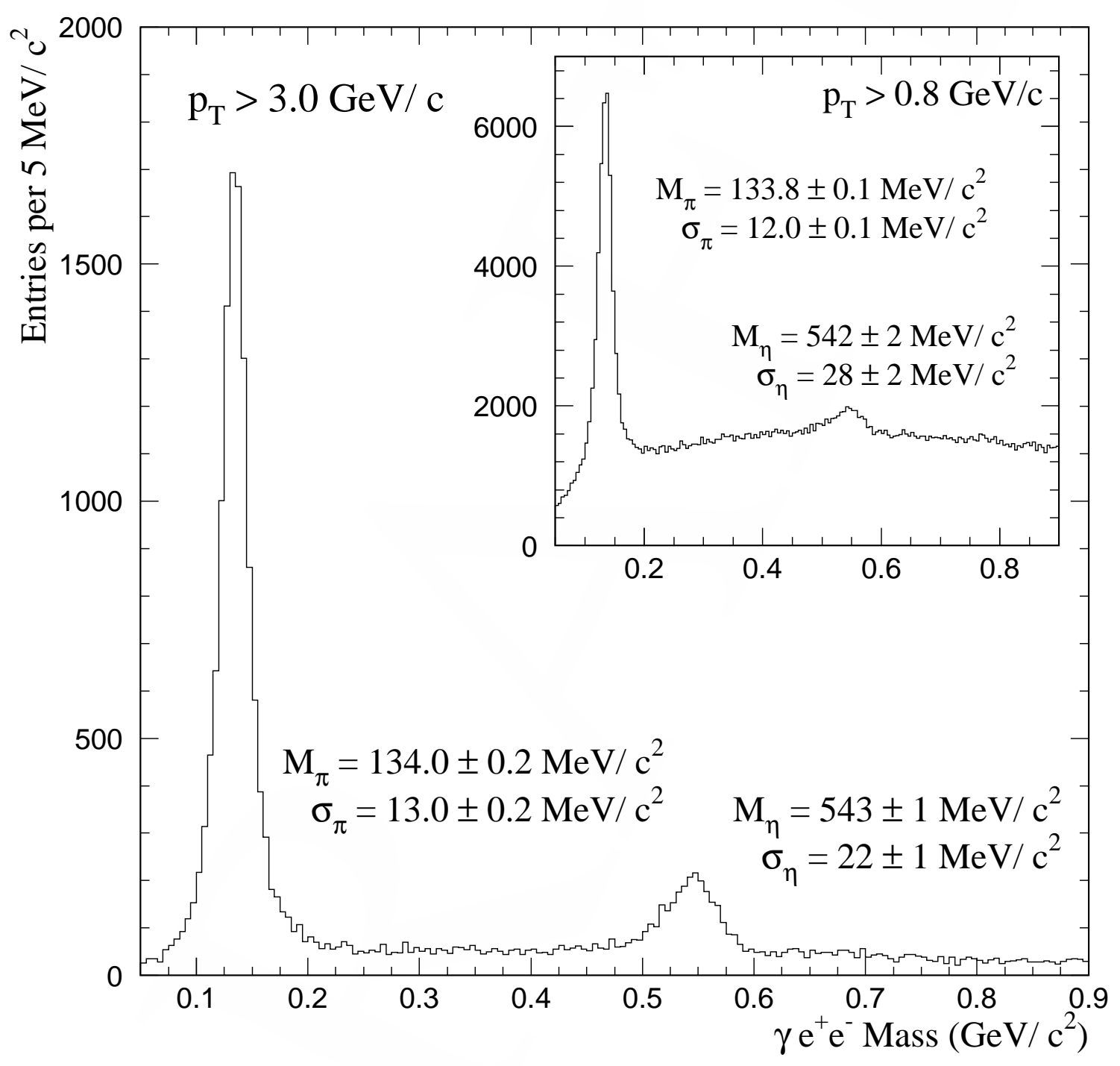

Figure 5.22 The $\gamma e^{+} e^{-}$mass distribution from the combined 1990 and 1991 data samples. 


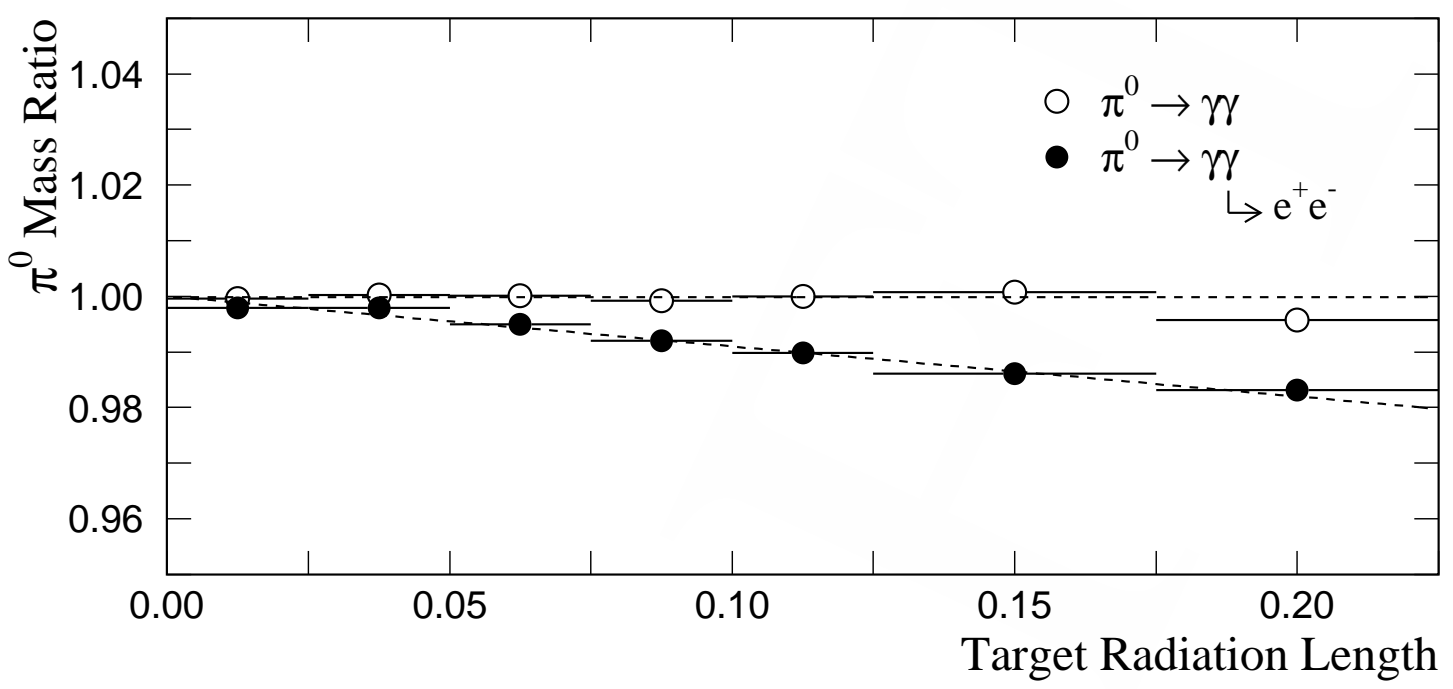

Figure 5.23 Ratio of the reconstructed $\gamma e^{+} e^{-}(\bullet)$ and $\gamma \gamma(\circ)$ masses to the $\pi^{0}$ world value versus the number of radiation lengths traversed in the target. The lines are fits to the data.

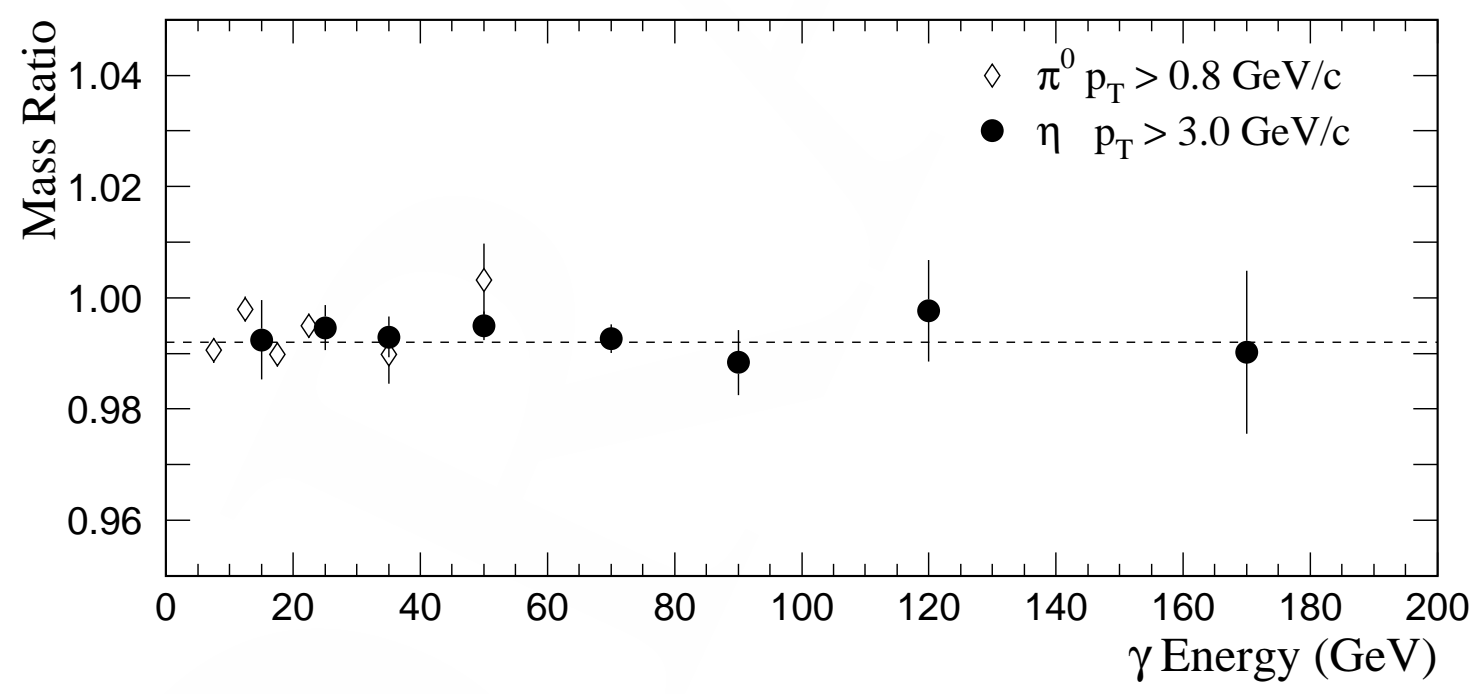

Figure 5.24 Ratio of the reconstructed $\gamma e^{+} e^{-}$mass to the $\eta$ and $\pi^{0}$ world values versus the energy of the unconverted photon. 


\subsection{Trigger Analysis}

The events used in this analysis were selected by the SINGLE LOCAL HIGH and SINGLE LOCAL LOW triggers in 1991, and the SINGLE LOCAL HIGH and LOCAL GLOBAL LOW triggers in $1990 .^{7}$ To correct for losses near the trigger threshold (see Figure 3.2), trigger corrections were determined for each trigger on an event by event basis. The determination of these corrections is described below.

\subsubsection{Trigger Corrections}

The efficiency of the local triggers was determined by the performance of the thirty-one (one for each sum-of-16) local discriminators in each octant. Ideally, these discriminators only issued a logic signal if the input signal (the trigger- $p_{T}$ ) exceeded the discriminator threshold. However, in practice, each discriminator had a small transition, or turn-on, region where the probability for the discriminator to fire changed from zero to one. Turn-on curves were measured as functions of the trigger- $p_{T}$ for each local discriminator. In addition, separate curves were measured for run regions in which the trigger response differed due to changes in the discriminator thresholds, replacement of hardware modules, etc.

To measure the turn-on curve for a given discriminator, a data sample that is unbiased with respect to the status of that discriminator must be chosen. For the LOCAL_HI discriminators, this sample consisted of events which satisfied the TWO GAMMA trigger (in the 1990 data), or the SINGLE LOCAL LOW trigger (in the 1991 data), in the octant of the given LOCAL_HI discriminator. These lower threshold triggers were generally fully efficient in the turn-on regions of the LOCAL_HI discriminators, and thus provided an unbiased sample. For the LOCAL_LO discriminators, an opposite octant sample was chosen. To obtain this

7 The LOCAL GLOBAL LOW trigger was used in 1990 because the SINGLE LOCAL LOW was only available during the later part of the run. 
sample, events that satisfied the SINGLE LOCAL HIGH trigger were selected, and the seven octants other than the octant that satisfied the SINGLE LOCAL HIGH trigger were ordered according to their respective $p_{T}$ depositions. The octant with the largest $p_{T}$ deposition was deemed the opposite octant and used to measure the LOCAL_LO turn-On.

Once the appropriate sample of events was selected, the turn-on curve was measured by taking the ratio of the trigger- $p_{T}$ distribution for the sample which fired the local discriminator to the trigger- $p_{T}$ distribution for the entire sample. The trigger- $p_{T}$ for a given sum-of-16 was calculated offline by taking the energy deposited in each strip, weighing it by the appropriate trigger gain, and then taking the sum of these weighted energies. Importantly, the energy in the strips were not corrected for the time dependence of the EMLAC response, as the discriminator thresholds did not scale with time. In Figure 5.25, typical LOCAL_HI and LOCAL_LO discriminator turn-on curves are shown for the 1991 data. Once the turn-on curves were determined, the probability for the LOCAL_LO Or LOCAL_HI signal to be issued in a given octant can be obtained from

$$
P=1-\prod_{i=1}^{31}\left(1-p_{i}\right)
$$

where $p_{i}$ is the probability that discriminator $i$ fired. The probabilities, $p_{i}$, were determined on an event-by-event basis by calculating the trigger- $p_{T}$ for each discriminator and obtaining the corresponding probability from the appropriate turn-on curve.

The efficiency of the GLOBAL_LO and PRETRIGGER HIGH discriminators was determined in a similar manner using the opposite octant event sample. However, the offline calculation of the input trigger- $p_{T}$ to the discriminators was more difficult due to the small threshold applied to the signals sent into the $p_{T}$ adder 

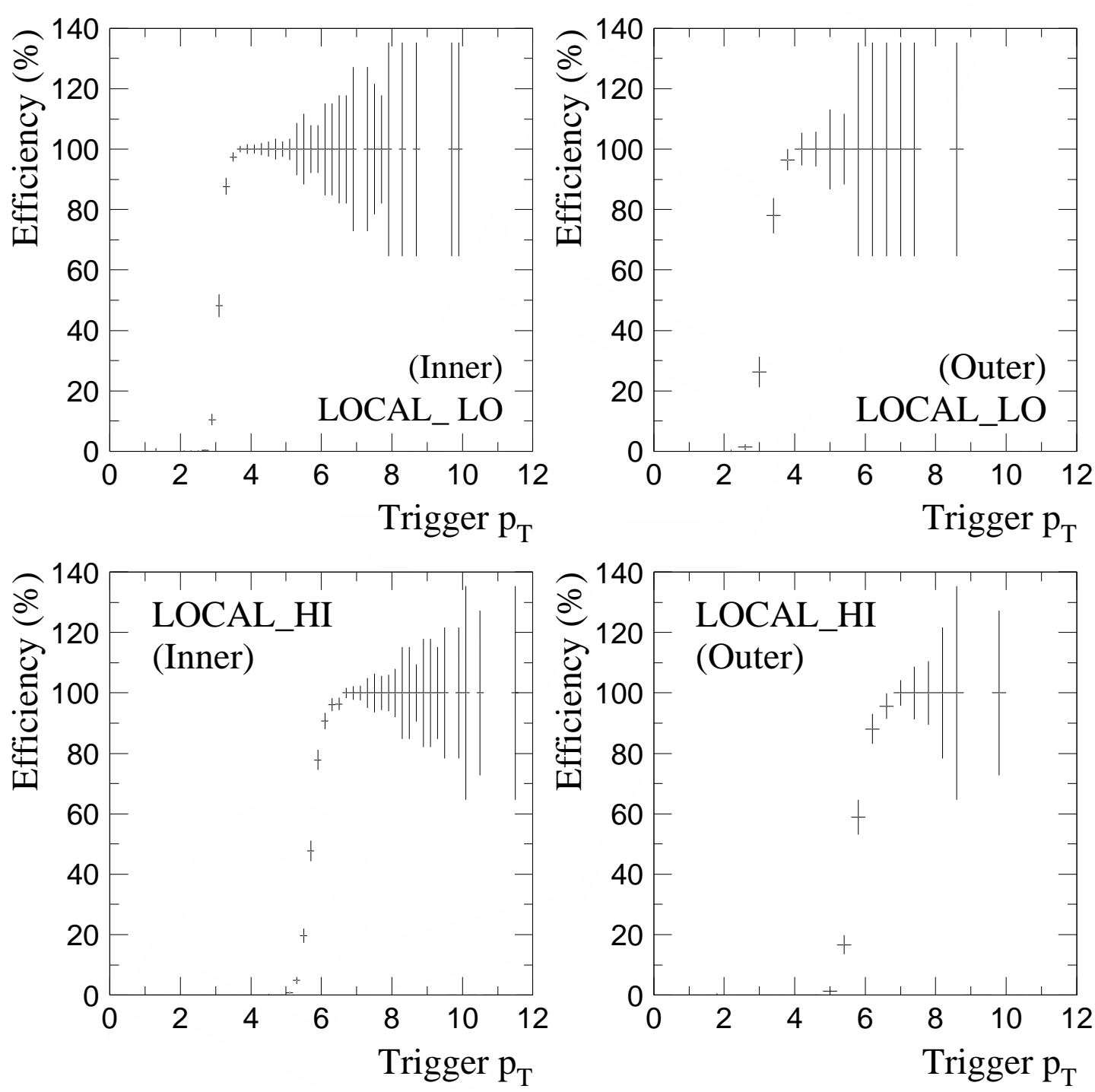

Figure 5.25 LOCAL_HI and LOCAL_LO discriminator turn-on curves for typical discriminators in the inner and outer regions of the EMLAC versus trigger- $p_{T}$ for the 1991 data. 
cards and the large number of strips contributing to the input. In addition, these efficiencies were found to be dependent upon the number of photons in the octant. Therefore, separate efficiency measurements were made depending upon the number of sums-of-eight contributing the trigger- $p_{T}$. For more details on the these analyses, the reader is referred to $[71,88]$.

\subsubsection{Trigger Selection}

The photons used in the final direct photon and neutral meson cross section analyses were required to land in octants where the trigger had a probability of firing of at least $10 \%$ to avoid excessively large trigger corrections. The corrections for losses below this cutoff was calculated by the Monte Carlo and was absorbed in the reconstruction efficiencies (see Section 6.3.5). However, at sufficiently low $p_{T}$ the correction for a given trigger becomes unreliable, and it becomes necessary to use a lower threshold trigger. The transition point between triggers was determined by comparing fully corrected cross sections measured using high and low threshold triggers in the turn-on region of the high threshold trigger. The point where the two measurements agreed was deemed the transition point. The transition point was different for $\pi^{0}$ 's, $\eta^{\prime}$ 's and direct photons, since a particular trigger's response is different for different particles. ${ }^{8}$ A composite trigger map was determined for each of these particles, and the transition point was rapidity dependent. The composite trigger map is shown for the $800 \mathrm{GeV} / c$ proton beam data in Figure 5.26. The transition points for the other data samples are similar.

${ }^{8}$ For example, the large separation of photons from $\eta$ decays relative to photons from $\pi^{0}$ decays (at a given $p_{T}$ ) tends to make the SINGLE LOCAL HIGH trigger less sensitive to $\eta$ 's. 

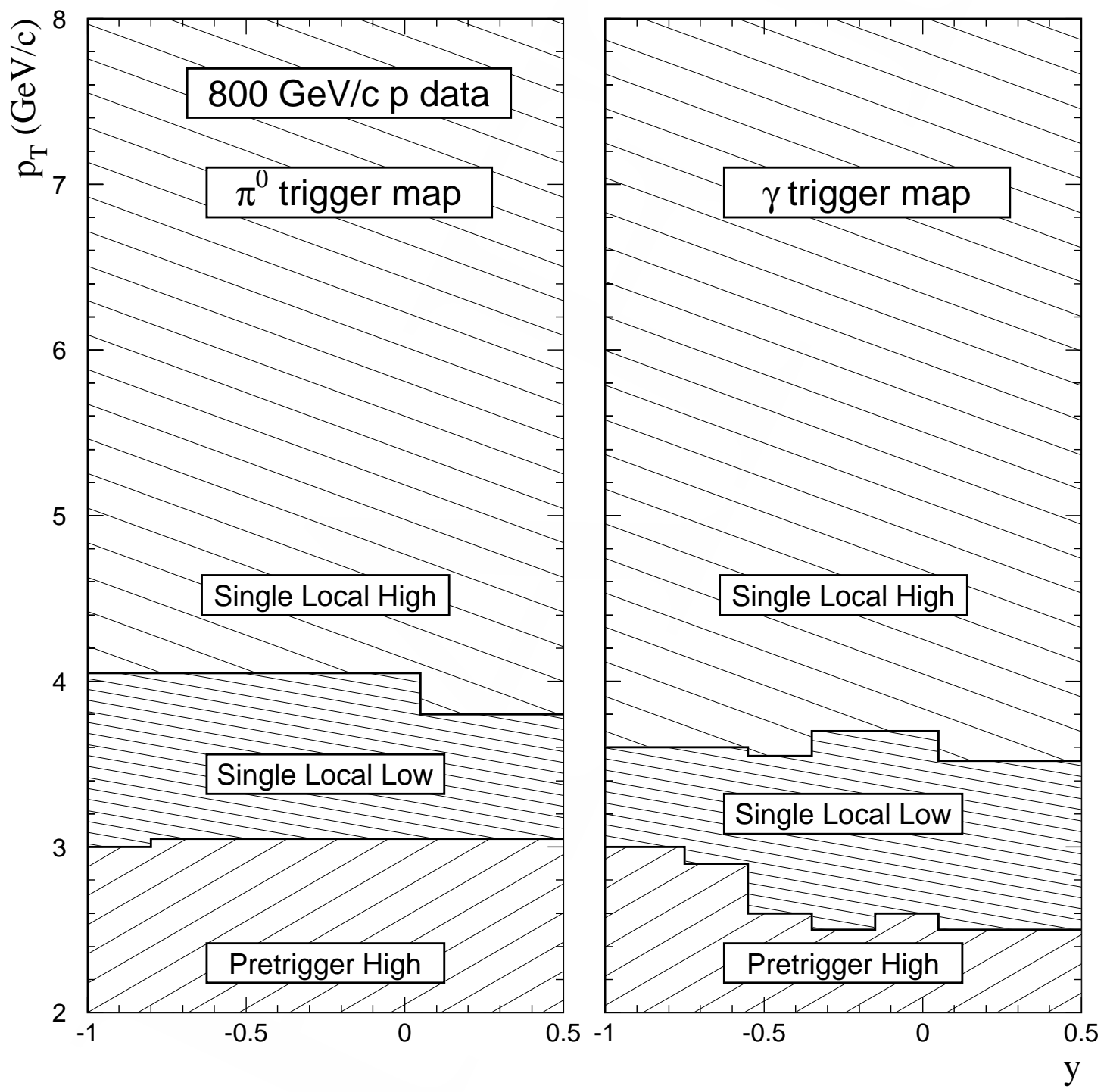

Figure 5.26 Composite trigger maps used for the $800 \mathrm{GeV} / c$ proton beam data. 
Table 5.2 Peak and sideband mass regions used in the $\pi^{0}$ and $\eta$ meson analysis.

\begin{tabular}{|c|c|c|c|}
\cline { 3 - 4 } Meson & \multirow{2}{*}{$\begin{array}{c}\text { Peak } \\
\left(\mathrm{MeV} / c^{2}\right)\end{array}$} & \multicolumn{2}{c|}{ Sidebands $\left(\mathrm{MeV} / c^{2}\right)$} \\
\cline { 3 - 4 } & $100-180$ & Low Mass & High Mass \\
\hline$\pi^{0}$ & $450-650$ & $350-450$ & $190-240$ \\
\hline$\eta$ & & $650-750$ \\
\hline
\end{tabular}

\section{$5.11 \pi^{0}$ and $\eta$ Signal Determination}

Invariant mass distributions for $\gamma \gamma$ pairs in the regions of the $\pi^{0}$ and $\eta$ are shown in Figures 5.27 and 5.28, respectively, for several bins in $\gamma \gamma p_{T}$. All meson candidate criteria have been applied to the showers contributing to the figure. For the $\gamma \gamma p_{T}$ bins where the background is linear $\left(p_{T}>2.0 \mathrm{GeV} / c\right)$, the background underneath the $\pi^{0}$ and $\eta$ peaks was determined using a sideband subtraction technique. In this technique, a peak region was defined for each meson. To each side of the peak region, sideband regions were defined. The range in mass spanned by the two sideband regions was chosen to be equal to the mass range spanned by the peak region. The physics distributions of interest (e.g. $p_{T}$, y) were made for $\gamma \gamma$ mass combinations in both the peak and sideband regions. The $\pi^{0}$ and $\eta$ signal distributions were then obtained by simply subtracting the sideband region distributions from the peak region distributions, since the combined width of the sideband regions is equal to the width of the peak region. The $\pi^{0}$ and $\eta$ peak and sideband regions used in this analysis are defined in Table 5.2. They are also shown graphically in Figure 5.29.

For the $\gamma \gamma p_{T}$ bins below $2.0 \mathrm{GeV} / c$, a fitting procedure was used to evaluate the background. The $\gamma \gamma$ mass distributions were fit using Gaussians for the signal, and second or third-order polynomials, depending upon the $p_{T}$ bin, for the background. The background was evaluated using the resultant fit parameters 


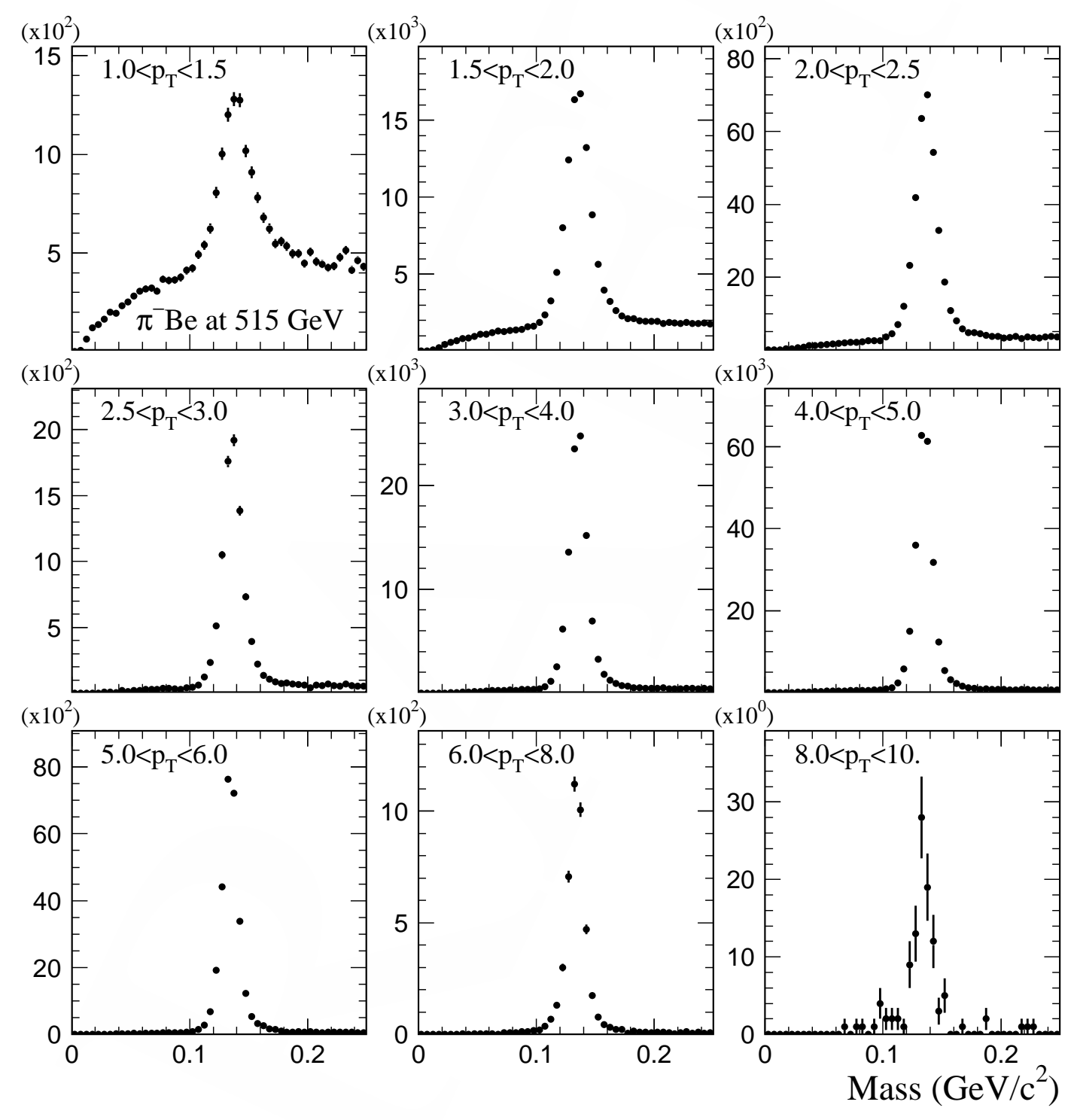

Figure 5.27 Invariant mass distributions for $\gamma \gamma$ pairs in the region of the $\pi^{0}$ for several $p_{T}$ bins. 


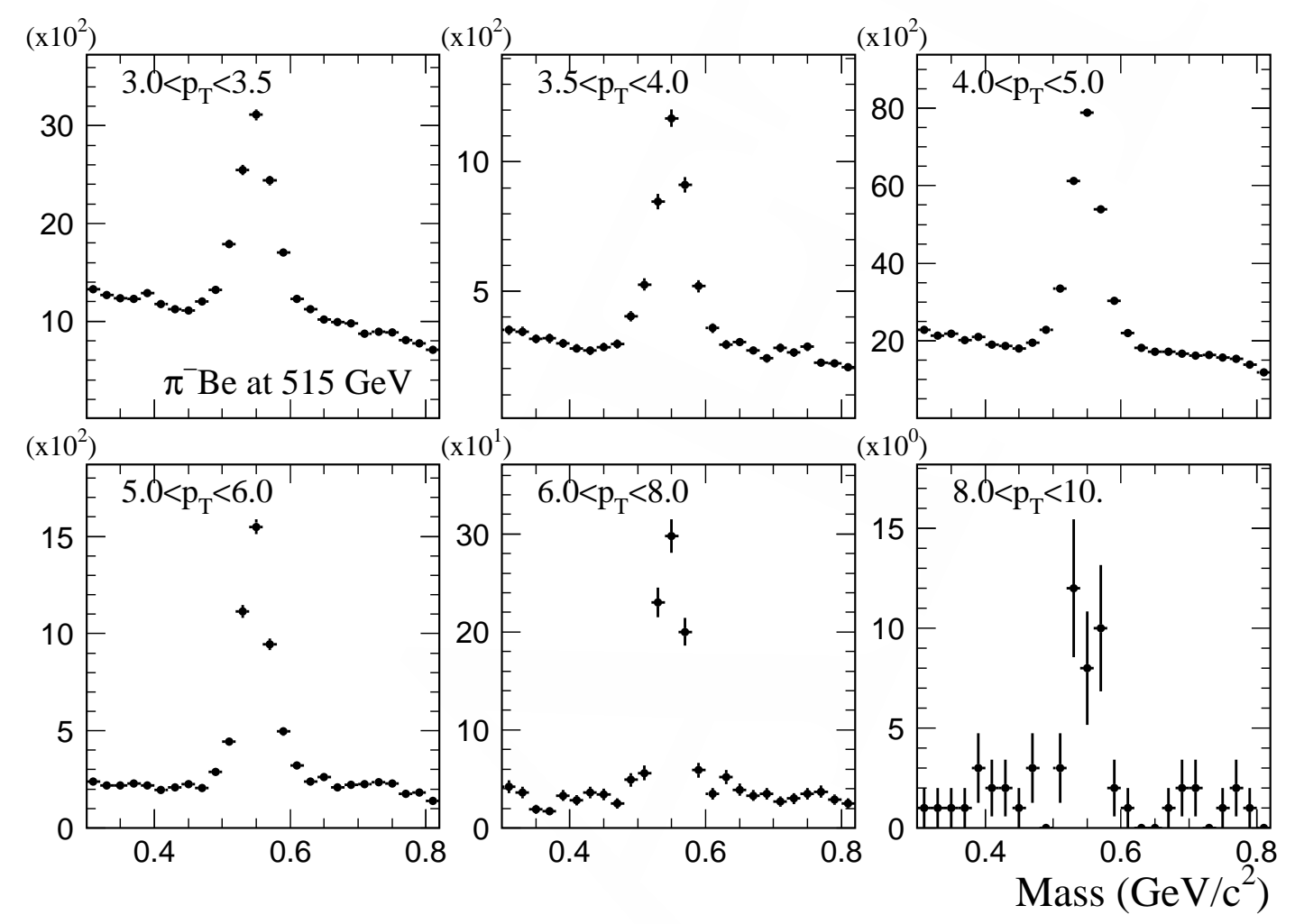

Figure 5.28 Invariant mass distributions for $\gamma \gamma$ pairs in the region of the $\eta$ for several $p_{T}$ bins.

and subtracted from the $\gamma \gamma$ mass distributions. The signal was then obtained by adding the counts within the peak region of the subtracted distribution. The peak regions for these low $p_{T}$ mass bins were defined to be somewhat wider than the peak regions used for the sideband subtraction techique to account for the wider signals in this regime. Several fits were performed for each $\gamma \gamma p_{T}$ bin in which the mass range of the fit and/or the order of the polynomial was varied. Examples of 


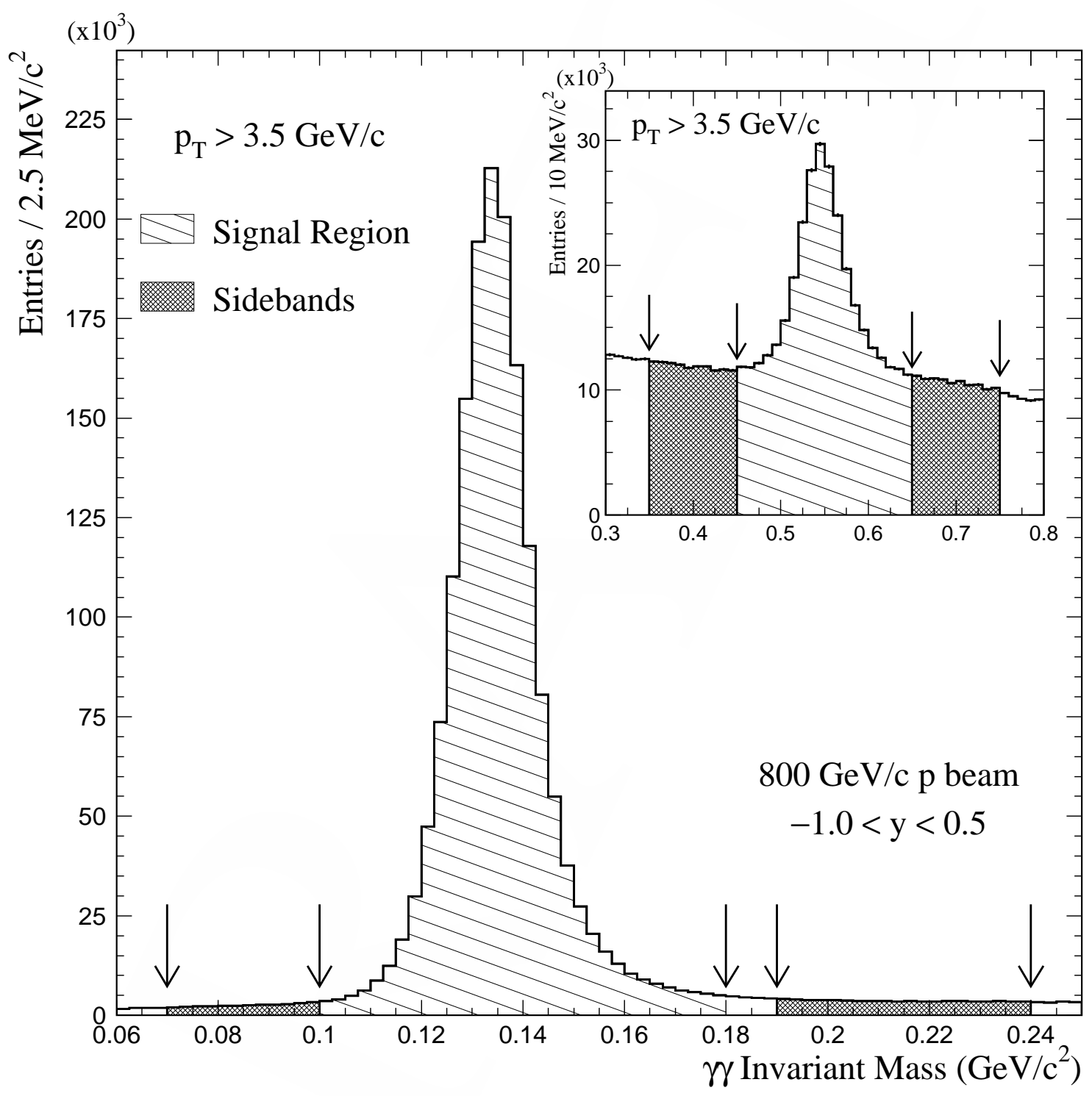

Figure 5.29 Invariant mass distributions for $\gamma \gamma$ pairs with $p_{T}>3.5 \mathrm{GeV} / c$ in the region of the $\pi^{0}$ and $\eta$. The arrows indicate the boundaries of the peak and sideband regions. 
these fits are shown in Figure 5.30. The result for the signal was taken from the average of the fits.

\subsection{Direct Photon Candidate Definition}

Only photons that passed the aforementioned hadron and muon rejection requirements and fell within the EMLAC fiducial volume were included in the direct photon candidate sample. Furthermore, to reduce the direct photon background resulting from the two photon decay modes of the $\pi^{0}$ and $\eta$, photons were rejected from the candidate sample if they combined with another photon in the same EMLAC octant to form a $\gamma \gamma$ pair with invariant mass in the $\pi^{0}$ or $\eta$ peak region and energy asymmetry less than some specified value. The specific value for the asymmetry cut depended upon the direct photon candidate definition. Three different direct photon candidate definitions, $75 \mathrm{~N}, 90 \mathrm{~N}$, and $75 \mathrm{~S}$, were used in this analysis. These definitions are described below.

In the $75 \mathrm{~N}(90 \mathrm{~N})$ definition, any photon which when considered in combination with another photon in the same EMLAC octant had $\gamma \gamma$ invariant mass in the $\pi^{0}$ peak region and energy asymmetry less than $0.75(0.90)$, was eliminated from the sample. A photon was also rejected by both the $75 \mathrm{~N}$ and $90 \mathrm{~N}$ definitions if it formed a $\gamma \gamma$ pair with another photon in the same octant with invariant mass in the $\eta$ peak region and the pair had energy asymmetry less than 0.8.

The third definition, $75 \mathrm{~S}$, rejected the same photons as the $75 \mathrm{~N}$ definition, but differed in that it also attempted to correct for losses due to true direct photons making accidental $\gamma \gamma$ mass combinations in the $\pi^{0}$ peak region. In this

definition, photons that formed mass combinations with other photons in the $\pi^{0}$ and $\eta$ sideband regions were weighted doubly - once for being outside the peak region and a second time to account for losses due to accidental combinations underneath the mass peaks. 


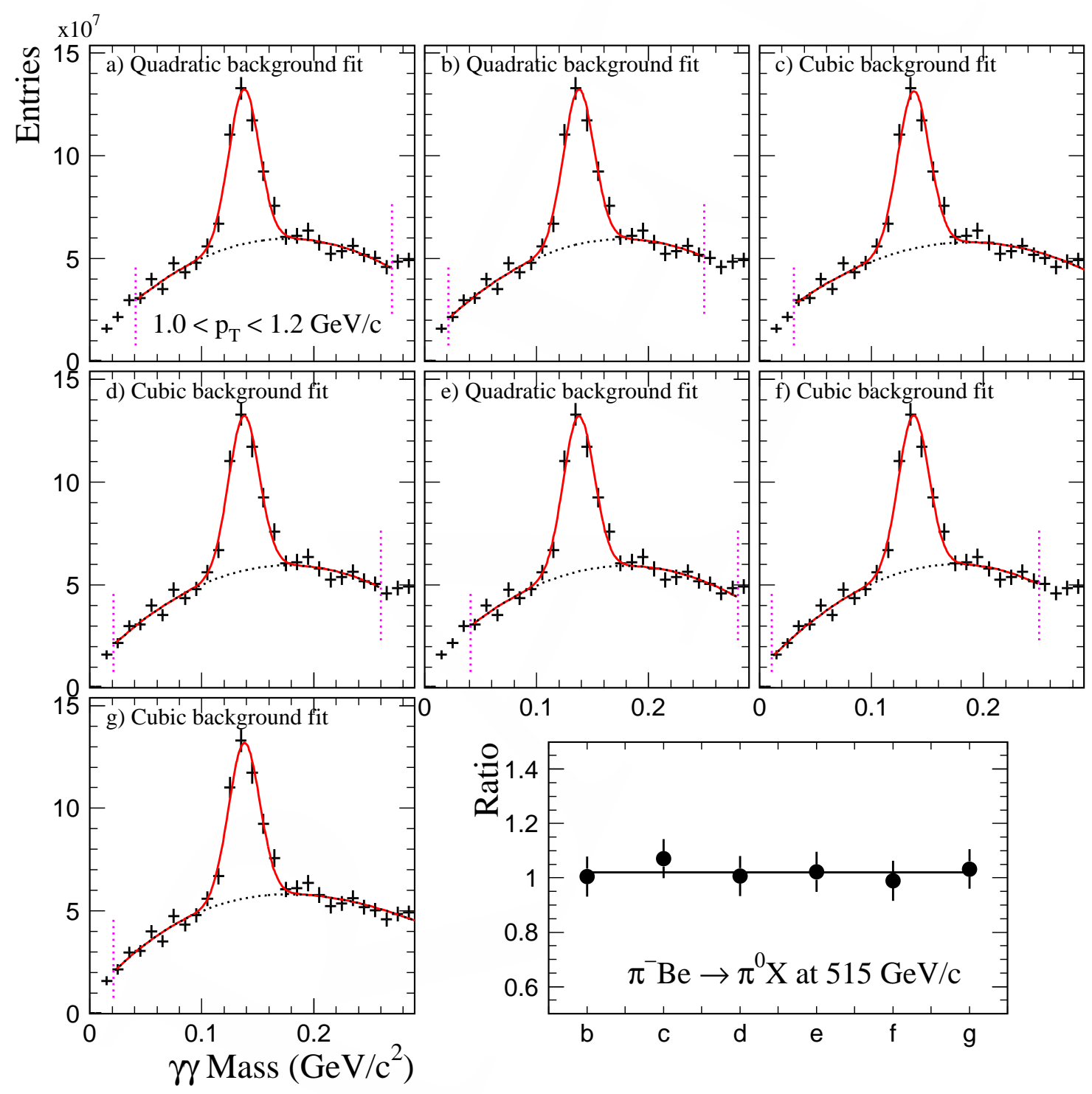

Figure 5.30 Illustration of the various fits and fit ranges used extract the signal in the bin $1.0<p_{T}<1.2 \mathrm{GeV} / c$. The resultant fits are drawn over the fit range of the histogram. 


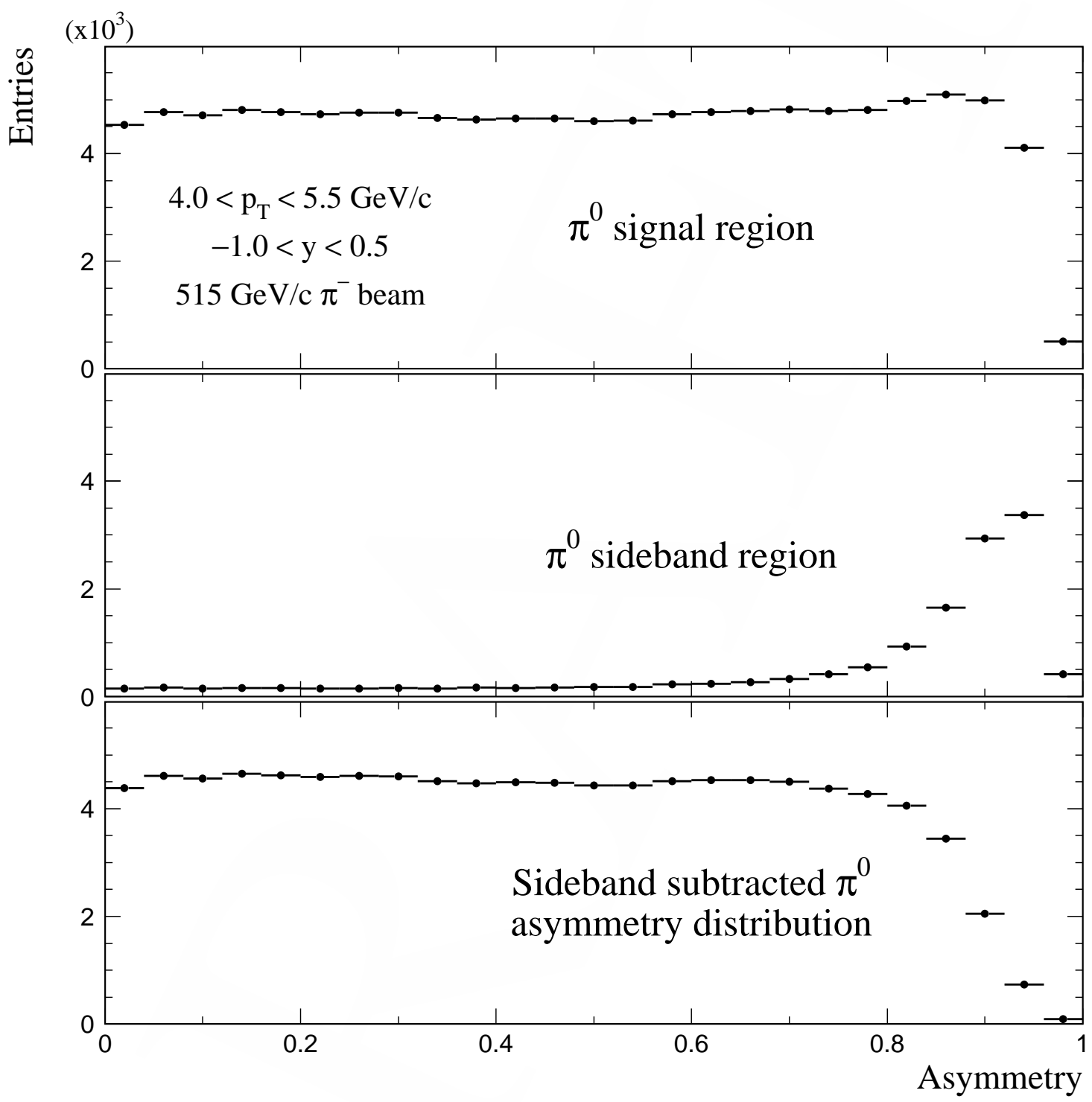

Figure 5.31 Asymmetry distributions for $\gamma \gamma$ mass combinations in the $\pi^{0}$ signal region, sideband region, and for the sideband subtracted $\pi^{0}$ signal. 
Each of these definitions have relative strengths and weaknesses. The $90 \mathrm{~N}$ definition rejects the most background. However, the residual background in this definition, which must be determined from the Monte Carlo simulation, is sensitive to how well the Monte Carlo reproduces the high end of the $\pi^{0}$ energy asymmetry distribution (Figure 5.31). In addition, losses of true direct photons are greatest in this definition, which again must be accounted for by the Monte Carlo simulation. The $75 \mathrm{~N}$ and $75 \mathrm{~S}$ definitions are less sensitive to the Monte Carlo simulation of low energy photons from $\pi^{0}$ decays, but have higher background contamination. The use of all three of these definitions provides a measure of the systematic uncertainties associated with the direct photon cross section measurement.

\subsection{Beam Normalization}

The number of beam particles incident on the target during the time in which the trigger and data acquisition system were ready to record data must be counted in order to calculate an absolutely normalized cross section. This quantity, called the live triggerable beam $\left(N_{L T B}\right)$, can be written as

$$
N_{\text {LTB }}=N_{\mathrm{BEAM} 1 \cdot \overline{\mathrm{BH}}} \times(\text { LiveFraction })
$$

where $N_{\text {BEAM1. } \overline{\mathrm{BH}}}$ is the number of isolated beam particles incident on the target that did not strike the beam hole counter and LiveFraction is the fraction of time the trigger and DA systems were live, or ready to take data. The LiveFraction can be expressed as:

$$
\begin{aligned}
\text { LiveFraction }=(\text { CompLiveFraction }) \times(\text { CleanIntFraction }) \times \\
\\
(\text { PretLiveFraction }) \times(\text { VetoLiveFraction }),
\end{aligned}
$$

where:

- CompLiveFraction is the fraction of interactions for which the data acquisition system was ready to accept data; 
- CleanIntFraction is the fraction of interactions not vetoed by the CLEANINT trigger requirement;

- PretLiveFraction is the fraction of time the PRETRIGGER logic was not busy evaluating another interaction;

- VetoLiveFraction is the fraction of interactions not vetoed by the presence of a signal in the veto wall, or a power supply noise spike, or early $p_{T}$.

To determine these quantities, the trigger system contained a number of electronic scalar units which counted various signal coincidences on a spill by spill basis.

The live triggerable beam count obtained from the scalers must be corrected for beam absorption in the target. This correction was calculated on an event-byevent basis from the formula:

$$
C_{a b s}=\prod_{i} e^{z_{i} / \lambda_{i}},
$$

where the product runs over the materials between the beam hodoscope and the interaction vertex, $\lambda_{i}$ is the absorption length of material $i$, and $z_{i}$ is the amount of material upstream of the interaction vertex. ${ }^{9}$ Average values for the absorption correction are tabulated in Table 5.3. Corrections were also applied to the live triggerable beam count to account for muon contamination within the beam [89], and for the fraction of beam incident on the target (Section 5.2).

The cross section normalization can be cross-checked using prescaled BEAM and INTERACTION trigger samples. In these samples, the normalization can be obtained independently of the scalers simply by counting events in the sample.

${ }^{9}$ For the nuclear targets, the values for $\lambda$ were obtained from [90]. For the liquid hydrogen target, $\lambda$ was obtained from [91]. 
Table 5.3 Average values of the beam absorption correction.

\begin{tabular}{|c|c|c|c|c|c|}
\hline & Beam & $\begin{array}{r}\text { Energy } \\
(\mathrm{GeV})\end{array}$ & $\mathrm{Be}$ & $\mathrm{Cu}$ & $H_{2}$ \\
\hline 1990 & $\pi^{-}$ & 515 & 1.06 & 1.02 & - \\
\hline \multirow{3}{*}{1991} & $\pi^{-}, \pi^{+}$ & 515 & 1.06 & 1.01 & 1.03 \\
& $\mathrm{p}$ & 530 & 1.08 & 1.02 & 1.04 \\
& $\mathrm{p}$ & 800 & 1.08 & 1.02 & 1.04 \\
\hline
\end{tabular}

Comparing the results using the two different normalization methods yielded a systematic uncertainty in the overall normalization of $\approx 10 \%$. 



\section{Chapter 6 Monte Carlo Simulation}

\subsection{Overview}

Monte Carlo computer simulations play an important role in the analysis of data from experiments investigating high energy particle interactions due to the complexity of these interactions and of the devices used to detect them. Typically, Monte Carlo simulations are used to evaluate, among other things, corrections for the detector acceptance and the detector response, particle reconstruction efficiencies, and backgrounds to the signals the experiments are attempting to extract. In this experiment, because the direct photon background can be substantial compared to the signal, significant effort was expended to ensure that the computer simulation provided precise and accurate information related to the direct photon background.

Technically, the Monte Carlo was used to calculate the production of photons from background sources, $\gamma_{b}$, relative to $\pi^{0}$ production. The direct photon background was then obtained by multiplying the ratio $\gamma_{b} / \pi^{0}$ by the measured $\pi^{0}$ cross section. Two different approaches were used to evaluate $\gamma_{b} / \pi^{0}$. In one approach, a highly parameterized single particle Monte Carlo simulation (PMC) was used. Only contributions from the major sources of background were evaluated in this simulation. Wherever possible, simple parameterizations were used to describe the effects of reconstruction and to evaluate contributions to $\gamma_{b} / \pi^{0}$. In the other approach, a more complicated Monte Carlo simulation was used. Multi-particle events from high $p_{T}$ hadronic interactions were generated and then processed through a sophisticated GEANT [92] simulation of the E706 spectrometer. This simulation modeled the interactions of the particles as they traveled through the spectrometer, as well as the response of the sensitive detector 
elements. The results of the detector simulation were written to output tapes in a format similar to that of the unpacked data. These output tapes were run through the same reconstruction software as the data, so any biases or inefficiencies introduced to the real data by the reconstruction programs and/or algorithms should also be represented in the simulated data and accounted for in the resulting corrections. This simulation is referred to as the detailed GEANT simulation (DGS).

This chapter presents a comprehensive description of both Monte Carlo simulations. Comparisons are shown between the real and simulated data for a variety of important distributions. Direct photon, $\pi^{0}$, and $\eta$ reconstruction efficiencies are presented. Also, a detailed analysis of $\gamma_{b} / \pi^{0}$ is given.

\subsection{Parameterized Monte Carlo Studies}

The PMC calculated the direct photon background resulting from the following decays: $\pi^{0} \rightarrow \gamma \gamma, \pi^{0} \rightarrow \gamma e^{+} e^{-}, \eta \rightarrow \gamma \gamma, \omega \rightarrow \pi^{0} \gamma, \eta^{\prime} \rightarrow \gamma \gamma$, and $\eta^{\prime} \rightarrow \rho^{0} \gamma$. These are expected to be the primary sources of direct photon background at high $p_{T}$. The contributions from other sources, such as from $\pi^{ \pm}$interactions in the EMLAC, neutrons, radiative decays of other hadrons, etc., were evaluted using the full shower Monte Carlo.

Parameterizations of the inclusive $\pi^{0}$ cross section as measured by the experiment were used to generate input $p_{T}$ and rapidity spectra for the PMC. A common expression used to fit inclusive hadronic cross sections is the phemomenological form $[56,93]$ :

$$
E \frac{d^{3} \sigma}{d p^{3}}=C \frac{\left(1-x_{D}\right)^{m}}{p_{T}^{n}}
$$

where:

$$
x_{D}=\left[x_{T}^{2}+\left(x_{F}-x_{O}\right)^{2}\right]^{1 / 2}, x_{T}=2 p_{T} / \sqrt{s}, x_{F}=2 p_{\| c m} / \sqrt{s},
$$


and $\mathrm{C}, \mathrm{m}, \mathrm{n}, x_{O}$ are free parameters and $p_{\| \mathrm{cm}}$ is the component of the $\pi^{0}$ momentum parallel to the beam direction in the center-of-mass frame of reference. However, it was found that this form did not provide a satisfactory fit over the full kinematic range spanned by the data. Therefore, the sum of two such forms was used to fit the measured $\pi^{0}$ cross sections. As shown in Figure 6.1, this provides a reasonable parameterization of the data. $\pi^{0}$ 's, $\eta$ 's, $\omega$ 's, and $\eta^{\prime \prime}$ s were all assumed to have the same input spectra, but were normalized so that $\eta / \pi^{0}$ in the PMC matched what was observed in the data (Figure 6.11), $\omega / \pi^{0}$ was $1.0[94,95]$ and $\eta^{\prime} / \pi^{0}$ was 0.85 [95]. The absolute $\pi^{0}$ normalization is unimportant, since only the ratio of background photons to $\pi^{0}$ 's is of interest. Once the above particles were generated, they were allowed to decay via the modes listed above according to their respective branching ratios [87]. The energies and positions of the resultant photons were then smeared using Gaussian distributions with widths determined from measurements of the EMLAC's intrinsic energy and position resolutions [27]. In Figure 6.2, the smeared $\gamma \gamma$ invariant mass distributions from $\pi^{0}$ and $\eta$ decays in the PMC are compared to the background subtracted $\gamma \gamma$ mass distributions from the data. The good agreement shows that this procedure yields a reasonable representation of the effects of the EMLAC resolution.

After the energies of the resulting photons were smeared, the photons were tested to see if they would contribute to the direct photon background. For photons from $\pi^{0} \rightarrow \gamma \gamma$ decays, the background contributions can be divided into two categories: background from $\pi^{0}$ 's with energy asymmetry greater than $A_{\text {cut }}$, where $A_{\text {cut }}$ is the value of the asymmetry cut used for a given direct photon candidate definition, and background from $\pi^{0}$ 's with energy asymmetry less than $A_{\text {cut }}$. Photons from $\pi^{0}$ 's with energy asymmetry greater than $A_{\text {cut }}$ were automatically considered as background to the direct photon signal since these photons would not be eliminated from the candidate sample via reconstruction of the $\pi^{0}$ mass with another photon. 


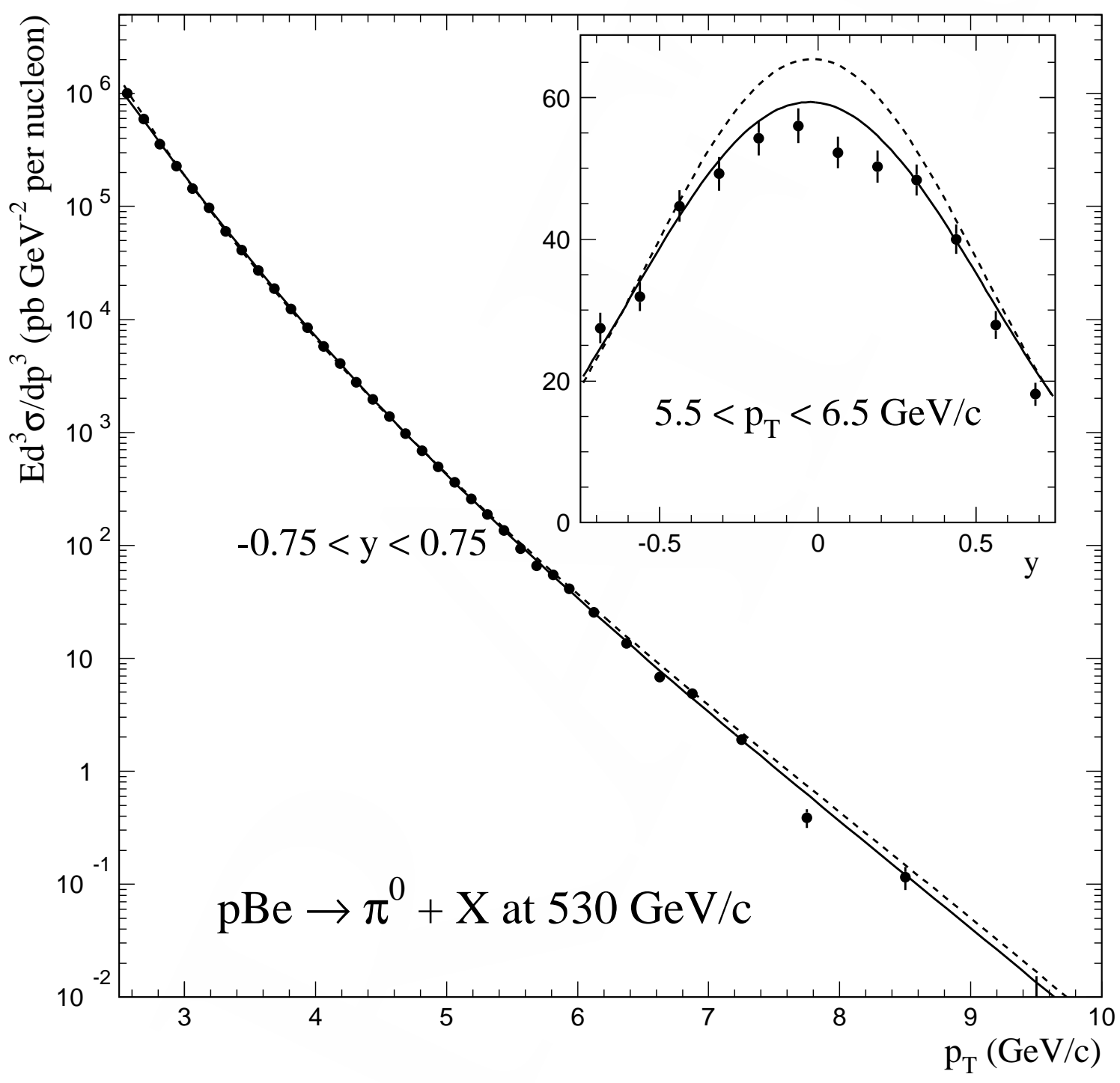

Figure 6.1 The $\pi^{0}$ cross section versus $p_{T}$ and rapidity (inset) for $530 \mathrm{GeV} / c$ proton beam incident on beryllium. The error bars are statistical only. Overlaid on the plots are the results of the fit used in the PMC (solid curve) and a fit to Equation 6.1 (dotted curve), integrated over the appropriate $p_{T}$ and rapidity ranges. 

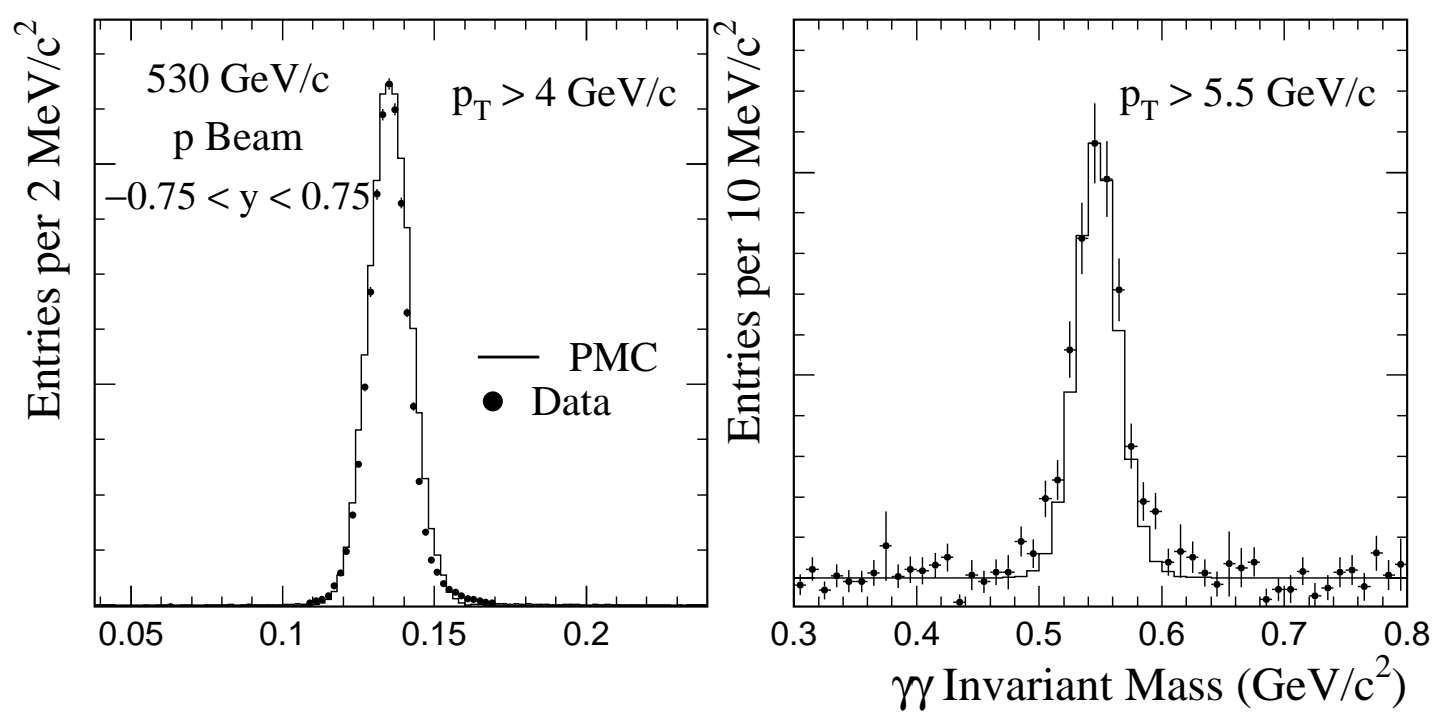

Figure 6.2 Background subtracted $\pi^{0}$ (left) and $\eta$ (right) mass distributions from the data compared to the energy resolution smeared $\pi^{0}$ and $\eta$ distributions from the PMC. Here, and in future plots where the $\mathrm{y}$-axis values are unspecified, the histograms are normalized to unity.

For $\pi^{0} \rightarrow \gamma \gamma$ decays with energy asymmetry less than $A_{c u t}$, direct photon background candidates result from the failure to simultaneously detect both photons from the $\pi^{0}$ decay. In the PMC analysis, the following possibilities for such failures were considered:

- one of the photons landed outside the EMLAC fiducial volume while the other photon landed inside it;

- the photons landed in different EMLAC octants;

- one of the photons converted into an $e^{+} e^{-}$pair; 
- one of the photons was lost due to detector inefficiency;

- the photons coalesced to form a single reconstructed shower.

The contribution to the direct photon background from the first two items listed above was straightforward to evaluate. The photons were projected to the front face of the EMLAC and checked to see if they fell within the EMLAC's fiducial volume. If one of the photons was inside the fiducial volume while the other photon was outside outside it, then the photon inside the fiducial volume became a direct photon background candidate. If the photons landed in different octants, then each became a candidate unless it landed outside the fiducial volume.

If both photons landed within the same octant and satisfied the EMLAC fiducial requirement, background to the direct photon signal could result from photon conversions, detector inefficiency or photon coalescence. The contributions to the direct photon background from these sources were determined using a weighting procedure. This procedure is outlined below.

The background from conversions were obtained by assigning a weight to each photon based upon the conversion probability of the other photon. The conversion probabilities were determined using Equation 5.2. To ensure that the photons in the PMC traversed the same amount of material as in the data, starting locations for the photons were assigned according to the observed primary vertex distributions in the data. Similarly, the background contributions due to detector inefficiency were obtained by weighing each photon from the $\pi^{0}$ decay by the non-detection probability of the other photon. The detection probabilities were determined using the efficiency function shown in Figure 5.16. The contributions from photon coalescence were obtained by assigning each generated $\pi^{0}$ a weight based upon its coalescence probability. This probability was determined using the DGS, since coalescence is dependent upon many factors, including the relative 
geometry of the two photons, the total and relative energies of the photons, and the reconstruction algorithm's ability to resolve the two photons. The probability of coalescence was significant in a very limited region of phase space, namely $p_{T} \gtrsim 7 \mathrm{GeV} / c$ and $y_{l a b} \gtrsim 3.8$.

It is possible to verify that the function shown in Figure 5.16 is a reasonable representation of the true detection efficiency by comparing $\pi^{0}$ energy asymmetry distributions in data and the PMC. This comparison is shown in Figure 6.3. If the detection efficiency was perfect, this distribution would be flat. However, due to losses of low energy photons, the distribution falls at high asymmetry. The good agreement indicates that the losses of low energy photons are adequately represented by the efficiency function.

The ratio of background photons from $\pi^{0} \rightarrow \gamma \gamma$ decays to generated $\pi^{0}$ 's is shown (without corrections for the effects of the EMLAC energy resolution (Sections 6.2.1 and 6.3.5)) on the left hand side of Figure 6.4. Also shown is the contribution from each of the sources described above. From the figure, it is evident that photons resulting from highly asymmetric $\pi^{0}$ decays are the dominant contributors to the direct photon background, although at very high $p_{T}$, the contribution due to photon coalescence becomes fairly significant.

The background contribution from $\eta \rightarrow \gamma \gamma$ decays was calculated in an analogous manner, except that in this case, $A_{c u t}=0.8$ for both photon definitions, and the background from coalescence could reliably be assumed to be negligible.

For the background contribution from the decays $\pi^{0} \rightarrow \gamma e^{+} e^{-}, \omega \rightarrow \pi^{0} \gamma$, and $\eta^{\prime} \rightarrow \rho^{0} \gamma$, the photon emanating directly from each of these decay vertices was automatically considered a direct photon background candidate, as no attempt was made to identify photons from these decays through reconstruction of the invariant mass. Photons arising from the subsequent decay of the $\pi^{0}$ in the 


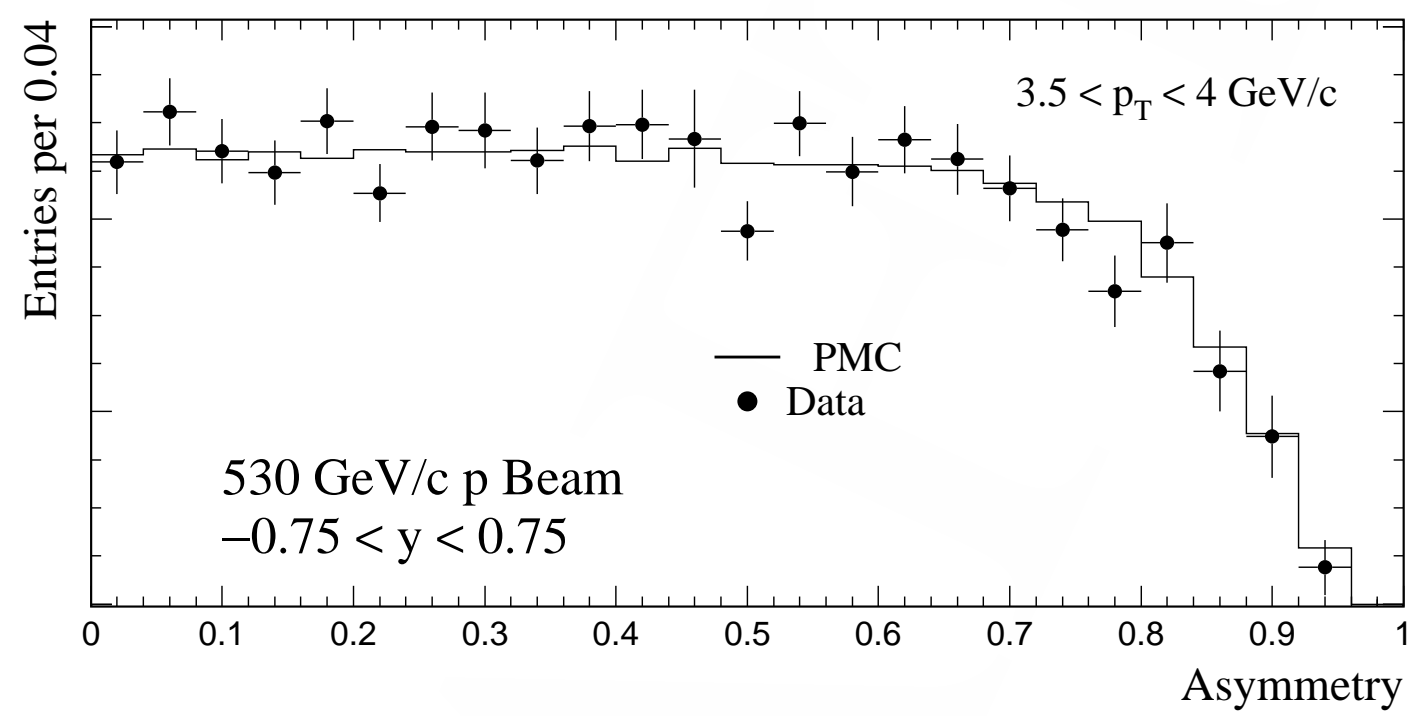

Figure 6.3 Comparison between the sideband subtracted $\pi^{0}$ energy asymmetry distribution in the data and the energy smeared PMC.

case of the $\omega$ and the $\rho^{0} \rightarrow \pi^{0} \pi^{0}$ decays are not considered in the direct photon background candidate sample, as their contribution to the background was already accounted for during the explicit consideration of the $\pi^{0}$. The contribution to the background from the $e^{+} e^{-}$pair in $\pi^{0} \rightarrow \gamma e^{+} e^{-}$decays was deemed to be negligible for two reasons: first, most of the electrons are removed from the candidate sample by the DTRK requirement, and second, since the energy of the $e^{+} e^{-}$pair is, on average, the same as the energy of one of the photons in the $\pi^{0} \rightarrow \gamma \gamma$ case, the electrons from these decays typically have much less energy, and hence $p_{T}$, than the parent $\pi^{0}$. Because of the steeply falling $p_{T}$ spectra, the background contributions 


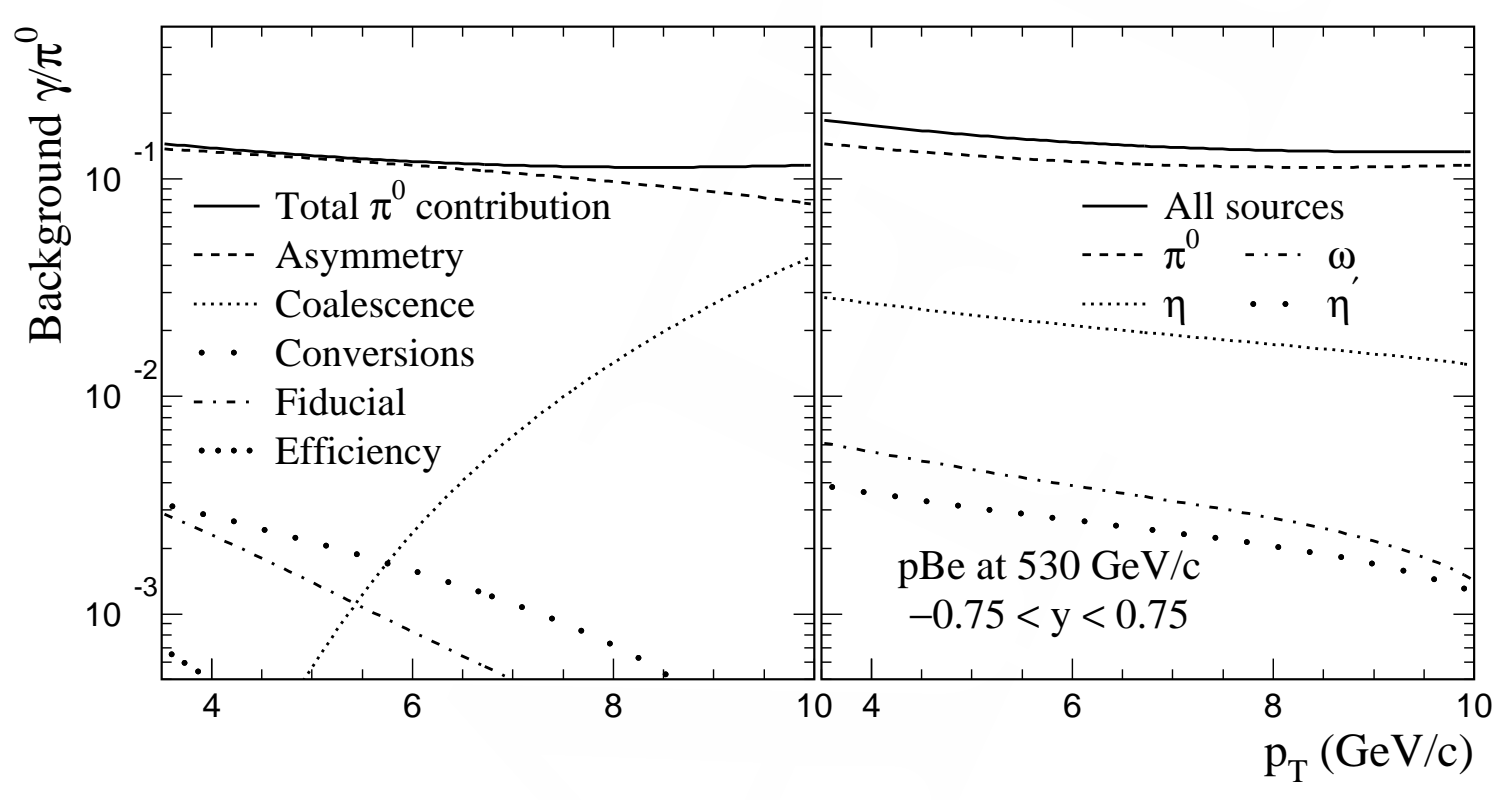

Figure 6.4 Left: Contribution to $\gamma_{b} / \pi^{0}$ from $\pi^{0}$ decays in the $75 \mathrm{~S}$ candidate photon definition. Also shown is the contribution from each of the sources described in the text. Right: Contribution to $\gamma_{b} / \pi^{0}$ from $\pi^{0}$ 's, $\eta$ 's, $\omega$ 's and $\eta^{\prime}$ 's. In these curves, the background photons have not been corrected for effects due to energy resolution.

from photons with $p_{T}$ significantly less than that of the parent $\pi^{0}$ are generally dwarfed in comparison to other contributions.

On the right hand side of Figure $6.4, \gamma_{b} / \pi^{0}$ (again without energy resolution corrections) in the PMC is shown. Also shown is the contribution to $\gamma_{b} / \pi^{0}$ from each of the particles considered in the PMC. The contribution from $\eta$ decays is $\approx 20 \%$ that of $\pi^{0}$ decays, which is roughly what is expected from simple considerations of the relative $\eta$ and $\pi^{0}$ production rates and their two photon branching ratios. 

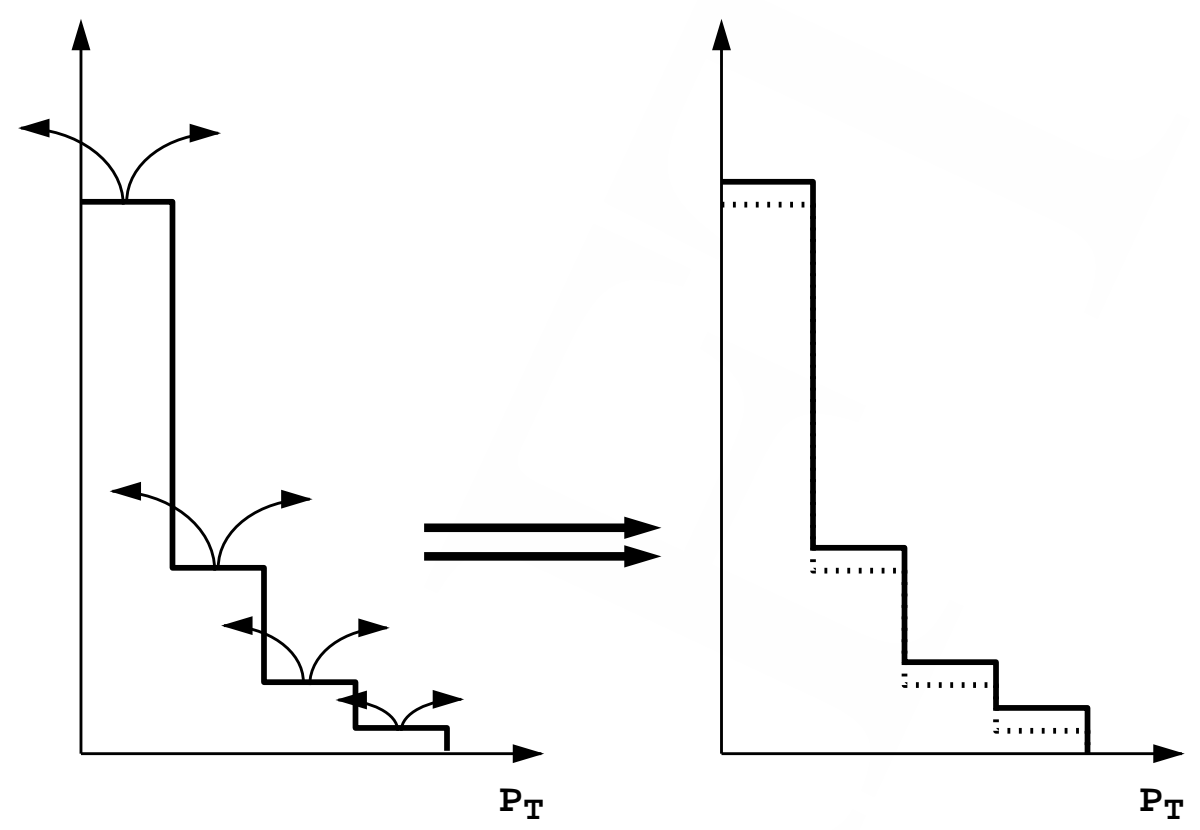

Figure 6.5 Illustration showing the effect of energy resolution on a steeply falling $p_{T}$ spectrum. For any given reconstructed $p_{T}$ bin, the number of entries entering from bins with lower true $p_{T}$ outnumber the losses out of the $p_{T}$ bin, leading to a net shift in the observed uncorrected $p_{T}$ spectrum.

\subsubsection{EMLAC Resolution Effects}

The energy resolution of the EMLAC affects the measurement of the inclusive cross section. The mean reconstructed $p_{T}$ tends to be shifted high relative to the true mean $p_{T}$, due to the steep $p_{T}$ dependence of the cross section. This effect is illustrated in Figure 6.5. In this section, the PMC is used to investigate how the EMLAC resolution smearing affects the analysis.

In Figure 6.6a, the ratio of the EMLAC resolution smeared $\pi^{0}$ energy, $E_{\text {smear }}$, to the unsmeared, or generated, energy, $E$, is plotted as a function of the smeared and unsmeared $p_{T}$. When plotted as a function of smeared $p_{T}, E_{\text {smear }} / E$ is greater than one, as expected from Figure 6.5. However, when plotted as a function of 
unsmeared $p_{T}, E_{\text {smear }} / E$ is equal to one, since the unsmeared $p_{T}$ is unaffected by resolution, and the energy is smeared symmetrically about the mean.

Resolution smearing affects the EMLAC energy calibration. Since the measured quantities are all smeared, the mean $\pi^{0}$ and $\eta$ energies, and hence the mean $\pi^{0}$ and $\eta$ masses, are shifted high relative to their true values. However, in the calibration procedure, the photon energies are set so that the mean $\pi^{0}$ and $\eta$ masses equal their world values. This procedure can be mimicked in the PMC by lowering the energies of the smeared photons so that the mean $\pi^{0}$ mass after energy resoluton smearing equals the world value. Once done, $E_{\text {smear }} / E$ plotted versus smeared $p_{T}$ is now $\approx 1$, as shown in Figure $6.6 \mathrm{~b}$. However, $E_{\text {smear }} / E$ plotted versus unsmeared $p_{T}$ is now shifted low. This means the mean reconstructed $p_{T}$ is shifted low relative to the true $p_{T}$, and hence the measured cross section is low. The DGS simulation of the detector was calibrated in the same manner as the energy scale in the data. Therefore, this feature is present in the Monte Carlo events, and was thus accounted for in the reconstruction efficiencies, which are described in Section 6.3.5.

\subsection{The Detailed GEANT Simulation}

The PMC evaluated $\gamma_{b} / \pi^{0}$ based upon relatively simple considerations of how direct photon background candidates may arise. However, only the major sources of background were evaluated by the PMC. In addition to contributions from decays other than those included in the PMC, the other particles created in these high $p_{T}$ interactions, as well as particles created from subsequent interactions within the detectors themselves, may effect $\gamma_{b} / \pi^{0}$ by confusing the reconstruction algorithms, affecting the trigger response, etc. Although these background contributions are expected to be minor, the detailed GEANT simulation (DGS) provided the means by which to fully evaluate their effects. The DGS was 


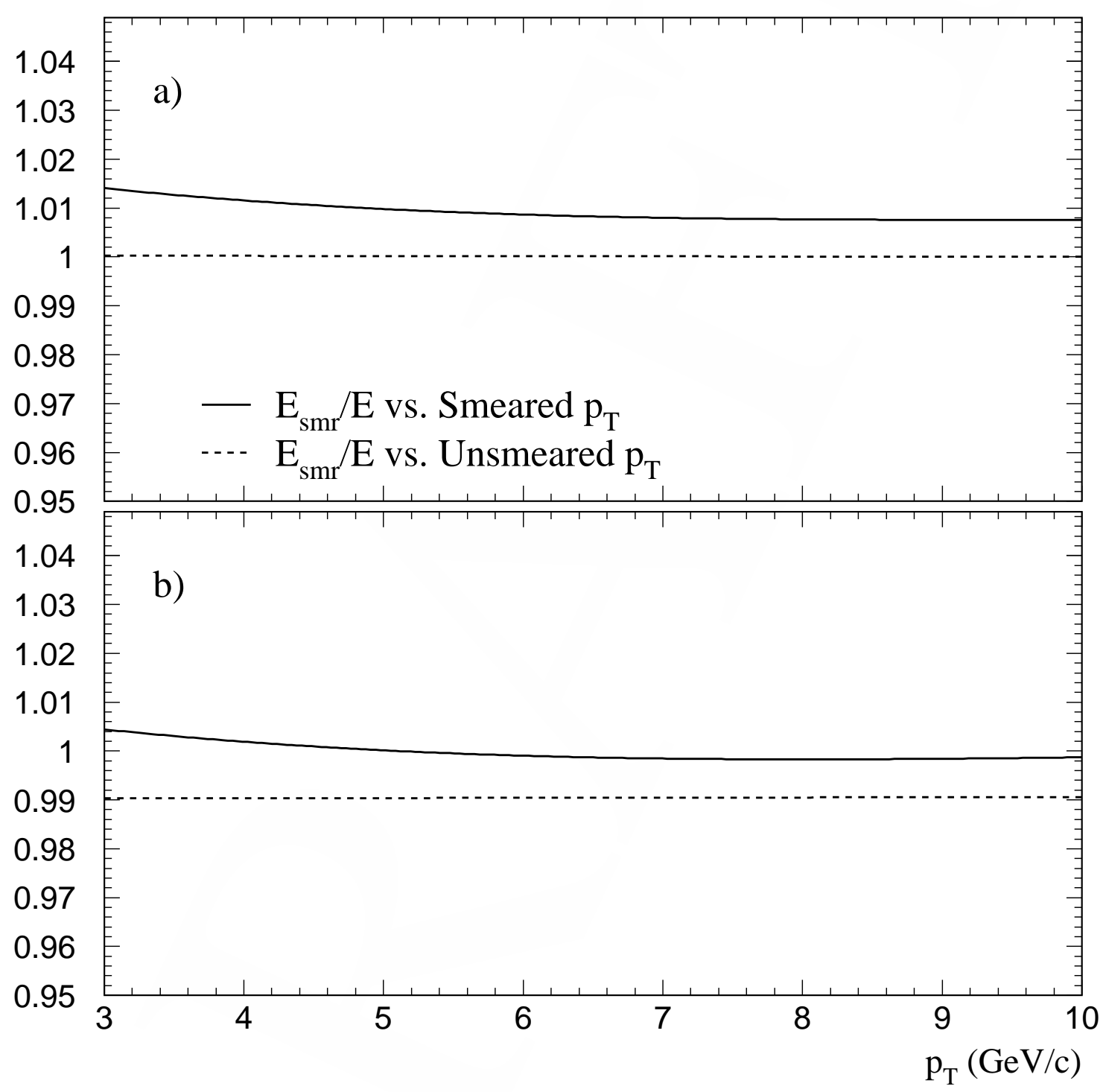

Figure 6.6 a) $E_{\text {smear }} / E$ versus $p_{T}$ in the PMC before rescaling the photon energies and $b$ ) after rescaling the photon energies. 
also responsible for the determination of the direct photon and neutral meson reconstruction efficiencies and the vertex reconstruction efficiency. Presented below is a description of the DGS.

\subsubsection{Event Simulation}

HERWIG [96] and PYTHIA [97] are two of the most frequently used generators of hadronic events. To choose between the two, a sample of events containing high $p_{T} \pi^{0}$ 's was produced using each of these generators. These samples were processed through the detector simulation, and then through the MAGIC reconstruction program. The outputs were compared to events containing high $p_{T} \pi^{0}$ 's in the data. In Figure 6.7, the number of reconstructed photons and charged tracks per event is shown for the data, the PYTHIA DGs, and the HERWIG DGs. ${ }^{1}$ Since the events generated by HERWIG match the data in these distributions better than those generated by PYTHIA, HERWIG was chosen as the primary event generator for the DGS.

A second sample of DGS events was also created to cross-check the HERWIG results. In this simulation, the reconstructed output from a selected sample of data events used as input to the GEANT simulation. This was called the DATA-DRIVEN Monte Carlo.

The simulation of electromagnetic showers in the EMLAC is highly CPU intensive. Therefore, several event selection algorithms were developed in order to reject uninteresting events before they entered this time consuming stage of the detector simulation. These algorithms typically required that the generated

\footnotetext{
1 Note that here and in future comparisons with the DGS, the data are represented by the histogram and the Monte Carlo by the points; opposite to the sense shown in the comparisons with the PMC. This is done so that the error bars are shown on the statistically limited sample.
} 

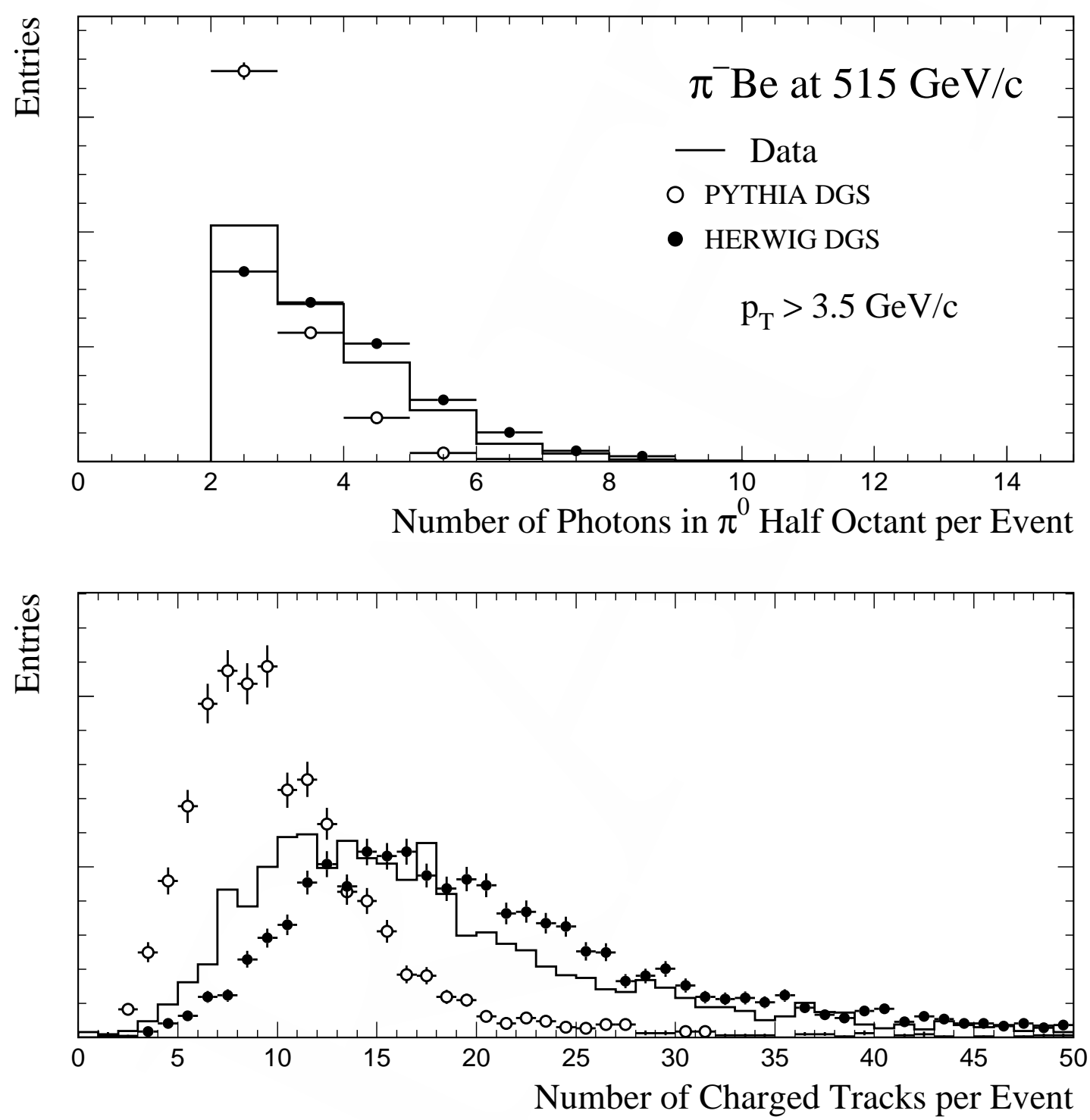

Figure 6.7 Comparison between the number of reconstructed photons in the triggering octant and the total number of reconstructed tracks in PYTHIA, HERWIG and the data for events containing $\pi^{0}$ 's with $p_{T}>3.5$ $\mathrm{GeV} / c$. The triggering octant is assumed to be the octant containing the $\pi^{0}$. 
event contain at least one user-defined particle with $p_{T}$ above some specified generation threshold, called $p_{T}^{G E N}$. Since the $p_{T}$ spectra of particles produced in strong interactions fall rapidly, several Monte Carlo samples were generated, each with a different value of $p_{T}^{G E N}$. This allowed the full range of $p_{T}$ spanned by the experiment to be adequately sampled, without investing huge amounts of resources populating the lower end of the $p_{T}$ spectrum. A description of the selection algorithms used by the DGS is presented below.

\section{HERWIG Event Selection Algorithms}

For the purpose of the evaluating $\gamma_{b} / \pi^{0}$, HERWIG was instructed to generate $2 \rightarrow 2$ QCD hard parton scatters. ${ }^{2}$ It is important to note that in these interactions, HERWIG does not generate any direct photons. Therefore, after processing the Monte Carlo data, any photons which satisfy the direct photon candidate criteria can be automatically considered as background to the direct photon signal. Also, since neutral mesons are natural products of these interactions, these samples were used to determine the $\pi^{0}$ and $\eta$ reconstruction efficiencies. For the evaluation of the direct photon reconstruction efficiency, a dedicated sample of HERWIG direct photon events was used. ${ }^{3}$

There were three different selection algorithms, or filters, used to select events for the evaluation of $\gamma_{b} / \pi^{0}$. These were called filters 3,2 , and 6 . The filter 3 algorithm proceeded as follows. After HERWIG generated a hard scattered interaction, a search was made over the final state particles in the interaction for either a $\pi^{0}, \gamma, e^{ \pm}$, or $K_{S}^{0}$ with $p_{T}>p_{T}^{G E N}$. These particles were chosen because either they themselves or their decay products readily produce electromagnetic showers in the EMLAC (and hence, background to the direct photon signal). If

$22 \rightarrow 2$ QCD hard scatters can be specified by setting the process identification number, variable IPROC in HERWIG, to 1500.

3 Direct photon events are generated by setting IPROC equal to 1800. 
the search was successful, then the event was accepted and processed through the full detector simulation, otherwise the event was rejected, and another hardscattered interaction was generated.

Although $\pi^{0}$ 's and $K_{S}^{0}$ 's are typically not considered final state particles, they were made final state particles by declaring them stable during the initialization of HERWIG. The decay of these particles was then handled during the detector simulation. The $\pi^{0}$ was explicitly held stable in HERWIG to ensure that the DGS also contained an unbiased sample of $\pi^{0}$ s $s$ for the evaluation of $\gamma_{b} / \pi^{0}$ and $\pi^{0}$ reconstruction efficiency calculations. ${ }^{4}$ The $K_{S}^{0}$ was held stable in HERWIG for a different reason. Due to its relatively long lifetime, the $p_{T}$ of the photons from the $K_{S}^{0}$ decay $^{5}$ tends to be mis-measured since the $p_{T}$ is calculated under the assumption that the photons originated at the primary vertex. By allowing the detector simulation to take care of the decay, this effect is included in the DGS. In general, short-lived particles such as the $\eta$ and the $\omega$ were decayed by HERWIG, while long-lived particles such as the $K_{S}^{0}$ were held stable and then decayed during the detector simulation.

Filter 2 was more sophisticated than filter 3. In addition to selecting events based upon particle $p_{T}$, it also selected events based upon the $p_{T}$ deposited in localized regions of the EMLAC. By selecting events in this manner, events in the data where multiple particles contributed to the satisfaction of a particular trigger definition were also included in the Monte Carlo simulation. In the final analysis, both filters were found to give consistent results for the ratio $\gamma_{b} / \pi^{0}$ and as a result, events from both filters were combined and used in the overall $\gamma_{b} / \pi^{0}$ determination.

\footnotetext{
${ }^{4}$ Otherwise, this filter would preferentially select events containing $\pi^{0}$ 's in which the $\pi^{0}$ decayed into a highly asymmetric photon pair.

5 These photons arise primarily from the decay chain $K_{S}^{0} \rightarrow \pi^{0} \pi^{0} \rightarrow \gamma \gamma \gamma \gamma$.
} 
The filter 2 algorithm proceeded in two stages. The first stage was similar to the algorithm used by filter 3, i.e., there was a search over the final state particles in the event for a high $p_{T}$ particle. However, in this case, the $\eta, \eta^{\prime}$, and $\omega$ were also declared stable in HERWIG, and the search for a high $p_{T}$ particle was over all final state particles with the exception of charged pions, protons, and antiprotons. Also, during this stage, the minimum particle $p_{T}$ requirement was set at $0.5 \mathrm{GeV} / c$ below the $p_{T}^{G E N}$ threshold. If the search was successful, then the first stage of the filter algorithm was satisfied and the event was sent to the detector simulation for the second stage of the filter algorithm.

In the second stage of filter 2 , the generated particles in the event were tracked by the detector simulation up to the ARMCO fire curtain, located just upstream of the LAC dewar. ${ }^{6}$ All the photons and electrons produced up to this point in the event were projected to the front face of the EMLAC, and "sums-of-8" 7 were calculated using the generated energies. Overlapping sums-of-16 were then calculated, and if the $p_{T}$ in any of the sums-of-16 was greater than $p_{T}^{G E N}$, or if the triggering particle was a $\pi^{0}, \gamma, \eta, \eta^{\prime}, \omega, K_{L}^{0}, K_{S}^{0}, e^{ \pm}$, or neutron and the total $p_{T}$ from photons and electrons in the trigger quadrant was greater than $p_{T}^{G E N}$, then the second stage of the filter was considered satisfied, and the detector simulation of the event was continued to completion.

Note that events containing high $p_{T}$ charged pions are not selected by either of the filters described above. These particles occasionally interact in the EMLAC and mimic an electromagnetic shower. Although this background is expected to be small since charged pions rarely deposit significant energy in the EMLAC, and the

6 The tracking of particles up to this point in the detector simulation is not prohibitively time consuming.

7 These sums-of- 8 are analogous to the sums-of- 8 used by the trigger (see Section 3.2). 
distance to nearest track and $E_{\text {front }} / E_{\text {total }}$ requirements imposed on direct photon candidates (see Section 5.5) will reject most of those that do, a sample of events containing high $p_{T} \pi^{ \pm}$'s was generated to explicitly evaluate this background. The filter used to produce this sample was called filter 6 . The filter 6 algorithm was very simple-requiring only that a $\pi^{+}$or a $\pi^{-}$with $p_{T}>p_{T}^{G E N}$ be found among the final state particles.

\section{$\underline{\text { Data-Driven Event Selection Algorithm }}$}

Events from the data that contained either a high $p_{T} \pi^{0}$ or $\eta$ candidate were used as input to the DATA-DRIVEn Monte Carlo. Two samples were generated, one with a low $p_{T}$ threshold and one with a high threshold. The low threshold sample consisted of events that satified the LOCAL GLOBAL LOW trigger and contained at least one $\pi^{0}$ or $\eta$ candidate with $p_{T}>3.0 \mathrm{GeV} / c$. The high threshold sample used

events that fired the SINGLE LOCAL HIGH trigger and had a $\pi^{0}$ or $\eta$ candidate with $p_{T}>3.5 \mathrm{GeV} / c$.

Photons which formed high $p_{T} \pi^{0}$ or $\eta$ candidates were removed from the input particle list. Substituted in their place was the reconstructed 4-vector of the meson candidate. In addition, photon pairs judged to be ZMP's were also removed and replaced by a single photon with the ZMP's 4-momenta. Photons that spatially matched with reconstructed tracks were replaced as either electrons, if they had high $E_{\text {front }} / E_{\text {total }}$, or as pions. Finally, since the data also contain true direct photons, any remaining photons that had $p_{T}$ greater than the generation threshold were removed from the input sample.

\subsubsection{Simulation of Detector Response}

The simulation of the spectrometer was performed using the GEANT software package developed at CERN. This is a package specifically designed to simulate 
the interactions of elementary particles with the detectors used in high energy physics experiments. GEANT provides a set of generic subroutines that enable the user to describe the shapes and properties of the various devices used by a given experiment. In addition, utilities are provided that allow the user to store various information during the simulation. This information can then later be used to digitize $^{8}$ the Monte Carlo data.

Understanding the response of the EMLAC to electromagnetic showers is critical in the study of direct photon and neutral meson production. The DGS played a large role in developing this understanding and so it was important to verify that GEANT accurately simulated the development of electromagnetic showers in the EMLAC. To this end, simulations of the development of electromagnetic showers in the EMLAC due to single electrons were performed, and the output was compared to a sample of high quality electron showers extracted from the $515 \mathrm{GeV} / c \pi^{-}$data. The input momentum spectrum of the Monte Carlo electrons was chosen to be the same as that observed for the electrons extracted from the data to eliminate ambiguities in the results due to differences in momenta spectra. The ratio of the electron energy measured in the EMLAC, $E$, to the electron momentum measured by the charged particle tracking system, $P$, is shown in Figure 6.8 as a function of $E$ for Monte Carlo and data. This distribution is sensitive to the electromagnetic shower shape, as well as to the amount of material in front of the EMLAC. The distribution from the Monte Carlo is in good agreement with the distribution from the real data. The falloff in the $E / P$ distribution at low energy is attributed to differences in the shower shape between electrons and photons. Electron-induced showers tend to be broader than photon-induced showers. However, the shower energies were determined from fits

8 Digitization refers to the process of taking the Monte Carlo event information and converting it into the hits registered by the various active detector elements. 
to shower shapes optimized for photon-induced showers (Section 4.3.3), which resulted in the underestimation of the energy of showers induced by low-energy electrons.

The tracking of the photons and electrons produced during the development of the showers in these simulations continued until their energies reached $1 \mathrm{MeV}$. Once this energy threshold was reached, the tracking of the particle stopped, and its remaining energy was deposited at that location. However, tracking photons and electrons in the EMLAC down to energies of $1 \mathrm{MeV}$ is a time consuming process. For example, using this energy cutoff, a single $70 \mathrm{GeV}$ photon shower took, on average, $\approx 3$ minutes of CPU time to fully develop on an SGI 4100 computer. Given the CPU resources available to the experiment, it was necessary to simulate electromagnetic shower development more efficiently.

Two important time-saving measures were taken. The first was to reduce the number of volumes ${ }^{9}$ needed to define the liquid argon calorimeter. In GEANT, a non-negligible fraction of time is spent calculating the probabilities for various physics processes to occur each time a particle encounters a new volume. A significant reduction of CPU time was achieved by combining several volumes into a single volume whose properties reflected the average of the combined volumes. To illustrate, in the original detector simulation, three volumes were necessary to define a copper clad G-10 board: two copper volumes and one G-10 volume. These three volumes were combined into a single homogenized volume of equivalent radiation length. In all, over 200 volumes were eliminated from the original GEANT simulation, and the CPU processing time per event was reduced by a factor of two.

The second measure taken was to increase the tracking cutoff energy from $1 \mathrm{MeV}$ to $10 \mathrm{MeV}$. This reduced the CPU time per event by a factor of 5 .

\footnotetext{
${ }^{9}$ In GEANT, the term volume refers to the separate elements of the detector.
} 


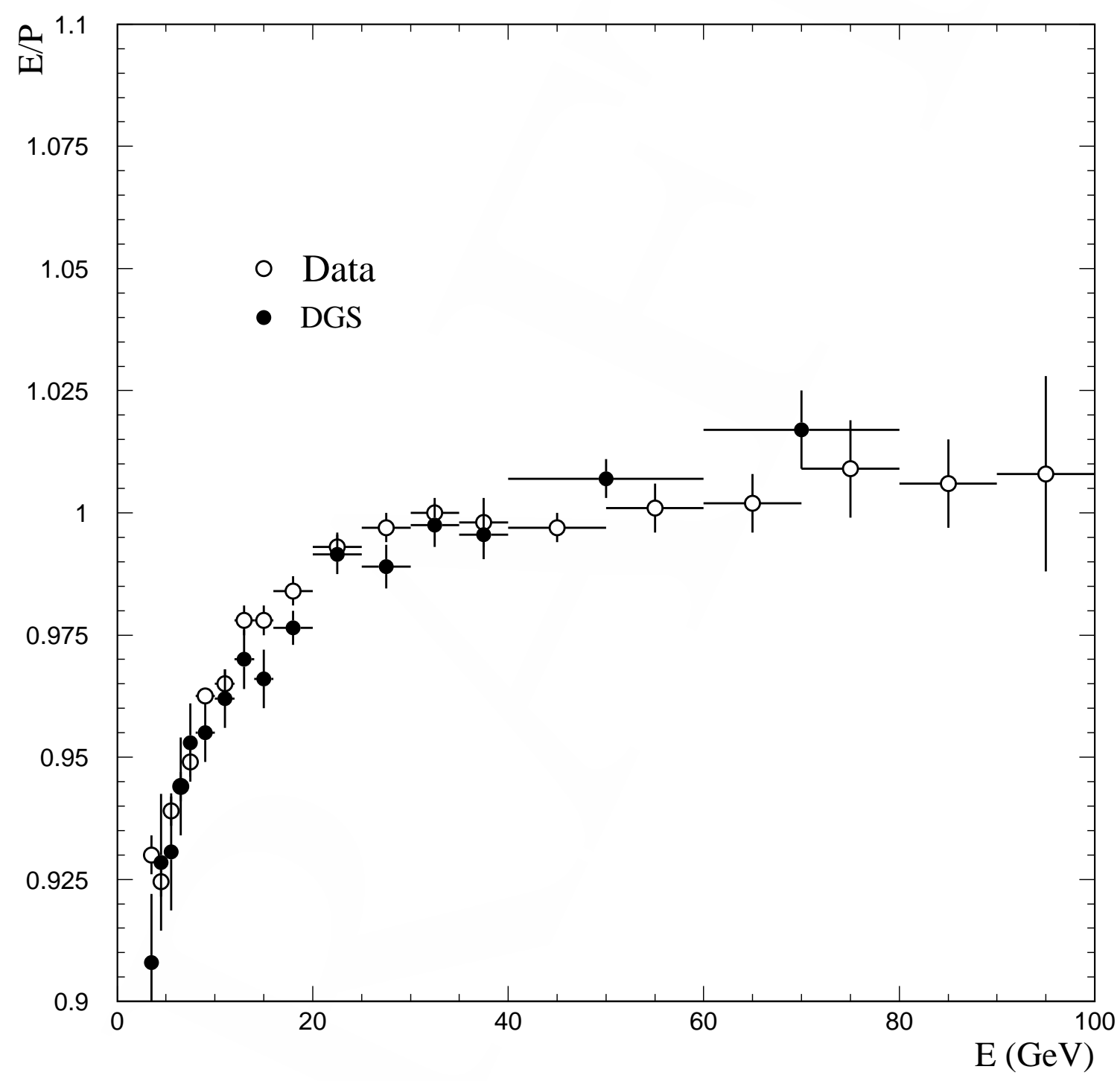

Figure 6.8 Comparison of $E / P$ distributions in the data and the Monte Carlo. 
Unfortunately, the generated shower shape suffered as a consequence. To rectify this, a simple model of electromagnetic shower development was implemented to continue the tracking after the $10 \mathrm{MeV}$ cutoff was reached in GEANT [72]. This model provided for photon conversion into $e^{+} e^{-}$pairs and energy loss due to ionization. Effects due to multiple scattering were also included in this model. The showers produced using this model and GEANT run with $10 \mathrm{MeV}$ energy cutoffs were found to be in reasonable agreement with the showers in the data in the transverse view. However, the longitudinal shower shape required additional tuning to reproduce the shower shape seen in the data.

Once this tuning was completed, the experiment generated the large sample of events needed to evaluate the direct photon background. These events were generated using several SGI and IBM computer clusters at Fermilab, and a cluster of SGI machines at the Physics Detector Simulation Facility (PDSF) in Dallas, Texas.

At the time of generation, the spectrometer elements were treated as "perfect" detectors-perfect in the sense that all channels were considered to be instrumented, fully efficient, and noiseless. Detector effects were then implemented prior to event reconstruction through the use of a preprocessing program called MCPREP. Among the detector effects modeled by the preprocessor were:

- noise and dead channels in the EMLAC;

- variations in the LACAMP gains;

- the intrinsic efficiency of the tracking system detectors;

- noise hits in the tracking system.

As many of these effects varied over time, MCPREP assigned run numbers from the data to the Monte Carlo events. The assignment of run numbers also allowed 
the simulation to account for the time dependence of the EMLAC response. The number of Monte Carlo events assigned per run number was proportional to the number of high $p_{T}$ triggers per run number in the data. This ensured the correct averaging of the run dependent effects.

In all, approximately 7.5 million HERWIG and DATA-DRIVEN Monte Carlo events were generated for the direct photon analysis. The breakdown, by beam type and $p_{T}^{G E N}$ threshold, of the HERWIG filter 2 and filter 3 direct photon background and HERWIG direct photon statistics is given in Tables 6.1 and 6.2, respectively. In addition, 55,000 filter 6 HERWIG events were generated for the purposes of evaluating the contribution from charged pions to $\gamma_{b} / \pi^{0}$. The DATADRIVEN Monte Carlo statistics numbered 0.3 million $p_{T}^{G E N}=3.0 \mathrm{GeV} / c$ and 1.0 million $p_{T}^{G E N}=3.5 \mathrm{GeV} / c 1990 \pi^{-}$beam events.

It may be noted that the Monte Carlo statistics for the analysis of the $1991 \pi^{-}$ and secondary proton beam data are very limited, particularly at low $p_{T}$. Time limitations prevented the generation of independent Monte Carlo statistics for these samples. Therefore, to obtain corrections for these beams, the $1990 \pi^{-}$beam Monte Carlo was rerun through MCPREP and the reconstructor using 1991 run numbers. In Figure 6.9, the number of charged tracks and the number of photons in the triggering half-octant ${ }^{10}$ per event are compared for the $515 \mathrm{GeV} / c \pi^{-}$beam data and the $530 \mathrm{GeV} / c$ proton beam data. Since these multiplicities do not differ significantly, using the $\pi^{-}$beam Monte Carlo to simulate the event structure in the secondary proton beam data is reasonable. Although the input particle spectra generated by the $\pi^{-}$beam HERWIG Monte Carlo are not appropriate for the 1991 secondary proton beam data, this problem was corrected through the use of special weighting functions that adjusted the input particle $p_{T}$ and rapidity spectra

10 In these comparisons, octants were divided into two at the inner-outer $\phi$ boundary $(\mathrm{R}=40.2 \mathrm{~cm})$. 
Table 6.1 Number of generated HERWIG filter 2 and filter 3 direct photon background Monte Carlo events (in thousands) as a function of the $p_{T}^{G E N}$ threshold.

\begin{tabular}{|c|c|c|c|c|}
\hline & 1990 & \multicolumn{3}{|c|}{1991} \\
\hline $\begin{array}{c}p_{T}^{G E N} \\
(\mathrm{GeV} / \mathrm{c})\end{array}$ & $\begin{array}{c}530 \mathrm{GeV} / \mathrm{c} \\
\pi^{-} \text {beam }\end{array}$ & $\begin{array}{c}800 \mathrm{GeV} / \mathrm{c} \\
\mathrm{p} \text { beam }\end{array}$ & $\begin{array}{c}530 \mathrm{GeV} / \mathrm{c} \\
\mathrm{p} \text { beam }\end{array}$ & $\begin{array}{c}530 \mathrm{GeV} / \mathrm{c} \\
\pi^{-} \text {beam }\end{array}$ \\
\hline 0.5 & 210 & 95 & - & - \\
\hline 1.5 & 220 & 330 & - & - \\
\hline 2.25 & 305 & - & - & - \\
\hline 2.5 & - & 195 & - & - \\
\hline 3.0 & 495 & 185 & - & - \\
\hline 3.5 & - & 850 & - & - \\
\hline 4.0 & 235 & - & - & - \\
\hline 5.0 & 200 & 265 & 110 & 95 \\
\hline 6.5 & 160 & 150 & - & - \\
\hline 7.0 & - & - & 100 & - \\
\hline 8.0 & 65 & 115 & 50 & - \\
\hline 9.0 & 40 & 35 & 25 & - \\
\hline
\end{tabular}

Table 6.2 Number of generated HERWIG Monte Carlo direct photon events (in thousands) as a function of the $p_{T}^{G E N}$ threshold.

\begin{tabular}{|c|c|c|c|c|}
\hline & 1990 & \multicolumn{3}{|c|}{1991} \\
\hline $\begin{array}{c}p_{T}^{G E N} \\
(\mathrm{GeV} / \mathrm{c})\end{array}$ & $\begin{array}{c}530 \mathrm{GeV} / \mathrm{c} \\
\pi^{-} \text {beam }\end{array}$ & $\begin{array}{c}800 \mathrm{GeV} / \mathrm{c} \\
\mathrm{p} \mathrm{beam}\end{array}$ & $\begin{array}{c}530 \mathrm{GeV} / \mathrm{c} \\
\mathrm{p} \text { beam }\end{array}$ & $\begin{array}{c}530 \mathrm{GeV} / \mathrm{c} \\
\pi^{-} \text {beam }\end{array}$ \\
\hline 3.0 & 440 & 347 & - & - \\
\hline 3.5 & - & 437 & - & - \\
\hline 5.0 & 203 & 210 & - & - \\
\hline 7.0 & 91 & 83 & - & - \\
\hline 8.5 & 10 & 48 & - & - \\
\hline
\end{tabular}


to the spectra observed in the data. These functions are described in the next section. Also, contributions to the direct photon background due to conversions were improperly calculated for these data samples, since the target configuration was different in the 1990 and 1991 runs. The PMC was run using the two target configurations to obtain a correction for this.

\subsubsection{Monte Carlo Spectrum Weighting}

The generated Monte Carlo $\pi^{0} p_{T}$ spectra were weighted to reproduce the spectra observed in the data, since the slope of the $p_{T}$ spectrum had effects on several aspects of the analysis (e.g. EMLAC calibration, evaluation of reconstruction efficiencies). The ratio $\gamma_{b} / \pi^{0}$ depends upon this slope also, since the number of background photons at a given $p_{T}$ depend upon the number of mesons at higher $p_{T}$.

The weighting functions were obtained through an iterative process. Originally, $\pi^{0}$ reconstruction efficiencies were determined and applied to the data with no weighting function. A weighting function was then determined, and applied to the Monte Carlo data. The reconstruction efficiencies were reevaluated, and updated cross sections were determined. This in turn led to new weighting functions and new efficiencies. This process was iterated several times until the results stabilized.

A separate function was determined for each $p_{T}^{G E N}$ sample in the DGS. For $p_{T}^{G E N}$ thresholds below $6.5 \mathrm{GeV} / c$, the weighting functions were functions of $p_{T}$ and rapidity. For $p_{T}^{G E N}$ thresholds $\geq 6.5 \mathrm{GeV} / c$, the weighting functions were functions of $p_{T}$ only, due to limited statistics at high $p_{T}$ in the data. A comparison

of the weighted and unweighted generated $\pi^{0} p_{T}$ spectra for the $1990 p_{T}^{\text {GEN }}=3.0$ $\mathrm{GeV} / c$ sample is shown in Figure 6.10. 

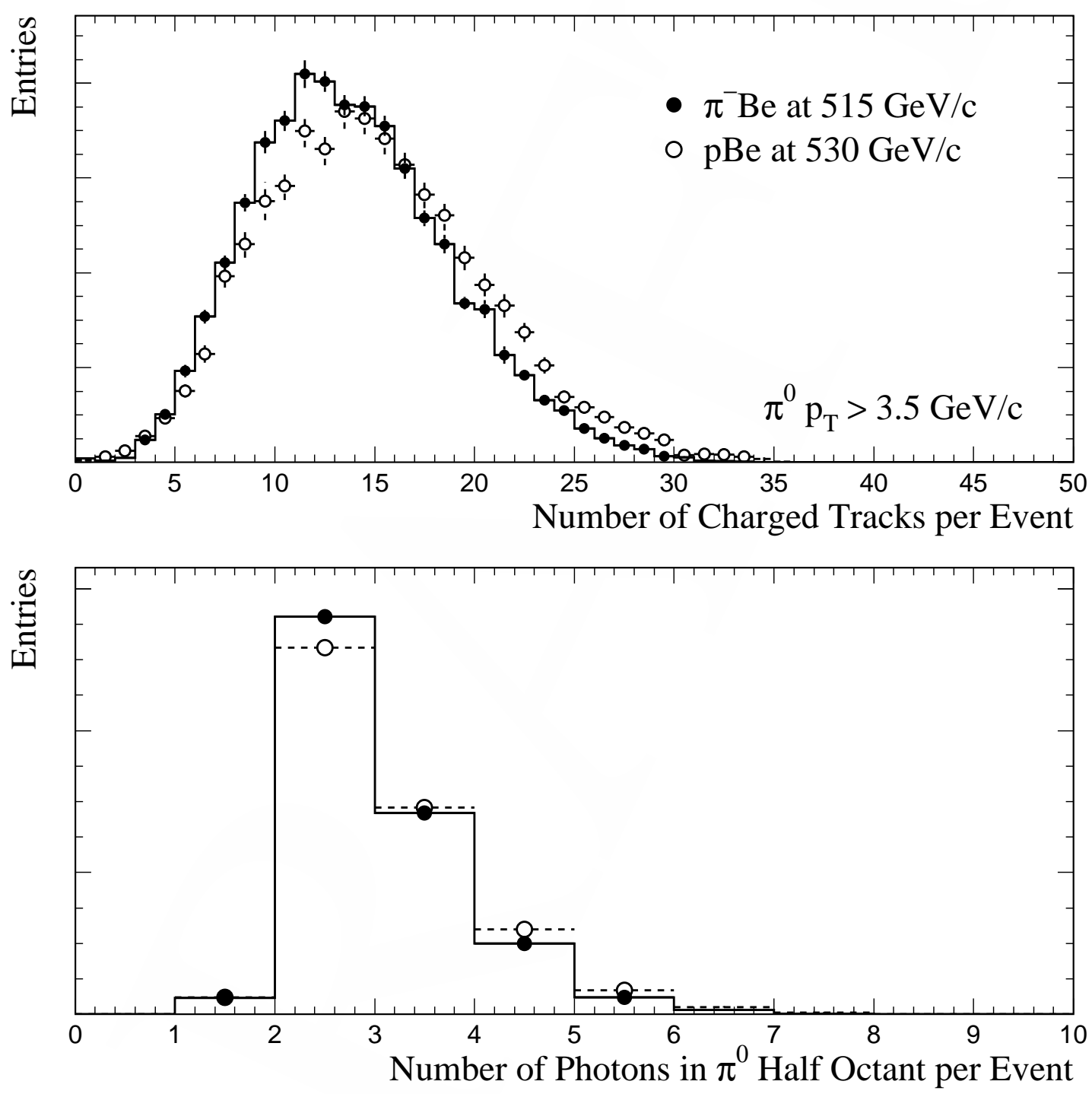

Figure 6.9 Comparison between the the total number of reconstructed tracks and the number of reconstructed photons in the triggering $1 / 2$ octant in the $515 \mathrm{GeV} / c \pi^{-}$beam data and the $530 \mathrm{GeV} / c$ proton beam data for events containing $\pi^{0}$ 's with $p_{T}>3.5 \mathrm{GeV} / c$. 


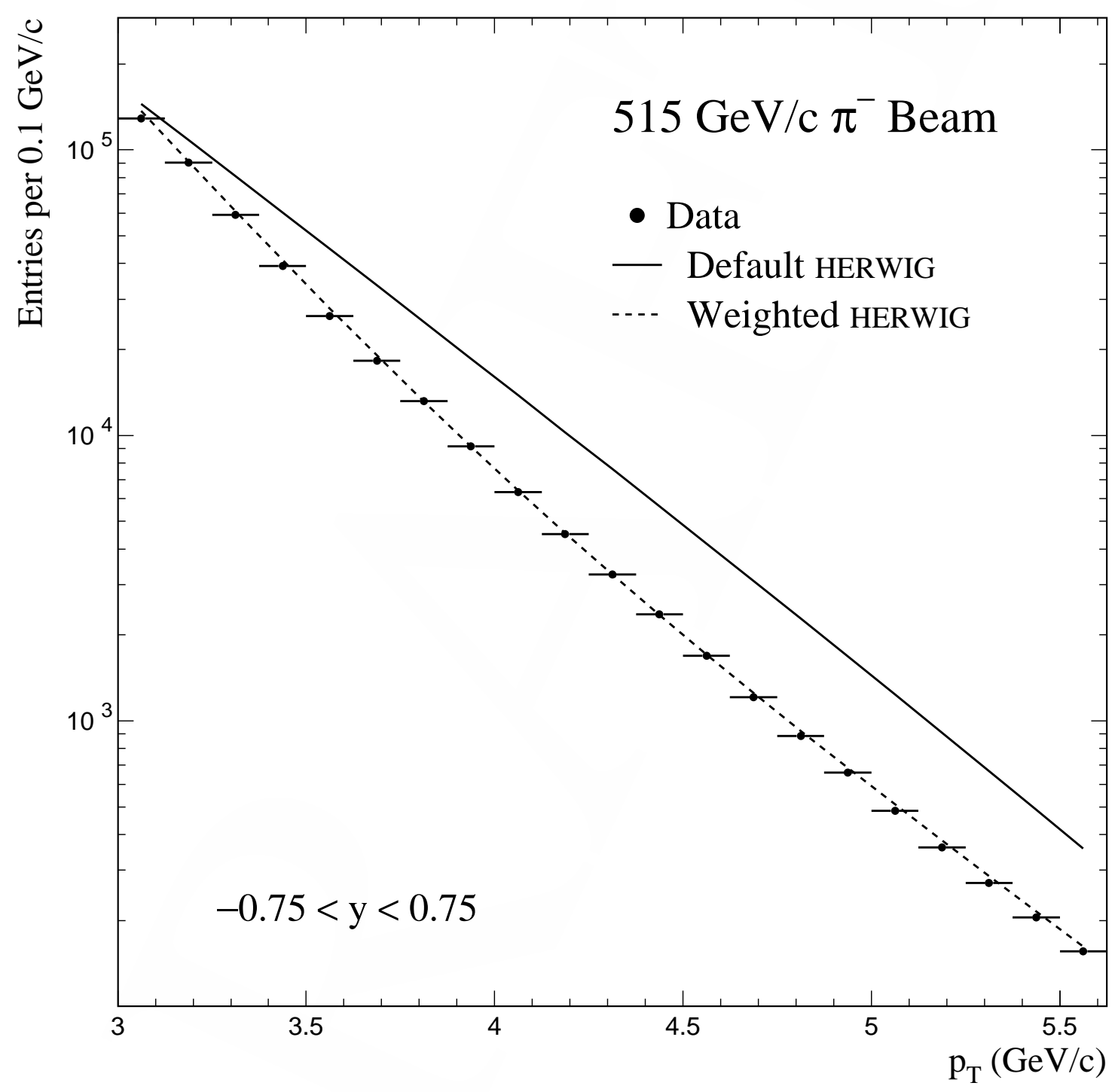

Figure 6.10 Comparison between the weighted and unweighted HERwiG $\pi^{0} p_{T}$ spectra for the $p_{T}^{G E N}=3.0 \mathrm{GeV} / c \pi^{-}$sample. The weighting surface is normalized to unity at $p_{T}=p_{T}^{G E N}, y_{c m}=0$. 
The DGS was also weighted so that $\eta / \pi^{0}$ was consistent with what was observed in the data, since $\eta$ 's contribute significantly to the direct photon background. In Figure 6.11 , the observed $\eta$ to $\pi^{0}$ production ratio is shown for the $515 \mathrm{GeV} / c \pi^{-}$ beam and the 530 and $800 \mathrm{GeV} / c$ proton beams. $\eta / \pi^{0}$ in HERWIG was adjusted to obtain this value. In principle, $\omega / \pi^{0}$ should also be weighted. However, in this case, the ratio was found to be consistent with data [94]. For other sources of direct photon background, it was assumed that HERWIG reproduced the ratio of these sources to $\pi^{0}$ 's correctly and therefore they were given the same weight as $\pi^{0}$ 's.

\subsubsection{Monte Carlo and Data Comparisons}

A variety of comparisons were made between the data and the DGS to verify that the E706 Monte Carlo provided an adequate simulation of the events seen in the data, and that it properly modeled the detector characteristics. These comparisons are discussed below.

As a cross-check of the DGS $p_{T}$ and rapidity weighting functions, comparisons were made between $\pi^{0}$ energy spectra in the DGS and data for each of the Monte Carlo samples. For example, Figure 6.12 shows the comparison between the data and the $p_{T}^{G E N}=3.0 \mathrm{GeV} / c$ DGS sample for $\pi^{0}$ 's with $p_{T}>3.5 \mathrm{GeV} / c$ in the 530 $\mathrm{GeV} / c$ proton data. The good agreement between the Monte Carlo and the data indicates the Monte Carlo $p_{T}$ and rapidity weighting function was appropriately evaluated.

It is important to verify that the DGS reproduces the resolution of the EMLAC. Since the EMLAC's $\mathrm{R}$ and $\phi$ boards are interleaved, the energy reconstructed in each view should be roughly equal, with the difference in energies due to fluctuations in the amount of deposited energy in each view. Hence the difference, $E_{R}-E_{\phi}$, provides a measure of the EMLAC's resolution. In Figure $6.13, E_{R}-E_{\phi}$ 


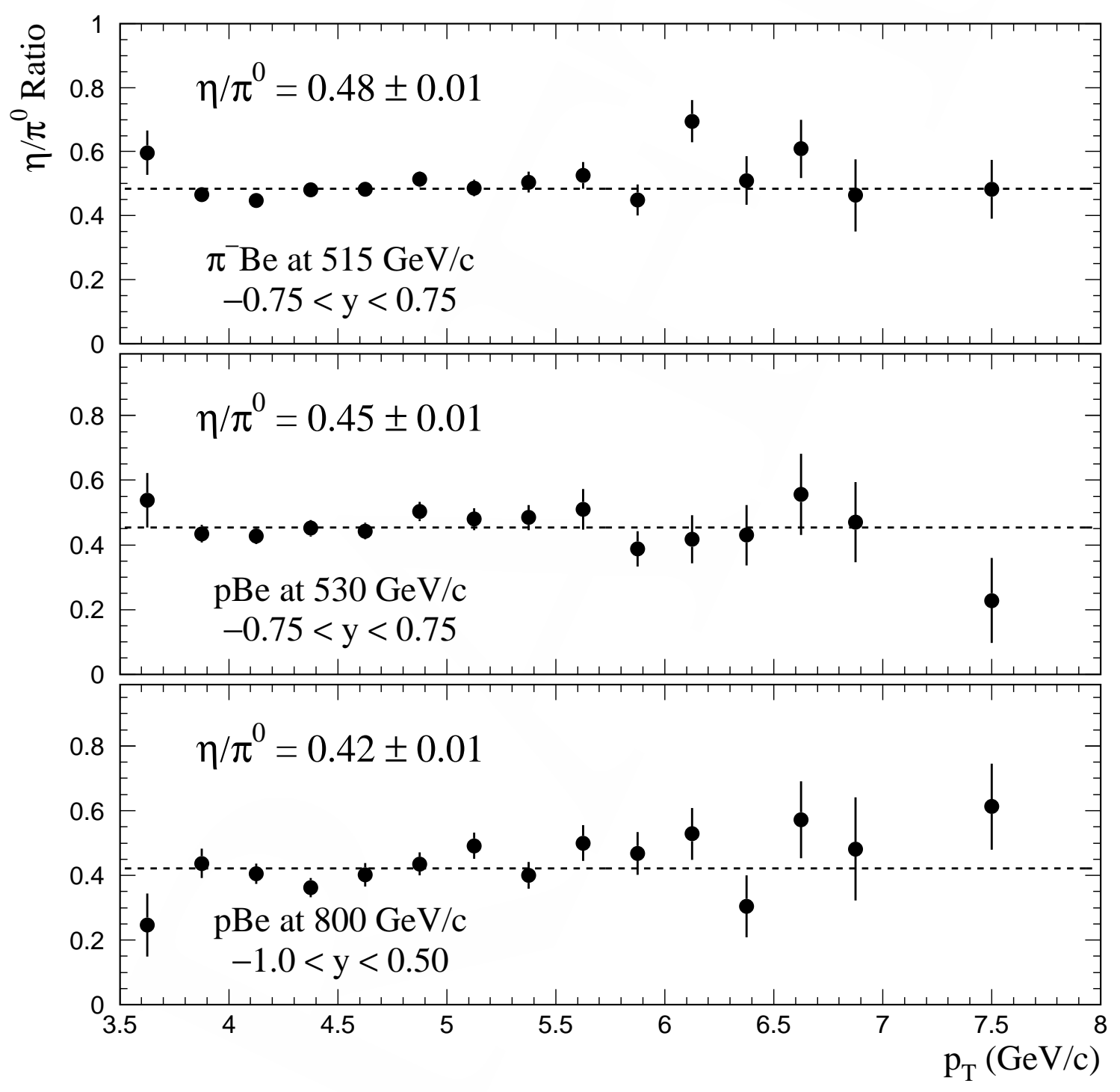

Figure 6.11 Ratio of the measured $\eta$ to $\pi^{0}$ production cross sections versus $p_{T}$ for $515 \mathrm{GeV} / c \pi^{-}$beam and 530 and $800 \mathrm{GeV} / c$ proton beams. The error bars represent only statistical errors. 


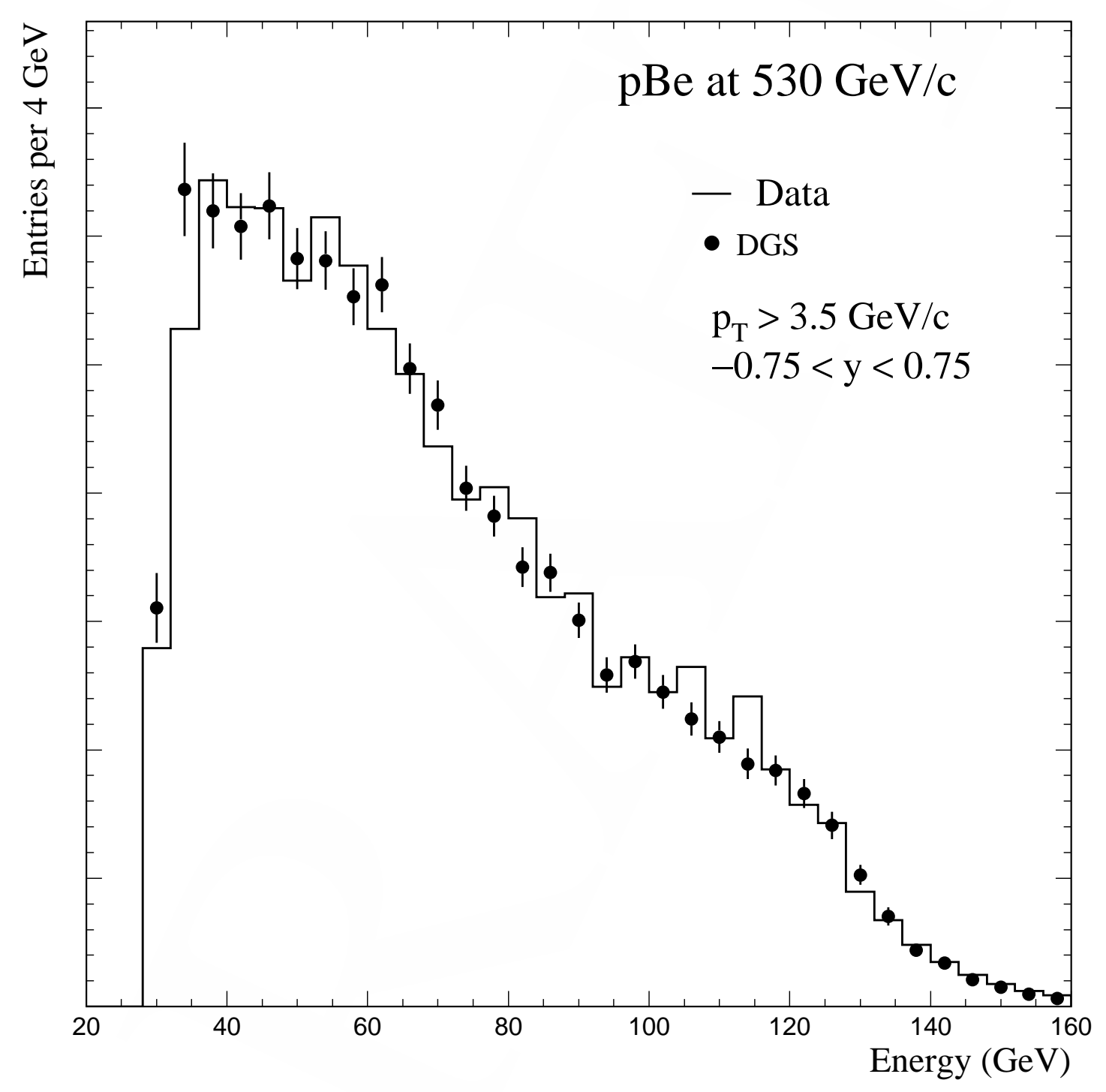

Figure 6.12 Comparison of $\pi^{0}$ energy distributions in the data and the DGS. The solid points are the DGS and the histogram is the data. 
is plotted for data and the DGS for several reconstructed energy intervals. The agreement is excellent over a large range of photon energies. Another distribution sensitive to the detector resolution is the reconstructed $\gamma \gamma$ mass distribution. Figure 6.14 shows a comparison between the reconstructed $\pi^{0}$ and $\eta$ masses in the data and the DGS. The width of these distributions is sensitive to both the position and energy resolution of the EMLAC. Also of interest in this plot is the amount of background underneath the $\pi^{0}$ and $\eta$ mass peaks. The good agreement between the backgrounds in data and the DGS indicates that the DGS is providing a reasonable representation of the underlying event structure.

The longitudinal development of electromagnetic showers can be checked by comparing distributions in $E_{\text {front }} / E_{\text {total }}$ for photons in the DGS and the data. These distributions are also sensitive to the amount of material located in front of the instrumented portion of the EMLAC. A comparison of $E_{\text {front }} / E_{\text {total }}$ distributions in data and the DGS is shown in Figure 6.15. Again, the agreement is excellent.

A comparison between the background subtracted energy asymmetry distributions for $\gamma \gamma$ pairs in the $\pi^{0}$ mass region in the DGS and the data is shown in Figure 6.16 for the $800 \mathrm{GeV} / c$ proton data. Since this distribution is sensitive to the loss of low energy photons, the good agreement between the data and the DGS indicates that the DGS simulated these losses well. Because the $\pi^{0}$ reconstruction efficiency was a function of rapidity as well as $p_{T}$, this comparison is also shown for several rapidity intervals in Figure 6.17. The agreement is good for each of these intervals.

It is worthwhile to compare the number of reconstructed photons in the DGS and the data since the response of the EMLAC may be affected by shower multiplicity. In Figure 6.18, the number of reconstructed photons in half-octants 


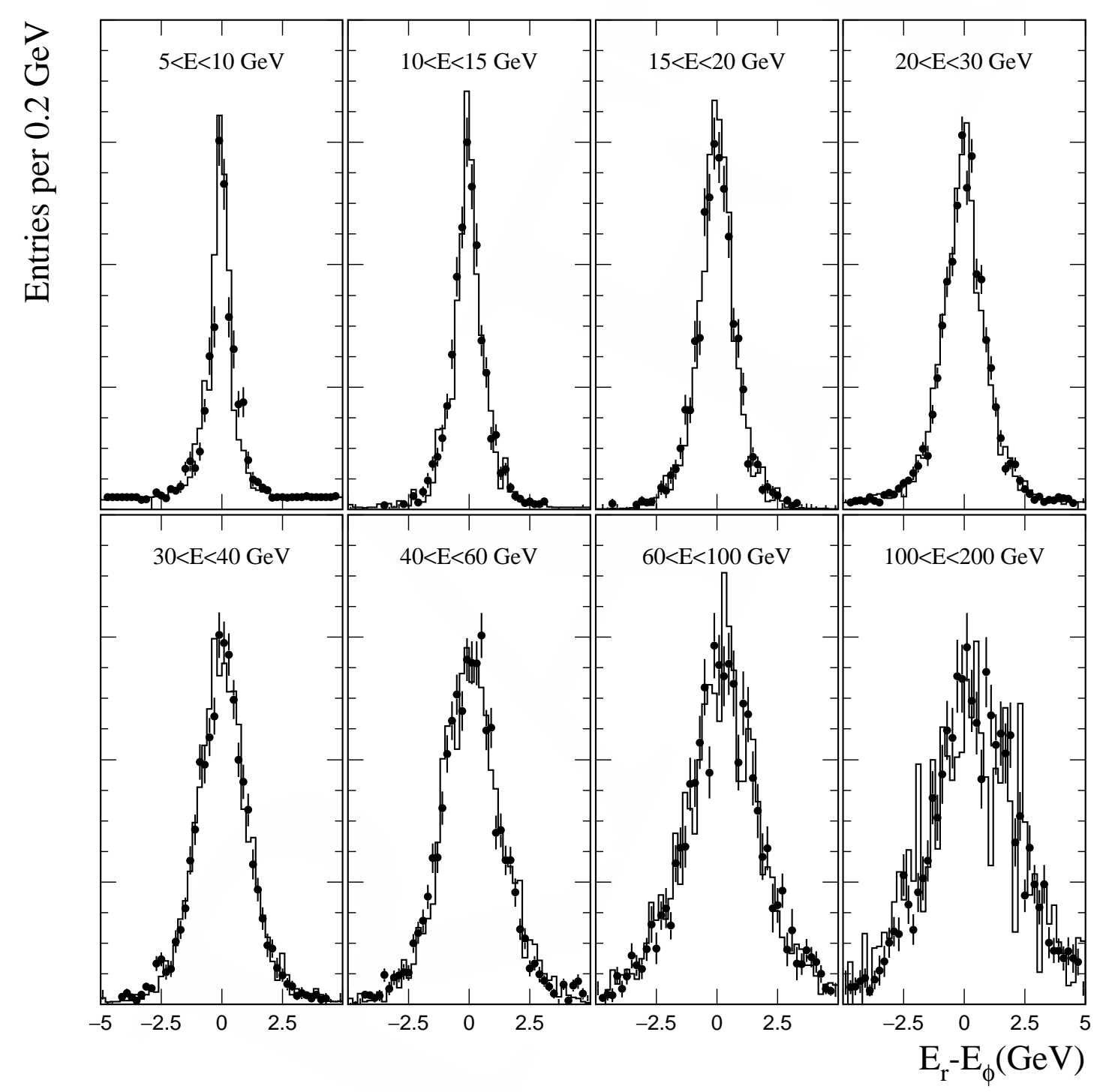

Figure 6.13 Comparison of $E_{R}-E_{\phi}$ distributions in the data and the DGS for the $530 \mathrm{GeV} / c$ proton beam. The black dots are the DGS and the histogram is the data. 


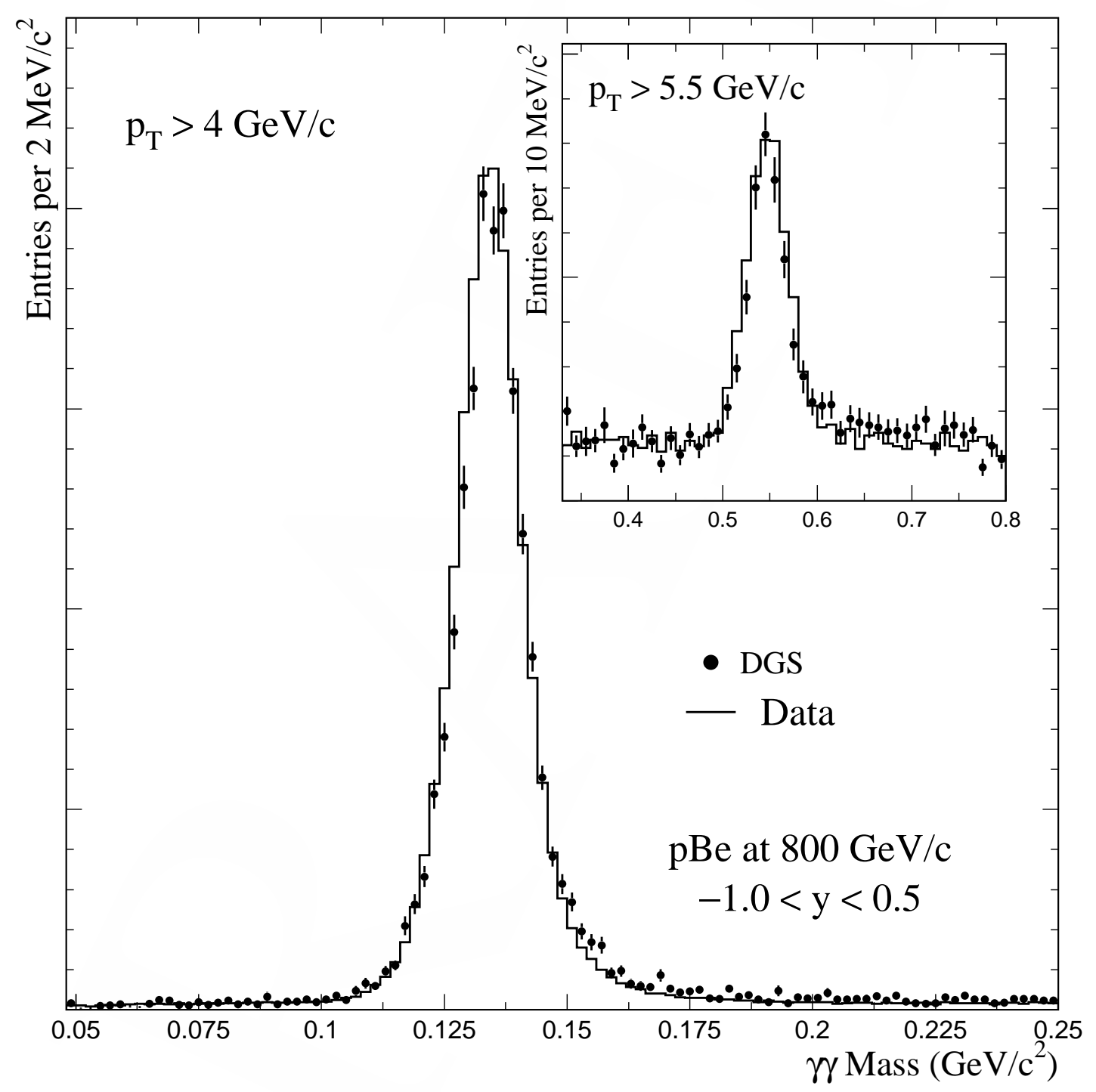

Figure 6.14 Comparison of the $\pi^{0}$ and $\eta$ mass distributions in the data (histogram) and the DGS $(\bullet)$ for the $800 \mathrm{GeV} / c$ p beam. 


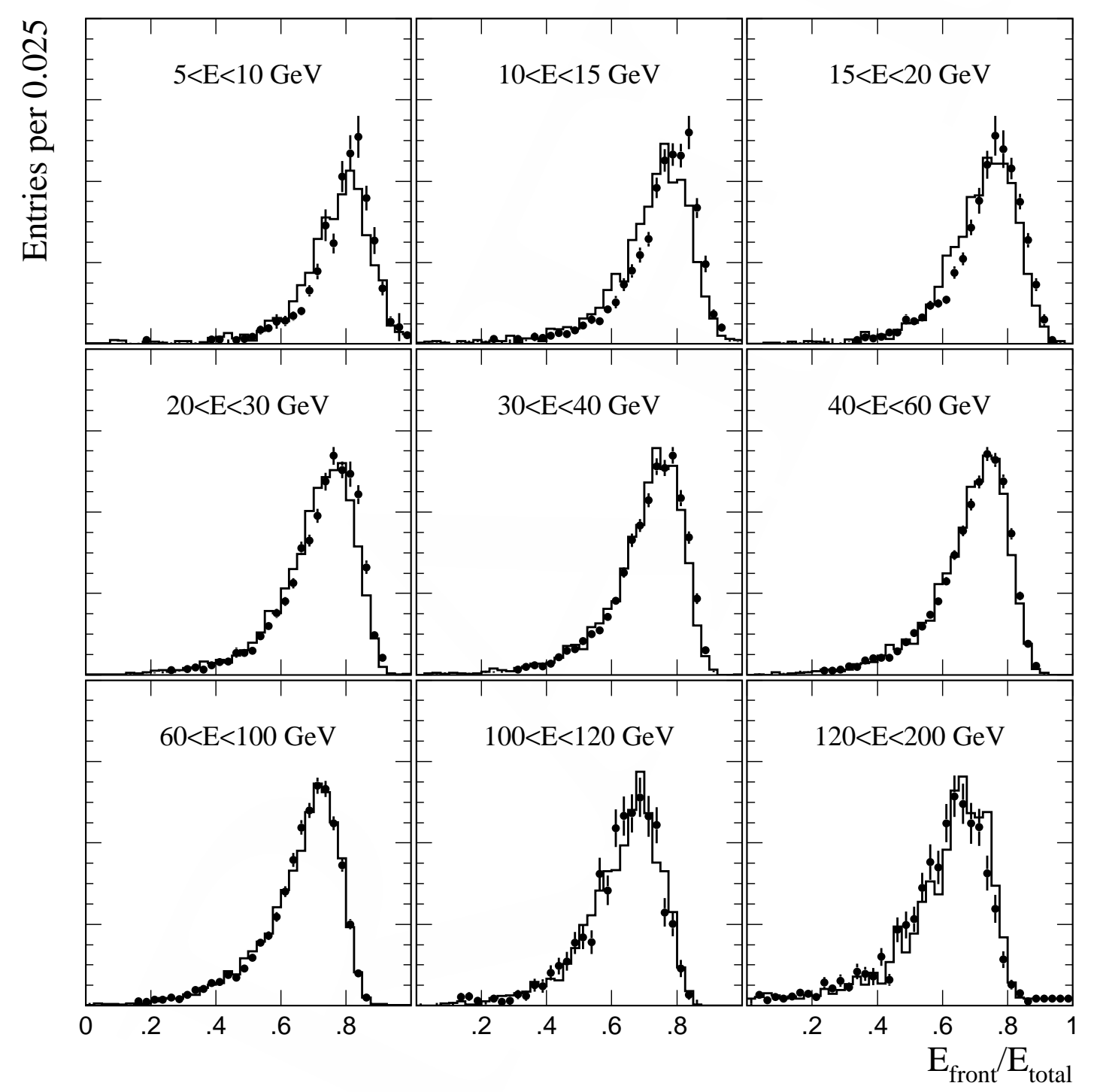

Figure 6.15 Comparison of $E_{\text {front }} / E_{\text {total }}$ distributions in the data and the DGS for the $515 \mathrm{GeV} / c \pi^{-}$beam. The histogram is the data, and the points are the DGS. 


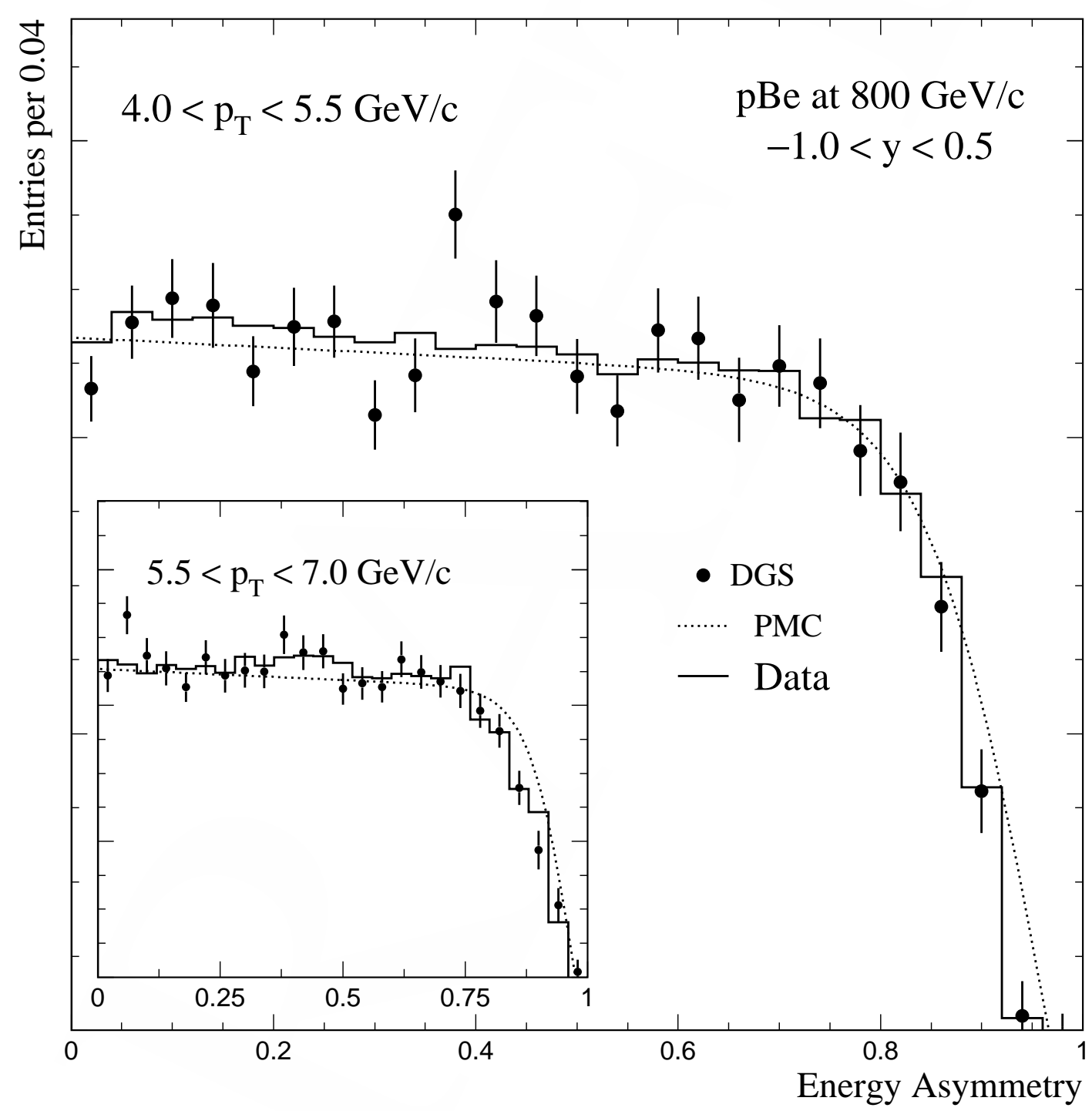

Figure 6.16 Comparisons of background subtracted $\pi^{0}$ energy asymmetry distributions in data (histogram) and the DGS $(\bullet)$. Comparisons are shown for two $p_{T}$ intervals, $4.0<p_{T}<5.5 \mathrm{GeV} / c$ and $5.5<p_{T}<7.0 \mathrm{GeV} / c$. Also shown are energy asymmetry distributions from the PMC (dotted curves). 


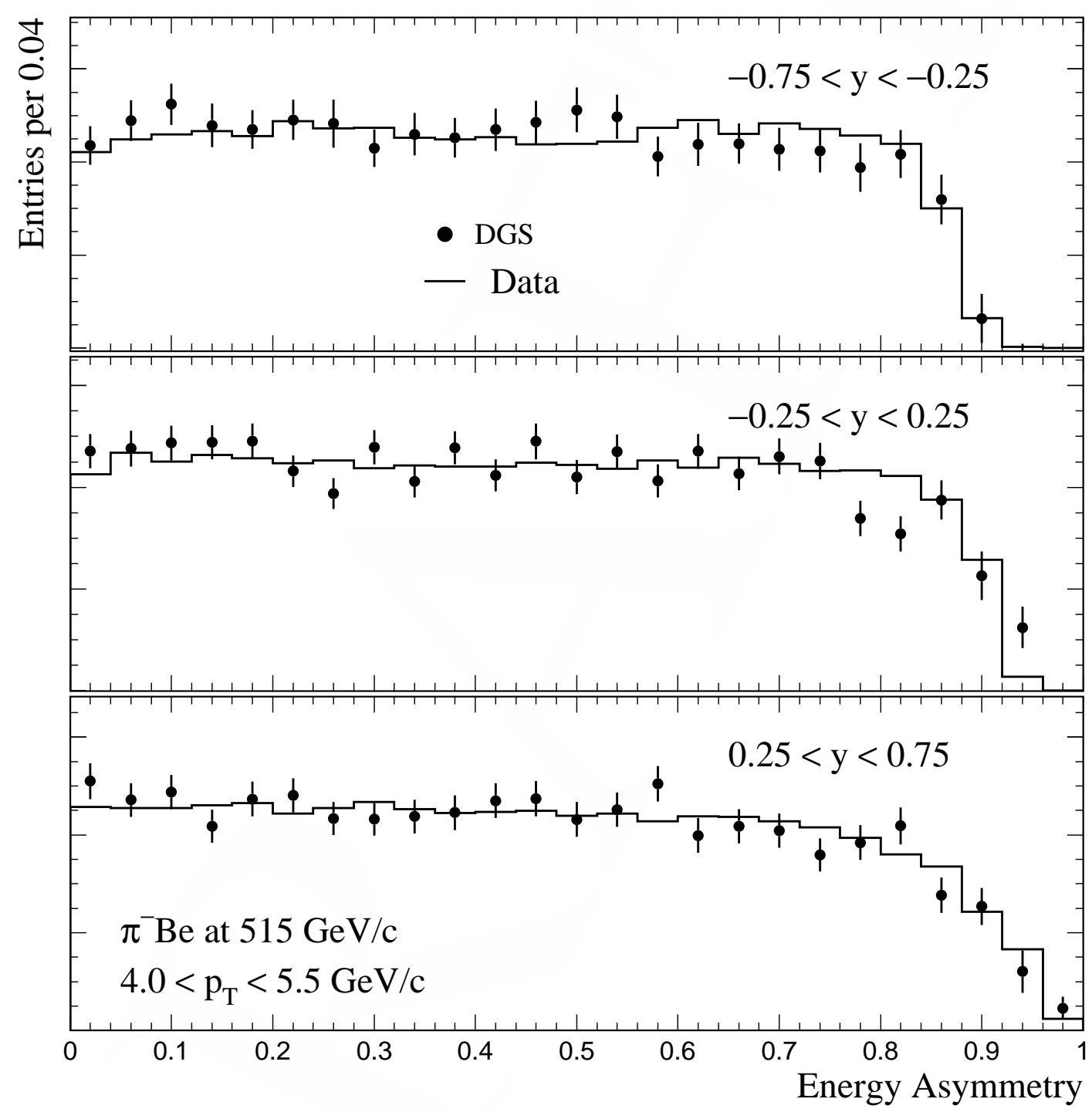

Figure 6.17 Comparison of background subtracted $\pi^{0}$ energy asymmetry distributions in the data (histogram) and the DGS $(\bullet)$ for backward, central, and forward rapidity ranges. 
containing a reconstructed $\pi^{0}$ candidate for the $800 \mathrm{GeV} / c$ proton beam are compared. The comparison is shown for over the full rapidity range and over several smaller ranges. The mean multiplicities in these plots agree to within 0.1 photons. Recall, this comparison was also made for the $515 \mathrm{GeV} / c \pi^{-}$beam in Figure 6.7. Although the agreement is still good there (the mean multiplicies are within 0.25 of each other), this may be an indication that HERWIG models the underlying event structure better in proton induced reactions than in $\pi^{-}$induced reactions.

\subsubsection{Evaluation of Reconstruction Efficiencies}

The DGS was used to evaluate the neutral meson and direct photon reconstruction efficiencies. These efficiencies, in addition to correcting for detector losses, also provided corrections for EMLAC resolution smearing effects (Section 6.2.1), the photon $E_{\text {front }} / E_{\text {total }}$ requirement (Section 5.4), and the trigger probability requirement (Section 5.10.2).

The reconstruction efficiencies were evaluated as functions of $p_{T}$ and rapidity, and were defined to be the ratio of the number of reconstructed particles to the number of generated particles. ${ }^{11}$ The reconstructed particles were binned according to their reconstructed $p_{T}$ and rapidity, while the generated particles were binned according to their generated $p_{T}$ and rapidity. By binning in this manner, the reconstruction efficiencies corrected for the effects of resolution smearing in the EMLAC.

The reconstructed photons used in the evaluation of the neutral meson and direct photon efficiencies had the $E_{\text {front }} / E_{\text {total }}$ cut imposed on them to correct for the $E_{\text {front }} / E_{\text {total }}$ requirement. Also, reconstructed entries were only included if

11 For the neutral meson efficiencies, the number of reconstructed particles was determined using the sideband subtraction technique described in Section 5.11. 


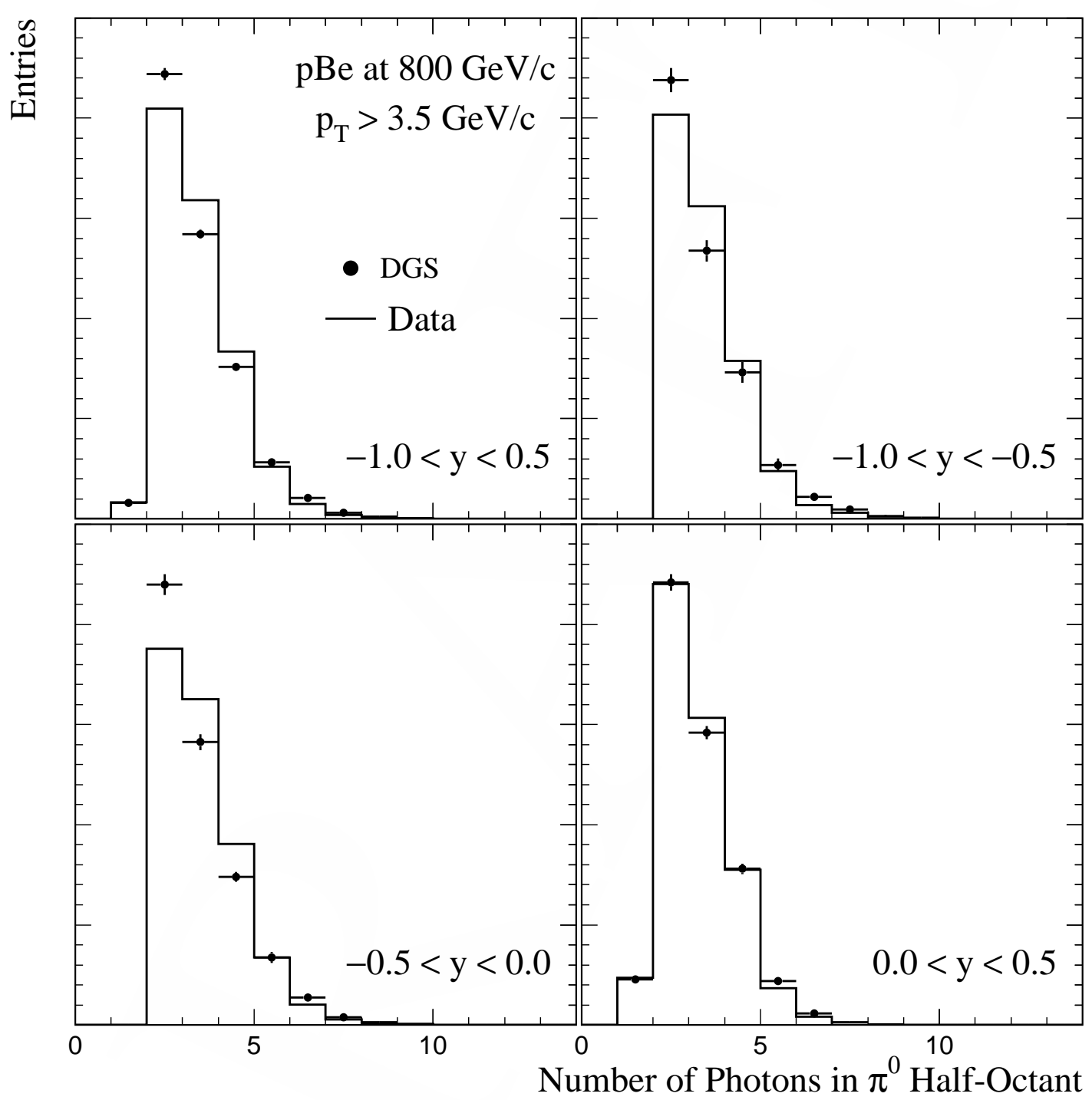

Figure 6.18 Comparison between the number of reconstructed photons in halfoctants containing a $\pi^{0}$ candidate with $p_{T}>3.5 \mathrm{GeV} / c$ in the DGS and the data. 
the octants they landed in had at least a $10 \%$ probabilty of firing a given trigger. This corrected for the minimum trigger probability requirement. Note that this implies each trigger definition had its own reconstruction efficiency function.

In addition, the following requirements were placed upon both the reconstructed and generated entries:

- a reconstructed vertex in the target region;

- the photons landed within the EMLAC's fiducial volume;

- the photons did not convert into $e^{ \pm}$pairs;

- for the neutral meson efficiencies, the energy asymmetry of the decay was less than 0.75 .

Each requirement listed above had its own independent correction. Imposing these requirements on both generated and reconstructed entries ensured the reconstruction efficiency did not correct for these requirements as well. Also, in order to fully reproduce the effects of resolution smearing, the reconstructed and generated particles were required to have $p_{T}$ at least $0.5 \mathrm{GeV} / c$ above the value of the $p_{T}^{G E N}$ threshold of the DGS sample.

Reconstruction efficiencies for $\pi^{0}$ mesons for the $1991530 \mathrm{GeV} / c$ proton beam are shown in Figure 6.19 for INTERACTION, SINGLE LOCAL LOW, and SINGLE LOCAL HIGH triggers. The falloff at low $p_{T}$ in the SINGLE LOCAL LOW and SINGLE LOCAL HIGH trigger efficiencies are due to the trigger probability requirement. The falloff at high $p_{T}$ and forward rapidity is due to the coalescence of the two photons from the $\pi^{0}$ decay. Real detector losses are seen at low $p_{T}$ and backward rapidity in the INTERACTION trigger efficiency. Also shown in the figure is the contribution to the efficiency from EMLAC resolution smearing. Note that away from the regions described above, resolution smearing is the dominant contributor to the inefficiency. 


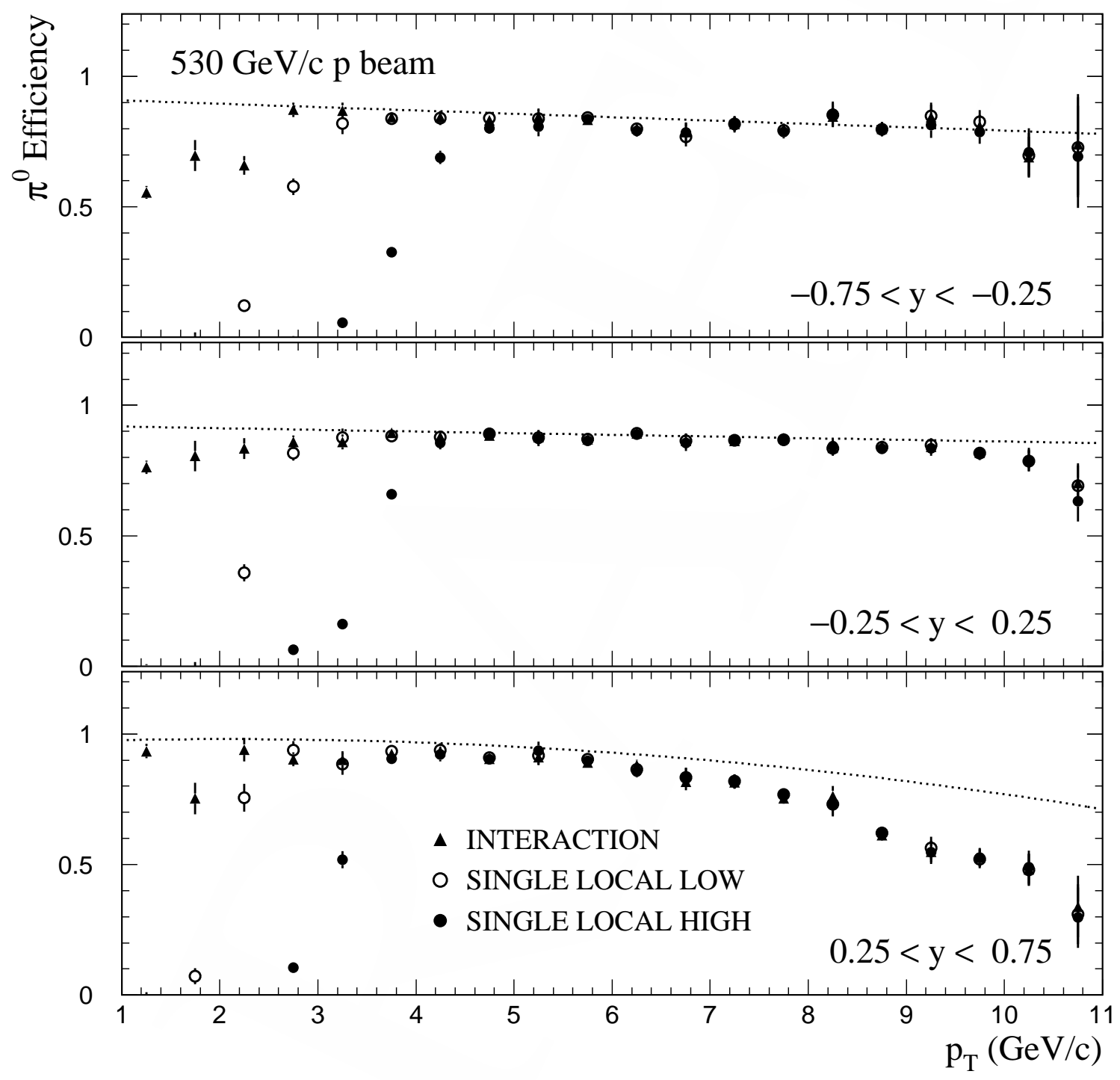

Figure 6.19 $\pi^{0}$ reconstruction efficiency for the $530 \mathrm{GeV} / c$ proton beam as a function of $p_{T}$ for several rapidity ranges. The dotted curves in the figure indicate the contribution from EMLAC resolution smearing alone. 


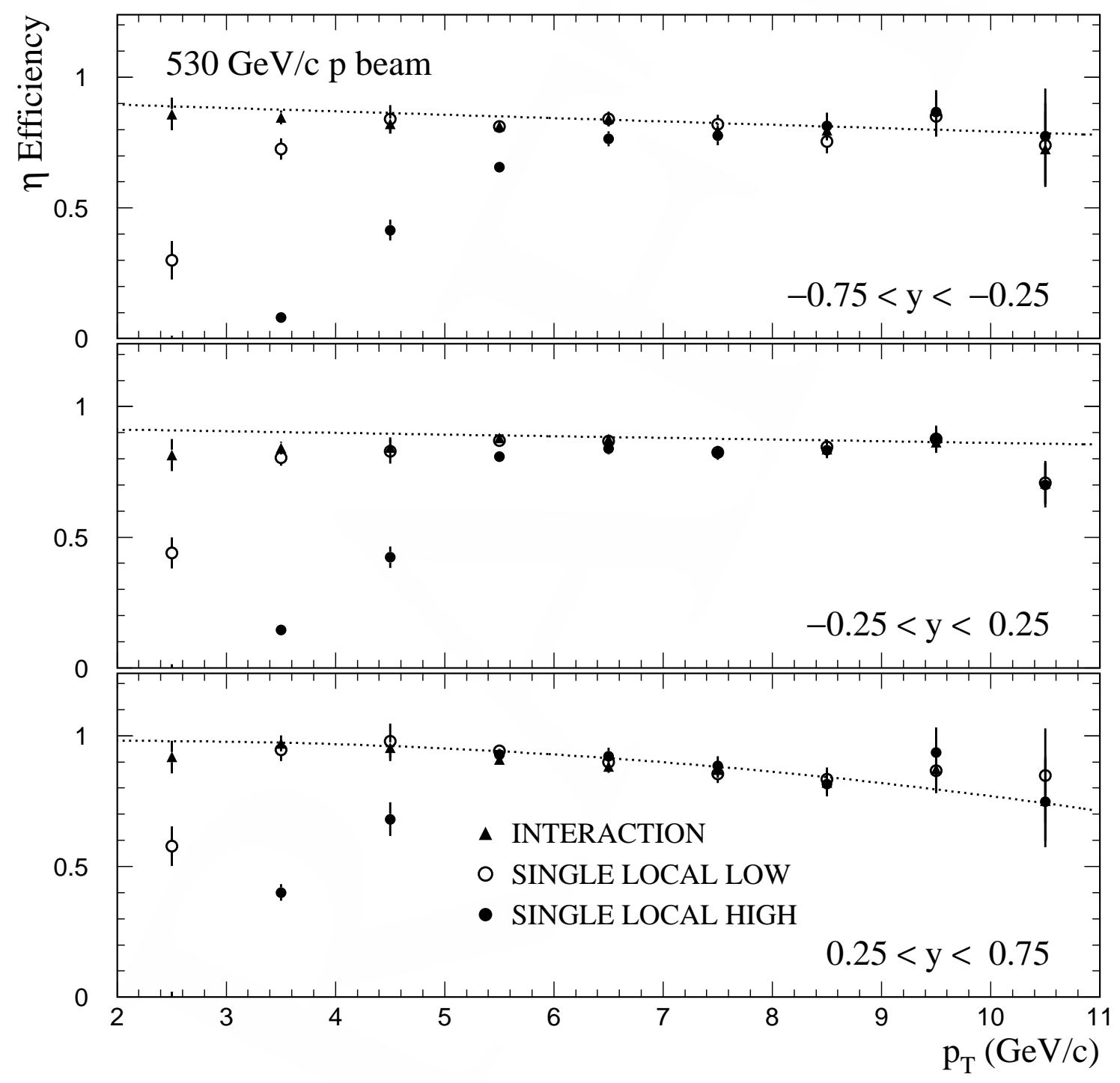

Figure 6.20 $\eta$ reconstruction efficiency for the $530 \mathrm{GeV} / c$ proton beam as a function of $p_{T}$ for several rapidity ranges. The dotted curves in the figure indicate the contribution from EMLAC resolution smearing alone. 
In Figure 6.20, corresponding efficiencies for the $\eta$ are shown. Note that in the case of the $\eta$, trigger effects are present at significantly higher $p_{T}$ 's than for the $\pi^{0}$, since the wider separation of the photons from the $\eta$ decay makes it more difficult to satisfy the trigger- $p_{T}$ threshold for a given sum-of-16. In addition, there is little evidence of coalescence at high $p_{T}$.

The SINGLE LOCAL HIGH direct photon efficiencies for the $1991530 \mathrm{GeV} / c$ proton data for the $90 \mathrm{~N}, 75 \mathrm{~N}$, and $75 \mathrm{~S}$ candidate definitions are shown as functions of $p_{T}$ in Figure 6.21. The $75 \mathrm{~S}$ definition has the highest efficiency since, in this definition, losses due to direct photons making $\gamma \gamma$ mass combinations in the $\pi^{0}$ signal region with random photons in the octant are compensated for (Section 5.12). These losses are greatest at low $p_{T}$, and are fractionally larger in the $90 \mathrm{~N}$ definition than in the $75 \mathrm{~N}$ definition since the combinatorial background underneath the $\pi^{0}$ grows with energy asymmetry (recall Figure 5.13). As was the case for the $\pi^{0}$, the majority of the direct photon inefficiency is due to energy resolution smearing.

$\underline{\text { Systematic Uncertainties in the Reconstruction Efficiencies }}$

The main contributors of systematic uncertainty in the reconstruction efficiencies were: statistical limitations in the Monte Carlo data, dependence of the efficiency on the modeling of the detector response, detector environment, and trigger response. To assess the systematic uncertainty arising from the Monte Carlo statistics, closure tests were performed in which the ratio between the efficiency-corrected number of reconstructed entries and the number of generated entries was calculated. These ratios were consistant with unity within $\pm 1 \%$.

To assess the uncertainty in the efficiency due to the modeling of the detector response, the smearing contribution to the efficiency was evaluated using the PMC and compared with the result from the full shower Monte Carlo. Comparisons 


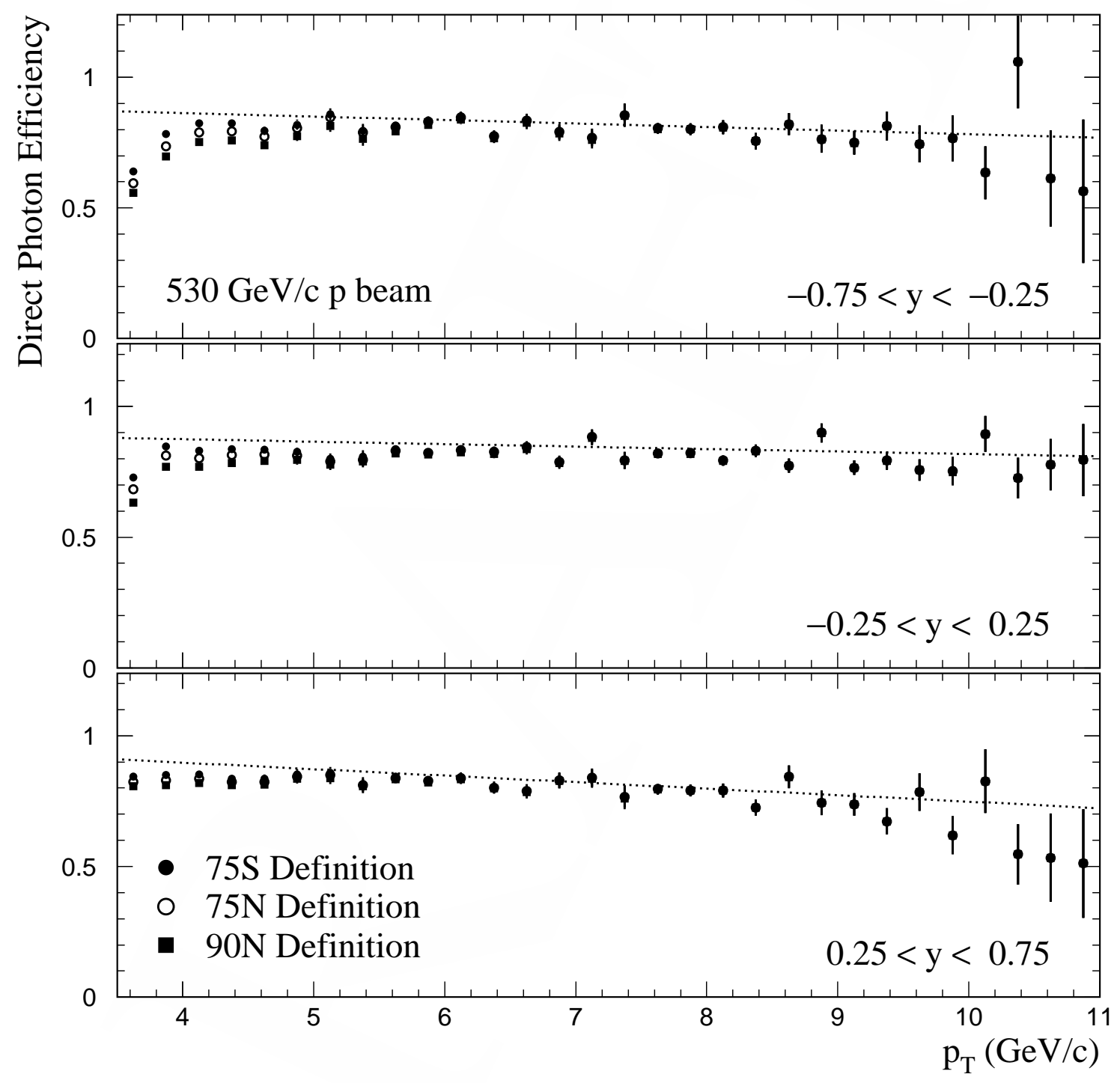

Figure 6.21 SINGLE LOCAL HIGH direct photon reconstruction efficiencies for the $90 \mathrm{~N}, 75 \mathrm{~N}$ and $75 \mathrm{~S}$ candidate definitions as functions of $p_{T}$ for several rapidity ranges. The dotted curves in the figure indicate the contribution from EMLAC resolution smearing alone. 
were made for direct photons, $\pi^{0}$ 's and $\eta$ 's and the results were typically found to agree to within $\pm 5 \%$. Therefore, a $\pm 5 \%$ systematic uncertainty was assigned for the modeling of the detector response.

The uncertainty in the efficiency due to the modeling of the detector environment was assessed by examining the reconstruction probability ${ }^{12}$ as a function of the number of generated photons in the $1 / 2$ octant containing the generated direct photon, $\pi^{0}$, or $\eta$. This is shown for $\pi^{0}$ 's in Figure 6.22. At low $p_{T}$, the reconstruction probability drops by $<2 \%$ for each additional generated photon in the $1 / 2$ octant. Since the mean number of reconstructed photons in the data and the DGS agree to better than 0.25 photons (recall the discussion in Section 6.3.4), a systematic uncertainty of $\pm 1 \%$ was assigned to account for the modeling of the detector environment. Another measure of the uncertainty in the modeling of the detector environment can be obtained by using the DATADRIVEN DGS to evaluate the reconstruction probability and comparing it to the reconstruction probability evaluted using the HERWIG DGS. This comparison is shown for both the LOCAL GLOBAL LOW and SINGLE LOCAL HIGH triggers in Figure 6.23. For the LOCAL GLOBAL LOW trigger, these probabilities are within $\pm 1 \%$ of each other. For the SINGLE LOCAL HIGH trigger, these probabilities also agree to within $\pm 1 \%$ for $p_{T}$ 's above $4.0 \mathrm{GeV} / c$. However, at lower $p_{T}$, the agreement is worse. This discrepancy between the two triggers is most likely due to the effect of the detector environment on the trigger response.

To assess the uncertainty in the trigger response near the trigger threshold, the ratio of the $\pi^{0}$ cross section measured using the SINGLE LOCAL HIGH trigger to the cross section measured with the SINGLE LOCAL LOW trigger (LOCAL GLOBAL

12 The reconstruction probability differs from the reconstruction efficiency in that the effects of smearing are removed from the reconstruction probability by binning the reconstructed entries according to their generated $p_{T}$ 's and rapidities. 


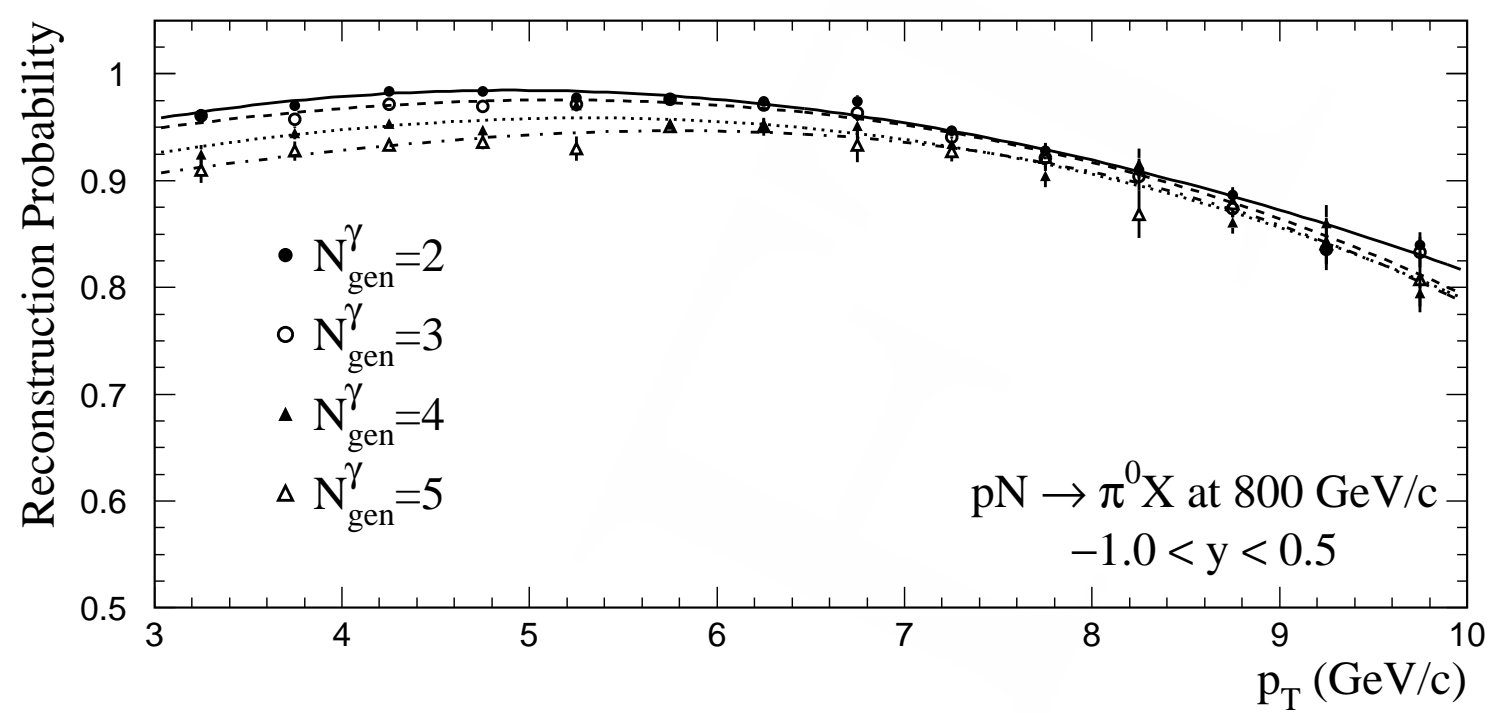

Figure 6.22 Reconstruction probability for $\pi^{0}$ 's as a function of the number of generated photons in the $1 / 2$ octant of the generated $\pi^{0}$. The curves represent fits to the Monte Carlo points. No trigger requirement has been placed on the Monte Carlo data.

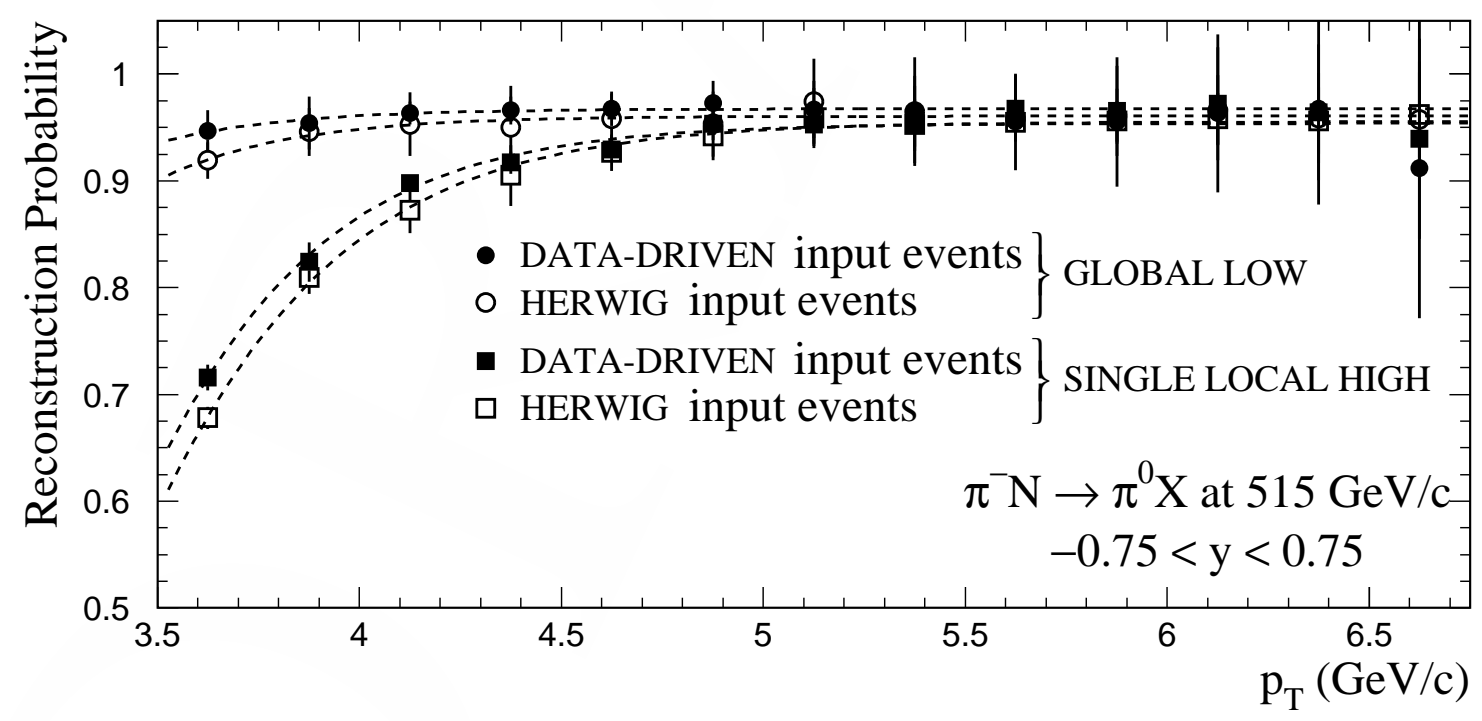

Figure 6.23 Comparison between reconstruction probabilities obtained using HERWIG and DATA-DRIVEN input to the DGS. 
LOW trigger in the $\pi^{-}$beam data) was examined. Because the low threshold triggers have relatively low statistics in the region of the SINGLE LOCAL HIGH turn-on, fits of the low threshold trigger data in the region of the turn-on were used. To constrain the fits at high $p_{T}\left(p_{T} \approx 5.0 \mathrm{GeV} / c\right)$, SINGLE LOCAL HIGH data was used. The transition point for the SINGLE LOCAL HIGH trigger, and the lower limit of the fit was varied in the fits to test sensitivity. In these ratios, the results were found to differ from unity by no more than $\approx 2 \%$, and thus a $2 \%$ uncertainty was assigned for the trigger response.

\subsubsection{Vertex Reconstruction Efficiency}

The DGS was also used to evaluate the primary vertex reconstruction efficiency. For this study, Monte Carlo samples were generated using HERWIG v6.1 [98], as the vertex distributions generated with this version were found to give much better agreement with the data than the samples generated with HERWIG v5.6. The transverse positions of the vertices in the DGS were chosen according to beam profiles observed in the data. The longitudinal positions were assigned using Monte Carlo methods based upon the absorption lengths of the materials in the target region. This approach gives rise to a number distribution for each target that varies approximately as $A^{2 / 3}$, where $\mathrm{A}$ is the atomic mass of the target. However, as described in Section 1.6, at high $p_{T}\left(p_{T} \gtrsim 3.0 \mathrm{GeV} / c\right)$ the number distribution is found to vary as $A^{\alpha}$, where $\alpha$ is around one. Therefore, for the purposes of evaluating the vertex efficiency, the vertices in the DGS were weighted to reproduce the values of $\alpha$ observed in the data. A comparison between the weighted DGS vertex distribution and the data is shown in Figure 6.24.

Separate reconstruction efficiencies were evaluated for the $\mathrm{Be}, \mathrm{Cu}$, and $\mathrm{H}_{2}$ targets. The reconstruction efficiency was defined as the number of reconstructed vertices divided by the number of generated vertices in each target's fiducial 

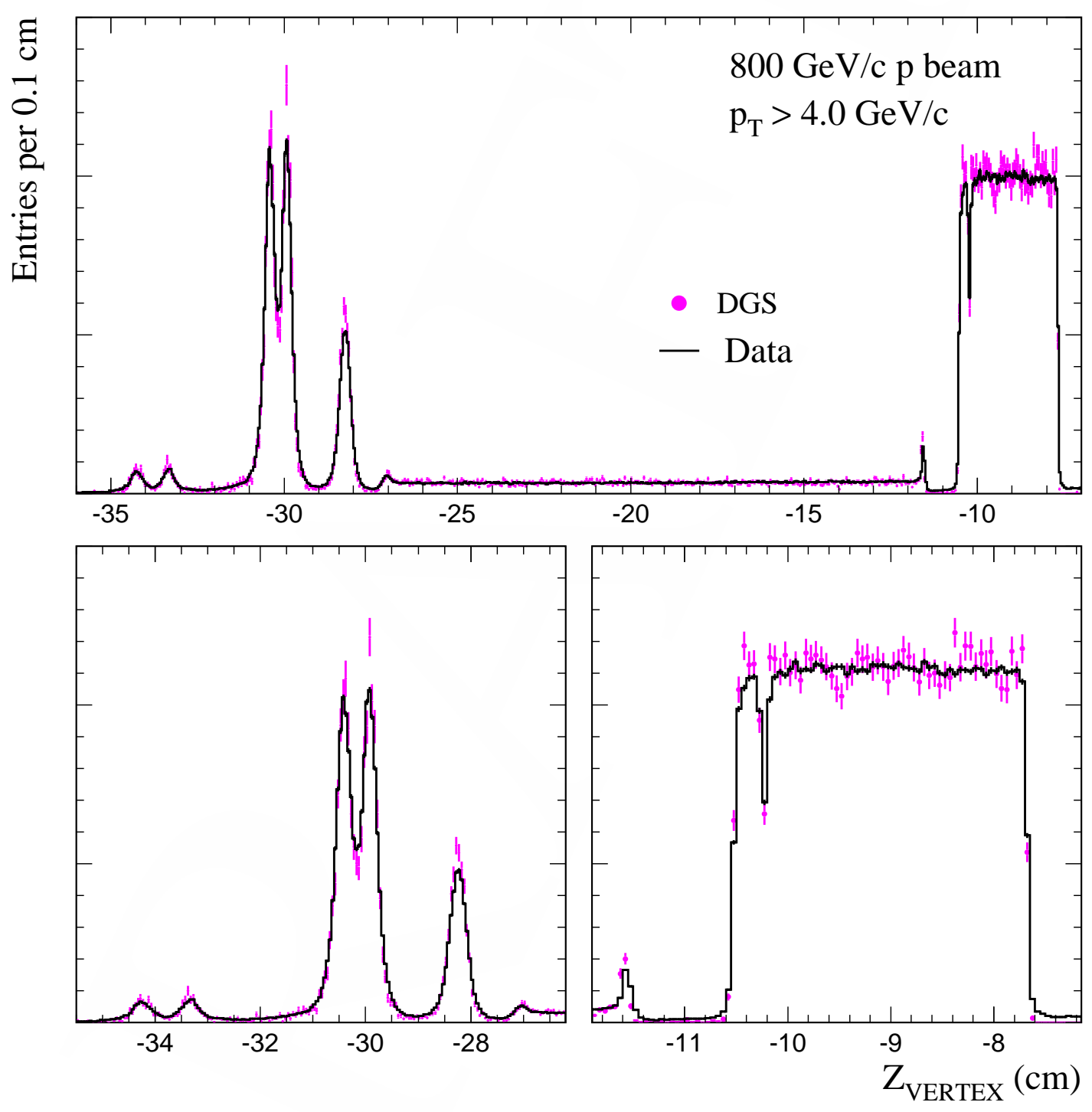

Figure 6.24 Comparison between the Z positions of primary vertices in the DGS (points) and the data (histogram) for events containing a $\pi^{0}$ candidate with $p_{T}>4.0 \mathrm{GeV} / c$. 
volume. The reconstructed entries used the reconstructed vertex position to determine if the vertex was in the fiducial volume while the generated entries used the generated vertex position. This allowed the efficiencies to also correct for resolution smearing of the reconstructed vertex positions. The reconstruction efficiencies for the $\mathrm{Cu}$ and Be targets for the 1990 target configuration and the $\mathrm{H}_{2}$ and downstream Be targets for the 1991-92 configuration were unity. The $\mathrm{Cu}$ and upstream Be efficiencies for the 1991-92 target were 0.96 and 0.97, respectively.

Additional beam particles occasionally interacted in the target material during the time the tracking system was sensitive to charged particles. For data taken using the relatively long target configuration of the 1991-92 run, such situations induced a reconstruction bias which favored interactions in the downstream target material over interactions in the upstream material. This bias was studied by comparing cross section measurements on the upstream and downstream pieces of Be, and with a dedicated DGS sample which included additional minimum bias interactions. The number of upstream $\mathrm{Be}$ and $\mathrm{Cu}$ vertices were corrected for this misreconstruction due to this confusion. The correction was 1.04 for the 1991$92 \pi^{-}$sample, 1.06 for the $530 \mathrm{GeV} / c$ p sample, and 1.12 for the $800 \mathrm{GeV} / c p$ sample. The systematic uncertainties in these corrections were dominated by the statistical uncertainties in the upstream Be cross section measurements. They were $\pm 2 \%$ for the $515 \mathrm{GeV} / c \pi^{-}$and $530 \mathrm{GeV} / c p$ beam samples, and $\pm 3 \%$ for the $800 \mathrm{GeV} / c$ p beam sample.

\subsubsection{Background Photon to $\pi^{0}$ Ratio}

After all the cross section corrections were evaluated, the filter 2 and filter 3 DGS Monte Carlo samples were run through the same analysis code as the real data, and $\gamma_{b}$ and $\pi^{0}$ "cross sections" were obtained. By dividing these cross sections, the nominal $\gamma_{b} / \pi^{0}$ were obtained. These ratios were fitted to surfaces 
in $p_{T}$ and rapidity. In Figure $6.25, \gamma_{b} / \pi^{0}$ are shown as functions of $p_{T}$ for the three major incident beams on beryllium. The fit results, integrated over the appropriate rapidity ranges, are indicated by the dotted lines Figure 6.25.

The ratio $\gamma_{b} / \pi^{0}$ for the $515 \mathrm{GeV} / c \pi^{-}$data and the $800 \mathrm{GeV} / c$ proton data at low to moderate values of $p_{T}$ are very similar. This is due to the fact that the slopes of the $\pi^{0} p_{T}$ spectra in these data are very similar. At high $p_{T}, \gamma_{b} / \pi^{0}$ is larger for the $800 \mathrm{GeV} / c$ proton data than it is for the $515 \mathrm{GeV} / c \pi^{-}$beam data. This is because the $800 \mathrm{GeV} / c \pi^{0}$ cross section is peaked more forward in rapidity than the $\pi^{-}$cross section, which allows for a larger background contribution from coalescence in the $800 \mathrm{GeV} / c$ data. The $530 \mathrm{GeV} / c$ proton beam $\gamma_{b} / \pi^{0}$ is smaller than in the other two beams. This is attributed to the slope of the $\pi^{0} p_{T}$ spectrum being significantly steeper in this sample. Also, $\gamma_{b} / \pi^{0}$ does not rise as sharply at high $p_{T}$ as in the other samples, since the $\pi^{0}$ rapidity distribution in this sample is the least forward.

In Figure 6.26, $\gamma_{b} / \pi^{0}$ is shown for the $530 \mathrm{GeV} / c$ proton data for several rapidity intervals. The background levels are quite similar in the backward and central rapidity regions. However, the background levels are significantly greater at high $p_{T}$ in the forward rapidity region due to contributions from coalescence.

Fits to $\gamma_{b} / \pi^{0}$ were only made for the beryllium target simulations due to the relatively poor full shower Monte Carlo statistics for interations in the copper and hydrogen targets. However, $\gamma_{b} / \pi^{0}$ is expected to be slightly different for each target due to the different amounts of target material the photons must traverse. Therefore, corrections to $\gamma_{b} / \pi^{0}$ for the copper and hydrogen target data were calculated using the PMC. In Figure 6.27 the differences between $\gamma_{b} / \pi^{0}$ in copper and beryllium and between hydrogen and beryllium are shown for the 800 $\mathrm{GeV} / c$ proton beam configuration. The differences for the other incident beams 


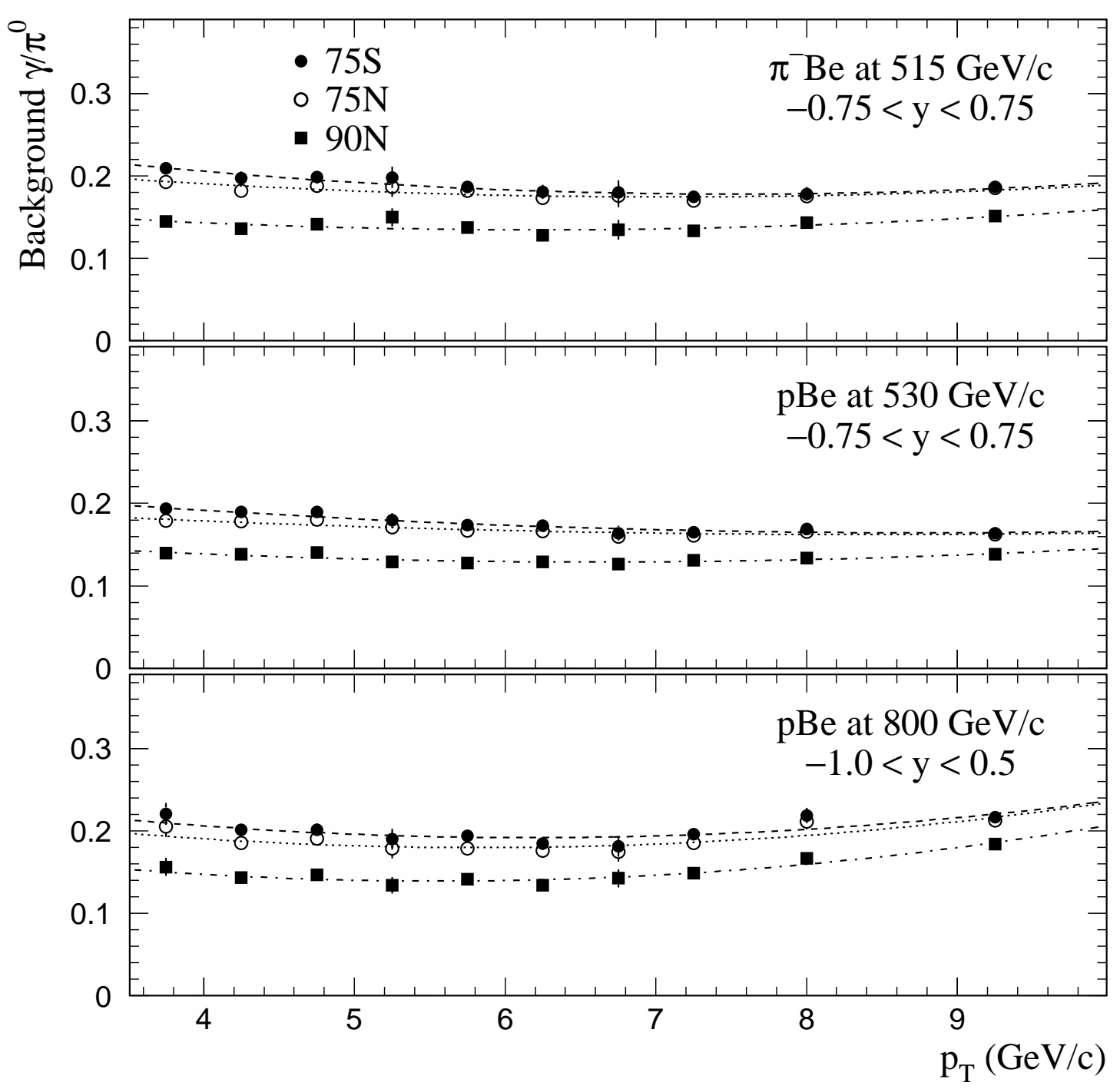

Figure 6.25 The ratio $\gamma_{b} / \pi^{0}$ for each direct photon candidate definition for the three major beam types as functions of $p_{T}$. The dotted curves represent the results of the background fits integrated over the indicated rapidity ranges. 


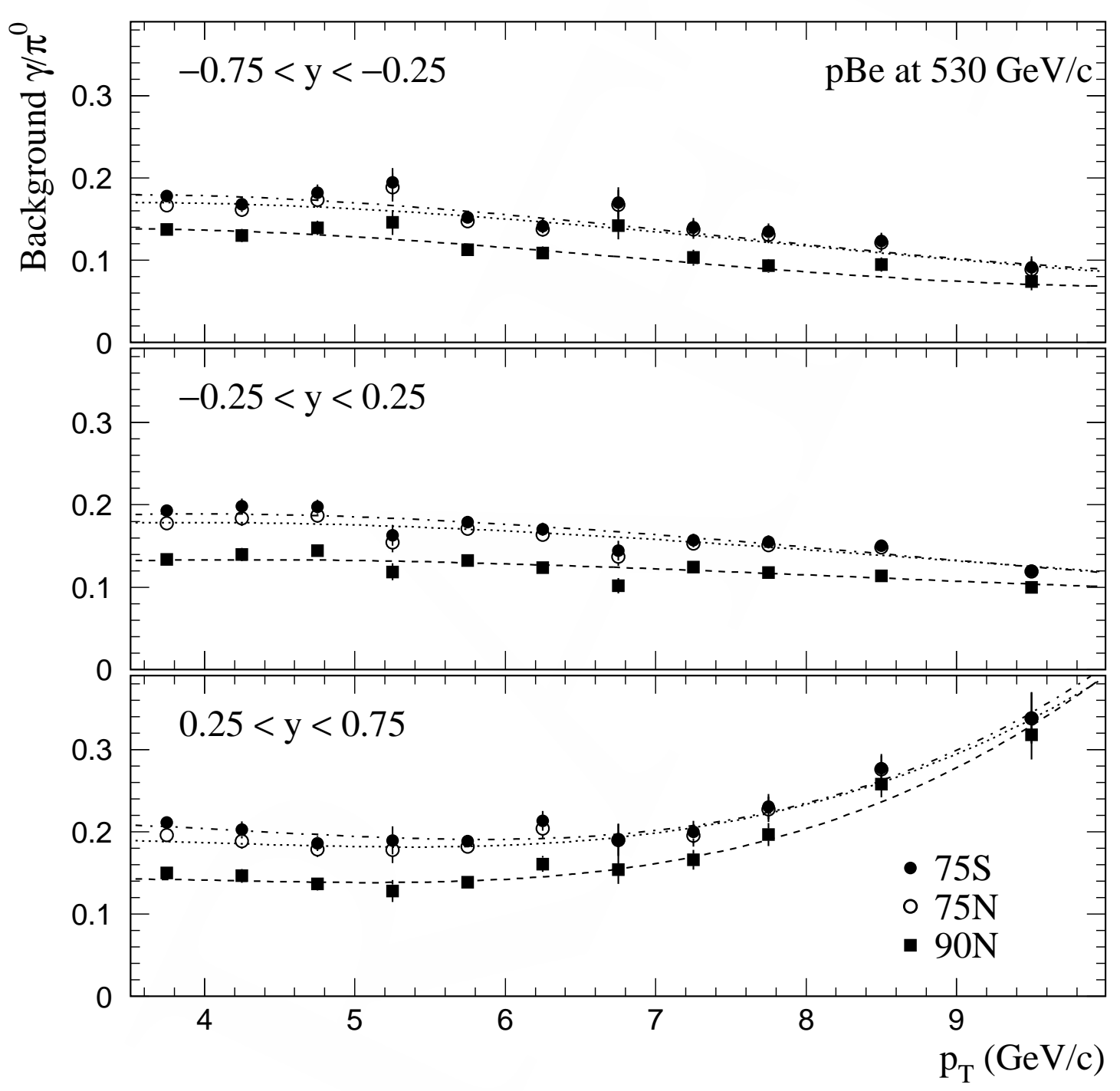

Figure 6.26 The ratio $\gamma_{b} / \pi^{0}$ as a function of $p_{T}$ for backward, central and forward rapidities for the $530 \mathrm{GeV} / c$ proton beam. The dotted curves represent the results of the background fits integrated over the indicated rapidity ranges. 


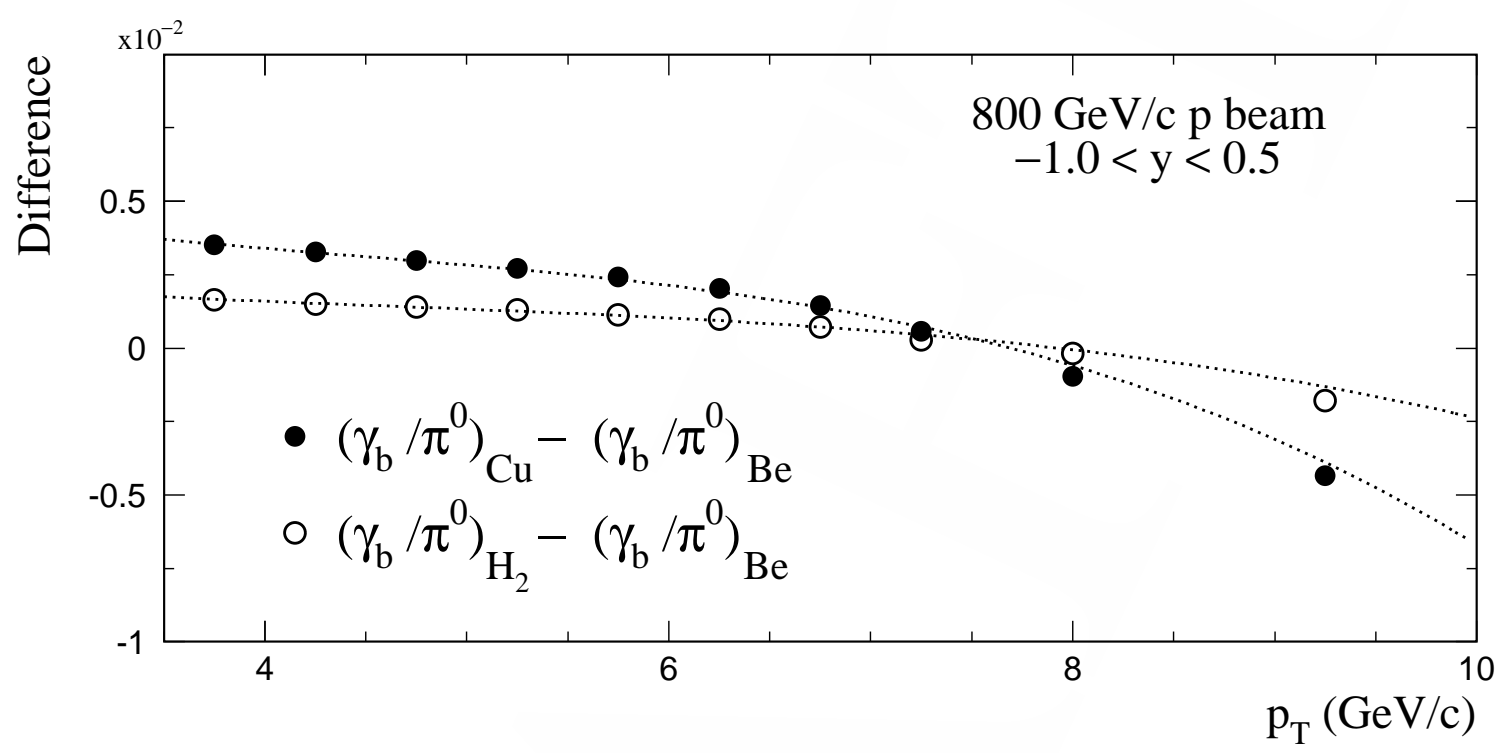

Figure 6.27 Difference between $\gamma_{b} / \pi^{0}$ in copper and beryllium versus $p_{T}$ for the $1991800 \mathrm{GeV} / c$ p beam data. Also shown is the difference between $\gamma_{b} / \pi^{0}$ in hydrogen and beryllium. The dotted lines represent fits to the differences, integrated over the rapidity range $-1.0<y<0.5$.

are similar. The difference is greater than zero at low $p_{T}$, which is expected since there should be more background photons resulting from $\gamma \rightarrow e^{+} e^{-}$conversions in interactions in the copper target. However, at high $p_{T}$ the correction becomes negative. This is because at high $p_{T}$, the contribution from coalescence, which is much more significant in this regime than the conversion contribution, is reduced due to the photon conversions. These differences were fit as functions of $p_{T}$ and rapidity for each incident beam type and used as additive corrections to the nominal fit to $\gamma_{b} / \pi^{0}$.

In addition, since the filter 2 and filter 3 Monte Carlo samples did not include contributions to the background from charged pions, the nominal $\gamma_{b} / \pi^{0}$ fits were 
adjusted to include these contributions. The filter 6 charged pion Monte Carlo was used to calculate this adjustment. As with the other Monte Carlo samples, the generated $\pi^{ \pm} p_{T}$ spectrum was weighted to reproduce the shape of the observed $\pi^{0}$ spectrum for each data sample. The ratio of background photons to charged pions from the filter 6 sample is shown in Figure 6.28 for the $515 \mathrm{GeV} / c \pi^{-}$data. The ratios for the other incident beams is similar. Fits to these ratios were made as functions of $p_{T}$. The fit results were then multiplied by a factor of two to convert the $\gamma_{b} / \pi^{ \pm}$ratios to $\gamma_{b} / \pi^{0}$ ratios. $^{13}$ The corrected $\gamma_{b} / \pi^{0}$ was obtained by taking this result and adding it to the result from the nominal $\gamma_{b} / \pi^{0}$ fit.

\section{Systematic Uncertainties in the $\gamma_{b} / \pi^{0}$ Ratio}

Statistical limitations in the DGS were a major source of systematic uncertainty in the $\gamma_{b} / \pi^{0}$. Closure tests were performed on the Monte Carlo data to check the statistical accuracy of the resultant fits to $\gamma_{b} / \pi^{0}$. From these tests, a $4 \%$ systematic uncertainty was assigned to the $\gamma_{b} / \pi^{0}$ due to the DGS statistics.

A measure of the systematic uncertainty in $\gamma_{b} / \pi^{0}$ due to the Monte Carlo model can be obtained by comparing results for the ratio from the PMC and the DGS, since the two give (nearly) independent determinations of $\gamma_{b} / \pi^{0}$. However, before making such a comparison, sources of background photons not included in the PMC must be removed from the DGS. Figure 6.29 shows comparisons between $\gamma_{b} / \pi^{0}$ from the PMC and the modified DGS for the three incident beam. From these comparisons, an $4 \%$ uncertainty was attributed to the Monte Carlo model.

The systematic uncertainty due to the modeling of the detector environment can be estimated by comparing DGS results using HERWIG for the input event to results using DATA-DRIVEN input. Again, in order to make a fair comparision

13 The factor of two comes from the assumption that the charged pion to $\pi^{0}$ ratio is $2: 1$. 


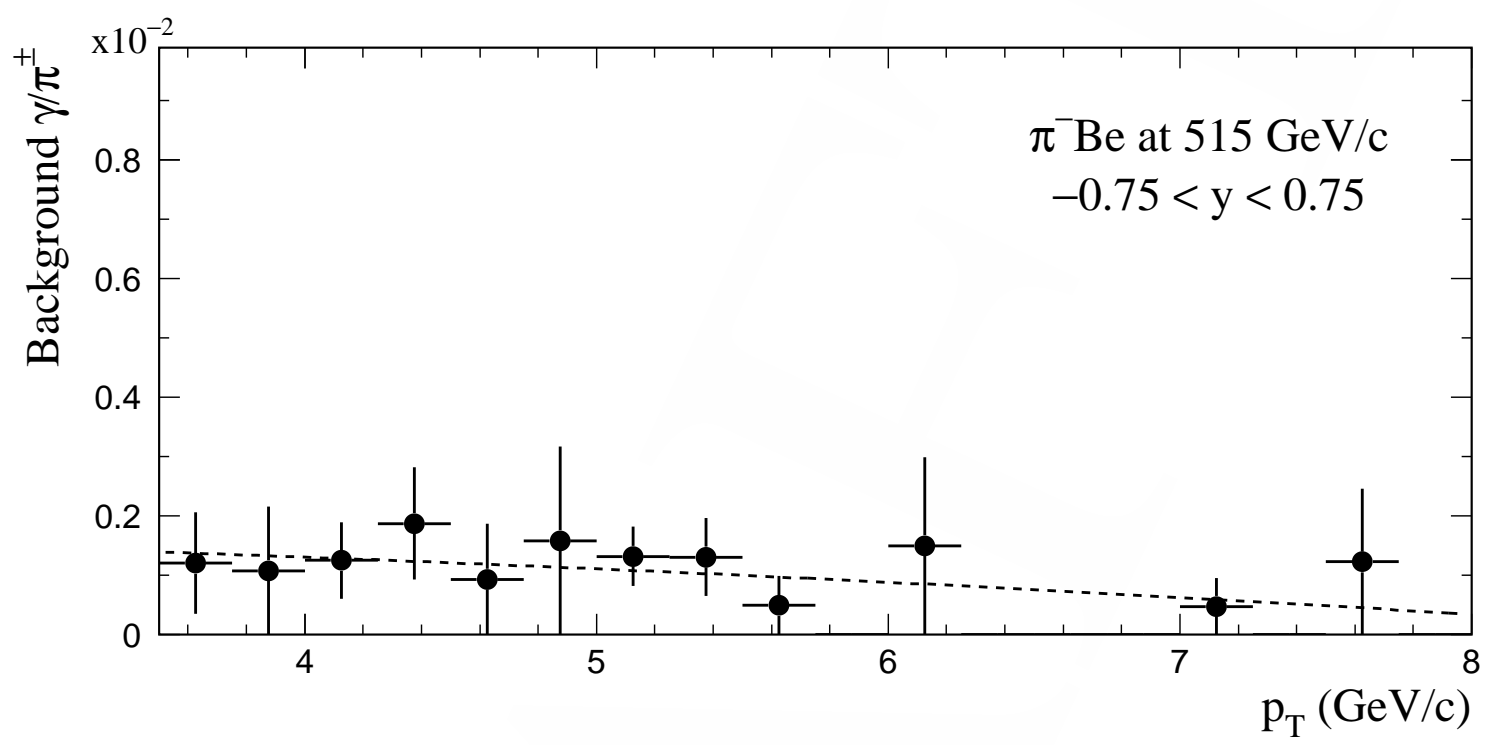

Figure 6.28 Ratio of background photons to charged pions for the $515 \mathrm{GeV} / c \pi^{-}$ data. The background photons are from interactions in the EMLAC by charged pions. The dotted line represents a fit to the Monte Carlo data. (Note $\gamma_{b} / \pi^{ \pm}$is $\lesssim 0.1 \%$.)

between the two Monte Carlo's, only sources of background included in both simulations were allowed in the comparison. This comparison is shown in Figure 6.30. The $\gamma_{b} / \pi^{0}$ values agree to within $5 \%$.

Other sources of systematic uncertainty which contributed at the $1-2 \%$ were the uncertainty in the $\eta / \pi^{0}$ and $\eta^{\prime} / \pi^{0}$ ratios and the uncertainty in the shape of the input $\pi^{0} p_{T}-y$ spectrum. The individual sources of uncertainty were added together in quadrature to obtain the total systematic uncertainty in the ratio $\gamma_{b} / \pi^{0}$, which was $\approx 8 \%$. 


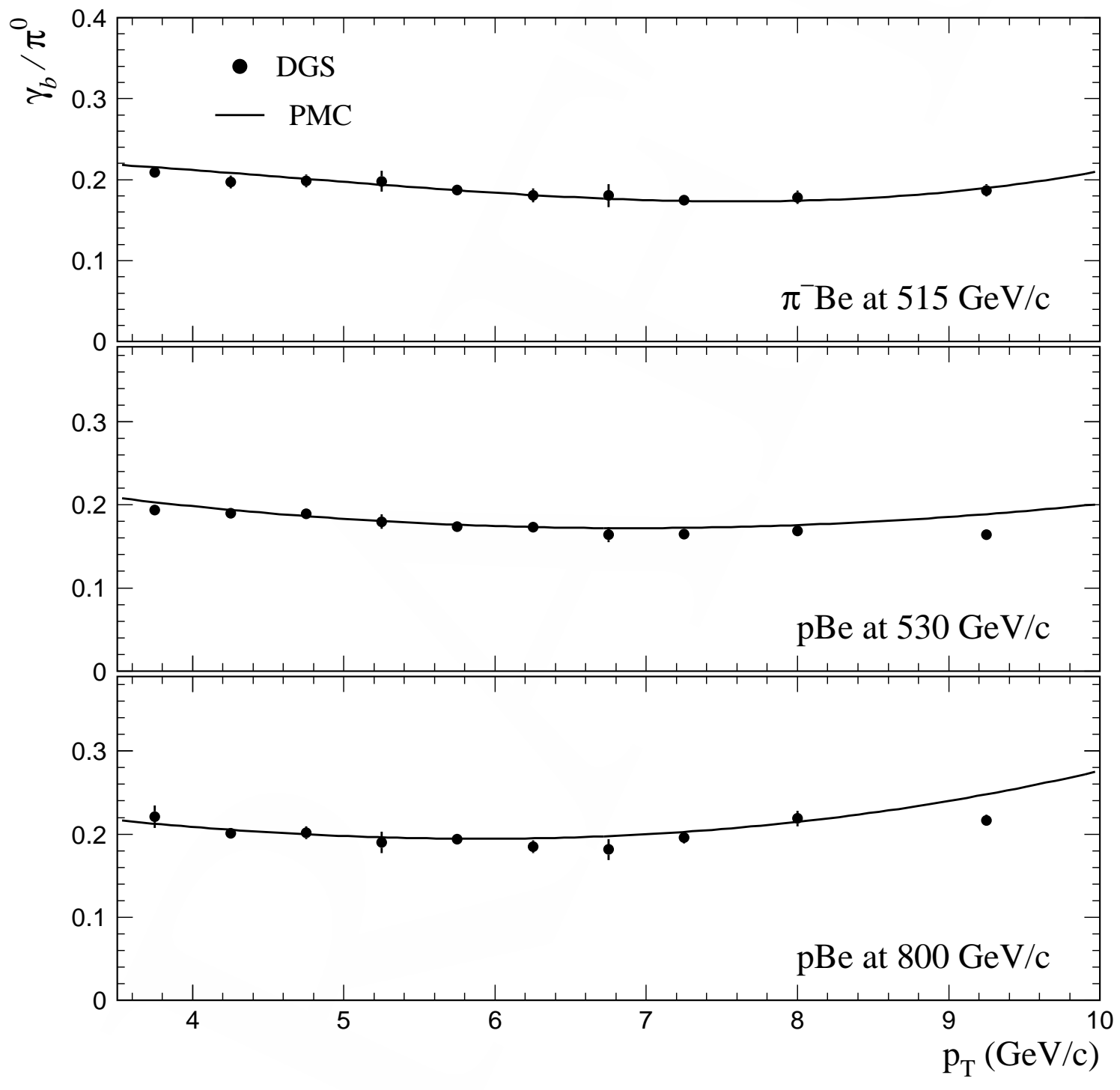

Figure 6.29 Comparison of $\gamma_{b} / \pi^{0}$ from the PMC and the DGS simulations for the $75 \mathrm{~S}$ photon candidate definition. 


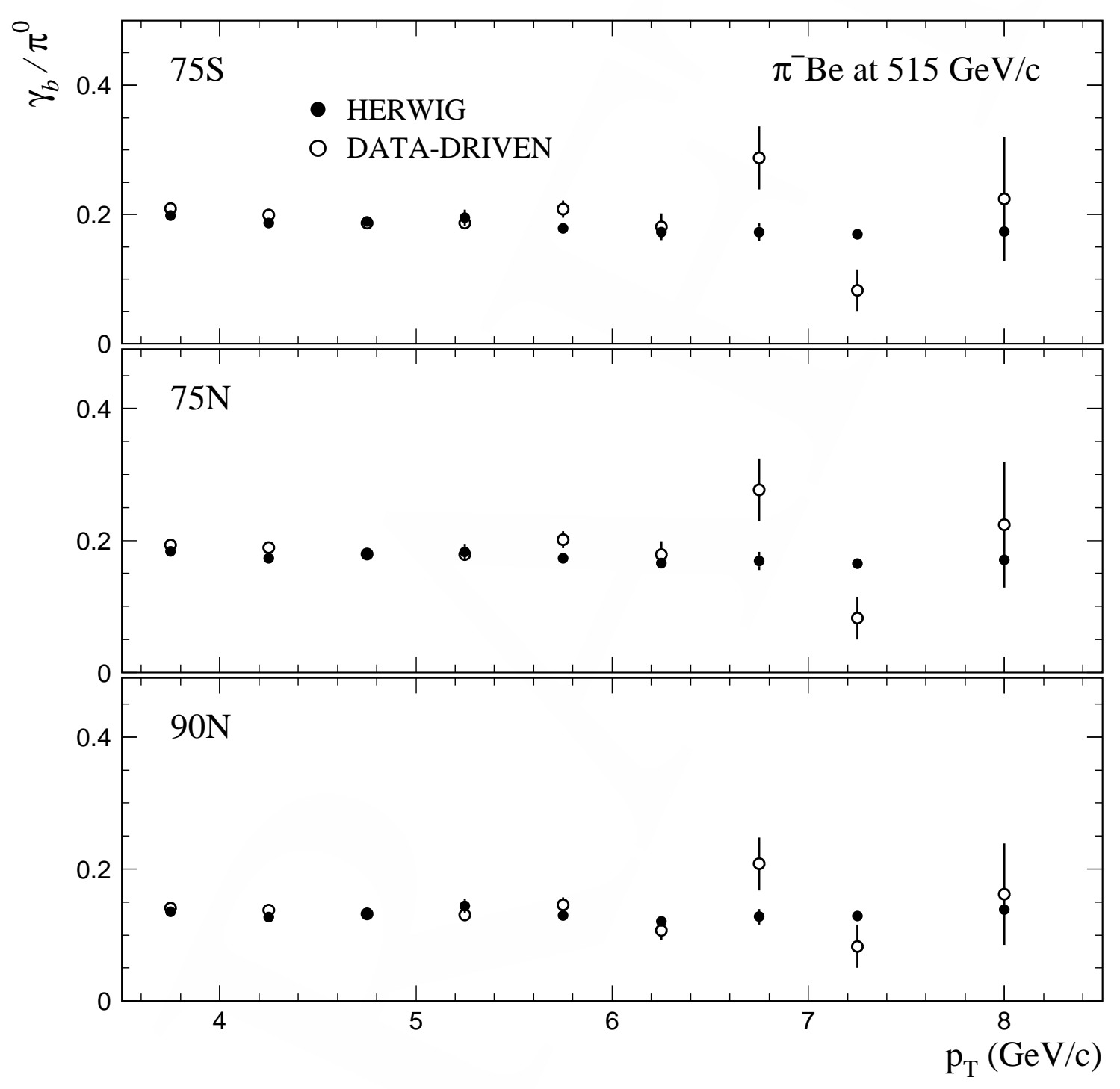

Figure 6.30 Comparison of $\gamma_{b} / \pi^{0}$ from the DGS using input events from HERWIG $(\bullet)$ and from data (o) for $90 \mathrm{~N}, 75 \mathrm{~N}$ and $75 \mathrm{~S}$ photon definitions. 


\section{Chapter 7 Results and Conclusions}

\section{$7.1 \quad$ Overview}

In this chapter, measurements of the production of direct photons and $\pi^{0}$ mesons by 530 and $800 \mathrm{GeV} / c$ proton and $515 \mathrm{GeV} / c \pi^{-}$beams on beryllium, copper, and liquid hydrogen targets are presented. The inclusive differential cross sections are shown as functions of $p_{T}$ and rapidity. The dependence of the production cross sections upon the nuclear target is shown and compared to theoretical expectations. The production cross sections are compared to nextto-leading order (NLO) pQCD predictions. Comparisons to NLO predictions are also made for measurements from several other experiments.

\subsection{Cross Section Calculation}

The invariant cross section per nucleon for inclusive particle production is given by

$$
E \frac{d \sigma}{d^{3} p}=\frac{1}{2 \pi p_{T} \Delta p_{T} \Delta y} \frac{1}{\mathcal{L}} \in N_{o b s}\left(p_{T}, y\right),
$$

where $\Delta p_{T}$ is the width of the transverse momentum bin, $\Delta y$ is the width of the center-of-mass rapidity bin, $\mathcal{L}$ is the integrated luminosity, $N_{\text {obs }}$ is the observed number of occurrences of the particle of interest in the specified $p_{T}$ and $y$ bin, and $\epsilon$ represents the combined product of the various corrections applied to the data. A summary of these corrections and their associated values is presented in Table 7.1. The luminosity is given by

$$
\mathcal{L}=N_{L T B} \rho L N_{A},
$$

where $N_{L T B}$ is the live triggerable beam (Section 5.13), $\rho$ is the target density, L is the target length, and $N_{A}$ is Avogadro's number. 
Table 7.1 Average value of corrections applied to the data.

\begin{tabular}{|c|c|c|c|}
\hline \multirow{2}{*}{ Correction } & \multicolumn{3}{|c|}{ Average Value or Range of Values } \\
\hline & Direct Photon & $\pi^{0}$ & $\eta$ \\
\hline Asymmetry Requirement & - & \multicolumn{2}{|c|}{1.33} \\
\hline Beam Absorption & \multicolumn{3}{|c|}{$1.015-1.08$} \\
\hline Beam Halo Rejection & \multicolumn{3}{|c|}{$1.02-1.10$} \\
\hline Beam Muon Contamination & \multicolumn{3}{|c|}{$1.005^{*}$} \\
\hline Branching Ratio & - & 1.012 & 2.55 \\
\hline Geometric Acceptance & \multicolumn{3}{|c|}{ See Figure 5.5} \\
\hline Hadron Rejection & $1.01-1.02$ & \multicolumn{2}{|c|}{$1.02-1.04$} \\
\hline Photon Conversions & \multicolumn{3}{|c|}{ See Figure 5.14} \\
\hline Reconstruction Efficiency & \multicolumn{3}{|c|}{ See Figures $6.19,6.20$ and 6.21} \\
\hline Transverse Target Fiducial Requirement & \multicolumn{3}{|c|}{$1.36^{\dagger}$} \\
\hline Trigger & $1.0-1.09$ & $1.0-1.12$ & $1.0-1.20$ \\
\hline Vertex Efficiency & \multicolumn{3}{|c|}{1.004} \\
\hline
\end{tabular}

* For the secondary beams; 1.0007 for the $800 \mathrm{GeV} / \mathrm{c}$ primary beam

$\dagger 1990$ target configuration only; 1.0 for 1991 target 


\section{3 $\pi^{0}$ Cross Sections}

The inclusive differential cross sections per nucleus ${ }^{1}$ for $\pi^{0}$ production are shown in Figures 7.1, 7.2, and 7.3 as functions of $p_{T}$ for 530 and $800 \mathrm{GeV} / c$ proton beams and $515 \mathrm{GeV} / c \pi^{-}$beam, respectively, incident upon copper, beryllium, and hydrogen targets. The inner error bars in the figures indicate the statistical uncertainties while the total error bars are the statistical and systematic uncertainties added in quadrature. ${ }^{2}$ Since the cross sections fall steeply, the data are plotted at abscissa values which correspond to the average values of the cross section in the appropriate $p_{T}$ bins assuming exponential $p_{T}$ spectra[99]. These cross section measurements are also presented in tabular form in Appendix A.

Differential $\pi^{0}$ cross sections are shown as functions of rapidity for several $p_{T}$ intervals in Figures 7.4, 7.5, and 7.6 for 530 and $800 \mathrm{GeV} / c$ proton beams and $515 \mathrm{GeV} / c \pi^{-}$beam, respectively. Results are shown for the beryllium target only. The corresponding cross sections for the other targets can be obtained from the tables in Appendix A.

\subsection{Direct Photon Cross Sections}

To calculate the direct photon cross sections, "single" photon cross sections were first evaluated using Equation 7.1. The direct photon cross sections were then obtained after statistical removal of the background using the formula:

$$
\sigma_{\gamma}^{\text {direct }}=\sigma_{\gamma}^{\text {single }}-\left(\gamma_{b} / \pi^{0}\right) \times \sigma_{\pi^{0}}
$$

where $\sigma_{\gamma}^{\text {single }}, \sigma_{\pi^{0}}$ are the measured "single" photon and $\pi^{0}$ cross sections. A measure of the direct photon signal-to-background ratio can be obtained by

1 The cross section per nucleus is obtained by multiplying Equation 7.1 by A, where $\mathrm{A}$ is the atomic weight of the target material.

2 A summary of the systematic errors associated with these measurements is presented in Section 7.5. 


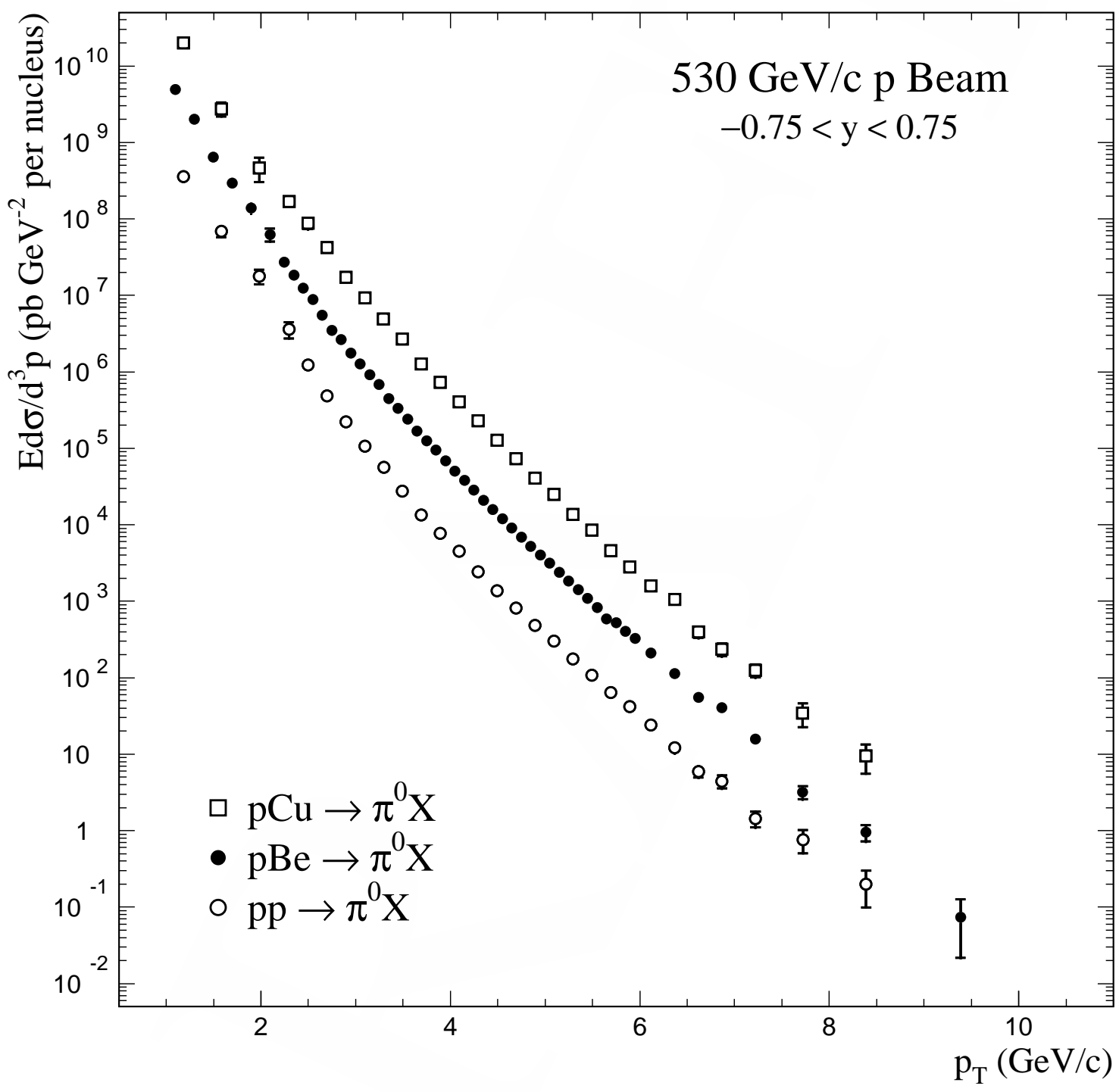

Figure 7.1 $\pi^{0}$ production cross sections per nucleus as functions of $p_{T}$ for $530 \mathrm{GeV} / c$ proton beam on copper, beryllium, and hydrogen targets. 


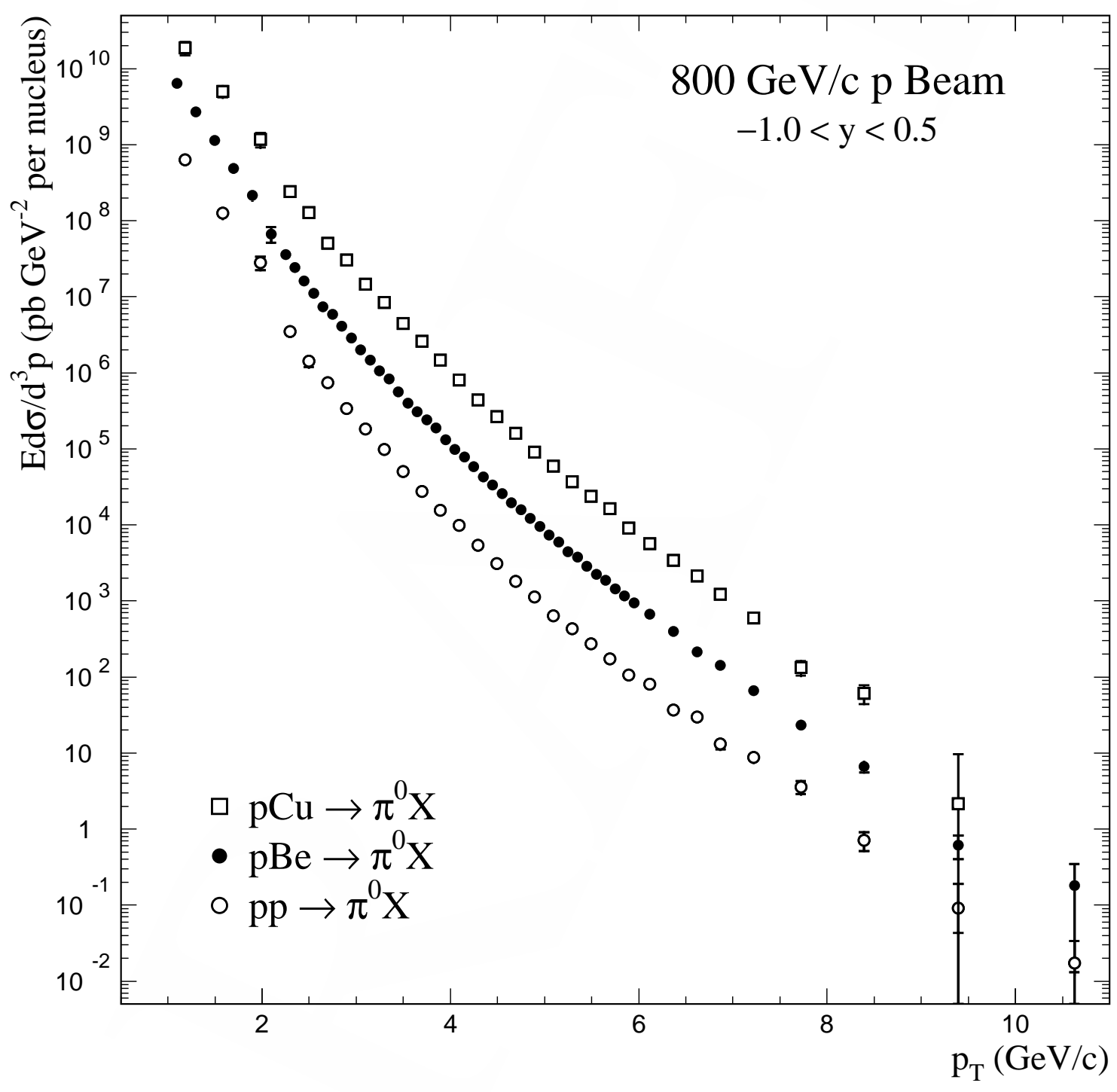

Figure 7.2 $\pi^{0}$ production cross sections per nucleus as functions of $p_{T}$ for $800 \mathrm{GeV} / c$ proton beam on copper, beryllium, and hydrogen targets. 


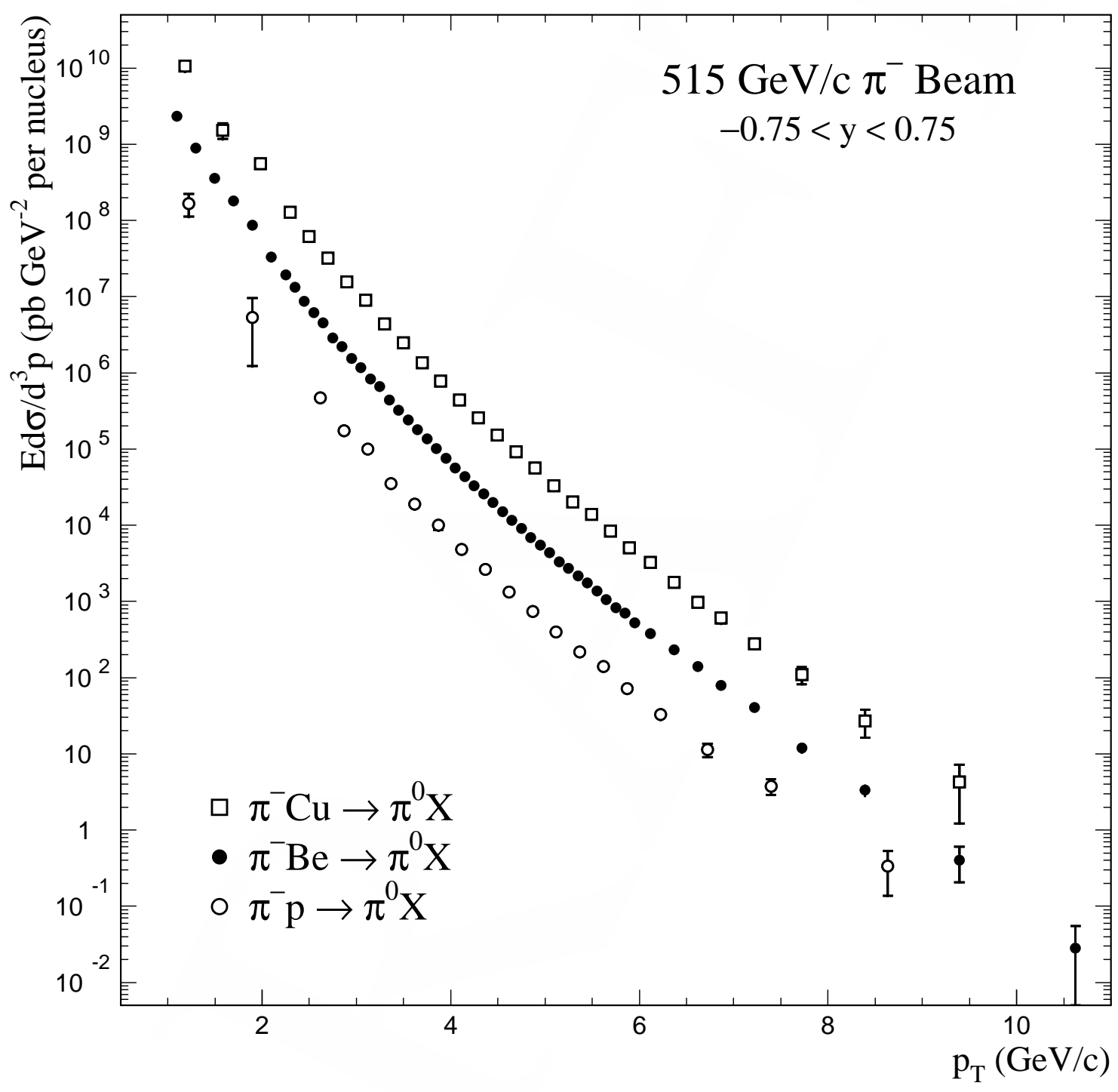

Figure 7.3 $\pi^{0}$ production cross sections per nucleus as functions of $p_{T}$ for $515 \mathrm{GeV} / c \pi^{-}$beam on copper, beryllium, and hydrogen targets. 


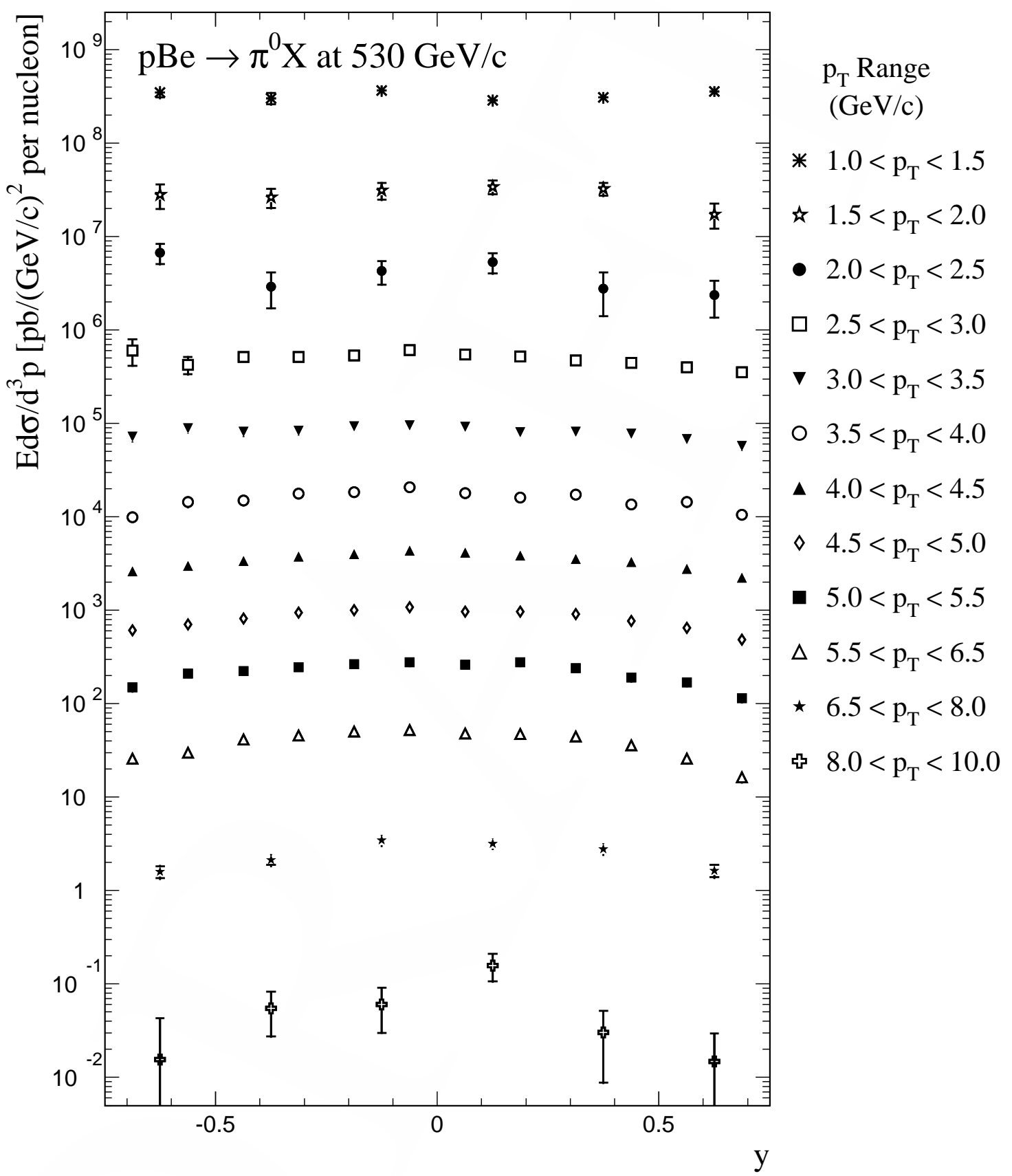

Figure $7.4 \pi^{0}$ cross section per nucleon as a function of rapidity for $530 \mathrm{GeV} / c$ proton beam on beryllium for several $p_{T}$ intervals. 


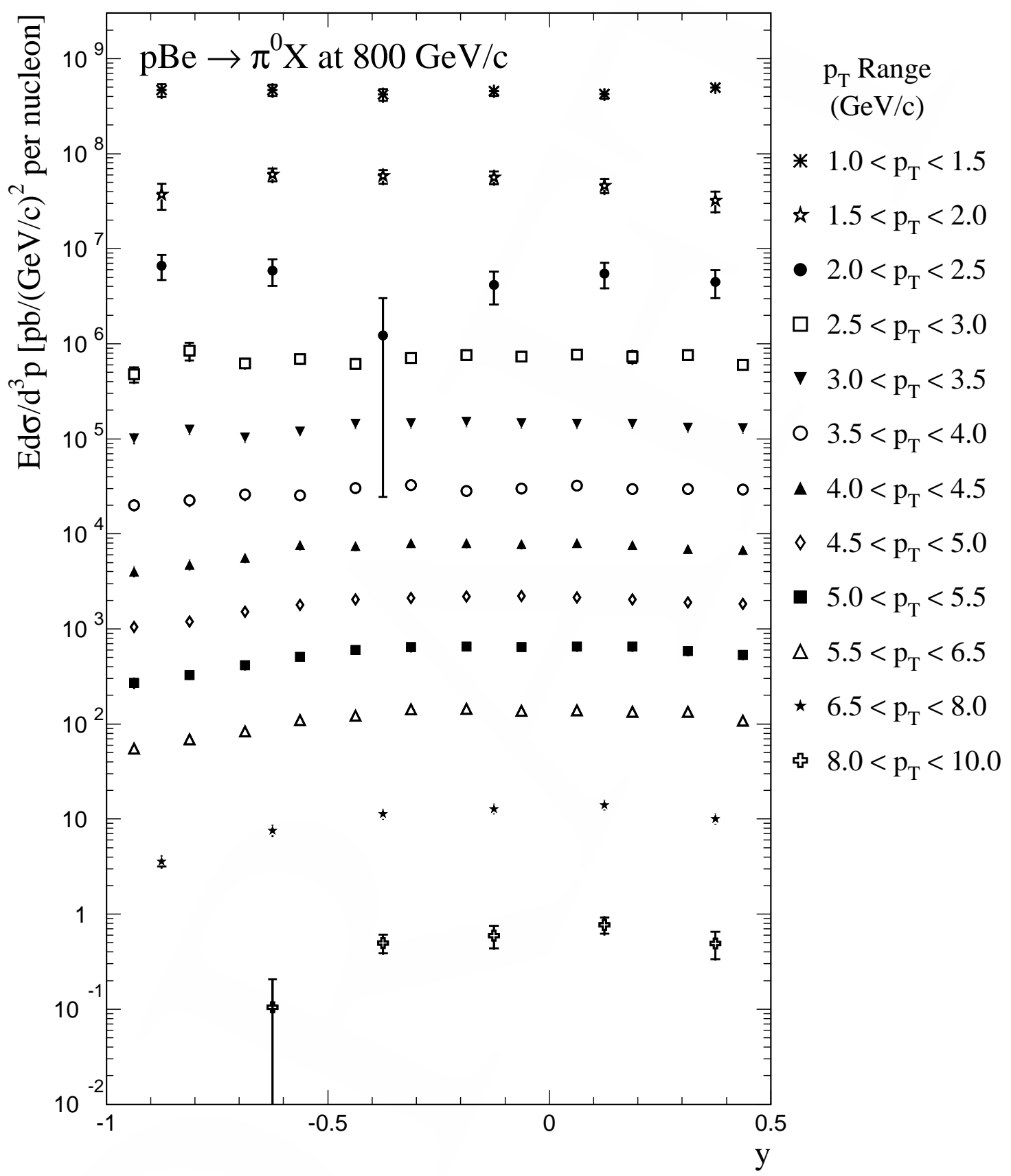

Figure 7.5 $\pi^{0}$ cross section per nucleon as a function of rapidity for $800 \mathrm{GeV} / c$ proton beam on beryllium for several $p_{T}$ intervals. 


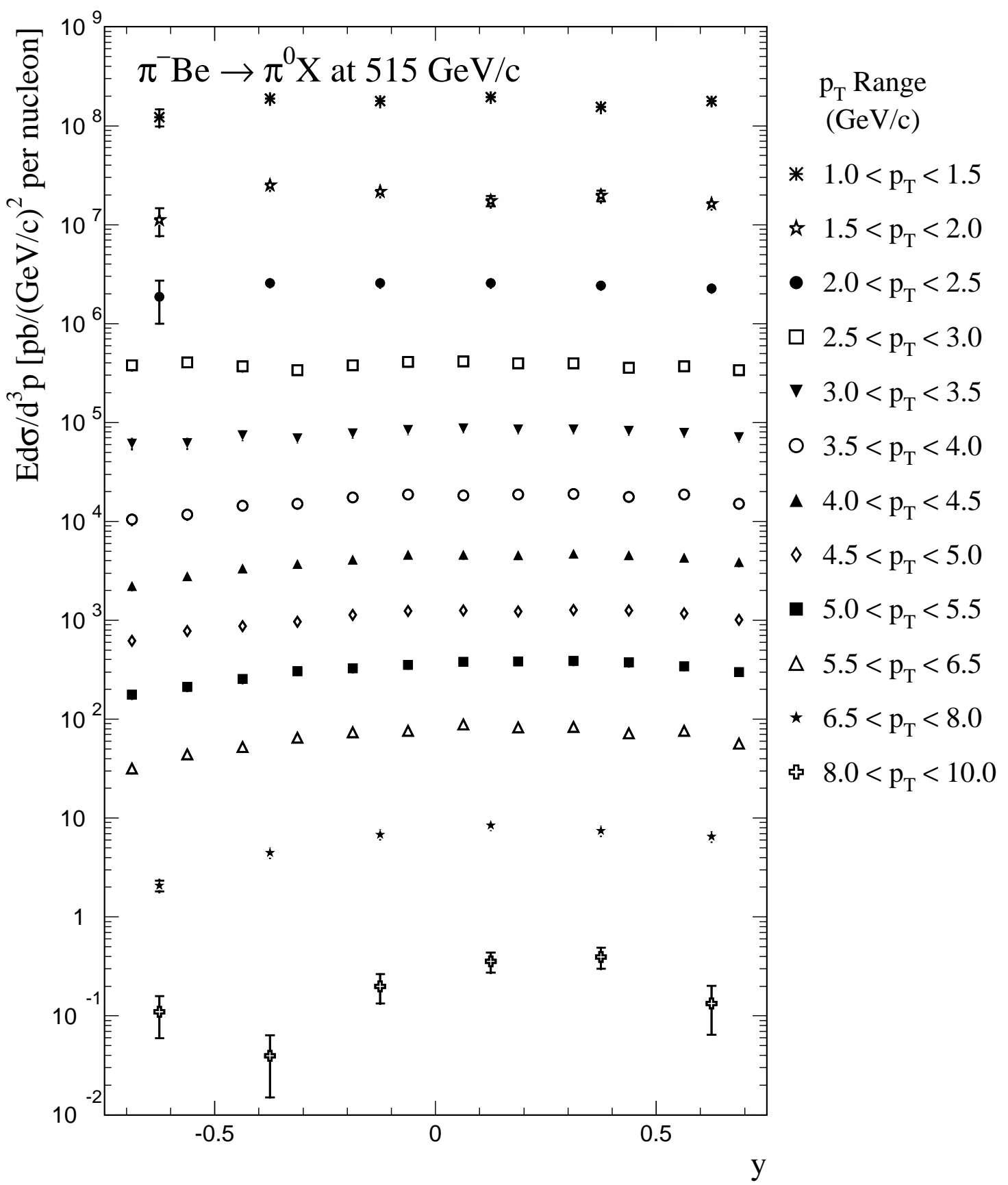

Figure 7.6 $\pi^{0}$ cross section per nucleon as a function of rapidity for $515 \mathrm{GeV} / c$ $\pi^{-}$beam on beryllium for several $p_{T}$ intervals. 
comparing the "single" photon to $\pi^{0}$ cross section ratio to $\gamma_{b} / \pi^{0}$ from the Monte Carlo. Such comparisons are shown in Figures 7.7, 7.8, and 7.9, for 530 and $800 \mathrm{GeV} / c$ pBe and $515 \mathrm{GeV} / c \pi^{-}$Be interactions, respectively. The $90 \mathrm{~N}$ photon definition is used in these comparisons; comparisons using the other photon definitions, or the other targets, are qualitatively similar. The thicknesses of the lines representing the $\gamma_{b} / \pi^{0}$ curves in these figures indicate the level of systematic uncertainty in these ratios.

The direct photon to $\pi^{0}$ ratio increases with increasing $p_{T}$ in all three data samples. As discussed in Section 1.5, this is the expected trend. Also, the signal is strongest in the $515 \mathrm{GeV} / c \pi^{-}$data sample. This is as expected since the presence of the valence anti-quark in the $\pi^{-}$allows for a much larger contribution to the cross section from the annihilation diagram.

The inclusive differential direct photon cross sections per nucleus are shown as functions of $p_{T}$ in Figures 7.10, 7.11, and 7.12 for 530 and $800 \mathrm{GeV} / c$ proton beams and $515 \mathrm{GeV} / c \pi^{-}$beam, respectively, incident upon copper, beryllium, and hydrogen targets. As in the case of the $\pi^{0}$ measurement, the error bars in the figures represent the statistical and systematic uncertainties added in quadrature, and the data are plotted at abscissa values corresponding to the average values of the cross section. Here, and elsewhere unless otherwise indicated, the cross sections are obtained using the $90 \mathrm{~N}$ photon candidate sample. The results obtained using the other photon definitions typically agree with these results to within $5 \%$, which is within the estimated systematic uncertainty. The direct photon cross sections are also presented in tabular form in Appendix B.

Direct photon cross sections versus rapidity for several $p_{T}$ intervals are shown in Figures 7.13, 7.14, and 7.15 for 530 and $800 \mathrm{GeV} / c$ proton beams and $515 \mathrm{GeV} / c$ $\pi^{-}$beam, respectively. Results are shown for the beryllium target only. The cross sections for the other targets may be obtained from the tables in Appendix B. 


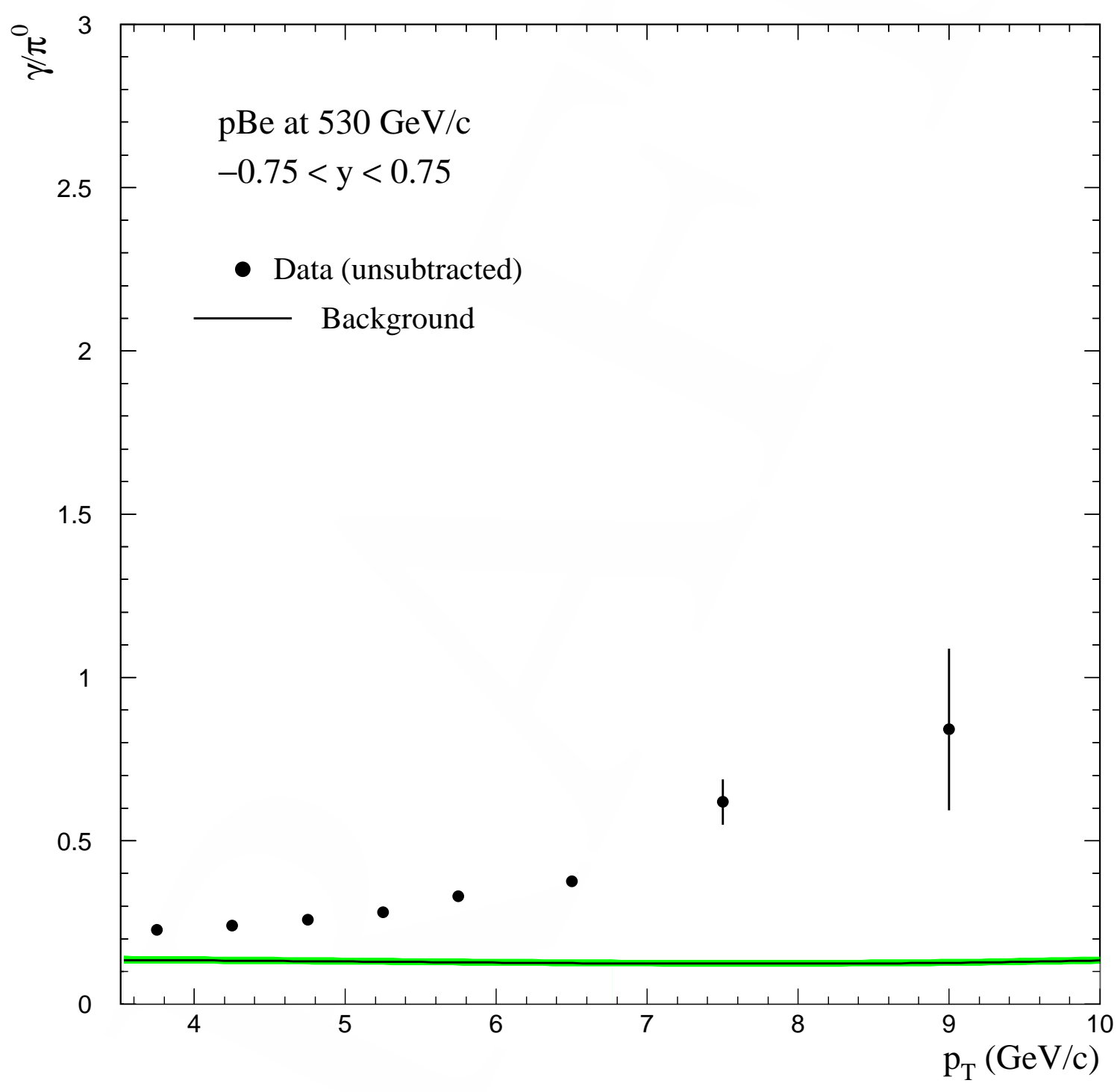

Figure 7.7 Single (unsubtracted) photon to $\pi^{0}$ ratio for $530 \mathrm{GeV} / c$ proton beam on beryllium. The error bars represent the statistical uncertainties only. Also shown is $\gamma_{b} / \pi^{0}$. 


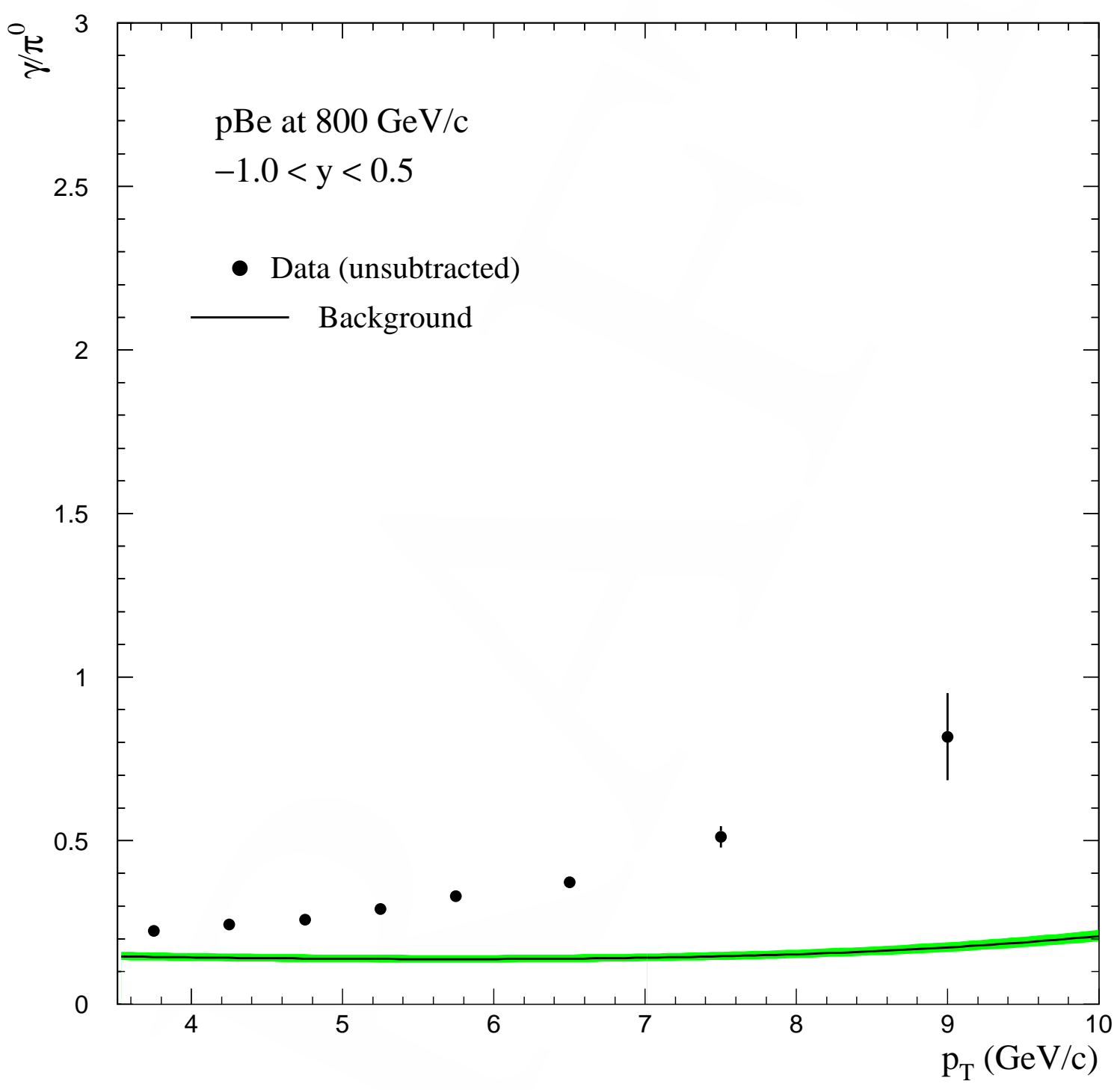

Figure 7.8 Single (unsubtracted) photon to $\pi^{0}$ ratio for $800 \mathrm{GeV} / c$ proton beam on beryllium. The error bars represent the statistical uncertainties only. Also shown is $\gamma_{b} / \pi^{0}$. 


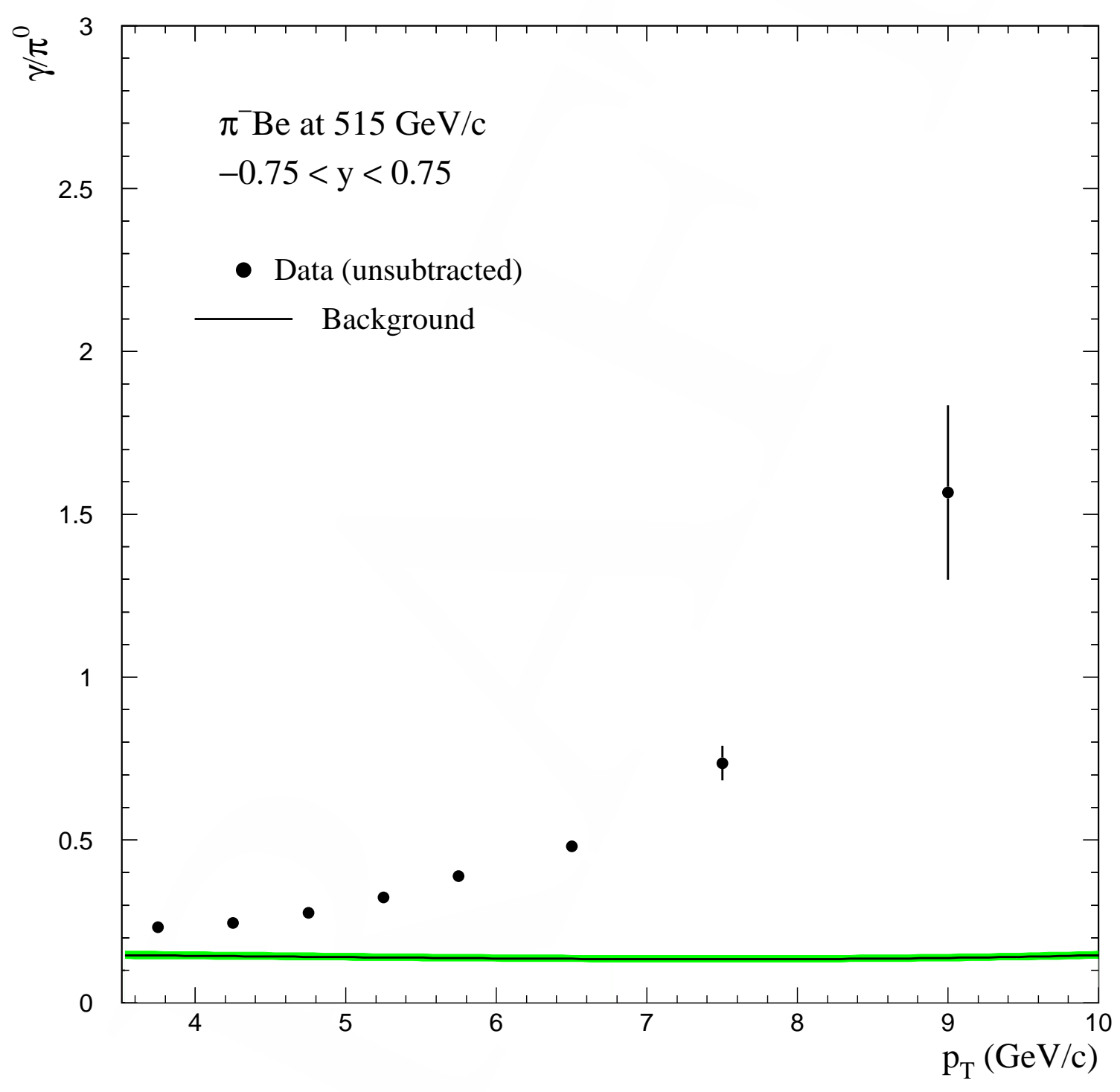

Figure 7.9 Single (unsubtracted) photon to $\pi^{0}$ ratio for $515 \mathrm{GeV} / c \pi^{-}$beam on beryllium. The error bars represent the statistical uncertainties only. Also shown is $\gamma_{b} / \pi^{0}$. 


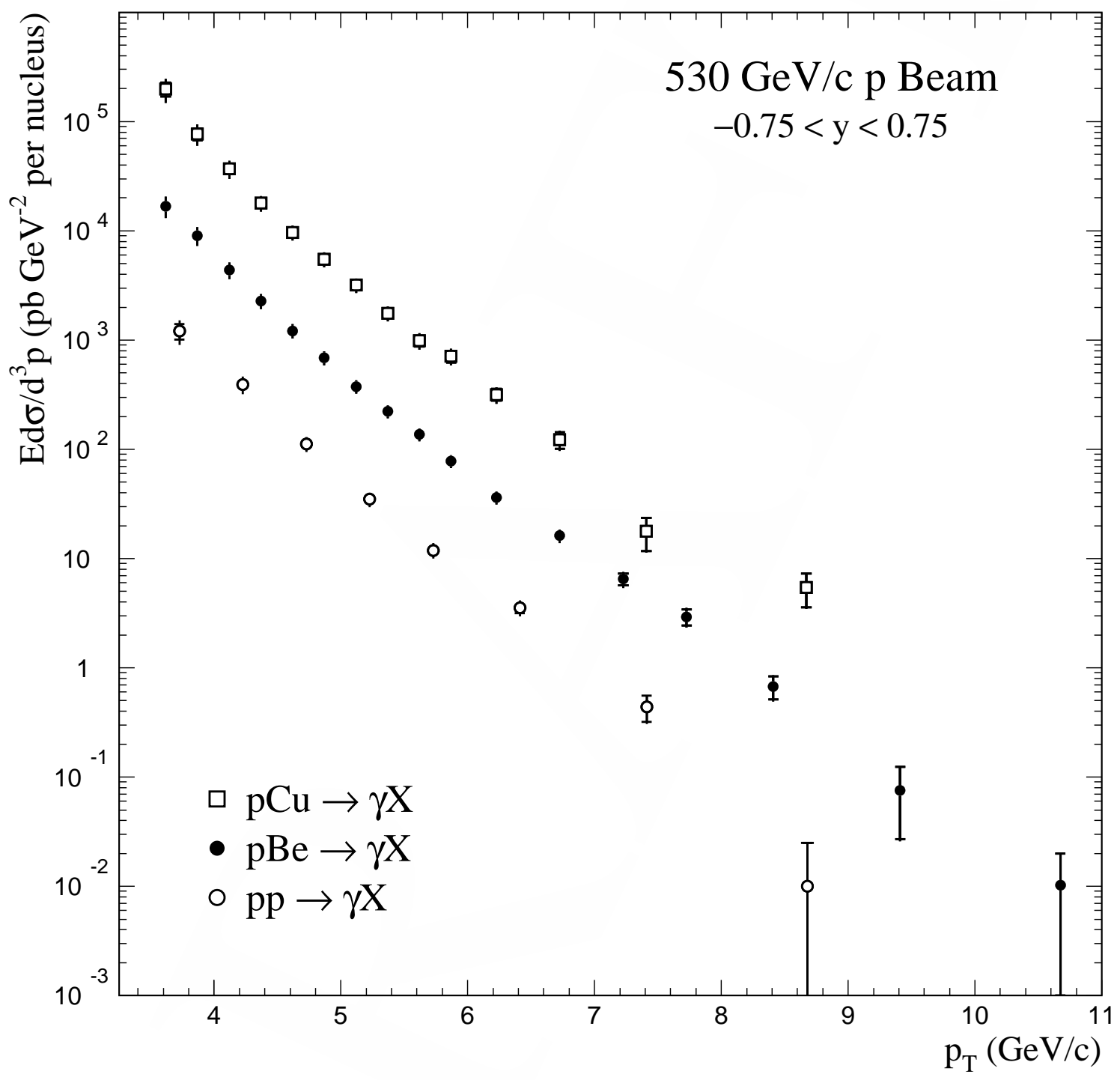

Figure 7.10 Direct photon cross sections per nucleus versus $p_{T}$ for $530 \mathrm{GeV} / c$ proton beam on copper, beryllium, and hydrogen targets. 


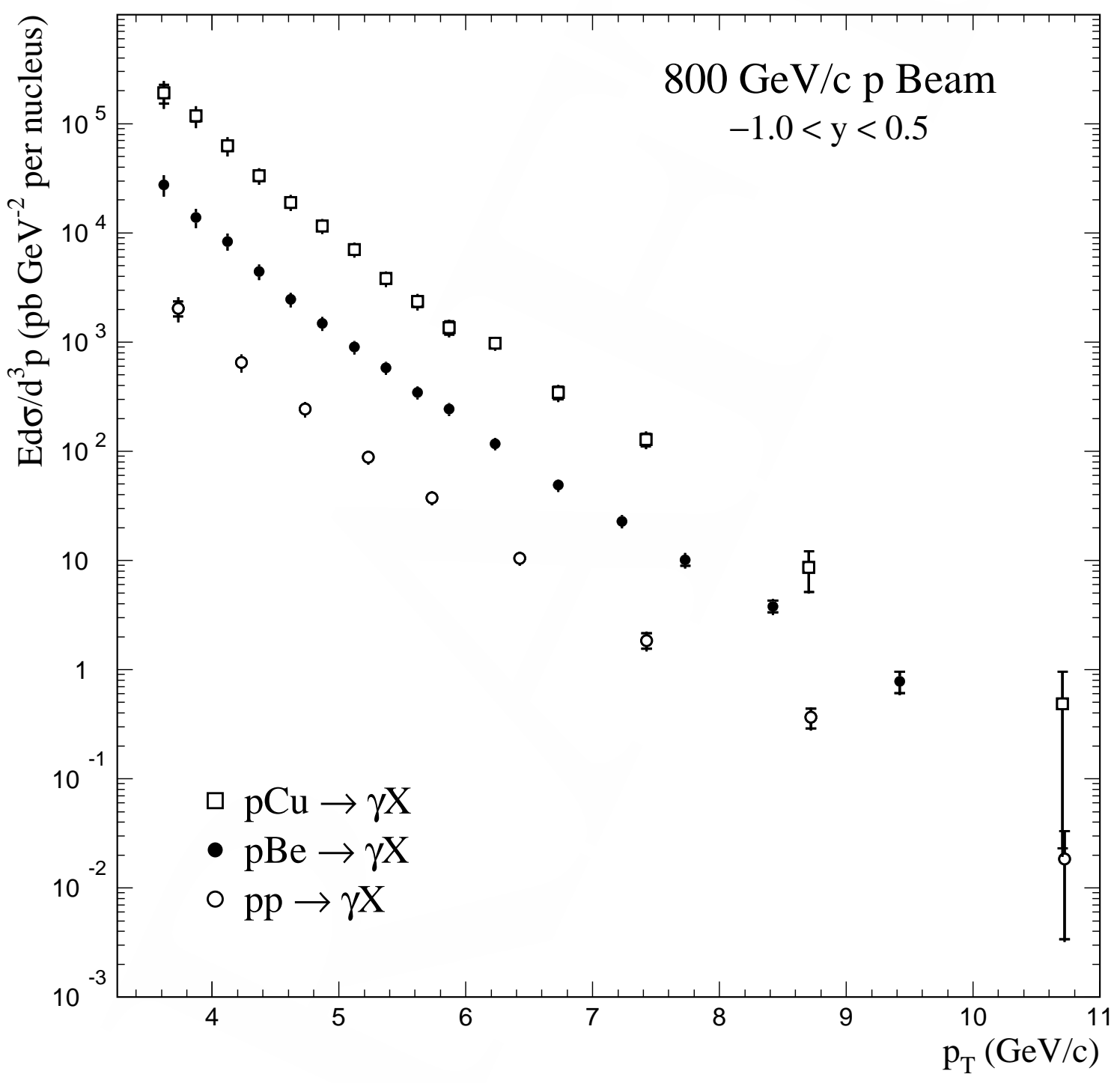

Figure 7.11 Direct photon cross sections per nucleus versus $p_{T}$ for $800 \mathrm{GeV} / c$ proton beam on copper, beryllium, and hydrogen targets. 


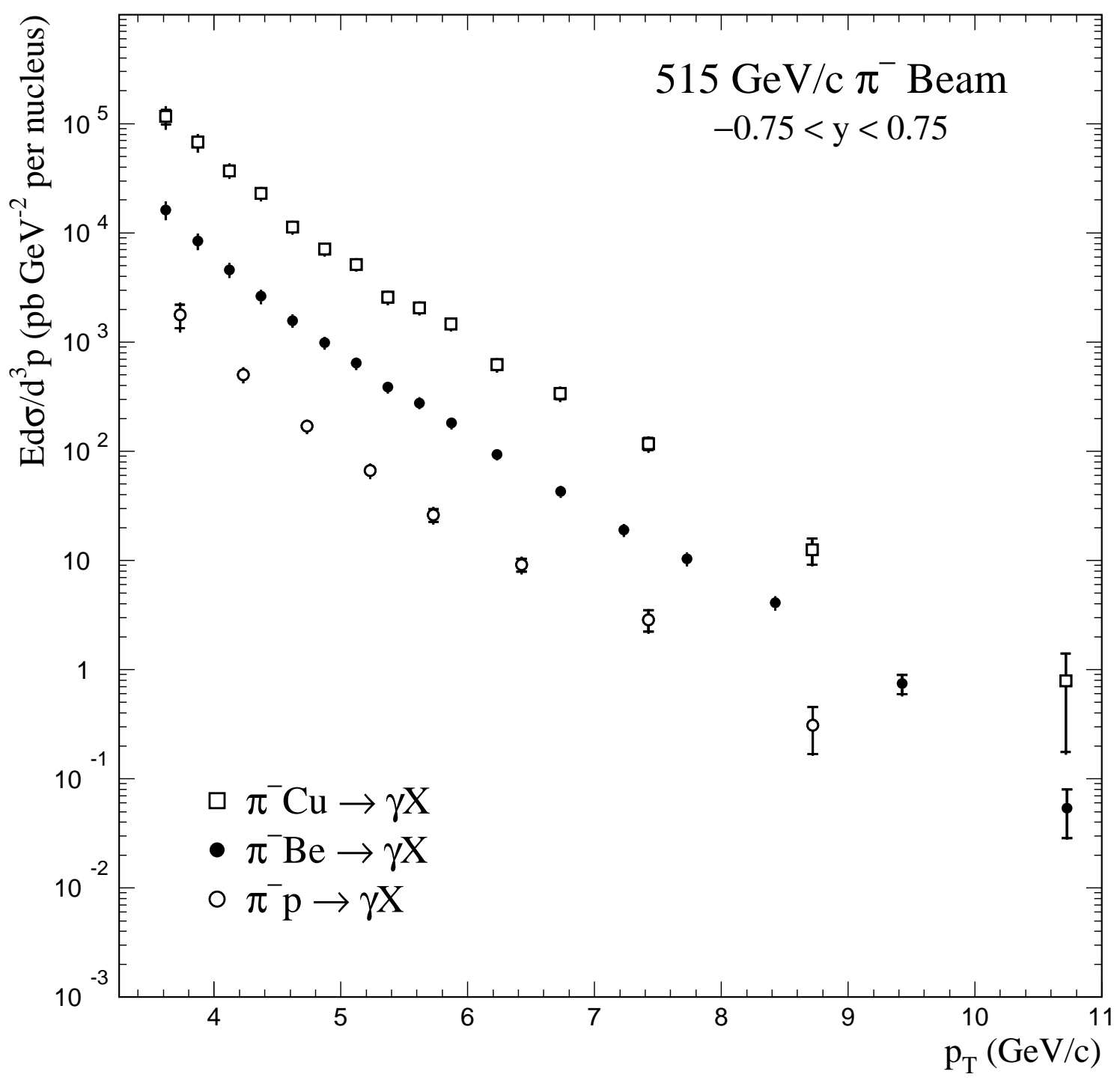

Figure 7.12 Direct photon cross sections per nucleus versus $p_{T}$ for $515 \mathrm{GeV} / c \pi^{-}$ beam on copper, beryllium, and hydrogen targets. 


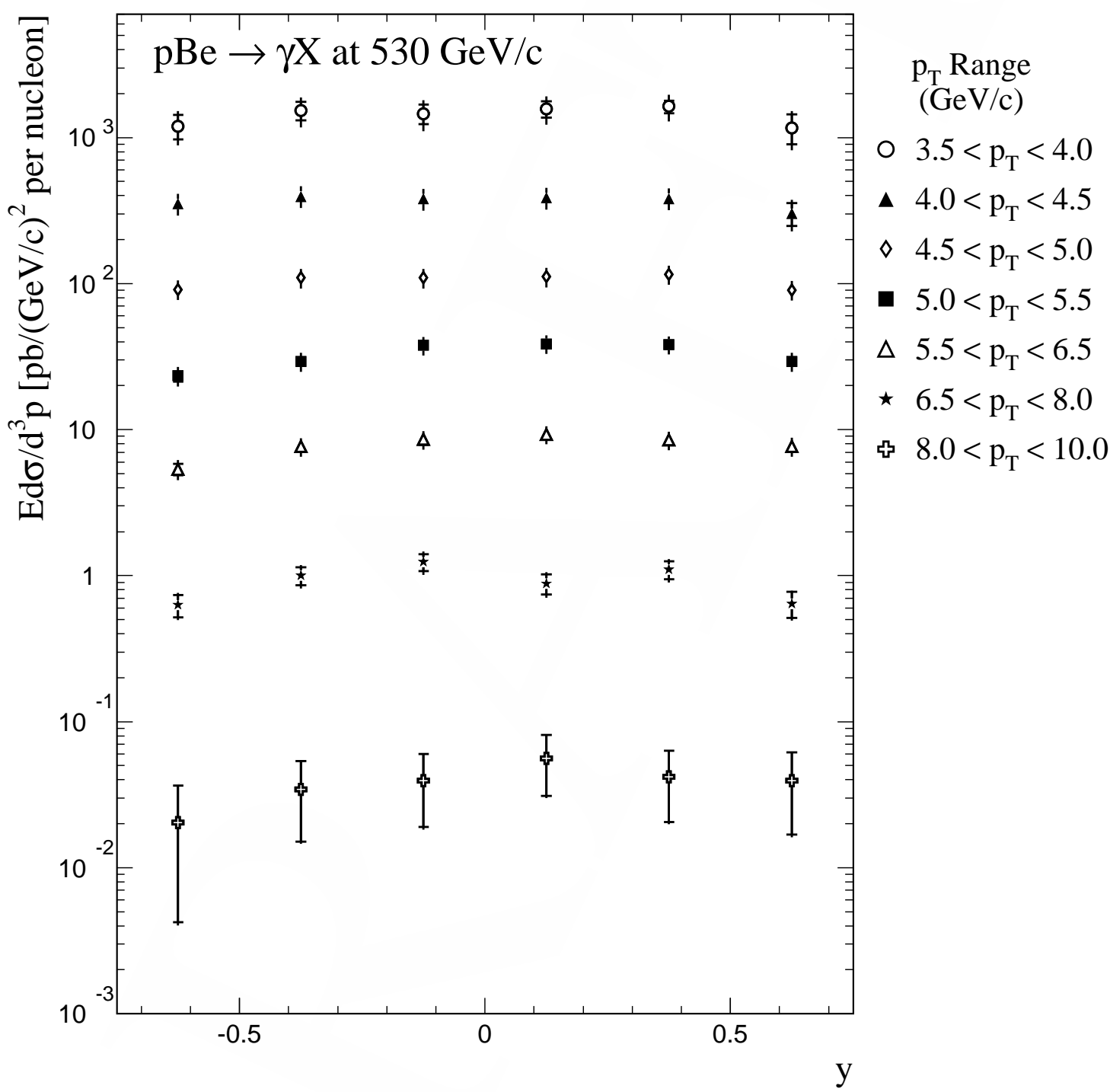

Figure 7.13 Direct photon cross section per nucleon as a function of rapidity for $530 \mathrm{GeV} / c$ proton beam on beryllium for several $p_{T}$ intervals. 


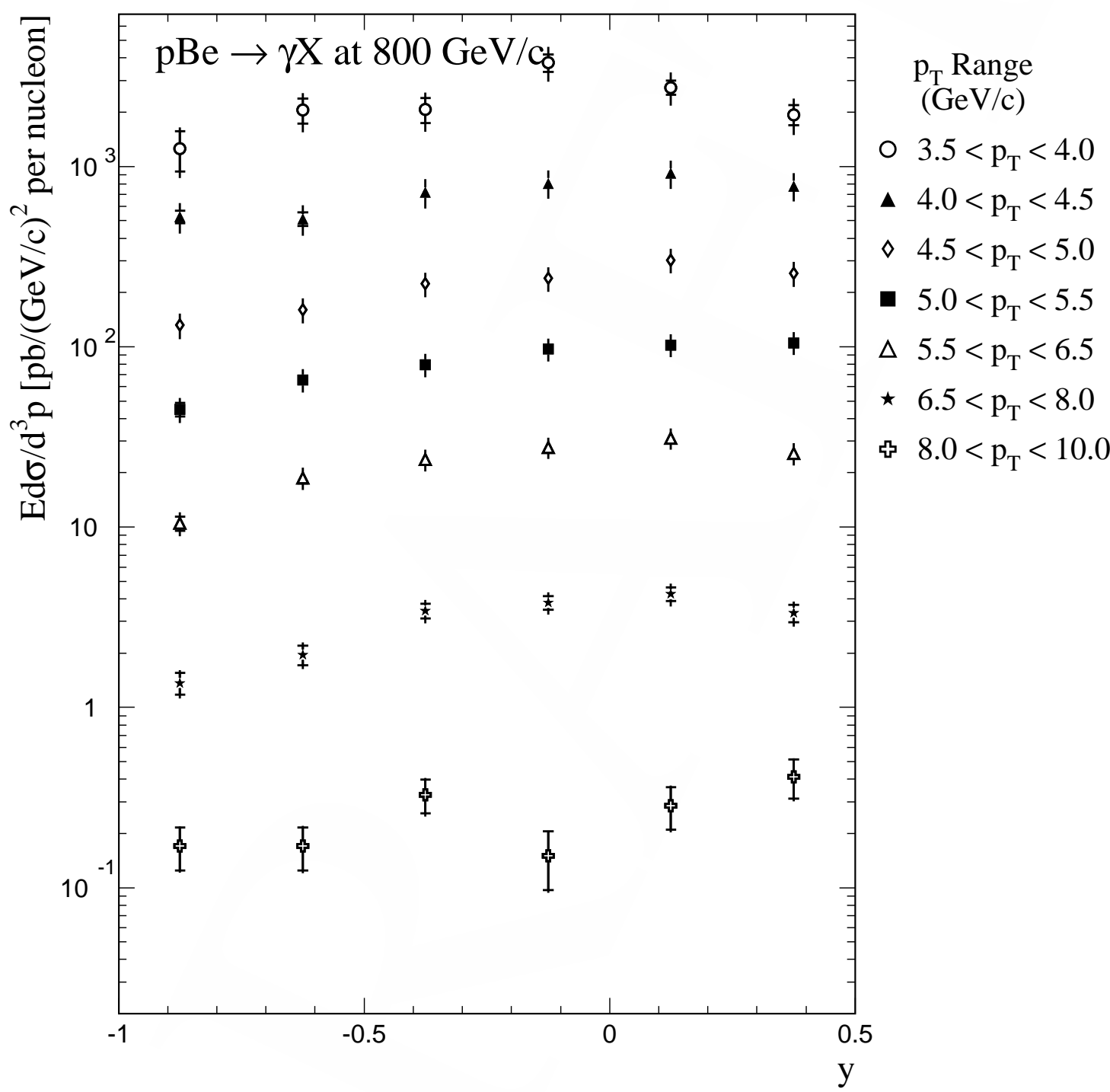

Figure 7.14 Direct photon cross section per nucleon as a function of rapidity for $800 \mathrm{GeV} / c$ proton beam on beryllium for several $p_{T}$ intervals. 


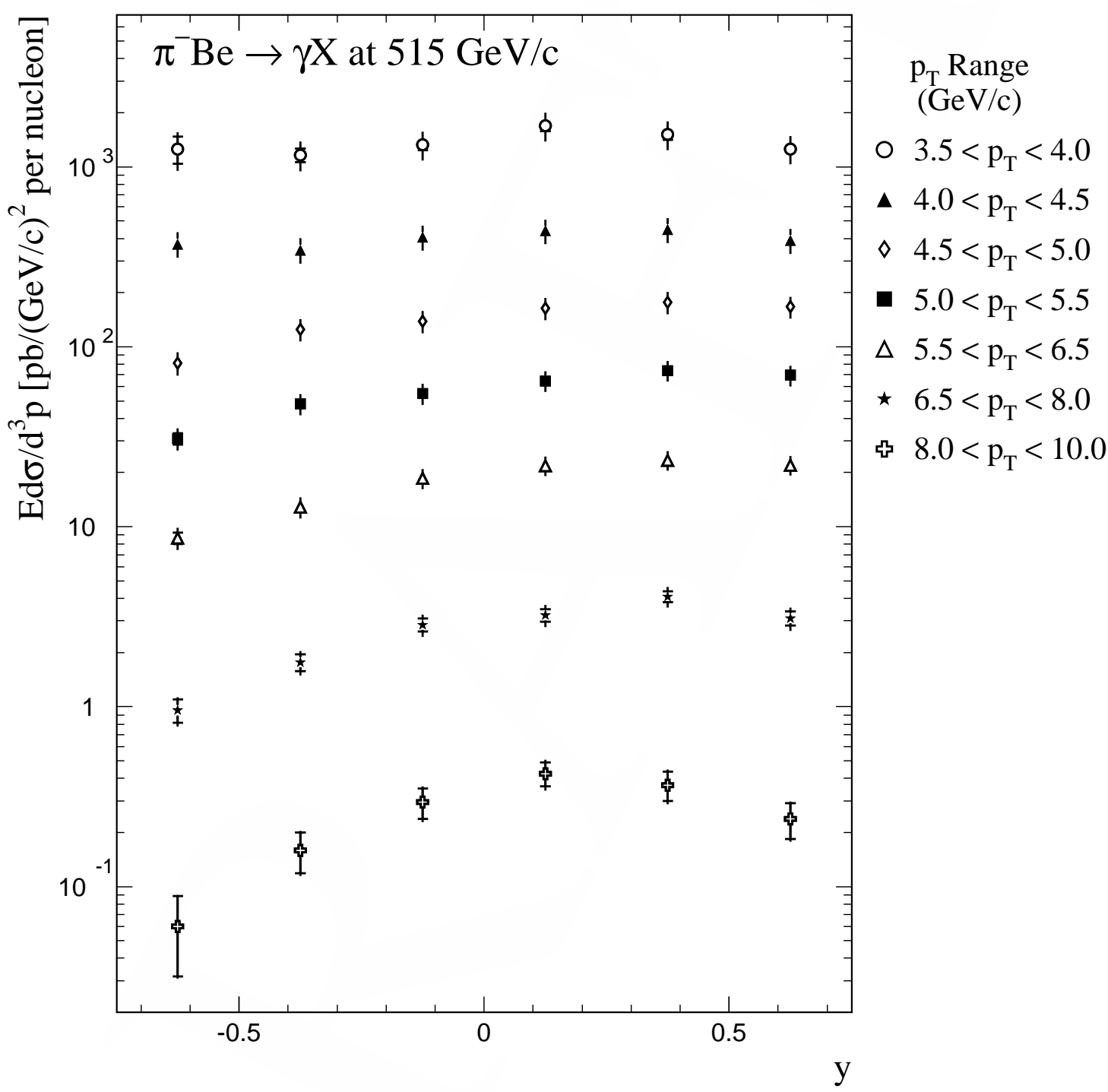

Figure 7.15 Direct photon cross section as a function of rapidity for $515 \mathrm{GeV} / \mathrm{c}$ $\pi^{-}$beam on beryllium for several $p_{T}$ intervals. 


\subsection{Summary of Systematic Uncertainties}

The primary sources of systematic uncertainty in the direct photon and $\pi^{0}$ cross section measurements are from the beam normalization, the reconstruction efficiency, the energy scale calibration, and, in the case of the direct photon cross section, the background subtraction.

The uncertainties in the beam normalization and reconstruction efficiency fold directly into the cross section uncertainty. Their magnitudes have been given in Section 5.13 and Section 6.3.5, respectively. To determine the effect of the energy scale uncertainty on the cross section measurements, the PMC was run using fits to the direct photon and $\pi^{0}$ cross sections as input and introducing a $\pm 0.5 \%$ shift to the output photon energies. ${ }^{3}$ By comparing the output cross sections obtained with the shifted photon energies to those with the proper photon energies, the systematic uncertainty in the cross sections due to the energy scale uncertainty was obtained. The effect of the background subtraction uncertainty was determined by shifting $\gamma_{b} / \pi^{0}$ by its uncertainty (Section 6.3.7) and substituting this value into Equation 7.3. The relative systematic uncertainties from each of these sources, as well as the total systematic uncertainties, are shown in Figure 7.16 as functions of $p_{T}$ for the direct photon and $\pi^{0}$ cross section measurements at $530 \mathrm{GeV} / c$. The corresponding uncertainties for the $515 \mathrm{GeV} / c \pi^{-}$beam and $800 \mathrm{GeV} / c$ proton beam measurements are qualitatively similar.

These uncertainties were cross-checked by comparing $515 \mathrm{GeV} / c \pi^{-}$beam results from data taken during the 1991-92 fixed target run to corresponding results from the 1990 run, as the majority of the corrections applied to these data were determined independently. ${ }^{4}$ Figure 7.17 shows ratios of results from

3 The uncertainty in the overall energy scale was determined to be less than $0.5 \%$ (Section 5.9).

4 Note that this only serves as a partial cross-check, as the same general techniques were used to obtain the corrections for both data samples. 


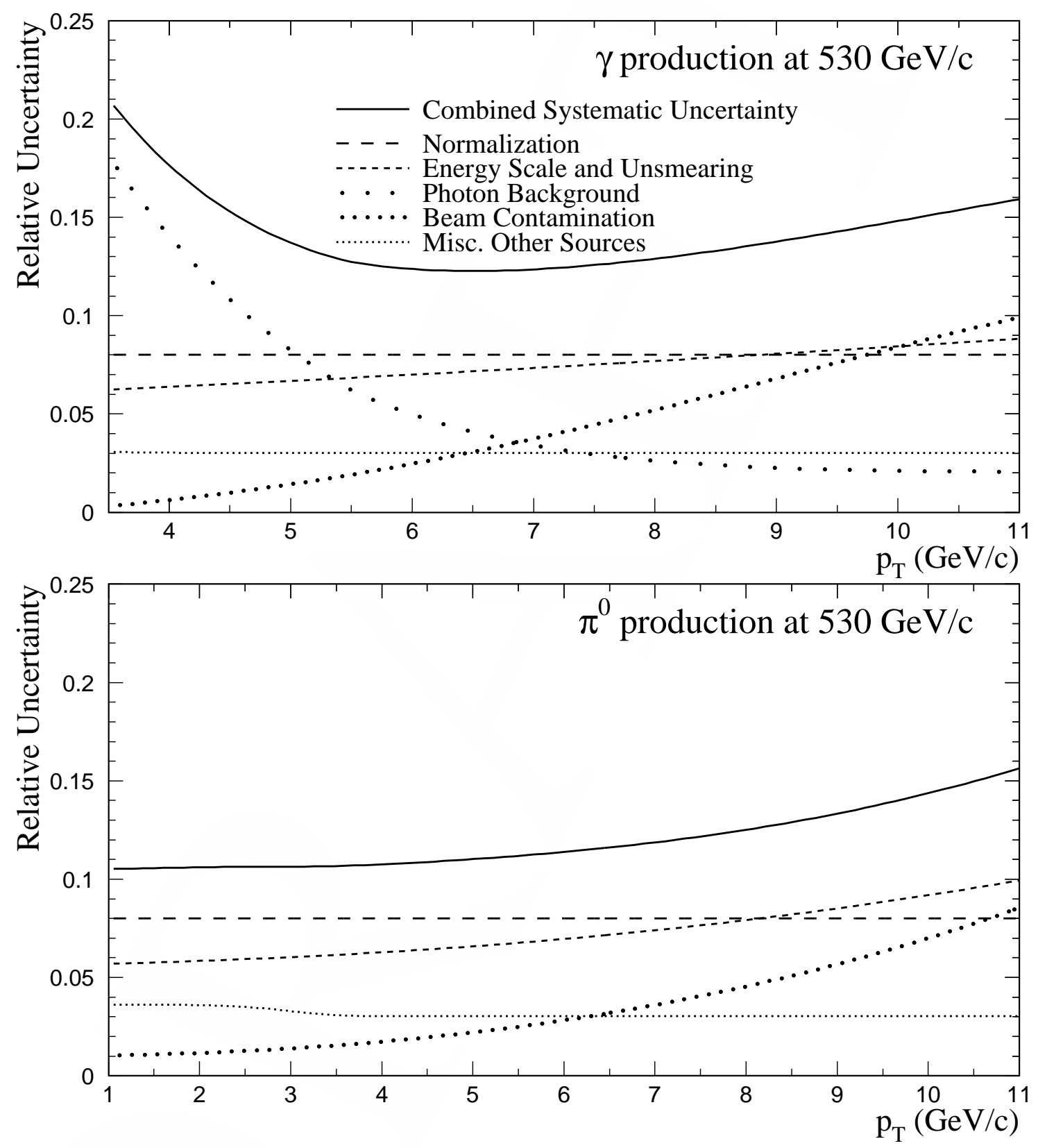

Figure 7.16 Relative systematic uncertainties for direct photon (top) and $\pi^{0}$ (bottom) production at $530 \mathrm{GeV} / c$ versus $p_{T}$. 


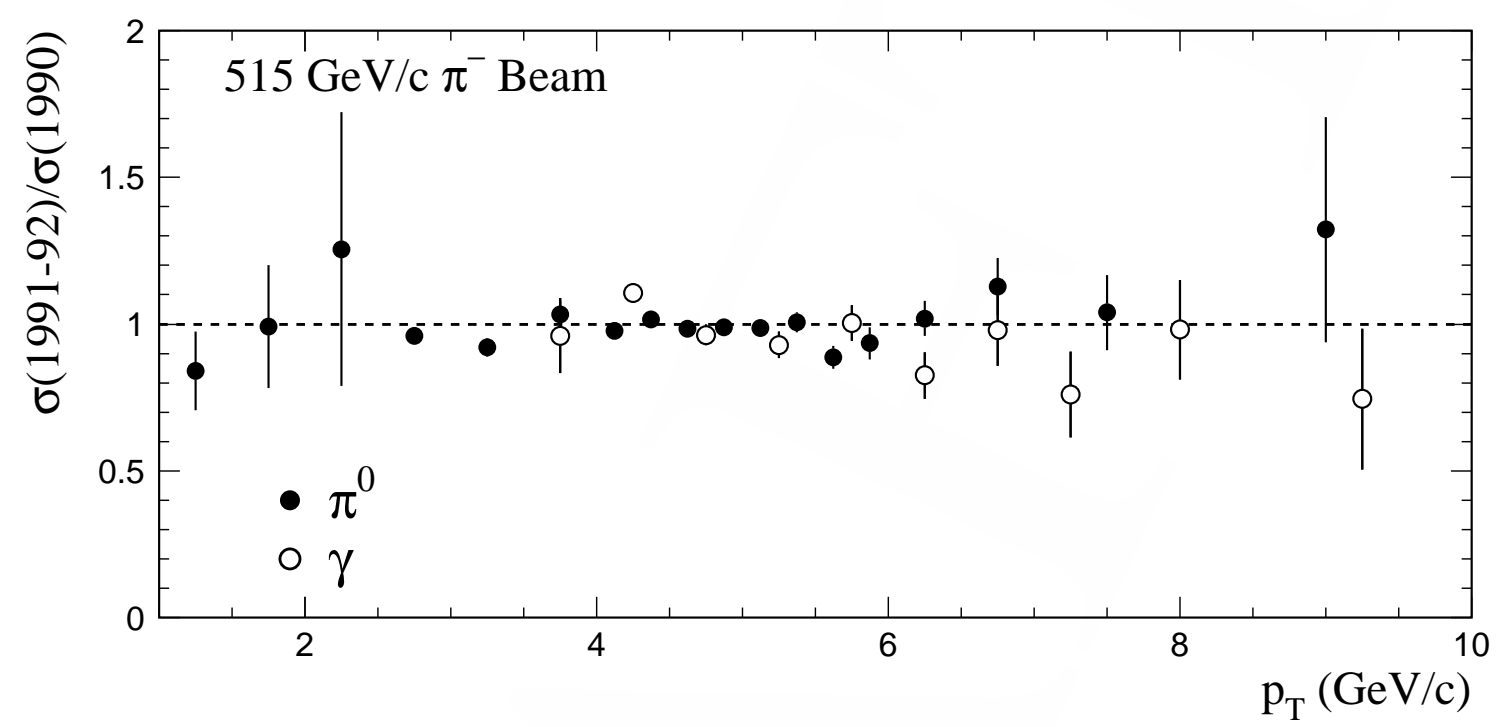

Figure 7.17 Ratios of direct photon and $\pi^{0}$ production cross sections by $515 \mathrm{GeV} / c \pi^{-}$beam on Be obtained from the 1991-92 fixed target run to those obtained from the 1990 run. The error bars reflect statistical uncertainties only.

the 1991-92 run to the 1990 run for the direct photon and $\pi^{0}$ production cross sections. Fits to these ratios over the range shown in the figure yield values of $0.985 \pm 0.006$ for the $\pi^{0}$ ratio, and $0.998 \pm 0.018$ for the direct photon ratio. These values are well within the quoted systematic uncertainties.

A cross-check of the systematic uncertainty on the direct photon results due to the uncertainty in the direct photon background determination can be made by examining the ratio of the cross sections obtained using the different direct photon candidate definitions. The ratio of the $90 \mathrm{~N}$ to $75 \mathrm{~S}$ and the $75 \mathrm{~N}$ to $75 \mathrm{~S}$ direct photon cross sections is shown for the three incident beams in Figure 7.18. 
These ratios differ from unity by $\approx 5 \%$, again well within the quoted systematic uncertainty.

\subsection{Nuclear Dependence}

As stated in Section 1.6, the cross section per nucleus for high- $p_{T}$ particle production is often parameterized as proportional to $A^{\alpha}$. The parameter $\alpha$ can be extracted from the $\mathrm{Cu}$ and Be cross sections per nucleon, $\sigma_{\mathrm{Cu}}$ and $\sigma_{\mathrm{Be}}$, using the relation

$$
\alpha=\frac{\ln \left(\sigma_{\mathrm{Cu}} / \sigma_{\mathrm{Be}}\right)}{\ln \left(A_{\mathrm{Cu}} / A_{\mathrm{Be}}\right)}+1
$$

The nuclear dependence parameters $\alpha$ for direct photon and $\pi^{0}$ production are shown as functions of $p_{T}$ for the three incident beams in Figure 7.19. Most of the experimental systematic uncertainties cancel in the ratio of the $\mathrm{Cu}$ and $\mathrm{Be}$ cross sections. The remaining uncertainties, indicated by the bands in the figure, are dominated by the target-related systematics described in Section 6.3.6. At low $p_{T}$, the values of $\alpha$ for $\pi^{0}$ production are below one - consistent with expectations that at low $p_{T}$ the interactions are occurring at the nuclear level rather than at the parton level. As the $p_{T}$ increases, the $\alpha$ values rise to, and exceed, one; the value expected for scatters occurring between the beam and target constituents. The excess above one is interpreted as due to multiple scattering of the partons in the nucleus. The values of $\alpha$ for direct photon production are clearly below the corresponding values for $\pi^{0}$ production, which may be expected since multiple scattering is limited to the initial state in the case of direct photon production. The solid lines represent fits to constants over the ranges indicated by the lines. The resultant values for $\alpha$ are presented in Table 7.2. In the table, the first uncertainty is statistical and the second is systematic. The dotted line overlaid on the $515 \mathrm{GeV} / c \pi^{-}$beam data represents a theoretical prediction for $\alpha$ in direct photon production from Guo and Qiu [26]. Also shown are $\alpha$ measurements for 


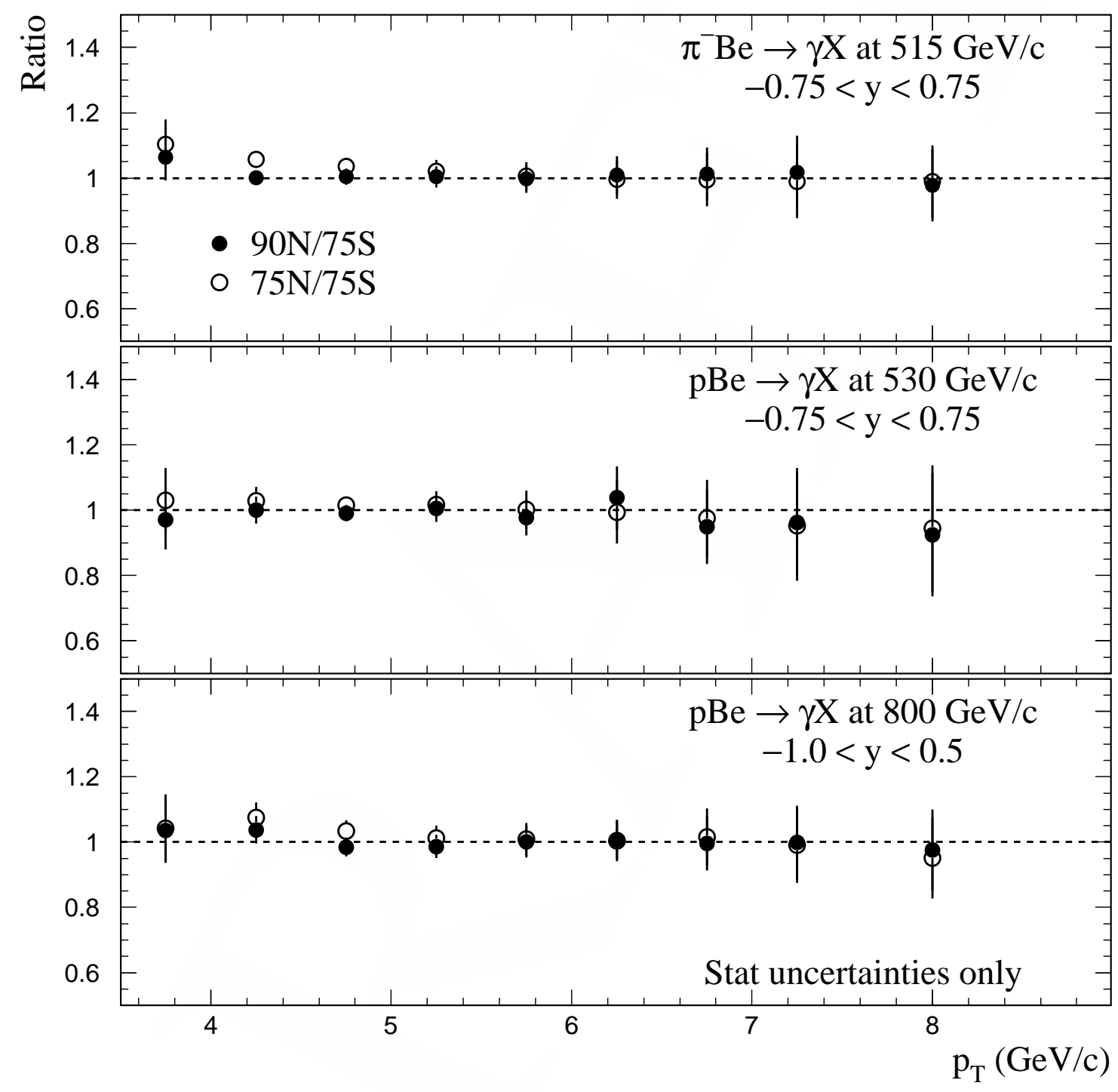

Figure 7.18 Ratio of $90 \mathrm{~N}$ to $75 \mathrm{~S}$ and $75 \mathrm{~N}$ to $75 \mathrm{~S}$ direct photon cross sections for the $515 \mathrm{GeV} / \mathrm{c} \pi^{-}$and 530 and $800 \mathrm{GeV} / \mathrm{c}$ proton beams. 
Table 7.2 Measured $\alpha$ values for $\pi^{0}$ and direct photon production as determined from the fits shown in Figure 7.19.

\begin{tabular}{|c|c|c|}
\hline Beam & $\pi^{0}$ & Direct photon \\
\hline $530 \mathrm{GeV} / c p$ & $1.123 \pm 0.007 \pm 0.011$ & $1.060 \pm 0.015 \pm 0.011$ \\
\hline $800 \mathrm{GeV} / c p$ & $1.129 \pm 0.011 \pm 0.017$ & $1.028 \pm 0.016 \pm 0.016$ \\
\hline $515 \mathrm{GeV} / c \pi^{-}$ & $1.109 \pm 0.007 \pm 0.011$ & $1.044 \pm 0.011 \pm 0.011$ \\
\hline
\end{tabular}

charged $\pi$ production by $200 \mathrm{GeV} / c \pi^{-}$beam by Fermilab experiment E258 [100]. The measurements are in good agreement with the $\alpha$ results for $\pi^{0}$ production at $515 \mathrm{GeV} / c$.

The results for the nuclear dependence of direct photon and $\pi^{0}$ production can be compared to theoretical expectations from the HIJING Monte Carlo-a program designed to simulate particle production in $p p, p \mathrm{~A}$, and AA collisions [101]. However, at these beam energies the normalization of the cross sections from HIJING are sensitive to the choice of the hard scatter $p_{T}$ threshold, and thus, only shape comparisons are possible. Comparisons for the ratio of $\pi^{0}$ and direct photon production cross sections on $\mathrm{Cu}$ target to those on Be target are shown in Figures 7.20 and 7.21, respectively, for the three incident beams. The HIJING normalizations were obtained by fitting the shapes obtained from HIJING to the data over the range indicated by the dashed lines. The shapes from HIJING for the incident proton beams are in good agreement with the data, while the shape of the HIJING prediction for the incident $\pi^{-}$beam has a bigger slope than is seen in the data.

Similar comparisons can also be made for the ratio of the inclusive $\pi^{0}$ and direct photon cross sections on Be target to those on $p$ target. These are shown in Figures 7.22 and 7.23. In these comparisons, the shapes from HIJING are seen to be in relatively good agreement with the data for all the data samples. 


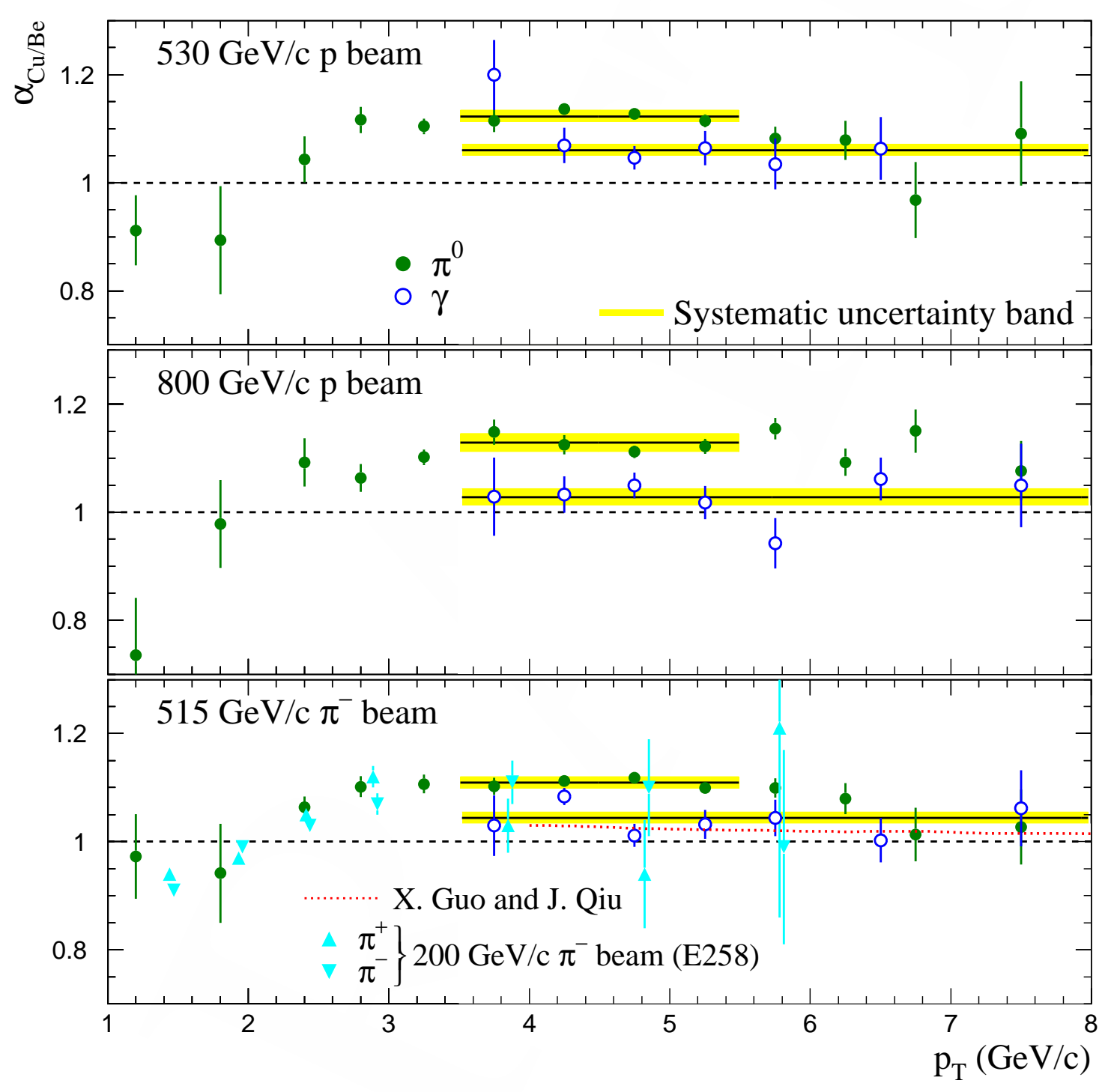

Figure 7.19 The nuclear dependence parameter $\alpha$ measured using $\mathrm{Cu}$ and $\mathrm{Be}$ targets versus $p_{T}$ for direct photon and $\pi^{0}$ production by incident 530 and $800 \mathrm{GeV} / c$ proton beams and incident $515 \mathrm{GeV} / c \pi^{-}$beam. 


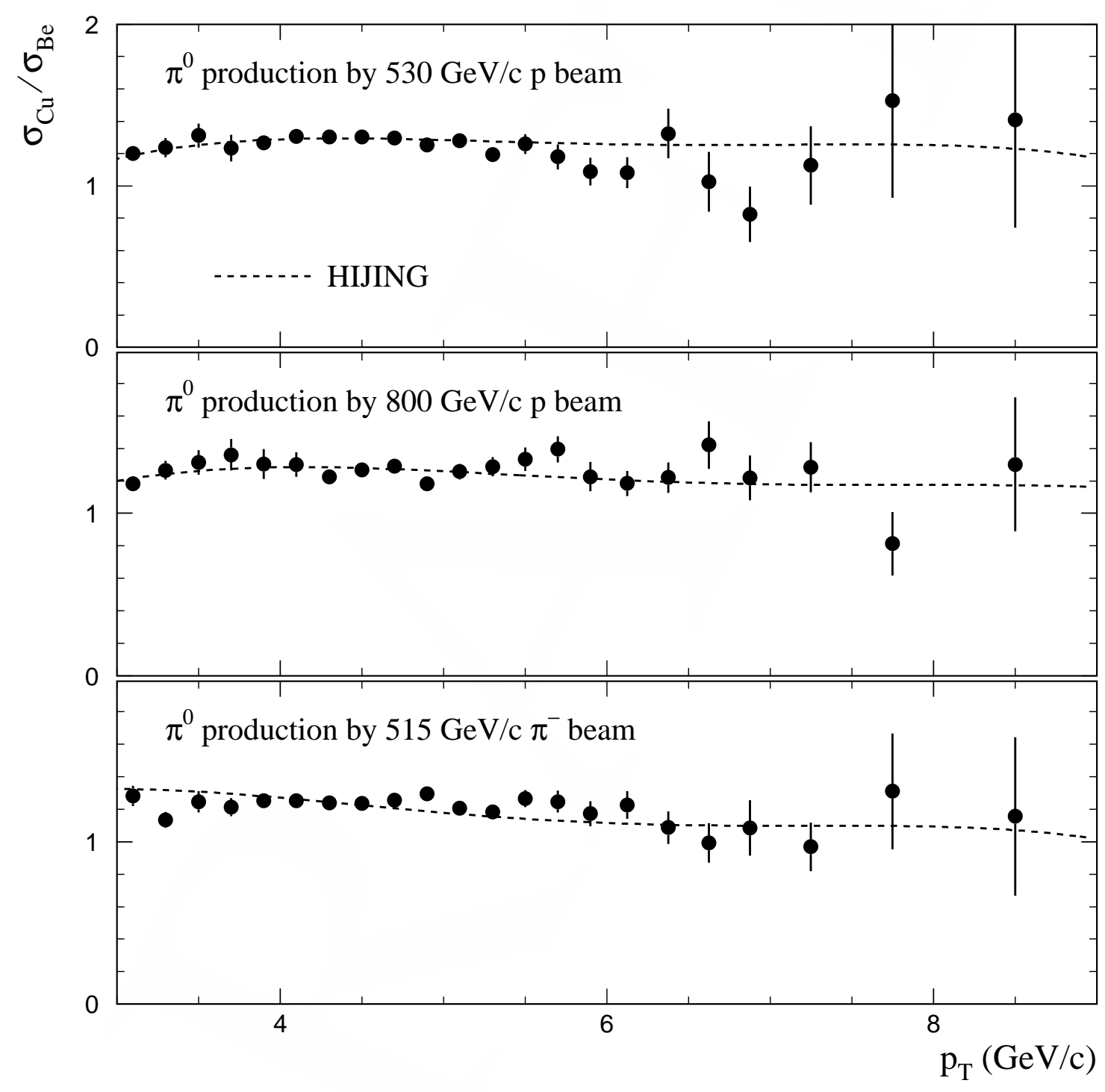

Figure 7.20 The ratio of inclusive $\pi^{0}$ production cross sections per nucleon on $\mathrm{Cu}$ to those on $\mathrm{Be}$ as functions of $\pi^{0} p_{T}$. Also shown are shape comparisons with HIJING. The error bars represent only statistical uncertainties. 


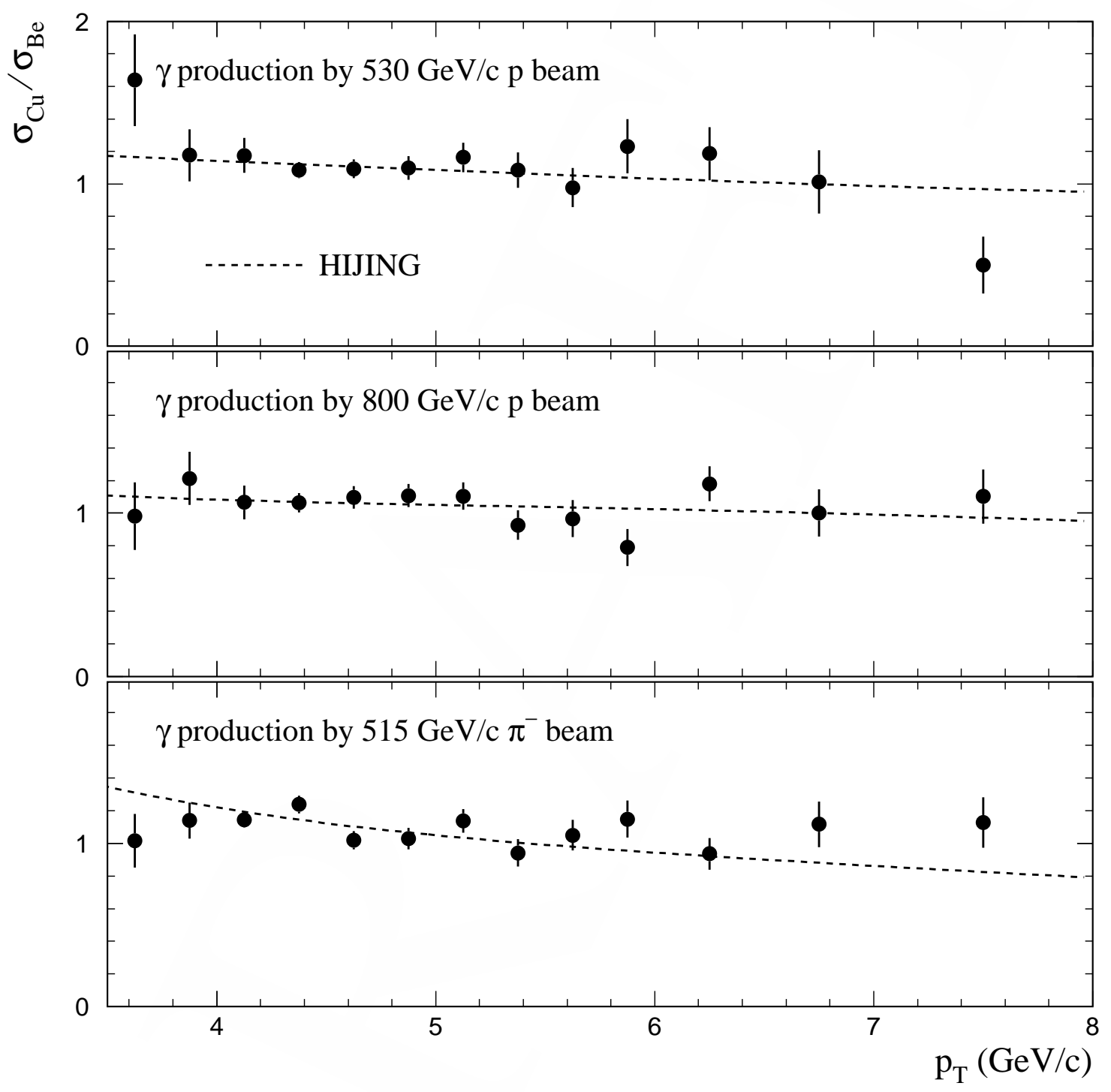

Figure 7.21 The ratio of inclusive direct photon production cross sections per nucleon on $\mathrm{Cu}$ to those on $\mathrm{Be}$ as functions of direct photon $p_{T}$. Also shown are shape comparisons with HIJING. The error bars represent only statistical uncertainties. 


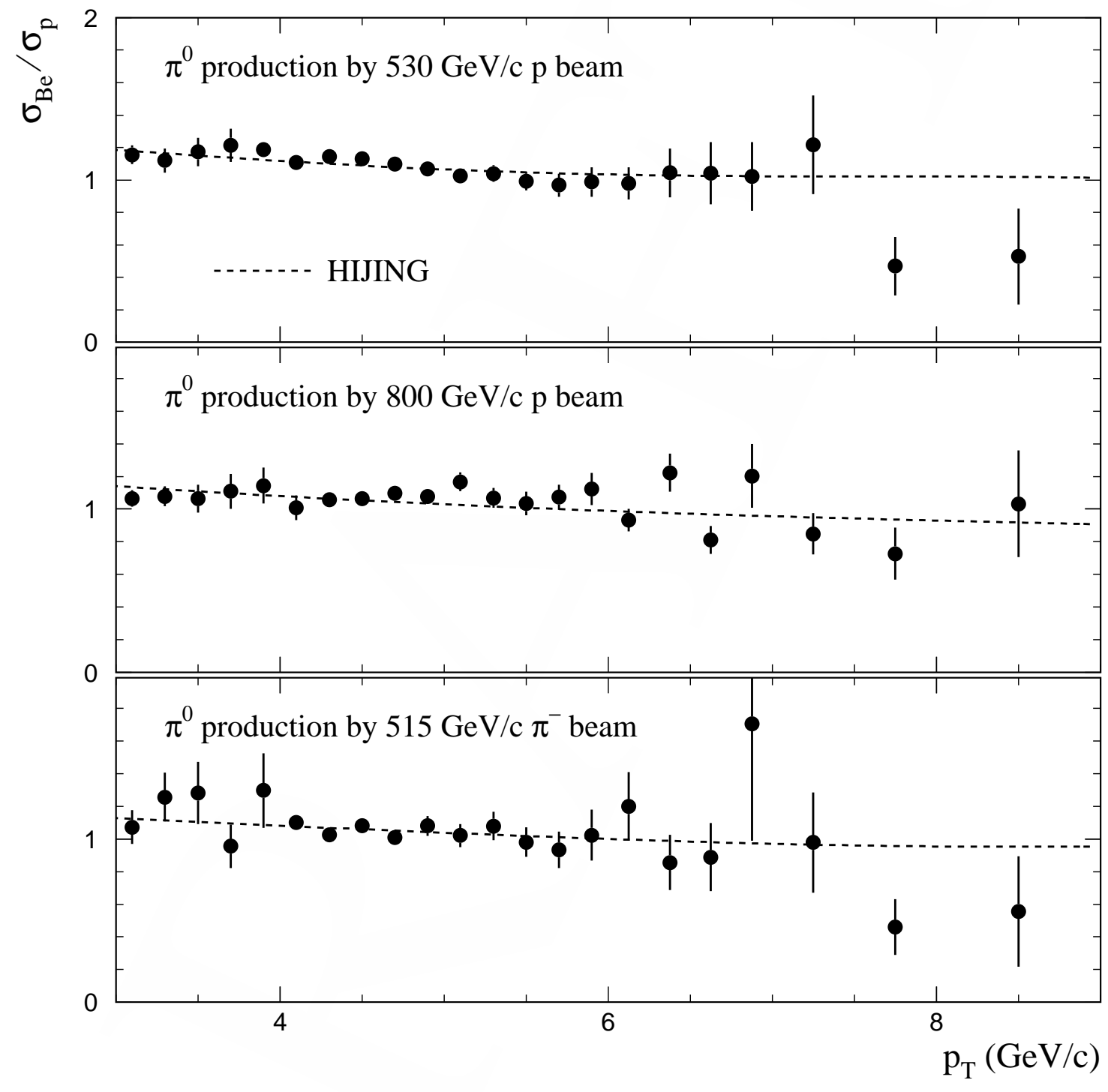

Figure 7.22 The ratio of inclusive $\pi^{0}$ cross sections per nucleon on Be target to those on $p$ target as functions of $\pi^{0} p_{T}$. Also shown are shape comparisons with HIJING. The error bars represent only statistical uncertainties. 


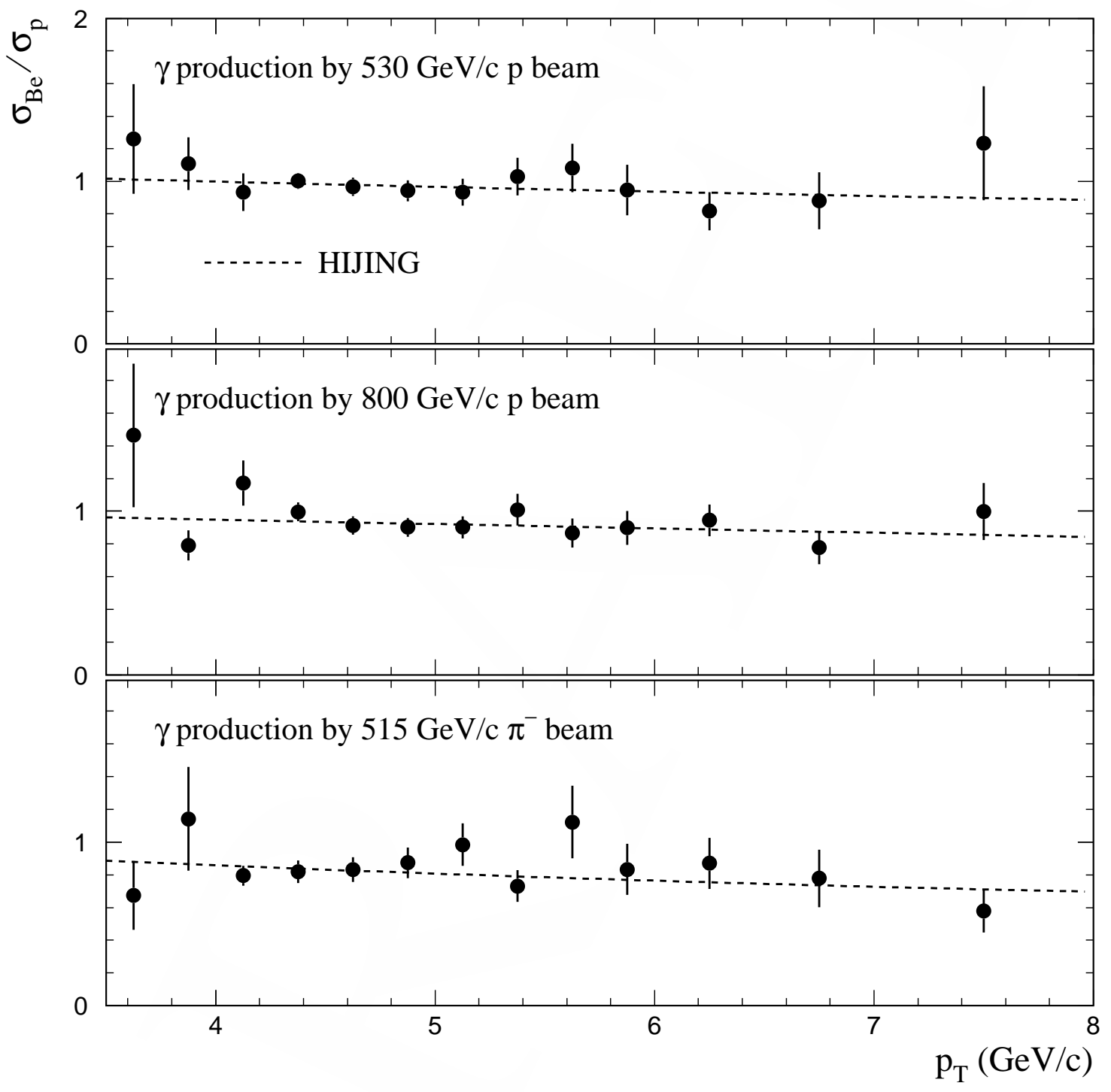

Figure 7.23 The ratio of inclusive direct photon cross sections per nucleon on Be target to those on $p$ target as function of direct photon $p_{T}$. Also shown are shape comparisons with HIJING. The error bars represent only statistical uncertainties. 


\subsection{Comparisons with NLO Calculations}

In this section, direct photon and $\pi^{0}$ cross section measurements are compared with perturbative predictions calculated to next-to-leading order precision. The NLO predictions for direct photon production are from Ref. [102], while the predictions for $\pi^{0}$ production are from Ref. [103]. In these comparisons, the theoretical scales, $\mu_{R}, \mu_{F}$, and $m_{F}$ (for the $\pi^{0}$ prediction), are all chosen to be equal. Also, for the $\pi^{0}$ comparisons, the calculations use KKP [19] fragmentation functions $(\mathrm{FF})$. Although the theoretical calculations account for the numbers of protons and neutrons in nuclear targets, they do not account for nuclear effects. Therefore, the theoretical calculations for the beryllium target have been adjusted using results from HIJING (Section 7.6).

In Figure 7.24, inclusive direct photon and $\pi^{0}$ cross sections per nucleon for $515 \mathrm{GeV} / c \pi^{-}$beam are compared to NLO pQCD results with scale choices of $\mu=\frac{1}{3} p_{T}, \frac{1}{2} p_{T}$, and $p_{T}{ }^{5}$. The discrepancy between the theory and the data is significant. Analogous comparisons between NLO calculations and the 530 and $800 \mathrm{GeV} / \mathrm{c} p$ beam data show similar discrepancies. In addition, the theory shows significant dependence on the scale choice. However, recent calculations which include the effects of soft-gluon resummation in direct photon production near the threshold limit $(x \rightarrow 1)$ [31] have significantly reduced scale dependence and, at E706 beam energies, are comparable to the bare NLO prediction with a scale choice of $\mu=\frac{1}{2} p_{T}$ (see Figure 7.25). This scale choice is employed in the following NLO comparisons with the data.

${ }^{5}$ The theoretical prediction for $\pi^{0}$ production with $\mu=\frac{1}{3} p_{T}$ is not shown below $p_{T}=4.25 \mathrm{GeV} / c$ because the scale is below the starting scale for the KKP fragmentation function. 


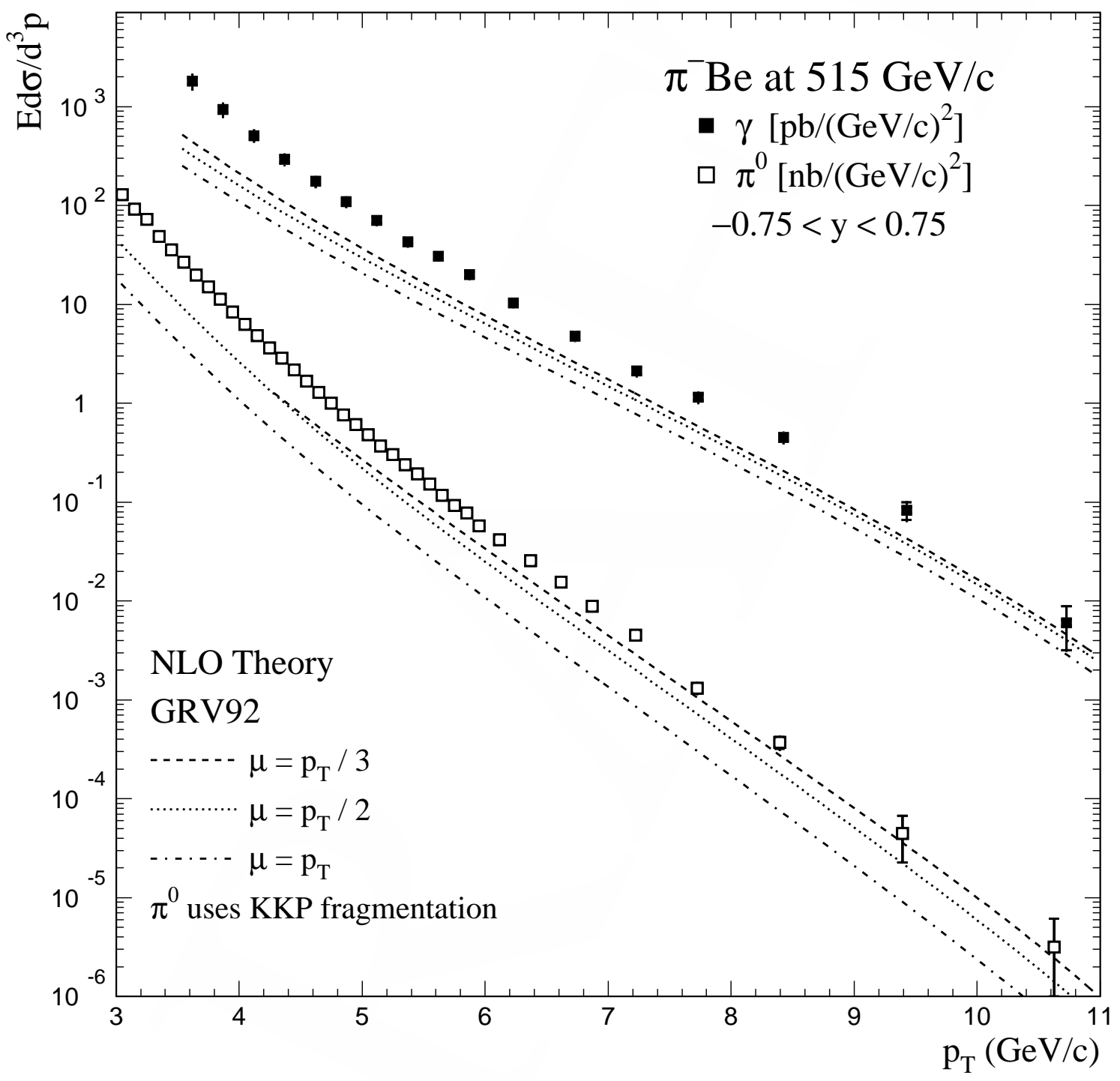

Figure 7.24 Direct photon cross section versus $p_{T}$ for $515 \mathrm{GeV} / c \pi^{-}$beam on beryllium compared to NLO pQCD results for several choices of scales. Also shown is the $\pi^{0}$ comparison (scaled down by a factor of 1000). 


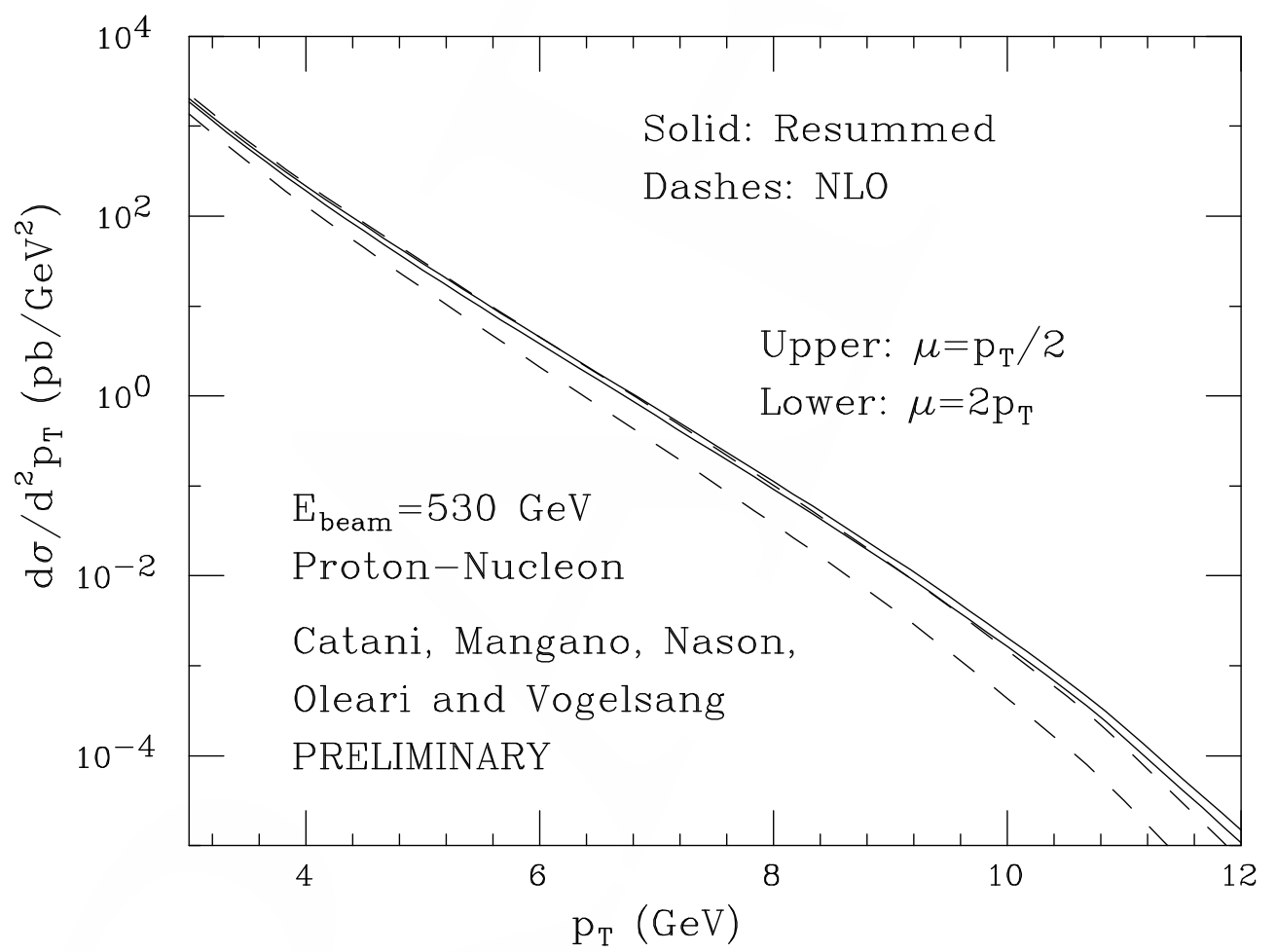

Figure 7.25 Comparison between a threshold resummed pQCD calculation and NLO pQCD for scale choices of $\frac{1}{2} p_{T}$ and $2 p_{T}$. Figure from Ref. [31]. 


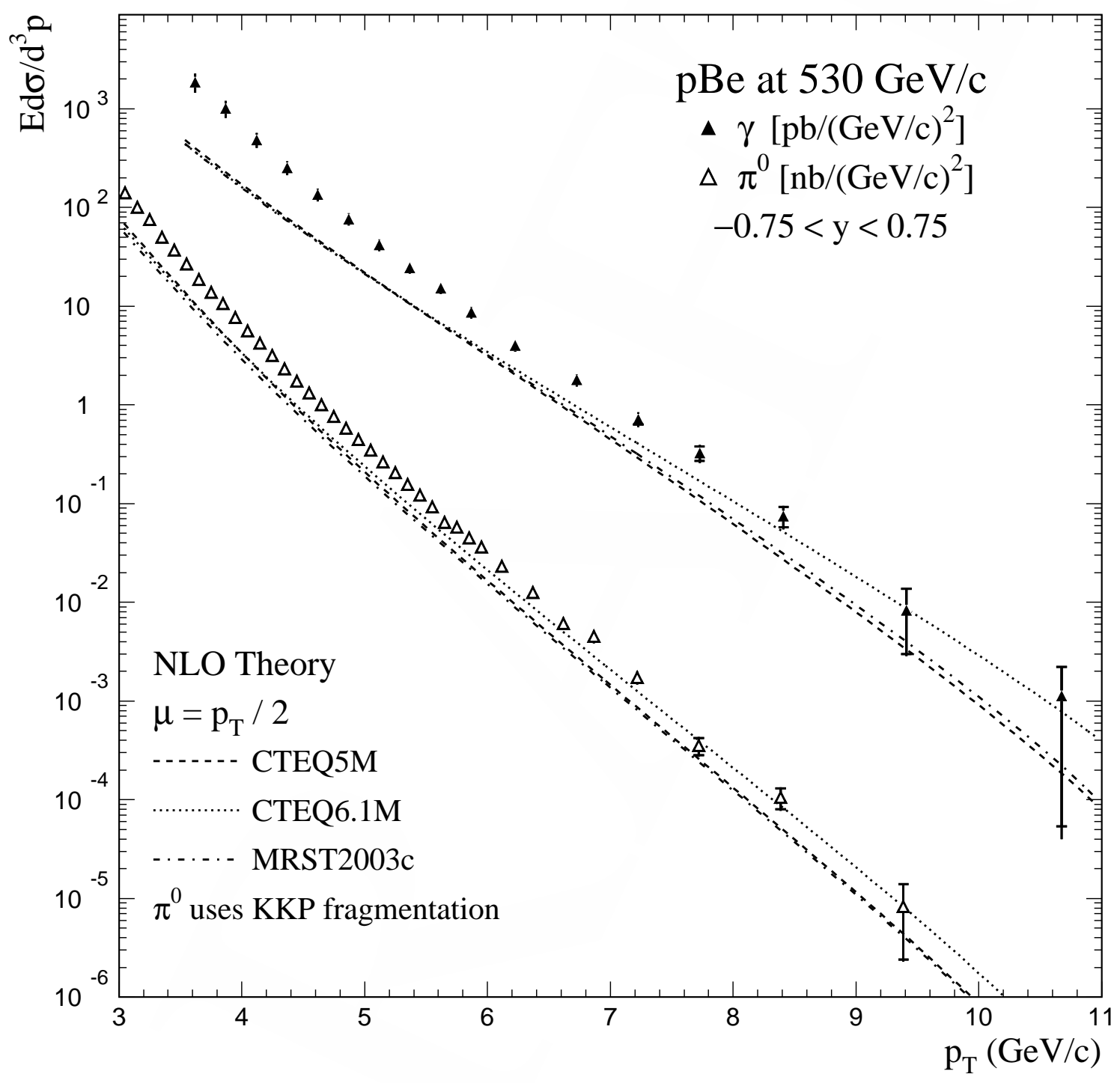

Figure 7.26 Direct photon and $\pi^{0}$ cross sections versus $p_{T}$ for $530 \mathrm{GeV} / c$ proton beam on beryllium compared to NLO pQCD results for several choices of parton distribution functions. 
In Figure 7.26, the direct photon and $\pi^{0}$ cross sections for $530 \mathrm{GeV} / c p$ beam are compared to NLO calculations using CTEQ5M[104], CTEQ6.1M[18] and MRST2003C[105] parton distribution functions (PDFs). Although the calculations show sensitivity to the choice of PDF, none of the PDF's bring the predictions into agreement with the data. PDF's from CTEQ6.1 and MRST2001E[106] also provide additional PDF sets which can be used to assess the uncertainty in the calculation due to the PDF uncertainty. In Figure 7.27, the direct photon and $\pi^{0}$ cross sections for $800 \mathrm{GeV} / c p$ beam data are compared to NLO calculations using MRST2001E PDF. The uncertainty in the calculation from the PDF is indictated by the shaded band in the figure. The uncertainty in the PDF is not large enough to account for the difference between the data and the theory.

\subsubsection{Evidence for Initial State Parton $k_{T}$}

As stated in Section 1.7, a possible cause for the large discrepancies between data and NLO calculations may lie in the effects of soft-gluon radiation in the initial state. Such soft-gluon emissions may not be fully accounted for in the NLO theory and may generate substantial amounts of transverse parton momenta $\left(k_{T}\right)$ in the initial state. Evidence for such $k_{T}$ can be found through the analysis of distributions of high-mass direct photon pairs in the data [27]. Distributions sensitive to $k_{T}$ include: the total $p_{T}$ of the two photons $\left(Q_{T}\right)$, the azimuthal angle between the photons $(\Delta \phi)$, and the out-of-plane momentum $\left(p_{O U T}\right)$, which is defined as the component of the momentum of one of the photons perpendicular to the plane formed by the incident beam direction and the direction of the other photon. These distributions are shown in Figure 7.28. Overlayed on the data are results from NLO pQCD (dashed) [107], resummed NLO (solid) [38], and 


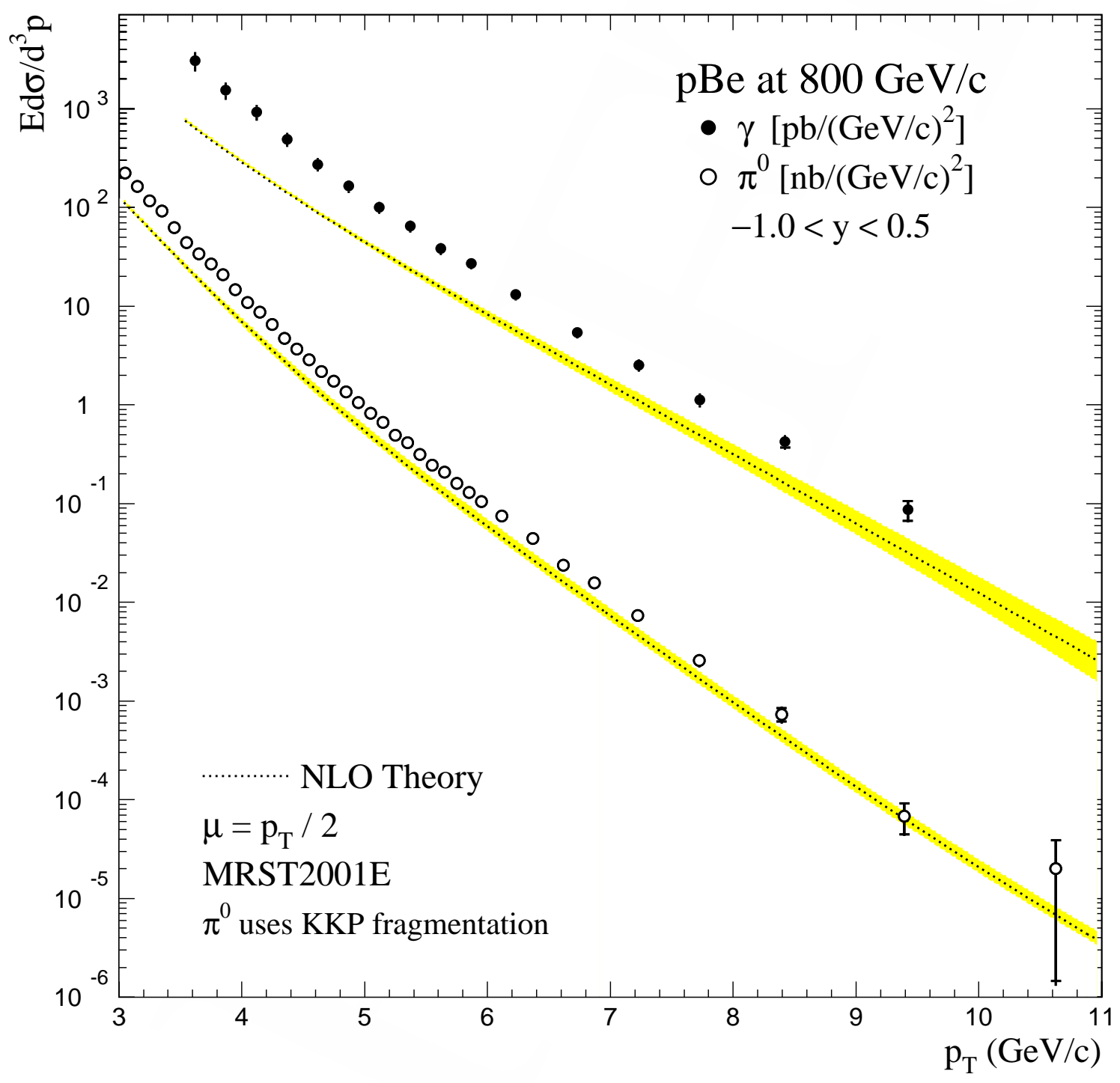

Figure 7.27 Direct photon and $\pi^{0}$ cross sections versus $p_{T}$ for $800 \mathrm{GeV} / c$ proton beam on beryllium compared to NLO pQCD results using MRST2001E PDF. The shaded band indicates the uncertainty associated with the PDF. 
$k_{T^{-}}$enhanced PYTHIA ${ }^{6}$ (dotted) calculations. The shape of the NLO prediction is inconsistent with the data. The resummed calculation, which accounts for multiple soft gluon emission, provides a reasonable match to the shape of the data. The shape of the $k_{T}$-enhanced PYTHIA distribution is also in good agreement with the data.

Similar comparisons can be made for high-mass $\pi^{0} \pi^{0}, \pi^{0} \eta$, and $\gamma \pi^{0}$ pairs. In Figure 7.29, $p_{O U T}$ distributions are shown for these, as well as for $\gamma \gamma$, pairs. The $\gamma \gamma$ results are compared to NLO pQCD (dashed line), resummed NLO (solid line) and $k_{T}$ enhanced PYTHIA (dotted line) calculations. The $\pi^{0} \pi^{0}$ and $\gamma \pi^{0}$ distributions are compared to LO theory with several choices of supplemental- $k_{T}$. Although fragmentation also contributes to the width of these distributions, the theoretical comparisons are greatly improved when $k_{T}$ effects are incorporated into the theory. The $\left\langle k_{T}\right\rangle$ values that provide the best agreement with the data are comparable to the $\left\langle k_{T}\right\rangle$ measured for $\gamma \gamma$ pairs. Also shown in the figure is the $p_{O U T}$ distribution for $\pi^{0} \eta$ pairs. Theoretical curves are not shown for this distribution, as fragmentation functions for $\eta$ production are not available. Therefore, the $\pi^{0} \pi^{0}$ distribution has been overlaid on the $\pi^{0} \eta$ distribution for comparison.

The presence of significant $k_{T}$ is also expected to affect other aspects of the data. In particular, consider the fragmentation of jets recoiling against high- $p_{T}$ photons. The fragmentation variable $z$ is defined as the longitudinal momentum fraction of the recoil jet momentum carried by particle $i, z \equiv \vec{p}_{i} \cdot \vec{p}_{j e t} /\left|\vec{p}_{j e t}\right|^{2}$. As the total recoil jet momentum is difficult to measure accurately, the momentum of the away-side direct photon can be used in its place. However, if the $\left\langle k_{T}\right\rangle$ is not negligible, such a procedure will affect the $z$ distribution. This is seen in Figure 7.30 [108], where the $z$ distribution of charged particles in jets recoiling

6 The effects of $k_{T}$ are approximated in PYTHIA using a Gaussian smearing prescription similar to the one described in Section 7.7.2. 

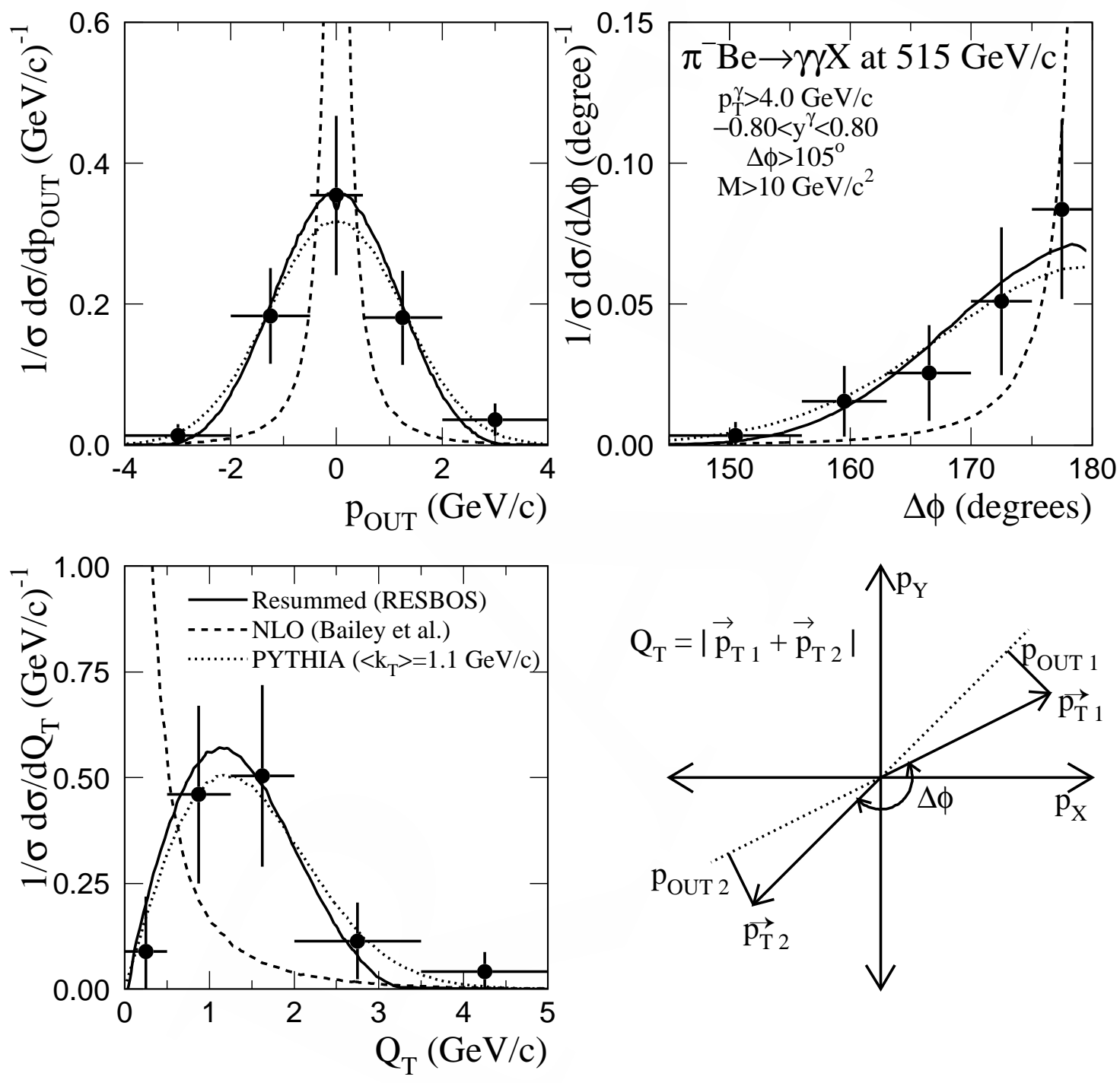

Figure 7.28 $p_{\text {OUT }}, \Delta \phi$ and $Q_{T}$ distributions for high-mass direct photon pairs in the $515 \mathrm{GeV} / c \pi^{-}$data. The data are compared to NLO pQCD (dashed), resummed NLO (solid) and $k_{T}$ enhanced PYTHIA (dotted) results. Figure from Ref. [27]. 


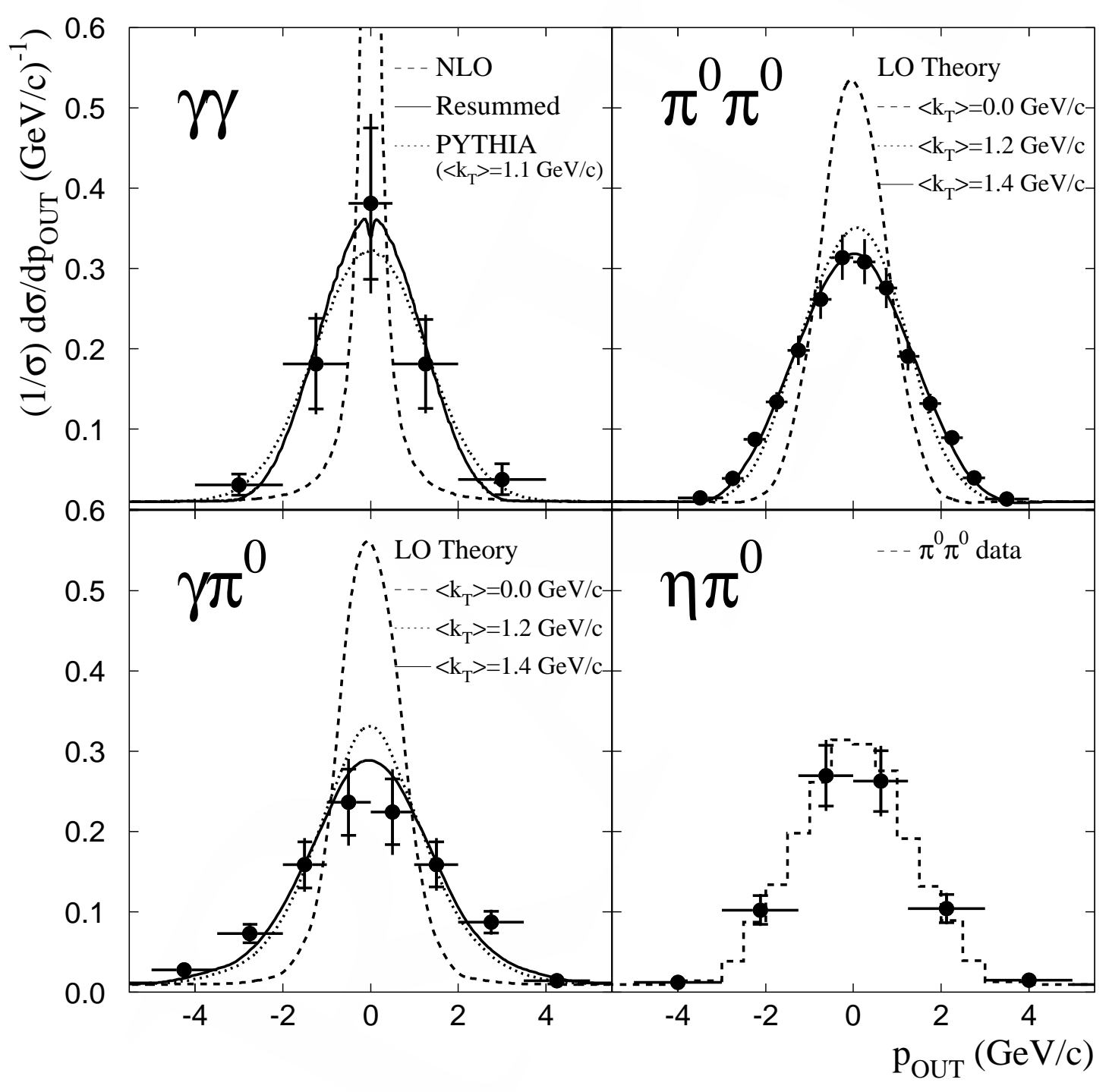

Figure 7.29 $p_{\text {OUT }}$ distributions for high-mass $\gamma \gamma, \pi^{0} \pi^{0}, \gamma \pi^{0}$, and $\pi^{0} \eta$ pairs in $\pi^{-}$Be interactions at $515 \mathrm{GeV} / c$. Figure from Ref. [27]. 
against isolated photons in the $800 \mathrm{GeV} / \mathrm{c}$ proton data is compared to theoretical expectations [15] for several choices of $\left\langle k_{T}\right\rangle$. The calculation with $\left\langle k_{T}\right\rangle$ comparable to the values found using the kinematic distributions discussed earlier is in good agreement with the data.

Finally, this experiment has also measured the cross section for the production of charm mesons at high $p_{T}[109]$. Figure 7.31 shows the differential $\mathrm{D}^{ \pm}$cross section compared to results from NLO pQCD calculations with and without supplemental- $k_{T}$ [110]. Again, the $k_{T}$-enhanced calculation accommodates the data better than the calculation without $k_{T}$.

\subsubsection{Comparisons with $k_{T}$-enhanced NLO Theory}

Higher-than-NLO calculations for direct photon production are currently being developed which simultaneously incorporate the threshold corrections cited earlier with corrections for the recoil from soft radiation before the hard scatter[111]. Such calculations are expected to account for the gluon emissions responsible for the generation of $k_{T}$. Although this work is still in its preliminary stages, early results are encouraging (see Figure 7.32).

Until these calculations are fully developed, $k_{T}$ effects may be implemented into NLO calculations using an intuitive phenomenological approach. An outline of this approach follows. A LO pQCD Monte Carlo calculation [15] imparts an effective $k_{T}$ to each of the colliding partons assuming a Gaussian $k_{T}$ distribution,

$$
g\left(\vec{k}_{T}\right)=\frac{e^{-k_{T}^{2} /\left\langle k_{T}^{2}\right\rangle}}{\pi\left\langle k_{T}^{2}\right\rangle}
$$

where $\left\langle k_{T}^{2}\right\rangle$ is the square of the 2-dimensional (2D) RMS width of the $k_{T}$

distribution for a single parton. It is related to $\left\langle k_{T}\right\rangle$ by the relation $\left\langle k_{T}^{2}\right\rangle=$ $4\left\langle k_{T}\right\rangle^{2} / \pi$. This LO calculation is used to generate $p_{T}$ dependent $k_{T}$-enhancement factors (K-factors), which are ratios of the LO calculation run with supplemental 


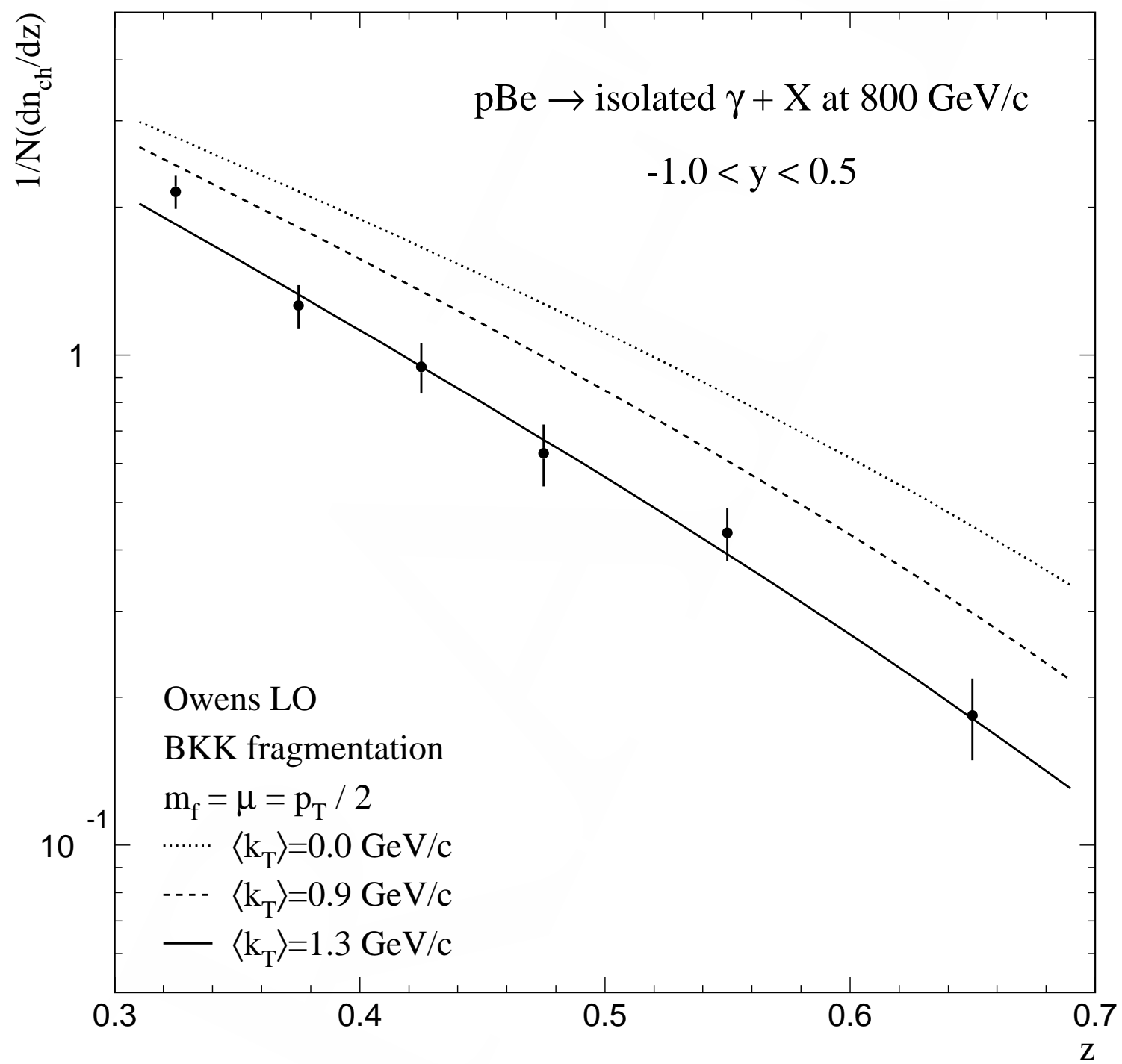

Figure 7.30 Away-side fragmentation function for jets recoiling against isolated $\gamma$ 's with $p_{T}>5.5 \mathrm{GeV} / c[108]$. 


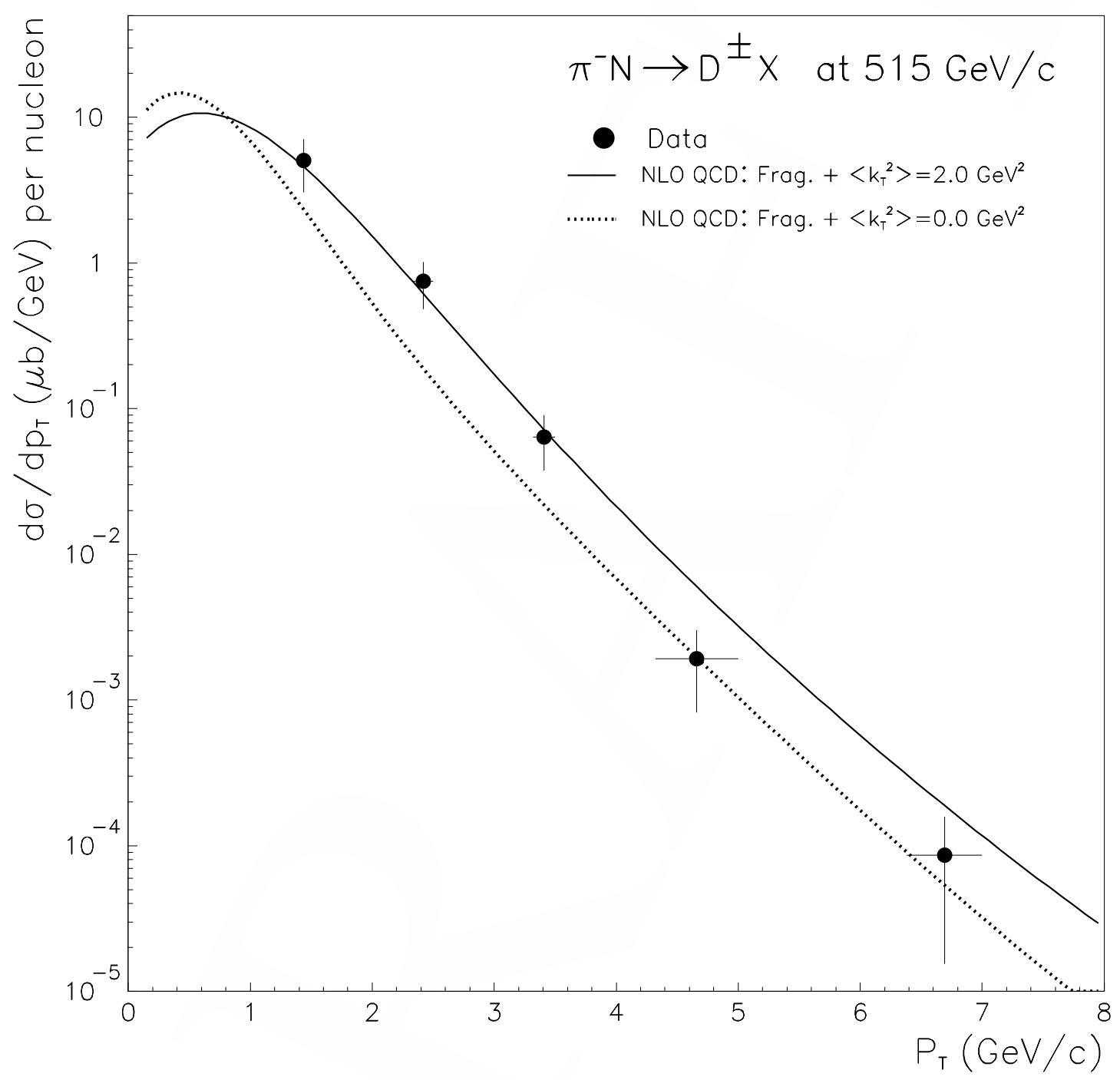

Figure 7.31 $\mathrm{D}^{ \pm}$cross section per nucleon versus $p_{T}$ for $515 \mathrm{GeV} / c \pi^{-}$-Nucleon collisions compared to NLO calculations with and without $k_{T}$. 


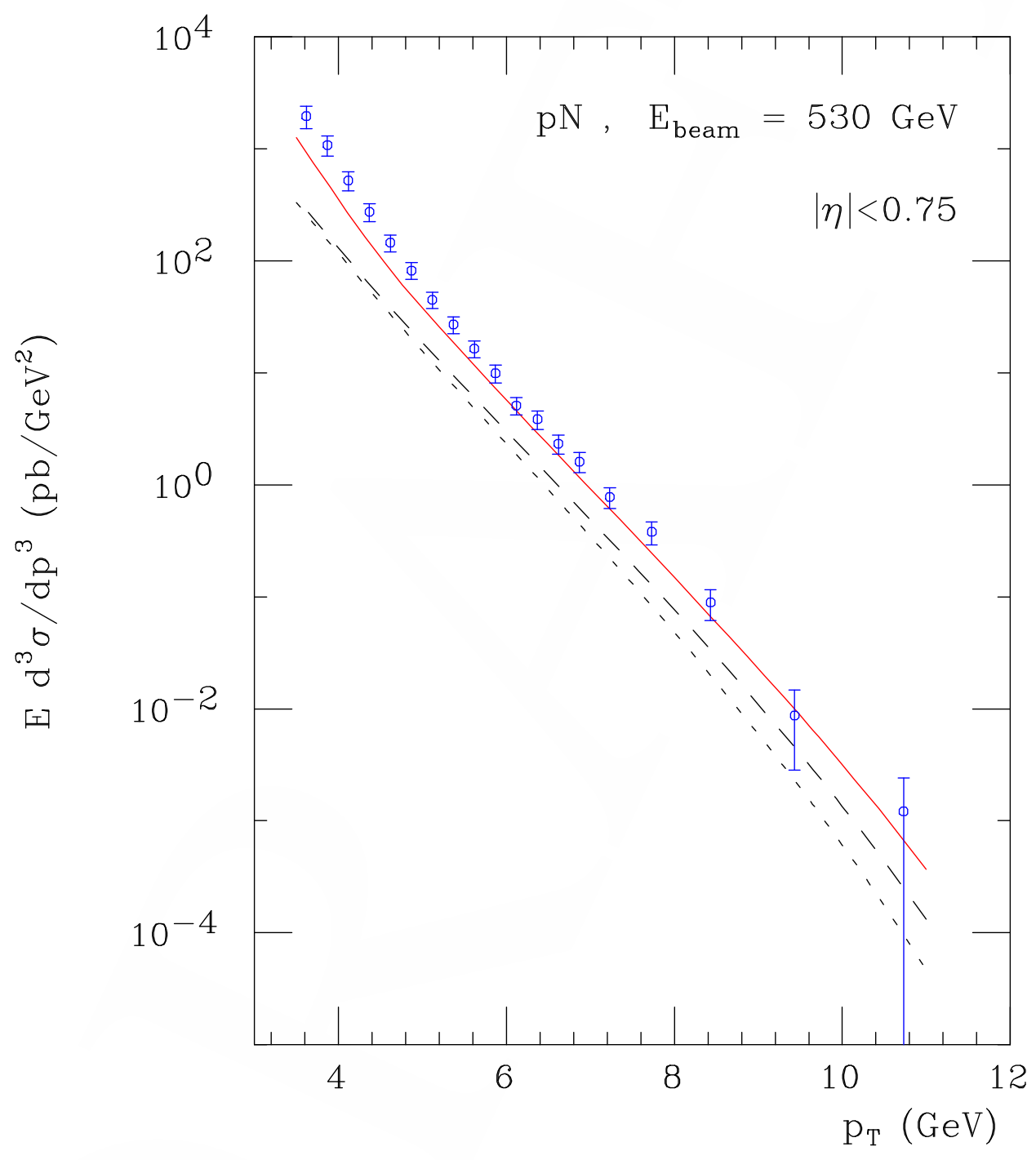

Figure 7.32 Direct photon cross section for pBe collisions at $530 \mathrm{GeV} / c$ compared to NLO (dotted), threshold resummed (dashed) and joint threshold and recoil resummed (solid) calculations. Figure from [111]. 
$k_{T}$ to the calculation run without supplemental $k_{T}$. The NLO calculation is then multiplied by the K-factor to obtain the $k_{T}$-enhanced NLO calculation. Although there may be some double-counting in this method since the NLO calculation contains diagrams that contribute to the effective $k_{T}$, the amount should be relatively small given the width of the bare NLO results illustrated in Figure 7.28. In Figure $7.33, k_{T}$-enhancement factors derived using this procedure are shown for direct photon and $\pi^{0}$ cross sections at $530 \mathrm{GeV} / c$ for several choices of $\left\langle k_{T}\right\rangle$. Note that there is significant $p_{T}$ dependence to the K-factors. This $p_{T}$ dependence is a result of the cross section changing slope over the $p_{T}$ range indicated. The K-factors are largest at the high and low ends of the $p_{T}$ spectrum, where the slope is steepest.

In Figures 7.34, 7.35 and 7.36, the direct photon and $\pi^{0}$ cross sections are compared to $k_{T}$-enhanced NLO calculations for 530 and $800 \mathrm{GeV} / c$ proton, and $515 \mathrm{GeV} / c \pi^{-}$beams, respectively. The $k_{T}$-enhanced calculations provide reasonable representations of both the normalization and shape of the data for each of the data samples. It is also notable that the $\left\langle k_{T}\right\rangle$ values obtained using the procedure described above are consistent with values obtained from the kinematic distributions of high mass $\gamma \gamma, \pi^{0} \pi^{0}$, and $\gamma \pi^{0}$ pairs described earlier.

\subsection{Comparisons Between Other Experiments and NLO Theory}

Direct comparisons between results from different experiments are frequently difficult due to the different beam energies and/or kinematic regimes explored by the experiments. However, NLO calculations can be employed as a baseline to facilitate such comparisons.

In Figure 7.37, direct photon cross section measurements for proton induced reactions from various experiments are compared to NLO predictions as a function of $x_{T}$. A similar comparison is shown for $\pi^{0}$ cross section measurements in 


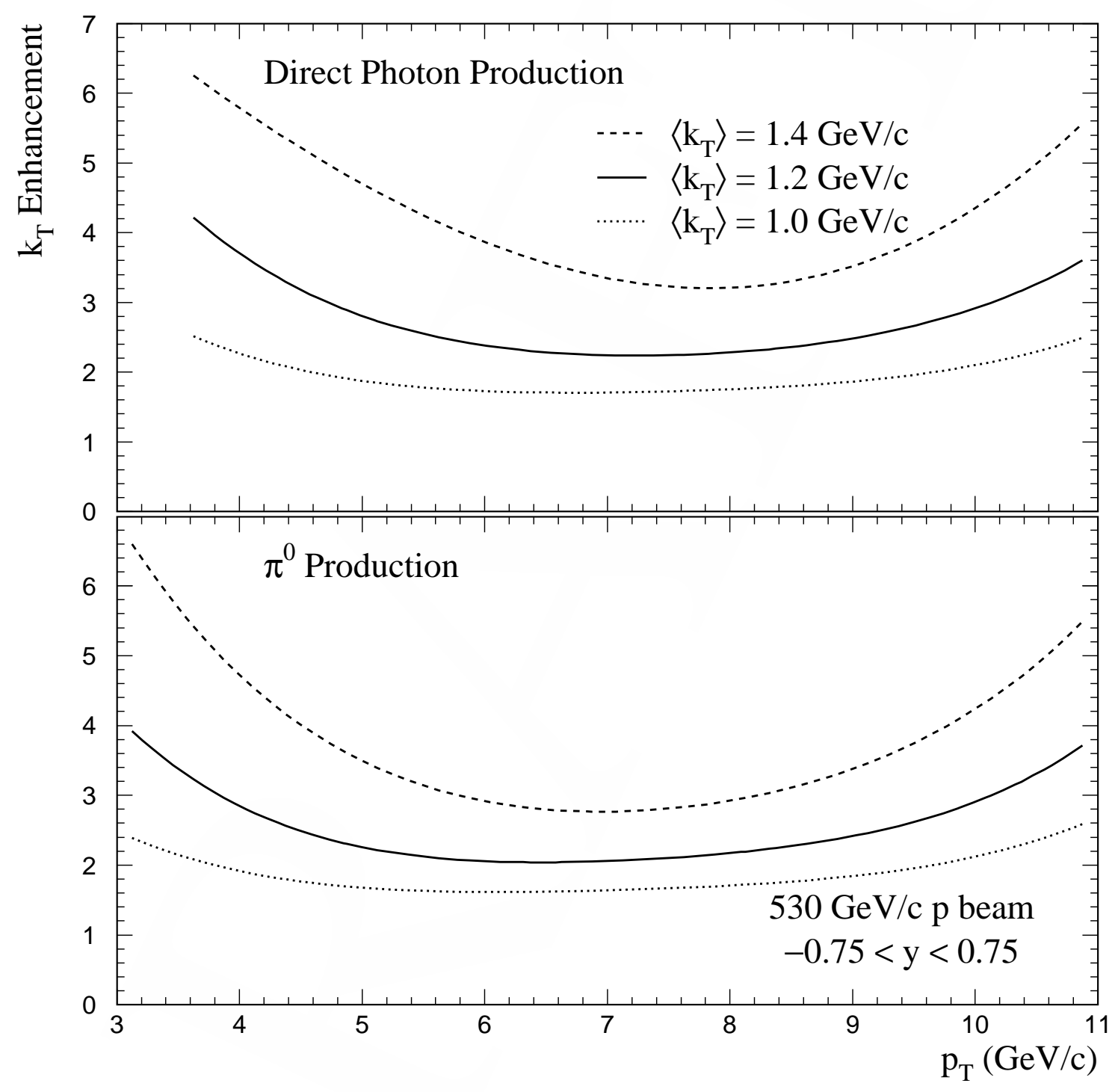

Figure 7.33 $k_{T^{-}}$enhancement factors for direct photon and $\pi^{0}$ production cross sections by $530 \mathrm{GeV} / c$ proton beam for several values of $\left\langle k_{T}\right\rangle$. 


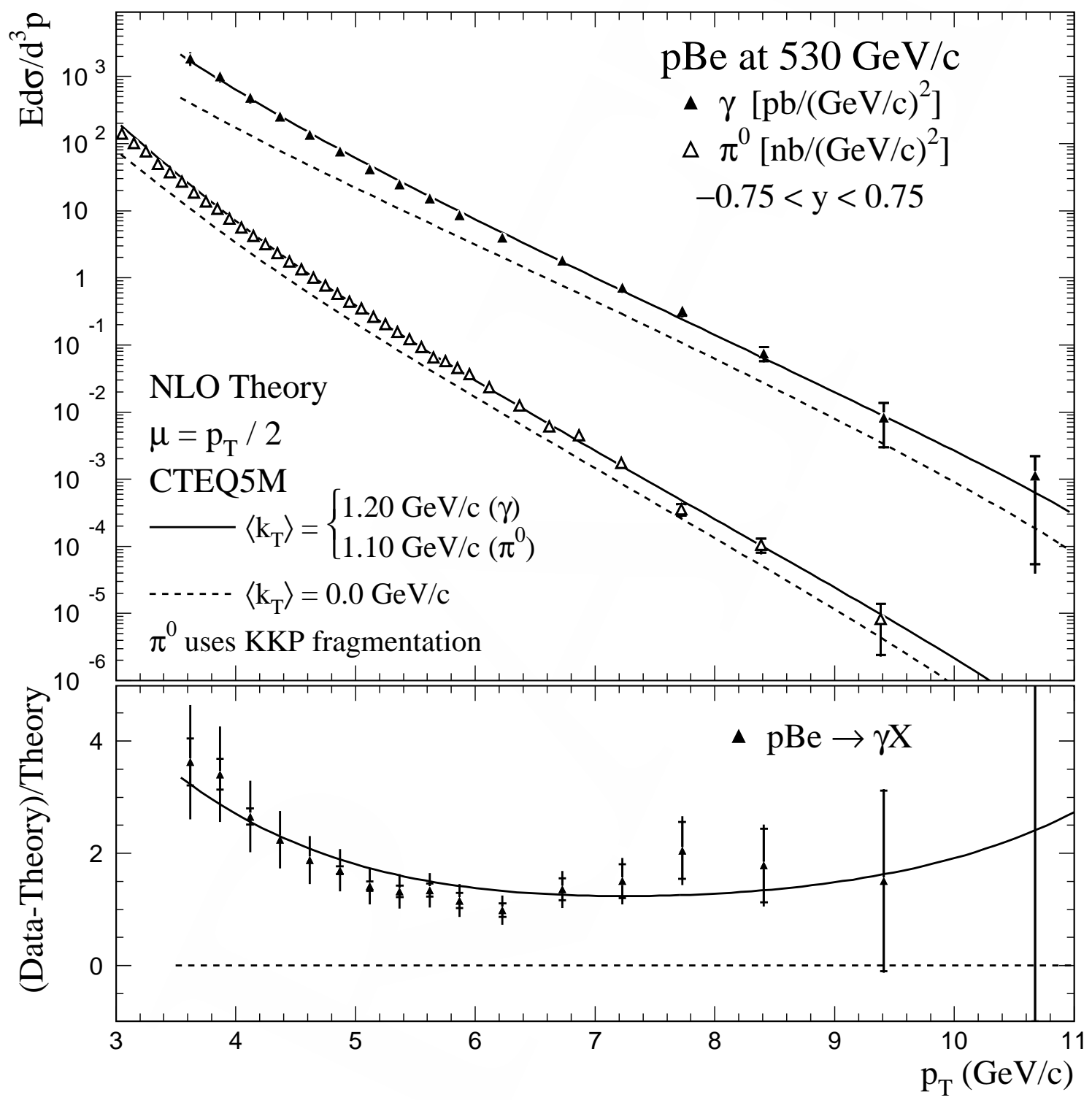

Figure 7.34 Direct photon and $\pi^{0}$ cross sections for $530 \mathrm{GeV} / c$ proton beam on beryllium compared to $k_{T}$-enhanced NLO pQCD calculations. Also shown is the quantity (Data - Theory)/Theory for the direct photon cross section. 


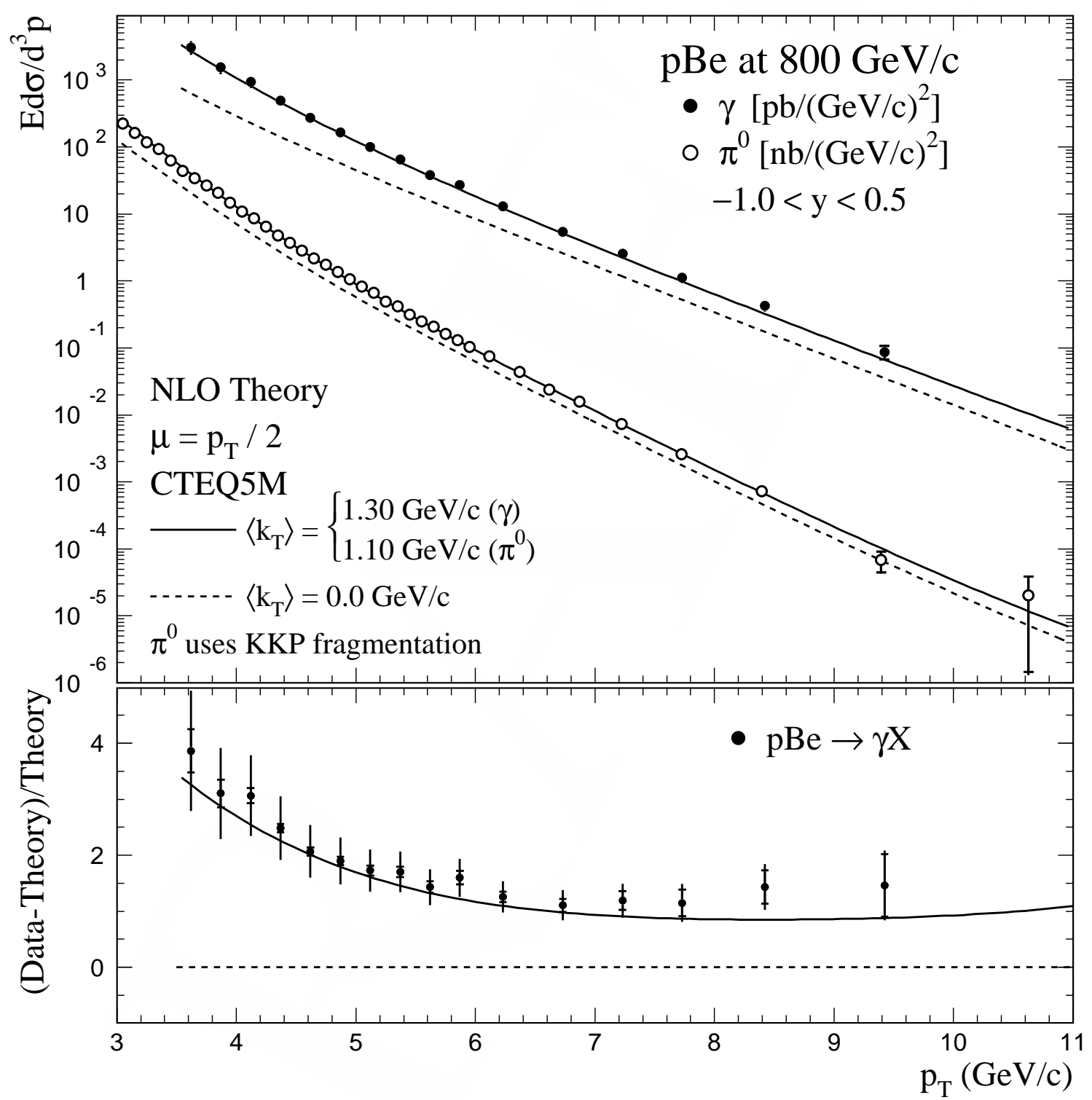

Figure 7.35 Direct photon and $\pi^{0}$ cross sections for $800 \mathrm{GeV} / c$ proton beam on beryllium compared to $k_{T}$-enhanced NLO pQCD calculations. Also shown is the quantity (Data - Theory)/Theory for the direct photon cross section. 


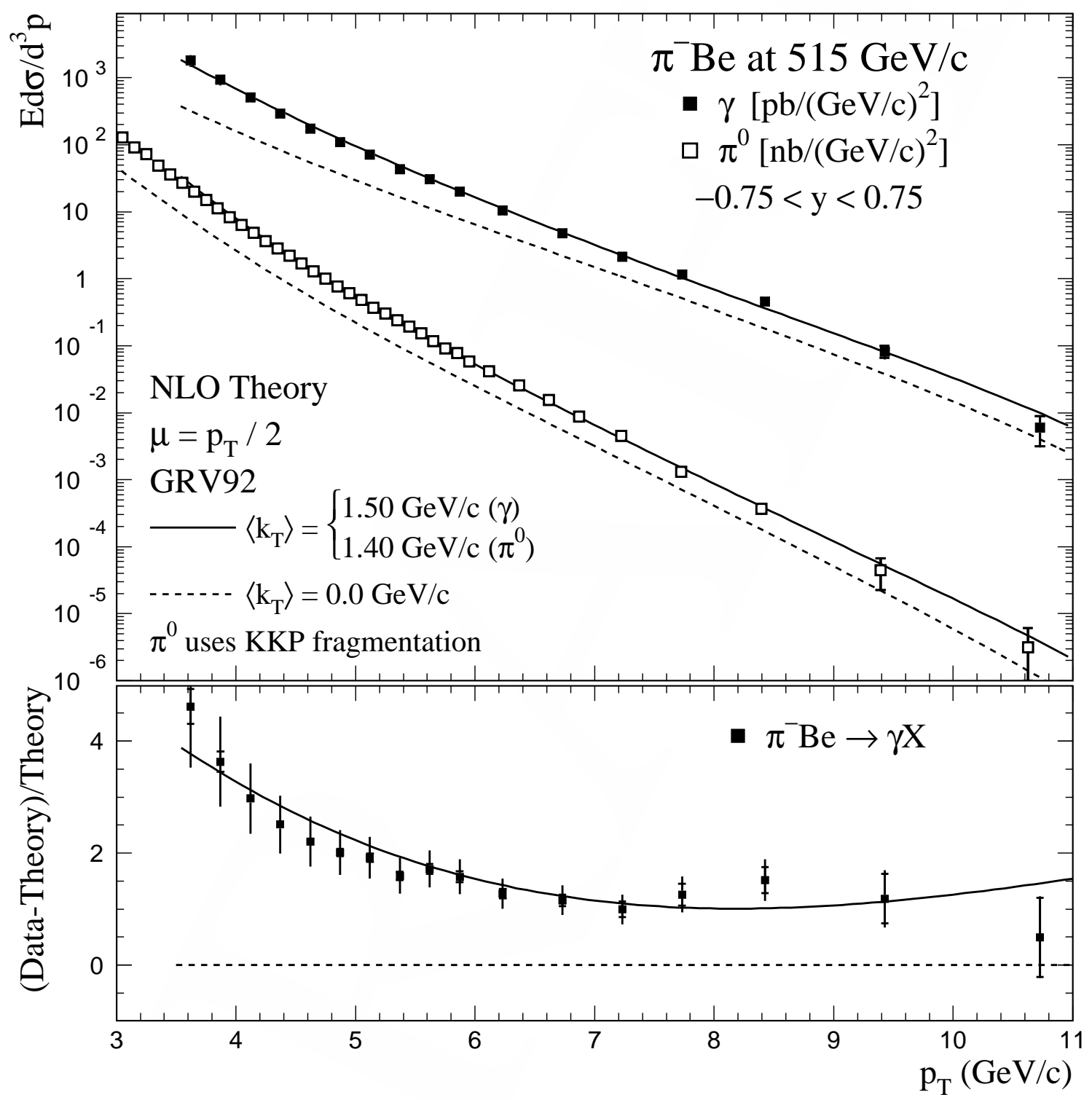

Figure 7.36 Direct photon and $\pi^{0}$ cross sections for $515 \mathrm{GeV} / c \pi^{-}$beam on beryllium compared to $k_{T}$-enhanced NLO pQCD calculations. Also shown is the quantity (Data - Theory)/Theory for the direct photon cross section. 
Figure 7.38. Although there is a large level of scatter between the various results, it is clear, particularly at large $x_{T}$, that large deviations exist between many of the data sets and the theory.

The most recent measurements for direct photon production at collision energies similar to those at E706 were made by the WA70 collaboration (using $p$ and $\pi^{-}$beams at $\left.280 \mathrm{GeV} / c\right)[58,59,93]$ and the UA6 collaboration (using $p$ and $\bar{p}$ beams at $315 \mathrm{GeV} / c$ ) [55]. In Figure 7.39, direct photon and $\pi^{0}$ cross sections from these experiments are compared to NLO expections with and without $k_{T}$ enhancements. The choice of $\left\langle k_{T}\right\rangle$ values shown in the figure is motivated from a study of kinematic distributions of direct photon pairs by WA70 [28], which measured an $\left\langle k_{T}\right\rangle$ of $0.9 \mathrm{GeV} / c$ in their $\pi^{-} p$ interactions. With the exception of the WA70 $p$ beam direct photon result, which appears to be reasonably represented by the bare NLO prediction, the measurements are better represented by $k_{T^{-}}$ enhanced calculations.

Direct photon cross sections were also measured for $p \bar{p}$ collisions at $\sqrt{s}=$ 1.8 TeV and $\sqrt{s}=0.63 \mathrm{TeV}$ at the Tevatron collider by the CDF [48] and D $\varnothing$ [49] collaborations. Comparisons of data from these experiments to NLO QCD calculations are shown in Figure 7.40. Also shown are curves representing the expected enhancement to the predictions from initial state parton- $k_{T}$ effects. The enhancement is only significant at the low end of the $p_{T}$ spectrum, where the $\left\langle k_{T}\right\rangle$ is comparable to the $p_{T}$. The result from $\mathrm{CDF}$ at $\sqrt{s}=1.8 \mathrm{TeV}$ has been scaled up by $10 \%$ to facilitate a shape comparison to the theory at low $p_{T}{ }^{7}$. The data from CDF and $\mathrm{D} \varnothing$ at both center-of-mass energies show an excess at low $p_{T}$ compared to bare NLO calculations, which is reasonably described by the $k_{T}$-enhanced calculations. The choices for the $\left\langle k_{T}\right\rangle$ are motivated by the measurements shown in Figure 1.7.

7 A shift of this magnitude is accommodated within the systematic uncertainties quoted for this measurement. 


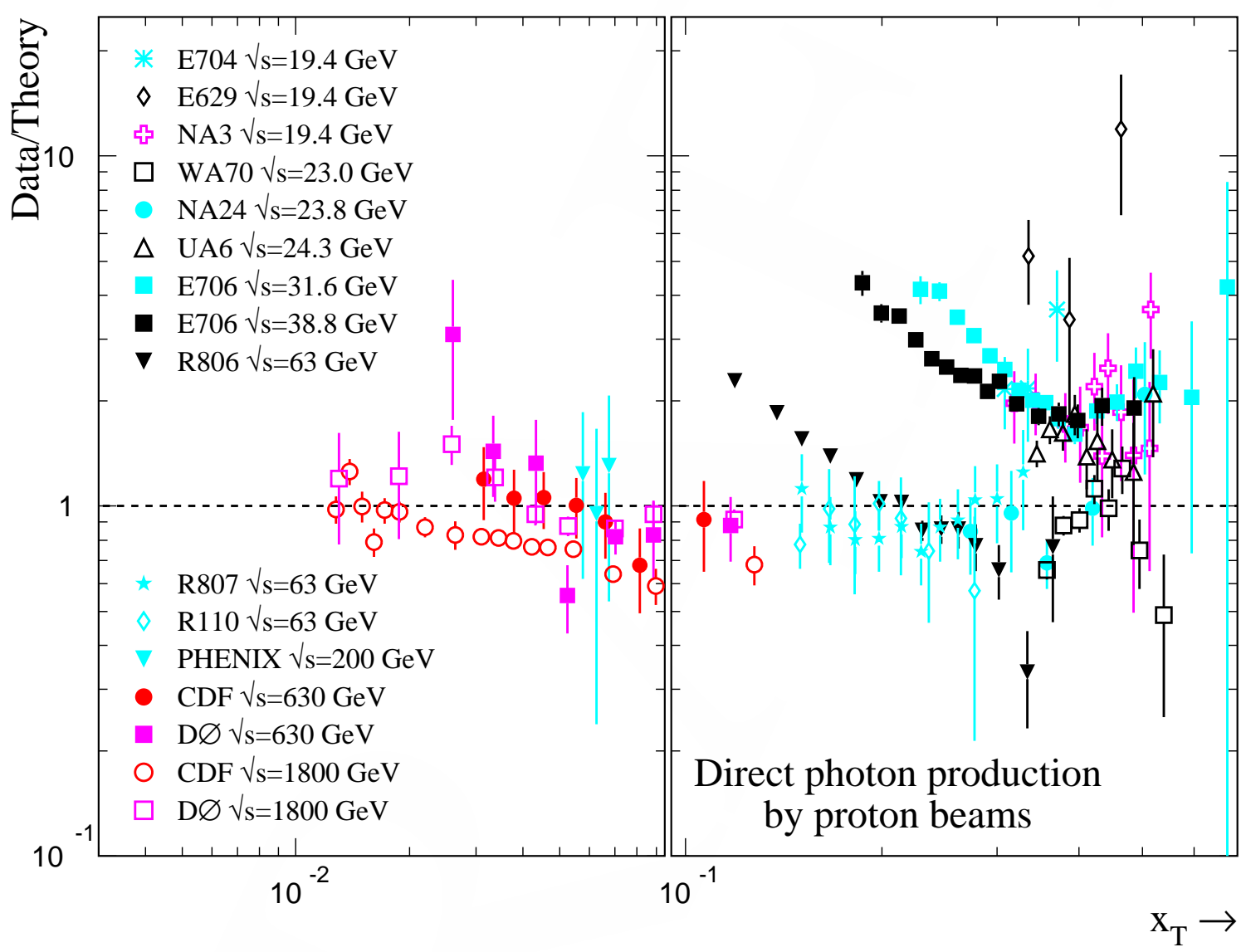

Figure 7.37 Data/Theory for proton induced direct photon data from various experiments as a function of $x_{T}$. The theory calculations use CTEQ5M PDF and scale $\mu=\frac{1}{2} p_{T}$. 


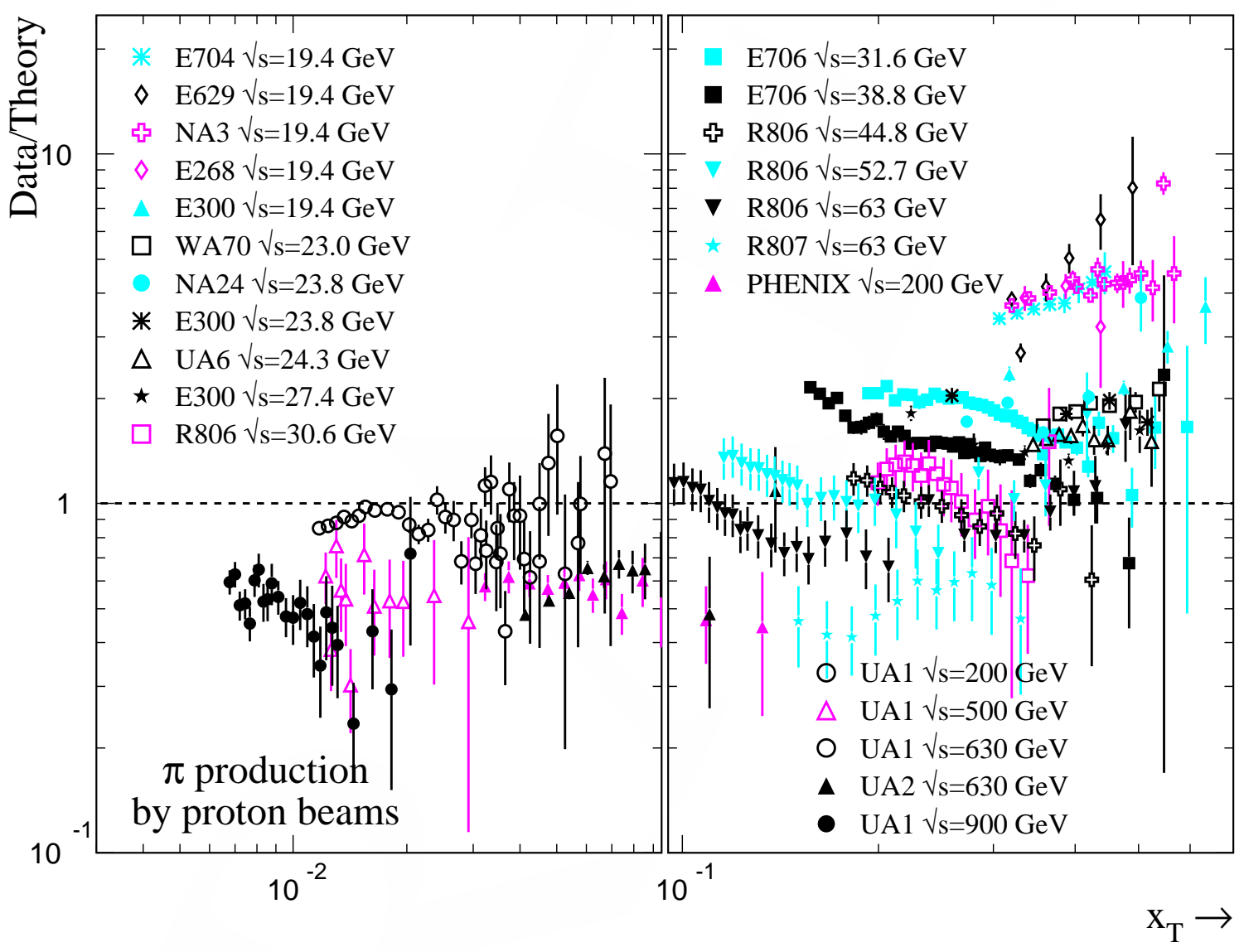

Figure 7.38 Data/Theory for proton induced $\pi^{0}$ data from various experiments as a function of $x_{T}$. The theory calculations use CTEQ5M PDF, KKP $\mathrm{FF}$, and scale $\mu=\frac{1}{2} p_{T}$. 

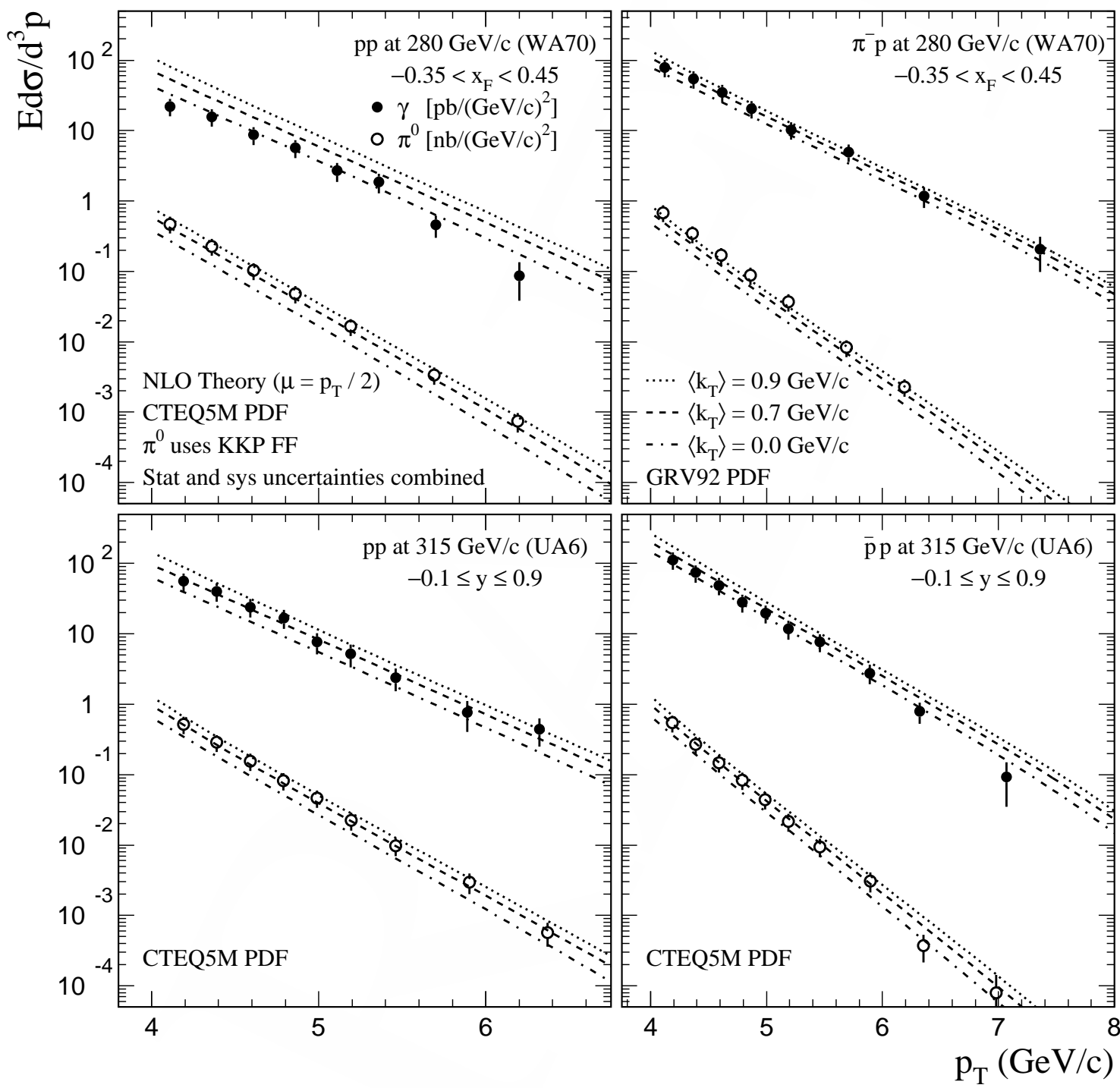

Figure 7.39 Direct-photon and $\pi^{0}$ cross sections from experiments WA70 and $\mathrm{UA6}$, compared to $k_{T}$-enhanced NLO calculations. 

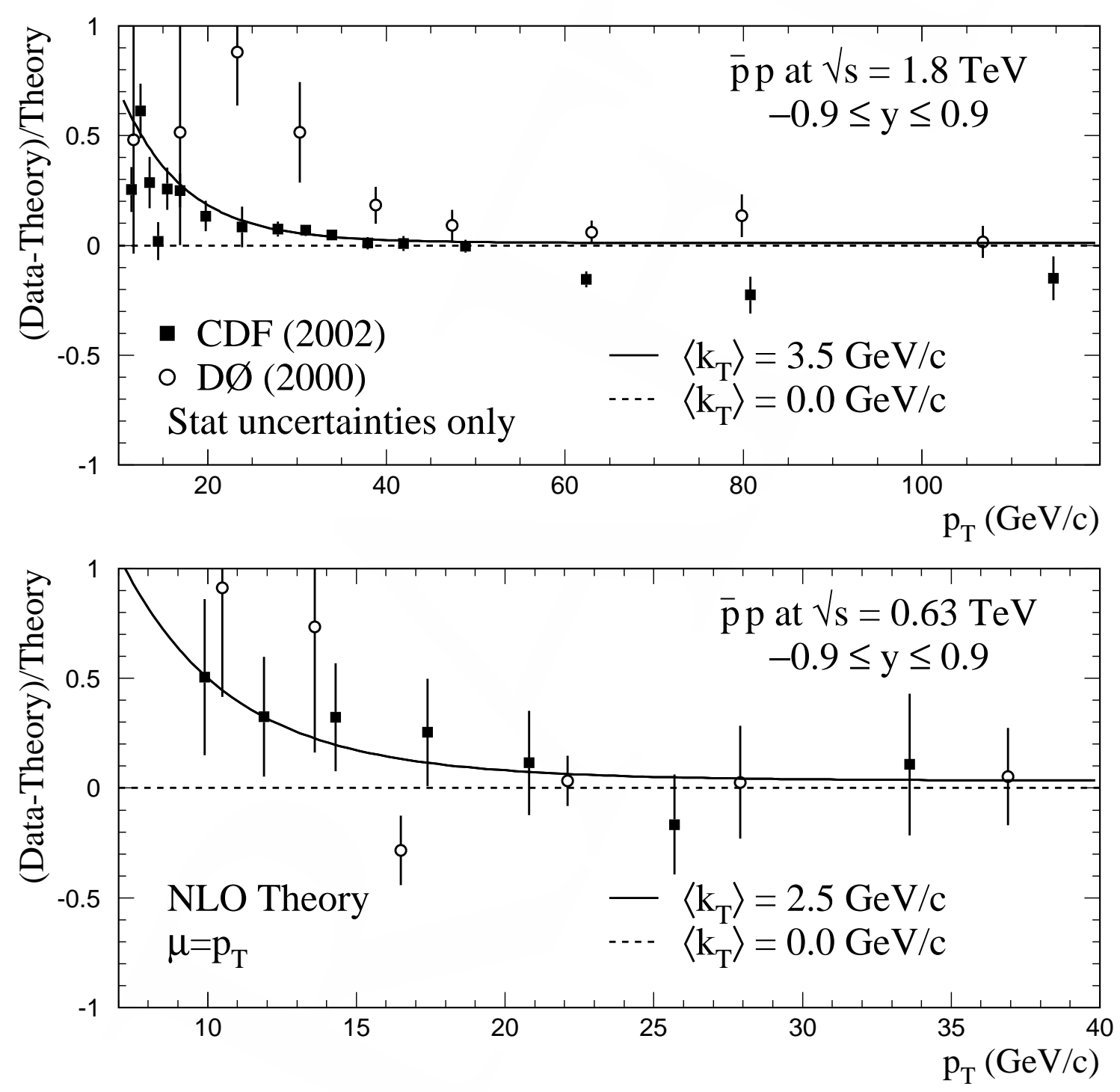

Figure 7.40 Isolated direct photon cross sections from $\mathrm{CDF}$ and $\mathrm{D} \emptyset$ at $\sqrt{s}=$ $1.8 \mathrm{TeV}$ (top) and $\sqrt{s}=0.63 \mathrm{TeV}$ (bottom) compared to NLO predictions. The predictions for CDF (DØ) use CTEQ5M (CTEQ4M) $\mathrm{PDF}$. The solid curves represent the expected enhancement to the predictions from parton- $k_{T}$. The data from CDF at $\sqrt{s}=1.8 \mathrm{TeV}$ have been scaled up by $10 \%$ to facilite a shape comparision. 
A related process to the hadroproduction of direct photons is the photoproduction of direct photons at electron-proton $(e p)$ colliders. The cross section for this process has been measured at the HERA ep collider by the ZEUS collaboration [112]. In Figure 7.41, results from this collaboration are compared to NLO predictions with and without $k_{T}$ enhancements. The choice of the $\left\langle k_{T}\right\rangle$ value is motivated by measurements of kinematic distributions made by this collaboration [113]. Note that in this reaction, the $k_{T}$-enhanced predictions are only $\approx 10 \%$ larger than the bare NLO predictions over most of the kinematic regime explored by the data. This relatively small enhancement, combined with the current level of experimental uncertainty, make it difficult to ascertain whether or not the data are better described by the $k_{T}$-enhanced predictions.

\subsection{Conclusions}

Differential cross sections for direct photon and $\pi^{0}$ production have been measured for 530 and $800 \mathrm{GeV} / c$ proton beams and $515 \mathrm{GeV} / c \pi^{-}$beams on beryllium, copper and hydrogen targets. NLO theoretical calculations for these cross sections with conventional scale choices lie significantly below the measured results. Many corresponding comparisons with other experimental results show similar disrepancies. A phenomenological $k_{T}$ model has been shown to improve these comparisons for many of the experimental results.

Many experimental and theoretical uncertainties cancel in a ratio between direct photon cross sections in proton-induced reactions at 800 and $530 \mathrm{GeV} / c$. A comparison of this ratio to $k_{T}$-enhanced predictions using MRST2003, CTEQ5M and CTEQ6.1M PDFs is shown in Figure 7.42 as a function of $p_{T}$. The CTEQ6.1M PDF has a gluon distribution that is much harder than the gluon distribution in the other sets; a result of the inclusion of inclusive jet results from Tevatron Run I in the global fit. However, the use of these data introduce the possibility 


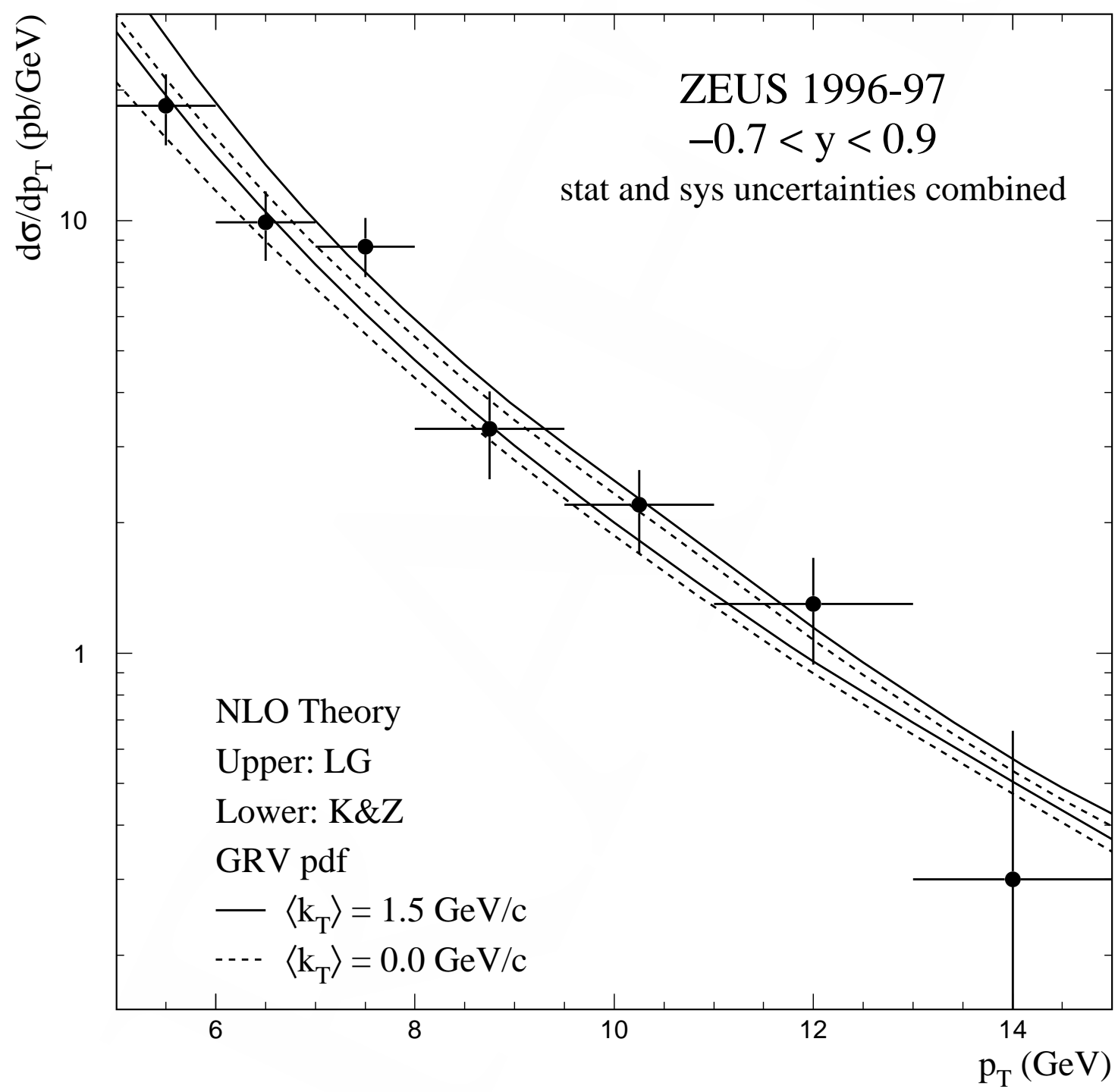

Figure 7.41 Isolated direct photon cross section from ZEUS. The NLO predictions are by Gordon (LG) and by Krawczyk and Zembruski (K\&W). 
that effects due to new physics phenomena may be contained within the current fit uncertainties and absorbed within the resultant PDFs. The E706 data, which appear to favor the calculations using the softer gluons, suggest such a possibility. 


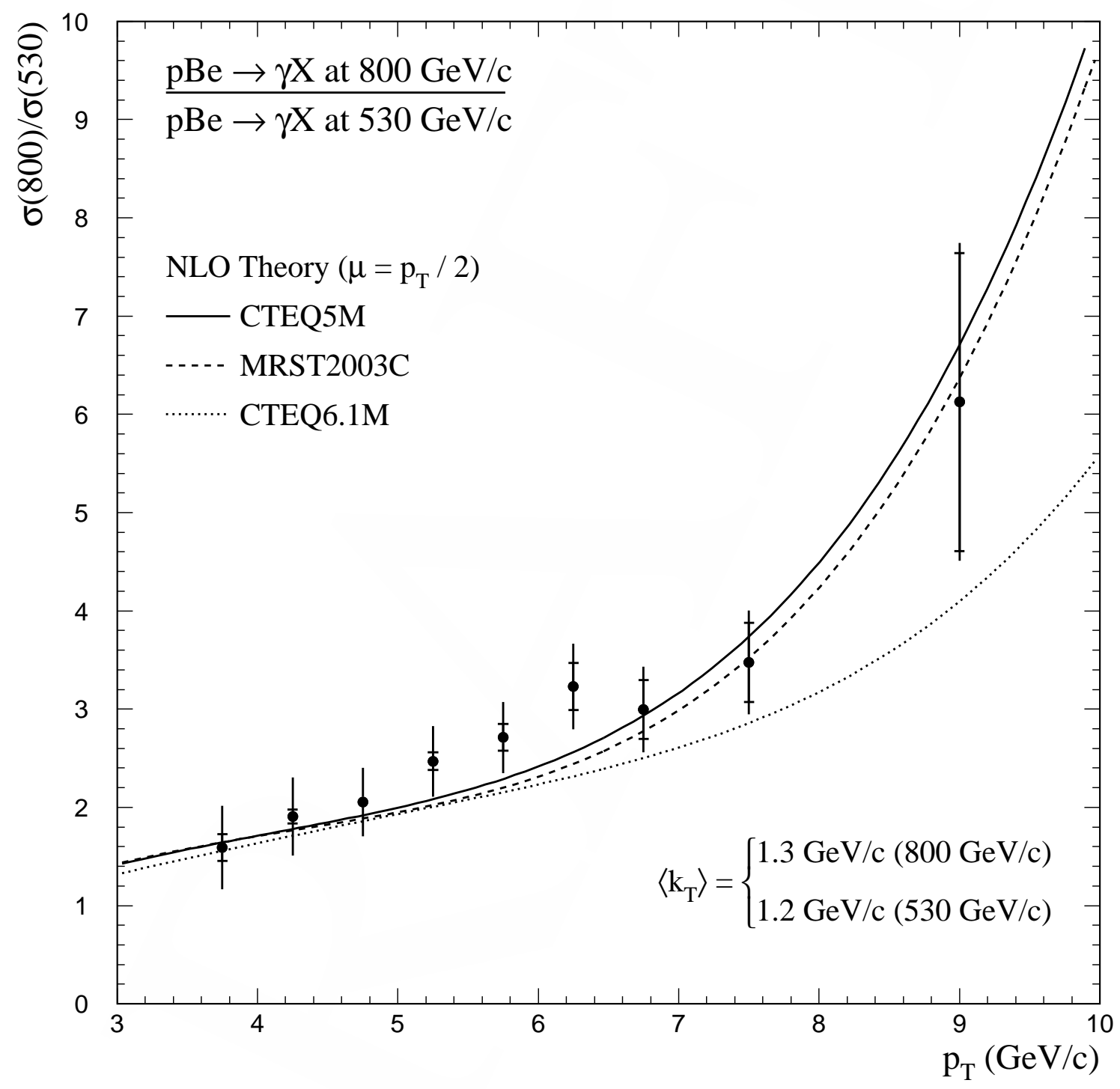

Figure 7.42 The ratio between $530 \mathrm{GeV} / \mathrm{c}$ and $800 \mathrm{GeV} / \mathrm{c}$ p beam direct photon cross sections as functions of $p_{T}$. Overlayed on the plots are NLO predictions with and without supplemental $k_{T}$. 



\section{Appendix A Tabulated $\pi^{0}$ Cross Sections}

This appendix contains the measured $\pi^{0}$ cross sections in tabular form. The results are presented in the form $A \pm B \pm C$, where $A$ is the measured value, and $B$ and $C$ represent the statistical and systematic uncertainties, respectively, on the value. For those cases where the systematic uncertainty is not given, the statistical and systematic uncertainties have been combined because of the large correlation between them (Section 5.11). 
Table A.1 Invariant differential cross sections per nucleon for $\pi^{0}$ production by 530 and $800 \mathrm{GeV} / c$ proton beams and $515 \mathrm{GeV} / c \pi^{-}$beam on Be targets, for $1.0<p_{T}<4.0 \mathrm{GeV} / c$.

\begin{tabular}{|c|c|c|c|}
\hline \multirow{2}{*}{$\begin{array}{c}p_{T} \text { Range } \\
(\mathrm{GeV} / c)\end{array}$} & $\begin{array}{c}|c| \\
\text { Be at } 530 \mathrm{GeV} / c \\
-0.75<y<0.75\end{array}$ & $\begin{array}{c}p \text { Be at } 800 \mathrm{GeV} / c \\
-1.0<y<0.5\end{array}$ & $\begin{array}{c}\pi^{-} \mathrm{Be} \text { at } 515 \mathrm{GeV} / c \\
-0.75<y<0.75\end{array}$ \\
\hline $1.00-1.20$ & $550 \pm 66$ & $706 \pm 95$ & $258 \pm 36$ \\
\hline $1.20-1.40$ & $223 \pm 28$ & $301 \pm 41$ & $98 \pm 14$ \\
\hline $1.40-1.60$ & $72 \pm 11$ & $125 \pm 18$ & $39.9 \pm 6.0$ \\
\hline $1.60-1.80$ & $32.7 \pm 5.3$ & $54.3 \pm 8.4$ & $20.0 \pm 2.6$ \\
\hline $1.80-2.00$ & $15.3 \pm 2.7$ & $23.9 \pm 4.1$ & $9.6 \pm 1.3$ \\
\hline $2.00-2.20$ & $6.9 \pm 1.3 \pm 0.7$ & $7.4 \pm 1.7 \pm 0.8$ & $3.68 \pm 0.36 \pm 0.43$ \\
\hline $2.20-2.30$ & $3.00 \pm 0.15 \pm 0.32$ & $3.95 \pm 0.24 \pm 0.44$ & $2.134 \pm 0.054 \pm 0.25$ \\
\hline $2.30-2.40$ & $2.02 \pm 0.13 \pm 0.22$ & $2.69 \pm 0.22 \pm 0.30$ & $1.475 \pm 0.038 \pm 0.17$ \\
\hline $2.40-2.50$ & $1.38 \pm 0.13 \pm 0.15$ & $1.78 \pm 0.14 \pm 0.20$ & $0.964 \pm 0.027 \pm 0.11$ \\
\hline & & $\left(\mathrm{nb} /(\mathrm{GeV} / c)^{2}\right)$ & \\
\hline $2.50-2.60$ & $982 \pm 87 \pm 100$ & $1221 \pm 96 \pm 140$ & $681 \pm 17 \pm 79$ \\
\hline $2.60-2.70$ & $614 \pm 17 \pm 65$ & $825 \pm 46 \pm 91$ & $502 \pm 14 \pm 58$ \\
\hline $2.70-2.80$ & $388 \pm 10 \pm 41$ & $653 \pm 33 \pm 72$ & $318.9 \pm 9.7 \pm 36$ \\
\hline $2.80-2.90$ & $291.1 \pm 9.2 \pm 31$ & $452 \pm 10 \pm 50$ & $246.4 \pm 7.3 \pm 28$ \\
\hline $2.90-3.00$ & $196.5 \pm 7.2 \pm 21$ & $319.8 \pm 7.3 \pm 35$ & $172.1 \pm 4.3 \pm 20$ \\
\hline $3.00-3.10$ & $141.0 \pm 3.2 \pm 15$ & $223.7 \pm 5.3 \pm 25$ & $128.8 \pm 3.3 \pm 15$ \\
\hline $3.10-3.20$ & $100.8 \pm 2.8 \pm 11$ & $163.2 \pm 4.6 \pm 18$ & $91.8 \pm 2.6 \pm 10$ \\
\hline $3.20-3.30$ & $75.7 \pm 2.4 \pm 8.1$ & $117.2 \pm 3.3 \pm 13$ & $72.9 \pm 1.9 \pm 8.2$ \\
\hline $3.30-3.40$ & $49.6 \pm 1.5 \pm 5.3$ & $92.4 \pm 3.2 \pm 10$ & $48.8 \pm 1.3 \pm 5.5$ \\
\hline $3.40-3.50$ & $37.2 \pm 1.5 \pm 4.0$ & $62.6 \pm 2.2 \pm 6.9$ & $35.7 \pm 1.0 \pm 4.0$ \\
\hline & & $\left(\mathrm{pb} /(\mathrm{GeV} / c)^{2}\right)$ & \\
\hline $3.50-3.60$ & $26800 \pm 1000 \pm 2900$ & $44200 \pm 1800 \pm 4900$ & $26810 \pm 660 \pm 3000$ \\
\hline $3.60-3.70$ & $18650 \pm 840 \pm 2000$ & $33800 \pm 1600 \pm 3700$ & $19940 \pm 500 \pm 2200$ \\
\hline $3.70-3.80$ & $13800 \pm 520 \pm 1500$ & $26600 \pm 1200 \pm 2900$ & $15030 \pm 360 \pm 1700$ \\
\hline $3.80-3.90$ & $10560 \pm 300 \pm 1100$ & $20800 \pm 1000 \pm 2300$ & $11260 \pm 300 \pm 1200$ \\
\hline $3.90-4.00$ & $7637 \pm 57 \pm 820$ & $14680 \pm 770 \pm 1600$ & $8370 \pm 110 \pm 920$ \\
\hline
\end{tabular}


Table A.2 Invariant differential cross sections per nucleon for $\pi^{0}$ production by 530 and $800 \mathrm{GeV} / c$ proton beams and $515 \mathrm{GeV} / c \pi^{-}$beam on Be targets, for $p_{T}>4.0 \mathrm{GeV} / c$.

\begin{tabular}{|c|c|c|c|}
\hline \multirow[b]{2}{*}{$\begin{array}{l}p_{T} \text { Range } \\
(\mathrm{GeV} / c)\end{array}$} & \multicolumn{3}{|c|}{$E d \sigma / d^{3} p\left(\mathrm{pb} /(\mathrm{GeV} / c)^{2}\right)$} \\
\hline & $\begin{array}{c}p \text { Be at } 530 \mathrm{GeV} / c \\
-0.75<y<0.75\end{array}$ & $\begin{array}{c}p \text { Be at } 800 \mathrm{GeV} / c \\
-1.0<y<0.5\end{array}$ & $\begin{array}{c}\pi^{-} \text {Be at } 515 \mathrm{GeV} / c \\
-0.75<y<0.75\end{array}$ \\
\hline $4.00-4.10$ & $5613 \pm 42 \pm 600$ & $10940 \pm 450 \pm 1200$ & $6286 \pm 40 \pm 690$ \\
\hline $4.10-4.20$ & $4203 \pm 33 \pm 450$ & $8660 \pm 120 \pm 960$ & $4820 \pm 34 \pm 530$ \\
\hline $4.20-4.30$ & $3177 \pm 27 \pm 340$ & $6500 \pm 97 \pm 720$ & $3642 \pm 27 \pm 400$ \\
\hline $4.30-4.40$ & $2318 \pm 22 \pm 250$ & $4746 \pm 73 \pm 530$ & $2855 \pm 23 \pm 310$ \\
\hline $4.40-4.50$ & $1748 \pm 18 \pm 190$ & $3687 \pm 68 \pm 410$ & $2189 \pm 19 \pm 240$ \\
\hline $4.50-4.60$ & $1327 \pm 15 \pm 140$ & $2856 \pm 54 \pm 320$ & $1672 \pm 15 \pm 180$ \\
\hline $4.60-4.70$ & $1005 \pm 12 \pm 110$ & $2178 \pm 46 \pm 240$ & $1290 \pm 13 \pm 140$ \\
\hline $4.70-4.80$ & $762 \pm 10 \pm 83$ & $1741 \pm 38 \pm 190$ & $1000 \pm 11 \pm 110$ \\
\hline $4.80-4.90$ & $577.2 \pm 8.6 \pm 63$ & $1353 \pm 25 \pm 150$ & $764.5 \pm 9.6 \pm 84$ \\
\hline $4.90-5.00$ & $443.2 \pm 7.4 \pm 49$ & $1057 \pm 22 \pm 120$ & $612.2 \pm 8.6 \pm 67$ \\
\hline $5.00-5.10$ & $348.0 \pm 6.5 \pm 38$ & $817 \pm 18 \pm 92$ & $480.9 \pm 7.3 \pm 53$ \\
\hline $5.10-5.20$ & $263.1 \pm 5.4 \pm 29$ & $664 \pm 17 \pm 75$ & $370.0 \pm 6.1 \pm 41$ \\
\hline $5.20-5.30$ & $205.1 \pm 4.9 \pm 23$ & $490 \pm 16 \pm 55$ & $301.3 \pm 5.5 \pm 33$ \\
\hline $5.30-5.40$ & $156.0 \pm 4.0 \pm 17$ & $415 \pm 13 \pm 47$ & $240.2 \pm 4.9 \pm 27$ \\
\hline $5.40-5.50$ & $121.2 \pm 3.5 \pm 14$ & $315 \pm 10 \pm 36$ & $193.4 \pm 4.3 \pm 21$ \\
\hline $5.50-5.60$ & $92.2 \pm 3.0 \pm 10$ & $246.4 \pm 7.9 \pm 28$ & $152.9 \pm 3.7 \pm 17$ \\
\hline $5.60-5.70$ & $64.7 \pm 2.6 \pm 7.3$ & $209.1 \pm 6.9 \pm 24$ & $117.7 \pm 3.2 \pm 13$ \\
\hline $5.70-5.80$ & $57.7 \pm 2.4 \pm 6.5$ & $160.8 \pm 6.2 \pm 18$ & $92.0 \pm 2.8 \pm 10$ \\
\hline $5.80-5.90$ & $45.1 \pm 2.1 \pm 5.1$ & $130.0 \pm 5.7 \pm 15$ & $78.0 \pm 2.6 \pm 8.7$ \\
\hline $5.90-6.00$ & $36.4 \pm 1.8 \pm 4.1$ & $104.2 \pm 5.4 \pm 12$ & $57.9 \pm 2.2 \pm 6.5$ \\
\hline $6.00-6.25$ & $23.28 \pm 0.89 \pm 2.7$ & $74.9 \pm 2.1 \pm 8.6$ & $41.7 \pm 1.2 \pm 4.7$ \\
\hline $6.25-6.50$ & $12.53 \pm 0.63 \pm 1.4$ & $44.2 \pm 1.6 \pm 5.1$ & $25.57 \pm 0.91 \pm 2.9$ \\
\hline $6.50-6.75$ & $6.11 \pm 0.43 \pm 0.71$ & $23.8 \pm 1.1 \pm 2.8$ & $15.43 \pm 0.68 \pm 1.8$ \\
\hline $6.75-7.00$ & $4.49 \pm 0.36 \pm 0.53$ & $15.68 \pm 0.84 \pm 1.8$ & $8.80 \pm 0.50 \pm 1.0$ \\
\hline $7.00-7.50$ & $1.74 \pm 0.16 \pm 0.21$ & $7.37 \pm 0.40 \pm 0.88$ & $4.53 \pm 0.25 \pm 0.53$ \\
\hline $7.50-8.00$ & $0.353 \pm 0.069 \pm 0.043$ & $2.58 \pm 0.25 \pm 0.31$ & $1.32 \pm 0.13 \pm 0.16$ \\
\hline $8.00-9.00$ & $0.105 \pm 0.025 \pm 0.013$ & $0.73 \pm 0.11 \pm 0.09$ & $0.370 \pm 0.050 \pm 0.046$ \\
\hline $9.00-10.00$ & $0.0082 \pm 0.0058 \pm 0.0011$ & $0.068 \pm 0.024 \pm 0.009$ & $0.045 \pm 0.022 \pm 0.006$ \\
\hline $10.00-12.00$ & - & $0.020 \pm 0.019 \pm 0.003$ & $0.0031 \pm 0.0031 \pm 0.0005$ \\
\hline
\end{tabular}


Table A.3 Invariant differential cross sections per nucleon for $\pi^{0}$ production by 530 and $800 \mathrm{GeV} / c$ proton beams and $515 \mathrm{GeV} / c \pi^{-}$beam on $\mathrm{Cu}$ targets.

\begin{tabular}{|c|c|c|c|}
\hline \multirow[b]{2}{*}{$\begin{array}{l}p_{T} \text { Range } \\
(\mathrm{GeV} / c)\end{array}$} & \multicolumn{3}{|c|}{$E d \sigma / d^{3} p\left(\mu \mathrm{b} /(\mathrm{GeV} / c)^{2}\right)$} \\
\hline & $\begin{array}{c}p \mathrm{Cu} \text { at } 530 \mathrm{GeV} / c \\
-0.75<y<0.75\end{array}$ & $\begin{array}{c}p \mathrm{Cu} \text { at } 800 \mathrm{GeV} / c \\
-1.0<y<0.5\end{array}$ & $\begin{array}{c}\pi^{-} \mathrm{Cu} \text { at } 515 \mathrm{GeV} / c \\
-0.75<y<0.75\end{array}$ \\
\hline $1.00-1.50$ & $252 \pm 40$ & $246 \pm 55$ & $135 \pm 26$ \\
\hline $1.50-2.00$ & $29.9 \pm 6.2$ & $51.9 \pm 10.0$ & $25.0 \pm 4.2$ \\
\hline \multirow[t]{2}{*}{$2.00-2.50$} & $4.1 \pm 1.2 \pm 0.5$ & $3.2 \pm 1.7 \pm 0.4$ & $2.409 \pm 0.067 \pm 0.29$ \\
\hline & \multicolumn{3}{|c|}{$\left(\mathrm{nb} /(\mathrm{GeV} / c)^{2}\right)$} \\
\hline $2.50-2.75$ & $920 \pm 57 \pm 99$ & $1260 \pm 110 \pm 140$ & $673 \pm 29 \pm 79$ \\
\hline $2.75-3.00$ & $319 \pm 13 \pm 35$ & $516 \pm 15 \pm 59$ & $268 \pm 12 \pm 31$ \\
\hline $3.00-3.25$ & $135.7 \pm 4.2 \pm 15$ & $212.2 \pm 6.9 \pm 24$ & $128.3 \pm 5.1 \pm 15$ \\
\hline \multirow[t]{2}{*}{$3.25-3.50$} & $62.7 \pm 2.4 \pm 6.8$ & $109.4 \pm 4.2 \pm 12$ & $58.8 \pm 2.3 \pm 6.7$ \\
\hline & \multicolumn{3}{|c|}{$\left(\mathrm{pb} /(\mathrm{GeV} / c)^{2}\right)$} \\
\hline $3.50-3.75$ & $26500 \pm 1300 \pm 2900$ & $50200 \pm 2600 \pm 5700$ & $26400 \pm 1000 \pm 3000$ \\
\hline $3.75-4.00$ & $12240 \pm 400 \pm 1300$ & $24700 \pm 1500 \pm 2800$ & $13410 \pm 410 \pm 1500$ \\
\hline $4.00-4.25$ & $6028 \pm 57 \pm 660$ & $11930 \pm 550 \pm 1400$ & $6555 \pm 66 \pm 730$ \\
\hline $4.25-4.50$ & $2881 \pm 33 \pm 320$ & $5700 \pm 120 \pm 660$ & $3310 \pm 40 \pm 370$ \\
\hline $4.50-4.75$ & $1424 \pm 21 \pm 160$ & $2976 \pm 83 \pm 340$ & $1742 \pm 25 \pm 190$ \\
\hline $4.75-5.00$ & $690 \pm 13 \pm 77$ & $1594 \pm 40 \pm 180$ & $946 \pm 17 \pm 110$ \\
\hline $5.00-5.25$ & $360.4 \pm 9.1 \pm 40$ & $887 \pm 27 \pm 100$ & $488 \pm 12 \pm 55$ \\
\hline $5.25-5.50$ & $187.1 \pm 6.3 \pm 21$ & $485 \pm 19 \pm 57$ & $282.5 \pm 8.6 \pm 32$ \\
\hline $5.50-5.75$ & $92.0 \pm 4.3 \pm 10$ & $305 \pm 13 \pm 36$ & $158.6 \pm 6.6 \pm 18$ \\
\hline $5.75-6.00$ & $47.1 \pm 3.0 \pm 5.4$ & $154.4 \pm 9.2 \pm 18$ & $83.6 \pm 4.3 \pm 9.5$ \\
\hline $6.00-6.50$ & $20.9 \pm 1.3 \pm 2.4$ & $71.3 \pm 3.2 \pm 8.5$ & $39.6 \pm 2.0 \pm 4.5$ \\
\hline $6.50-7.00$ & $4.99 \pm 0.63 \pm 0.59$ & $26.4 \pm 1.8 \pm 3.2$ & $12.4 \pm 1.1 \pm 1.4$ \\
\hline $7.00-8.00$ & $1.25 \pm 0.21 \pm 0.15$ & $5.78 \pm 0.56 \pm 0.71$ & $3.08 \pm 0.39 \pm 0.37$ \\
\hline $8.00-10.00$ & $0.074 \pm 0.030 \pm 0.010$ & $0.49 \pm 0.15 \pm 0.06$ & $0.244 \pm 0.088 \pm 0.031$ \\
\hline
\end{tabular}


Table A.4 Invariant differential cross sections for $\pi^{0}$ production by 530 and 800 $\mathrm{GeV} / c$ proton beams and $515 \mathrm{GeV} / c \pi^{-}$beam on proton targets.

\begin{tabular}{|c|c|c|c|}
\hline \multirow[b]{2}{*}{$\begin{array}{l}p_{T} \text { Range } \\
(\mathrm{GeV} / c)\end{array}$} & \multicolumn{3}{|c|}{$E d \sigma / d^{3} p\left(\mu \mathrm{b} /(\mathrm{GeV} / c)^{2}\right)$} \\
\hline & $\begin{array}{l}p p \text { at } 530 \mathrm{GeV} / c \\
-0.75<y<0.75\end{array}$ & $\begin{array}{c}p p \text { at } 800 \mathrm{GeV} / c \\
-1.0<y<0.5\end{array}$ & $\begin{array}{c}\pi^{-} p \text { at } 515 \mathrm{GeV} / c \\
-0.75<y<0.75\end{array}$ \\
\hline $1.00-1.40$ & $355 \pm 63$ & $630 \pm 110$ & $164 \pm 70$ \\
\hline $1.40-1.80$ & $68 \pm 14$ & $124 \pm 23$ & $33 \pm 16$ \\
\hline $1.80-2.20$ & $17.5 \pm 3.7 \pm 2.0$ & $27.6 \pm 5.5 \pm 3.3$ & $4.4 \pm 3.2 \pm 0.6$ \\
\hline \multirow[t]{2}{*}{$2.20-2.40$} & $3.54 \pm 0.84 \pm 0.40$ & $3.44 \pm 0.45 \pm 0.40$ & - \\
\hline & \multicolumn{3}{|c|}{$\left(\mathrm{nb} /(\mathrm{GeV} / c)^{2}\right)$} \\
\hline $2.40-2.60$ & $1220 \pm 150 \pm 140$ & $1410 \pm 230 \pm 170$ & $290 \pm 32 \pm 35$ \\
\hline $2.60-2.80$ & $479 \pm 23 \pm 54$ & $732 \pm 60 \pm 86$ & $351 \pm 31 \pm 43$ \\
\hline $2.80-3.00$ & $219 \pm 16 \pm 25$ & $334 \pm 14 \pm 39$ & $153 \pm 14 \pm 18$ \\
\hline $3.00-3.20$ & $104.6 \pm 4.8 \pm 12$ & $181.6 \pm 8.2 \pm 21$ & $103.0 \pm 9.7 \pm 12$ \\
\hline \multirow[t]{2}{*}{$3.20-3.40$} & $56.0 \pm 3.5 \pm 6.4$ & $97.1 \pm 4.9 \pm 11$ & $48.6 \pm 5.7 \pm 5.8$ \\
\hline & \multicolumn{3}{|c|}{$\left(\mathrm{pb} /(\mathrm{GeV} / c)^{2}\right)$} \\
\hline $3.40-3.60$ & $27300 \pm 1900 \pm 3100$ & $50200 \pm 3700 \pm 5900$ & $24500 \pm 3600 \pm 2900$ \\
\hline $3.60-3.80$ & $13400 \pm 1000 \pm 1500$ & $27300 \pm 2500 \pm 3200$ & $18300 \pm 2500 \pm 2200$ \\
\hline $3.80-4.00$ & $7660 \pm 250 \pm 880$ & $15500 \pm 1400 \pm 1800$ & $7600 \pm 1300 \pm 890$ \\
\hline $4.00-4.20$ & $4427 \pm 63 \pm 510$ & $9710 \pm 690 \pm 1100$ & $5040 \pm 150 \pm 590$ \\
\hline $4.20-4.40$ & $2398 \pm 39 \pm 280$ & $5320 \pm 160 \pm 630$ & $3170 \pm 100 \pm 370$ \\
\hline $4.40-4.60$ & $1357 \pm 29 \pm 160$ & $3080 \pm 100 \pm 360$ & $1786 \pm 72 \pm 210$ \\
\hline $4.60-4.80$ & $803 \pm 19 \pm 93$ & $1785 \pm 68 \pm 210$ & $1134 \pm 48 \pm 130$ \\
\hline $4.80-5.00$ & $477 \pm 14 \pm 56$ & $1118 \pm 38 \pm 130$ & $637 \pm 35 \pm 74$ \\
\hline $5.00-5.20$ & $297 \pm 10 \pm 35$ & $635 \pm 29 \pm 76$ & $417 \pm 28 \pm 49$ \\
\hline $5.20-5.40$ & $173.5 \pm 8.0 \pm 20$ & $423 \pm 22 \pm 51$ & $251 \pm 20 \pm 29$ \\
\hline $5.40-5.60$ & $107.4 \pm 5.7 \pm 13$ & $271 \pm 18 \pm 33$ & $177 \pm 16 \pm 21$ \\
\hline $5.60-5.80$ & $63.2 \pm 4.2 \pm 7.5$ & $172 \pm 11 \pm 21$ & $112 \pm 13 \pm 13$ \\
\hline $5.80-6.00$ & $41.2 \pm 3.5 \pm 4.9$ & $104.3 \pm 8.4 \pm 13$ & $66.4 \pm 9.9 \pm 7.9$ \\
\hline $6.00-6.25$ & $23.8 \pm 2.2 \pm 2.9$ & $80.2 \pm 5.6 \pm 9.8$ & $34.8 \pm 6.0 \pm 4.2$ \\
\hline $6.25-6.50$ & $12.0 \pm 1.6 \pm 1.5$ & $36.1 \pm 3.2 \pm 4.4$ & $29.9 \pm 5.8 \pm 3.6$ \\
\hline $6.50-6.75$ & $5.87 \pm 1.00 \pm 0.72$ & $29.3 \pm 2.7 \pm 3.6$ & $17.4 \pm 4.0 \pm 2.1$ \\
\hline $6.75-7.00$ & $4.39 \pm 0.84 \pm 0.55$ & $13.0 \pm 2.0 \pm 1.6$ & $5.2 \pm 2.1 \pm 0.6$ \\
\hline $7.00-7.50$ & $1.43 \pm 0.33 \pm 0.18$ & $8.7 \pm 1.2 \pm 1.1$ & $4.6 \pm 1.4 \pm 0.6$ \\
\hline $7.50-8.00$ & $0.75 \pm 0.25 \pm 0.10$ & $3.55 \pm 0.70 \pm 0.45$ & $2.9 \pm 1.0 \pm 0.4$ \\
\hline $8.00-9.00$ & $0.199 \pm 0.100 \pm 0.027$ & $0.71 \pm 0.20 \pm 0.09$ & - \\
\hline $9.00-10.00$ & - & $0.09 \pm 0.10 \pm 0.01$ & - \\
\hline $10.00-12.00$ & - & $0.017 \pm 0.017 \pm 0.003$ & - \\
\hline
\end{tabular}


Table A.5 Invariant differential cross section per nucleon for $\pi^{0}$ production by $530 \mathrm{GeV} / c$ proton beam on a Be target as a function of $p_{T}$ and rapidity.

\begin{tabular}{|c|c|c|c|c|}
\hline \multirow[b]{2}{*}{ Rapidity } & \multicolumn{4}{|c|}{$p_{T}(\mathrm{GeV} / c)$} \\
\hline & $\begin{array}{c}1.00-1.50 \\
\mu \mathrm{b} /(\mathrm{GeV} / c)^{2}\end{array}$ & $\begin{array}{c}1.50-2.00 \\
\mu \mathrm{b} /(\mathrm{GeV} / c)^{2}\end{array}$ & $\begin{array}{c}2.00-2.50 \\
\mu \mathrm{b} /(\mathrm{GeV} / c)^{2}\end{array}$ & $\begin{array}{c}2.50-3.00 \\
\mathrm{nb} /(\mathrm{GeV} / \mathrm{c})^{2}\end{array}$ \\
\hline $\begin{array}{l}-0.750--0.625 \\
-0.625--0.500\end{array}$ & $350 \pm 57$ & $27.9 \pm 8.7$ & $6.7 \pm 1.7 \pm 0.7$ & $\begin{array}{c}600 \pm 190 \pm 60 \\
425 \pm 89 \pm 45\end{array}$ \\
\hline $\begin{array}{l}-0.500--0.375 \\
-0.375--0.250 \\
\end{array}$ & $303 \pm 52$ & $26.4 \pm 6.8$ & $2.9 \pm 1.2 \pm 0.3$ & $\begin{array}{l}514 \pm 24 \pm 55 \\
512 \pm 15 \pm 54 \\
\end{array}$ \\
\hline $\begin{array}{c}-0.250--0.125 \\
-0.125-0.000\end{array}$ & $364 \pm 51$ & $31.2 \pm 7.2$ & $4.3 \pm 1.2 \pm 0.5$ & $\begin{array}{l}532 \pm 12 \pm 57 \\
609 \pm 13 \pm 65\end{array}$ \\
\hline $\begin{array}{l}0.000-0.125 \\
0.125-0.250\end{array}$ & $289 \pm 43$ & $34.1 \pm 6.9$ & $5.3 \pm 1.3 \pm 0.6$ & $\begin{array}{c}547 \pm 10 \pm 58 \\
520.4 \pm 8.5 \pm 55\end{array}$ \\
\hline $\begin{array}{l}0.250-0.375 \\
0.375-0.500\end{array}$ & $308 \pm 44$ & $32.6 \pm 6.1$ & $2.8 \pm 1.4 \pm 0.3$ & $\begin{array}{l}471.4 \pm 7.9 \pm 50 \\
443.2 \pm 7.7 \pm 47\end{array}$ \\
\hline $\begin{array}{l}0.500-0.625 \\
0.625-0.750\end{array}$ & $359 \pm 48$ & $17.3 \pm 5.5$ & $2.4 \pm 1.0 \pm 0.3$ & $\begin{array}{l}401.2 \pm 8.3 \pm 43 \\
353.8 \pm 8.0 \pm 38\end{array}$ \\
\hline & $\begin{array}{c}3.00-3.50 \\
\mathrm{nb} /(\mathrm{GeV} / \mathrm{c})^{2}\end{array}$ & $\begin{array}{c}3.50-4.00 \\
\mathrm{nb} /(\mathrm{GeV} / \mathrm{c})^{2}\end{array}$ & $\begin{array}{c}4.00-4.50 \\
\mathrm{nb} /(\mathrm{GeV} / \mathrm{c})^{2}\end{array}$ & $\begin{array}{c}4.50-5.00 \\
\mathrm{pb} /(\mathrm{GeV} / \mathrm{c})^{2}\end{array}$ \\
\hline $\begin{array}{l}-0.750--0.625 \\
-0.625--0.500 \\
\end{array}$ & $\begin{array}{l}71.8 \pm 4.8 \pm 7.6 \\
88.6 \pm 5.7 \pm 9.4\end{array}$ & $\begin{array}{c}9.96 \pm 0.68 \pm 1.1 \\
14.4 \pm 1.3 \pm 1.5\end{array}$ & $\begin{array}{l}2.624 \pm 0.068 \pm 0.28 \\
2.982 \pm 0.064 \pm 0.32\end{array}$ & $\begin{array}{l}607 \pm 21 \pm 66 \\
703 \pm 20 \pm 77\end{array}$ \\
\hline $\begin{array}{l}-0.500--0.375 \\
-0.375--0.250 \\
\end{array}$ & $\begin{array}{l}81.9 \pm 5.3 \pm 8.7 \\
83.8 \pm 3.3 \pm 8.9\end{array}$ & $\begin{array}{l}14.9 \pm 1.1 \pm 1.6 \\
17.7 \pm 1.1 \pm 1.9\end{array}$ & $\begin{array}{l}3.383 \pm 0.055 \pm 0.36 \\
3.743 \pm 0.053 \pm 0.40\end{array}$ & $\begin{array}{c}812 \pm 18 \pm 89 \\
943 \pm 19 \pm 100\end{array}$ \\
\hline $\begin{array}{c}-0.250--0.125 \\
-0.125-0.000\end{array}$ & $\begin{array}{c}93.2 \pm 3.4 \pm 9.9 \\
94.9 \pm 3.4 \pm 10\end{array}$ & $\begin{array}{l}18.4 \pm 1.1 \pm 2.0 \\
20.7 \pm 1.2 \pm 2.2\end{array}$ & $\begin{array}{l}4.014 \pm 0.043 \pm 0.43 \\
4.350 \pm 0.043 \pm 0.47\end{array}$ & $\begin{array}{c}999 \pm 17 \pm 110 \\
1078 \pm 18 \pm 120\end{array}$ \\
\hline $\begin{array}{l}0.000-0.125 \\
0.125-0.250\end{array}$ & $\begin{array}{l}91.8 \pm 3.0 \pm 9.8 \\
80.4 \pm 2.5 \pm 8.6\end{array}$ & $\begin{array}{l}17.94 \pm 0.99 \pm 1.9 \\
16.04 \pm 0.75 \pm 1.7\end{array}$ & $\begin{array}{l}4.151 \pm 0.040 \pm 0.45 \\
3.864 \pm 0.036 \pm 0.42\end{array}$ & $\begin{array}{l}961 \pm 16 \pm 110 \\
965 \pm 16 \pm 110\end{array}$ \\
\hline $\begin{array}{l}0.250-0.375 \\
0.375-0.500\end{array}$ & $\begin{array}{l}81.9 \pm 2.6 \pm 8.7 \\
77.4 \pm 2.6 \pm 8.2\end{array}$ & $\begin{array}{l}17.40 \pm 0.88 \pm 1.9 \\
13.62 \pm 0.79 \pm 1.5\end{array}$ & $\begin{array}{l}3.549 \pm 0.033 \pm 0.38 \\
3.268 \pm 0.035 \pm 0.35 \\
\end{array}$ & $\begin{array}{l}905 \pm 15 \pm 99 \\
769 \pm 15 \pm 84 \\
\end{array}$ \\
\hline $\begin{array}{l}0.500-0.625 \\
0.625-0.750\end{array}$ & $\begin{array}{l}67.7 \pm 2.7 \pm 7.2 \\
57.5 \pm 2.7 \pm 6.1\end{array}$ & $\begin{aligned} 14.4 & \pm 1.1 \pm 1.5 \\
10.52 & \pm 0.92 \pm 1.1\end{aligned}$ & $\begin{array}{l}2.769 \pm 0.034 \pm 0.30 \\
2.238 \pm 0.032 \pm 0.24\end{array}$ & $\begin{array}{l}650 \pm 14 \pm 71 \\
486 \pm 13 \pm 53\end{array}$ \\
\hline & $\begin{array}{c}5.00-5.50 \\
\mathrm{pb} /(\mathrm{GeV} / \mathrm{c})^{2}\end{array}$ & $\begin{array}{r}5.50-6.50 \\
\mathrm{pb} /(\mathrm{GeV} / \mathrm{c})^{2}\end{array}$ & $\begin{array}{c}6.50-8.00 \\
\mathrm{pb} /(\mathrm{GeV} / c)^{2}\end{array}$ & \\
\hline $\begin{array}{l}-0.750--0.625 \\
-0.625--0.500\end{array}$ & $\begin{array}{l}150.0 \pm 7.9 \pm 17 \\
210.3 \pm 8.9 \pm 23\end{array}$ & $\begin{array}{l}25.8 \pm 2.1 \pm 2.9 \\
29.9 \pm 2.0 \pm 3.4\end{array}$ & $1.59 \pm 0.23 \pm 0.19$ & \\
\hline $\begin{array}{l}-0.500--0.375 \\
-0.375--0.250 \\
\end{array}$ & $\begin{array}{l}224.3 \pm 8.0 \pm 25 \\
246.1 \pm 8.1 \pm 27\end{array}$ & $\begin{array}{l}41.6 \pm 2.1 \pm 4.7 \\
45.5 \pm 2.3 \pm 5.2 \\
\end{array}$ & $2.11 \pm 0.24 \pm 0.25$ & \\
\hline $\begin{array}{c}-0.250--0.125 \\
-0.125-0.000\end{array}$ & $\begin{array}{l}263.4 \pm 8.2 \pm 29 \\
278.9 \pm 8.5 \pm 31\end{array}$ & $\begin{array}{l}50.2 \pm 2.3 \pm 5.7 \\
52.2 \pm 2.4 \pm 5.9\end{array}$ & $3.43 \pm 0.31 \pm 0.41$ & \\
\hline $\begin{array}{l}0.000-0.125 \\
0.125-0.250\end{array}$ & $\begin{array}{l}260.5 \pm 7.6 \pm 29 \\
278.7 \pm 7.9 \pm 31\end{array}$ & $\begin{array}{l}47.8 \pm 2.1 \pm 5.4 \\
47.2 \pm 2.2 \pm 5.4\end{array}$ & $3.17 \pm 0.28 \pm 0.38$ & \\
\hline $\begin{array}{l}0.250-0.375 \\
0.375-0.500\end{array}$ & $\begin{array}{l}238.8 \pm 7.5 \pm 27 \\
189.8 \pm 6.9 \pm 21\end{array}$ & $\begin{array}{l}44.3 \pm 2.1 \pm 5.0 \\
36.0 \pm 1.9 \pm 4.1\end{array}$ & $2.78 \pm 0.29 \pm 0.33$ & \\
\hline $\begin{array}{l}0.500-0.625 \\
0.625-0.750\end{array}$ & $\begin{array}{l}169.6 \pm 6.8 \pm 19 \\
114.9 \pm 5.9 \pm 13\end{array}$ & $\begin{array}{l}25.8 \pm 1.9 \pm 2.9 \\
16.3 \pm 1.5 \pm 1.8\end{array}$ & $1.63 \pm 0.24 \pm 0.19$ & \\
\hline
\end{tabular}


Table A.6 Invariant differential cross section per nucleon for $\pi^{0}$ production by $800 \mathrm{GeV} / c$ proton beam on a Be target as a function of $p_{T}$ and rapidity.

\begin{tabular}{|c|c|c|c|c|}
\hline \multirow[b]{2}{*}{ Rapidity } & \multicolumn{4}{|c|}{$\bar{p}(\mathrm{GeV} / c)$} \\
\hline & $\begin{array}{c}1.00-1.50 \\
\mu \mathrm{b} /(\mathrm{GeV} / c)^{2}\end{array}$ & $\begin{array}{c}1.50-2.00 \\
\mu \mathrm{b} /(\mathrm{GeV} / \mathrm{c})^{2}\end{array}$ & $\begin{array}{c}2.00-2.50 \\
\mu \mathrm{b} /(\mathrm{GeV} / c)^{2}\end{array}$ & $\begin{array}{c}2.50-3.00 \\
\mathrm{nb} /(\mathrm{GeV} / \mathrm{c})^{2}\end{array}$ \\
\hline $\begin{array}{c}-1.00--0.875 \\
-0.875--0.750\end{array}$ & $464 \pm 88$ & $37 \pm 12$ & $6.6 \pm 1.9 \pm 0.7$ & $\begin{array}{c}479 \pm 87 \pm 53 \\
850 \pm 180 \pm 90\end{array}$ \\
\hline $\begin{array}{l}-0.750--0.625 \\
-0.625--0.500\end{array}$ & $469 \pm 83$ & $60 \pm 11$ & $5.9 \pm 1.8 \pm 0.7$ & $\begin{array}{l}621 \pm 56 \pm 69 \\
690 \pm 50 \pm 76\end{array}$ \\
\hline $\begin{array}{l}-0.500--0.375 \\
-0.375--0.250\end{array}$ & $419 \pm 76$ & $58 \pm 12$ & $1.2 \pm 1.8 \pm 0.1$ & $\begin{array}{l}615 \pm 51 \pm 68 \\
712 \pm 52 \pm 79\end{array}$ \\
\hline $\begin{array}{c}-0.250--0.125 \\
-0.125-0.000\end{array}$ & $457 \pm 74$ & $56 \pm 11$ & $4.2 \pm 1.6 \pm 0.5$ & $\begin{array}{l}765 \pm 45 \pm 85 \\
737 \pm 46 \pm 81\end{array}$ \\
\hline $\begin{array}{l}0.000-0.125 \\
0.125-0.250\end{array}$ & $423 \pm 66$ & $46.2 \pm 9.4$ & $5.5 \pm 1.6 \pm 0.6$ & $\begin{array}{c}773 \pm 48 \pm 85 \\
730 \pm 100 \pm 80\end{array}$ \\
\hline $\begin{array}{l}0.250-0.375 \\
0.375-0.500\end{array}$ & $495 \pm 71$ & $32.0 \pm 8.6$ & $4.5 \pm 1.5 \pm 0.5$ & $\begin{array}{l}757 \pm 49 \pm 84 \\
597 \pm 43 \pm 66\end{array}$ \\
\hline & $\begin{array}{c}3.00-3.50 \\
\mathrm{nb} /(\mathrm{GeV} / \mathrm{c})^{2}\end{array}$ & $\begin{array}{c}3.50-4.00 \\
\mathrm{nb} /(\mathrm{GeV} / \mathrm{c})^{2}\end{array}$ & $\begin{array}{c}4.00-4.50 \\
\mathrm{nb} /(\mathrm{GeV} / \mathrm{c})^{2}\end{array}$ & $\begin{array}{c}4.50-5.00 \\
\mathrm{pb} /(\mathrm{GeV} / \mathrm{c})^{2}\end{array}$ \\
\hline $\begin{array}{c}-1.00--0.875 \\
-0.875--0.750\end{array}$ & $\begin{array}{l}101.1 \pm 8.1 \pm 11 \\
125.4 \pm 7.7 \pm 14\end{array}$ & $\begin{array}{l}20.1 \pm 2.0 \pm 2.2 \\
22.5 \pm 2.2 \pm 2.5\end{array}$ & $\begin{array}{l}4.03 \pm 0.28 \pm 0.45 \\
4.75 \pm 0.37 \pm 0.53\end{array}$ & $\begin{array}{l}1046 \pm 36 \pm 120 \\
1190 \pm 37 \pm 130\end{array}$ \\
\hline $\begin{array}{l}-0.750--0.625 \\
-0.625--0.500\end{array}$ & $\begin{array}{l}103.2 \pm 6.2 \pm 11 \\
119.3 \pm 5.2 \pm 13\end{array}$ & $\begin{array}{l}25.9 \pm 2.5 \pm 2.9 \\
25.4 \pm 1.9 \pm 2.8\end{array}$ & $\begin{array}{l}5.63 \pm 0.31 \pm 0.62 \\
7.68 \pm 0.45 \pm 0.85\end{array}$ & $\begin{array}{l}1525 \pm 38 \pm 170 \\
1801 \pm 43 \pm 200\end{array}$ \\
\hline$-0.500--0.375$ & $144.1 \pm 6.5 \pm 16$ & $30.2 \pm 2.1 \pm 3.3$ & $7.47 \pm 0.40 \pm 0.83$ & $2046 \pm 37 \pm 230$ \\
\hline$-0.375--0.250$ & $145.6 \pm 5.8 \pm 16$ & $32.6 \pm 2.1 \pm 3.6$ & $8.06 \pm 0.36 \pm 0.89$ & $2120 \pm 35 \pm 240$ \\
\hline$-0.250--0.125$ & $150.5 \pm 5.8 \pm 17$ & $28.3 \pm 2.2 \pm 3.1$ & $7.97 \pm 0.32 \pm 0.88$ & $2187 \pm 36 \pm 240$ \\
\hline$-0.125-0.000$ & $145.6 \pm 5.9 \pm 16$ & $30.1 \pm 2.3 \pm 3.3$ & $7.84 \pm 0.38 \pm 0.87$ & $2211 \pm 38 \pm 250$ \\
\hline $0.000-0.125$ & $143.3 \pm 4.8 \pm 16$ & $32.4 \pm 1.9 \pm 3.6$ & $8.01 \pm 0.29 \pm 0.89$ & $2145 \pm 87 \pm 240$ \\
\hline $0.125-0.250$ & $144.4 \pm 4.6 \pm 16$ & $29.8 \pm 1.7 \pm 3.3$ & $7.68 \pm 0.29 \pm 0.85$ & $2040 \pm 100 \pm 230$ \\
\hline $\begin{array}{l}0.250-0.375 \\
0.375-0.500\end{array}$ & $\begin{array}{l}130.6 \pm 4.9 \pm 14 \\
128.6 \pm 5.6 \pm 14\end{array}$ & $\begin{array}{l}29.7 \pm 1.8 \pm 3.3 \\
29.3 \pm 2.1 \pm 3.2\end{array}$ & $\begin{array}{l}6.95 \pm 0.22 \pm 0.77 \\
6.81 \pm 0.24 \pm 0.75\end{array}$ & $\begin{array}{l}1896 \pm 87 \pm 210 \\
1842 \pm 85 \pm 210\end{array}$ \\
\hline & $\begin{array}{c}5.00-5.50 \\
\mathrm{pb} /(\mathrm{GeV} / \mathrm{c})^{2}\end{array}$ & $\begin{array}{c}5.50-6.50 \\
\mathrm{pb} /(\mathrm{GeV} / c)^{2}\end{array}$ & $\begin{array}{c}6.50-8.00 \\
\mathrm{pb} /(\mathrm{GeV} / \mathrm{c})^{2}\end{array}$ & \\
\hline $\begin{array}{c}-1.00--0.875 \\
-0.875--0.750\end{array}$ & $\begin{array}{l}270 \pm 25 \pm 30 \\
329 \pm 16 \pm 37\end{array}$ & $\begin{array}{l}54.7 \pm 3.7 \pm 6.3 \\
68.6 \pm 4.3 \pm 7.9\end{array}$ & $3.58 \pm 0.42 \pm 0.42$ & \\
\hline $\begin{array}{l}-0.750--0.625 \\
-0.625--0.500\end{array}$ & $\begin{array}{l}415 \pm 16 \pm 47 \\
509 \pm 19 \pm 57\end{array}$ & $\begin{array}{l}83.5 \pm 4.2 \pm 9.6 \\
110.3 \pm 5.7 \pm 13\end{array}$ & $7.58 \pm 0.64 \pm 0.90$ & \\
\hline $\begin{array}{l}-0.500--0.375 \\
-0.375--0.250\end{array}$ & $\begin{array}{l}600 \pm 18 \pm 68 \\
642 \pm 17 \pm 72\end{array}$ & $\begin{array}{l}121.5 \pm 4.9 \pm 14 \\
142.7 \pm 5.3 \pm 16\end{array}$ & $11.25 \pm 0.70 \pm 1.3$ & \\
\hline $\begin{array}{c}-0.250--0.125 \\
-0.125-0.000\end{array}$ & $\begin{array}{l}651 \pm 18 \pm 73 \\
646 \pm 19 \pm 73\end{array}$ & $\begin{array}{l}143.5 \pm 5.1 \pm 16 \\
136.7 \pm 5.3 \pm 16\end{array}$ & $12.81 \pm 0.75 \pm 1.5$ & \\
\hline $\begin{array}{l}0.000-0.125 \\
0.125-0.250\end{array}$ & $\begin{array}{l}650 \pm 28 \pm 73 \\
652 \pm 38 \pm 74\end{array}$ & $\begin{array}{l}139.1 \pm 6.2 \pm 16 \\
134.0 \pm 7.4 \pm 15\end{array}$ & $14.08 \pm 0.78 \pm 1.7$ & \\
\hline $\begin{array}{l}0.250-0.375 \\
0.375-0.500\end{array}$ & $\begin{array}{l}588 \pm 29 \pm 66 \\
531 \pm 27 \pm 60\end{array}$ & $\begin{array}{l}134.9 \pm 6.5 \pm 16 \\
108.0 \pm 6.0 \pm 12\end{array}$ & $10.04 \pm 0.74 \pm 1.2$ & \\
\hline
\end{tabular}


Table A.7 Invariant differential cross section per nucleon for $\pi^{0}$ production by $515 \mathrm{GeV} / c \pi^{-}$beam on a Be target as a function of $p_{T}$ and rapidity.

\begin{tabular}{|c|c|c|c|c|}
\hline \multirow[b]{2}{*}{ Rapidity } & \multicolumn{4}{|c|}{$p_{T}(\mathrm{GeV} / c)$} \\
\hline & $\begin{array}{c}1.00-1.50 \\
\mu \mathrm{b} /(\mathrm{GeV} / c)^{2}\end{array}$ & $\begin{array}{c}1.50-2.00 \\
\mu \mathrm{b} /(\mathrm{GeV} / c)^{2}\end{array}$ & $\begin{array}{c}2.00-2.50 \\
\mu \mathrm{b} /(\mathrm{GeV} / c)^{2}\end{array}$ & $\begin{array}{c}2.50-3.00 \\
\mathrm{nb} /(\mathrm{GeV} / \mathrm{c})^{2}\end{array}$ \\
\hline $\begin{array}{l}-0.750--0.625 \\
-0.625--0.500\end{array}$ & $123 \pm 29$ & $11.2 \pm 3.8$ & $1.87 \pm 0.86 \pm 0.22$ & $\begin{array}{l}377 \pm 26 \pm 43 \\
404 \pm 24 \pm 46\end{array}$ \\
\hline $\begin{array}{l}-0.500--0.375 \\
-0.375--0.250 \\
\end{array}$ & $189 \pm 30$ & $25.2 \pm 3.8$ & $2.577 \pm 0.083 \pm 0.30$ & $\begin{array}{l}370 \pm 24 \pm 42 \\
339 \pm 16 \pm 39 \\
\end{array}$ \\
\hline $\begin{array}{c}-0.250--0.125 \\
-0.125-0.000\end{array}$ & $178 \pm 28$ & $21.7 \pm 3.3$ & $2.555 \pm 0.055 \pm 0.30$ & $\begin{array}{l}380 \pm 16 \pm 44 \\
408 \pm 15 \pm 47\end{array}$ \\
\hline $\begin{array}{l}0.000-0.125 \\
0.125-0.250\end{array}$ & $195 \pm 29$ & $17.4 \pm 2.9$ & $2.554 \pm 0.045 \pm 0.30$ & $\begin{array}{l}414 \pm 14 \pm 47 \\
396 \pm 12 \pm 45\end{array}$ \\
\hline $\begin{array}{l}0.250-0.375 \\
0.375-0.500\end{array}$ & $155 \pm 24$ & $19.7 \pm 3.5$ & $2.430 \pm 0.049 \pm 0.28$ & $\begin{array}{l}398 \pm 12 \pm 46 \\
358 \pm 12 \pm 41\end{array}$ \\
\hline $\begin{array}{l}0.500-0.625 \\
0.625-0.750\end{array}$ & $179 \pm 26$ & $16.3 \pm 2.5$ & $2.267 \pm 0.052 \pm 0.27$ & $\begin{array}{l}372 \pm 13 \pm 43 \\
338 \pm 12 \pm 39\end{array}$ \\
\hline & $\begin{array}{c}3.00-3.50 \\
\mathrm{nb} /(\mathrm{GeV} / \mathrm{c})^{2}\end{array}$ & $\begin{array}{c}3.50-4.00 \\
\mathrm{nb} /(\mathrm{GeV} / \mathrm{c})^{2}\end{array}$ & $\begin{array}{c}4.00-4.50 \\
\mathrm{nb} /(\mathrm{GeV} / \mathrm{c})^{2}\end{array}$ & $\begin{array}{c}4.50-5.00 \\
\mathrm{pb} /(\mathrm{GeV} / \mathrm{c})^{2} \\
\end{array}$ \\
\hline $\begin{array}{l}-0.750--0.625 \\
-0.625--0.500 \\
\end{array}$ & $\begin{array}{l}61.4 \pm 5.7 \pm 6.9 \\
61.6 \pm 5.8 \pm 6.9\end{array}$ & $\begin{array}{l}10.48 \pm 0.98 \pm 1.2 \\
11.71 \pm 0.96 \pm 1.3\end{array}$ & $\begin{array}{l}2.221 \pm 0.072 \pm 0.24 \\
2.795 \pm 0.057 \pm 0.31\end{array}$ & $\begin{array}{l}620 \pm 22 \pm 68 \\
778 \pm 19 \pm 85\end{array}$ \\
\hline $\begin{array}{l}-0.500--0.375 \\
-0.375--0.250 \\
\end{array}$ & $\begin{array}{l}74.1 \pm 4.1 \pm 8.3 \\
69.4 \pm 3.4 \pm 7.8\end{array}$ & $\begin{array}{l}14.42 \pm 0.47 \pm 1.6 \\
15.11 \pm 0.57 \pm 1.7\end{array}$ & $\begin{array}{l}3.343 \pm 0.045 \pm 0.37 \\
3.706 \pm 0.041 \pm 0.41\end{array}$ & $\begin{array}{c}872 \pm 16 \pm 96 \\
965 \pm 17 \pm 110\end{array}$ \\
\hline $\begin{array}{c}-0.250--0.125 \\
-0.125-0.000\end{array}$ & $\begin{array}{l}78.0 \pm 2.9 \pm 8.8 \\
84.1 \pm 2.4 \pm 9.4\end{array}$ & $\begin{array}{l}17.49 \pm 0.66 \pm 1.9 \\
18.75 \pm 0.53 \pm 2.1\end{array}$ & $\begin{array}{l}4.109 \pm 0.038 \pm 0.45 \\
4.609 \pm 0.040 \pm 0.51\end{array}$ & $\begin{array}{l}1129 \pm 18 \pm 120 \\
1242 \pm 19 \pm 140\end{array}$ \\
\hline $\begin{array}{l}0.000-0.125 \\
0.125-0.250\end{array}$ & $\begin{array}{l}87.5 \pm 1.7 \pm 9.8 \\
85.5 \pm 1.7 \pm 9.6\end{array}$ & $\begin{array}{l}18.33 \pm 0.54 \pm 2.0 \\
18.77 \pm 0.61 \pm 2.1\end{array}$ & $\begin{array}{l}4.582 \pm 0.038 \pm 0.50 \\
4.574 \pm 0.038 \pm 0.50\end{array}$ & $\begin{array}{l}1258 \pm 18 \pm 140 \\
1231 \pm 17 \pm 140\end{array}$ \\
\hline $\begin{array}{l}0.250-0.375 \\
0.375-0.500\end{array}$ & $\begin{array}{l}85.4 \pm 1.7 \pm 9.6 \\
82.6 \pm 1.9 \pm 9.3\end{array}$ & $\begin{array}{l}18.84 \pm 0.61 \pm 2.1 \\
17.71 \pm 0.63 \pm 2.0\end{array}$ & $\begin{array}{l}4.723 \pm 0.040 \pm 0.52 \\
4.578 \pm 0.041 \pm 0.50\end{array}$ & $\begin{array}{l}1276 \pm 18 \pm 140 \\
1255 \pm 19 \pm 140\end{array}$ \\
\hline $\begin{array}{l}0.500-0.625 \\
0.625-0.750\end{array}$ & $\begin{array}{l}78.9 \pm 1.8 \pm 8.9 \\
70.9 \pm 1.8 \pm 8.0\end{array}$ & $\begin{array}{l}18.72 \pm 0.58 \pm 2.1 \\
15.11 \pm 0.51 \pm 1.7\end{array}$ & $\begin{array}{l}4.307 \pm 0.041 \pm 0.47 \\
3.861 \pm 0.040 \pm 0.42\end{array}$ & $\begin{array}{l}1171 \pm 19 \pm 130 \\
1016 \pm 18 \pm 110\end{array}$ \\
\hline & $\begin{array}{c}5.00-5.50 \\
\mathrm{pb} /(\mathrm{GeV} / \mathrm{c})^{2}\end{array}$ & $\begin{array}{r}5.50-6.50 \\
\mathrm{pb} /(\mathrm{GeV} / \mathrm{c})^{2}\end{array}$ & $\begin{array}{c}6.50-8.00 \\
\mathrm{pb} /(\mathrm{GeV} / c)^{2}\end{array}$ & \\
\hline $\begin{array}{l}-0.750--0.625 \\
-0.625--0.500\end{array}$ & $\begin{array}{l}177.2 \pm 8.5 \pm 20 \\
213.0 \pm 7.5 \pm 24\end{array}$ & $\begin{array}{l}31.8 \pm 2.1 \pm 3.6 \\
44.1 \pm 2.2 \pm 4.9\end{array}$ & $2.08 \pm 0.26 \pm 0.24$ & \\
\hline $\begin{array}{l}-0.500--0.375 \\
-0.375--0.250 \\
\end{array}$ & $\begin{array}{l}253.5 \pm 8.2 \pm 28 \\
306.6 \pm 9.0 \pm 34\end{array}$ & $\begin{array}{l}52.1 \pm 2.3 \pm 5.8 \\
65.3 \pm 2.6 \pm 7.3 \\
\end{array}$ & $4.47 \pm 0.35 \pm 0.52$ & \\
\hline $\begin{array}{c}-0.250--0.125 \\
-0.125-0.000\end{array}$ & $\begin{array}{l}326.9 \pm 8.8 \pm 36 \\
356.2 \pm 9.0 \pm 39\end{array}$ & $\begin{array}{l}73.4 \pm 2.8 \pm 8.2 \\
75.8 \pm 2.8 \pm 8.5\end{array}$ & $6.84 \pm 0.43 \pm 0.79$ & \\
\hline $\begin{array}{l}0.000-0.125 \\
0.125-0.250\end{array}$ & $\begin{array}{l}381.2 \pm 9.0 \pm 42 \\
383.9 \pm 8.9 \pm 42\end{array}$ & $\begin{array}{l}88.5 \pm 2.9 \pm 9.9 \\
82.5 \pm 2.8 \pm 9.2\end{array}$ & $8.44 \pm 0.46 \pm 0.98$ & \\
\hline $\begin{array}{l}0.250-0.375 \\
0.375-0.500\end{array}$ & $\begin{array}{l}387.9 \pm 9.2 \pm 43 \\
375.3 \pm 9.6 \pm 41\end{array}$ & $\begin{array}{l}83.2 \pm 2.8 \pm 9.3 \\
72.0 \pm 2.9 \pm 8.1\end{array}$ & $7.42 \pm 0.46 \pm 0.86$ & \\
\hline $\begin{array}{l}0.500-0.625 \\
0.625-0.750\end{array}$ & $\begin{array}{l}343.7 \pm 9.4 \pm 38 \\
299.5 \pm 8.9 \pm 33\end{array}$ & $\begin{array}{l}76.1 \pm 2.9 \pm 8.5 \\
57.0 \pm 2.6 \pm 6.4\end{array}$ & $6.49 \pm 0.49 \pm 0.75$ & \\
\hline
\end{tabular}


Table A.8 Invariant differential cross section for $\pi^{0}$ production by $530 \mathrm{GeV} / \mathrm{c}$ proton beam on a $\mathrm{Cu}$ target as a function of $p_{T}$ and rapidity.

\begin{tabular}{|c|c|c|c|}
\hline Rapidity & $\begin{array}{c}1.00-2.50 \\
\mu \mathrm{b} /(\mathrm{GeV} / c)^{2}\end{array}$ & $\begin{array}{c}p_{T}(\mathrm{GeV} / c) \\
2.50-3.00 \\
\mathrm{nb} /(\mathrm{GeV} / c)^{2}\end{array}$ & $\begin{array}{c}3.00-3.50 \\
\mathrm{nb} /(\mathrm{GeV} / c)^{2}\end{array}$ \\
\hline $\begin{array}{l}-0.750--0.625 \\
-0.625--0.500\end{array}$ & $90 \pm 31 \pm 9.0$ & $\begin{array}{c}710 \pm 240 \pm 80 \\
990 \pm 240 \pm 110\end{array}$ & $\begin{array}{l}83 \pm 12 \pm 9.0 \\
109 \pm 13 \pm 12\end{array}$ \\
\hline $\begin{array}{l}-0.500--0.375 \\
-0.375--0.250\end{array}$ & $112 \pm 29 \pm 12$ & $\begin{array}{l}545 \pm 54 \pm 58 \\
614 \pm 38 \pm 65\end{array}$ & $\begin{array}{l}98.3 \pm 8.5 \pm 11 \\
99.6 \pm 7.6 \pm 11\end{array}$ \\
\hline $\begin{array}{c}-0.250--0.125 \\
-0.125-0.000\end{array}$ & $91 \pm 24 \pm 10.0$ & $\begin{array}{l}604 \pm 26 \pm 64 \\
737 \pm 36 \pm 78\end{array}$ & $\begin{array}{l}111.1 \pm 8.0 \pm 12 \\
120.5 \pm 7.8 \pm 13\end{array}$ \\
\hline $\begin{array}{l}0.000-0.125 \\
0.125-0.250\end{array}$ & $90 \pm 22 \pm 9.0$ & $\begin{array}{l}652 \pm 24 \pm 69 \\
621 \pm 20 \pm 66\end{array}$ & $\begin{array}{c}107.0 \pm 7.1 \pm 11 \\
97.8 \pm 6.4 \pm 10\end{array}$ \\
\hline $\begin{array}{l}0.250-0.375 \\
0.375-0.500\end{array}$ & $71 \pm 23 \pm 7.0$ & $\begin{array}{l}551 \pm 20 \pm 59 \\
511 \pm 18 \pm 54\end{array}$ & $\begin{array}{l}110.3 \pm 6.7 \pm 12 \\
92.1 \pm 6.8 \pm 9.8\end{array}$ \\
\hline \multirow[t]{2}{*}{$\begin{array}{l}0.500-0.625 \\
0.625-0.750\end{array}$} & $78 \pm 19 \pm 8.0$ & $\begin{array}{l}491 \pm 21 \pm 52 \\
404 \pm 19 \pm 43\end{array}$ & $\begin{array}{l}77.9 \pm 6.8 \pm 8.3 \\
84.0 \pm 7.5 \pm 8.9\end{array}$ \\
\hline & $\begin{array}{c}3.50-4.00 \\
\mathrm{nb} /(\mathrm{GeV} / \mathrm{c})^{2}\end{array}$ & $\begin{array}{c}4.00-4.50 \\
\mathrm{nb} /(\mathrm{GeV} / \mathrm{c})^{2}\end{array}$ & $\begin{array}{c}4.50-5.00 \\
\mathrm{pb} /(\mathrm{GeV} / \mathrm{c})^{2}\end{array}$ \\
\hline $\begin{array}{l}-0.750--0.625 \\
-0.625--0.500\end{array}$ & $\begin{array}{l}15.6 \pm 2.4 \pm 1.7 \\
19.1 \pm 2.2 \pm 2.0\end{array}$ & $\begin{array}{l}3.44 \pm 0.17 \pm 0.37 \\
4.06 \pm 0.15 \pm 0.44\end{array}$ & $\begin{array}{c}780 \pm 45 \pm 85 \\
961 \pm 54 \pm 110\end{array}$ \\
\hline $\begin{array}{l}-0.500--0.375 \\
-0.375--0.250\end{array}$ & $\begin{array}{l}18.3 \pm 2.4 \pm 2.0 \\
24.8 \pm 2.8 \pm 2.7\end{array}$ & $\begin{array}{l}4.32 \pm 0.13 \pm 0.47 \\
5.07 \pm 0.13 \pm 0.55\end{array}$ & $\begin{array}{l}1070 \pm 44 \pm 120 \\
1227 \pm 47 \pm 130\end{array}$ \\
\hline $\begin{array}{c}-0.250--0.125 \\
-0.125-0.000\end{array}$ & $\begin{array}{l}22.6 \pm 2.6 \pm 2.4 \\
23.4 \pm 2.9 \pm 2.5\end{array}$ & $\begin{array}{l}5.39 \pm 0.11 \pm 0.58 \\
5.70 \pm 0.11 \pm 0.62\end{array}$ & $\begin{array}{l}1258 \pm 43 \pm 140 \\
1325 \pm 45 \pm 150\end{array}$ \\
\hline $\begin{array}{l}0.000-0.125 \\
0.125-0.250\end{array}$ & $\begin{array}{l}20.8 \pm 2.4 \pm 2.2 \\
22.9 \pm 2.2 \pm 2.4\end{array}$ & $\begin{array}{l}5.475 \pm 0.099 \pm 0.59 \\
5.037 \pm 0.092 \pm 0.54\end{array}$ & $\begin{array}{l}1191 \pm 39 \pm 130 \\
1258 \pm 41 \pm 140\end{array}$ \\
\hline $\begin{array}{l}0.250-0.375 \\
0.375-0.500\end{array}$ & $\begin{array}{l}19.5 \pm 2.0 \pm 2.1 \\
20.3 \pm 2.1 \pm 2.2\end{array}$ & $\begin{array}{l}4.507 \pm 0.083 \pm 0.49 \\
4.094 \pm 0.090 \pm 0.44\end{array}$ & $\begin{array}{l}1108 \pm 38 \pm 120 \\
1049 \pm 38 \pm 120\end{array}$ \\
\hline \multirow[t]{2}{*}{$0.625-0.750$} & $\begin{array}{l}13.6 \pm 2.3 \pm 1.5 \\
11.7 \pm 2.2 \pm 1.2\end{array}$ & $\begin{array}{l}3.614 \pm 0.088 \pm 0.39 \\
2.748 \pm 0.080 \pm 0.30\end{array}$ & $\begin{array}{l}795 \pm 35 \pm 87 \\
665 \pm 33 \pm 73\end{array}$ \\
\hline & $\begin{array}{c}5.00-5.50 \\
\mathrm{pb} /(\mathrm{GeV} / \mathrm{c})^{2}\end{array}$ & $\begin{array}{c}5.50-6.50 \\
\mathrm{pb} /(\mathrm{GeV} / \mathrm{c})^{2}\end{array}$ & $\begin{array}{c}6.50-8.00 \\
\mathrm{pb} /(\mathrm{GeV} / \mathrm{c})^{2}\end{array}$ \\
\hline $\begin{array}{l}-0.750--0.625 \\
-0.625--0.500 \\
\end{array}$ & $\begin{array}{l}237 \pm 21 \pm 26 \\
200 \pm 18 \pm 22 \\
\end{array}$ & $\begin{array}{l}25.9 \pm 4.7 \pm 2.9 \\
34.2 \pm 5.3 \pm 3.9 \\
\end{array}$ & $1.62 \pm 0.65 \pm 0.19$ \\
\hline $\begin{array}{l}-0.500--0.375 \\
-0.375--0.250\end{array}$ & $\begin{array}{l}257 \pm 20 \pm 29 \\
297 \pm 20 \pm 33\end{array}$ & $\begin{array}{l}55.0 \pm 5.9 \pm 6.2 \\
43.7 \pm 4.7 \pm 5.0\end{array}$ & $3.17 \pm 0.65 \pm 0.38$ \\
\hline $\begin{array}{c}-0.250--0.125 \\
-0.125-0.000\end{array}$ & $\begin{array}{l}343 \pm 20 \pm 38 \\
328 \pm 21 \pm 36\end{array}$ & $\begin{array}{l}53.8 \pm 5.3 \pm 6.1 \\
68.7 \pm 6.1 \pm 7.8\end{array}$ & $4.23 \pm 0.76 \pm 0.50$ \\
\hline $\begin{array}{l}0.000-0.125 \\
0.125-0.250\end{array}$ & $\begin{array}{l}361 \pm 20 \pm 40 \\
337 \pm 20 \pm 37\end{array}$ & $\begin{array}{l}63.8 \pm 5.6 \pm 7.2 \\
57.3 \pm 5.2 \pm 6.5\end{array}$ & $3.53 \pm 0.68 \pm 0.42$ \\
\hline $\begin{array}{l}0.250-0.375 \\
0.375-0.500\end{array}$ & $\begin{array}{l}301 \pm 19 \pm 33 \\
234 \pm 17 \pm 26\end{array}$ & $\begin{array}{l}56.2 \pm 5.3 \pm 6.4 \\
38.6 \pm 4.6 \pm 4.4\end{array}$ & $1.45 \pm 0.53 \pm 0.17$ \\
\hline $\begin{array}{l}0.500-0.625 \\
0.625-0.750\end{array}$ & $\begin{array}{l}228 \pm 17 \pm 25 \\
164 \pm 15 \pm 18\end{array}$ & $\begin{array}{l}28.5 \pm 4.1 \pm 3.2 \\
17.2 \pm 3.4 \pm 2.0\end{array}$ & $0.97 \pm 0.40 \pm 0.12$ \\
\hline
\end{tabular}


Table A.9 Invariant differential cross section for $\pi^{0}$ production by $800 \mathrm{GeV} / c$ proton beam on a $\mathrm{Cu}$ target as a function of $p_{T}$ and rapidity.

\begin{tabular}{|c|c|c|c|}
\hline Rapidity & $\begin{array}{c}1.00-2.50 \\
\mu \mathrm{b} /(\mathrm{GeV} / c)^{2}\end{array}$ & $\begin{array}{c}p_{T}(\mathrm{GeV} / c) \\
2.50-3.00 \\
\mathrm{nb} /(\mathrm{GeV} / c)^{2}\end{array}$ & $\begin{array}{c}3.00-3.50 \\
\mathrm{nb} /(\mathrm{GeV} / c)^{2}\end{array}$ \\
\hline $\begin{array}{l}-1.00--0.875 \\
-0.875--0.750 \\
\end{array}$ & $103 \pm 49 \pm 11$ & $\begin{array}{c}390 \pm 170 \pm 40 \\
860 \pm 290 \pm 100\end{array}$ & $\begin{array}{c}90 \pm 16 \pm 10.0 \\
158 \pm 17 \pm 17\end{array}$ \\
\hline $\begin{array}{l}-0.750--0.625 \\
-0.625--0.500\end{array}$ & $66 \pm 45 \pm 7.0$ & $\begin{array}{c}840 \pm 210 \pm 90 \\
870 \pm 110 \pm 100\end{array}$ & $\begin{array}{l}117 \pm 13 \pm 13 \\
184 \pm 15 \pm 20\end{array}$ \\
\hline $\begin{array}{l}-0.500--0.375 \\
-0.375--0.250\end{array}$ & $100 \pm 39 \pm 11$ & $\begin{array}{c}600 \pm 110 \pm 70 \\
673 \pm 78 \pm 74\end{array}$ & $\begin{array}{l}159 \pm 13 \pm 17 \\
187 \pm 13 \pm 21\end{array}$ \\
\hline $\begin{array}{c}-0.250--0.125 \\
-0.125-0.000 \\
\end{array}$ & $146 \pm 37 \pm 16$ & $\begin{array}{l}950 \pm 150 \pm 100 \\
920 \pm 120 \pm 100\end{array}$ & $\begin{array}{l}186 \pm 15 \pm 20 \\
174 \pm 15 \pm 19\end{array}$ \\
\hline $\begin{array}{l}0.000-0.125 \\
0.125-0.250 \\
\end{array}$ & $93 \pm 32 \pm 10$ & $\begin{array}{l}1030 \pm 110 \pm 110 \\
1050 \pm 140 \pm 120 \\
\end{array}$ & $\begin{array}{l}196 \pm 13 \pm 22 \\
167 \pm 11 \pm 18 \\
\end{array}$ \\
\hline \multirow[t]{2}{*}{$\begin{array}{l}0.250-0.375 \\
0.375-0.500\end{array}$} & $206 \pm 31 \pm 23$ & $\begin{array}{c}1520 \pm 420 \pm 170 \\
940 \pm 120 \pm 100\end{array}$ & $\begin{array}{l}158 \pm 12 \pm 17 \\
153 \pm 13 \pm 17\end{array}$ \\
\hline & $\begin{array}{c}3.50-4.00 \\
\mathrm{nb} /(\mathrm{GeV} / \mathrm{c})^{2}\end{array}$ & $\begin{array}{c}4.00-4.50 \\
\mathrm{nb} /(\mathrm{GeV} / \mathrm{c})^{2}\end{array}$ & $\begin{array}{c}4.50-5.00 \\
\mathrm{pb} /(\mathrm{GeV} / c)^{2}\end{array}$ \\
\hline $\begin{array}{c}-1.00--0.875 \\
-0.875--0.750\end{array}$ & $\begin{array}{l}16.1 \pm 3.4 \pm 1.8 \\
26.6 \pm 4.6 \pm 2.9\end{array}$ & $\begin{array}{l}4.00 \pm 0.42 \pm 0.44 \\
5.61 \pm 0.50 \pm 0.62\end{array}$ & $\begin{array}{l}1390 \pm 110 \pm 160 \\
1530 \pm 110 \pm 170\end{array}$ \\
\hline $\begin{array}{l}-0.750--0.625 \\
-0.625--0.500\end{array}$ & $\begin{array}{l}40.4 \pm 5.8 \pm 4.5 \\
41.0 \pm 5.9 \pm 4.5\end{array}$ & $\begin{array}{c}9.3 \pm 1.8 \pm 1.0 \\
8.67 \pm 0.64 \pm 0.96\end{array}$ & $\begin{array}{c}1855 \pm 88 \pm 210 \\
2350 \pm 110 \pm 260\end{array}$ \\
\hline $\begin{array}{l}-0.500--0.375 \\
-0.375--0.250 \\
\end{array}$ & $\begin{array}{l}39.0 \pm 5.6 \pm 4.3 \\
46.5 \pm 5.8 \pm 5.1 \\
\end{array}$ & $\begin{array}{l}11.0 \pm 1.1 \pm 1.2 \\
10.6 \pm 1.2 \pm 1.2 \\
\end{array}$ & $\begin{array}{l}2447 \pm 88 \pm 270 \\
2721 \pm 91 \pm 300 \\
\end{array}$ \\
\hline $\begin{array}{c}-0.250--0.125 \\
-0.125-0.000 \\
\end{array}$ & $\begin{array}{l}35.2 \pm 5.2 \pm 3.9 \\
43.0 \pm 5.7 \pm 4.7 \\
\end{array}$ & $\begin{array}{l}10.1 \pm 1.2 \pm 1.1 \\
10.9 \pm 1.4 \pm 1.2 \\
\end{array}$ & $\begin{array}{l}2753 \pm 92 \pm 310 \\
2796 \pm 96 \pm 310 \\
\end{array}$ \\
\hline $\begin{array}{l}0.000-0.125 \\
0.125-0.250 \\
\end{array}$ & $\begin{array}{l}45.4 \pm 5.0 \pm 5.0 \\
42.2 \pm 4.4 \pm 4.6\end{array}$ & $\begin{array}{c}9.43 \pm 0.56 \pm 1.1 \\
8.19 \pm 0.59 \pm 0.91 \\
\end{array}$ & $\begin{array}{l}2670 \pm 230 \pm 300 \\
2630 \pm 280 \pm 290 \\
\end{array}$ \\
\hline \multirow[t]{2}{*}{$0.375-0.500$} & $\begin{array}{l}36.8 \pm 4.6 \pm 4.0 \\
36.9 \pm 5.4 \pm 4.1\end{array}$ & $\begin{array}{c}9.14 \pm 0.59 \pm 1.0 \\
8.78 \pm 0.59 \pm 0.97\end{array}$ & $\begin{array}{l}2150 \pm 220 \pm 240 \\
2130 \pm 220 \pm 240\end{array}$ \\
\hline & $\begin{array}{c}5.00-5.50 \\
\mathrm{pb} /(\mathrm{GeV} / c)^{2}\end{array}$ & $\begin{array}{c}5.50-6.50 \\
\mathrm{pb} /(\mathrm{GeV} / c)^{2}\end{array}$ & $\begin{array}{c}6.50-8.00 \\
\mathrm{pb} /(\mathrm{GeV} / c)^{2}\end{array}$ \\
\hline $\begin{array}{r}-1.00--0.875 \\
-0.875--0.750 \\
\end{array}$ & $\begin{array}{l}310 \pm 34 \pm 35 \\
322 \pm 29 \pm 36 \\
\end{array}$ & $\begin{aligned} & 67.7 \pm 9.9 \pm 7.8 \\
& 99 \pm 12 \pm 11 \\
&\end{aligned}$ & $3.38 \pm 0.79 \pm 0.40$ \\
\hline $\begin{array}{l}-0.750--0.625 \\
-0.625--0.500 \\
\end{array}$ & $\begin{array}{l}547 \pm 43 \pm 62 \\
575 \pm 47 \pm 65\end{array}$ & $\begin{array}{l}107 \pm 11 \pm 12 \\
120 \pm 15 \pm 14\end{array}$ & $8.3 \pm 1.7 \pm 1.0$ \\
\hline $\begin{array}{l}-0.500--0.375 \\
-0.375--0.250 \\
\end{array}$ & $\begin{array}{l}768 \pm 47 \pm 87 \\
792 \pm 46 \pm 89\end{array}$ & $\begin{array}{l}144 \pm 13 \pm 16 \\
200 \pm 14 \pm 23\end{array}$ & $13.8 \pm 1.8 \pm 1.6$ \\
\hline $\begin{array}{c}-0.250--0.125 \\
-0.125-0.000\end{array}$ & $\begin{array}{c}844 \pm 47 \pm 95 \\
883 \pm 49 \pm 100\end{array}$ & $\begin{array}{l}175 \pm 14 \pm 20 \\
204 \pm 13 \pm 23\end{array}$ & $20.0 \pm 2.0 \pm 2.4$ \\
\hline $\begin{array}{l}0.000-0.125 \\
0.125-0.250\end{array}$ & $\begin{array}{c}931 \pm 75 \pm 110 \\
756 \pm 87 \pm 85\end{array}$ & $\begin{array}{l}191 \pm 17 \pm 22 \\
187 \pm 20 \pm 21\end{array}$ & $16.8 \pm 1.9 \pm 2.0$ \\
\hline $\begin{array}{l}0.250-0.375 \\
0.375-0.500\end{array}$ & $\begin{array}{l}878 \pm 80 \pm 99 \\
622 \pm 73 \pm 70\end{array}$ & $\begin{array}{l}167 \pm 18 \pm 19 \\
145 \pm 16 \pm 17\end{array}$ & $13.7 \pm 1.9 \pm 1.6$ \\
\hline
\end{tabular}


Table A.10 Invariant differential cross section for $\pi^{0}$ production by $515 \mathrm{GeV} / c$ $\pi^{-}$beam on a $\mathrm{Cu}$ target as a function of $p_{T}$ and rapidity.

\begin{tabular}{|c|c|c|c|c|}
\hline \multirow[b]{2}{*}{ Rapidity } & \multicolumn{4}{|c|}{$p_{T}(\mathrm{GeV} / c)$} \\
\hline & $\begin{array}{c}2.50-3.00 \\
\mathrm{nb} /(\mathrm{GeV} / c)^{2}\end{array}$ & $\begin{array}{c}3.00-3.50 \\
\mathrm{nb} /(\mathrm{GeV} / c)^{2}\end{array}$ & $\begin{array}{c}3.50-4.00 \\
\mathrm{nb} /(\mathrm{GeV} / c)^{2}\end{array}$ & $\begin{array}{c}4.00-4.50 \\
\mathrm{nb} /(\mathrm{GeV} / c)^{2}\end{array}$ \\
\hline $\begin{array}{l}-0.750--0.625 \\
-0.625--0.500\end{array}$ & $497 \pm 55 \pm 57$ & $82 \pm 11 \pm 9.0$ & $10.7 \pm 1.6 \pm 1.2$ & $\begin{array}{l}2.77 \pm 0.21 \pm 0.30 \\
3.51 \pm 0.16 \pm 0.39\end{array}$ \\
\hline $\begin{array}{l}-0.500--0.375 \\
-0.375--0.250\end{array}$ & $426 \pm 39 \pm 49$ & $77.0 \pm 7.0 \pm 8.6$ & $20.2 \pm 1.4 \pm 2.2$ & $\begin{array}{l}4.02 \pm 0.13 \pm 0.44 \\
4.66 \pm 0.12 \pm 0.51\end{array}$ \\
\hline $\begin{array}{c}-0.250--0.125 \\
-0.125-0.000\end{array}$ & $492 \pm 28 \pm 56$ & $105.7 \pm 5.6 \pm 12$ & $22.9 \pm 1.2 \pm 2.5$ & $\begin{array}{l}5.07 \pm 0.11 \pm 0.56 \\
5.93 \pm 0.12 \pm 0.65\end{array}$ \\
\hline $\begin{array}{l}0.000-0.125 \\
0.125-0.250\end{array}$ & $502 \pm 26 \pm 58$ & $99.9 \pm 3.5 \pm 11$ & $22.1 \pm 1.2 \pm 2.5$ & $\begin{array}{l}5.56 \pm 0.11 \pm 0.61 \\
5.74 \pm 0.11 \pm 0.63\end{array}$ \\
\hline $\begin{array}{l}0.250-0.375 \\
0.375-0.500\end{array}$ & $413 \pm 21 \pm 47$ & $100.0 \pm 3.7 \pm 11$ & $21.0 \pm 1.2 \pm 2.3$ & $\begin{array}{l}5.98 \pm 0.11 \pm 0.66 \\
5.73 \pm 0.12 \pm 0.63\end{array}$ \\
\hline $\begin{array}{l}0.500-0.625 \\
0.625-0.750\end{array}$ & $390 \pm 23 \pm 45$ & $91.6 \pm 3.9 \pm 10$ & $21.6 \pm 1.2 \pm 2.4$ & $\begin{array}{l}5.47 \pm 0.12 \pm 0.60 \\
4.72 \pm 0.12 \pm 0.52\end{array}$ \\
\hline & $\begin{array}{c}4.50-5.00 \\
\mathrm{pb} /(\mathrm{GeV} / c)^{2}\end{array}$ & $\begin{array}{c}5.00-5.50 \\
\mathrm{pb} /(\mathrm{GeV} / c)^{2}\end{array}$ & $\begin{array}{c}5.50-6.50 \\
\mathrm{pb} /(\mathrm{GeV} / c)^{2}\end{array}$ & $\begin{array}{c}6.50-8.00 \\
\mathrm{pb} /(\mathrm{GeV} / c)^{2}\end{array}$ \\
\hline $\begin{array}{l}-0.750--0.625 \\
-0.625--0.500\end{array}$ & $\begin{array}{l}634 \pm 48 \pm 70 \\
899 \pm 48 \pm 99\end{array}$ & $\begin{array}{l}234 \pm 25 \pm 26 \\
262 \pm 22 \pm 29\end{array}$ & $\begin{array}{l}43.7 \pm 6.1 \pm 4.9 \\
50.7 \pm 7.7 \pm 5.7\end{array}$ & $2.05 \pm 0.69 \pm 0.24$ \\
\hline $\begin{array}{l}-0.500--0.375 \\
-0.375--0.250\end{array}$ & $\begin{array}{l}1078 \pm 48 \pm 120 \\
1218 \pm 48 \pm 130\end{array}$ & $\begin{array}{l}332 \pm 26 \pm 37 \\
350 \pm 23 \pm 39\end{array}$ & $\begin{array}{l}68.4 \pm 7.3 \pm 7.7 \\
72.5 \pm 7.0 \pm 8.1\end{array}$ & $4.91 \pm 0.97 \pm 0.57$ \\
\hline $\begin{array}{c}-0.250--0.125 \\
-0.125-0.000\end{array}$ & $\begin{array}{l}1426 \pm 51 \pm 160 \\
1634 \pm 55 \pm 180\end{array}$ & $\begin{array}{l}412 \pm 25 \pm 45 \\
380 \pm 24 \pm 42\end{array}$ & $\begin{array}{l}84.0 \pm 7.8 \pm 9.4 \\
86.4 \pm 7.8 \pm 9.7\end{array}$ & $7.3 \pm 1.1 \pm 0.8$ \\
\hline $\begin{array}{l}0.000-0.125 \\
0.125-0.250\end{array}$ & $\begin{array}{l}1655 \pm 52 \pm 180 \\
1518 \pm 50 \pm 170\end{array}$ & $\begin{array}{l}488 \pm 27 \pm 54 \\
439 \pm 25 \pm 48\end{array}$ & $\begin{array}{c}99.2 \pm 7.7 \pm 11 \\
120.3 \pm 8.5 \pm 14\end{array}$ & $8.2 \pm 1.2 \pm 1.0$ \\
\hline $\begin{array}{l}0.250-0.375 \\
0.375-0.500\end{array}$ & $\begin{array}{l}1685 \pm 55 \pm 190 \\
1593 \pm 55 \pm 180\end{array}$ & $\begin{array}{l}521 \pm 27 \pm 57 \\
465 \pm 28 \pm 51\end{array}$ & $\begin{array}{c}98.6 \pm 8.1 \pm 11 \\
85.7 \pm 7.7 \pm 9.6\end{array}$ & $7.7 \pm 1.3 \pm 0.9$ \\
\hline $\begin{array}{l}0.500-0.625 \\
0.625-0.750\end{array}$ & $\begin{array}{l}1523 \pm 57 \pm 170 \\
1265 \pm 51 \pm 140\end{array}$ & $\begin{array}{l}400 \pm 27 \pm 44 \\
315 \pm 23 \pm 35\end{array}$ & $\begin{array}{l}79.5 \pm 7.8 \pm 8.9 \\
73.0 \pm 7.8 \pm 8.2\end{array}$ & $5.9 \pm 1.3 \pm 0.7$ \\
\hline
\end{tabular}


Table A.11 Invariant differential cross section for $\pi^{0}$ production by $530 \mathrm{GeV} / c$ proton beam on a $p$ target as a function of $p_{T}$ and rapidity.

\begin{tabular}{|c|c|c|c|}
\hline Rapidity & $\begin{array}{c}1.00-2.50 \\
\mu \mathrm{b} /(\mathrm{GeV} / c)^{2}\end{array}$ & $\begin{array}{c}p_{T}(\mathrm{GeV} / c) \\
2.50-3.00 \\
\mathrm{nb} /(\mathrm{GeV} / c)^{2}\end{array}$ & $\begin{array}{r}3.00-3.50 \\
\mathrm{nb} /(\mathrm{GeV} / \mathrm{c})^{2}\end{array}$ \\
\hline $\begin{array}{l}-0.750--0.625 \\
-0.625--0.500 \\
\end{array}$ & $186 \pm 41 \pm 20$ & $\begin{aligned} 450 & \pm 210 \pm 50 \\
1000 & \pm 340 \pm 110\end{aligned}$ & $\begin{array}{l}72 \pm 11 \pm 8.0 \\
69 \pm 16 \pm 7.0 \\
\end{array}$ \\
\hline $\begin{array}{l}-0.500--0.375 \\
-0.375--0.250 \\
\end{array}$ & $124 \pm 35 \pm 13$ & $\begin{array}{l}390 \pm 43 \pm 41 \\
466 \pm 41 \pm 50 \\
\end{array}$ & $\begin{array}{c}66 \pm 11 \pm 7.0 \\
69.2 \pm 7.6 \pm 7.4 \\
\end{array}$ \\
\hline $\begin{array}{c}-0.250--0.125 \\
-0.125-0.000\end{array}$ & $79 \pm 33 \pm 8.0$ & $\begin{array}{l}484 \pm 28 \pm 52 \\
566 \pm 34 \pm 60\end{array}$ & $\begin{array}{l}81.0 \pm 7.3 \pm 8.6 \\
87.1 \pm 7.7 \pm 9.3\end{array}$ \\
\hline $\begin{array}{l}0.000-0.125 \\
0.125-0.250\end{array}$ & $121 \pm 28 \pm 13$ & $\begin{array}{l}484 \pm 21 \pm 51 \\
449 \pm 20 \pm 48\end{array}$ & $\begin{array}{l}83.0 \pm 6.8 \pm 8.8 \\
71.9 \pm 5.7 \pm 7.6\end{array}$ \\
\hline $\begin{array}{l}0.250-0.375 \\
0.375-0.500\end{array}$ & $111 \pm 28 \pm 12$ & $\begin{array}{l}420 \pm 19 \pm 45 \\
390 \pm 18 \pm 42\end{array}$ & $\begin{array}{l}67.6 \pm 6.1 \pm 7.2 \\
67.6 \pm 6.3 \pm 7.2\end{array}$ \\
\hline $\begin{array}{l}0.500-0.625 \\
0.625-0.750\end{array}$ & $138 \pm 29 \pm 15$ & $\begin{array}{l}366 \pm 19 \pm 39 \\
319 \pm 19 \pm 34\end{array}$ & $\begin{array}{l}60.6 \pm 6.5 \pm 6.4 \\
52.1 \pm 6.2 \pm 5.5\end{array}$ \\
\hline & $\begin{array}{c}3.50-4.00 \\
\mathrm{nb} /(\mathrm{GeV} / \mathrm{c})^{2}\end{array}$ & $\begin{array}{c}4.00-4.50 \\
\mathrm{nb} /(\mathrm{GeV} / c)^{2}\end{array}$ & $\begin{array}{c}4.50-5.00 \\
\mathrm{pb} /(\mathrm{GeV} / c)^{2}\end{array}$ \\
\hline $\begin{array}{l}-0.750--0.625 \\
-0.625--0.500\end{array}$ & $\begin{array}{c}9.2 \pm 1.4 \pm 1.0 \\
10.6 \pm 1.5 \pm 1.1\end{array}$ & $\begin{array}{l}2.14 \pm 0.15 \pm 0.23 \\
2.60 \pm 0.15 \pm 0.28\end{array}$ & $\begin{array}{l}652 \pm 66 \pm 71 \\
544 \pm 49 \pm 59\end{array}$ \\
\hline $\begin{array}{l}-0.500--0.375 \\
-0.375--0.250\end{array}$ & $\begin{array}{l}13.1 \pm 2.9 \pm 1.4 \\
15.8 \pm 2.4 \pm 1.7\end{array}$ & $\begin{array}{l}2.91 \pm 0.12 \pm 0.31 \\
3.54 \pm 0.13 \pm 0.38\end{array}$ & $\begin{array}{l}686 \pm 39 \pm 75 \\
886 \pm 48 \pm 97\end{array}$ \\
\hline $\begin{array}{c}-0.250--0.125 \\
-0.125-0.000\end{array}$ & $\begin{array}{l}16.4 \pm 2.6 \pm 1.8 \\
15.5 \pm 2.6 \pm 1.7\end{array}$ & $\begin{array}{c}3.518 \pm 0.096 \pm 0.38 \\
3.78 \pm 0.10 \pm 0.41\end{array}$ & $\begin{array}{c}858 \pm 40 \pm 94 \\
974 \pm 42 \pm 110 \\
\end{array}$ \\
\hline $\begin{array}{l}0.000-0.125 \\
0.125-0.250\end{array}$ & $\begin{array}{l}14.5 \pm 2.4 \pm 1.6 \\
14.3 \pm 1.8 \pm 1.5\end{array}$ & $\begin{array}{l}3.780 \pm 0.097 \pm 0.41 \\
3.512 \pm 0.088 \pm 0.38\end{array}$ & $\begin{array}{l}857 \pm 36 \pm 94 \\
896 \pm 39 \pm 98\end{array}$ \\
\hline $\begin{array}{l}0.250-0.375 \\
0.375-0.500\end{array}$ & $\begin{array}{l}11.9 \pm 1.7 \pm 1.3 \\
15.0 \pm 2.1 \pm 1.6\end{array}$ & $\begin{array}{l}3.153 \pm 0.077 \pm 0.34 \\
2.958 \pm 0.083 \pm 0.32\end{array}$ & $\begin{array}{l}767 \pm 35 \pm 84 \\
723 \pm 35 \pm 79\end{array}$ \\
\hline $\begin{array}{l}0.500-0.625 \\
0.625-0.750\end{array}$ & $\begin{array}{c}11.8 \pm 2.3 \pm 1.3 \\
8.2 \pm 1.7 \pm 0.9\end{array}$ & $\begin{array}{l}2.501 \pm 0.080 \pm 0.27 \\
2.037 \pm 0.073 \pm 0.22\end{array}$ & $\begin{array}{l}659 \pm 36 \pm 72 \\
476 \pm 31 \pm 52\end{array}$ \\
\hline & $\begin{array}{c}5.00-5.50 \\
\mathrm{pb} /(\mathrm{GeV} / c)^{2}\end{array}$ & $\begin{array}{c}5.50-6.50 \\
\mathrm{pb} /(\mathrm{GeV} / c)^{2}\end{array}$ & $\begin{array}{c}6.50-8.00 \\
\mathrm{pb} /(\mathrm{GeV} / c)^{2}\end{array}$ \\
\hline $\begin{array}{l}-0.750--0.625 \\
-0.625--0.500\end{array}$ & $\begin{array}{l}110 \pm 13 \pm 12 \\
175 \pm 23 \pm 19\end{array}$ & $\begin{array}{l}29.2 \pm 4.9 \pm 3.3 \\
22.2 \pm 4.4 \pm 2.5\end{array}$ & $2.12 \pm 0.60 \pm 0.25$ \\
\hline $\begin{array}{l}-0.500--0.375 \\
-0.375--0.250 \\
\end{array}$ & $\begin{array}{l}219 \pm 20 \pm 24 \\
204 \pm 18 \pm 23\end{array}$ & $\begin{array}{l}43.0 \pm 5.7 \pm 4.9 \\
42.4 \pm 5.2 \pm 4.8 \\
\end{array}$ & $2.77 \pm 0.70 \pm 0.33$ \\
\hline $\begin{array}{c}-0.250--0.125 \\
-0.125-0.000\end{array}$ & $\begin{array}{l}245 \pm 19 \pm 27 \\
252 \pm 20 \pm 28\end{array}$ & $\begin{array}{l}43.8 \pm 5.5 \pm 5.0 \\
66.4 \pm 6.8 \pm 7.5\end{array}$ & $3.16 \pm 0.73 \pm 0.38$ \\
\hline $\begin{array}{l}0.000-0.125 \\
0.125-0.250\end{array}$ & $\begin{array}{l}282 \pm 20 \pm 31 \\
265 \pm 19 \pm 29\end{array}$ & $\begin{array}{l}55.0 \pm 5.8 \pm 6.2 \\
55.3 \pm 5.9 \pm 6.3\end{array}$ & $3.84 \pm 0.74 \pm 0.46$ \\
\hline $\begin{array}{l}0.250-0.375 \\
0.375-0.500 \\
\end{array}$ & $\begin{array}{l}249 \pm 19 \pm 28 \\
211 \pm 18 \pm 23 \\
\end{array}$ & $\begin{array}{l}40.8 \pm 5.1 \pm 4.6 \\
33.0 \pm 4.6 \pm 3.7 \\
\end{array}$ & $1.79 \pm 0.52 \pm 0.21$ \\
\hline $\begin{array}{l}0.500-0.625 \\
0.625-0.750\end{array}$ & $\begin{array}{l}189 \pm 18 \pm 21 \\
124 \pm 15 \pm 14\end{array}$ & $\begin{array}{l}26.8 \pm 4.4 \pm 3.0 \\
23.1 \pm 4.3 \pm 2.6\end{array}$ & $0.96 \pm 0.43 \pm 0.11$ \\
\hline
\end{tabular}


Table A.12 Invariant differential cross section for $\pi^{0}$ production by $800 \mathrm{GeV} / c$ proton beam on a $p$ target as a function of $p_{T}$ and rapidity.

\begin{tabular}{|c|c|c|c|}
\hline Rapidity & $\begin{array}{c}1.00-2.50 \\
\mu \mathrm{b} /(\mathrm{GeV} / c)^{2}\end{array}$ & $\begin{array}{c}p_{T}(\mathrm{GeV} / c) \\
2.50-3.00 \\
\mathrm{nb} /(\mathrm{GeV} / c)^{2}\end{array}$ & $\begin{array}{c}3.00-3.50 \\
\mathrm{nb} /(\mathrm{GeV} / c)^{2}\end{array}$ \\
\hline $\begin{array}{c}-1.00--0.875 \\
-0.875--0.750\end{array}$ & $159 \pm 65 \pm 18$ & $\begin{aligned} 1060 & \pm 630 \pm 120 \\
430 & \pm 120 \pm 50\end{aligned}$ & $\begin{array}{c}87 \pm 17 \pm 10.0 \\
115 \pm 15 \pm 13\end{array}$ \\
\hline $\begin{array}{l}-0.750--0.625 \\
-0.625--0.500\end{array}$ & $238 \pm 62 \pm 26$ & $\begin{array}{c}444 \pm 66 \pm 49 \\
610 \pm 110 \pm 70\end{array}$ & $\begin{array}{l}106 \pm 15 \pm 12 \\
117 \pm 13 \pm 13\end{array}$ \\
\hline $\begin{array}{l}-0.500--0.375 \\
-0.375--0.250\end{array}$ & $286 \pm 57 \pm 32$ & $\begin{array}{l}600 \pm 120 \pm 70 \\
640 \pm 100 \pm 70\end{array}$ & $\begin{array}{l}153 \pm 15 \pm 17 \\
138 \pm 14 \pm 15\end{array}$ \\
\hline $\begin{array}{c}-0.250--0.125 \\
-0.125-0.000\end{array}$ & $265 \pm 52 \pm 29$ & $\begin{array}{l}800 \pm 180 \pm 90 \\
680 \pm 120 \pm 80\end{array}$ & $\begin{array}{l}123 \pm 15 \pm 14 \\
124 \pm 13 \pm 14\end{array}$ \\
\hline $\begin{array}{l}0.000-0.125 \\
0.125-0.250\end{array}$ & $199 \pm 44 \pm 22$ & $\begin{array}{l}707 \pm 100 \pm 78 \\
760 \pm 110 \pm 80\end{array}$ & $\begin{array}{l}134 \pm 11 \pm 15 \\
137 \pm 11 \pm 15\end{array}$ \\
\hline $\begin{array}{l}0.250-0.375 \\
0.375-0.500\end{array}$ & $236 \pm 43 \pm 26$ & $\begin{array}{c}540 \pm 88 \pm 60 \\
740 \pm 100 \pm 80\end{array}$ & $\begin{array}{l}122 \pm 12 \pm 13 \\
108 \pm 13 \pm 12\end{array}$ \\
\hline & $\begin{array}{c}3.50-4.00 \\
\mathrm{nb} /(\mathrm{GeV} / \mathrm{c})^{2}\end{array}$ & $\begin{array}{c}4.00-4.50 \\
\mathrm{nb} /(\mathrm{GeV} / \mathrm{c})^{2}\end{array}$ & $\begin{array}{c}4.50-5.00 \\
\mathrm{pb} /(\mathrm{GeV} / \mathrm{c})^{2}\end{array}$ \\
\hline $\begin{array}{c}-1.00--0.875 \\
-0.875--0.750\end{array}$ & $\begin{array}{l}20.2 \pm 7.8 \pm 2.2 \\
19.8 \pm 4.3 \pm 2.2\end{array}$ & $\begin{array}{l}3.20 \pm 0.26 \pm 0.36 \\
4.92 \pm 0.69 \pm 0.55\end{array}$ & $\begin{array}{c}1250 \pm 130 \pm 140 \\
1015 \pm 77 \pm 110\end{array}$ \\
\hline $\begin{array}{l}-0.750--0.625 \\
-0.625--0.500\end{array}$ & $\begin{array}{l}38.8 \pm 7.2 \pm 4.3 \\
19.0 \pm 3.8 \pm 2.1\end{array}$ & $\begin{array}{l}9.0 \pm 2.3 \pm 1.0 \\
7.8 \pm 1.6 \pm 0.9\end{array}$ & $\begin{array}{l}1427 \pm 90 \pm 160 \\
1590 \pm 98 \pm 180\end{array}$ \\
\hline $\begin{array}{l}-0.500--0.375 \\
-0.375--0.250\end{array}$ & $\begin{array}{l}28.2 \pm 5.8 \pm 3.1 \\
39.2 \pm 6.3 \pm 4.3\end{array}$ & $\begin{array}{l}6.75 \pm 0.65 \pm 0.75 \\
7.27 \pm 0.65 \pm 0.81\end{array}$ & $\begin{array}{l}1911 \pm 92 \pm 210 \\
2013 \pm 86 \pm 230\end{array}$ \\
\hline $\begin{array}{c}-0.250--0.125 \\
-0.125-0.000\end{array}$ & $\begin{array}{l}32.9 \pm 5.5 \pm 3.6 \\
21.8 \pm 4.4 \pm 2.4\end{array}$ & $\begin{array}{l}6.34 \pm 0.21 \pm 0.70 \\
6.49 \pm 0.37 \pm 0.72\end{array}$ & $\begin{array}{l}2075 \pm 87 \pm 230 \\
1984 \pm 86 \pm 220\end{array}$ \\
\hline $\begin{array}{l}0.000-0.125 \\
0.125-0.250\end{array}$ & $\begin{array}{l}25.1 \pm 4.8 \pm 2.8 \\
27.9 \pm 4.3 \pm 3.1\end{array}$ & $\begin{array}{c}8.7 \pm 1.1 \pm 1.0 \\
6.55 \pm 0.60 \pm 0.73\end{array}$ & $\begin{array}{l}1580 \pm 140 \pm 180 \\
1810 \pm 230 \pm 200\end{array}$ \\
\hline \multirow[t]{2}{*}{$0.375-0.500$} & $\begin{array}{l}23.8 \pm 3.8 \pm 2.6 \\
23.7 \pm 5.4 \pm 2.6\end{array}$ & $\begin{array}{l}7.49 \pm 0.60 \pm 0.83 \\
6.01 \pm 0.54 \pm 0.67\end{array}$ & $\begin{array}{l}1770 \pm 210 \pm 200 \\
1920 \pm 220 \pm 210\end{array}$ \\
\hline & $\begin{array}{c}5.00-5.50 \\
\mathrm{pb} /(\mathrm{GeV} / \mathrm{c})^{2}\end{array}$ & $\begin{array}{c}5.50-6.50 \\
\mathrm{pb} /(\mathrm{GeV} / \mathrm{c})^{2}\end{array}$ & $\begin{array}{c}6.50-8.00 \\
\mathrm{pb} /(\mathrm{GeV} / \mathrm{c})^{2}\end{array}$ \\
\hline $\begin{array}{c}-1.00--0.875 \\
-0.875--0.750\end{array}$ & $\begin{array}{l}297 \pm 49 \pm 33 \\
229 \pm 27 \pm 26\end{array}$ & $\begin{array}{c}32.1 \pm 5.5 \pm 3.7 \\
73 \pm 14 \pm 8.0\end{array}$ & $4.4 \pm 1.1 \pm 0.5$ \\
\hline $\begin{array}{l}-0.750--0.625 \\
-0.625--0.500\end{array}$ & $\begin{array}{l}350 \pm 36 \pm 39 \\
448 \pm 38 \pm 51\end{array}$ & $\begin{array}{c}63.8 \pm 8.8 \pm 7.3 \\
105 \pm 12 \pm 12\end{array}$ & $9.3 \pm 1.7 \pm 1.1$ \\
\hline $\begin{array}{l}-0.500--0.375 \\
-0.375--0.250\end{array}$ & $\begin{array}{l}507 \pm 44 \pm 57 \\
547 \pm 41 \pm 62\end{array}$ & $\begin{array}{c}90 \pm 10 \pm 10 \\
132 \pm 12 \pm 15\end{array}$ & $14.8 \pm 2.0 \pm 1.7$ \\
\hline $\begin{array}{c}-0.250--0.125 \\
-0.125-0.000\end{array}$ & $\begin{array}{l}629 \pm 43 \pm 71 \\
585 \pm 41 \pm 66\end{array}$ & $\begin{array}{l}117 \pm 11 \pm 13 \\
148 \pm 14 \pm 17\end{array}$ & $14.6 \pm 1.8 \pm 1.7$ \\
\hline $\begin{array}{l}0.000-0.125 \\
0.125-0.250\end{array}$ & $\begin{array}{l}643 \pm 72 \pm 73 \\
600 \pm 93 \pm 68\end{array}$ & $\begin{array}{l}159 \pm 18 \pm 18 \\
118 \pm 15 \pm 13\end{array}$ & $13.4 \pm 2.2 \pm 1.6$ \\
\hline $\begin{array}{l}0.250-0.375 \\
0.375-0.500\end{array}$ & $\begin{array}{l}487 \pm 65 \pm 55 \\
530 \pm 67 \pm 60\end{array}$ & $\begin{array}{l}103 \pm 13 \pm 12 \\
138 \pm 18 \pm 16\end{array}$ & $10.2 \pm 1.9 \pm 1.2$ \\
\hline
\end{tabular}


Table A.13 Invariant differential cross section for $\pi^{0}$ production by $515 \mathrm{GeV} / c$ $\pi^{-}$beam on a $p$ target as a function of $p_{T}$ and rapidity.

\begin{tabular}{|c|c|c|c|c|}
\hline \multirow[b]{2}{*}{ Rapidity } & \multicolumn{4}{|c|}{$p_{T}(\mathrm{GeV} / c)$} \\
\hline & $\begin{array}{c}2.50-3.00 \\
\mathrm{nb} /(\mathrm{GeV} / c)^{2}\end{array}$ & $\begin{array}{c}3.00-3.50 \\
\mathrm{nb} /(\mathrm{GeV} / c)^{2}\end{array}$ & $\begin{array}{c}3.50-4.00 \\
\mathrm{nb} /(\mathrm{GeV} / c)^{2}\end{array}$ & $\begin{array}{c}4.00-4.50 \\
\mathrm{nb} /(\mathrm{GeV} / c)^{2}\end{array}$ \\
\hline $\begin{array}{l}-0.750--0.625 \\
-0.625--0.500\end{array}$ & $293 \pm 71 \pm 34$ & $51 \pm 15 \pm 6.0$ & $5.7 \pm 3.7 \pm 0.6$ & $\begin{array}{l}1.97 \pm 0.29 \pm 0.22 \\
2.96 \pm 0.34 \pm 0.33\end{array}$ \\
\hline $\begin{array}{l}-0.500--0.375 \\
-0.375--0.250\end{array}$ & $300 \pm 62 \pm 34$ & $51.1 \pm 9.5 \pm 5.7$ & $4.0 \pm 2.9 \pm 0.4$ & $\begin{array}{l}3.61 \pm 0.35 \pm 0.40 \\
3.85 \pm 0.29 \pm 0.42\end{array}$ \\
\hline $\begin{array}{c}-0.250--0.125 \\
-0.125-0.000\end{array}$ & $297 \pm 32 \pm 34$ & $77 \pm 12 \pm 9.0$ & $16.2 \pm 3.0 \pm 1.8$ & $\begin{array}{l}3.61 \pm 0.29 \pm 0.40 \\
3.91 \pm 0.21 \pm 0.43\end{array}$ \\
\hline $\begin{array}{l}0.000-0.125 \\
0.125-0.250\end{array}$ & $351 \pm 31 \pm 40$ & $77 \pm 10 \pm 9.0$ & $22.9 \pm 4.0 \pm 2.5$ & $\begin{array}{l}4.66 \pm 0.23 \pm 0.51 \\
4.10 \pm 0.21 \pm 0.45\end{array}$ \\
\hline $\begin{array}{l}0.250-0.375 \\
0.375-0.500\end{array}$ & $373 \pm 26 \pm 43$ & $68.1 \pm 9.3 \pm 7.7$ & $20.9 \pm 3.3 \pm 2.3$ & $\begin{array}{l}4.26 \pm 0.20 \pm 0.47 \\
4.02 \pm 0.22 \pm 0.44\end{array}$ \\
\hline $\begin{array}{l}0.500-0.625 \\
0.625-0.750\end{array}$ & $291 \pm 28 \pm 33$ & $73 \pm 11 \pm 8.0$ & $16.8 \pm 4.6 \pm 1.9$ & $\begin{array}{l}3.92 \pm 0.25 \pm 0.43 \\
3.34 \pm 0.24 \pm 0.37\end{array}$ \\
\hline & $\begin{array}{c}4.50-5.00 \\
\mathrm{pb} /(\mathrm{GeV} / c)^{2}\end{array}$ & $\begin{array}{c}5.00-5.50 \\
\mathrm{pb} /(\mathrm{GeV} / c)^{2}\end{array}$ & $\begin{array}{c}5.50-6.50 \\
\mathrm{pb} /(\mathrm{GeV} / c)^{2}\end{array}$ & $\begin{array}{c}6.50-8.00 \\
\mathrm{pb} /(\mathrm{GeV} / c)^{2}\end{array}$ \\
\hline $\begin{array}{l}-0.750--0.625 \\
-0.625--0.500\end{array}$ & $\begin{array}{c}493 \pm 91 \pm 54 \\
680 \pm 110 \pm 70\end{array}$ & $\begin{array}{l}119 \pm 30 \pm 13 \\
263 \pm 68 \pm 29\end{array}$ & $\begin{array}{l}46 \pm 15 \pm 5.0 \\
69 \pm 18 \pm 8.0\end{array}$ & $3.2 \pm 1.9 \pm 0.4$ \\
\hline $\begin{array}{l}-0.500--0.375 \\
-0.375--0.250\end{array}$ & $\begin{array}{c}910 \pm 100 \pm 100 \\
905 \pm 95 \pm 99\end{array}$ & $\begin{array}{l}322 \pm 55 \pm 36 \\
279 \pm 46 \pm 31\end{array}$ & $\begin{array}{l}77 \pm 17 \pm 9.0 \\
72 \pm 17 \pm 8.0\end{array}$ & $2.2 \pm 1.3 \pm 0.3$ \\
\hline $\begin{array}{c}-0.250--0.125 \\
-0.125-0.000\end{array}$ & $\begin{array}{c}1129 \pm 96 \pm 120 \\
1170 \pm 100 \pm 130\end{array}$ & $\begin{array}{l}368 \pm 50 \pm 41 \\
410 \pm 55 \pm 45\end{array}$ & $\begin{array}{l}62 \pm 15 \pm 7.0 \\
62 \pm 13 \pm 7.0\end{array}$ & $7.5 \pm 2.5 \pm 0.9$ \\
\hline $\begin{array}{l}0.000-0.125 \\
0.125-0.250\end{array}$ & $\begin{array}{c}1122 \pm 95 \pm 120 \\
1450 \pm 110 \pm 160\end{array}$ & $\begin{array}{l}326 \pm 46 \pm 36 \\
342 \pm 49 \pm 38\end{array}$ & $\begin{array}{c}89 \pm 16 \pm 10.0 \\
70 \pm 14 \pm 8.0\end{array}$ & $9.3 \pm 2.7 \pm 1.1$ \\
\hline $\begin{array}{l}0.250-0.375 \\
0.375-0.500\end{array}$ & $\begin{array}{l}1290 \pm 100 \pm 140 \\
1107 \pm 100 \pm 120\end{array}$ & $\begin{array}{l}382 \pm 51 \pm 42 \\
362 \pm 51 \pm 40\end{array}$ & $\begin{array}{l}103 \pm 18 \pm 12 \\
59 \pm 15 \pm 7.0\end{array}$ & $7.6 \pm 2.4 \pm 0.9$ \\
\hline $\begin{array}{l}0.500-0.625 \\
0.625-0.750\end{array}$ & $\begin{array}{c}1100 \pm 110 \pm 120 \\
930 \pm 110 \pm 100\end{array}$ & $\begin{array}{l}255 \pm 48 \pm 28 \\
224 \pm 47 \pm 25\end{array}$ & $\begin{array}{l}57 \pm 14 \pm 6.0 \\
59 \pm 16 \pm 7.0\end{array}$ & $7.7 \pm 2.7 \pm 0.9$ \\
\hline
\end{tabular}




\section{Appendix B Tabulated Direct Photon Cross Sections}

In this appendix, the measured direct photon cross sections are presented in

tabular form. The entries in the tables are given in the form $A \pm B \pm C$, where $\mathrm{A}$ is the cross section measurement, and $\mathrm{B}$ and $\mathrm{C}$ represent the statistical and systematic uncertainties, respectively, on the measurement. 
Table B.1 Invariant differential cross sections per nucleon for direct photon production by 530 and $800 \mathrm{GeV} / c$ proton beams and $515 \mathrm{GeV} / c \pi^{-}$ beam on Be targets.

\begin{tabular}{|c|c|c|c|}
\hline \multirow{2}{*}{$\begin{array}{c}p_{T} \text { Range } \\
(\mathrm{GeV} / c)\end{array}$} & $p$ Be at $530 \mathrm{GeV} / c$ & $p$ Be at $800 \mathrm{GeV} / c$ & $\pi^{-}$Be at $515 \mathrm{GeV} / c$ \\
& $-0.75<y<0.75$ & $-1.0<y<0.5$ & $-0.75<y<0.75$ \\
\hline $3.50-3.75$ & $1860 \pm 170 \pm 370$ & $3070 \pm 240 \pm 640$ & $1810 \pm 100 \pm 340$ \\
\hline $3.75-4.00$ & $1010 \pm 10 \pm 190$ & $1540 \pm 10 \pm 290$ & $935 \pm 10 \pm 160$ \\
\hline $4.00-4.25$ & $484 \pm 10 \pm 82$ & $927 \pm 10 \pm 163$ & $511 \pm 1 \pm 81$ \\
\hline $4.25-4.50$ & $254 \pm 1 \pm 40$ & $492 \pm 10 \pm 80$ & $292 \pm 1 \pm 43$ \\
\hline $4.50-4.75$ & $135 \pm 1 \pm 20$ & $273 \pm 1 \pm 42$ & $175 \pm 1 \pm 24$ \\
\hline $4.75-5.00$ & $76.4 \pm 2.1 \pm 10.8$ & $165 \pm 1 \pm 24$ & $109 \pm 1 \pm 14$ \\
\hline $5.00-5.25$ & $41.7 \pm 1.4 \pm 5.6$ & $100 \pm 1 \pm 14$ & $71.2 \pm 1.8 \pm 9.0$ \\
\hline $5.25-5.50$ & $24.6 \pm 1.0 \pm 3.2$ & $64.7 \pm 2.3 \pm 8.5$ & $42.9 \pm 1.3 \pm 5.3$ \\
\hline $5.50-5.75$ & $15.3 \pm 0.7 \pm 1.9$ & $38.3 \pm 1.6 \pm 4.9$ & $30.8 \pm 1.0 \pm 3.7$ \\
\hline $5.75-6.00$ & $8.67 \pm 0.55 \pm 1.08$ & $27.1 \pm 1.3 \pm 3.4$ & $20.1 \pm 0.8 \pm 2.4$ \\
\hline $6.00-6.50$ & $4.00 \pm 0.25 \pm 0.49$ & $13.0 \pm 0.5 \pm 1.6$ & $10.4 \pm 0.4 \pm 1.2$ \\
\hline $6.50-7.00$ & $1.80 \pm 0.15 \pm 0.22$ & $5.43 \pm 0.30 \pm 0.64$ & $4.75 \pm 0.23 \pm 0.55$ \\
\hline $7.00-7.50$ & $0.72 \pm 0.09 \pm 0.09$ & $2.54 \pm 0.19 \pm 0.29$ & $2.12 \pm 0.15 \pm 0.25$ \\
\hline $7.50-8.00$ & $0.33 \pm 0.05 \pm 0.04$ & $1.12 \pm 0.12 \pm 0.13$ & $1.15 \pm 0.10 \pm 0.13$ \\
\hline $8.00-9.00$ & $0.08 \pm 0.02 \pm 0.010$ & $0.42 \pm 0.05 \pm 0.05$ & $0.45 \pm 0.04 \pm 0.05$ \\
\hline $9.00-10.00$ & $0.008 \pm 0.005 \pm 0.001$ & $0.09 \pm 0.02 \pm 0.01$ & $0.08 \pm 0.02 \pm 0.01$ \\
\hline $10.00-12.00$ & $0.001 \pm 0.001 \pm 0.0002$ & & $0.006 \pm 0.003 \pm 0.0008$ \\
\hline
\end{tabular}


Table B.2 Invariant differential cross sections per nucleon for direct photon production by 530 and $800 \mathrm{GeV} / c$ proton beams and $515 \mathrm{GeV} / c \pi^{-}$ beam on $\mathrm{Cu}$ targets.

\begin{tabular}{|c|c|c|c|}
\hline \multirow{2}{*}{$\begin{array}{c}p_{T} \text { Range } \\
(\mathrm{GeV} / c)\end{array}$} & $p \mathrm{4}$ at $530 \mathrm{GeV} / c$ & $p \mathrm{Cu}$ at $800 \mathrm{GeV} / c$ & $\pi^{-} \mathrm{Cu}$ at $515 \mathrm{GeV} / c$ \\
& $-0.75<y<0.75$ & $-1.0<y<0.5$ & $-0.75<y<0.75$ \\
\hline $3.50-3.75$ & $3110 \pm 450 \pm 630$ & $3010 \pm 590 \pm 630$ & $1840 \pm 280 \pm 340$ \\
\hline $3.75-4.00$ & $1210 \pm 150 \pm 220$ & $1870 \pm 230 \pm 360$ & $1070 \pm 23 \pm 180$ \\
\hline $4.00-4.25$ & $583 \pm 23 \pm 10$ & $989 \pm 23 \pm 176$ & $585 \pm 23 \pm 93$ \\
\hline $4.25-4.50$ & $282 \pm 23 \pm 45$ & $523 \pm 23 \pm 87$ & $361 \pm 23 \pm 54$ \\
\hline $4.50-4.75$ & $152 \pm 2 \pm 23$ & $299 \pm 23 \pm 47$ & $178 \pm 2 \pm 25$ \\
\hline $4.75-5.00$ & $86.5 \pm 5.2 \pm 12.3$ & $182 \pm 23 \pm 27$ & $112 \pm 2 \pm 15$ \\
\hline $5.00-5.25$ & $50.2 \pm 3.5 \pm 6.8$ & $111 \pm 2 \pm 16$ & $81.0 \pm 4.8 \pm 10.4$ \\
\hline $5.25-5.50$ & $27.7 \pm 2.5 \pm 3.6$ & $59.9 \pm 5.5 \pm 8.1$ & $40.4 \pm 3.4 \pm 5.0$ \\
\hline $5.50-5.75$ & $15.6 \pm 1.8 \pm 2.0$ & $37.0 \pm 4.1 \pm 4.8$ & $32.3 \pm 2.7 \pm 3.9$ \\
\hline $5.75-6.00$ & $11.2 \pm 1.3 \pm 1.4$ & $21.4 \pm 2.9 \pm 2.7$ & $23.1 \pm 2.1 \pm 2.8$ \\
\hline $6.00-6.50$ & $4.99 \pm 0.62 \pm 0.62$ & $15.4 \pm 1.3 \pm 1.9$ & $9.74 \pm 0.95 \pm 1.15$ \\
\hline $6.50-7.00$ & $1.93 \pm 0.34 \pm 0.24$ & $5.43 \pm 0.72 \pm 0.66$ & $5.30 \pm 0.60 \pm 0.62$ \\
\hline $7.00-8.00$ & $0.28 \pm 0.09 \pm 0.04$ & $2.01 \pm 0.27 \pm 0.24$ & $1.84 \pm 0.23 \pm 0.22$ \\
\hline $8.00-10.00$ & $0.09 \pm 0.03 \pm 0.01$ & $0.14 \pm 0.06 \pm 0.02$ & $0.20 \pm 0.05 \pm 0.02$ \\
\hline
\end{tabular}


Table B.3 Invariant differential cross sections for direct photon production by 530 and $800 \mathrm{GeV} / c$ proton beams and $515 \mathrm{GeV} / c \pi^{-}$beam on proton targets.

\begin{tabular}{|c|c|c|c|}
\hline \multirow{2}{*}{$\begin{array}{c}p_{T} \text { Range } \\
(\mathrm{GeV} / c)\end{array}$} & $p p$ at $530 \mathrm{GeV} / c$ & $p p$ at $800 \mathrm{GeV} / c$ & $\pi^{-} p$ at $515 \mathrm{GeV} / c$ \\
& $-0.75<y<0.75$ & $-1.0<y<0.5$ & $-0.75<y<0.75$ \\
\hline $3.50-4.00$ & $1200 \pm 200 \pm 240$ & $2020 \pm 320 \pm 410$ & $1750 \pm 420 \pm 320$ \\
\hline $4.00-4.50$ & $388 \pm 42 \pm 66$ & $642 \pm 42 \pm 112$ & $500 \pm 42 \pm 79$ \\
\hline $4.50-5.00$ & $111 \pm 4 \pm 17$ & $241 \pm 42 \pm 37$ & $168 \pm 42 \pm 24$ \\
\hline $5.00-5.50$ & $34.6 \pm 2.2 \pm 4.8$ & $87.5 \pm 4.7 \pm 12.3$ & $65.6 \pm 6.0 \pm 8.6$ \\
\hline $5.50-6.00$ & $11.8 \pm 1.2 \pm 1.5$ & $37.2 \pm 2.6 \pm 4.9$ & $25.8 \pm 3.5 \pm 3.2$ \\
\hline $6.00-7.00$ & $3.53 \pm 0.38 \pm 0.46$ & $10.4 \pm 0.8 \pm 1.3$ & $9.03 \pm 1.26 \pm 1.11$ \\
\hline $7.00-8.00$ & $0.43 \pm 0.12 \pm 0.06$ & $1.83 \pm 0.30 \pm 0.22$ & $2.83 \pm 0.62 \pm 0.35$ \\
\hline $8.00-10.00$ & $0.010 \pm 0.02 \pm 0.001$ & $0.36 \pm 0.07 \pm 0.04$ & $0.31 \pm 0.14 \pm 0.04$ \\
\hline $10.00-12.00$ & - & $0.02 \pm 0.01 \pm 0.002$ & \\
\hline
\end{tabular}


Table B.4 Invariant differential cross section per nucleon for the inclusive reaction $\mathrm{pBe} \rightarrow \gamma \mathrm{X}$ at $530 \mathrm{GeV} / c$ as a function of rapidity for several $p_{T}$ bins.

\begin{tabular}{|c|c|c|c|c|}
\hline \multirow{2}{*}{ Rapidity } & \multicolumn{4}{|c|}{$p_{T}(\mathrm{GeV} / c)$} \\
& $\begin{array}{c}3.50-4.00 \\
\mathrm{pb} /(\mathrm{GeV} / c)^{2}\end{array}$ & $\begin{array}{c}4.00-4.50 \\
\mathrm{pb} /(\mathrm{GeV} / c)^{2}\end{array}$ & $\begin{array}{c}4.50-5.00 \\
\mathrm{pb} /(\mathrm{GeV} / c)^{2}\end{array}$ & $\begin{array}{c}5.00-5.50 \\
\mathrm{pb} /(\mathrm{GeV} / c)^{2}\end{array}$ \\
\hline$-0.75--0.50$ & $1200 \pm 230 \pm 220$ & $352 \pm 14 \pm 59$ & $91.1 \pm 4.4 \pm 13$ & $23.3 \pm 1.9 \pm 3.1$ \\
$-0.50--0.25$ & $1530 \pm 220 \pm 280$ & $396 \pm 12 \pm 67$ & $109.4 \pm 4.6 \pm 16$ & $29.2 \pm 2.1 \pm 3.9$ \\
\hline$-0.25-0.00$ & $1450 \pm 220 \pm 270$ & $380 \pm 11 \pm 64$ & $109.5 \pm 4.8 \pm 16$ & $37.7 \pm 2.4 \pm 5.0$ \\
$0.00-0.25$ & $1570 \pm 200 \pm 290$ & $386 \pm 10 \pm 65$ & $111.3 \pm 4.4 \pm 16$ & $38.6 \pm 2.3 \pm 5.1$ \\
\hline $0.25-0.50$ & $1630 \pm 160 \pm 300$ & $381.9 \pm 9.5 \pm 64$ & $115.6 \pm 4.3 \pm 17$ & $38.1 \pm 2.2 \pm 5.1$ \\
$0.50-0.75$ & $1170 \pm 270 \pm 210$ & $302 \pm 53 \pm 51$ & $90.4 \pm 4.1 \pm 13$ & $29.1 \pm 2.0 \pm 3.9$ \\
\hline & $5.50-6.50$ & $6.50-8.00$ & $8.00-10.00$ & \\
\hline$-0.75--0.50$ & $5.35 \pm 0.50 \pm 0.67$ & $0.63 \pm 0.11 \pm 0.08$ & $0.020 \pm 0.016 \pm 0.003$ & \\
$-0.50--0.25$ & $7.65 \pm 0.62 \pm 0.95$ & $1.00 \pm 0.14 \pm 0.12$ & $0.034 \pm 0.019 \pm 0.005$ & \\
\hline$-0.25-0.00$ & $8.54 \pm 0.70 \pm 1.1$ & $1.24 \pm 0.17 \pm 0.15$ & $0.039 \pm 0.021 \pm 0.005$ & \\
$0.00-0.25$ & $9.26 \pm 0.67 \pm 1.2$ & $0.88 \pm 0.14 \pm 0.11$ & $0.056 \pm 0.025 \pm 0.007$ & \multicolumn{1}{|c}{} \\
\hline $0.25-0.50$ & $8.47 \pm 0.65 \pm 1.1$ & $1.10 \pm 0.16 \pm 0.14$ & $0.042 \pm 0.021 \pm 0.006$ & \\
$0.50-0.75$ & $7.67 \pm 0.62 \pm 0.96$ & $0.64 \pm 0.13 \pm 0.08$ & $0.039 \pm 0.023 \pm 0.005$ &
\end{tabular}

Table B.5 Invariant differential cross section per nucleon for the inclusive reaction $\mathrm{pBe} \rightarrow \gamma \mathrm{X}$ at $800 \mathrm{GeV} / c$ as a function of rapidity for several $p_{T}$ bins

\begin{tabular}{|c|c|c|c|c|}
\hline \multirow{2}{*}{ Rapidity } & \multicolumn{5}{|c|}{$p_{T}(\mathrm{GeV} / c)$} & $4.50-5.00$ & $5.00-5.50$ \\
& $\begin{array}{c}3.50-4.00 \\
\mathrm{pb} /(\mathrm{GeV} / c)^{2}\end{array}$ & $\begin{array}{c}4.00-4.50 \\
\mathrm{pb} /(\mathrm{GeV} / c)^{2}\end{array}$ & $\mathrm{pb} /(\mathrm{GeV} / c)^{2}$ & $\mathrm{pb} /(\mathrm{GeV} / c)^{2}$ \\
\hline$-1.0--0.75$ & $1260 \pm 320 \pm 240$ & $526 \pm 43 \pm 92$ & $131.7 \pm 7.5 \pm 20$ & $44.9 \pm 3.8 \pm 6.1$ \\
$-0.75--0.50$ & $2050 \pm 320 \pm 390$ & $511 \pm 44 \pm 89$ & $160.5 \pm 8.2 \pm 24$ & $65.5 \pm 4.1 \pm 8.9$ \\
\hline$-0.50--0.25$ & $2070 \pm 320 \pm 390$ & $718 \pm 44 \pm 130$ & $223.4 \pm 9.0 \pm 34$ & $79.4 \pm 4.6 \pm 11$ \\
$-0.25-0.00$ & $3770 \pm 420 \pm 710$ & $807 \pm 39 \pm 140$ & $239.3 \pm 8.6 \pm 36$ & $97.0 \pm 4.6 \pm 13$ \\
\hline $0.00-0.25$ & $2740 \pm 250 \pm 520$ & $916 \pm 38 \pm 160$ & $302 \pm 12 \pm 46$ & $102.2 \pm 5.5 \pm 14$ \\
$0.25-0.50$ & $1940 \pm 250 \pm 370$ & $779 \pm 35 \pm 140$ & $255 \pm 12 \pm 39$ & $105.2 \pm 5.6 \pm 14$ \\
\hline & $5.50-6.50$ & $6.50-8.00$ & $8.00-10.00$ & \\
\hline$-1.0--0.75$ & $10.51 \pm 0.94 \pm 1.3$ & $1.36 \pm 0.19 \pm 0.16$ & $0.170 \pm 0.045 \pm 0.020$ & \\
\hline$-0.75--0.50$ & $18.7 \pm 1.3 \pm 2.3$ & $1.96 \pm 0.24 \pm 0.23$ & $0.171 \pm 0.046 \pm 0.020$ & \\
\hline$-0.50--0.25$ & $23.7 \pm 1.5 \pm 2.9$ & $3.44 \pm 0.33 \pm 0.40$ & $0.328 \pm 0.070 \pm 0.038$ & \\
\hline$-0.25-0.00$ & $27.6 \pm 1.4 \pm 3.4$ & $3.81 \pm 0.33 \pm 0.45$ & $0.151 \pm 0.054 \pm 0.017$ & \\
\hline $0.00-0.25$ & $31.1 \pm 1.6 \pm 3.9$ & $4.25 \pm 0.37 \pm 0.50$ & $0.286 \pm 0.076 \pm 0.033$ \\
$0.25-0.50$ & $25.6 \pm 1.6 \pm 3.2$ & $3.34 \pm 0.37 \pm 0.39$ & $0.41 \pm 0.10 \pm 0.05$ \\
\hline
\end{tabular}


Table B.6 Invariant differential cross section per nucleon for the inclusive reaction $\pi^{-} \mathrm{Be} \rightarrow \gamma \mathrm{X}$ at $515 \mathrm{GeV} / c$ as a function of rapidity for several $p_{T}$ bins

\begin{tabular}{|c|c|c|c|c|}
\hline \multirow[b]{2}{*}{ Rapidity } & \multicolumn{4}{|c|}{$p_{T}(\mathrm{GeV} / c)$} \\
\hline & $\begin{array}{c}3.50-4.00 \\
\mathrm{pb} /(\mathrm{GeV} / \mathrm{c})^{2}\end{array}$ & $\begin{array}{c}4.00-4.50 \\
\mathrm{pb} /(\mathrm{GeV} / \mathrm{c})^{2}\end{array}$ & $\begin{array}{c}4.50-5.00 \\
\mathrm{pb} /(\mathrm{GeV} / c)^{2}\end{array}$ & $\begin{array}{c}5.00-5.50 \\
\mathrm{pb} /(\mathrm{GeV} / \mathrm{c})^{2}\end{array}$ \\
\hline$-0.75--0.50$ & $1260 \pm 220 \pm 210$ & $373 \pm 15 \pm 58$ & $81.2 \pm 5.0 \pm 11$ & $30.9 \pm 2.2 \pm 3.9$ \\
\hline$-0.50--0.25$ & $1160 \pm 100 \pm 200$ & $346 \pm 11 \pm 54$ & $125.3 \pm 4.7 \pm 17$ & $48.3 \pm 2.5 \pm 6.1$ \\
\hline$-0.25-0.00$ & $1329 \pm 88 \pm 230$ & $406 \pm 11 \pm 63$ & $138.6 \pm 5.1 \pm 19$ & $54.9 \pm 2.7 \pm 6.9$ \\
\hline $0.00-0.25$ & $1690 \pm 120 \pm 290$ & $441 \pm 11 \pm 69$ & $164.0 \pm 5.1 \pm 23$ & $64.5 \pm 2.7 \pm 8.1$ \\
\hline $0.25-0.50$ & $1510 \pm 100 \pm 260$ & $449 \pm 11 \pm 70$ & $176.6 \pm 5.4 \pm 24$ & $73.6 \pm 2.9 \pm 9.2$ \\
\hline \multirow[t]{2}{*}{$0.50-0.75$} & $1258 \pm 67 \pm 210$ & $392 \pm 11 \pm 61$ & $166.8 \pm 5.4 \pm 23$ & $69.5 \pm 3.0 \pm 8.7$ \\
\hline & $\begin{array}{c}5.50-6.50 \\
\mathrm{pb} /(\mathrm{GeV} / \mathrm{c})^{2}\end{array}$ & $\begin{array}{c}6.50-8.00 \\
\mathrm{pb} /(\mathrm{GeV} / \mathrm{c})^{2}\end{array}$ & $\begin{array}{r}8.00-10.00 \\
\mathrm{pb} /(\mathrm{GeV} / \mathrm{c})^{2}\end{array}$ & \\
\hline$-0.75--0.50$ & $8.66 \pm 0.64 \pm 1.0$ & $0.96 \pm 0.14 \pm 0.11$ & $0.060 \pm 0.029 \pm 0.007$ & \\
\hline$-0.50--0.25$ & $12.86 \pm 0.79 \pm 1.5$ & $1.77 \pm 0.19 \pm 0.20$ & $0.159 \pm 0.040 \pm 0.019$ & \\
\hline$-0.25-0.00$ & $18.59 \pm 0.93 \pm 2.2$ & $2.85 \pm 0.24 \pm 0.33$ & $0.294 \pm 0.056 \pm 0.035$ & \\
\hline $0.00-0.25$ & $21.81 \pm 0.95 \pm 2.6$ & $3.23 \pm 0.25 \pm 0.37$ & $0.425 \pm 0.065 \pm 0.051$ & \\
\hline $0.25-0.50$ & $23.38 \pm 0.99 \pm 2.8$ & $4.10 \pm 0.29 \pm 0.48$ & $0.367 \pm 0.068 \pm 0.044$ & \\
\hline $0.50-0.75$ & $22.0 \pm 1.0 \pm 2.6$ & $3.10 \pm 0.28 \pm 0.36$ & $0.238 \pm 0.053 \pm 0.029$ & \\
\hline
\end{tabular}

Table B.7 Invariant differential cross section per nucleon for the inclusive reaction $\mathrm{pCu} \rightarrow \gamma \mathrm{X}$ at $530 \mathrm{GeV} / c$ as a function of rapidity for several $p_{T}$ bins.

\begin{tabular}{|c|c|c|c|}
\hline Rapidity & $\begin{array}{c}3.50-4.00 \\
\mathrm{pb} /(\mathrm{GeV} / c)^{2}\end{array}$ & $\begin{array}{c}p_{T}(\mathrm{GeV} / c) \\
4.00-4.50 \\
\mathrm{pb} /(\mathrm{GeV} / c)^{2}\end{array}$ & $\begin{array}{c}4.50-5.00 \\
\mathrm{pb} /(\mathrm{GeV} / c)^{2}\end{array}$ \\
\hline $\begin{array}{l}-0.75--0.50 \\
-0.50--0.25\end{array}$ & $\begin{array}{l}1990 \pm 640 \pm 370 \\
2780 \pm 620 \pm 510\end{array}$ & $\begin{array}{l}466 \pm 35 \pm 79 \\
426 \pm 31 \pm 72\end{array}$ & $\begin{array}{l}100 \pm 11 \pm 15 \\
126 \pm 12 \pm 19\end{array}$ \\
\hline $\begin{array}{c}-0.25-0.00 \\
0.00-0.25\end{array}$ & $\begin{array}{l}2200 \pm 560 \pm 410 \\
2180 \pm 540 \pm 400\end{array}$ & $\begin{array}{l}426 \pm 28 \pm 72 \\
444 \pm 25 \pm 75\end{array}$ & $\begin{array}{l}126 \pm 12 \pm 19 \\
141 \pm 11 \pm 21\end{array}$ \\
\hline $\begin{array}{l}0.25-0.50 \\
0.50-0.75\end{array}$ & $\begin{array}{l}1110 \pm 310 \pm 200 \\
2720 \pm 740 \pm 500\end{array}$ & $\begin{array}{c}465 \pm 24 \pm 79 \\
370 \pm 130 \pm 60\end{array}$ & $\begin{array}{c}141 \pm 11 \pm 21 \\
80.1 \pm 9.8 \pm 12\end{array}$ \\
\hline & $\begin{array}{c}5.00-5.50 \\
\mathrm{pb} /(\mathrm{GeV} / c)^{2}\end{array}$ & $\begin{array}{c}5.50-6.50 \\
\mathrm{pb} /(\mathrm{GeV} / c)^{2}\end{array}$ & $\begin{array}{c}6.50-8.00 \\
\mathrm{pb} /(\mathrm{GeV} / c)^{2}\end{array}$ \\
\hline $\begin{array}{l}-0.75--0.50 \\
-0.50--0.25\end{array}$ & $\begin{array}{l}27.1 \pm 4.3 \pm 3.6 \\
45.2 \pm 5.4 \pm 6.0\end{array}$ & $\begin{array}{l}5.8 \pm 1.2 \pm 0.7 \\
8.4 \pm 1.5 \pm 1.1\end{array}$ & $\begin{array}{l}0.39 \pm 0.20 \pm 0.05 \\
0.58 \pm 0.27 \pm 0.07\end{array}$ \\
\hline $\begin{array}{c}-0.25-0.00 \\
0.00-0.25\end{array}$ & $\begin{array}{l}39.7 \pm 5.8 \pm 5.3 \\
42.8 \pm 5.6 \pm 5.7\end{array}$ & $\begin{array}{c}10.1 \pm 1.7 \pm 1.3 \\
8.8 \pm 1.6 \pm 1.1\end{array}$ & $\begin{array}{l}1.18 \pm 0.39 \pm 0.15 \\
1.37 \pm 0.38 \pm 0.17\end{array}$ \\
\hline $\begin{array}{l}0.25-0.50 \\
0.50-0.75\end{array}$ & $\begin{array}{l}49.7 \pm 5.6 \pm 6.6 \\
29.2 \pm 5.1 \pm 3.9\end{array}$ & $\begin{array}{c}12.4 \pm 1.7 \pm 1.5 \\
9.6 \pm 1.6 \pm 1.2\end{array}$ & $\begin{array}{l}0.66 \pm 0.28 \pm 0.08 \\
0.78 \pm 0.32 \pm 0.10\end{array}$ \\
\hline
\end{tabular}


Table B.8 Invariant differential cross section per nucleon for the inclusive reaction $\mathrm{pCu} \rightarrow \gamma \mathrm{X}$ at $800 \mathrm{GeV} / c$ as a function of rapidity for several $p_{T}$ bins.

\begin{tabular}{|c|c|c|c|}
\hline Rapidity & $\begin{array}{c}3.50-4.00 \\
\mathrm{pb} /(\mathrm{GeV} / c)^{2}\end{array}$ & $\begin{array}{c}p_{T}(\mathrm{GeV} / c) \\
4.00-4.50 \\
\mathrm{pb} /(\mathrm{GeV} / c)^{2} \\
\end{array}$ & $\begin{array}{c}4.50-5.00 \\
\mathrm{pb} /(\mathrm{GeV} / c)^{2}\end{array}$ \\
\hline $\begin{array}{r}-1.0--0.75 \\
-0.75--0.50 \\
\end{array}$ & $\begin{array}{l}1600 \pm 570 \pm 300 \\
1280 \pm 790 \pm 240 \\
\end{array}$ & $\begin{array}{l}768 \pm 79 \pm 130 \\
440 \pm 150 \pm 80 \\
\end{array}$ & $\begin{array}{l}143 \pm 19 \pm 22 \\
206 \pm 20 \pm 31 \\
\end{array}$ \\
\hline $\begin{array}{c}-0.50--0.25 \\
-0.25-0.00 \\
\end{array}$ & $\begin{array}{c}1860 \pm 810 \pm 350 \\
4500 \pm 1000 \pm 900 \\
\end{array}$ & $\begin{array}{c}500 \pm 130 \pm 90 \\
800 \pm 140 \pm 140 \\
\end{array}$ & $\begin{array}{l}261 \pm 22 \pm 40 \\
257 \pm 21 \pm 39 \\
\end{array}$ \\
\hline $\begin{array}{l}0.00-0.25 \\
0.25-0.50\end{array}$ & $\begin{array}{l}2700 \pm 680 \pm 510 \\
2660 \pm 650 \pm 500\end{array}$ & $\begin{array}{c}1105 \pm 83 \pm 190 \\
929 \pm 90 \pm 160\end{array}$ & $\begin{array}{l}284 \pm 33 \pm 43 \\
294 \pm 31 \pm 44\end{array}$ \\
\hline & $\begin{array}{c}5.00-5.50 \\
\mathrm{pb} /(\mathrm{GeV} / c)^{2}\end{array}$ & $\begin{array}{c}5.50-6.50 \\
\mathrm{pb} /(\mathrm{GeV} / c)^{2}\end{array}$ & $\begin{array}{c}6.50-8.00 \\
\mathrm{pb} /(\mathrm{GeV} / c)^{2}\end{array}$ \\
\hline $\begin{array}{l}-1.0--0.75 \\
-0.75--0.50\end{array}$ & $\begin{array}{c}49.5 \pm 7.3 \pm 6.7 \\
85 \pm 10 \pm 12\end{array}$ & $\begin{array}{l}12.7 \pm 2.5 \pm 1.6 \\
17.3 \pm 2.9 \pm 2.2\end{array}$ & $\begin{array}{l}1.62 \pm 0.43 \pm 0.19 \\
2.83 \pm 0.63 \pm 0.33\end{array}$ \\
\hline $\begin{array}{c}-0.50--0.25 \\
-0.25-0.00 \\
\end{array}$ & $\begin{array}{l}84 \pm 11 \pm 11 \\
93 \pm 11 \pm 13 \\
\end{array}$ & $\begin{array}{l}23.9 \pm 3.5 \pm 3.0 \\
30.3 \pm 3.6 \pm 3.8 \\
\end{array}$ & $\begin{array}{l}3.26 \pm 0.73 \pm 0.38 \\
2.29 \pm 0.72 \pm 0.27\end{array}$ \\
\hline $\begin{array}{l}0.00-0.25 \\
0.25-0.50\end{array}$ & $\begin{array}{c}109 \pm 14 \pm 15 \\
91 \pm 14 \pm 12\end{array}$ & $\begin{array}{l}23.8 \pm 3.8 \pm 3.0 \\
25.8 \pm 4.0 \pm 3.2\end{array}$ & $\begin{array}{l}3.69 \pm 0.83 \pm 0.43 \\
5.24 \pm 0.99 \pm 0.61\end{array}$ \\
\hline
\end{tabular}

Table B.9 Invariant differential cross section per nucleon for the inclusive reaction $\pi^{-} \mathrm{Cu} \rightarrow \gamma \mathrm{X}$ at $515 \mathrm{GeV} / c$ as a function of rapidity for several $p_{T}$ bins.

\begin{tabular}{|c|c|c|c|}
\hline \multirow{2}{*}{ Rapidity } & \multicolumn{3}{|c|}{$p_{T}(\mathrm{GeV} / c)$} \\
& $\begin{array}{c}3.50-4.00 \\
\mathrm{pb} /(\mathrm{GeV} / c)^{2}\end{array}$ & $\begin{array}{c}4.00-4.50 \\
\mathrm{pb} /(\mathrm{GeV} / c)^{2}\end{array}$ & $\begin{array}{c}4.50-5.00 \\
\mathrm{pb} /(\mathrm{GeV} / c)^{2}\end{array}$ \\
\hline$-0.75--0.50$ & $2180 \pm 590 \pm 370$ & $459 \pm 42 \pm 72$ & $124 \pm 13 \pm 17$ \\
$-0.50--0.25$ & $820 \pm 280 \pm 140$ & $427 \pm 30 \pm 67$ & $142 \pm 13 \pm 20$ \\
\hline$-0.25-0.00$ & $1580 \pm 270 \pm 270$ & $450 \pm 30 \pm 70$ & $142 \pm 14 \pm 20$ \\
$0.00-0.25$ & $1410 \pm 290 \pm 240$ & $552 \pm 30 \pm 86$ & $150 \pm 14 \pm 21$ \\
\hline $0.25-0.50$ & $2020 \pm 260 \pm 340$ & $468 \pm 31 \pm 73$ & $166 \pm 15 \pm 23$ \\
$0.50-0.75$ & $1030 \pm 210 \pm 170$ & $461 \pm 32 \pm 72$ & $143 \pm 14 \pm 20$ \\
\hline & $5.00-5.50$ & $5.50-6.50$ & $6.50-8.00$ \\
& $\mathrm{pb} /(\mathrm{GeV} / c)^{2}$ & $\mathrm{pb} /(\mathrm{GeV} / c)^{2}$ & $\mathrm{pb} /(\mathrm{GeV} / c)^{2}$ \\
\hline$-0.75--0.50$ & $37.9 \pm 6.0 \pm 4.8$ & $9.4 \pm 1.8 \pm 1.1$ & $1.20 \pm 0.38 \pm 0.14$ \\
$-0.50--0.25$ & $39.5 \pm 6.3 \pm 5.0$ & $15.8 \pm 2.2 \pm 1.9$ & $2.03 \pm 0.51 \pm 0.24$ \\
\hline$-0.25-0.00$ & $64.0 \pm 7.3 \pm 8.0$ & $18.7 \pm 2.4 \pm 2.2$ & $2.48 \pm 0.58 \pm 0.29$ \\
$0.00-0.25$ & $65.8 \pm 7.3 \pm 8.3$ & $19.7 \pm 2.5 \pm 2.3$ & $4.90 \pm 0.73 \pm 0.57$ \\
\hline $0.25-0.50$ & $63.4 \pm 7.6 \pm 8.0$ & $20.1 \pm 2.5 \pm 2.4$ & $3.44 \pm 0.69 \pm 0.40$ \\
$0.50-0.75$ & $89.6 \pm 8.3 \pm 11$ & $26.8 \pm 2.8 \pm 3.2$ & $3.57 \pm 0.71 \pm 0.41$ \\
\hline
\end{tabular}


Table B.10 Invariant differential cross section for the inclusive reaction $p p \rightarrow \gamma \mathrm{X}$ at $530 \mathrm{GeV} / c$ as a function of rapidity for several $p_{T}$ bins.

\begin{tabular}{|c|c|c|c|}
\hline \multirow[b]{2}{*}{ Rapidity } & \multicolumn{3}{|c|}{$p_{T}(\mathrm{GeV} / c)$} \\
\hline & $\begin{array}{c}3.50-4.00 \\
\mathrm{pb} /(\mathrm{GeV} / c)^{2}\end{array}$ & $\begin{array}{c}4.00-4.50 \\
\mathrm{pb} /(\mathrm{GeV} / \mathrm{c})^{2}\end{array}$ & $\begin{array}{c}4.50-5.00 \\
\mathrm{pb} /(\mathrm{GeV} / c)^{2}\end{array}$ \\
\hline$-0.75--0.50$ & $2350 \pm 600 \pm 430$ & $378 \pm 34 \pm 64$ & $98 \pm 12 \pm 14$ \\
\hline$-0.50--0.25$ & $1250 \pm 510 \pm 230$ & $385 \pm 29 \pm 65$ & $118 \pm 12 \pm 17$ \\
\hline$-0.25-0.00$ & $900 \pm 440 \pm 160$ & $437 \pm 27 \pm 74$ & $135 \pm 12 \pm 20$ \\
\hline $0.00-0.25$ & $1170 \pm 410 \pm 210$ & $336 \pm 24 \pm 57$ & $116 \pm 11 \pm 17$ \\
\hline $0.25-0.50$ & $850 \pm 250 \pm 150$ & $348 \pm 23 \pm 59$ & $110 \pm 10 \pm 16$ \\
\hline \multirow[t]{2}{*}{$0.50-0.75$} & $590 \pm 550 \pm 110$ & $410 \pm 170 \pm 70$ & $77.9 \pm 9.7 \pm 12$ \\
\hline & $\begin{array}{c}5.00-5.50 \\
\mathrm{pb} /(\mathrm{GeV} / \mathrm{c})^{2}\end{array}$ & $\begin{array}{c}5.50-6.50 \\
\mathrm{pb} /(\mathrm{GeV} / c)^{2}\end{array}$ & $\begin{array}{c}6.50-8.00 \\
\mathrm{pb} /(\mathrm{GeV} / \mathrm{c})^{2}\end{array}$ \\
\hline$-0.75--0.50$ & $26.0 \pm 5.3 \pm 3.5$ & $5.9 \pm 1.2 \pm 0.7$ & $0.26 \pm 0.21 \pm 0.03$ \\
\hline$-0.50--0.25$ & $37.7 \pm 5.2 \pm 5.0$ & $9.7 \pm 1.6 \pm 1.2$ & $0.89 \pm 0.34 \pm 0.11$ \\
\hline$-0.25-0.00$ & $40.2 \pm 5.8 \pm 5.4$ & $7.3 \pm 1.7 \pm 0.9$ & $0.93 \pm 0.36 \pm 0.11$ \\
\hline $0.00-0.25$ & $38.0 \pm 5.6 \pm 5.1$ & $11.0 \pm 1.8 \pm 1.4$ & $1.06 \pm 0.37 \pm 0.13$ \\
\hline $0.25-0.50$ & $35.2 \pm 5.3 \pm 4.7$ & $10.6 \pm 1.7 \pm 1.3$ & $1.75 \pm 0.43 \pm 0.22$ \\
\hline $0.50-0.75$ & $25.7 \pm 5.0 \pm 3.4$ & $4.2 \pm 1.4 \pm 0.5$ & $0.63 \pm 0.29 \pm 0.08$ \\
\hline
\end{tabular}

Table B.11 Invariant differential cross section for the inclusive reaction $p p \rightarrow \gamma \mathrm{X}$ at $800 \mathrm{GeV} / c$ as a function of rapidity for several $p_{T}$ bins.

\begin{tabular}{|c|c|c|c|}
\hline \multirow[b]{2}{*}{ Rapidity } & \multicolumn{3}{|c|}{$p_{T}(\mathrm{GeV} / c)$} \\
\hline & $\begin{array}{c}3.50-4.00 \\
\mathrm{pb} /(\mathrm{GeV} / \mathrm{c})^{2}\end{array}$ & $\begin{array}{r}4.00-4.50 \\
\mathrm{pb} /(\mathrm{GeV} / \mathrm{c})^{2}\end{array}$ & $\begin{array}{c}4.50-5.00 \\
\mathrm{pb} /(\mathrm{GeV} / \mathrm{c})^{2}\end{array}$ \\
\hline$-1.0--0.75$ & $2140 \pm 920 \pm 400$ & $516 \pm 76 \pm 90$ & $174 \pm 21 \pm 26$ \\
\hline$-0.75--0.50$ & $1430 \pm 830 \pm 270$ & $160 \pm 210 \pm 30$ & $225 \pm 21 \pm 34$ \\
\hline$-0.50--0.25$ & $1080 \pm 830 \pm 200$ & $796 \pm 87 \pm 140$ & $234 \pm 22 \pm 35$ \\
\hline$-0.25-0.00$ & $2460 \pm 860 \pm 460$ & $855 \pm 54 \pm 150$ & $223 \pm 20 \pm 34$ \\
\hline $0.00-0.25$ & $2670 \pm 600 \pm 500$ & $860 \pm 110 \pm 150$ & $351 \pm 28 \pm 53$ \\
\hline \multirow[t]{2}{*}{$0.25-0.50$} & $2350 \pm 580 \pm 440$ & $666 \pm 84 \pm 120$ & $237 \pm 31 \pm 36$ \\
\hline & $\begin{array}{c}5.00-5.50 \\
\mathrm{pb} /(\mathrm{GeV} / \mathrm{c})^{2}\end{array}$ & $\begin{array}{c}5.50-6.50 \\
\mathrm{pb} /(\mathrm{GeV} / \mathrm{c})^{2}\end{array}$ & $\begin{array}{c}6.50-8.00 \\
\mathrm{pb} /(\mathrm{GeV} / \mathrm{c})^{2}\end{array}$ \\
\hline$-1.0--0.75$ & $47.0 \pm 8.1 \pm 6.4$ & $13.5 \pm 2.4 \pm 1.7$ & $1.78 \pm 0.52 \pm 0.21$ \\
\hline$-0.75--0.50$ & $84 \pm 10 \pm 11$ & $22.1 \pm 3.2 \pm 2.7$ & $2.32 \pm 0.68 \pm 0.27$ \\
\hline$-0.50--0.25$ & $89 \pm 11 \pm 12$ & $26.5 \pm 3.5 \pm 3.3$ & $3.21 \pm 0.84 \pm 0.38$ \\
\hline$-0.25-0.00$ & $90 \pm 11 \pm 12$ & $32.3 \pm 3.7 \pm 4.0$ & $3.97 \pm 0.84 \pm 0.46$ \\
\hline $0.00-0.25$ & $118 \pm 14 \pm 16$ & $33.4 \pm 4.0 \pm 4.1$ & $5.6 \pm 1.0 \pm 0.7$ \\
\hline $0.25-0.50$ & $97 \pm 13 \pm 13$ & $25.1 \pm 4.1 \pm 3.1$ & $4.44 \pm 1.00 \pm 0.52$ \\
\hline
\end{tabular}


Table B.12 Invariant differential cross section for the inclusive reaction $\pi^{-} p \rightarrow \gamma \mathrm{X}$ at $515 \mathrm{GeV} / c$ as a function of rapidity for several $p_{T}$ bins.

\begin{tabular}{|c|c|c|c|}
\hline \multirow[b]{2}{*}{ Rapidity } & \multicolumn{3}{|c|}{$\overline{p_{T}(\mathrm{GeV} / c)}$} \\
\hline & $\begin{array}{c}3.50-4.00 \\
\mathrm{pb} /(\mathrm{GeV} / c)^{2}\end{array}$ & $\begin{array}{c}4.00-4.50 \\
\mathrm{pb} /(\mathrm{GeV} / \mathrm{c})^{2}\end{array}$ & $\begin{array}{c}4.50-5.00 \\
\mathrm{pb} /(\mathrm{GeV} / c)^{2}\end{array}$ \\
\hline$-0.75--0.50$ & $-999 \pm 790 \pm 40$ & $483 \pm 76 \pm 75$ & $158 \pm 26 \pm 22$ \\
\hline$-0.25-0.00$ & $4300 \pm 1600 \pm 700$ & $575 \pm 71 \pm 90$ & $192 \pm 30 \pm 26$ \\
\hline $0.00-0.25$ & $730 \pm 770 \pm 120$ & $521 \pm 64 \pm 81$ & $139 \pm 26 \pm 19$ \\
\hline $0.25-0.50$ & $880 \pm 490 \pm 150$ & $551 \pm 57 \pm 86$ & $210 \pm 29 \pm 29$ \\
\hline \multirow[t]{2}{*}{$0.50-0.75$} & $240 \pm 710 \pm 40$ & $409 \pm 67 \pm 64$ & $147 \pm 29 \pm 20$ \\
\hline & $\begin{array}{c}5.00-5.50 \\
\mathrm{pb} /(\mathrm{GeV} / \mathrm{c})^{2}\end{array}$ & $\begin{array}{c}5.50-6.50 \\
\mathrm{pb} /(\mathrm{GeV} / \mathrm{c})^{2}\end{array}$ & $\begin{array}{c}6.50-8.00 \\
\mathrm{pb} /(\mathrm{GeV} / \mathrm{c})^{2}\end{array}$ \\
\hline$-0.75--0.50$ & $43 \pm 12 \pm 5.0$ & $6.7 \pm 3.5 \pm 0.8$ & $2.2 \pm 1.0 \pm 0.3$ \\
\hline$-0.50--0.25$ & $52 \pm 14 \pm 7.0$ & $14.3 \pm 4.7 \pm 1.7$ & $2.9 \pm 1.2 \pm 0.3$ \\
\hline$-0.25-0.00$ & $74 \pm 16 \pm 9.0$ & $20.8 \pm 5.1 \pm 2.5$ & $4.0 \pm 1.5 \pm 0.5$ \\
\hline $0.00-0.25$ & $66 \pm 14 \pm 8.0$ & $26.1 \pm 5.4 \pm 3.1$ & $4.7 \pm 1.6 \pm 0.5$ \\
\hline $0.25-0.50$ & $85 \pm 16 \pm 11$ & $25.4 \pm 5.5 \pm 3.0$ & $4.7 \pm 1.6 \pm 0.5$ \\
\hline $0.50-0.75$ & $73 \pm 16 \pm 9.0$ & $19.9 \pm 5.5 \pm 2.4$ & $5.0 \pm 1.9 \pm 0.6$ \\
\hline
\end{tabular}





\section{Appendix C $\eta$ Cross Sections}

In Figures C.1, C.2, and C.3, inclusive $\eta$ cross sections per nucleus are shown as functions of $p_{T}$ for 530 and $800 \mathrm{GeV} / c$ proton beams and $515 \mathrm{GeV} / c \pi^{-}$ beam, respectively, incident upon copper, beryllium, and liquid hydrogen targets. Since the cross sections fall steeply, the data are plotted at abscissa values which correspond to the average values of the cross section in the appropriate $p_{T}$ bins assuming exponential $p_{T}$ spectra[99]. These results are also presented in tabular form in Tables C.1 - C.3. In addition, the cross sections as functions of rapidity for several $p_{T}$ intervals are presented in Tables C.4 - C.12. 


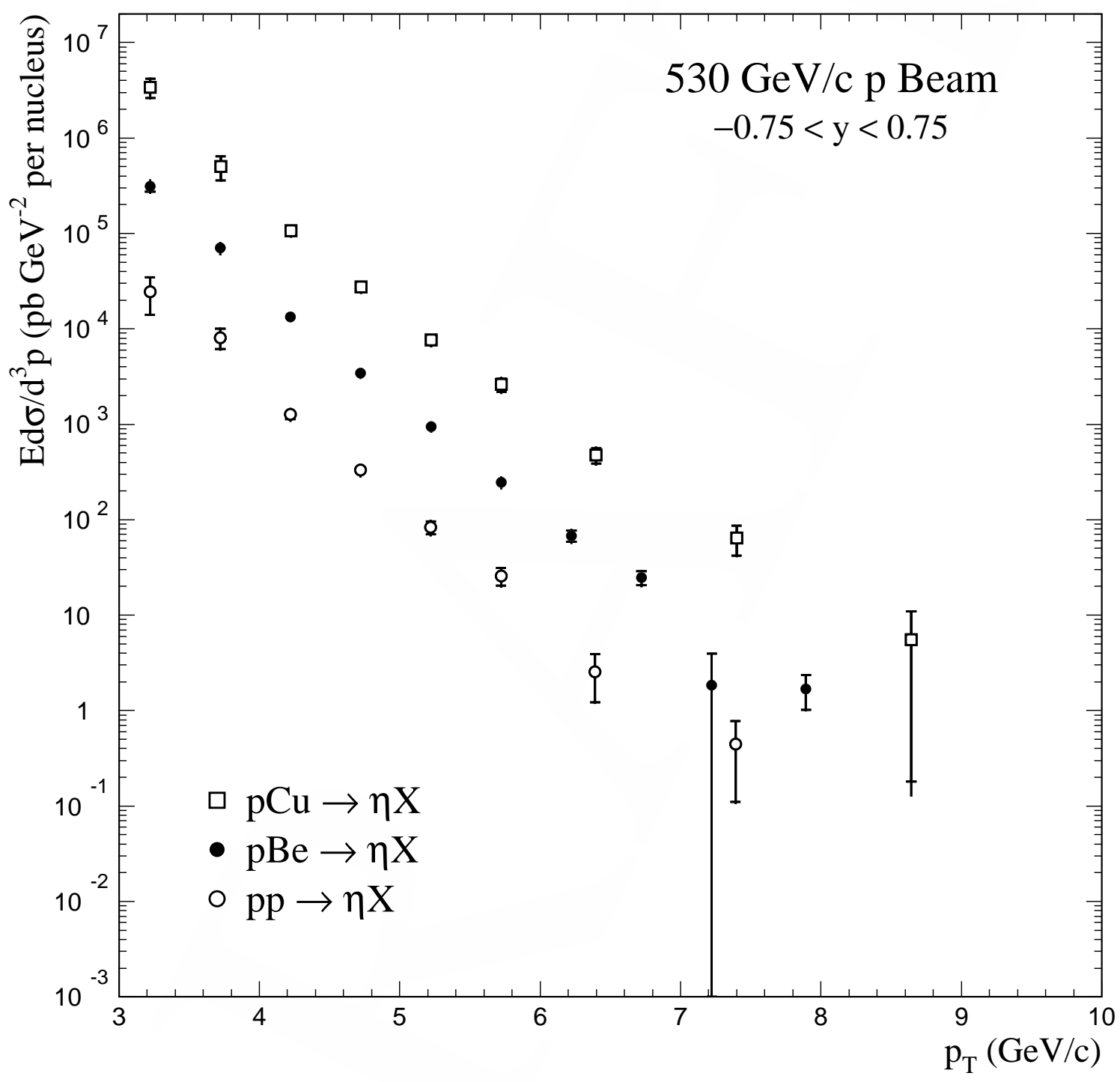

Figure C.1 $\eta$ production cross sections per nucleus as functions of $p_{T}$ for 530 $\mathrm{GeV} / c$ proton beam on copper, beryllium, and liquid hydrogen targets. 


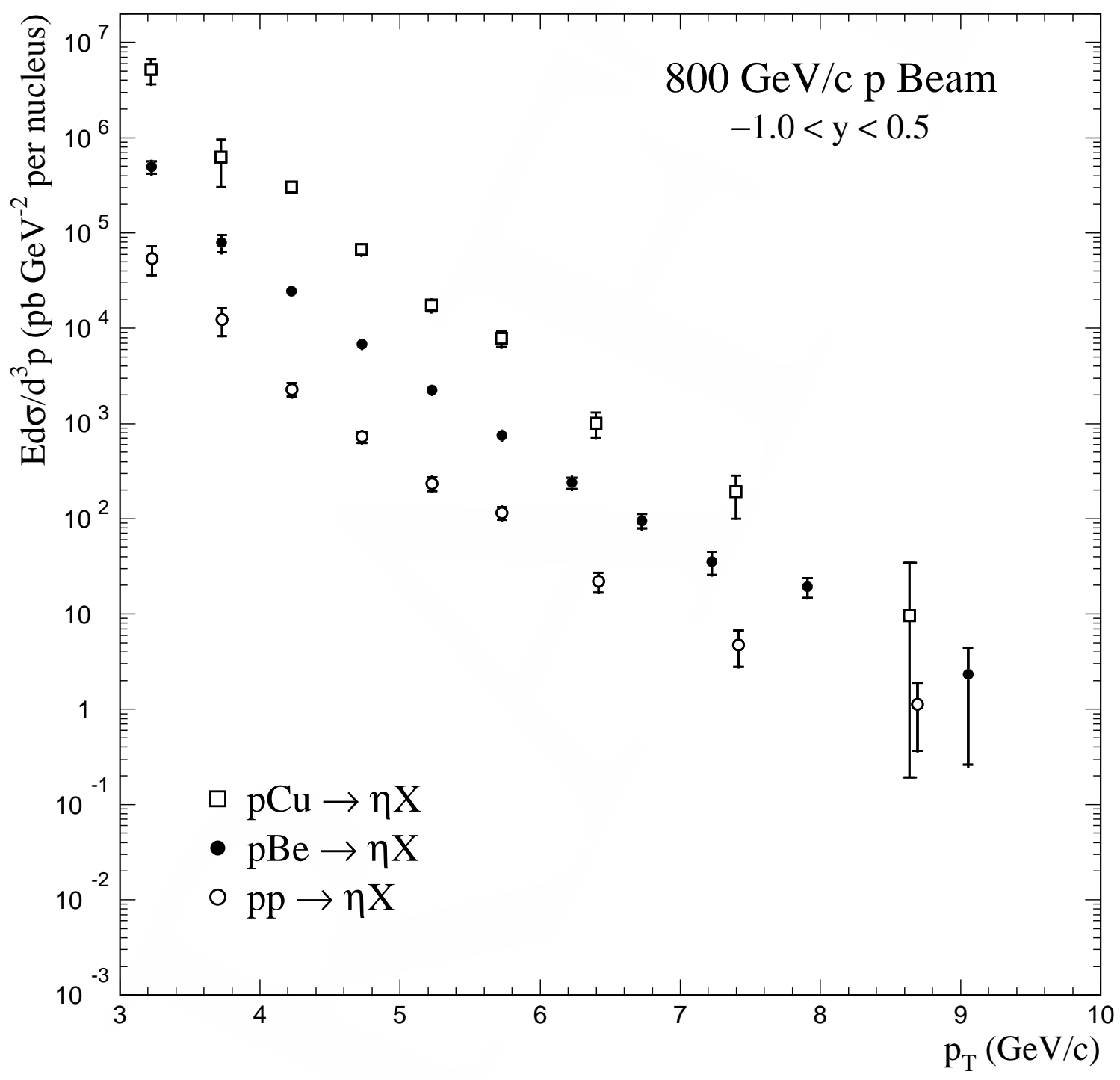

Figure C.2 $\eta$ production cross sections per nucleus as functions of $p_{T}$ for 800 $\mathrm{GeV} / c$ proton beam on copper, beryllium, and liquid hydrogen targets. 


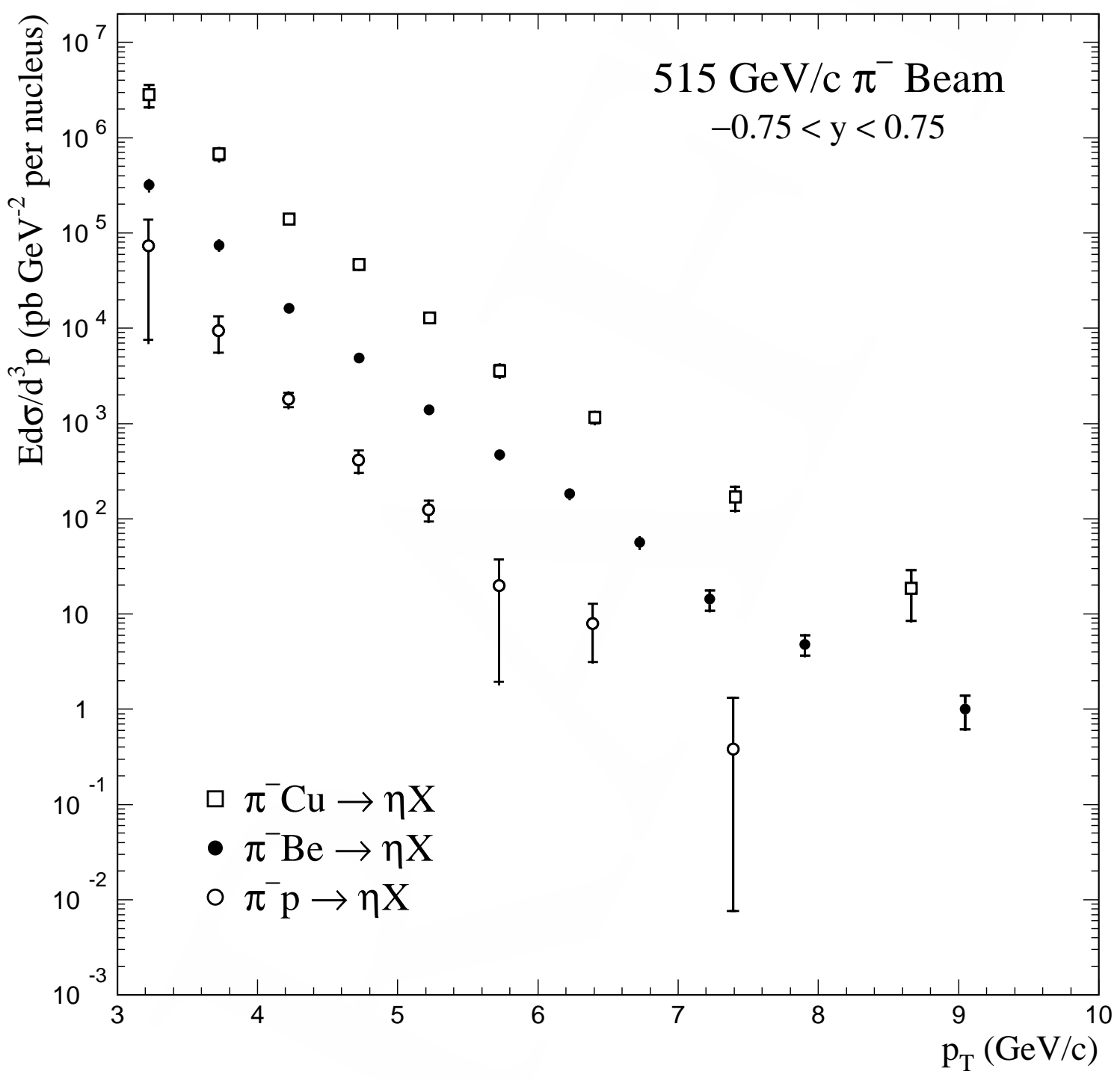

Figure C.3 $\eta$ production cross sections per nucleus as functions of $p_{T}$ for 515 $\mathrm{GeV} / c \pi^{-}$beam on copper, beryllium, and liquid hydrogen targets. 
Table C.1 Invariant differential cross sections per nucleon for $\eta$ production by 530 and 800 $\mathrm{GeV} / c$ proton beams and $515 \mathrm{GeV} / c \pi^{-}$beam on Be targets.

\begin{tabular}{|c|c|c|c|}
\hline \multirow{2}{*}{$\begin{array}{c}p_{T} \text { Range } \\
(\mathrm{GeV} / \mathrm{c})\end{array}$} & $\begin{array}{c}|c| \\
\end{array}$ & $-0.75<y<d^{3} p\left(\mathrm{pb} /(\mathrm{GeV} / c)^{2}\right)$ \\
\hline $3.00-3.50$ & $34800 \pm 4300 \pm 4200$ & $54800 \pm 8000 \pm 6800$ & $35300 \pm 3500 \pm 4400$ \\
\hline $3.50-4.00$ & $7810 \pm 890 \pm 900$ & $8760 \pm 1800 \pm 1000$ & $8290 \pm 660 \pm 990$ \\
\hline $4.00-4.50$ & $1480 \pm 66 \pm 170$ & $2700 \pm 160 \pm 320$ & $1810 \pm 16 \pm 210$ \\
\hline $4.50-5.00$ & $379 \pm 16 \pm 44$ & $761 \pm 16 \pm 90$ & $537 \pm 16 \pm 62$ \\
\hline $5.00-5.50$ & $105 \pm 1 \pm 12$ & $248 \pm 16 \pm 29$ & $154 \pm 1 \pm 18$ \\
\hline $5.50-6.00$ & $27.4 \pm 2.6 \pm 3.2$ & $83.0 \pm 7.1 \pm 10.0$ & $51.9 \pm 2.8 \pm 6.1$ \\
\hline $6.00-6.50$ & $7.52 \pm 1.01 \pm 0.91$ & $26.5 \pm 3.6 \pm 3.2$ & $20.3 \pm 1.5 \pm 2.4$ \\
\hline $6.50-7.00$ & $2.74 \pm 0.45 \pm 0.34$ & $10.6 \pm 1.8 \pm 1.3$ & $6.30 \pm 0.73 \pm 0.76$ \\
\hline $7.00-7.50$ & $0.21 \pm 0.24 \pm 0.03$ & $3.93 \pm 1.07 \pm 0.49$ & $1.59 \pm 0.38 \pm 0.19$ \\
\hline $7.50-8.50$ & $0.19 \pm 0.07 \pm 0.02$ & $2.15 \pm 0.51 \pm 0.27$ & $0.53 \pm 0.13 \pm 0.07$ \\
\hline $8.50-10.00$ & - & $0.26 \pm 0.23 \pm 0.03$ & $0.11 \pm 0.04 \pm 0.01$ \\
\hline $10.00-12.00$ & - & $0.07 \pm 0.05 \pm 0.01$ & \\
\hline
\end{tabular}

Table C.2 Invariant differential cross sections per nucleon for $\eta$ production by 530 and 800 $\mathrm{GeV} / c$ proton beams and $515 \mathrm{GeV} / c \pi^{-}$beam on $\mathrm{Cu}$ targets.

\begin{tabular}{|c|c|c|c|}
\hline \multirow{2}{*}{$\begin{array}{c}p_{T} \text { Range } \\
(\mathrm{GeV} / \mathrm{c})\end{array}$} & $\begin{array}{c}\mid 3 \\
\mathrm{Cu} \text { at } 530 \mathrm{GeV} / c \\
-0.75<y<0.75\end{array}$ & $\begin{array}{c}p \mathrm{Cu} \text { at } 800 \mathrm{GeV} / c \\
-1.0<y<0.5\end{array}$ & $\begin{array}{c}\pi^{-} \mathrm{Cu} \text { at } 515 \mathrm{GeV} / c \\
-0.75<y<0.75\end{array}$ \\
\hline $3.00-3.50$ & $53400 \pm 12000 \pm 6500$ & $81800 \pm 25000 \pm 10000$ & $44500 \pm 12000 \pm 5700$ \\
\hline $3.50-4.00$ & $7880 \pm 2200 \pm 920$ & $9900 \pm 5100 \pm 1200$ & $10600 \pm 1600 \pm 1300$ \\
\hline $4.00-4.50$ & $1680 \pm 170 \pm 200$ & $4780 \pm 470 \pm 580$ & $2210 \pm 170 \pm 260$ \\
\hline $4.50-5.00$ & $434 \pm 17 \pm 51$ & $1060 \pm 120 \pm 130$ & $730 \pm 12 \pm 86$ \\
\hline $5.00-5.50$ & $120 \pm 12 \pm 14$ & $274 \pm 12 \pm 34$ & $203 \pm 12 \pm 24$ \\
\hline $5.50-6.00$ & $41.0 \pm 6.6 \pm 4.9$ & $123 \pm 12 \pm 15$ & $56.6 \pm 8.0 \pm 6.7$ \\
\hline $6.00-7.00$ & $7.48 \pm 1.42 \pm 0.92$ & $15.8 \pm 4.7 \pm 2.0$ & $18.2 \pm 2.3 \pm 2.2$ \\
\hline $7.00-8.00$ & $1.01 \pm 0.35 \pm 0.13$ & $3.02 \pm 1.46 \pm 0.39$ & $2.66 \pm 0.76 \pm 0.33$ \\
\hline $8.00-10.00$ & $0.09 \pm 0.08 \pm 0.01$ & $0.15 \pm 0.41 \pm 0.02$ & $0.29 \pm 0.16 \pm 0.04$ \\
\hline
\end{tabular}


Table C.3 Invariant differential cross sections for $\eta$ production by 530 and $800 \mathrm{GeV} / c$ proton beams and $515 \mathrm{GeV} / c \pi^{-}$beam on proton targets.

\begin{tabular}{|c|c|c|c|}
\hline \multirow{2}{*}{$\begin{array}{c}p_{T} \text { Range } \\
(\mathrm{GeV} / \mathrm{c})\end{array}$} & $\begin{array}{c}\mid 4 \\
\end{array}$ & $-0.75<y<d^{3} p\left(\mathrm{pb} /(\mathrm{GeV} / c)^{2}\right)$ \\
\hline $3.00-3.50$ & $24100 \pm 10000 \pm 3100$ & $53400 \pm 18000 \pm 7000$ & $72200 \pm 65000 \pm 9500$ \\
\hline $3.50-4.00$ & $8030 \pm 2000 \pm 980$ & $12200 \pm 3900 \pm 1500$ & $9350 \pm 3900 \pm 1200$ \\
\hline $4.00-4.50$ & $1270 \pm 150 \pm 150$ & $2250 \pm 360 \pm 280$ & $1780 \pm 310 \pm 220$ \\
\hline $4.50-5.00$ & $329 \pm 31 \pm 40$ & $717 \pm 31 \pm 89$ & $407 \pm 108 \pm 50$ \\
\hline $5.00-5.50$ & $82.3 \pm 12.7 \pm 10.2$ & $232 \pm 10 \pm 29$ & $124 \pm 10 \pm 15$ \\
\hline $5.50-6.00$ & $25.5 \pm 5.3 \pm 3.2$ & $114 \pm 10 \pm 14$ & $19.5 \pm 17.6 \pm 2.4$ \\
\hline $6.00-7.00$ & $2.54 \pm 1.33 \pm 0.32$ & $21.8 \pm 5.1 \pm 2.8$ & $7.88 \pm 4.79 \pm 0.99$ \\
\hline $7.00-8.00$ & $0.44 \pm 0.33 \pm 0.06$ & $4.68 \pm 1.93 \pm 0.61$ & $0.38 \pm 0.97 \pm 0.05$ \\
\hline $8.00-10.00$ & - & $1.12 \pm 0.76 \pm 0.15$ & \\
\hline
\end{tabular}


Table C.4 Invariant differential cross section per nucleon for $\eta$ production by $530 \mathrm{GeV} / c$ proton beam on Be target as a function of $p_{T}$ and rapidity.

\begin{tabular}{|c|c|c|c|}
\hline Rapidity & $\begin{array}{c}3.00-4.00 \\
\mathrm{nb} /(\mathrm{GeV} / c)^{2}\end{array}$ & $\begin{array}{c}p_{T}(\mathrm{GeV} / c) \\
4.00-4.50 \\
\mathrm{nb} /(\mathrm{GeV} / c)^{2}\end{array}$ & $\begin{array}{c}4.50-5.00 \\
\mathrm{pb} /(\mathrm{GeV} / c)^{2}\end{array}$ \\
\hline $\begin{array}{l}-0.750--0.625 \\
-0.625--0.500\end{array}$ & $15.4 \pm 9.4 \pm 1.8$ & $\begin{array}{l}1.09 \pm 0.32 \pm 0.12 \\
1.00 \pm 0.33 \pm 0.11\end{array}$ & $\begin{array}{l}256 \pm 64 \pm 29 \\
329 \pm 91 \pm 38\end{array}$ \\
\hline $\begin{array}{l}-0.500--0.375 \\
-0.375--0.250\end{array}$ & $25.6 \pm 5.2 \pm 3.0$ & $\begin{array}{l}1.47 \pm 0.29 \pm 0.17 \\
1.80 \pm 0.30 \pm 0.21\end{array}$ & $\begin{array}{l}390 \pm 70 \pm 45 \\
440 \pm 63 \pm 51\end{array}$ \\
\hline $\begin{array}{c}-0.250--0.125 \\
-0.125-0.000\end{array}$ & $22.1 \pm 4.0 \pm 2.6$ & $\begin{array}{l}1.98 \pm 0.26 \pm 0.23 \\
1.80 \pm 0.21 \pm 0.21\end{array}$ & $\begin{array}{l}503 \pm 59 \pm 58 \\
534 \pm 58 \pm 62\end{array}$ \\
\hline $\begin{array}{l}0.000-0.125 \\
0.125-0.250\end{array}$ & $27.4 \pm 3.5 \pm 3.3$ & $\begin{array}{l}1.38 \pm 0.18 \pm 0.16 \\
2.14 \pm 0.17 \pm 0.24\end{array}$ & $\begin{array}{l}502 \pm 53 \pm 58 \\
379 \pm 47 \pm 44\end{array}$ \\
\hline $\begin{array}{l}0.250-0.375 \\
0.375-0.500\end{array}$ & $23.5 \pm 3.4 \pm 2.8$ & $\begin{array}{l}1.53 \pm 0.12 \pm 0.17 \\
1.45 \pm 0.11 \pm 0.17\end{array}$ & $\begin{array}{l}345 \pm 41 \pm 40 \\
354 \pm 37 \pm 41\end{array}$ \\
\hline \multirow[t]{2}{*}{$\begin{array}{l}0.500-0.625 \\
0.625-0.750\end{array}$} & $13.9 \pm 4.2 \pm 1.6$ & $\begin{array}{c}1.27 \pm 0.11 \pm 0.15 \\
0.863 \pm 0.077 \pm 0.099\end{array}$ & $\begin{array}{l}334 \pm 37 \pm 39 \\
193 \pm 29 \pm 22\end{array}$ \\
\hline & $\begin{array}{c}5.00-5.50 \\
\mathrm{pb} /(\mathrm{GeV} / c)^{2}\end{array}$ & $\begin{array}{c}5.50-6.50 \\
\mathrm{pb} /(\mathrm{GeV} / c)^{2}\end{array}$ & $\begin{array}{c}6.50-8.00 \\
\mathrm{pb} /(\mathrm{GeV} / c)^{2}\end{array}$ \\
\hline $\begin{array}{l}-0.750--0.625 \\
-0.625--0.500\end{array}$ & $\begin{array}{l}66 \pm 26 \pm 8.0 \\
99 \pm 21 \pm 12\end{array}$ & $\begin{array}{c}4.8 \pm 7.2 \pm 0.6 \\
14.0 \pm 4.1 \pm 1.7\end{array}$ & $0.37 \pm 0.34 \pm 0.05$ \\
\hline $\begin{array}{l}-0.500--0.375 \\
-0.375--0.250\end{array}$ & $\begin{array}{l}104 \pm 27 \pm 12 \\
112 \pm 18 \pm 13\end{array}$ & $\begin{array}{c}7.9 \pm 7.1 \pm 0.9 \\
16.9 \pm 4.5 \pm 2.0\end{array}$ & $1.07 \pm 0.48 \pm 0.13$ \\
\hline $\begin{array}{c}-0.250--0.125 \\
-0.125-0.000\end{array}$ & $\begin{array}{l}139 \pm 20 \pm 16 \\
129 \pm 18 \pm 15\end{array}$ & $\begin{array}{l}21.8 \pm 4.8 \pm 2.6 \\
20.2 \pm 4.6 \pm 2.4\end{array}$ & $1.65 \pm 0.48 \pm 0.20$ \\
\hline $\begin{array}{l}0.000-0.125 \\
0.125-0.250\end{array}$ & $\begin{array}{l}126 \pm 19 \pm 15 \\
102 \pm 15 \pm 12\end{array}$ & $\begin{array}{l}22.4 \pm 4.4 \pm 2.7 \\
20.9 \pm 4.1 \pm 2.5\end{array}$ & $1.74 \pm 0.47 \pm 0.22$ \\
\hline $\begin{array}{l}0.250-0.375 \\
0.375-0.500\end{array}$ & $\begin{array}{l}133 \pm 21 \pm 15 \\
97 \pm 15 \pm 11\end{array}$ & $\begin{array}{l}24.6 \pm 4.0 \pm 2.9 \\
20.7 \pm 4.0 \pm 2.5\end{array}$ & $0.85 \pm 0.42 \pm 0.10$ \\
\hline $\begin{array}{l}0.500-0.625 \\
0.625-0.750\end{array}$ & $\begin{array}{l}94 \pm 15 \pm 11 \\
57 \pm 12 \pm 7.0\end{array}$ & $\begin{array}{l}19.2 \pm 3.4 \pm 2.3 \\
15.0 \pm 3.3 \pm 1.8\end{array}$ & $0.71 \pm 0.35 \pm 0.09$ \\
\hline
\end{tabular}


Table C.5 Invariant differential cross section per nucleon for $\eta$ production by $800 \mathrm{GeV} / c$ proton beam on Be target as a function of $p_{T}$ and rapidity.

\begin{tabular}{|c|c|c|c|}
\hline Rapidity & $\begin{array}{c}3.00-4.00 \\
\mathrm{nb} /(\mathrm{GeV} / c)^{2}\end{array}$ & $\begin{array}{c}p_{T}(\mathrm{GeV} / c) \\
4.00-4.50 \\
\mathrm{nb} /(\mathrm{GeV} / c)^{2}\end{array}$ & $\begin{array}{c}4.50-5.00 \\
\mathrm{pb} /(\mathrm{GeV} / c)^{2}\end{array}$ \\
\hline $\begin{array}{l}-1.00--0.875 \\
-0.875--0.750\end{array}$ & $13 \pm 16 \pm 2.0$ & $\begin{array}{l}0.92 \pm 0.58 \pm 0.11 \\
1.36 \pm 0.63 \pm 0.16\end{array}$ & $500 \pm 240 \pm 60$ \\
\hline $\begin{array}{l}-0.750--0.625 \\
-0.625--0.500\end{array}$ & $47 \pm 11 \pm 6.0$ & $\begin{array}{l}2.00 \pm 0.64 \pm 0.23 \\
1.27 \pm 0.58 \pm 0.15\end{array}$ & $\begin{array}{l}560 \pm 130 \pm 70 \\
560 \pm 160 \pm 70\end{array}$ \\
\hline $\begin{array}{l}-0.500--0.375 \\
-0.375--0.250\end{array}$ & $17.6 \pm 8.1 \pm 2.1$ & $\begin{array}{l}2.35 \pm 0.55 \pm 0.27 \\
3.88 \pm 0.54 \pm 0.45\end{array}$ & $\begin{array}{l}640 \pm 120 \pm 80 \\
710 \pm 150 \pm 80\end{array}$ \\
\hline $\begin{array}{c}-0.250--0.125 \\
-0.125-0.000\end{array}$ & $48.5 \pm 8.8 \pm 5.9$ & $\begin{array}{l}3.54 \pm 0.57 \pm 0.41 \\
4.17 \pm 0.52 \pm 0.49\end{array}$ & $\begin{array}{c}570 \pm 130 \pm 70 \\
950 \pm 140 \pm 110\end{array}$ \\
\hline $\begin{array}{l}0.000-0.125 \\
0.125-0.250\end{array}$ & $35.2 \pm 6.8 \pm 4.3$ & $\begin{array}{l}4.10 \pm 0.60 \pm 0.48 \\
2.86 \pm 0.52 \pm 0.33\end{array}$ & $\begin{array}{l}1200 \pm 160 \pm 140 \\
1240 \pm 190 \pm 150\end{array}$ \\
\hline \multirow[t]{2}{*}{$\begin{array}{l}0.250-0.375 \\
0.375-0.500\end{array}$} & $29.3 \pm 7.1 \pm 3.6$ & $\begin{array}{l}3.71 \pm 0.35 \pm 0.43 \\
2.25 \pm 0.26 \pm 0.26\end{array}$ & $\begin{array}{l}1180 \pm 130 \pm 140 \\
1090 \pm 110 \pm 130\end{array}$ \\
\hline & $\begin{array}{c}5.00-5.50 \\
\mathrm{pb} /(\mathrm{GeV} / c)^{2}\end{array}$ & $\begin{array}{c}5.50-6.50 \\
\mathrm{pb} /(\mathrm{GeV} / c)^{2}\end{array}$ & $\begin{array}{c}6.50-8.00 \\
\mathrm{pb} /(\mathrm{GeV} / c)^{2}\end{array}$ \\
\hline $\begin{array}{l}-1.00--0.875 \\
-0.875--0.750\end{array}$ & $\begin{array}{l}115 \pm 47 \pm 14 \\
88 \pm 52 \pm 10\end{array}$ & $\begin{array}{c}9 \pm 10 \pm 1.0 \\
22.5 \pm 9.1 \pm 2.7\end{array}$ & \\
\hline $\begin{array}{r}-0.750--0.625 \\
-0.625--0.500 \\
\end{array}$ & $\begin{array}{l}159 \pm 44 \pm 19 \\
260 \pm 48 \pm 31 \\
\end{array}$ & $\begin{array}{l}52 \pm 16 \pm 6.0 \\
41 \pm 11 \pm 5.0\end{array}$ & $6.8 \pm 2.0 \pm 0.8$ \\
\hline $\begin{array}{l}-0.500--0.375 \\
-0.375--0.250\end{array}$ & $\begin{array}{l}302 \pm 49 \pm 36 \\
255 \pm 56 \pm 30\end{array}$ & $\begin{array}{l}34 \pm 12 \pm 4.0 \\
51 \pm 11 \pm 6.0\end{array}$ & $4.3 \pm 1.6 \pm 0.5$ \\
\hline $\begin{array}{c}-0.250--0.125 \\
-0.125-0.000\end{array}$ & $\begin{array}{l}240 \pm 48 \pm 28 \\
293 \pm 54 \pm 35\end{array}$ & $\begin{array}{c}87 \pm 16 \pm 10 \\
80 \pm 13 \pm 10.0\end{array}$ & $6.5 \pm 1.5 \pm 0.8$ \\
\hline $\begin{array}{l}0.000-0.125 \\
0.125-0.250\end{array}$ & $\begin{array}{l}315 \pm 64 \pm 37 \\
390 \pm 76 \pm 46\end{array}$ & $\begin{array}{l}94 \pm 17 \pm 11 \\
71 \pm 19 \pm 9.0\end{array}$ & $8.3 \pm 2.2 \pm 1.0$ \\
\hline $\begin{array}{l}0.250-0.375 \\
0.375-0.500\end{array}$ & $\begin{array}{l}277 \pm 58 \pm 33 \\
284 \pm 49 \pm 34\end{array}$ & $\begin{array}{l}62 \pm 15 \pm 7.0 \\
54 \pm 13 \pm 6.0\end{array}$ & $7.5 \pm 1.8 \pm 0.9$ \\
\hline
\end{tabular}


Table C.6 Invariant differential cross section per nucleon for $\eta$ production by $515 \mathrm{GeV} / c \pi^{-}$ beam on Be target as a function of $p_{T}$ and rapidity.

\begin{tabular}{|c|c|c|c|}
\hline Rapidity & $\begin{array}{c}3.00-4.00 \\
\mathrm{nb} /(\mathrm{GeV} / c)^{2}\end{array}$ & $\begin{array}{c}p_{T}(\mathrm{GeV} / c) \\
4.00-4.50 \\
\mathrm{nb} /(\mathrm{GeV} / c)^{2}\end{array}$ & $\begin{array}{c}4.50-5.00 \\
\mathrm{pb} /(\mathrm{GeV} / c)^{2}\end{array}$ \\
\hline $\begin{array}{l}-0.750--0.625 \\
-0.625--0.500\end{array}$ & $14.2 \pm 6.3 \pm 1.7$ & $\begin{array}{l}0.69 \pm 0.26 \pm 0.08 \\
0.89 \pm 0.23 \pm 0.10\end{array}$ & $\begin{array}{l}290 \pm 89 \pm 34 \\
281 \pm 62 \pm 33\end{array}$ \\
\hline $\begin{array}{l}-0.500--0.375 \\
-0.375--0.250\end{array}$ & $17.2 \pm 5.7 \pm 2.1$ & $\begin{array}{l}1.31 \pm 0.18 \pm 0.15 \\
1.83 \pm 0.18 \pm 0.21\end{array}$ & $\begin{array}{l}343 \pm 52 \pm 40 \\
461 \pm 45 \pm 53\end{array}$ \\
\hline $\begin{array}{c}-0.250--0.125 \\
-0.125-0.000\end{array}$ & $27.4 \pm 4.6 \pm 3.4$ & $\begin{array}{l}1.73 \pm 0.15 \pm 0.20 \\
2.23 \pm 0.14 \pm 0.26\end{array}$ & $\begin{array}{l}587 \pm 53 \pm 68 \\
562 \pm 47 \pm 65\end{array}$ \\
\hline $\begin{array}{l}0.000-0.125 \\
0.125-0.250\end{array}$ & $27.8 \pm 2.4 \pm 3.4$ & $\begin{array}{l}1.77 \pm 0.13 \pm 0.21 \\
2.43 \pm 0.14 \pm 0.28\end{array}$ & $\begin{array}{l}584 \pm 47 \pm 68 \\
657 \pm 55 \pm 76\end{array}$ \\
\hline $\begin{array}{l}0.250-0.375 \\
0.375-0.500\end{array}$ & $23.9 \pm 2.5 \pm 2.9$ & $\begin{array}{l}2.39 \pm 0.15 \pm 0.28 \\
2.30 \pm 0.14 \pm 0.27\end{array}$ & $\begin{array}{l}690 \pm 52 \pm 80 \\
695 \pm 51 \pm 80\end{array}$ \\
\hline \multirow[t]{2}{*}{$\begin{array}{l}0.500-0.625 \\
0.625-0.750\end{array}$} & $18.3 \pm 2.2 \pm 2.3$ & $\begin{array}{l}2.05 \pm 0.12 \pm 0.24 \\
2.09 \pm 0.12 \pm 0.24\end{array}$ & $\begin{array}{l}657 \pm 47 \pm 76 \\
601 \pm 44 \pm 70\end{array}$ \\
\hline & $\begin{array}{c}5.00-5.50 \\
\mathrm{pb} /(\mathrm{GeV} / c)^{2}\end{array}$ & $\begin{array}{c}5.50-6.50 \\
\mathrm{pb} /(\mathrm{GeV} / c)^{2}\end{array}$ & $\begin{array}{c}6.50-8.00 \\
\mathrm{pb} /(\mathrm{GeV} / c)^{2}\end{array}$ \\
\hline $\begin{array}{l}-0.750--0.625 \\
-0.625--0.500\end{array}$ & $\begin{array}{l}37 \pm 15 \pm 4.0 \\
79 \pm 22 \pm 9.0\end{array}$ & $\begin{array}{c}6.9 \pm 4.6 \pm 0.8 \\
23.1 \pm 4.9 \pm 2.7\end{array}$ & $0.49 \pm 0.49 \pm 0.06$ \\
\hline $\begin{array}{l}-0.500--0.375 \\
-0.375--0.250\end{array}$ & $\begin{array}{l}142 \pm 22 \pm 17 \\
126 \pm 17 \pm 15\end{array}$ & $\begin{array}{l}18.7 \pm 4.3 \pm 2.2 \\
32.5 \pm 5.7 \pm 3.8\end{array}$ & $1.32 \pm 0.49 \pm 0.16$ \\
\hline $\begin{array}{c}-0.250--0.125 \\
-0.125-0.000\end{array}$ & $\begin{array}{l}158 \pm 17 \pm 18 \\
145 \pm 20 \pm 17\end{array}$ & $\begin{array}{l}37.0 \pm 4.5 \pm 4.4 \\
43.0 \pm 5.6 \pm 5.1\end{array}$ & $3.67 \pm 0.72 \pm 0.44$ \\
\hline $\begin{array}{l}0.000-0.125 \\
0.125-0.250\end{array}$ & $\begin{array}{l}139 \pm 19 \pm 16 \\
200 \pm 22 \pm 23\end{array}$ & $\begin{array}{l}33.7 \pm 5.3 \pm 4.0 \\
46.1 \pm 5.5 \pm 5.4\end{array}$ & $3.60 \pm 0.78 \pm 0.44$ \\
\hline $\begin{array}{l}0.250-0.375 \\
0.375-0.500\end{array}$ & $\begin{array}{l}227 \pm 22 \pm 26 \\
220 \pm 23 \pm 26\end{array}$ & $\begin{array}{l}48.4 \pm 5.4 \pm 5.7 \\
46.5 \pm 6.5 \pm 5.5\end{array}$ & $4.40 \pm 0.86 \pm 0.53$ \\
\hline $\begin{array}{l}0.500-0.625 \\
0.625-0.750\end{array}$ & $\begin{array}{l}186 \pm 21 \pm 22 \\
175 \pm 18 \pm 20\end{array}$ & $\begin{array}{l}41.9 \pm 5.9 \pm 4.9 \\
36.2 \pm 5.2 \pm 4.3\end{array}$ & $3.90 \pm 0.78 \pm 0.47$ \\
\hline
\end{tabular}


Table C.7 Invariant differential cross section per nucleon for $\eta$ production by $530 \mathrm{GeV} / c$ proton beam on $\mathrm{Cu}$ target as a function of $p_{T}$ and rapidity.

\begin{tabular}{|c|c|c|c|}
\hline Rapidity & $\begin{array}{c}4.00-5.00 \\
\mathrm{pb} /(\mathrm{GeV} / c)^{2}\end{array}$ & $\begin{array}{c}p_{T}(\mathrm{GeV} / c) \\
5.00-6.00 \\
\mathrm{pb} /(\mathrm{GeV} / c)^{2}\end{array}$ & $\begin{array}{c}6.00-8.00 \\
\mathrm{pb} /(\mathrm{GeV} / c)^{2}\end{array}$ \\
\hline $\begin{array}{l}-0.75--0.50 \\
-0.50--0.25\end{array}$ & $\begin{array}{c}580 \pm 300 \pm 70 \\
1190 \pm 290 \pm 140\end{array}$ & $\begin{array}{l}58 \pm 18 \pm 7.0 \\
86 \pm 21 \pm 10\end{array}$ & $3.6 \pm 1.5 \pm 0.4$ \\
\hline $\begin{array}{c}-0.25-0.00 \\
0.00-0.25\end{array}$ & $\begin{array}{l}1390 \pm 230 \pm 160 \\
1320 \pm 170 \pm 150\end{array}$ & $\begin{array}{c}95 \pm 18 \pm 11 \\
112 \pm 18 \pm 13\end{array}$ & $3.7 \pm 1.1 \pm 0.5$ \\
\hline $\begin{array}{l}0.25-0.50 \\
0.50-0.75\end{array}$ & $\begin{array}{c}1170 \pm 110 \pm 130 \\
710 \pm 90 \pm 81\end{array}$ & $\begin{array}{l}72 \pm 19 \pm 8.0 \\
59 \pm 12 \pm 7.0\end{array}$ & $5.4 \pm 1.2 \pm 0.7$ \\
\hline
\end{tabular}

Table C.8 Invariant differential cross section per nucleon for $\eta$ production by $800 \mathrm{GeV} / c$ proton beam on $\mathrm{Cu}$ target as a function of $p_{T}$ and rapidity.

\begin{tabular}{|c|c|c|c|}
\hline Rapidity & $\begin{array}{c}4.00-5.00 \\
\mathrm{pb} /(\mathrm{GeV} / \mathrm{c})^{2}\end{array}$ & $\begin{array}{c}p_{T}(\mathrm{GeV} / c) \\
5.00-6.00 \\
\mathrm{pb} /(\mathrm{GeV} / c)^{2}\end{array}$ & $\begin{array}{c}6.00-8.00 \\
\mathrm{pb} /(\mathrm{GeV} / c)^{2}\end{array}$ \\
\hline $\begin{array}{c}-1.0--0.75 \\
-0.75--0.50\end{array}$ & $\begin{array}{l}3240 \pm 950 \pm 380 \\
2460 \pm 580 \pm 290\end{array}$ & $\begin{array}{l}47 \pm 31 \pm 6.0 \\
136 \pm 49 \pm 16\end{array}$ & $9.7 \pm 4.1 \pm 1.2$ \\
\hline $\begin{array}{c}-0.50--0.25 \\
-0.25-0.00\end{array}$ & $\begin{array}{l}2550 \pm 480 \pm 300 \\
3490 \pm 570 \pm 410\end{array}$ & $\begin{array}{l}233 \pm 45 \pm 28 \\
235 \pm 52 \pm 28\end{array}$ & $6.6 \pm 3.6 \pm 0.8$ \\
\hline $\begin{array}{l}0.00-0.25 \\
0.25-0.50\end{array}$ & $\begin{array}{l}3170 \pm 520 \pm 370 \\
2600 \pm 320 \pm 300\end{array}$ & $\begin{array}{l}310 \pm 78 \pm 37 \\
230 \pm 54 \pm 27\end{array}$ & $11.8 \pm 5.1 \pm 1.4$ \\
\hline
\end{tabular}

Table C.9 Invariant differential cross section per nucleon for $\eta$ production by $515 \mathrm{GeV} / c \pi^{-}$ beam on $\mathrm{Cu}$ target as a function of $p_{T}$ and rapidity.

\begin{tabular}{|c|c|c|c|}
\hline Rapidity & $\begin{array}{c}4.00-5.00 \\
\mathrm{pb} /(\mathrm{GeV} / c)^{2}\end{array}$ & $\begin{array}{c}p_{T}(\mathrm{GeV} / c) \\
5.00-6.00 \\
\mathrm{pb} /(\mathrm{GeV} / c)^{2}\end{array}$ & $\begin{array}{c}6.00-8.00 \\
\mathrm{pb} /(\mathrm{GeV} / c)^{2}\end{array}$ \\
\hline $\begin{array}{l}-0.75--0.50 \\
-0.50--0.25\end{array}$ & $\begin{aligned} 630 & \pm 350 \pm 70 \\
1350 & \pm 260 \pm 160\end{aligned}$ & $\begin{array}{l}47 \pm 24 \pm 5.0 \\
74 \pm 20 \pm 9.0\end{array}$ & $6.8 \pm 1.9 \pm 0.8$ \\
\hline $\begin{array}{c}-0.25-0.00 \\
0.00-0.25\end{array}$ & $\begin{array}{l}1770 \pm 170 \pm 210 \\
1560 \pm 160 \pm 180\end{array}$ & $\begin{array}{l}134 \pm 20 \pm 16 \\
156 \pm 24 \pm 18\end{array}$ & $13.0 \pm 2.3 \pm 1.6$ \\
\hline $\begin{array}{l}0.25-0.50 \\
0.50-0.75\end{array}$ & $\begin{array}{l}1960 \pm 170 \pm 230 \\
1520 \pm 140 \pm 180\end{array}$ & $\begin{array}{l}187 \pm 23 \pm 22 \\
174 \pm 23 \pm 20\end{array}$ & $11.7 \pm 2.2 \pm 1.4$ \\
\hline
\end{tabular}


Table C.10 Invariant differential cross section for $\eta$ production by $530 \mathrm{GeV} / c$ proton beam on $p$ target as a function of $p_{T}$ and rapidity.

\begin{tabular}{|c|c|c|c|}
\hline Rapidity & $\begin{array}{c}4.00-5.00 \\
\mathrm{pb} /(\mathrm{GeV} / c)^{2}\end{array}$ & $\begin{array}{c}p_{T}(\mathrm{GeV} / c) \\
5.00-6.00 \\
\mathrm{pb} /(\mathrm{GeV} / c)^{2}\end{array}$ & $\begin{array}{c}6.00-8.00 \\
\mathrm{pb} /(\mathrm{GeV} / c)^{2}\end{array}$ \\
\hline $\begin{array}{l}-0.75--0.50 \\
-0.50--0.25\end{array}$ & $\begin{array}{l}580 \pm 270 \pm 70 \\
750 \pm 240 \pm 90\end{array}$ & $\begin{array}{l}31 \pm 20 \pm 4.0 \\
56 \pm 17 \pm 7.0\end{array}$ & $1.7 \pm 1.1 \pm 0.2$ \\
\hline $\begin{array}{c}-0.25-0.00 \\
0.00-0.25\end{array}$ & $\begin{array}{c}1230 \pm 200 \pm 140 \\
780 \pm 160 \pm 90\end{array}$ & $\begin{array}{l}62 \pm 17 \pm 7.0 \\
74 \pm 19 \pm 9.0\end{array}$ & $1.4 \pm 1.1 \pm 0.2$ \\
\hline $\begin{array}{l}0.25-0.50 \\
0.50-0.75\end{array}$ & $\begin{array}{c}850 \pm 110 \pm 100 \\
600 \pm 77 \pm 69\end{array}$ & $\begin{array}{l}49 \pm 13 \pm 6.0 \\
52 \pm 14 \pm 6.0\end{array}$ & $1.4 \pm 1.3 \pm 0.2$ \\
\hline
\end{tabular}

Table C.11 Invariant differential cross section for $\eta$ production by $800 \mathrm{GeV} / c$ proton beam on $p$ target as a function of $p_{T}$ and rapidity.

\begin{tabular}{|c|c|c|c|}
\hline Rapidity & $\begin{array}{c}4.00-5.00 \\
\mathrm{pb} /(\mathrm{GeV} / c)^{2}\end{array}$ & $\begin{array}{c}p_{T}(\mathrm{GeV} / c) \\
5.00-6.00 \\
\mathrm{pb} /(\mathrm{GeV} / c)^{2}\end{array}$ & $\begin{array}{c}6.00-8.00 \\
\mathrm{pb} /(\mathrm{GeV} / c)^{2}\end{array}$ \\
\hline $\begin{array}{r}-1.0--0.75 \\
-0.75--0.50\end{array}$ & $\begin{array}{c}1120 \pm 460 \pm 130 \\
380 \pm 550 \pm 40\end{array}$ & $\begin{array}{l}76 \pm 53 \pm 9.0 \\
102 \pm 51 \pm 12\end{array}$ & $6.3 \pm 4.2 \pm 0.8$ \\
\hline $\begin{array}{c}-0.50--0.25 \\
-0.25-0.00\end{array}$ & $\begin{array}{l}2040 \pm 440 \pm 240 \\
2000 \pm 470 \pm 230\end{array}$ & $\begin{array}{l}206 \pm 42 \pm 25 \\
271 \pm 50 \pm 32\end{array}$ & $19.5 \pm 5.1 \pm 2.4$ \\
\hline $\begin{array}{l}0.00-0.25 \\
0.25-0.50\end{array}$ & $\begin{array}{l}2090 \pm 500 \pm 240 \\
1290 \pm 260 \pm 150\end{array}$ & $\begin{array}{l}174 \pm 58 \pm 21 \\
208 \pm 53 \pm 25\end{array}$ & $13.9 \pm 4.8 \pm 1.7$ \\
\hline
\end{tabular}

Table C.12 Invariant differential cross section per nucleon for $\eta$ production by $515 \mathrm{GeV} / c \pi^{-}$ beam on $p$ target as a function of $p_{T}$ and rapidity.

\begin{tabular}{|c|c|c|c|}
\hline Rapidity & $\begin{array}{c}4.00-5.00 \\
\mathrm{pb} /(\mathrm{GeV} / c)^{2}\end{array}$ & $\begin{array}{c}p_{T}(\mathrm{GeV} / c) \\
5.00-6.00 \\
\mathrm{pb} /(\mathrm{GeV} / c)^{2}\end{array}$ & $\begin{array}{c}6.00-8.00 \\
\mathrm{pb} /(\mathrm{GeV} / c)^{2}\end{array}$ \\
\hline $\begin{array}{l}-0.75--0.50 \\
-0.50--0.25\end{array}$ & $\begin{array}{c}550 \pm 550 \pm 60 \\
960 \pm 460 \pm 110\end{array}$ & $76 \pm 43 \pm 9.0$ & $1.6 \pm 1.6 \pm 0.2$ \\
\hline $\begin{array}{c}-0.25-0.00 \\
0.00-0.25\end{array}$ & $\begin{array}{l}1190 \pm 360 \pm 140 \\
1850 \pm 440 \pm 220\end{array}$ & $\begin{array}{l}40 \pm 39 \pm 5.0 \\
149 \pm 48 \pm 17\end{array}$ & $6.7 \pm 4.4 \pm 0.8$ \\
\hline $\begin{array}{l}0.25-0.50 \\
0.50-0.75\end{array}$ & $\begin{array}{c}1030 \pm 270 \pm 120 \\
980 \pm 220 \pm 110\end{array}$ & $\begin{array}{l}155 \pm 51 \pm 18 \\
45 \pm 43 \pm 5.0\end{array}$ & $4.1 \pm 5.6 \pm 0.5$ \\
\hline
\end{tabular}





\section{REFERENCES}

[1] L. Apanasevich et al., Production of $\pi^{0}$ and $\eta$ mesons at large transverse momenta in pp and $p B e$ interactions at 530 and $800 \mathrm{GeV} / \mathrm{c}$, Phys. Rev. D 68, $052001(2003)$.

[2] L. Apanasevich et al., Production of $\pi^{0}$ and $\eta$ mesons at large transverse momenta in $\pi^{-} p$ and $\pi^{-}$Be interactions at $515 \mathrm{GeV} / \mathrm{c}$, Phys. Rev. D 69, 032003 (2004).

[3] L. Apanasevich et al., Measurement of direct photon production at Tevatron fixed target energies, Phys. Rev. D 70, 092009 (2004).

[4] E. Bloom et al., High-energy inelastic e-p scattering at $6^{\circ}$ and $10^{\circ}$, Phys. Rev. Lett. 23 (1969).

[5] M. Breidenbach et al., Observed behavior of highly inelastic electron-proton scattering, Phys. Rev. Lett. 23 (1969).

[6] J. D. Bjorken and E. A. Paschos, Inelastic electron-proton and $\gamma$-proton scattering and the structure of the nucleon, Phys. Rev. 185, 1975 (1969).

[7] R. P. Feynman, Very high-energy collisions of hadrons, Phys. Rev. Lett. 23, 1415 (1969).

[8] H. Fritzsch, M. Gell-man, and H. Leutwyler, Advantages of the color octet gluon picture, Phys. Lett. 47B, 365 (1973).

[9] R. D. Field, Applications of Perturbative QCD (Addison-Wesley, New York, 1989).

[10] LEPS Collaboration, T. Nakano et al., Observation of $s=+1$ baryon resonance in photo-production from neutron, Phys. Rev. Lett. 91, 012002 (2003), hep-ex/0301020.

[11] A. J. Buras, Asymptotic freedom in deep inelastic processes in the leading order and beyond, Rev. Mod. Phys. 52, 199 (1980).

[12] I. Aitchison and A. Hey, Gauge Theories in Particle Physics (IOP Publishing Ltd, New York, 1989).

[13] R. D. Field and R. P. Feynman, Quark elastic scattering as a source of high-transverse-momentum mesons, Phys. Rev. D15, 2590 (1977).

[14] CTEQ Collaboration, G. Sterman et al., Handbook of perturbative QCD, Rev. Mod. Phys. 67, 157 (1995).

[15] J. Owens, Large-momentum-transfer production of direct photons, jets, and particles, Rev. Mod. Phys. 59, 465 (1987). 
[16] P. Stevenson, Optimized perturbation theory, Phys. Rev. D23, 2916 (1981).

[17] H. Politzer, Stevenson's optimized perturbation theory applied to factorization and mass scheme dependence, Nucl. Phys. B194, 493 (1982); P. Stevenson and H. Politzer, Optimized perturbation theory applied to factorization scheme dependence, ibid, B277, 758 (1986).

[18] D. Stump et al., Inclusive jet production, parton distributions, and the search for new physics, JHEP 10, 046 (2003).

[19] B. A. Kniehl, G. Kramer, and B. Pötter, Fragmentation functions for pions, kaons, and protons at next-to-leading order, Nucl. Phys. B582, 514 (2000).

[20] Yu. L. Dokshitser, Sov. Phys. JETP 46, 641 (1977); V. N. Gribov and L. N. Lipatov, Sov. J. Nucl. Phys. 15, 438 (1972); G. Altarelli and G. Parisi, Nucl. Phys. B126, 298 (1977).

[21] H. Fritzsch and P. Minkowski, Measuring QCD compton effects, Phys. Lett. 69B, 316 (1977).

[22] L. Cormell and J. F. Owens, High-p production of direct photons and jets in quantum chromodynamics, Phys. Rev. D22, 1609 (1980).

[23] F. Halzen, M. Dechantsreiter, and D. M. Scott, Structure of direct-photon events, Phys. Rev. D22, 1617 (1980).

[24] J. Owens, On the $Q^{2}$ dependence of parton fragmentation functions, Phys. Lett. 76B, 85 (1978).

[25] J. Cronin et al., Production of hadrons at large transverse momentum at 200, 300, and 400 GeV, Phys. Rev. D11, 3105 (1975).

[26] X. Guo and J. Qiu, Nuclear dependence in direct photon production, Phys. Rev. D53, 6144 (1996).

[27] M. Begel, Production of High-Mass Pairs of Direct Photons and Neutral Mesons in a Tevatron Fixed-Target Experiment, Ph.D. thesis, University of Rochester, 1999.

[28] WA70 Collaboration, E. Bonvin et al., Intrinsic transverse momentum in the $\pi^{-} p \longrightarrow \gamma \gamma X$ reaction at $280 \mathrm{GeV} / \mathrm{c}$, Phys. Lett. 236B, 523 (1990).

[29] CDF Collaboration, F. Abe et al., Measurement of the cross section for the production of two isolated prompt photons in $p \bar{p}$ collisions at $\sqrt{s}=1.8 \mathrm{TeV}$, Phys. Rev. Lett. 70, 2232 (1993).

[30] WA11 Collaboration, R. Barate et al., Phys. Rev. Lett. 21, 1541 (1979); WA12 Collaboration, M. J. Corden et al., Phys. Lett. 76B, 226 (1978); 
R209 Collaboration, D. Antreasyan et al., Phys. Rev. Lett. 47, 12 (1981); NA3 Collaboration, J. Badier et al., Phys. Lett. 117B, 372 (1982); E288 Collaboration, A. S. Ito et al., Phys Rev. D23, 604 (1981); E300 Collaboration, D. Antreaysan et al., Phys. Rev. Lett. 39, 906 (1977); E444 Collaboration, K. J. Anderson et al., Phys. Rev. Lett. 42, 944 (1979); E537 Collaboration, E. Anassontzis et al., Phys Rev. D38, 1377 (1988); E605 Collaboration, G. Moreno et al., Phys Rev. D43, 2815 (1991); E615 Collaboration, S. Palestini et al., Phys. Rev. Lett. 55, 2649 (1985); E615 Collaboration, J. S. Conway et al., Phys Rev. D39, 92 (1989); E687 Collaboration, J. Alspector et al., Phys. Lett. 81B, 397 (1979); E395 Collaboration, M. D. Corcoran et al., Phys. Rev. D21, 641 (1980); R702 Collaboration, A. G. Clark et al., Nucl. Phys. B160, 397 (1979); CCOR (R108) Collaboration, A. L. S. Angelis et al., Phys. Scripta 19, 116 (1979); CCOR (R108) Collaboration, A. L. S. Angelis et al., Phys. Lett. 97B, 163 (1980).

[31] S. Catani, M. L. Mangano, and P. Nason, Sudakov resummation for prompt photon production in hadron collisions, JHEP 07, 024 (1998).

[32] Hung-Liang Lai and Hsiang-nan Li, Origin of the $k_{T}$ smearing in direct photon production, Phys. Rev. D58, 114020 (1998).

[33] R. Bonciani, S. Catani, M. L. Mangano, and P. Nason, Sudakov resummation of multiparton qcd cross sections, Phys. Lett. B575, 268 (2003).

[34] P. Bolzoni, S. Forte, and G. Ridolfi, Renormalization group approach to sudakov resummation in prompt photon production, (2005), hep-ph/0504115.

[35] A. D. Martin, R. G. Roberts, W. J. Stirling, and R. S. Thorne, Parton distributions: A new global analysis, Eur. Phys. J. C4, 463 (1998).

[36] G. Sterman, Summation of large corrections to short-distance hadronic cross sections, Nucl. Phys. B281, 310 (1987).

[37] S. Catani and L. Trentadue, Summation of the QCD perturbative series for hard processes, Nucl. Phys. B327, 323 (1989).

[38] C. Balazs, E. L. Berger, S. Mrenna, and C. P. Yuan, Photon pair production with soft gluon resummation in hadronic interactions, Phys. Rev. D57, 6934 (1998).

[39] P. Chiappetta, R. Fergani, and J. P. Guillet, Double prompt photon production from hadronic collisions, Phys. Lett. B348, 646 (1995).

[40] R412 (ISR) Collaboration, P. Darriulat et al., Direct production of high $p_{T}$ single photons in pp collisions at the CERN ISR, Nucl. Phys. B110, 365 (1976). 
[41] R107 (ISR) Collaboration, E. Amaldi et al., Search for single photon direct production in $p+p$ collisions at $\sqrt{s}=53.2 \mathrm{GeV}$, Phys. Lett. 77B, 240 (1978).

[42] ISR Collaboration, M. Diakonou et al., Direct production of high $p_{T}$ single photons in pp collisions at the CERN ISR, Phys. Lett. 87B, 292 (1979).

[43] CCOR (R108) Collaboration, A. L. S. Angelis et al., Search for direct singlephoton production at large $p_{T}$ in proton-proton collisions at $\sqrt{s}=62.4 \mathrm{GeV}$, Phys. Lett. 94B, 106 (1980).

[44] CMOR (R110) Collaboration, A. L. S. Angelis et al., Direct photon production at the CERN ISR, Nucl. Phys. B327, 541 (1989).

[45] T. Ferbel and W. Molzen, Direct-photon production in high-energy collisions, Rev. Mod. Phys. 56, 181 (1984).

[46] UA1 Collaboration, C. Albajar et al., Direct photon production at the CERN proton-antiproton collider, Phys. Lett. 209B, 385 (1988).

[47] UA2 Collaboration, J. Alitti et al., A measurement of the direct photon production cross-section at the CERN $\bar{p} p$ collider, Phys. Lett. 263B, 544 (1991).

[48] CDF Collaboration, D. Acosta et al., Comparison of the isolated direct

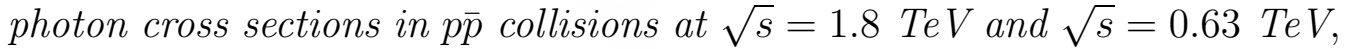
Phys. Rev. D 65, 112003 (2002).

[49] D $\emptyset$ Collaboration, B. Abbott et al., Isolated photon cross section in $p \bar{p}$ collisions at $\sqrt{s}=1.8$ TeV, Phys. Rev. Lett. 84, 2786 (2000).

[50] R806 Collaboration, E. Anassontzis et al., High-p $p_{T}$ direct photon production in pp collisions, Z. Phys. C13, 277 (1982).

[51] AFS (R807) Collaboration, T. Åkesson et al., High $p_{T}$ photon and $\pi^{0}$ production, inclusive and with a recoil jet, in pp collisions at $\sqrt{s}=63 \mathrm{GeV}$, Sov. J. Nucl. Phys. 51, 836 (1990).

[52] AFS (R808) Collaboration, T. Åkesson et al., A comparison of direct photon, $\pi^{0}$, and $\eta$ production in $p \bar{p}$ and $p p$ interactions at the CERN ISR, Phys. Lett. 158B, 282 (1985).

[53] E629 Collaboration, M. McLaughlin et al., Inclusive production of direct photons in $200 \mathrm{GeV} / \mathrm{c}$ collisions, Phys. Rev. Lett. 51, 971 (1983).

[54] E704 Collaboration, D. Adams et al., Measurement of single spin asymmetry for direct photon production in pp collisions at $200 \mathrm{GeV} / \mathrm{c}$, Phys. Lett. B345, 569 (1995). 
[55] UA6 Collaboration, G. Ballocchi et al., Direct photon cross sections in proton-proton and antiproton-proton interactions at $\sqrt{s}=24.3 \mathrm{GeV}$, Phys. Lett. B436, 222 (1998).

[56] NA3 Collaboration, J. Badier et al., Direct photon production from pions and protons at $200 \mathrm{GeV} / \mathrm{c}$, Z. Phys. C31, 341 (1986).

[57] NA24 Collaboration, C. D. Marzo et al., A measurement of direct photon production at large transverse momentum in $\pi^{-} p, \pi^{+} p$ and $p p$ collisions at $300 \mathrm{GeV} / \mathrm{c}$, Phys. Rev. D36, 8 (1987).

[58] WA70 Collaboration, M. Bonesini et al., Production of high transverse momentum prompt photons and neutral pions in proton-proton interactions at $280 \mathrm{GeV} / \mathrm{c}$, Z. Phys. C38, 371 (1988).

[59] WA70 Collaboration, M. Bonesini et al., High transverse momentum prompt photon production by $\pi^{-}$and $\pi^{+}$on protons at $280 \mathrm{GeV} / \mathrm{c}$, Z. Phys. C37, 535 (1988).

[60] E672 Collaboration, A. Gribushin et al., Production of $J / \psi$ and $\psi(2 S)$ mesons in $\pi^{-}$Be collisions at 515 GeV/c, Phys. Rev. D53, 4723 (1996).

[61] D. Striley, Large Transverse Momentum $\pi^{0}$ Meson Production by $0.5 \mathrm{TeV} / \mathrm{c}$ $p, \pi^{+}$and $K^{+}$Incident on Beryllium, Ph.D. thesis, University of MissouriColumbia, 1996.

[62] Nanometric Systems Inc., Oak Park, Illinois, MultiWire Proportional Chamber Readout System.

[63] CAMAC Instrumentation and Interface Standards, IEEE 1982, ISBN 0-47189737.

[64] S. Mani, A Silicon Microvertex Detector for Studying QCD Jets Associated with Direct Photons, Ph.D. thesis, University of Pittsburgh, 1986.

[65] FNAL Computer Department, Fermilab, Batavia, Ill., PN-267, ZIPTRACK.

[66] D. Brown, A Comparison of High Transverse Momentum Direct Photon and Neutral Pion Events in Negative Pion and Proton-Nucleus Collisions at 31.5 GeV Center of Mass Energy, Ph.D. thesis, Michigan State University, 1992.

[67] LeCroy Corp., 700 Chestnut Ridge Rd., Chestnut Ridge, NY 10977 ; Model 1879 .

[68] W. DeSoi, Construction and Performance of a Liquid Argon Calorimeter for Use in E-706 at the Fermi National Accelerator Laboratory, Ph.D. thesis, University of Rochester, 1990. 
[69] G. Drake et al., CDF front end electronics: The Rabbit system, Nucl. Instrum. Methods A269, 68 (1988).

[70] R. Benson, Characteristics of Forward Energy Production in Proton-Nucleus and Pion-Nucleus Collisions at $530 \mathrm{GeV} / \mathrm{c}$, Ph.D. thesis, University of Minnesota, 1989.

[71] L. Sorrell, Measurement of the Nuclear Dependence of Direct Photon and Neutral Meson Production at High Transverse Momentum by Negative 515 GeV/c Pions Incident on Beryllium and Copper Targets, Ph.D. thesis, Michigan State University, 1995.

[72] R. Roser, Eta Production at High Transverse Momentum by Negative 520 GeV/c Pions Incident on Beryllium and Copper Targets, Ph.D. thesis, University of Rochester, 1994.

[73] N. Varelas, $\pi^{0}$ Production at High Transverse Momenta from $\pi^{-}$Collisions at $520 \mathrm{GeV} / \mathrm{c}$ on $\mathrm{Be}$ and $\mathrm{Cu}$ Targets, Ph.D. thesis, University of Rochester, 1994.

[74] ANSI/IEEE Std 960-1986, IEEE Standard FASTBUS Modular HighSpeed Data Aquisition and Control System (The Institute of Electrical and Electronics Engineers, Inc., New York, 1985).

[75] FNAL Computer Department, Fermilab, Batavia, Ill., PN-252, VAXONLINE Users Guide.

[76] G. Alverson and E. Pothier, E706 Internal Note 139, 1985.

[77] R. Brun et al., ZEBRA User's Guide, CERN Computer Center Program Library, DD/EE/85-6 Q100, or FNAL Software Note PU-0046.

[78] H. Klein and J.Zoll, PATCHY reference manual, CERN Computer Center Program Library, FNAL Software Note PU-0013.

[79] A. Sinanidis, Particles Produced in Association with High Transverse Momentum Single Photons and $\pi^{0} s$ in Hadronic Collisions, Ph.D. thesis, Northeastern University, 1989.

[80] S. Blusk, Measurement of the Charm Cross Section in High $p_{T}$ Collisions of $515 \mathrm{GeV} / \mathrm{c} \pi^{-}$Beams Incident on a Beryllium and Copper Targets, Ph.D. thesis, University of Pittsburgh, 1995.

[81] P. Chang, Massive $\pi^{0} \pi^{0}, \pi^{0} \pi^{-}$and $\pi^{0} \pi^{+}$Production from $515 \mathrm{GeV} / \mathrm{c} \pi^{-}$ Collisions with Beryllium and Copper Targets, Ph.D. thesis, Northeastern University, 1994.

[82] P. Weerasundara, A Study of Large Transverse Momentum Direct Photon plus Away-Side Jet Production using $500 \mathrm{GeV} / \mathrm{c}$ proton and $\pi^{-}$Beams 
Incident on a Beryllium Target, Ph.D. thesis, University of Pittsburgh, 1993.

[83] W. Dlugosz, The Production of High $p_{T} \pi^{0}$ Mesons in $515 \mathrm{GeV} / \mathrm{c} \pi^{-}$Nucleus Collisions, Ph.D. thesis, Northeastern University, 1994.

[84] L. Apanasevich et al., Calibration and performance of the Er06 lead and liquid-argon electromagnetic calorimeter, Nucl. Instrum. Methods A417, 50 (1998).

[85] J. Motz et al., Pair production by photons, Rev. Mod. Phys. 41, 581 (1969).

[86] E706 Collaboration, N. Varelas et al., Calibration of the Fermilab E706 liquid argon electromagnetic calorimeter, Nucl. Phys. B. (Proc. Suppl.) 44, 153 (1995).

[87] R. Barnett et al., Review of particle properties, Phys. Rev. D54, 1 (1996).

[88] G. Osborne, Direct Photon and Neutral Meson Production at High Transverse Momentum by Negative $515 \mathrm{GeV} / \mathrm{c}$ Pions and $530 \mathrm{GeV} / \mathrm{c}$ Protons Incident upon Hydrogen, Beryllium and Copper Targets, Ph.D. thesis, University of Rochester, 1996.

[89] G. Osborne, A Measurement of the Muon Content of the 1991 MWest Beam, E706 Note 197.

[90] A. S. Carroll et al., Absorption cross sections of $\pi^{ \pm}, K^{ \pm}, p$ and $\bar{p}$ on nuclei between 60 and $280 \mathrm{GeV} / \mathrm{c}$, Phys. Lett. 80B, 319 (1979).

[91] A. Baldini et al., Total cross-sections for reactions of high energy particles, in Numerical Data and Functional Relationships in Science and Technology, edited by H. Schoppper, Vol.12, New York, 1988, Springer-Verlag.

[92] F. Carminati et al., GEANT: Detector Description and Simulation Tool, CERN Program Library Long Writeup W5013, 1993.

[93] WA70 Collaboration, M. Bonesini et al., High transverse momentum $\pi^{0}$ production by $\pi^{-}$and $\pi^{+}$on protons at $280 \mathrm{GeV} / \mathrm{c}$, Z. Phys. C37, 39 (1987).

[94] L. de Barbaro, Omega Production at High Transverse Momentum by Negative 515 GeV/c Pions Incident on Beryllium and Copper Targets, Ph.D. thesis, University of Rochester, 1995.

[95] M. Diakonou et al., Inclusive high- $p_{T} \omega^{0}$ and $\eta^{\prime}$ production at the ISR, Phys. Lett. 89B, 432 (1979).

[96] G. Marchesini, I.G. Knowles, M.H. Seymour, and B.R. Webber, HERWIG Reference Manual, V5.6. 
[97] T. Sjostrand, Pythia 5.7 and Jetset 7.4 Physics Manual, 1993.

[98] G. Corcella et al., HERWIG 6: an event generator for hadron emission reactions with interfering gluons (including supersymmetric processes), V6.1, 2001.

[99] G. D. Lafferty and T. R. Wyatt, Where to stick your data points: The treatment of measurements within wide bins, Nucl. Instrum. Methods A355, 541 (1995).

[100] H. Frisch et al., Phys. Rev. D 27, 1001 (1983).

[101] X.-N. Wang, High p(t) hadron spectra in high-energy heavy-ion collisions, Nucl. Phys. A661, 609 (1999).

[102] P. Aurenche et al., Prompt photon production at large $p_{T}$ in $Q C D$ beyond the leading order, Phys. Lett. 140B, 87 (1984).

[103] F. Aversa et al., QCD corrections to parton-parton scattering processes, Nucl. Phys. B327, 105 (1989).

[104] H. L. Lai et al., Global QCD analysis of parton structure of the nucleon: CTEQ5 parton distributions, Eur. Phys. J. C12, 375 (2000).

[105] A. D. Martin, R. G. Roberts, W. J. Stirling, and R. S. Thorne, Mrst partons and uncertainties, (2003), hep-ph/0307262.

[106] A. D. Martin, R. G. Roberts, W. J. Stirling, and R. S. Thorne, Mrst2001: Partons and alpha(s) from precise deep inelastic scattering and tevatron jet data, Eur. Phys. J. C23, 73 (2002).

[107] B. Bailey, J. F. Owens, and J. Ohnemus, Order $\alpha_{s}$ Monte Carlo calculation of hadronic double-photon production, Phys. Rev. D46, 2018 (1992).

[108] A. Maul, private communication.

[109] E706 Collaboration, L. Apanasevich et al., Production of charm mesons at high transverse momentum in 515-GeV/c $\pi^{-}$nucleon collisions, Phys. Rev. D56, 1391 (1997).

[110] M. L. Mangano, P. Nason, and G. Ridolfi, Fixed-target hadroproduction of heavy quarks, Nucl. Phys. B405, 507 (1993).

[111] E. Laenen, G. Sterman and W. Vogelsang, Higher-order QCD corrections in prompt photon production., Phys. Rev. Lett. 84, 4296 (2000).

[112] ZEUS Collaboration, J. Breitweg et al., Measurement of inclusive prompt photon photoproduction at HERA, Phys. Lett. 472B, 175 (2000).

[113] ZEUS Collaboration, Proceedings of the XXXth International Conference on High Energy Physics, 2000. 